Physics Division

Physics Division Physics Division Physics Division Physics Division Physics Division Physics Division

\title{
Physics Division Annual Report 1998
}

Physics Division

Physics-Division Physics Division Physics Division Physics Division Physics Division Physics Divisjog Physics Division Physics DivisiónPhysics. Bivision Priysies Division Physics Division Physics Division Physics Division Physics Division? Physics Division Physics Division Physics Division Physics Division Physics Division Physics Division Physics Division Physics Diyision Physics Division Physics Division Physics Division Physics/Division Physics Division Physics Dipision Physics Division Physics Division Physics Division Physics Division Physics Division

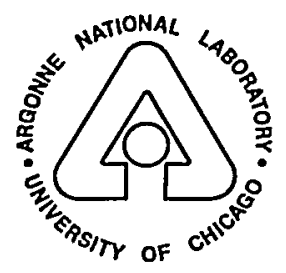

Argonne National Laboratory operated by The University of Chicago for the United States Department of Energy under Contract W-31-109-Eng-38

Physics Division Physics Division
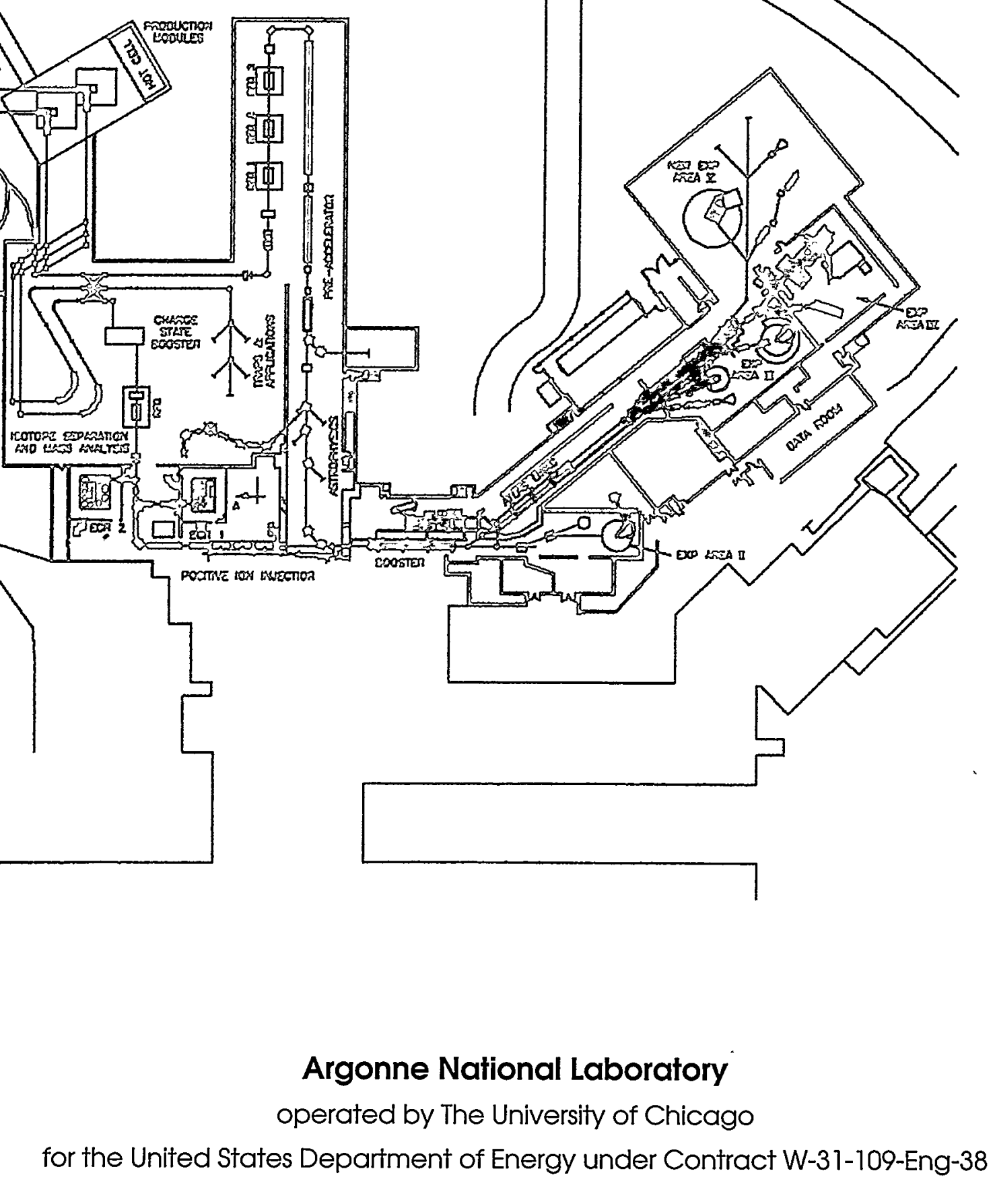
Argonne National Laboratory, with facilities in the states of Illinois and Idaho, is owned by the United States government, and operated by The University of Chicago under the provisions of a contract with the Department of Energy.

\section{DISCLAIMER}

This report was prepared as an account of work sponsored by an agency of the United States Government. Neither the United States Government nor any agency thereof, nor any of their employees, makes any warranty, express or implied, or assumes any legal liability or responsibility for the accuracy, completeness, or usefulness of any information, apparatus, product, or process disclosed, or represents that its use would not infringe privately owned rights. Reference herein to any specific commercial product, process, or service by trade name, trademark, manufacturer, or otherwise, does not necessarily constitute or imply its endorsement, recommendation, or favoring by the United States Government or any agency thereof. The views and opinions of authors expressed herein do not necessarily state or reflect those of the United States Government or any agency thereof.

Reproduced from the best available copy.

Available to DOE and DOE contractors from the Office of Scientific and Technical Information

P.O. Box 62

Oak Ridge, TN 37831

Prices available from (423) 576-8401

Available to the public from the

National Technical Information Service

U.S. Department of Commerce 5285 Port Royal Road Springfield, VA 22161 


\section{DISCLAIMER}

Portions of this document may be illegible in electronic image products. Images are produced from the best available original document. 


\begin{abstract}
ANL-99/12
ARGONNE NATIONAL LABORATORY

9700 S. Cass Avenue

Argonne, Illinois 60439-4801

\section{PHYSICS DIVISION ANNUAL REPORT \\ 1998}

Walter F. Henning

Director

August 1999

Preceding Annual Reports

ANL-96/15 April 1995-March 1996

ANL-97/14 1996

ANL-98/24 1997 
Edited by Karen J. Thayer 


\section{FOREWORD}

The past year saw two basic thrusts in the Argonne Physics Division's research activities: the first focused on furthering the science that is presently being pursued with key instrumentation developed recently. The second focused on the Division's major future initiative: the development, design and construction of a major facility for accelerating short-lived nuclei.

In the medium energy nuclear physics program, spin-structure functions and semi-inclusive asymmetries for the proton and neutron were studied in the HERMES experiment, as well as exclusive rho-meson electroproduction; new results were obtained on the energy dependence of nucleon propagation in nuclei, on electroproduction of kaons and light hypernuclei, and on longitudinal charged-pion electroproduction in experiments at Jefferson Lab.

Research at ATLAS included studies of reactions of astrophysical importance utilizing beams of short-lived nuclei; studies of nuclear structure at the limits of stability with Gammasphere coupled with the FMA fragment analyzer, including gamma-ray spectroscopy of a nobelium $(Z=102)$ isotope; demonstration of high-precision mass resolution $\left(\sim 10^{-8}\right)$ with the Penning trap; spectroscopy of proton-emitters including fine structure; various aspects of superdeformation; and the evolution with rotational frequency of an octupole vibration into a deformed octupole rotation in plutonium nuclei.

The theoretical work made new strides in the areas of QCD calculations based on the Dyson-Schwinger approach; chiral perturbation theory and the chiral constituent quark model; quantum Monte Carlo calculations of nuclei with realistic nucleon-nucleon interactions and three-body forces; structure of heavy nuclei in mean-field calculations; and nuclear reactions of astrophysical interest.

The operation of ATLAS set a new milestone: for the 1998 calendar year it provided close to 6200 hours beam on target for research! This included beams of short-lived ${ }^{17} \mathrm{~F},{ }^{21} \mathrm{Na},{ }^{25} \mathrm{Na}$, and ${ }^{56} \mathrm{Ni}$ for $15 \%$ of the beamtime. The excellent reliability of the accelerator also played a major role in the successful completion of 43 experiments with Gammasphere during the calendar year.

Major progress was made in developing the technology for the future advanced facility for beams of shortlived nuclei: development of the fragmentation/high-pressure gas stopper cell, which provides a conceptual breakthrough in terms of fast extraction and chemical independence; accelerating-field demonstration of the superconducting prototype spoke resonator for intermediate $(\beta=0.4)$ ion velocities; beam tests of the $12 \mathrm{MHz} \mathrm{CW} R \mathrm{RQ}$; target designs based on liquid-lithium fragmentation-target/cooling technology.

Research in atomic physics focused on benchmark measurements at the Argonne 7-GeV Advanced Photon Source of $\mathrm{x}$-ray photo-ionization and scattering on simple gas targets (rare gases) which serve as a testbed for the development of theoretical methods that can be extended to complex systems. The persistence of electron-correlation effects at high photon energies is of particular theoretical interest. Research in fewelectron systems continued at the ATLAS and GSI facilities. Progress was made in testing relativisticmany-body theories by measuring the spectral shapes of two-photon decay at intermediate and high nuclear charge.

This will be my last Foreword for the Division's Annual Report as Director of the Physics Division. It has been a privilege to serve this group of outstanding and enthusiastic scientists and technical and administrative support staff. To the staff, as well as to the many students, postdocs and visitors who have contributed to the intellectual climate of the Division, I want to express my thanks and extend my best wishes for the future.

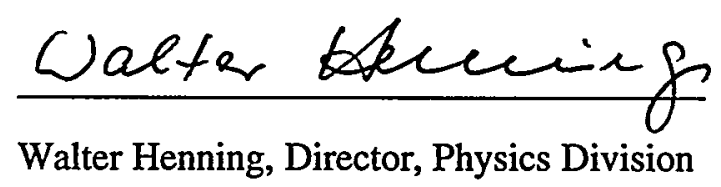




\section{TABLE OF CONTENTS}

Page

\section{HEAVY-ION NUCLEAR PHYSICS RESEARCH......1}

A. EXPERIMENTS WITH SECONDARY BEAMS ..........................

a.1. Study of the ${ }^{8} \mathrm{~B}$ Neutrino SpectrumThrough the ${ }^{8} \mathrm{~B}\left(\beta^{+}\right)^{8} \mathrm{Be}(2 \alpha)$ Decay

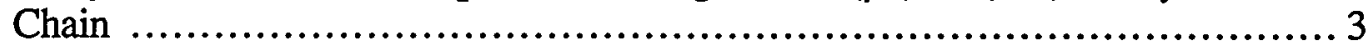

a.2. Study of the ${ }^{14} \mathrm{O}+\alpha \rightarrow p+{ }^{17} \mathrm{~F}$ Reaction: A Gateway in Nucleosynthesis...... 4

a.3. Progress in Producing Radioactive Beams with the Gas Cell Setup at

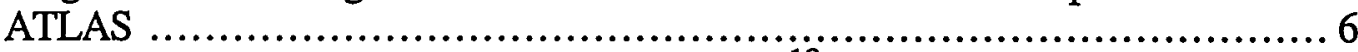

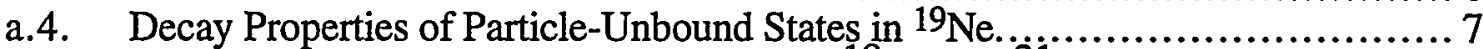

a.5. Breakout from the Hot CNO Cycle Via the ${ }^{18} \mathrm{Ne}(\alpha, \mathrm{p})^{2} 1_{\mathrm{Na}}$ Reaction ............ 8

a.6. Production of an $25 \mathrm{Al}$ Beam............................................................ 9

a.7. Development of $\mathrm{a}^{44}$ Ti Beam ................................................ 9

a.8. Study of the ${ }^{44} \mathrm{Ti}(\alpha, p){ }^{47} \mathrm{~V}$ Reaction at Energies of Astrophysical Interest ........10

a.9. The Measurement of Spectroscopic Factors in ${ }^{57} \mathrm{Cu}$ with Radioactive ${ }^{56} \mathrm{Ni}$

Beams ......................................................................11

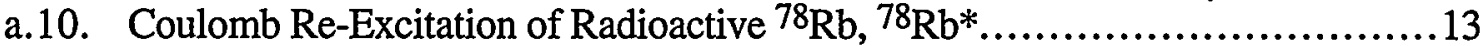

a.11. Production Rate Calculations for an Advanced Exotic Beam Facility .............13

B. STRUCTURE OF NUCLEI AT THE LIMITS OF STABILITY ............18

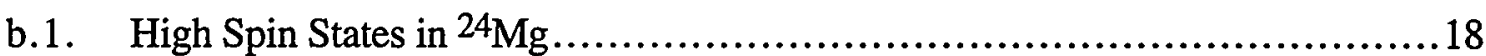

b.2. Deformed Negative-Parity Excitations in ${ }^{71}$ As................................19

b.3. The Structure of the Mirror Pair ${ }^{79} \mathrm{Y}$ and ${ }^{79} \mathrm{Zr} \quad 19$

b.4. In-Beam $\gamma$-Ray Spectroscopy of the Proton Emitter ${ }^{109}$ I........................20

b.5. In-Beam $\gamma$-Ray Spectroscopy in the Ground-State Proton Emitter ${ }^{113}$ Cs ........21

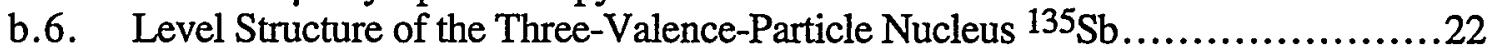

b.7. Yrast Spectroscopy of $\mathrm{N}=82,83$ Isotopes ${ }^{136} \mathrm{Xe}$ and ${ }^{137} \mathrm{Xe}$ from ${ }^{248} \mathrm{Cm}$

Fission Studies ............................................................................................23

b.8. In-Beam Studies of the Deformed Proton Emitter ${ }^{141}$ Ho .........................24

b.9. Spectroscopy of $\mathrm{N}=84$ Nuclei at the Proton Drip Line ..................................25

b.10. Study of Excited States in the Proton-Emitter ${ }^{167}$ Ir and its Neighbors ..........27

b.11. Search for Enhanced Deformation in Light Hg Isotopes.........................28

b.12. Spectroscopy of ${ }^{183} \mathrm{Tl}$ with Recoil-Mass and Z Identification....................29

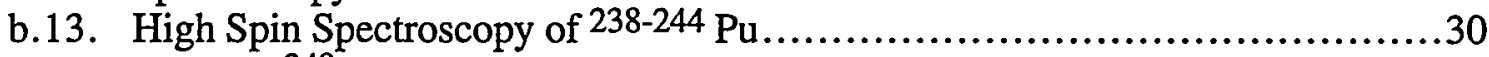

b.14. Study of $248 \mathrm{Cm}$ Fission Products with Gammasphere...........................32

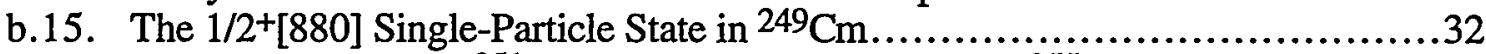

b.16. Nuclear Structure of ${ }^{251} \mathrm{Cf}$ from the Decay of the $20-\mathrm{h} 255 \mathrm{Fm} . \ldots \ldots \ldots \ldots \ldots \ldots . \ldots . \ldots 33$

b.17. Ground-State Band and Deformation of the $Z=102$ Isotope 254 No .............34

b.18. Formation Mechanism of the $Z=102$ Isotope ${ }^{254}$ No and the Fission Barrier

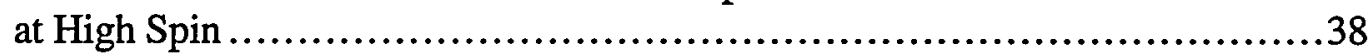

b.19. Jyväskylä Experiment on Excited States in ${ }^{254}$ No ............................ 40

b.20. New Actinide Signature Measurements .....................................41

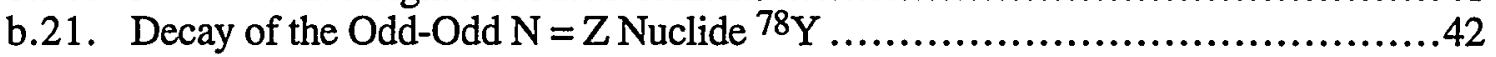


b.22. Radioactive Decay of ${ }^{80} \mathrm{Y}$ and Low-Lying States in ${ }^{80} \mathrm{Sr} \ldots \ldots \ldots \ldots \ldots \ldots \ldots \ldots . \ldots 42$

b.23. ${ }^{80} \mathrm{Y}$ Isomers and Decay.......................................................43

b.24. Fine Structure in the Proton Decay of the Highly Deformed Nuclei ${ }^{131} \mathrm{Eu}$

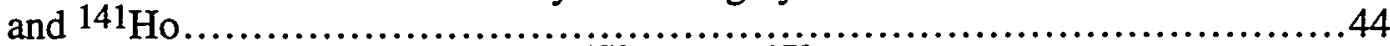

b.25. Decay Properties of New Isotopes ${ }^{172} \mathrm{Hg}$ and ${ }^{173} \mathrm{Hg}$............................45

b.26. Identification of ${ }^{180} \mathrm{Tl} \alpha$ Decay .............................................45

C. SUPERDEFORMATION AND OTHER SPECTROSCOPY TOPICS.

c.1. Decay Out of the Doubly Magic Superdeformed Band in the $\mathrm{N}=\mathrm{Z}$ Nucleus ${ }^{60} \mathrm{Zn}$.........................................................46

c.2. Level Structures at High Spins in Nuclei near and at $N=52 \ldots \ldots \ldots \ldots \ldots \ldots \ldots . .47$

c.3. Quadrupole Moments and Identical Superdeformed Bands in ${ }^{149} \mathrm{~Tb}$............48

c.4. Differential Quadrupole Moment Determinations in Superdeformed

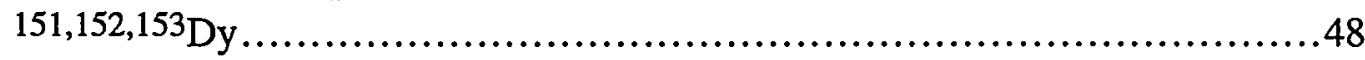

c.5. Search for Hyper-Deformation in 151,152 Dy .................................49

c.6. Second Proton and Neutron Crossings in the Doubly-Odd $154,156 \mathrm{~Tb}$ Nuclei, High-Spin Structure of ${ }^{155} \mathrm{~Tb}$..............................................50

c.7. Search for Hyper-Deformation and Triaxial Superdeformed Bands in Hf

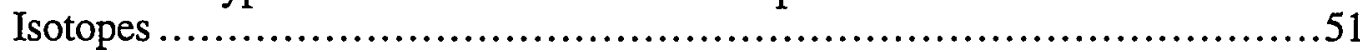

c.8. High-K Isomers in Neutron-Rich Hafnium Nuclei ........................51

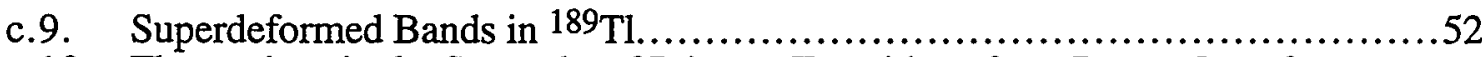

c.10. Fluctuations in the Strengths of Primary Transitions from Decay Out of a

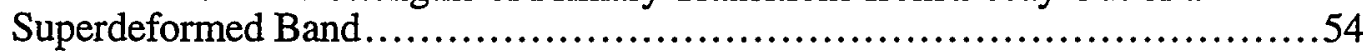

c.11. Spins and Excitation Energy of the Yrast Superdeformed Band in ${ }^{191} \mathrm{Hg}$.........54

c.12. Determination of Spin and Excitation Energy of Superdeformed Bands in

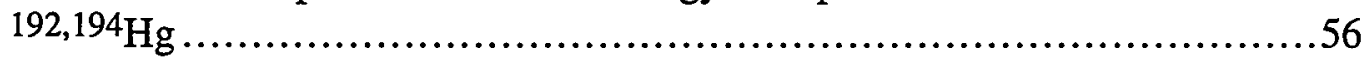

c.13. Quasicontinuum Spectrum of Gamma Rays Which Depopulate SD States in

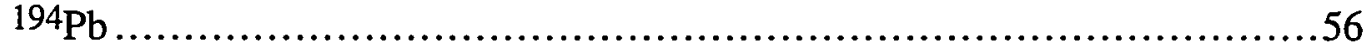

c.14. Intensity Distribution of Primary Transitions in Decay from the

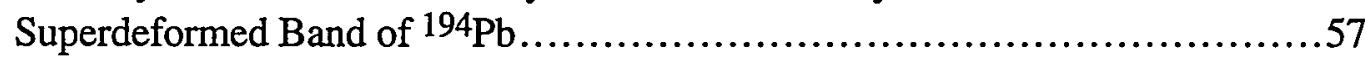

c.15. Search for the Two-Phonon Octupole Vibrational States in ${ }^{208} \mathrm{~Pb} . . \ldots \ldots \ldots \ldots \ldots . . . .58$

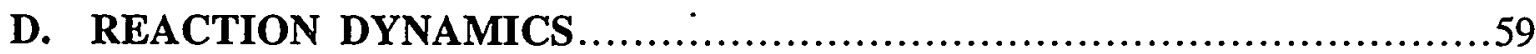

d.1. Studies of $\mathrm{Au}+\mathrm{Au}$ Collisions at $6,8,10.8 \mathrm{GeV} /$ nucleon at the AGS ............59

d.2. Reaction Plane Determination in Au + Au Collisions - Experiment E917..........69

d.3. The PHOBOS Experiment at RHIC ............................................70 
e.1. Progress at the Canadian Penning Trap Mass Spectrometer.....................79

e.2. Development of a Large Accelerated Gas Cell System for the Collection of Fast Recoiling Radioactive Ions............................................8 82

e.3. Temperature, Ordering and Equilibrium in an RF Ion Trap .....................85

e.4. A Long Measurement of the ${ }^{4 t}$ Ti Half-Life.......................................8

e.5. A Proposed Method for Measuring the Electric Dipole Moment of the Neutron by a Large Improvement of the Shull Method ........................88

F . EQUIPMENT DEVELOPMENT AT THE ATLAS FACILITY ..............89

f.1. Installation and First Tests of Gammasphere ...................................89

f.2. Gammasphere Operation................................................ 90

f.3. Maintenance of Gammasphere's Germanium Detectors..........................90

f.4. Gammasphere Software Developments ....................................91

f.5. Calibration of Gammasphere as a Gamma-Ray Calorimeter and Multiplicity Counter..................................................................91

f.6. New Improved and Generalized Background Subtraction Procedure .............92

f.7. Neutron Detectors..................................................... 94

f.8. Status of Channel-Plate-Based Heavy-Ion Detectors...........................94

f.9. Prolonging the Useful Life of Gammasphere/FMA Targets under

Bombardment with Intense Beams ...........................................95

f.10. Performance of Planar Germanium Detectors for Low-Energy Gamma-Rays ....96

f.11. Development of HpGeDSSD Planar Germanium Wafer Technology ............97

f.12. Electronics Development of General-Purpose, High-Speed Readout Module.....97

f.13. Large Efficiency Heavy Ion Detector - LEPPEX ............................. 98

f.14. Design of a Temperature Sensing System-LEPPEX ........................ 100

f.15. Nuclear Target Development ............................................. 101

f.16. Physics Computing Facilities.......................................... 102

f.17. Data-Acquisition Systems.......................................... 102

G. ASSISTANCE TO OUTSIDE USERS OF ATLAS .................... 103

a. Experiments Involving Outside Users.................................. 104

b. Outside Users of ATLAS During the Period 10/1/97 - 9/30/98 ................. 109

c. Summaries of the Continuing User Programs for FY 1998 .................. 112

\section{OPERATION AND DEVELOPMENT OF ATLAS. 113}

\section{A. OPERATION OF THE ACCELERATOR .............................. 114}

a. Status of the new 14-GHz ECR Ion Source.................................. 116

b. $\quad$ Project to Upgrade the ATLAS PII-ECR Ion Source......................... 117

c. First Tests of a Traveling-Wave Chopper for ATLAS ....................... 117

d. ATLAS Cryogenic System .............................................. 117

e. ATLAS Control System.............................................. 118 
a. In-Flight Production of Radioactive Beams at ATLAS........................ 119

b. Timing Synchronization for Low-Intensity Beams ............................ 120

c. A Vibration Damper for a Low Velocity Four Gap Accelerating Structure ...... 121

d. Superconducting Accelerating Structures for Low-Beta Heavy Ions............. 121

C. R \& D RELATED TO A FUTURE ADVANCED EXOTIC BEAM

FACILITY................................................................. 123

a. Introduction....................................................... 123

b. Radioisotope Production and High-Power Targetry............................ 124

b.1. Fragmentation/high-pressure He gas stopper cell ....................... 125

b.2. Two-step, neutron-generator target ................................... 127

b.3. Direct irradiation spallation target...................................... 127

c. Superconducting Driver Linac Studies and Cavity Development .............. 128

d. The Post-Accelerator ................................................... 130

d.1. Ion sources............................................................. 130

d.2. Isobar separator..................................................... 131

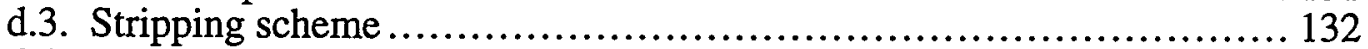

d.4. A 12-Mhz CW RFQ for Injecting Low-Charge-State Ions into ATLAS... 133

d.5. Superconducting linacs ............................................. 134

e. Experimental Areas and Systems................................................... 134

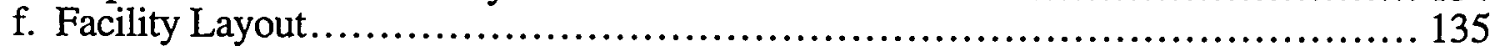

\section{MEDIUM-ENERGY NUCLEAR PHYSICS}

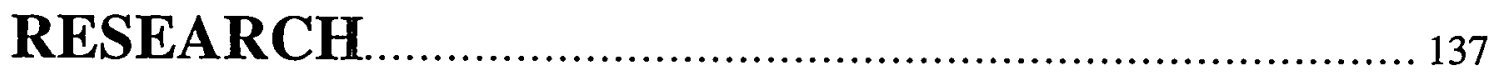

A. SUBNUCLEONIC EFFECTS IN NUCLEI................................... 139

a.1. HERMES, Measurements of Spin-Structure Functions and Semi-Inclusive Asymmetries for the Proton and Neutron at HERA .......................... 139

a.2. Nuclear- and Spin-dependent Cross Section for Exclusive $\rho^{\circ}$

Electroproduction.

a.3. A Dual Radiator Ring Imaging Cerenkov Counter for HERMES.................. 142

a.4. The Energy Dependence of Nucleon Propagation in Nuclei as Measured in the $\left(e, e^{\prime} p\right)$ Reaction ....................................................... 144

a.5. Electroproduction of Kaons and Light Hypernuclei .............................. 146

a.6. Momentum Transfer Dependence of $\mathrm{H}\left(\mathrm{e}, \mathrm{e}^{\prime} \mathrm{K}^{+}\right) \mathrm{Y}$ Reactions ................. 147

a.7. Measurements of Inclusive Cross Section and $R=\sigma_{\mathrm{L}} / \sigma_{\mathrm{T}}$ in the Nucleon Resonance Region ................................................... 147

a.8. Measurements of Deuteron Photo-disintegration up to $4.0 \mathrm{GeV} \ldots \ldots \ldots \ldots \ldots \ldots . . . . .148$

a.9. A Study of Longitudinal Charged-Pion Electroproduction in $\mathrm{D},{ }^{3} \mathrm{He}$, and ${ }^{4} \mathrm{He}$

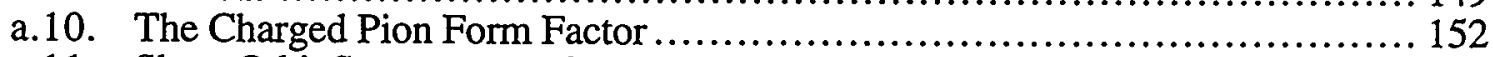

a.11. Short-Orbit Spectrometer for Hall C at TJNAF ........................... 152

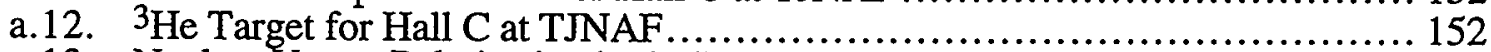

a.13. Nuclear Vector Polarization in the Laser Driven Polarized Target at IUCF and Measurement of $\mathbf{d}(\overline{\mathbf{p}}, \mathbf{p p}) \mathbf{n}$ Reaction 
a.14. An Ultra-Sensitive Trace Analyzer of Krypton-81 and Krypton-85 Atoms ..... 153

a.15. Nuclear Moments and Radii of Unstable Cesium Isotopes ................... 154

a.16. Testing Time-Reversal-Symmetry in Radon Atoms ......................... 155

a.17. Di-lepton Production with $800-\mathrm{GeV}$ Protons Measurement of $\overline{\mathbf{d}} / \mathbf{\mathbf { u }}$ in the Nucleon

a.18. $\Upsilon$ and $\mathrm{J} / \psi$ Production from $800-\mathrm{GeV}$ Protons Incident on $\mathrm{D}_{2}$ and $\mathrm{H}_{2}$ Targets .156

a.19. Di-lepton Production With $120-\mathrm{GeV}$ Protons to Extend the Measurement

of $\overline{\mathbf{d}} / \overline{\mathbf{u}}$ in the Nucleon.

A. NUCLEAR DYNAMICS WITH SUBNUCLEONIC DEGREES OF FREEDOM.

a.1. Pion Mass and Decay Constant ......................................... 161

a.2. Heavy- to Light-Meson Transition Form Factors .......................... 161

a.3. Thermodynamic Properties of a Simple, Confining Model....................... 161

a.4. Chemical Potential Dependence of $\pi$ and $\rho$ properties ........................ 162

a.5. Deconfinement at Finite Chemical Potential .................................. 162

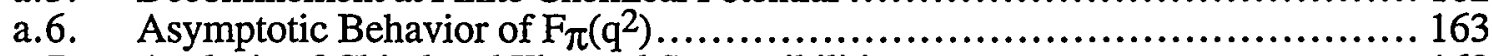

a.7. Analysis of Chiral and Thermal Susceptibilities.............................. 163

a.8. Infrared Behavior of Propagators and Vertices.................................... 163

a.9. A Dynamical, Confining Model and Hot Quark Stars........................ 164

a.10. Mean Field Exponents and Small Quark Masses.............................. 164

a.11. Survey of Heavy-meson Observables.......................................... 165

a.12. Diquark Condensation at High Density ................................... 166

a.13. Electromagnetic Nucleon Form Factors …................................ 167

a.14. Non-perturbative Gluon and Ghost Propagators in QCD ....................... 167

a.15. Dynamical Chiral Symmetry Breaking in QCD .............................. 167

a.16. Electromagnetic Production of Mesons and Nucleon Resonances............... 168

a.17. Study of the N $\Delta$ Interactions With d(e,e' $\pi$ ) Reactions....................... 169

a.18. Structure of the Vector Meson Photoproduction Amplitude at a Few GeV ...... 169

a.19. Non-perturbative Chiral Approach to $\mathrm{K}^{-} \mathrm{p} \rightarrow \gamma \mathrm{Y}$ Reactions.................... 170

a.20. Soft-core Hyperon-nucleon and Hyperon-hyperon Potentials ................. 171

a.21. Strange Hadron Matter and SU(3) Symmetry ............................. 171

a.22. Study of Hyperon-Nucleon Interactions with $\mathrm{d}\left(\mathrm{e}, \mathrm{e}^{\mathrm{K}} \mathrm{K}\right)$ Reactions............... 172

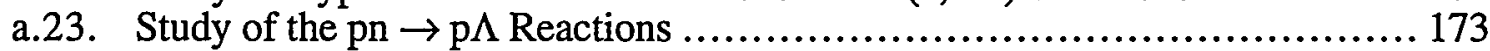

a.24. Two-frequency Shell Model for Hypernuclei ................................ 173

a.25. Covariant Quark Model for the Baryons .................................... 174

a.26. Poincaré Covariant Quark Models of Baryon Form Factors....................... 174

a.27. Null-Plane Invariance of Hamiltonian Null-Plane Dynamics ................. 175

B . NUCLEAR FORCES AND NUCLEAR SYSTEMS .......................... 175

b.1. Variational Monte Carlo Calculations of Light p-shell Nuclei .................. 176

b.2. Green's Function Monte Carlo Calculations of Light $p$-shell Nuclei ............... 179

b.3. Studies of Three-Nucleon Interactions in Nuclear Systems ..................... 180

b.4. Microscopic Calculation of ${ }^{6} \mathrm{Li}$ Elastic and Transition Form Factors............. 181

b.5. Nuclear Structure Studies With the ${ }^{7} \operatorname{Li}\left(e, e^{\prime} p\right)$ Reaction.......................... 182

b.6. Weak Capture of Protons by Protons ........................................ 183 
b.7. Radiative Capture Reactions for Astrophysical Applications .................. 183

b.8. $\Lambda$ Single-Particle Energies.............................................. 184

b.9. Core-Nucleus Distortion in Hypernuclei................................... 184

b.10. Charge-Symmetry Breaking $\Lambda$-Nucleon Interaction....................... 185

\section{NUCLEAR STRUCTURE AND HEAVY-ION REACTIONS................ 185}

c.1. Momentum Distributions of ${ }^{7} \mathrm{Be}$ Fragments in Breakup Reactions of ${ }^{8} \mathrm{~B}$....... 186

c.2. Nuclear-Induced Breakup of Halo Nuclei ................................ 187

c.3. Breakup Reactions of $8 \mathrm{~B}$ at Low Energies................................. 188

c.4. Coupled-Channels Calculations of ${ }^{17} \mathrm{~F}+{ }^{208} \mathrm{pb}$ Fusion Reactions ............ 189

c.5. Many-Body Wave Functions........................................... 190

c.6. Very Extended Shapes in Nuclei......................................... 191

c.7. Single-Particle States in the Heaviest Elements ........................... 192

c.8. Studies of Nuclear Energy Surfaces ..................................... 193

c.9. Nuclear Structure Near the Proton Drip Line.............................. 194

\section{ATOMIC THEORY AND FUNDAMENTAL QUANTUM}

MECHANICS

d.1. Interactions of High-Energy Photons with Matter ........................... 195

d.2. Interactions of Fast Charged Particles with Matter............................ 195

d.3. Electron Energy-loss Spectrum of Nanowires ................................. 196

d.4. Quantum Robots ................................................... 196

d.5. Definitions of Truth, Validity, Consistency and Completeness in Quantum Mechanics................................................................... 197

d.6. Force-Free Interactions and Nondispersive Phase Shifts in Interferometry .... 197

d.7. A No-go Theorem for Matter-Wave Interferometry, with Application to Neutron Electric-Dipole Moment Experiments ............................... 198

E. OTHER ACTIVITIES ................................................... 198

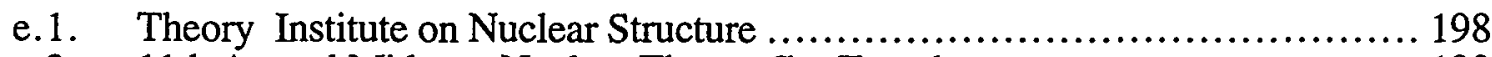

e.2. 11th Annual Midwest Nuclear Theory Get-Together......................... 199

V. ATOMIC AND MOLECULAR PHYSICS RESEARCH...................................................... 201

a. Forward-Backward Asymmetries of Atomic Photoelectrons................... 203

b. Electron Pair Correlation Functions from X-ray Scattering..................... 204

c. Double Ionization of Helium by X-Ray Compton Scattering.................. 205

d. Observation of Double K-Ionization by X-Ray Photoionization of

Molybdenum ................................................................. 207

e. Two-Photon Decay ................................................... 208

f. K-Shell Excitation in Ion-Atom Collisions ................................. 210

g. Laser-Cooled Li Target for Investigation of Ionization Processes ............ 211 


\section{OTHER EDUCATIONAL ACTIVITIES IN THE PHYSICS DIVISION}

a. Enhancement of Minority Involvement in DOE Nuclear Physics Programs............ 213

b. Nuclear Physics Award for Faculty in Undergraduate Institutions .....................213

c. Scientific Support of SciTech Museum Exhibits and.Outreach Programs .............. 214

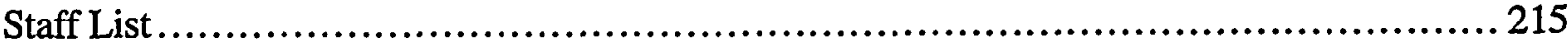

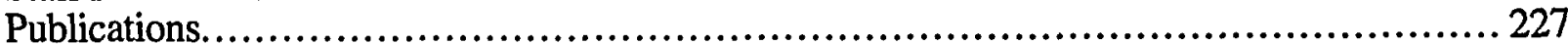




\section{HEAVY-ION NUCLEAR PHYSICS RESEARCH}

\section{OVERVIEW}

The Heavy-Ion program in the Argonne Physics Division addresses key questions about the structure and dynamics of the nuclear many-body system. Nuclear structure and reactions are studied in collisions between complex nuclei with heavyion beams mostly from the Argonne Tandem-Linac Accelerator System (ATLAS), a national heavy-ion users facility. Some important studies are performed at forefront facilities elsewhere. The major thrusts of this program are three-fold: a) the understanding of the nucleus as a many-body system built of protons and neutrons and governed by the strong force, $b$ ) the exploration of the origin of the chemical elements and their role in shaping the reactions that occur in the cataclysmic events of the cosmos, and c) tests of the limits of validity of the Standard Model, the fundamental theory that currently best represents our understanding of the laws and fundamental symmetries of Nature. The specific current research topics include the development and acceleration of short-lived nuclei and their use in measurements of cross-sections of astrophysics interests as well as in nuclear structure and reaction dynamics studies; the production and study of nuclei at the very limits of stability, in particular the discovery of new proton emitters near the drip line and the study of the level structure of very heavy elements $(Z>100)$; the study of exotic nuclear shapes, including superdeformation; the delineation of the essential parameters governing dynamics of reactions between heavy nuclei such as fusion, fission, transfer and deep inelastic reactions; tests of current descriptions of the weak force;precision mass measurements with a Penning ion-trap. Smaller scale efforts complementary to the main research program focus on the behavior of cooled ions confined in storage rings and ions traps, and on the study of collisions between heavy ions at relativistic energies. These efforts are based on forefront instrumentation available at ATLAS which includes Gammasphere, the Fragment Mass Analyzer (both systems are also used in combination), the Canadian Penning Trap, and exploit the unique capabilities of the accelerator to produce radioactive beams either through reactions in a production target or directly from the ion source. Participation to the PHOBOS detector construction is at the core of the research with relativistic heavy ions. 
Some of the main goals of the program can be summarized as follows:

Develop and utilize short-lived nuclear beams (1) to measure reactions of astrophysics interest $\left({ }^{17}, 18 \mathrm{~F},{ }^{21} \mathrm{Na}\right.$ and the break-out of the hot $\mathrm{CNO}$ cycle, ${ }^{25} \mathrm{Al}$, ${ }^{44} \mathrm{Ti}$ and ${ }^{56} \mathrm{Ni}$ and the rp process), (2) to study the properties of proton-rich nuclei (single particle states near the unstable doubly-magic nucleus $56 \mathrm{Ni}$ ), and (3) to investigate aspects of reaction dynamics (the impact of weakly bound states on fusion).

Discover new isotopes and determine the properties of these nuclei at the limits of proton-stability by measuring (1) groundstate quantum numbers, (2) particle decay. modes (proton, alpha and beta decay) and (3) gamma decay of excited states taking advantage of the resolving power of Gammasphere and the selectivity offered by the Fragment Mass Analyzer (FMA).

Study the importance of shell effects on nuclear structure as a function of mass, excitation energy and spin. In particular, understand the properties of heavy nuclei $(Z>82)$, of superdeformed nuclei, and of light $(A<30)$ nuclei (in the regime where particle and gamma decay compete) by comprehensive measurements with the highest detection sensitivity (Gammasphere).

Determine time scales of dissipation processes in nuclei through the characterization of the gamma decay of giant dipole resonances (utilizing the LEPPEX array of $\mathrm{BaF}_{2}$ detectors) with mass or angular momentum selection.

Measure with high precision nuclear masses with the CPT, in particular the mass of super-allowed $0^{+}$to $0^{+}$beta emitters $(50<A<100)$. These mass determinations have a direct impact on the determination of the fundamental weak vector coupling constant and the unitarity test of the top row of the Cabbibo-Kobayashi-Maskawa matrix.

Use the high quality beams of ATLAS to pursue unique applications with energetic heavy-ion beams such as defects in high $\mathrm{T}_{c}$ superconductors, and accelerator mass spectrometry of heavy (transactinide) radioisotopes.

Understand the behavior of nuclear matter at high density by exploring collisions between relativistic heavy ions. Studies focus on particle production, phenomena associated with collective flow and in-medium modification of meson masses. 


\section{A. EXPERIMENTS WITH SECONDARY BEAMS}

Over the last few years, a number of secondary (e.g. radioactive) beams of short-lived nuclei were produced and accelerated at ATLAS. To the ${ }^{8} \mathrm{~B},{ }^{17} \mathrm{~F},{ }^{18} \mathrm{~F},{ }^{56} \mathrm{Co}$ and ${ }^{56} \mathrm{Ni}$ beams developed in the past, new beams of ${ }^{21} \mathrm{Na},{ }^{25} \mathrm{Al}$ and ${ }^{44} \mathrm{Ti}$ were added this year. The experiments being performed cover issues of interest in nuclear astrophysics and in nuclear structure. Another approach being pursued is one where nuclei, produced at the target location of the FMA and transported by the instrument, are being excited via Coulomb excitation behind the focal plane of the instrument. This technique of Coulomb re-excitation is being applied to the case of $78 \mathrm{Rb}$ where the excitation of states built on the groundstate and two isomers was investigated. Finally, this section also contains a description of the production rate calculations being performed for the Advanced Exotic Beam Facility described elsewhere in this report.

\section{a.1. Study of the ${ }^{8} \mathrm{~B}$ Neutrino SpectrumThrough the ${ }^{8} \mathrm{~B}\left(\beta^{+}\right)^{8} \mathrm{Be}(2 \alpha)$ Decay Chain (K. E. Rehm, B. Harss, R. V. F. Janssens, C. L. Jiang, S. J. Freedman,*R. C. Pardo, G. Savard,J. P. Schiffer, B. Fujikawa,* and M. Paul, $\dagger$ )}

The main source of the high energy neutrinos from the sun is the beta decay of $8_{B}$ that is produced by nucleosynthesis deep in the solar interior. The neutrinos from ${ }^{8} \mathrm{~B}$ were observed in three different solar neutrino detectors: The $\mathrm{CCl}_{4}$ detector at the Homestake mine, Kamiokande and Super-Kamiokande. All three observations are below the neutrino flux expected from the Standard Solar model which uses nuclear reaction cross sections as input. It is anticipated that Super-Kamiokande and the SNO detector will provide detailed measurements of the shape of the spectrum of solar ${ }^{8} \mathrm{~B}$ neutrinos at the earth. A detailed examination of the spectral shape could provide evidence whether matter-enhanced solar-neutrino oscillations via the MSW effect are the cause of the low total flux. To realize this goal it is essential to know the reference neutrino spectrum of the unmodified ${ }^{8} \mathrm{~B}$ decay. Although this decay is allowed, the final state is broad with the decay proceeding $100 \%$ to the unbound firstexcited state of ${ }^{8} \mathrm{Be}$ at $2.9 \mathrm{MeV}$. The large width of this state causes both the neutrino and positron spectra to be different from that of a simple allowed shape and this distortion depends sensitively on the precise shape of the broad resonance and the overlap of the continuum wave function with the ground state of ${ }^{8} \mathrm{~B}$.

The available experimental data on ${ }^{8} \mathrm{~B}$ decay were recently reviewed with an attempt to predict the ${ }^{8} \mathrm{~B}$ neutrino spectrum and establish the uncertainty with which it is known. There are two types of experimental input: the singles spectra of delayed alpha particles and the beta decay spectrum. It was noted that the 5 existing measurements of the alpha particle spectrum are in poor agreement. The basic shape of the alpha singles spectrum seems to be reproduced in the various experiments, but the spectra are shifted relative to one another, suggesting a systematic error in the reported energy scales on the order of $80 \mathrm{keV}$. In all cases studied previously, the ${ }^{8} \mathrm{~B}$ source is produced by the ${ }^{6} \mathrm{Li}\left({ }^{3} \mathrm{He}, \mathrm{n}\right){ }^{8} \mathrm{~B}$ reaction and the recoiling ${ }^{8} \mathrm{~B}$ are implanted in thin metallic foils. While careful attention to energy loss effects was claimed in each of these experiments it is likely that the discrepancy is due to a failure to properly correct for alpha-particle straggling in the catcher foil and/or the detector dead layer. The availability of radioactive ${ }^{8} \mathrm{~B}\left(\mathrm{~T}_{1 / 2}=770 \mathrm{~ms}\right)$ beams produced via the in-flight technique at ATLAS together with large-area $\mathrm{Si}$ detectors provide a unique opportunity for determining the shape of the ${ }^{8} \mathrm{~B}$ neutrino spectrum with the required accuracy.

In a first test experiment ${ }^{8} \mathrm{~B}$ beams with energies of 35 $\mathrm{MeV}$ were produced via the ${ }^{3} \mathrm{He}\left({ }^{6} \mathrm{Li}, 8 \mathrm{~B}\right) \mathrm{n}$ reaction using a pulsed ${ }^{6} \mathrm{Li}$ beam $\left(2 \mathrm{sec}\right.$ beam on, 2 sec beam off). The ${ }^{8} \mathrm{~B}$ particles were implanted into a $100-\mu$ thick $\mathrm{Si}$ detector located in the focal plane of the Enge Split-Pole spectrograph and the subsequent decay into 2 alphas was measured in the beam-off periods. This technique eliminates the catcher foil and uncertainties associated with the energy loss in the detector dead layer. The main difficulty encountered in the first test experiment came from the large amount of primary ${ }^{6} \mathrm{Li}$ beam that also hit the Si detector. With the improved in-flight production setup described in Section a. 3 which allows us to use a rebunching resonator after the production target we expect to reduce the amount of primary beam considerably. Together with a cooled Si detector array we hope to reduce the uncertainties associated with the ${ }^{8} \mathrm{~B}$ decay by about an order of magnitude.

*University of California at Berkeley and Lawrence Berkeley National Laboratory, †Hebrew University, Jerusalem, Israel 
a.2. Study of the ${ }^{14} \mathrm{O}+\alpha \rightarrow p+{ }^{17} \mathbf{F}$ Reaction: A Gateway in Nucleosynthesis (B. Harss, * J. P. Greene, D. Henderson, R. V. F. Janssens, C. L. Jiang, J. Nolen, R. C. Pardo, K. E. Rehm, J. P. Schiffer, A. A. Sonzogni, J. Uusitalo, I. Wiedenhöver, M. Paul, † T. F. Wang, $\ddagger$ F. Borasi,§ R. E. Segel,§ J. Blackmon,II M. Smith, I A. Chen,॥ and P. Parkerll)

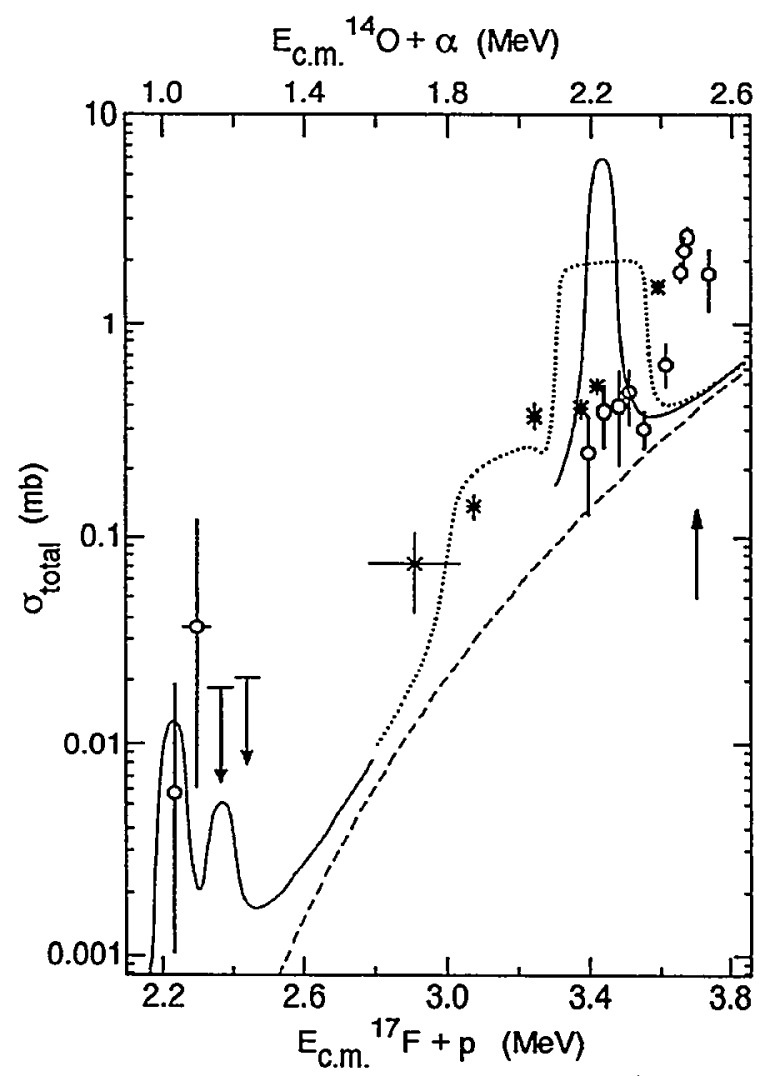

Fig. I-1. Measured cross sections for the $p\left({ }^{17} F,{ }^{14} O\right) \alpha$ reaction (top). The circles represent measurements with thin $\left(100 \mu \mathrm{g} / \mathrm{cm}^{2}\right)$ targets, while the crosses are for thicker $\left(500 \mu \mathrm{g} / \mathrm{cm}^{2}\right)$ ones. The horizontal bars, shown for one point only indicate the energy interval corresponding to the target thickness. The dotted line represents the expected thick-target yield and the solid line that for the thinner targets using the resonance parameters of Ref. 5. The dashed line is the direct component estimated in Ref. 9. The arrow indicates the location of the state in 18 Ne that was listed in Ref. 5 , but not included in the estimates. The energy scales at the top and bottom are for the entrance and exit channels involved in the reaction.

The development of techniques for producing beams of short-lived nuclei is beginning to allow the measurement of reactions of interest in nuclear structure and in nuclear astrophysics, where reactions involving these nuclei play an important role in explosive astrophysical events such as X-ray bursts and novae.
This report describes the first measurement of this reaction with the newly-developed, high-efficiency silicon array LUDWIG II, via its time-reversed process, ${ }^{17} \mathrm{~F}(\mathrm{p}, \alpha){ }^{14} \mathrm{O}$, using a beam of ${ }^{17} \mathrm{~F}\left(\mathrm{~T}_{1 / 2}=64 \mathrm{~s}\right)$ interacting with a hydrogen $\left(\mathrm{CH}_{2}\right)$ target in the energy range corresponding to stellar temperatures of $T \geq 1 \times$ $10^{9} \mathrm{~K}$.

Prior to the present measurements, estimates of the absolute value and energy dependence of the cross section for the ${ }^{14} \mathrm{O}(\alpha, p){ }^{17} \mathrm{~F}$ reaction were made from indirect information. Since the reaction is mainly resonant, it depends on the properties of unbound states in the compound nucleus $18 \mathrm{Ne}$ in the region of excitation energy between 6 and $8 \mathrm{MeV}$. The goal of the present work was to obtain information relevant to the ${ }^{14} \mathrm{O}(\alpha, \mathrm{p})^{17_{\mathrm{F}}}$ reaction in the c.m. energy range 1.6 $2.6 \mathrm{MeV}$ (for the $\alpha+{ }^{14} \mathrm{O}$ system) corresponding to $E_{\mathrm{X}}$ $=6.7-7.7 \mathrm{MeV}$ in ${ }^{18} \mathrm{Ne}$.

The ${ }^{17} \mathrm{~F}$ beam was produced via the $\mathrm{d}\left({ }^{16} \mathrm{O},{ }^{17} \mathrm{~F}\right) \mathrm{n}$ using our gas cell setup described elsewhere in this report. Two sets of measurements of the $\mathrm{p}\left({ }^{17} \mathrm{~F}, \alpha\right)^{14} \mathrm{O}$ reaction were performed. First, the reaction was measured with a thick $480-\mu \mathrm{g} / \mathrm{cm}^{2} \mathrm{CH}_{2}$ target at laboratory energies of $54,57,60,63$ and $66 \mathrm{MeV}$. In a second run, a thinner target $\left(105-\mu \mathrm{g} / \mathrm{cm}^{2} \mathrm{CH}_{2} \Delta \mathrm{E}_{\mathrm{lab}} \approx\right.$ $700 \mathrm{keV}$ ) was used to measure the excitation function in $\sim 700 \mathrm{keV}$ steps $\left(\Delta \mathrm{E}_{\mathrm{cm}} \approx 39 \mathrm{keV}\right)$ between 61.5 and 67 $\mathrm{MeV}$. The measurements covered the energy region of $\mathrm{E}_{\mathrm{cm}}=2.7-3.7 \mathrm{MeV}$ in the $17 \mathrm{~F}+\mathrm{p}$ system, corresponding to an excitation energy range of 6.7- 7.7 $\mathrm{MeV}$ in ${ }^{18} \mathrm{Ne}$.

Coplanar kinematic coincidences between the $\alpha$ particle in the first detector and the ${ }^{14} \mathrm{O}$ residual nucleus in the second counter were used to identify events from the $\mathrm{p}\left({ }^{17} \mathrm{~F}, \alpha\right){ }^{14} \mathrm{O}$ reaction. The measured total cross sections are presented in Fig. I-1 as a function of the center of mass energies in both channels, $E_{\mathrm{cm}}\left({ }^{17} \mathrm{~F}+\right.$ $p)$ and $E_{\mathrm{cm}}\left({ }^{14} \mathrm{O}+\alpha\right)$. The uncertainties in the cross section values shown are purely statistical. The detection efficiency was $\sim 65 \%$, obtained from a Monte Carlo calculation assuming an isotropic angular distribution, and including the effects of a finite beam spot and multiple scattering in the target. For absolute cross-section determination, the integrated beam intensity was obtained from the $17 \mathrm{~F}$ ions elastically 
scattered off ${ }^{12} \mathrm{C}$ in the $\mathrm{CH}_{2}$ target. The cross sections presented here are averaged over the energy interval covered by the target, and the (smaller) energy spread of the beam. The angular range covered by the detector corresponds to center-of-mass angles of 40 to 120 degrees. The total systematic uncertainties are estimated to be $\sim 20 \%$. The results of the thin- and thick-target measurements are consistent within the statistical uncertainties.

Previous estimates of the ${ }^{14} \mathrm{O}(\alpha, \mathrm{p})^{17} \mathrm{~F}$ reaction cross section $^{2-4}$ utilized the known levels of ${ }^{18} \mathrm{O}$ and the expected Coulomb shifts to obtain assignments to states in ${ }^{18} \mathrm{Ne}$ with $\mathrm{E}_{\mathrm{X}}>5 \mathrm{MeV}$. In Ref. 2 the partial widths of resonances were taken from the widths of the corresponding $18 \mathrm{O}$ states or from systematics. A comparison of the results of this estimate with our data (Fig. I-1) shows distinct discrepancies.

The thin-target measurement reveals a strong resonance at a center-of-mass energy of $\mathrm{E}_{\mathrm{cm}}\left({ }^{\left.17_{\mathrm{F}}+\mathrm{p}\right)}=3.7 \mathrm{MeV}\right.$ $\left(E_{x}=7.60 \pm 0.05 \mathrm{MeV}\right)$. This resonance was not included in the excitation function derived in Ref. 2 because insufficient information about its properties was available. The value of the strength parameter $\omega \gamma$ for this resonance is $(300 \pm 100) \mathrm{eV}$, derived independently from both the thin- and thick-target measurements.

In the energy region $\mathrm{E}_{\mathrm{cm}}\left({ }^{17} \mathrm{~F}+\mathrm{p}\right)=3.4-3.55 \mathrm{MeV}$ $\left(E_{X}=7.3-7.5 \mathrm{MeV}\right)$, the thin-target data show a small structure that is attributed to a weak resonance. Assuming a single level, a value of $\omega \gamma=(20 \pm 10) \mathrm{eV}$ is measured for this resonance. Another factor-of-three increase in cross sections is seen between the thick target points centered at $E_{\mathrm{cm}}\left({ }^{17} \mathrm{~F}+\mathrm{p}\right)$ of 3.06 and $3.24 \mathrm{MeV}$, suggesting the presence of an additional resonance at an excitation energy of $\sim 7.16 \mathrm{MeV}$ with $\omega \gamma=(75 \pm 20) \mathrm{eV}$. At even lower energies, the thicktarget yield drops, but not as fast as expected from the estimated energy dependence of penetrabilities. This might be interpreted as being due to yet another resonance around $2.9 \mathrm{MeV}$ or, possibly, to a direct contribution about twice as large as estimated in Ref. 4 . The resonance at $E_{X}=(7.60 \pm 0.05) \mathrm{MeV}$ is consistent with a level seen at $7.62 \mathrm{MeV}$ in ${ }^{18} \mathrm{Ne}$ in the ${ }^{12} \mathrm{C}\left({ }^{12} \mathrm{C},{ }^{6} \mathrm{He}\right)^{18} \mathrm{Ne}$ reaction. ${ }^{2}$ The weaker structure at $(7.37 \pm 0.06) \mathrm{MeV}$ is consistent with a level seen in both the $\left({ }^{12} \mathrm{C},{ }^{6} \mathrm{He}\right)$ and the ${ }^{16} \mathrm{O}\left({ }^{3} \mathrm{He}, \mathrm{n}\right)$ reactions at $7.35 \mathrm{MeV}$. The resonance deduced from the thick target measurement at $\mathrm{E}_{\mathrm{X}}=(7.16 \pm 15) \mathrm{MeV}$ could be attributed to a level at $7.12 \mathrm{MeV}$, also listed in Ref. 2 .

The parameters for the three resonances found in the present work are summarized in Table I-I, which also includes the parameters used in Ref. 2 for the excitation energy region of interest here. In the table, the possible spin-parity assignments have been restricted by the limits on energy differences. Another constraint is that the $\alpha$-particle width implied by a spin assignment be less than the reduced $\alpha$-particle width $\Theta^{2} \equiv \Gamma \alpha / \Gamma \alpha^{\text {s.p. }}$. The remaining options and constraints from the known widths of some of the levels in $18 \mathrm{O}$ are also shown in Table 1. By isospin symmetry, the $\alpha$-particle widths for the decay of excited levels in $18 \mathrm{O}$ should be related to the widths for alpha emission from the corresponding levels in ${ }^{18} \mathrm{Ne}$.

With these criteria, the $7.16-\mathrm{MeV}$ resonance in $18_{\mathrm{Ne}}$ is consistent only with a $1^{-}$assignment, and corresponds to the $7.62-\mathrm{MeV}, 1^{-}$level in ${ }^{18} \mathrm{O}$. The $7.37-\mathrm{MeV}$ level can be assigned either a $1^{-}$or a $4^{+}$value. With the first assignment, this state would be the partner of the 8.04$\mathrm{MeV} 1^{-}$level in $18 \mathrm{O}$, with an energy difference near the limit. The $4^{+}$assignment would make the state the partner of the $7.13-\mathrm{MeV} 4^{+}$level in 180 with the widths in rather good agreement. Finally, the strong 7.60-MeV resonance could have $\mathrm{J}^{\pi}=1^{-}, 2^{+}$or $3^{-}$. A $\mathrm{J} \pi=3^{-}$assignment, however, agrees poorly with the $\alpha$ width of the corresponding state in 180 . Since there are only two $1^{-}$states known in ${ }^{18} \mathrm{O}$, only the assignments of $\mathrm{J}^{\pi}\left(1^{-} ; 4^{+} ; 1^{-}\right),\left(1^{-} ; 4^{+} ; 2^{+}\right)$or $\left(1^{-} ; 1^{-} ; 2^{+}\right)$for the observed resonances at $7.16 \mathrm{MeV}, 7.37 \mathrm{MeV}$ and 7.60 respectively remain.

*Argonne National Laboratory and Technical University of Münich, Germany, †Hebrew University, Jerusalem,

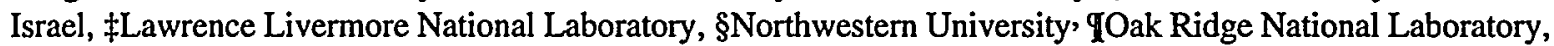
IIYale University

'A. E. Champagne and M. Wiescher, Ann. Rev. Nucl. Part. Sci. 42, 39 (1992).

${ }^{2}$ K. I. Hahn et al., Phys. Rev. C 54, 1999 (1996).

${ }^{3}$ M. Wiescher, J. Görres and F.-K. Thielmann, Astrophys. J. 316, 384 (1988).

${ }^{4}$ C. Funck, B. Grund and K. Langanke, Z. Phys. A332, 109 (1989).

${ }^{5}$ F. Ajzenberg-Selove, Nucl. Phys. A475, 1 (1987).

${ }^{6}$ J. A. Weinman and E. Silverstein, Phys. Rev. 111, 277 (1958).

${ }^{7}$ K. E. Rehm et al., Phys. Rev. C 52, R460 (1995).

${ }^{8}$ R. Coszach et al., Phys. Lett. B353, 184 (1995). 
Table I-I. Resonance parameters for various states in $18 \mathrm{Ne}$. The first three rows are from the present experiment, while the last six lines were taken from Ref. 5.

\begin{tabular}{|c|c|c|c|c|c|c|c|c|}
\hline $\mathrm{E}_{\mathrm{X}}$ & $\mathrm{E}_{\mathrm{cm}}$ & $\omega \gamma$ & $\mathrm{J} \pi$ & $\Gamma_{\mathrm{a}}^{\exp }$ & $\theta_{\mathrm{a}}^{2}$ & $\mathrm{E}_{\mathrm{X}}$ & $\theta_{\mathrm{a}}^{2}$ & $E_{x}\left({ }^{18} O\right)-$ \\
\hline$(18 \mathrm{Ne})$ & $(p, \alpha)$ & $(p, \alpha)$ & & & $\left({ }^{18} \mathrm{Ne}\right)$ & $\left({ }^{18} \mathrm{O}\right)$ & $\left({ }^{18} \mathrm{O}\right)$ & $\mathrm{E}_{\mathrm{x}}\left({ }^{18 \mathrm{Ne}}\right)$ \\
\hline$[\mathrm{MeV}]$ & {$[\mathrm{MeV}]$} & {$[\mathrm{eV}]$} & & {$[\mathrm{keV}]$} & & {$[\mathrm{MeV}]$} & & {$[\mathrm{keV}]$} \\
\hline $7.16 \pm 0.15$ & 3.24 & $75 \pm 20$ & $1^{-}$ & 0.3 & 0.016 & 7.62 & $0.012^{\mathrm{a}}$ & 460 \\
\hline \multirow[t]{2}{*}{$7.37 \pm 0.06$} & 3.48 & $23 \pm 12$ & $1^{-}$ & 0.08 & 0.002 & 8.04 & $<0.1^{b}$ & 670 \\
\hline & & & $4^{+}$ & 0.03 & 0.09 & 7.11 & $0.11^{\mathrm{a}}$ & -250 \\
\hline \multirow[t]{3}{*}{$7.60 \pm 0.05$} & 3.69 & $300 \pm 40$ & $1^{-}$ & 1.2 & 0.015 & 8.04 & $<0.03^{b}$ & 440 \\
\hline & & & $2^{+}$ & 0.7 & 0.026 & 8.21 & $0.03 \pm 0.02^{c}$ & 610 \\
\hline & & & $3^{-}$ & 0.5 & 0.09 & 8.28 & $0.72 \pm 0.04^{\mathrm{C}}$ & 680 \\
\hline 6.15 & 2.23 & 0.55 & $\left(1^{-}\right)$ & $2.2 \times 10-3$ & $(6.20)$ & & & \\
\hline 6.29 & 2.36 & 0.2 & $\left(3^{-}\right)$ & $3.4 \times 10-4$ & $(6.40)$ & & & \\
\hline 7.05 & 3.13 & 36 & $\left(4^{+}\right)$ & $4.8 \times 10-2$ & (7.11) & & & \\
\hline 7.12 & 3.20 & - & - & - & & & & \\
\hline 7.35 & 3.43 & 375 & $\left(1^{-}\right)$ & 1.7 & & $(7.62)$ & & \\
\hline 7.62 & 3.70 & - & - & - & & & & \\
\hline
\end{tabular}

$\mathrm{a}[15], b_{[14],}^{c}[16]$

\section{a.3. Progress in Producing Radioactive Beams with the Gas Cell Setup at} ATLAS (B. Harss, R. C. Pardo, C. L. Jiang, B. Nardi, J. Nolen, K. E. Rehm, J. Specht, and B. Zabransky)

The capability of producing secondary radioactive heavy ion beams at ATLAS was further improved. The general setup was already described in the 1998 Physics Division Annual Report. Using a primary gas target in combination with a superconducting solenoid and two ATLAS debunching resonators, one of them located three meters downstream from the primary target, beams of $17 \mathrm{~F},{ }^{25} \mathrm{Al}$ and ${ }^{21} \mathrm{Na}$ were successfully produced. The intensities for the ${ }^{25} \mathrm{Al}$ and ${ }^{21} \mathrm{Na}$ beams were $2-5 \times$ $10^{3}$ ions $/ \mathrm{sec}$ for $1 \mathrm{pnA}$ of incident primary beam, allowing for maximum intensities of $2-5 \times 10^{5} \mathrm{pps}$ on target. While the ${ }^{17} \mathrm{~F}$ beams produced previously showed an energy spread of more than $1 \mathrm{MeV}$ at energies around $60 \mathrm{MeV}$, the resonators allowed us to reduce the width to less than $400 \mathrm{keV}$ (Fig. I-2).

The procedure of beam tuning was also improved. After discovering that a reliable tune requires the full primary beam intensity on the primary target, a remotelycontrolled 1:1000 attenuator was installed between the 
primary and the secondary (physics) target. This allows the use of the ATLAS Enge split-pole spectrograph at $0^{\circ}$ as an diagnostic tool. The signals from its focal plane detector were sampled, histogrammed and transmitted to the control room by a custom-built module described elsewhere in this Report.

Important improvements in the cooling system of the setup were achieved. A buffered liquid nitrogen supply system was installed and the liquid helium filling system of the solenoid dewar was completely automated. The new cooling scheme was used since it was installed for several hundred hours of experiments without any malfunction, thereby greatly improving the overall reliability of the production setup.

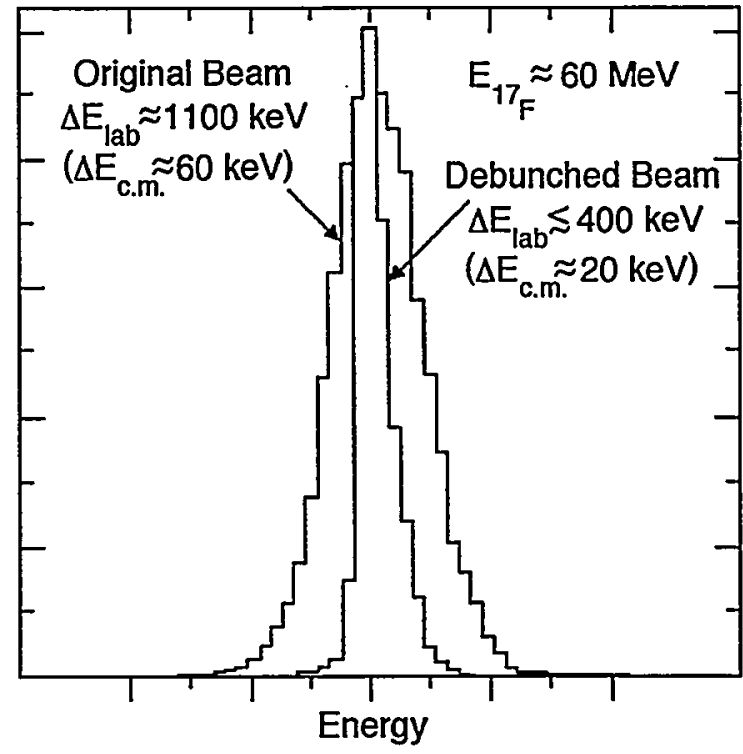

Fig. I-2. The normalized energy distributions of two ${ }^{17} \mathrm{~F}$ beams of $\sim 60 \mathrm{MeV}$ with intensities of $\sim 1 \times 10^{6}$ particles.

\section{a.4. Decay Properties of Particle-Unbound States in ${ }^{19} \mathrm{Ne}$ (F. Borasi, K. E. Rehm,} B. Harss, R. V. F. Janssens, C. L. Jiang, J. P. Schiffer, R. Siemssen, A. Sonzogni, J. Uusitalo, I. Wiedenhöver, A. H. Wuosmaa, M. Paul,* and R. E. Segel, $\dagger$ )

The breakout from the hot $\mathrm{CNO}$ cycle into the so-called rapid-proton capture (rp) process via the ${ }^{15} \mathrm{O}(\alpha, \gamma){ }^{19} \mathrm{Ne}$ reaction is strongly determined by the alpha widths of particle-unbound states in $19 \mathrm{Ne}$ with the main contribution expected to come from the $4.033 \mathrm{MeV}$ $3 / 2^{+}$state in ${ }^{19} \mathrm{Ne}$ which is the first level above the $15 \mathrm{O}+\alpha$ threshold and is unbound to alpha particle emission by $504 \mathrm{keV}$.

Due to its importance for nuclear astrophysics there were several attempts to determine the critical reaction parameters of this state in the past. However, no direct measurement of the ${ }^{15} \mathrm{O}(\alpha, \gamma)$ reaction rate has been performed so far. An alpha width $\mathrm{T}_{\alpha}=9 \mu \mathrm{eV}$ for the $3 / 2^{+}$state was estimated based on the width of the mirror state in ${ }^{19} \mathrm{~F}$. However, since the spectroscopic factor of this state is quite small $\left(\Theta_{\alpha} \sim 0.06\right)$ the validity of estimating the alpha width from the $\left({ }^{6} \mathrm{Li}, \mathrm{d}\right)$ reaction is subject to considerable uncertainty.

We plan to measure the alpha width of this state using the inverse $d\left({ }^{20} \mathrm{Ne}, \mathrm{t}\right)^{19} \mathrm{Ne}$ reaction for populating the $3 / 2^{+}$state in ${ }^{19} \mathrm{Ne}$. A deuterium gas target will be bombarded with a $20 \mathrm{Ne}$ beam of $\sim 250 \mathrm{MeV}$. The outgoing tritons and the coincident $15 \mathrm{O}$ ions from the decay of ${ }^{19} \mathrm{Ne}$ will be detected in the Enge split-pole spectrograph at $0^{\circ}$. The simultaneous detection of these particles requires two independently operating detectors. The tritons will be detected in a large area positionsensitive $\mathrm{Si}$ detector telescope mounted in the focal plane of the spectrograph while the ${ }^{15} \mathrm{O}$ ions will be identified with respect to mass and $\mathrm{Z}$ with the existing hybrid focal plane detector.

As deuterium target we will use the new gas target which consists of a gas cell with two HAVAR windows separated by a distance which is variable between $1 \mathrm{~mm}$ and $10 \mathrm{~mm}$. Filling the gas cell (length $5 \mathrm{~mm}$ ) with 700 mbar of deuterium and cooling it down to liquid $\mathrm{N}_{2}$ temperatures produces a deuterium target with a thickness of $220 \mu \mathrm{g} / \mathrm{cm}^{2}$. The entrance foils of this gas target were shown to withstand beam currents up to 100 pnA.

A first test experiment to study the particle identification with this setup was performed.

*Hebrew University, Jerusalem, Israel, $\uparrow$ Northwestern University 


\section{a.5. Breakout from the Hot CNO Cycle Via the ${ }^{18} \mathrm{Ne}(\alpha, p)^{21} \mathrm{Na}$ Reaction (K. E. Rehm, B. Harss, R. V. F. Janssens, C. L. Jiang, J. Nolen, R. C. Pardo, J. P. Schiffer, A. Sonzogni, J. Uusitalo, I. Wiedenhöver, N. Bateman, * F. Borasi, $\dagger$ P. Parker, $\ddagger$ M. Paul,§ and R. E. Segel $\dagger$ )}

The neon isotope ${ }^{19} \mathrm{Ne}$ is an important gateway for the production of heavy nuclei via the rapid proton (rp) process. The (rp) process is a network of $(p, \gamma)$ and $(p, \alpha)$ reactions occurring in a hot, hydrogen-rich stellar environment where starting from CNO material, heavy nuclei up to ${ }^{56} \mathrm{Ni}$ and beyond are produced. ${ }^{19} \mathrm{Ne}$ represents a 'barrier' in this process, because, once nuclei beyond ${ }^{19} \mathrm{Ne}$ are produced, they will not be recycled back into normal CNO material. There are three reaction paths which can pass the ${ }^{19} \mathrm{Ne}$ "barrier": 1. ${ }^{18} \mathrm{~F}(\mathrm{p}, \gamma){ }^{19} \mathrm{Ne}, 2 .{ }^{15} \mathrm{O}(\alpha, \gamma){ }^{19} \mathrm{Ne}$, 3. ${ }^{19} \mathrm{Ne}(\alpha, \mathrm{p})^{21} \mathrm{Na}$

The first of these reactions was shown to have a low astrophysical reaction rate, because ${ }^{18} \mathrm{~F}$ produced in the hot CNO cycle is efficiently recycled back into ${ }^{15} \mathrm{O}$ via the ${ }^{18} \mathrm{~F}(\mathrm{p}, \alpha)^{15} \mathrm{O}$ reaction.

The second reaction path has not yet been measured and only estimates of the reaction rates were obtained which are based on the properties of the mirror nucleus ${ }^{15} \mathrm{~N}$. Since this path involves radiative capture of an alpha particle it occurs at higher temperatures. Network calculations show that this reaction path is stronger than the first path, but due to the higher Coulomb barrier the breakout occurs at temperatures above $\mathrm{T}_{9} \sim$ 0.6 .

The third reaction path, which involves only one $(\alpha, p)$ reaction, can compete despite the higher Coulomb barrier in the system ${ }^{18} \mathrm{Ne}+\alpha$. If levels with appropriate spin values in the compound nucleus $22 \mathrm{Mg}$ are available, the $(\alpha, p)$ reaction should have cross sections that are about 1000 times larger than that of a typical $(\mathrm{p}, \gamma)$ reaction.
A direct measurement of the ${ }^{18} \mathrm{Ne}(\alpha, \mathrm{p})^{21} \mathrm{Na}$ reaction is difficult, since it requires a ${ }^{18} \mathrm{Ne}$ beam and a ${ }^{4} \mathrm{He}$ gas target. The use of a ${ }^{4} \mathrm{He}$ gas target can be avoided if one measures the inverse ${ }^{21} \mathrm{Na}(\mathrm{p}, \alpha)^{18} \mathrm{Ne}$ reaction with a solid $\mathrm{CH}_{2}$ target. The reaction rate for the $(\alpha, p)$ reaction will then be obtained by using the principle of detailed balance.

To test the intensities that can be obtained for a radioactive ${ }^{21} \mathrm{Na}$ beam using the in-flight technique we have studied the $\mathrm{d}\left({ }^{20} \mathrm{Ne},{ }^{21} \mathrm{Na}\right) \mathrm{n}$ and $\mathrm{p}\left({ }^{21} \mathrm{Ne},{ }^{21} \mathrm{Na}\right) \mathrm{n}$ reactions. $20,21 \mathrm{Ne}$ beams from the positive ion injector of ATLAS bombarded a gas cell mounted in front of a focusing solenoid and a debunching resonator (see contribution a.3). The secondary ${ }^{21} \mathrm{Na}$ beam was identified with respect to mass $A$ and charge $Z$ in the focal plane of the Enge Split-Pole spectrograph. For both incident beams $20 \mathrm{Ne}$ and $21_{\mathrm{Ne}}$ intensities of $7 x$ $10^{321} \mathrm{Na} / \mathrm{sec} / \mathrm{pnA}$ were measured in the spectrograph. The energy of the secondary ${ }^{21} \mathrm{Na}$ beam was $114 \mathrm{MeV}$ in both cases. For a 150 pnA primary beam, intensities of $10^{621} \mathrm{Na} / \mathrm{sec}$ can be expected.

A measurement of the $\mathrm{p}\left({ }^{21} \mathrm{Na}, \alpha\right){ }^{18} \mathrm{Ne}$ reaction by detecting ${ }^{18} \mathrm{Ne}$ and $\alpha$ particles in kinematic coincidence requires a position-sensitive detector capable of identifying ${ }^{18} \mathrm{Ne}$ with respect to energy and $\mathrm{Z}$ at small scattering angles. We are presently constructing a cylindrical position-sensitive ionization chamber which, together with an annular Si detector for the detection of the alpha particle, will allow a measurement the $\mathrm{p}\left({ }^{21} \mathrm{Na}, \alpha\right){ }^{18} \mathrm{Ne}$ cross section in the important excitation energy region $\mathrm{E}_{\mathrm{X}}(22 \mathrm{Mg})=8.5-10.5 \mathrm{MeV}$.

*TRIUMF, Vancouver, Canada, †Northwestern University, $\$$ Yale University, §Hebrew University, Jerusalem, Israel 


\section{a.6. Production of an ${ }^{25} \mathrm{Al}$ Beam (J. Greene, B. Harss, R. V. F. Janssens, C. L. Jiang, R. Pardo, K. E. Rehm, A. Sonzogni, J. Uusitalo, I. Wiedenhöver, A. E. Champagne, * J. C. Blackmon, $\dagger$ F. Borasi,§ A. Chen, $\ddagger$ P. D. Parker, $\ddagger$ M. S. Smith $\nmid \dagger$ and T. F. Wang, I)}

The origin of ${ }^{26} \mathrm{Al}$ is a long-standing mystery in nuclear astrophysics with implications for galactic and stellar evolution, and for the origin of the solar system. Nova explosions should be efficient producers of ${ }^{26} \mathrm{Al}$, but observations of galactic gamma-ray emissions suggest otherwise. One possibility to bypass ${ }^{26} \mathrm{Al}$ would be a strong yield for the ${ }^{25} \mathrm{Al}(\mathrm{p}, \gamma)^{26} \mathrm{Si}$ reaction which is faster than the $\beta^{+}$decay of ${ }^{25} \mathrm{Al}$. A direct measurement of this reaction is not feasible with current technologies. However, constraints on the astrophysical reaction rate can be obtained from a measurement of the resonances which dominate this reaction. The most important contribution to the reaction rate comes from a single $3^{+}$state (s wave resonance) in ${ }^{26} \mathrm{Si}$ located about $450 \mathrm{keV}$ above the $(p, \gamma)$ threshold. This state which so far is only predicted by shell-model calculations has not yet been observed experimentally and carries an uncertainty in its excitation energy of $\sim 100 \mathrm{keV}$. In order to better determine the excitation energy of this state we propose to populate the $3^{+}$state via the ${ }^{25} \mathrm{Al}(\mathrm{d}, \mathrm{n})^{26}$ Si reaction with a radioactive ${ }^{25} \mathrm{Al}$ beam and detecting the decay of this state using Gammasphere.
As a first step we have investigated possible production mechanisms for generating a $25 \mathrm{Al}$ beam using the $\mathrm{d}\left({ }^{24} \mathrm{Mg},{ }^{25} \mathrm{Al}\right) \mathrm{n}$ and the $\mathrm{p}\left({ }^{25} \mathrm{Mg},{ }^{25} \mathrm{Al}\right) \mathrm{n}$ reactions. Low intensity $24,25 \mathrm{Mg}$ beams bombarded a cooled ( $\mathrm{T}=77$ $\mathrm{K})$ gas cell loaded with deuterium or hydrogen at pressures of $700 \mathrm{mb}$. The secondary ${ }^{25} \mathrm{Al} 13+$ beams were focused by a superconducting solenoid located $\sim 50$ $\mathrm{cm}$ behind the gas cell and transported into the scattering chamber of the Enge Split Pole spectrograph. In the focal plane of the spectrograph the particles were identified with respect to mass and nuclear charge by a position sensitive hybrid focal plane detector. Production rates for ${ }^{25} \mathrm{Al}$ were measured at incident energies of $\mathrm{E}\left({ }^{24} \mathrm{Mg}\right)=243 \mathrm{MeV}$ and $\mathrm{E}\left({ }^{25} \mathrm{Mg}\right)=220$ and $250 \mathrm{MeV}$. The largest yields $\left(\sim 2 \times 10^{3}\right.$ $\left.{ }^{25} \mathrm{Al} / \mathrm{sec} / \mathrm{pnA}\right)$ were measured with the $\mathrm{p}\left(25_{\mathrm{Mg}}{ }^{25} \mathrm{Al}\right) \mathrm{n}$ reaction at $\mathrm{E}\left({ }^{25} \mathrm{Mg}\right)=220 \mathrm{MeV}$. With the $\mathrm{d}\left({ }^{24} \mathrm{Mg},{ }^{25} \mathrm{Al}\right) \mathrm{n}$ reaction the production rate was smaller by a factor of two. Thus, with a $100 \mathrm{pnA}$ primary beam intensities of $2 \times 10^{5} 25 \mathrm{Al}$ on target can be expected which should be sufficient for a study of the ${ }^{25} \mathrm{Al}(\mathrm{d}, \mathrm{n})^{26} \mathrm{Si}$ reaction.

*University of North Carolina, †Oak Ridge National Laboratory, $\ddagger$ Yale University, §Northwestern University, ILawrence Livermore National Laboratory

a.7. Development of a ${ }^{44}$ Ti Beam (A. A. Sonzogni, K. E. Rehm, I. Ahmad, P. R. Crotty, C. N. Davids, J. P. Greene, B. Harss, D. J. Henderson, W. F. Henning, R. V. F. Janssens, C. L. Jiang, J. A. Nolen, R. C. Pardo, P. Reiter, J. P. Schiffer, J. Schwartz, D. Seweryniak, S. Siem, R. H. Siemssen, J. Uusitalo, I. Wiedenhover, A. H. Wuosmaa, B. J. Zabransky, F. Borasi, * F. Brumwell, $\uparrow$ G. McMichael, $\uparrow$ P. Parker, $¥$ M. Paul, $\S$ and R. E. Segel*)

As explained in the next contribution, the study of the ${ }^{44} \mathrm{Ti}(\alpha, p)$ reaction is crucial to obtain a better understanding of the production of ${ }^{44} \mathrm{Ti}$ in supernovae. Since it was concluded that the performance of the experiment in inverse kinematics would give cleaner results, a key part of the entire project is the development of a ${ }^{44} \mathrm{Ti}$ beam. Within our current technical capabilities, two different methods can be used to produce beams of radioactive species. One of them, the so-called "in-flight" technique was used in the production of ${ }^{17} \mathrm{~F},{ }^{21} \mathrm{Na}$ and $25_{\mathrm{Al}}{ }^{1}$, while the other, often referred as "two-stage acceleration" was used for longer lived isotopes such as $18 \mathrm{~F}$ and ${ }^{56} \mathrm{Ni}^{2}$. For ${ }^{44} \mathrm{Ti}$, we concluded that the second method would yield higher intensities.

Nuclei of ${ }^{44} \mathrm{Ti}$ were produced by the ${ }^{45} \mathrm{Sc}(\mathrm{p}, 2 \mathrm{n})$ reaction, using a $50-\mathrm{MeV}$ proton beam from the Argonne Intense Pulsed Neutron Source (IPNS) facility, with intensities of up to $20 \mu \mathrm{A}$. The target, a 5-mm thick slab of Sc, was mounted in a cooled $\mathrm{Cu}$ holder, the front of the which was made of graphite, acting as 
both collimator and degrader. After the irradiation, the Sc sample will be chemically treated to separate the ${ }^{44} \mathrm{Ti}$ material from Sc. This method to produce and separate ${ }^{44} \mathrm{Ti}$ was tested at Jülich and TRIUMF in the past $^{3}$. The purified $\mathrm{Ti}$ material will be then inserted into a negative-ion sputter ion source and accelerated through ATLAS. Tests with natural Ti samples were already performed. It was observed that the extraction of TiO from the ion source gives the highest yield. Combining all the efficiencies together, an intensity of about $2.5 \times 10^{644} \mathrm{Ti} / \mathrm{sec}$ on target can be estimated. The first attempt to accelerate ${ }^{44} \mathrm{Ti}$ is scheduled for April 1999.

*Northwestern University, †Intense Pulsed Neutron Source, ANL, $\ddagger$ Yale University, §Hebrew University, Jerusalem, Israel

${ }_{1}$ K. E. Rehm et al., Phys. Rev. Lett. 81, 3341 (1998).

${ }^{2}$ K. E. Rehm et al., Phys. Rev. Lett. 80, 676 (1998).

${ }^{3}$ D. Frekers et al., Phys. Rev. C 28, 1756 (1983). R. Lange et al., to be published.

\section{a.8. Study of the ${ }^{44} \mathrm{Ti}(\alpha, \mathrm{p})^{47} \mathrm{~V}$ Reaction at Energies of Astrophysical Interest}

(A. A. Sonzogni, K. E. Rehm, I. Ahmad, P. R. Crotty, C. N. Davids, J. P. Greene, B. Harss, D. J. Henderson, W. F. Henning, R. V. F. Janssens, C. L. Jiang, J. A. Nolen, R. C. Pardo, P. Reiter, J. P. Schiffer, J. Schwartz, D. Seweryniak, J. Schwartz, S. Siem, R. H. Siemssen, J. Uusitalo, I. Wiedenhöver, A. H. Wuosmaa, F. Borasi,* F. Brumwell, $\uparrow$ G. McMichael, $\uparrow$ P. Parker,$\ddagger$ M. Paul, $\S$ and R. E. Segel*)

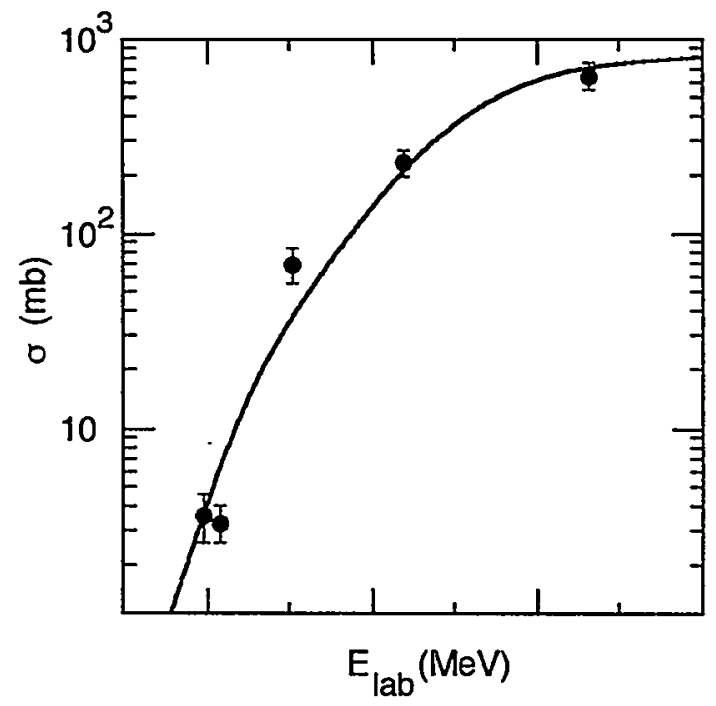

Fig. I-3. Excitation functions for the ${ }^{40} \mathrm{Ca}(\alpha, p)^{43} \mathrm{SC}$ reaction measured in this experiment (open points). The solid line is the result from a previous experiment.

The nucleus ${ }^{44} \mathrm{Ti}$ is of great importance in nuclear astrophysics ${ }^{1,2}$. The large natural abundance of ${ }^{44} \mathrm{Ca}$ was among the first indications that considerable amounts of ${ }^{44} \mathrm{Ti}$ must be produced in stellar environments. It is thought that supernovae are the site for ${ }^{44} \mathrm{Ti}$ production, which in turn makes ${ }^{44} \mathrm{Ti}$ a most valuable nucleus in gamma-ray astronomy, since its half-life $(59.2 \mathrm{y})$ is very suitable to reveal individual galactic supernova which may have taken place a few centuries ago and escaped optical observation ${ }^{3}$.
For a type II supernova, the Clemson University group has made a thorough analysis of the nuclear reactions involved in the production of ${ }^{44} \mathrm{Ti}^{4}$. They concluded that the ${ }^{44} \mathrm{Ti}(\alpha, \mathrm{p})$ reaction plays the most significant role, since the calculated final yield of ${ }^{44} \mathrm{Ti}$ is most sensitive to variations in the ${ }^{44} \mathrm{Ti}(\alpha, p)$ cross sections. Due to the importance of the ${ }^{44} \mathrm{Ti}(\alpha, \mathrm{p})$ reaction, we decided to measure its cross section at energies of astrophysical interest. The choice of the experimental technique is not trivial. Due to the radioactive nature of ${ }^{44} \mathrm{Ti}$ and the small center-of-mass energies $(4-7 \mathrm{MeV})$ at which the measurement should be performed, the reaction products will be more easily distinguished in inverse kinematics conditions, i.e. bombarding a ${ }^{4} \mathrm{He}$ target with a ${ }^{44} \mathrm{Ti}$ beam.

In order to investigate this detection technique, test runs were performed with beams of ${ }^{40} \mathrm{Ca}$ and ${ }^{46} \mathrm{Ti}$. For ${ }^{44} \mathrm{Ti}$, the $(\alpha, p)$ reaction is the dominant channel at low energies, resembling in this sense ${ }^{40} \mathrm{Ca}$ and for which a very precise measurement exists. For ${ }^{46} \mathrm{Ti}$ both $(\alpha, \mathrm{p})$ and $(\alpha, n)$ channels are expected to be open, however measurements only exist for the second one. The target consisted of a liquid-nitrogen-cooled ${ }^{4} \mathrm{He}$ gas cell with an effective thickness of $25 \mu \mathrm{g} / \mathrm{cm}^{2}$. The FMA was used to separate the nuclei of interest with respect to M/q. A combination of a PGAC (Parallel Grid Avalanche Counter), an ion chamber and a Si detector at 
the focal plane were used to identify the reaction products, which could be separated from beam particles down to the mb region. An excitation function of the ${ }^{40} \mathrm{Ca}(\alpha, p)$ reaction can be seen in Fig. I-3. The full line represents the results from a previous measurement ${ }^{5}$, a good agreement is observed between both sets. We expect to complete the measurements with the radioactive ${ }^{44} \mathrm{Ti}$ beam during this year.

*Northwestern University, $\nmid$ Intense Pulsed Neutron Source, ANL, $\ddagger$ Yale University, §Hebrew University, Jerusalem, Israel

${ }_{1}^{1}$ Supernovae and Nucleosynthesis by David Arnett, Princeton University Press (1996).

${ }^{2}$ M. D. Leising, Nucl. Phys. A621, 71c (1997); C. Dupraz et al., Astron. Astrophys. 324, 683 (1997).

${ }^{3}$ A. F. Iyudin et al., Nature 396, 142 (1998).

${ }^{4}$ L.-S. The et al., Astrophys. Journal 504, 500 (1998).

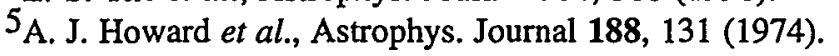

\section{a.9. The Measurement of Spectroscopic Factors in ${ }^{57} \mathrm{Cu}$ with Radioactive ${ }^{56} \mathrm{Ni}$}

Beams (C. L. Jiang, K. E. Rehm, I. Ahmad, J. Greene, B. Harss, D. Henderson, W. F. Henning, R. V. F. Janssens, R. C. Pardo, P. Reiter, J. P. Schiffer, D.

Seweryniak, A. Sonzogni, J. Uusitalo, I. Wiedenhoever, A. H. Wuosmaa, B. Zabransky, F. Brumwell,* G. McMichael,* M. Paul, $†$ R. E. Segel, $\ddagger$ J. Gorres,§ M. Wiescher,§ and D. AckermannID

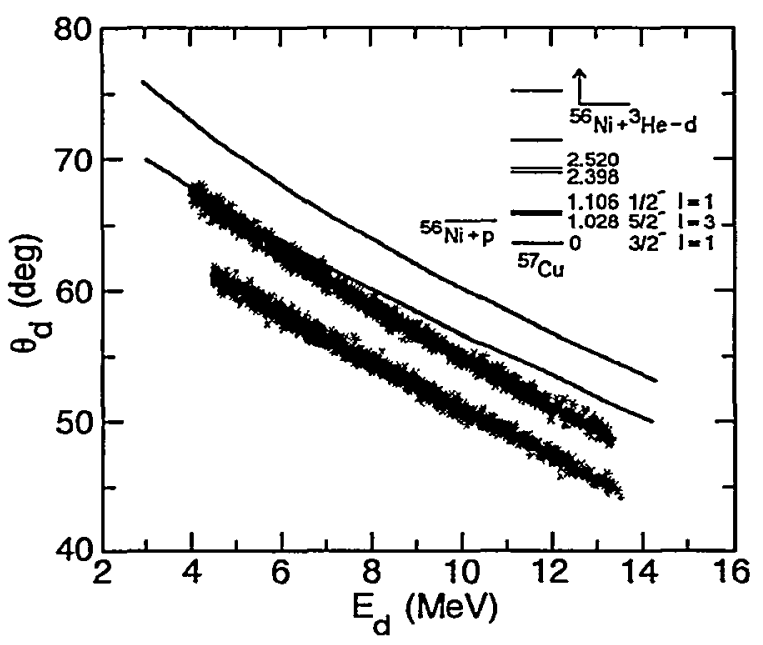

Fig. I-4 A two dimensional plot, deuteron energies versus angle for transitions to ground and first excited states of $57 \mathrm{Cu}$. Monte Carlo simulations, include contributions from the energy loss, energy and angular straggling in the gas target. Two thin lines are the kinematic curves expected without straggling effects. Insert: Level scheme of low-lying levels of ${ }^{57} \mathrm{Cu}^{4}$.

The nuclear structure of ${ }^{57} \mathrm{Cu}$ plays a key role in both shell-model study, and in the nucleosynthesis of heavy proton-rich nuclei. ${ }^{1}$ In a previous study of the reaction $\mathrm{d}\left({ }^{56} \mathrm{Ni}, \mathrm{p}\right){ }^{57} \mathrm{Ni}^{2}$, it was found that the first three states in $57 \mathrm{Ni}$, at $0.0,0.768$, and $1.113 \mathrm{MeV}$ are well characterized as $2 \mathrm{p}_{3 / 2}, 2 \mathrm{p}_{1 / 2}$, and $1 \mathrm{f}_{5 / 2}$, single-particle states. With this information the astrophysical interesting yield for the $\left.56_{\mathrm{Ni}} \mathrm{p}, \gamma\right)$ reaction to the mirror nucleus ${ }^{57} \mathrm{Cu}$ was then calculated assuming charge symmetry.

The spectroscopic factors of some low laying states in ${ }^{57} \mathrm{Cu}$ can be measured directly with the ${ }^{3} \mathrm{He}\left({ }^{56} \mathrm{Ni}, \mathrm{d}\right){ }^{57} \mathrm{Cu}$ reaction using a recently developed ${ }^{3} \mathrm{He}$ gas target. ${ }^{3}$ The technique for producing ${ }^{56} \mathrm{Ni}$ beam was the same as described in Ref. 2 . The beam energy was $250 \mathrm{MeV}$ and the beam intensity on target was $\sim 7 \times 10^{456} \mathrm{Ni} / \mathrm{sec}$ with a contaminant ${ }^{56} \mathrm{Co}$ beam of about $3 \times 10^{556} \mathrm{Co} / \mathrm{sec}$. The ${ }^{3} \mathrm{He}$ target consists of a $\sim 1.5-\mathrm{mm}$ long gas cell with $1-2 \mathrm{mg} / \mathrm{cm}^{2}$ thick titanium windows. By cooling to liquid nitrogen temperature a target thickness of about $50 \mu \mathrm{g} / \mathrm{cm}^{2}$-was achieved. The target was located at the center of the scattering chamber of the Argonne Fragment Mass Analyzer (FMA), which was used to identify the mass 57 reaction products ${ }^{57} \mathrm{Cu}$ and ${ }^{57} \mathrm{Co}$. A gold foil with thickness of about $4 \mathrm{mg} / \mathrm{cm}^{2}$ served as a passive absorber to improve the $Z$ identification and to decrease the energy of ${ }^{57} \mathrm{Cu}$ to about $150 \mathrm{MeV}$, which is within the E/q acceptance range of FMA. Since the ground state Q-value of ${ }^{3} \mathrm{He}\left({ }^{56} \mathrm{Ni}, \mathrm{d}\right){ }^{57} \mathrm{Cu}$ reaction is -4.7 $\mathrm{MeV}$, the outgoing projectile-like particle ${ }^{57} \mathrm{Cu}$ concentrated in a cone with an opening angle was of less than $2^{\circ}$ which is within the angle acceptance of FMA. The technique for separating and identifying 


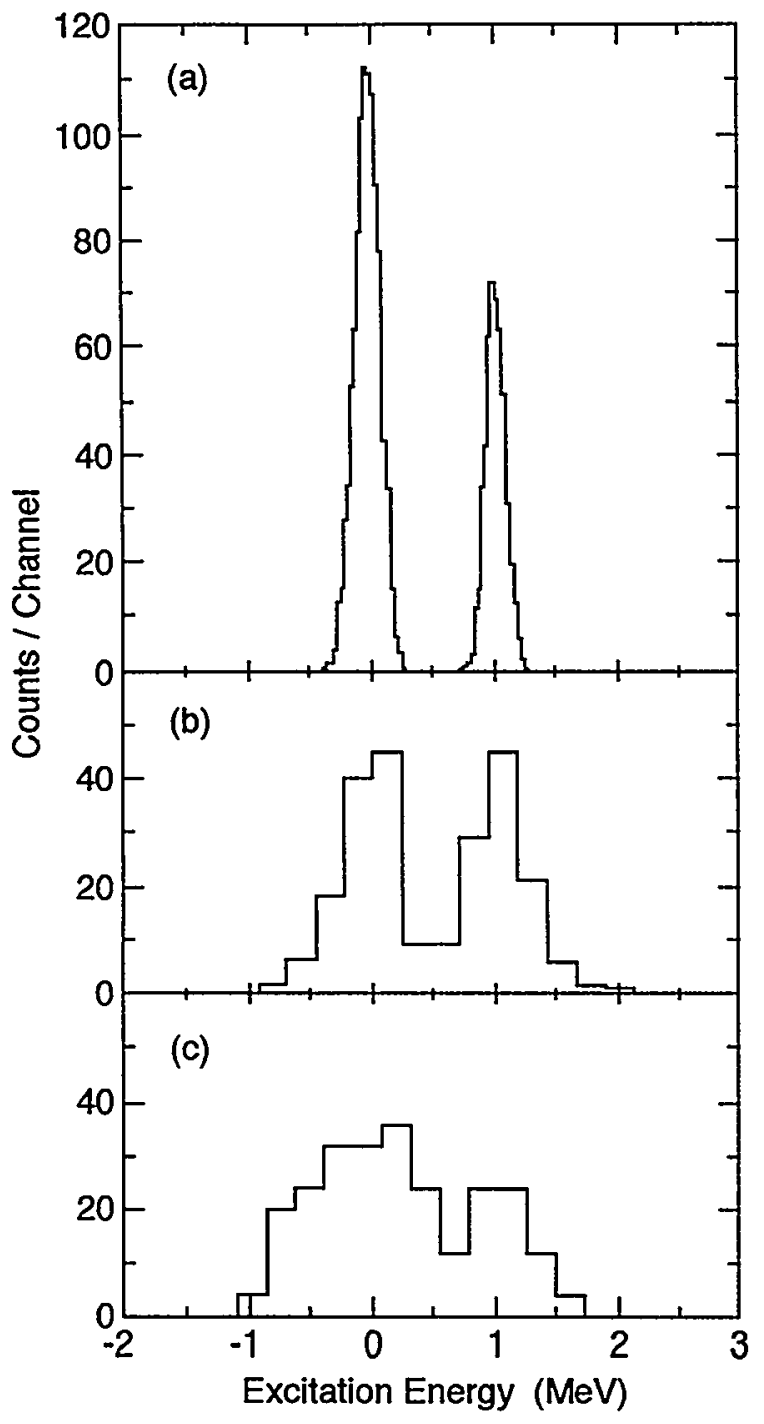

Fig. 1-5. Q-value spectra of these two deuteron groups. a. From Monte Carlo simulations in Fig. I-4. b. Monte Carlo simulations including the effect of detector geometry. c. Experimental results

${ }^{57} \mathrm{Cu}$ from scattered ${ }^{56} \mathrm{Ni},{ }^{56} \mathrm{Co}$ particles, and ${ }^{57} \mathrm{Ni}$ reaction products is very similar to the one used in the $\mathrm{d}\left({ }^{56} \mathrm{Ni}, \mathrm{p}\right){ }^{57} \mathrm{Ni}$ experiments described in Ref. 2.
The outgoing deuterons were detected with a Si-detector array, consisting of six $5 \times 5 \mathrm{~cm}^{2}$ strip detectors (strip width $1 \mathrm{~mm}$ ), covering an angular range of 45 to $76^{\circ}$, corresponding to a solid angle of about $2.9 \mathrm{sr}$. The coincidence between deuterons in the Si-detector and ${ }^{57} \mathrm{Cu}$ in the FMA selected the good events.

The excitation energies of low-lying levels of ${ }^{57} \mathrm{Cu}$ shown in the insert of Fig. I-4 were measured with the ${ }^{1} \mathrm{H}\left({ }^{58} \mathrm{Ni},{ }^{57} \mathrm{Cu}-\gamma\right) 2 \mathrm{n}$ reaction recently. ${ }^{4}$ Because of the small proton separation energy of ${ }^{57} \mathrm{Cu}(750 \mathrm{keV})$, all excited states of ${ }^{57} \mathrm{Cu}$ are unbound. Only the $5 / 2^{-}$state at $\mathrm{E}_{\mathrm{X}}=1.028 \mathrm{MeV}$ has a lifetime which is long enough that the ${ }^{57} \mathrm{Cu}$ reaches the focal plane of the FMA. Then only transitions to the ground state and to the $1.0285 / 2^{-}$state can be studied by this technique.

Shown in Fig. I-4 are Monte Carlo simulations, deuteron energies versus angle for transitions to these two states. The simulations include contributions from the energy loss, energy and angular straggling in the gas target. The two thin lines correspond to the kinematic curves expected without straggling effects. The Q-value spectrum of these two deuteron groups is shown in Fig. I-5a. Because of the finite strip size and the geometry of the detector array, each unit (every two strips were connected together as a unit) measures a certain angular range, for example for the middle unit it is $3.3^{\circ}$. Monte Carlo simulations for the Q-value spectrum including the detector geometry are shown in Fig. I-5b. The experimental results are shown in Fig. I-5c. Although other effects such as intrinsic energy resolution and effect of a finite beam spot deteriorate the Q-value resolution, transitions to the two states in ${ }^{57} \mathrm{Cu}$ can be separated.

The transport efficiency of ${ }^{57} \mathrm{Cu}$ through FMA was calculated with the program GIOS $^{5}$, taking the kinematics, small angle and energy straggling, and charge state distributions into account. Corrections due to the break up of the first excited state in ${ }^{57} \mathrm{Cu}$ were also included.

A further comparison of the result with DWBA calculations is in progress.

*Intense Pulsed Neutron Source, ANL, †Hebrew University, Jerusalem, Israel, $\ddagger$ Northwestern University, §University of Notre Dame, TLegnaro National Laboratory, Italy

${ }^{1}$ E. M. Burbidge, G. R. Burbidge, W. A. Fowler, and F. Hoyle, Rev. Mod. Phys. 29, 547 (1957); R. K. Wallace

and S. E. Woosely, Astrophys. J. Suppl. 45, 389 (1981).

2K. E. Rehm et al., Phys. Rev. Lett. 80, 676 (1998).

${ }^{3} \mathrm{~K}$. E. Rehm et al., for gas target, this annual report.

${ }^{4}$ X. G. Zhou et al., Phys. Rev. C 53, 982 (1996).

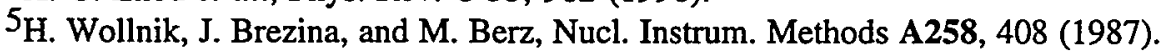




\section{a.10. Coulomb Re-Excitation of Radioactive ${ }^{78} \mathbf{R b},{ }^{78} \mathbf{R b} *$ (J. Schwartz, C. J. Lister, D. Henderson, S. Fischer, R. V. F. Janssens, P. Reiter, D. Seweryniak, S. Vincent,* A. Aprahamian, $\dagger$ and R. DeHaan $\dagger$ )}

The study of nuclei near the driplines has become a central theme of nuclear structure research. For neutronrich nuclei, beams of radioactive particles are essential to extend our knowledge, and for proton-rich nuclei they will be very useful. One of the most promising tools for spectroscopy near the driplines is Coulomb Excitation of the radioactive projectiles. This technique can extend the power and precision of electromagnetic excitation to any nuclide which is sufficiently intense and long-lived to form into a beam. In a preparatory study, to explore the challenges of radioactive beam Coulomb excitation, we used the FMA to produce a near-parallel secondary beam of radioactive particles. We performed a successful Coulomb re-excitation experiment on $\mathrm{A}=78$ isobars with beams of less than $10^{4}$ particles per second.

These data form the body of information for the Ph.D. thesis of J. Schwartz (Yale University). It is clear that information on the quadrupole moments of the ${ }^{78} \mathrm{Rb} \mathrm{J}^{\pi}$ $=0^{+}$groundstate and on the $\mathrm{J}^{\pi}=4^{+}$and $\mathrm{J}^{\pi}=4^{-}$isomers can be measured, both relative to atomic spectroscopic studies of rubidium isotopes and to ${ }^{78} \mathrm{Kr}$. It is also clear that the analysis is very sensitive to the mean $\mathrm{K}$ value of the bandhead states, a quantity which is difficult to extract from "inbeam" measurement. The intense excitation of the $J=4$ isomers can only be explained if $\mathrm{K}=3$.

A final analysis of the data is in progress. It is hoped the thesis work will be finished in the spring and a paper submitted by summer. A follow-up experiment, perhaps on neutron deficient $\mathrm{Ba}$ and $\mathrm{Xe}$ isotopes, is being considered when Gammasphere leaves ANL and high primary currents can be used to be prepare intense secondary beams.

*University of Surrey, United Kingdom, †University of Notre Dame

\section{a.11. Production Rate Calculations for an Advanced Exotic Beam Facility (C. L. Jiang, K. E. Rehm, B. Back, J. Nolen and G. Savard)}

The capabilities of an advanced exotic beam facility depend strongly on the production yields for exotic nuclei produced by the primary beams provided by such a facility. While predictions of production yields should be based on experimental data as much as possible, in many cases experimental cross sections are not available, and the yields need to be estimated using theoretical calculations. Calculations are also helpful in estimating the optimum energy of the primary beams, while taking production yields and cost effectiveness into consideration. The Argonne proposal for a next generation exotic beam facility will provide primary beams of both light and heavy ions, with energies of about $100 \mathrm{MeV} / \mathrm{u}$.

As illustrated in the Concept for an Advanced Exotic Beam Facility based on ATLAS (1995) and in the ATLAS Newsletter (March 1999), the two most important nuclear reaction mechanisms utilized in our design concept are heavy-ion fragmentation and neutroninduced fission. Other mechanisms used for specific cases are spallation, fission induced by heavy ions, fusion-evaporation and pre-equilibrium reactions. The production yields from these different reaction mechanisms were calculated using a variety of codes.

To check the accuracy of these codes, comparisons between experimental data and calculations were made. Here we discuss the results of charged-particle reactions. The yields obtained from neutron-induced fission processes will be presented in a separate contribution.

\section{1) ISAPACE, EPAX}

ISAPACE and EPAX are two well-known codes developed for fragmentation reaction calculations. 1,2 ISAPACE is a hybrid Monte Carlo-type code which incorporates a microscopic internuclear cascade model (INC), represented by the ISABEL code and a statistical model code PACE, which follows the subsequent deexcitation process. The original code was developed for a VAX machine for reactions involving heavier projectiles. In order to increase the computation speed a new version of ISAPACE developed at GSI was transferred to the alpha-cluster. ${ }^{4}$ 
The code EPAX calculates fragmentation yields using an empirical parametrization of the cross sections which were measured at high energies ${ }^{2}$. In many cases it gives good estimates of fragmentation cross sections at these energies. EPAX runs very fast and, contrary to the Monte Carlo code ISAPACE, can calculate processes with very small cross sections. Since the EPAX calculations are energy-independent, the integrated yields were calculated using the energy dependence obtained from ISAPACE for strong reaction channels. A comparison of experimental cross sections measured for the system ${ }^{18} \mathrm{O}+{ }^{27} \mathrm{Al}^{5}$ with the results of ISAPACE and EPAX calculations is shown in Fig. I-6. The agreement between theory and experiment for $\mathrm{Li}, \mathrm{Be}, \mathrm{B}, \mathrm{N}$ and $\mathrm{C}$ isotopes is generally within a factor of five.
${ }^{11} \mathrm{Li}$ is another important exotic nucleus for which experimental cross sections were measured by Kubo et al. ${ }^{6}$ At RIKEN ${ }^{11} \mathrm{Li}$ was produced by a $100-\mathrm{MeV} / \mathrm{u}$ ${ }^{18} \mathrm{O}$ beam bombarding a $1.1-\mathrm{mg} / \mathrm{cm}^{2} \mathrm{Be}$ target and secondary beams of $2.8 \times 10^{4} 11_{\mathrm{Li}} / \mathrm{sec}, 4.4 \times 10^{3}$ ${ }^{14} \mathrm{Be} / \mathrm{sec}$ and $1.7 \times 10^{5} 8 \mathrm{He} / \mathrm{sec}$, were obtained at the exit of the mass separator RIPS. The total production yields for these beams calculated with a transport efficiency of RIPS of $6 \%^{7}$ are summarized in Table I-II. Also included are the production yields calculated with ISAPACE and EPAX. The agreement between the data and the ISAPACE calculation is quite remarkable. The yields calculated by the EPAX code are usually a factor of 10 smaller than the experimental values.

Table I-II. Total production yields for ${ }^{11}{ }_{L i},{ }^{14}$ Be and ${ }^{8} \mathrm{He}$ from experiments and from calculations.

$\begin{array}{lll}\text { Nucleus } & \begin{array}{l}\text { Intensity } \\ \text { observed }\end{array} & \begin{array}{l}\text { Intensity } \\ \text { after target* }\end{array} \\ { }^{11_{\mathrm{Li}}} & 2.8 \times 10^{4} / \mathrm{sec} & 4.7 \times 10^{5} / \mathrm{sec} \\ { }^{14} \mathrm{Be} & 4.4 \times 10^{3} / \mathrm{sec} & 7.3 \times 10^{4} / \mathrm{sec} \\ { }^{8} \mathrm{He} & 1.7 \times 10^{5} / \mathrm{sec} & 2.8 \times 10^{6} / \mathrm{sec}\end{array}$

*Using a transport efficiency of $6 \%{ }^{7}$

ISAPACE

calculations

$3.0 \times 10^{5} / \mathrm{sec}$

$6.0 \times 10^{4} / \mathrm{sec}$

$4.0 \times 10^{6} / \mathrm{sec}$
EPAX

calculations

$7.1 \times 10^{4} / \mathrm{sec}$

$7.1 \times 10^{3} / \mathrm{sec}$

$1.0 \times 10^{5} / \mathrm{sec}$
Fission processes are also included in the ISAPACE code. A comparison of the ISAPACE predictions for neutron-rich $\mathrm{Rb}$ isotopes produced by $750-\mathrm{MeV} / \mathrm{u}$ uranium beams bombarding a ${ }^{9} \mathrm{Be}$ target with measurements in the same system at $\mathrm{GSI}^{8}$ is given in Fig. I-7. The agreement again is typically better than a factor of five. The short computation times for EPAX allow for large scale searches for the optimum projectile-target combination for the production of exotic nuclei via the fragmentation process. The production yields (atoms $/ \mathrm{p} \mu \mathrm{C}$ ) for various exotic nuclei are shown in a plot in the Z-N plane in Fig. I-8. While the accuracy of these yields is estimated to be within an order of magnitude it is interesting to observe that many exotic nuclei can be produced with the fragmentation method which are difficult to reach by other reactions.

\section{2) LAHET}

LAHET is a well-known Monte Carlo Code, developed first at Oak Ridge and later at Los Alamos. ${ }^{9}$ It can incorporate complex target geometries and has many powerful features like following the transport of neutrons and protons through the actual target. LAHET restricts the choice on the incident particle to be either hydrogen or $\mathrm{He}$. We have used this code to calculate cross sections and yields for the incident particles $\mathrm{p}, \mathrm{d}$, ${ }^{3} \mathrm{He}$, and ${ }^{4} \mathrm{He}$.

Some LAHET calculations for producing $\mathrm{Cs}$ and $\mathrm{Fr}$ isotopes are shown in Fig. I-9. Also shown are experimental data from CERN 10,11 and the predictions from the ISAPACE and EPAX calculations. The agreement between the data and the predictions for these cases is again within a factor of five.

\section{3) LPACE and ALICE}

LPACE $^{12}$ and ALICE ${ }^{13}$ are two commonly used codes for calculating low-energy fusion-evaporation reactions. Because the range of low-energy projectiles in the target material is quite short and fusion reactions often peak at low energies it is difficult to obtain high yields from this reaction mode. However, for some select cases (e.g. ${ }^{56} \mathrm{Ni},{ }^{100} \mathrm{Sn}, 14$ or nuclei which can be reached via $(\mathrm{p}, \mathrm{xn})$ or $(\mathrm{d}, \mathrm{xn})$ processes), fusion-evaporation reactions are a quite competitive production technique. ${ }^{14}$ 


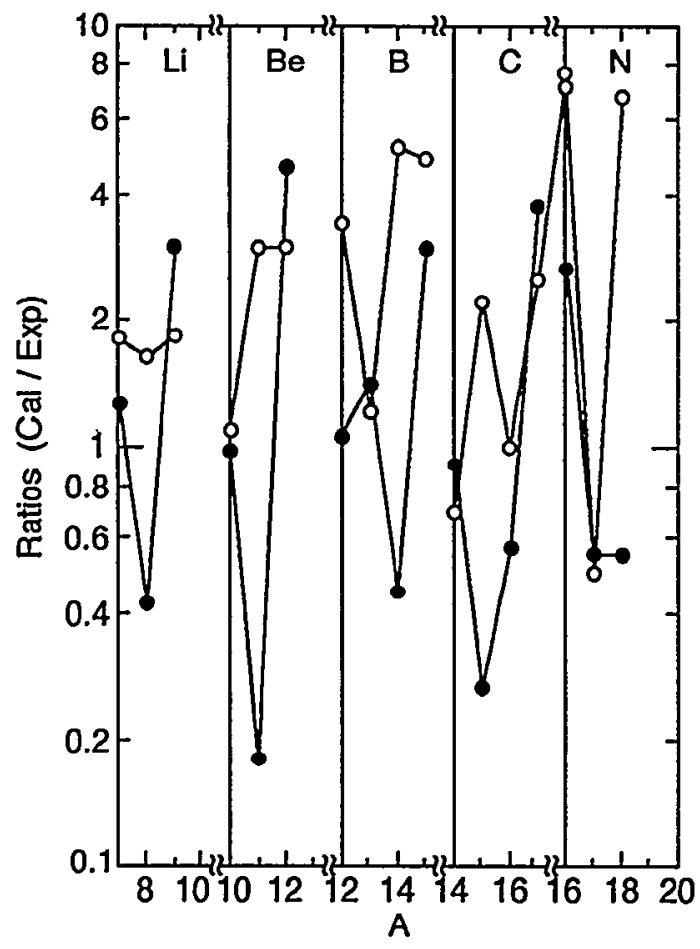

Fig. I-6. A comparison of experimental cross sections measured for the system $18 O+27_{A l^{5}}$ with the results of ISAPACE (filled circles) calculations.

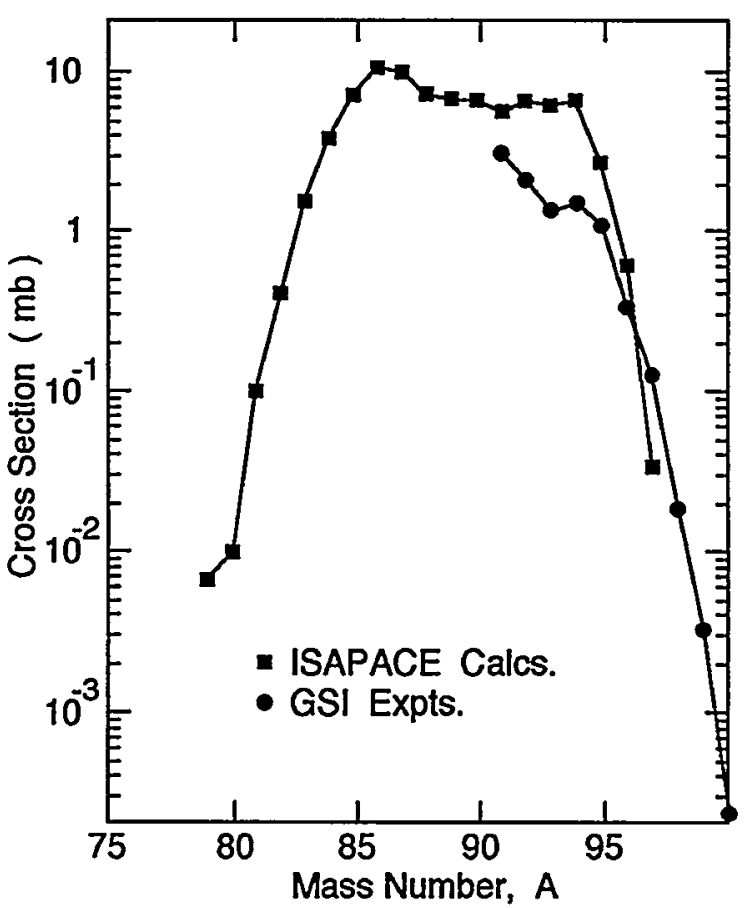

Fig. I-7. A comparison of the ISAPACE predictions for neutron-rich Rb isotopes produced by $750 \mathrm{MeV} / \mathrm{u}$ uranium beams bombarding a ${ }^{9}$ Be target with measurements at GSI. ${ }^{8}$ 


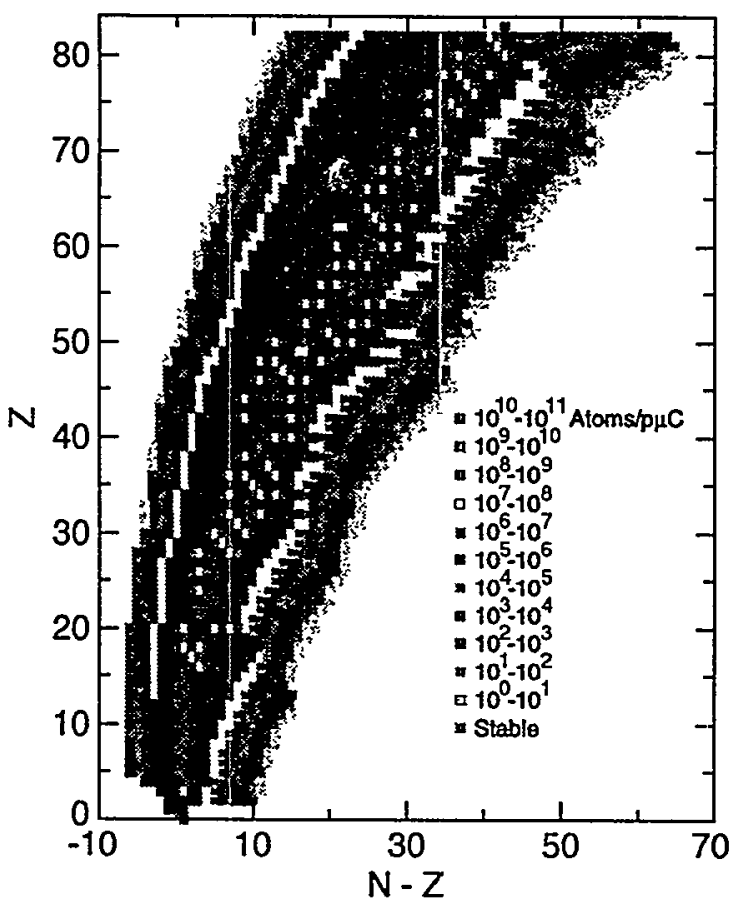

Fig. I-8. The production yields for various exotic nuclei which can be obtained with the optimum projectile-target combination via the fragmentation reaction.

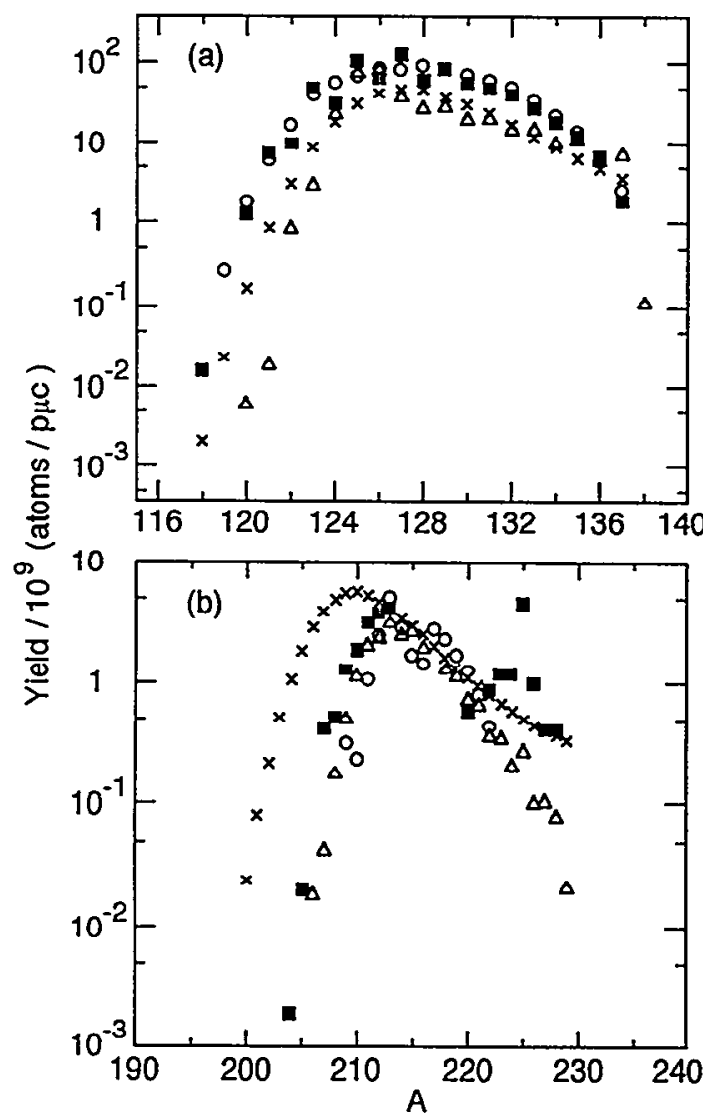

Fig. I-9. The production yields for $\mathrm{Cs}(a)$ and $\mathrm{Fr}(b)$ isotopes by $600-\mathrm{MeV}$ proton beams bombarding La and ${ }^{232}$ Th targets respectively, experiments (squares), LAHET calculations (triangles), ISAPACE calculations (circles) and EPAX calculations (crosses). 


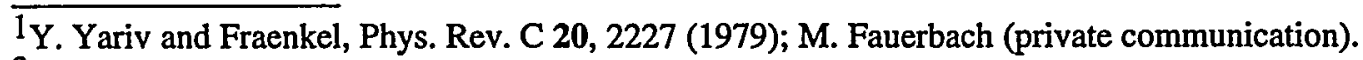

${ }^{2}$ K. Summerer, W. Bruchle, D. J. Morrissey, M. Schadel, B. Szweryn, and Y. Weifan, Phys. Rev. C 42, 2546 (1990).

${ }^{3}$ For example, B. Blank et al., Phys. Rev. C 50, 2398 (1994).

${ }^{4}$ Private communication, M. Fauerbach, 1998.

$5_{G}$. A. Souliots et al., Phys. Rev. C 49, 3301 (1994).

${ }^{6} \mathrm{~T}$. Kubo et al., Proceedings of the Second International Conference on Radioactive Nuclear Beams, Louvain-laNeuve, Belgium, 1991, pp 65.

7 T. Kubo et al., Proceedings of the First International Conference on Radioactive Nuclear Beams (World Scientific), 1989 , pp 563.

${ }^{8}$ A dissertation at GSI.

${ }^{9}$ R. G. Alsmiller et al., Nucl. Instrum. Methods A278, 713 (1989); R. E. Price and H. Lichtenstein, Los Alamos National Laboratory Report, LA-UR-89-3104 (1989).

${ }^{10}$ T. Bjornstad et al., Z. Phys. A - Atoms and Nuclei 303, 227 (1981), and Nucl. Instrum. Methods 186, 391 (1981).

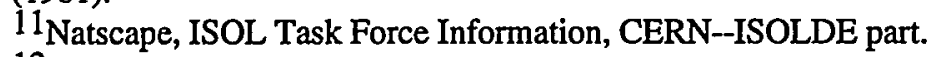

12 A. Gavron, Phys. Rev. C 21, 230 (1980).

13 E. J. Friedland et al., Z. Phys. A328, 333 (1987); Z. Phys. A328, 445 (1987).

${ }^{14}$ W. Mitag et al., Nucl. Phys. A616, 329 (1997); M. Lewitowicz, et al., Phys. Lett. B332, 20 (1994). 


\section{B. STRUCTURE OF NUCLEI AT THE LIMITS OF STABILITY}

The study of the properties of nuclei at the very limits of stability is currently a subject of great interest. Gammasphere was installed at the target position of the FMA late in 1997. A vigorous research program taking advantage of the unique capabilities brought about by the coupling of the two devices developed since then, accounting for a larger fraction of the available beam time. In particular, the technique of recoil decay tagging was used with success to investigate nuclei near or at the proton drip line. Spectroscopy information is now available on states produced at the submicrobarn level.

This section presents mostly the results of studies where Gammasphere was used in conjunction with the FMA, although measurements performed with Gammasphere alone such as Coulomb excitation of radioactive, actinide targets and studies of neutron-rich nuclei via prompt spectroscopy following fission are included as well. Finally, this section ends with contributions where the FMA was used alone to either search for new proton or alpha emitters or to perform decay studies at the focal plane of the instrument.

b.1. High Spin States in ${ }^{24} \mathrm{Mg}$ (C. J. Lister, A. Wuosmaa, R. R. Betts, M. P. Carpenter, C. N. Davids, D. Henderson, R. V. F. Janssens, T. Lauritsen, P. Reiter, D. Seweryniak, J. Schwartz, I. Wiedenhöver, and W. Catford*)

Light nuclei in the p- and sd- shells still pose some of the most interesting and direct challenges to our understanding of nuclear structure physics, as both the experimental data and our theoretical interpretations are most complete for these nuclei. Comparison of the data to the nuclear models, and comparisons of the models themselves, lead to deeper understanding of nuclear structure in general. One of the most studied nuclei in this region is ${ }^{24} \mathrm{Mg}$, where there are extensive data and theoretical calculations of all types. ${ }^{1}$ This nucleus is, for example, ideal for investigating the microscopic origin of nuclear collective rotation, through comparing the predictions of deformed and spherical shell models. ${ }^{2}$ At the higher spins, clustering is known to play a key role in the structure, ${ }^{3}$, and states of extremely high deformation predicted both in ${ }^{24} \mathrm{Mg}$, and neighboring
${ }^{32} \mathrm{~S}$, but the relationships between the deformed states and low-lying excitations are not clear.

We performed a high-statistics gamma-ray study of ${ }^{24} \mathrm{Mg}$ using the ${ }^{12} \mathrm{C}\left({ }^{16} \mathrm{O}, \alpha\right){ }^{24} \mathrm{Mg}$ reaction at 52 and $62 \mathrm{MeV}$ and Gammasphere triggered by the Fragment Mass Analyzer (FMA). The FMA was used as a timeof-flight spectrometer to select the excitation energy in ${ }^{24} \mathrm{Mg}$. The beam was bunched with a FWHM of $<300$ ps, which combined with the $8.8 \mathrm{~m}$ FMA flight path, should give state-selection at the 100 's $\mathrm{keV}$ level. In practice, to increase yield, a relatively thick (120 $\mu \mathrm{g} / \mathrm{cm}^{2}$ ) target was used, degrading the excitation energy resolution to about $2 \mathrm{MeV}$. Data analysis is in progress.

*University of Surrey, United Kingdom

${ }^{1}$ Table of Isotopes, Vol. 1, Ed. V. S. Shirley (Wiley 1996); P. M. Endt and C. Van der Leun, Nucl. Phys. A310, 1 (1978).

${ }_{2}^{2}$. K. Sheline, I. Ragnarsson, and S. Aberg, J. Phys. G 14, 1201 (1988).

${ }^{3}$ See, for example, A. H. Wuosmaa et al., Ann. Rev. Nucl. Sci. 45, 89 (1995). 


\section{b.2. Deformed Negative-Parity Excitations in ${ }^{\mathbf{7 1}} \mathrm{As}$ (M. P. Carpenter, C. N. Davids, R. V. F. Janssens, C. J. Lister, D. Seweryniak, T. L. Khoo, T. Lauritsen, D. Nisius, P. Reiter, J. Uusitalo, I. Wiedenhoever, N. Fotiades,* J. A. Cizewski,* A. O. Macchiavelli, $\dagger$ and R. W. MacLeod $\ddagger$ )}

The nuclei in the $A=70$ mass region exhibit a complicated interplay between single-particle and collective degrees of freedom, reflecting the influence of competing shell gaps in the single-particle levels. As a result, gamma sequences built on configurations corresponding to different shapes are observed in the same nucleus e.g. ${ }^{72} \mathrm{Se}^{1}$ The same interplay is expected to be present in the isotone ${ }^{71} \mathrm{As}$ as is suggested by theoretical calculations of the total Routhian surface (TRS). ${ }^{2}$ These calculations for the negative-parity states predict shallow minima at prolate and non-collective oblate deformations at low rotational frequencies which become more deformed as a function of frequency and triaxial above $\hbar \omega=0.5 \mathrm{MeV}$.

High-spin states in ${ }^{71}$ As were investigated using the $16 \mathrm{O}+58 \mathrm{Ni}$ reaction at a beam energy of $59.5 \mathrm{MeV}$. The 160 beam was supplied by the ATLAS accelerator at Argonne National Laboratory. Gamma rays at the target position were detected by the Gammasphere array which was coupled to the Fragment Mass Analyzer
(FMA) in order to separate evaporation residues from other reaction products. Mass 71 and 72 were the strongest evaporation residue channels observed in this reaction. In addition to ${ }^{71} \mathrm{As}(3 p),{ }^{(68)} \mathrm{Ge}(2 p \alpha),{ }^{71} \mathrm{Se}$ $(2 p n),{ }^{72} \mathrm{Se}(2 p)$ and ${ }^{72} \mathrm{Br}(p n)$ have also been identified in this data set.

The previous reported level structure ${ }^{3}$ for ${ }^{71}$ As was confirmed and extended in this experiment. In addition, a new sequence of negative-parity levels were observed at moderate excitation. This sequence consists of two rotational bands connected to each other by dipole transitions which compete favorably with the quadrupole cross-over transitions. Based on the extracted $B(\mathrm{M} 1) / B(\mathrm{E} 2)$ ratios, the band was given a $7 / 2^{-}$ [303] (f/2) assignment with $\varepsilon_{2} \sim 0.37$. This is the first observation of a deformed proton $f_{7 / 2}$ configuration in the $\mathrm{A}=70$ mass region.

A paper reporting the results from this experiment was recently published in Phys. Rev. C.4-

*Rutgers University, †Lawrence Berkeley National Laboratory, $¥$ Thomas Jefferson National Accelerator Laboratory

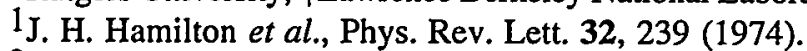

${ }^{2}$ W. Nazarewicz et al., Nucl. Phys. A435, 397 (1985).

${ }^{3}$ R. S. Zighelboim et al., Phys. Rev. C 50, 716 (1994).

${ }^{4}$ N. Fotiades et al., Phys. Rev. C 59, 2919 (1999).

\section{b.3. The Structure of the Mirror Pair ${ }^{79} \mathrm{Y}$ and ${ }^{79} \mathrm{Zr}$ (C. J. Lister, M. P. Carpenter, R. V. F. Janssens, T. Lauritsen, D. Seweryniak, D. Henderson, M. Leddy, S. J. Freeman,* B. J. Varley,* D. L. Durrell,* L. Bernstein, $\dagger$ and K. Hauschild $\dagger$ )}

The study of mirror pairs of nuclei, with conjugated neutron and proton number has long been a precision tool for studying the symmetries of nuclear forces and effective interactions. The charge-symmetry of the forces is established, so now the small observed spectral differences in the energy levels of the mirror pairs is interpreted as arising form small changes in Coulomb shifts of the respective wavefunctions. Recently, precise measurements of mirror pairs in the fp-shell nuclei ${ }^{1}$ were made and used as a delicate tool for appraising energy shifts at the $50-\mathrm{keV}$ level. This is an unusually sensitive probe of nuclear wavefunctions.
This "mirror-pair" technique gains new relevance at the proton dripline, where the symmetry of the pair is broken as the $T=-1 / 2$ partner becomes unbound. Using Gammasphere and the FMA, several projects were initiated to approach this limit, in $\mathrm{A}=51,2,65,3$ and $79^{4}$ systems. The heaviest pair, at $\mathrm{A}=79$ is experimentally challenging, but the nuclear structure is very stable, so should be relatively easy to interpret. The "easier" member of the pair, ${ }^{79} \mathrm{Y}$ with $\mathrm{T}=+1 / 2$ has a production cross section of about $2 \mathrm{mb}$ in the reaction ${ }^{58} \mathrm{Ni}\left({ }^{24} \mathrm{Mg}, \mathrm{p} 2 \mathrm{n}\right){ }^{79} \mathrm{Y}$. Using a reaction with inverse kinematics, quite good Z-resolution $(\mathrm{dZZ}$ $1 / 40$ ) can be, and was, achieved. At the onset of this project nothing was published on this nucleus, though 
two recent papers have appeared 5,6 reporting states in the groundstate band. A large data set was acquired, far superior to previous experiments. The groundstate band has been extended and a candidate negative parity band found. The ${ }^{79} \mathrm{Y}$ gated $\gamma-\gamma$ matrix had many thousands of counts in each of the photopeaks in the gsb, so the data set is probably big enough to allow a realistic search for members of the ${ }^{79} \mathrm{Zr}$ groundstate band (which has a predicted production cross-section of 5-10 microbarns). A carefully optimized sort of the data to seek states in ${ }^{79} \mathrm{Zr}$ is now in progress.

*University of Manchester, United Kingdom, †Lawrence Livermore National Laboratory

${ }^{1}$ C. D. O'Leary et al., Phys. Rev. Lett. 79, 4349 (1997).

2 ATLAS proposal \#684

3 ATLAS proposal $\# 730$

${ }^{4}$ ATLAS proposal \#739

$5_{\mathrm{M}}$. Leddy et al., Phys. Rev. C 58, 1438 (1998).

${ }^{6}$ S. D. Paul et al., Phys. Rev. C 58, R3038 (1998).

b.4. In-Beam $\gamma$-Ray Spectroscopy of the Proton Emitter ${ }^{109} \mathrm{I}$ (M. P. Carpenter, C. N. Davids, R. V. F. Janssens, C. J. Lister, D. Seweryniak, J. Uusitalo, C. H.Yu,* A. Galindo-Uribarri,* S. D. Paul, ${ }^{*}$, and B. D. McDonald $\ddagger$ )

The recoil decay tagging (RDT) technique proved to be a powerful tool in the study of proton-rich nuclei. With the placement of Gammasphere in front of the FMA, this technique was successfully used to probe excited states in nuclei which lie at the edges of stability. In the last year a number of RDT measurements were performed to study excited states in proton emitters, including ${ }^{109} \mathrm{I}$ which was previously studied at Daresbury using the EUROGAM I array and the Daresbury Recoil Mass Spectrometer.

In a recent experiment at Gammasphere, excited states in ${ }^{109} \mathrm{I}$ were populated using the ${ }^{54} \mathrm{Fe}\left({ }^{58} \mathrm{Ni}, p 2 n\right)$ reaction at a beam energy of $220 \mathrm{MeV}$. Gamma rays in ${ }^{109}$ I were identified using the RDT technique. The analysis of the $\gamma-\gamma$ data yielded the yrast sequence in 109 I which was interpreted as an excitation built on the $\mathrm{h}_{11 / 2}$ proton orbital. Interestingly, the sequence of gamma-rays assigned to this band in ${ }^{109}$ I is different than that reported in Ref. 1. Since the Gammasphere assignments are based on a significantly larger number of gamma-proton correlated events, we believe that the sequence reported in Ref. 1 is incorrect.

Previous systematic analyses of proton emitters showed that most known proton emitters have spectroscopic factors close to unity. ${ }^{109}$ I stands out as one of the few which have very small spectroscopic factors. The proton emitting ground state of ${ }^{109} \mathrm{I}$ was assigned to the $d_{5 / 2}$ configuration, however, its spectroscopic factor is very small $(S=0.055)$, and this fact was cited as evidence that the ground state has substantial prolate deformation. Unfortunately, the current experiment was unable to identify states associated with the ground state configuration, and thus, no information on the deformation of the ground-state could be extracted. The trend of the $h_{11 / 2}$ sequence indicates a decrease in deformation with decreasing $\mathrm{N}$ which is supported by theoretical predictions.

A paper reporting the results of this work was recently published in Physical Review C. ${ }^{2}$

*Oak Ridge National Laboratory, †Oak Ridge Institute for Science and Education, $\ddagger$ Georgia Institute of Technology

${ }_{1}^{1}$ E. S. Paul, P. J. Woods, et al., Phys. Rev. C 51, 78 (1995).

${ }^{2}$ C. H.-Yu et al., Phys. Rev. C 59, R1834 (1999). 


\section{b.5. In-Beam $\gamma$-Ray Spectroscopy in the Ground-State Proton Emitter ${ }^{113} \mathbf{C s}$} (M. P. Carpenter, C. N. Davids, R. V. F. Janssens, C. J. Lister, D. Seweryniak, J. Uusitalo, C. J. Gross, $* \dagger$ Y. A. Akovali, $\uparrow$ C. Baktash, $\uparrow$ J. C. Batchelder,* C. R. Bingham, $\ddagger$ T. Davinson, $\S$ D. Ellis, $\ddagger$ A. Galindo-Uribarri, $\uparrow$ T. N. Ginter, $\mathbb{R}$ R. Grzywacz, $\div$ J. W. Johnson,* J. F. Liang, $\dagger$ J. Mas, $\|$ B. D. MacDonald,** S. D. Paul, $\dagger$ A. Piechaczek, $\uparrow \uparrow$ D. C. Radford, $\uparrow$ W. Reviol, $\ddagger$ K. Rykaczewski, $\uparrow$ W. Satula, $\|$ D. Shapira, $\dagger$ K. S. Toth, $\uparrow$ W. Weintraub, $\ddagger$ P. J. Woods, $\S$ C.-H. Yu, $\uparrow$ and E. F. Zganjar $\dagger \dagger)$

The lifetimes of the majority of proton emitters observed between $\mathrm{Z}=51$ and $\mathrm{Z}=85$ can be reproduced using simple approaches such as the WKB method (see for example Ref. 1). There are, however, a few proton emitters such as ${ }^{113} \mathrm{Cs}$ which have anomalous halflives that cannot be described using the spherical WKB approach. In the case of ${ }^{113} \mathrm{Cs}$, it was suggested that the failure of the WKB approach results from the fact that this nucleus is deformed in its ground state. Using the approach of Bugrov and Kadmenskii ${ }^{2}$ which includes configuration mixing associated with deformation, the half-life of the ${ }^{113} \mathrm{Cs}$ proton decay is best reproduced with the $3 / 2^{+}$[422] assignment at a deformation of $\beta_{2}=0.2$. An independent confirmation of the deformation of the ground state can be obtained by measuring the sequence of excited states lying above it and comparing the energy spacings to well established criteria for establishing collectivity.

Two experiments were performed to identify excited states in ${ }^{113} \mathrm{Cs}$ using the ${ }^{58} \mathrm{Ni}\left({ }^{58} \mathrm{Ni}, \mathrm{p} 2 \mathrm{n}\right)$ reaction at a beam energy of $230 \mathrm{MeV}$. Both experiments utilized the Recoil Tagging Technique (RDT) in order to correlate $\gamma$ rays with the proton decay of ${ }^{113} \mathrm{Cs}$. The first experiment was performed at the Holifield Radioactive Beam Facility where $\gamma$ rays at the target were detected with 6 Clover and 5 Duet Ge detectors in coincidence with recoils at the focal plane of the Recoil
Mass Spectrometer. The second experiment was performed at the ATLAS facility where recoil $-\gamma-\gamma$ coincidences were detected using Gammasphere coupled to the Fragment Mass Analyzer (FMA). In both experiments, correlations between $\gamma$ rays and proton decays were obtained by placing a doubled-sided silicon strip detector (DSSD) behind the focal plane detectors and allowing the residues to be implanted into the DSSD.

In the ORNL experiment, the $\gamma$-ray spectrum correlated with the proton decay of ${ }^{113} \mathrm{Cs}$ identified $12 \gamma$-ray transitions in this nucleus. In the Argonne experiment, the same transitions were observed, however, the increased efficiency of Gammasphere allowed for a gamma-ray coincidence analysis to be performed. This analysis revealed a cascade relationship between the 384 596-737-814 keV transitions. Based on the energy level systematics for the $h_{11 / 2}$ bands in the cesium isotopes, this cascade was tentatively assigned to the $h_{11 / 2}$ configuration. Unfortunately, the data is not of sufficient quality to allow for the delineation of the decay of this band to the proton-emitting ground state. As a result, no direct information is available concerning the deformation of the ground state. However, analysis is still ongoing. A paper reporting on the results of this work can be found in Ref. 3 .

*Oak Ridge Institute for Science and Education, †Oak Ridge National Laboratory, łUniversity of Tennessee, §University of Edinburgh, United Kingdom, IVanderbilt University, ||Joint Institute for Heavy Ion Research, **Georgia Institute of Technology, $\dagger \dagger$ Louisiana State University

${ }^{1}$ P. J. Woods and C. N. Davids, Ann. Rev. Nucl. Part. Sci. 47, 505 (1997).

2V. P. Bugrov and S. G. Kadmenskii, Sov. J. Phys. 49, 967 (1989).

${ }^{3}$ C. J. Gross et al., ENAM98: Exotic Nuclei and Atomic Masses, ed. by B. M Sherrill, D. J. Morrissey and C. N. Davids, (1998) pg. 444-449. 


\section{b.6. Level Structure of the Three-Valence-Particle Nucleus ${ }^{135} \mathrm{Sb}$ (I. Ahmad, T. Lauritsen, L. R. Morss,* C. P. Bhattacharyya, $\uparrow$ P. J. Daly, $\dagger$ B. Fornal,$\dagger$ Z. W. Grabowski, $\uparrow$ C. T. Zhang, $\dagger$ W. R. Phillips, $\ddagger$ J. L. Durell $\ddagger$ M. J. Leddy, $\ddagger$

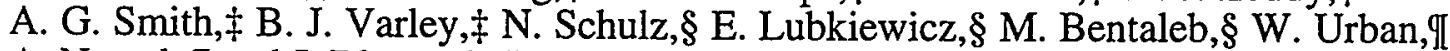 A. Nowak, Il and J. Blomqvistll)}

Spectroscopic studies of few-valence-particle nuclei around the doubly-magic ${ }^{132} \mathrm{Sn}$ provide information on nucleon-nucleon interactions and effective charges in an important section of the nuclear chart. Data from the experiment at EUROGAM2 with a ${ }^{248} \mathrm{Cm}$ fission source were analyzed and the structures of several nuclei in this mass region including ${ }^{132} \mathrm{Sn},{ }^{134} \mathrm{Sn},{ }^{134} \mathrm{Te}$, ${ }^{135} \mathrm{I}$, and ${ }^{134} \mathrm{Sb}$ were determined. These results have already been published.

Last year we were able to deduce the level structure of ${ }^{135} \mathrm{Sb}$ which was not known before. The gamma-ray transitions deexciting levels in ${ }^{135} \mathrm{Sb}$ were identified by gating on gamma peaks in the complementary $R h$ fragments. By combining results from spectra obtained by various gating conditions we were able to deduce the level structure shown in Fig. I-10. The ground state of ${ }^{135} \mathrm{Sb}$ is known from its $\beta$ decay to be $7 / 2^{+}$. The excited states of ${ }^{134} \mathrm{Sn}$ were previously observed and were interpreted as $2^{+}, 4^{+}$, and $6^{+}$states of $v f_{7 / 2}^{2}$ character. The low-lying states in ${ }^{135}$ Sb may be considered as a $\pi g_{7 / 2} v f_{7 / 2}^{2}$ configuration and hence the 707-, 1118- and 1343-keV levels are given as $11 / 2^{+}$, $15 / 2^{+}$and $19 / 2^{+}$assignments. The $1972-\mathrm{keV}$ level is given a tentative assignment of $\left(\pi g_{7 / 2} v f_{7 / 2} h_{9 / 2}\right) 23 / 2^{+}$. The present interpretation relies on the results of shell model calculations performed with the OXBASH code. The results of the calculations are included in Fig. I-10. As can be seen, the calculations reproduce the experimental data fairly well and thus provide support for the present assignments. The results of this study were published. ${ }^{1}$

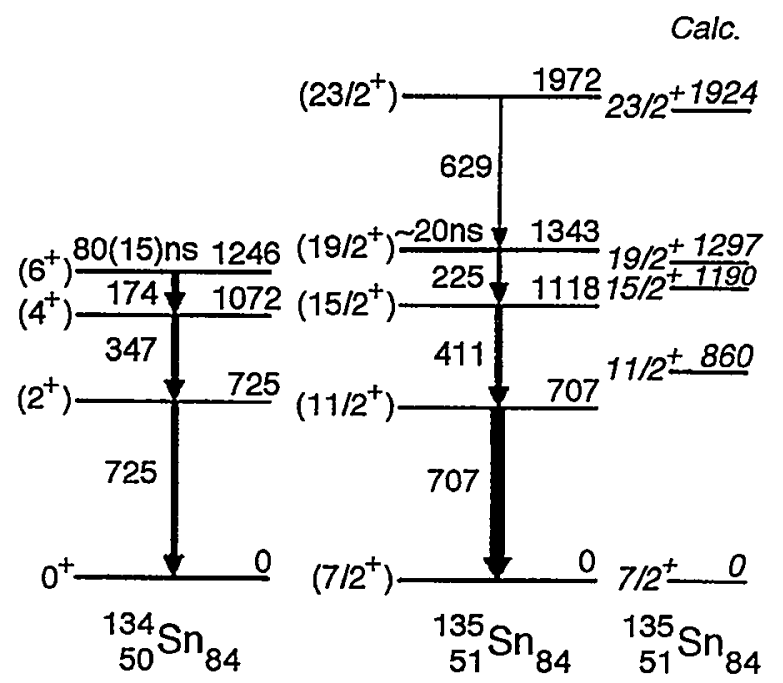

Fig. 1-10. The proposed level diagram of ${ }^{135} \mathrm{Sb}$. The experimental level scheme of ${ }^{134} \mathrm{Sn}$ and results of a shell model calculations for ${ }^{135} \mathrm{Sb}$ are also included.

*Chemistry Division, ANL, †Purdue University, $\ddagger$ University of Manchester, United Kingdom, §IRS, Strasbourg, France, IW arsaw University, Poland, URoyal Institute of Technology, Stockholm, Sweden

${ }^{1}$ Eur. Phys. J. A3, 109 (1998). 


\section{b.7. Yrast Spectroscopy of $\mathrm{N}=82,83$ Isotopes ${ }^{136} \mathrm{Xe}$ and ${ }^{137} \mathrm{Xe}$ from ${ }^{248} \mathrm{Cm}$}

Fission Studies (I. Ahmad, T. Lauritsen, L. R. Morss, * P. J. Daly, $\dagger$

P. Bhattacharyya, $\uparrow$ R. Broda, $\uparrow$ C. T. Zhang, $\uparrow$ B. Fornal, $\uparrow Z$ Z. W. Grabowski, $\uparrow$

W. R. Phillips,

E. Lubkiewicz,§ M. Bentaleb,§ W. Urban, II and J. Blomqvistll)

As discussed in the previous contribution, spectroscopy of nuclei around the doubly-magic ${ }^{132} \mathrm{Sn}$ provides nucleon-nucleon interactions and effective charges. We have studied the level structures of the four-valence proton $\mathrm{N}=82$ nucleus ${ }^{136} \mathrm{Xe}$ and its neighbor ${ }^{137} \mathrm{Xe}$ from the analysis of the data obtained in the measurement with the Eurogam2 array and a ${ }^{248} \mathrm{Cm}$ fission source.

The level structures of ${ }^{136} \mathrm{Xe}$ and ${ }^{137} \mathrm{Xe}$ are shown in Fig. I-11. In ${ }^{136} \mathrm{Xe}$, levels with spin 6 and excitation energy $2262 \mathrm{keV}$ were known from previous studies. Structure on top of the $2262-\mathrm{keV}$ level was deduced from the present work by usual coincidence gating techniques. In the case of ${ }^{137} \mathrm{Xe}, 314-, 400$ - and 1221 $\mathrm{keV}$ gamma rays were known from previous work; the additional levels are identified in this study. The multinucleon configuration assignments are based on a comparison of the data with the results of shell model calculations. The results of this investigation have been submitted for publication in Phys. Rev. C.

*Chemistry Division, ANL, †Purdue University, $¥$ University of Manchester, United Kingdom, §IRS Strasbourg, France, IWWarsaw University, Poland, IIRoyal Institute of Technology, Stockholm, Sweden

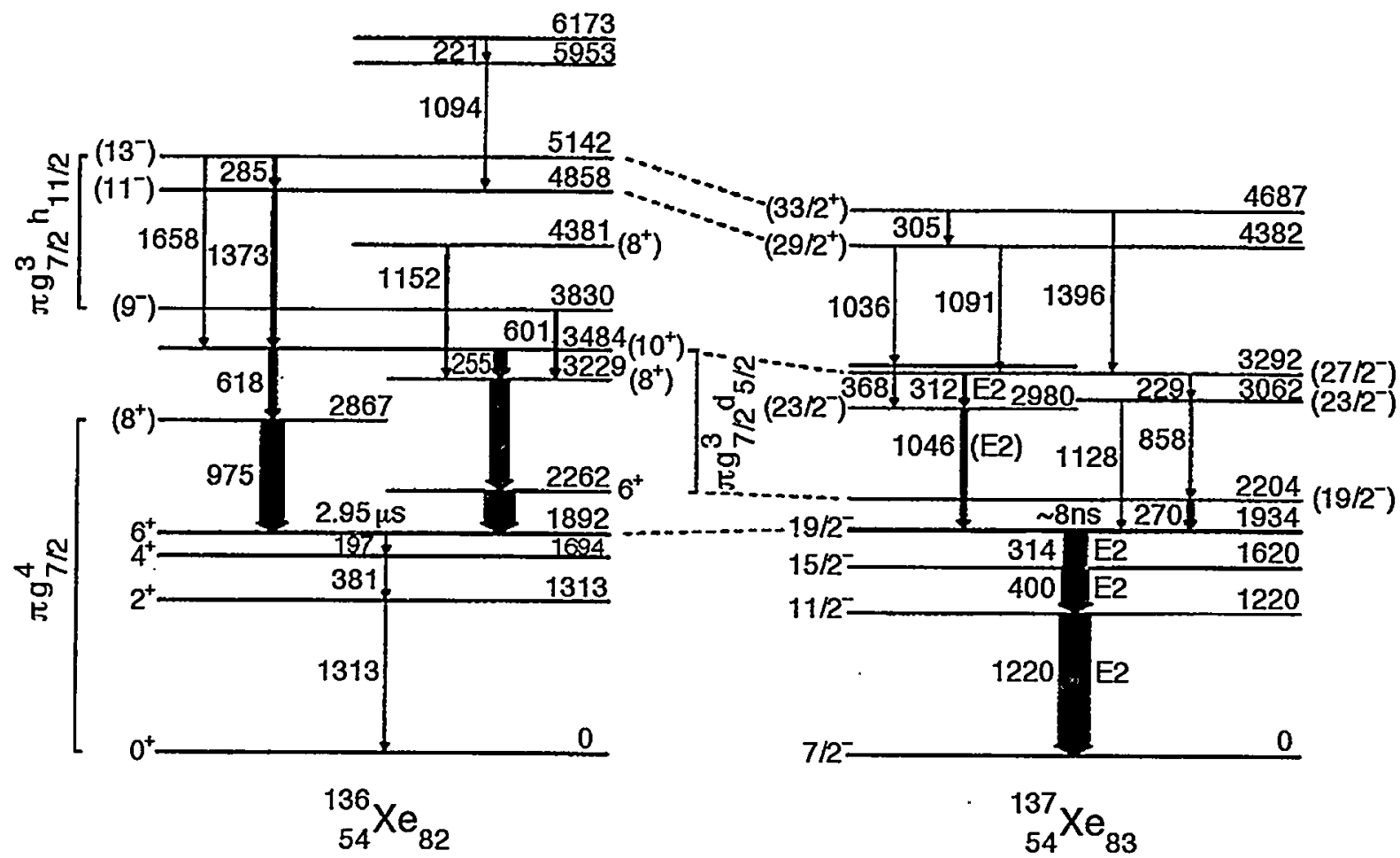

Fig. I-II. The ${ }^{136}$ Xe and ${ }^{137}$ Xe level scemes with dominant shell-model configurations indicated. 
b.8. In-Beam Studies of the Deformed Proton Emitter ${ }^{141 H o}$ (D. Seweryniak, C. N. Davids, A. Sonzogni, J. Uusitalo, M. P. Carpenter, A. Heinz, R. V. F. Janssens, T. L. Khoo, F. Kondev, C. J. Lister, P. Reiter, I. Wiedenhoever, T. Davinson, * J. Ressler, $†$ W. B. Walters, $\uparrow$ P. J. Woods, * and J. A. Cizewski $\ddagger$ )

The Recoil-Decay Tagging method was used to study excited states in the proton emitter ${ }^{141}$ Ho. In the first experiment the ${ }^{92} \mathrm{Mo}\left({ }^{54} \mathrm{Fe}, \mathrm{p} 4 \mathrm{n}\right){ }^{141} \mathrm{Ho}$ reaction was used to populate states in $141_{\mathrm{Ho}}$. In a second run the same reaction in inverse kinematics was used to obtain data with better statistics by taking advantage of the larger FMA transmission. Gammasphere was used to detect prompt gamma-ray transitions and the FMA equipped with the DSSD behind its focal plane supplied the proton tag. Gamma rays feeding both the ground state and the isomeric state in ${ }^{141} \mathrm{Ho}$ were identified and are shown in Fig. I-12. A preliminary partial level scheme proposed for ${ }^{141}$ Ho is shown in Fig. I-13. The spins and parities shown in Fig. I-13 are based on the assumption that the ground state is the 7/2-[523] state and the $1 / 2^{+}[411]$ orbital forms the isomer, both giving rise to strongly coupled bands. The decrease in the intensity of the ground state band possibly indicates the existence of a signature partner band. The $3 / 2^{+} \rightarrow 1 / 2^{+}$ transition feeding the isomer was not observed because of its low energy. The proposed rotational bands confirm that ${ }^{141}$ Ho is deformed as inferred from previous proton-decay studies at ANL. A deformation of beta $\sim 0.3$ can be estimated from the energies of the observed gamma-ray transitions. In addition, the data analysis did not reveal any evidence for a proton decay branch to the $2^{+}$state in the daughter nucleus for both the decay of the ground state and the isomeric state. It is worth noting that the cross section for populating the ground state is $300 \mathrm{nb}$ and for the isomer is about $50 \mathrm{nb}$. These are the weakest channels ever studied using in-beam gamma-ray spectroscopic methods.

*University of Edinburgh, United Kingdom, †University of Maryland, $¥$ Rutgers University

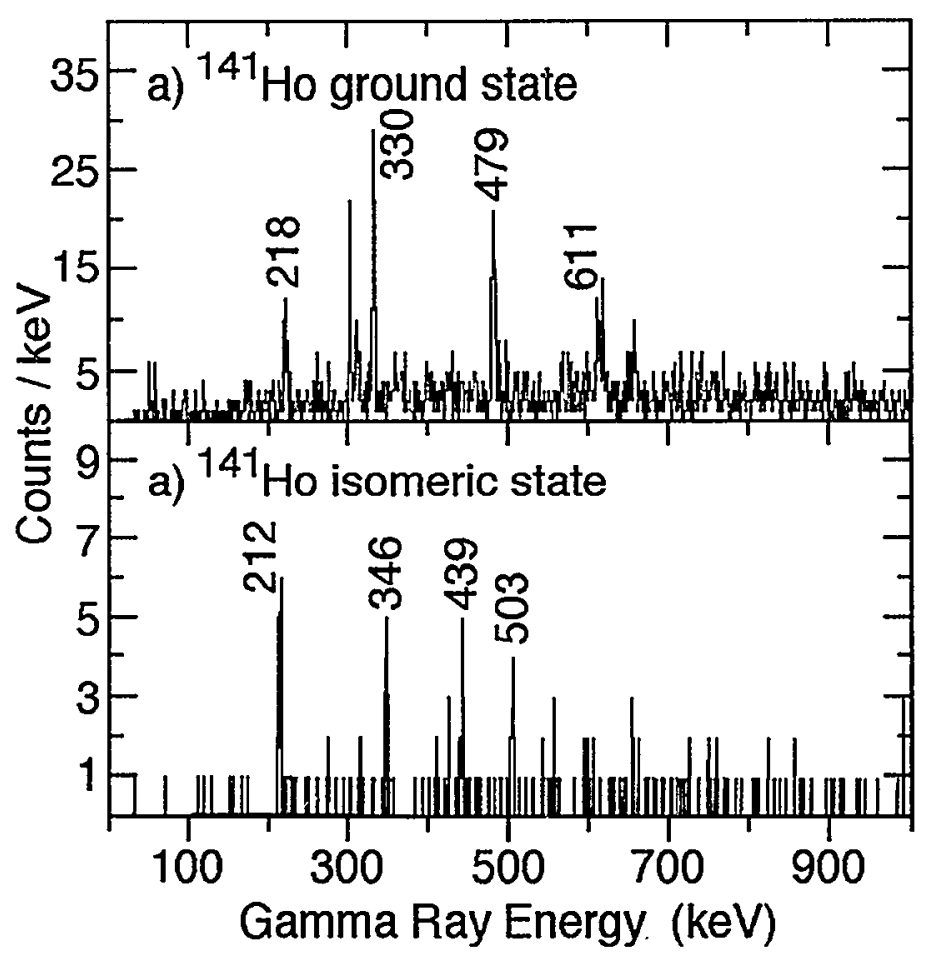

Fig. I-12. Gamma ray spectra tagged on (a) the ground state and (b) the isomeric state protons in ${ }^{141}$ Ho. 

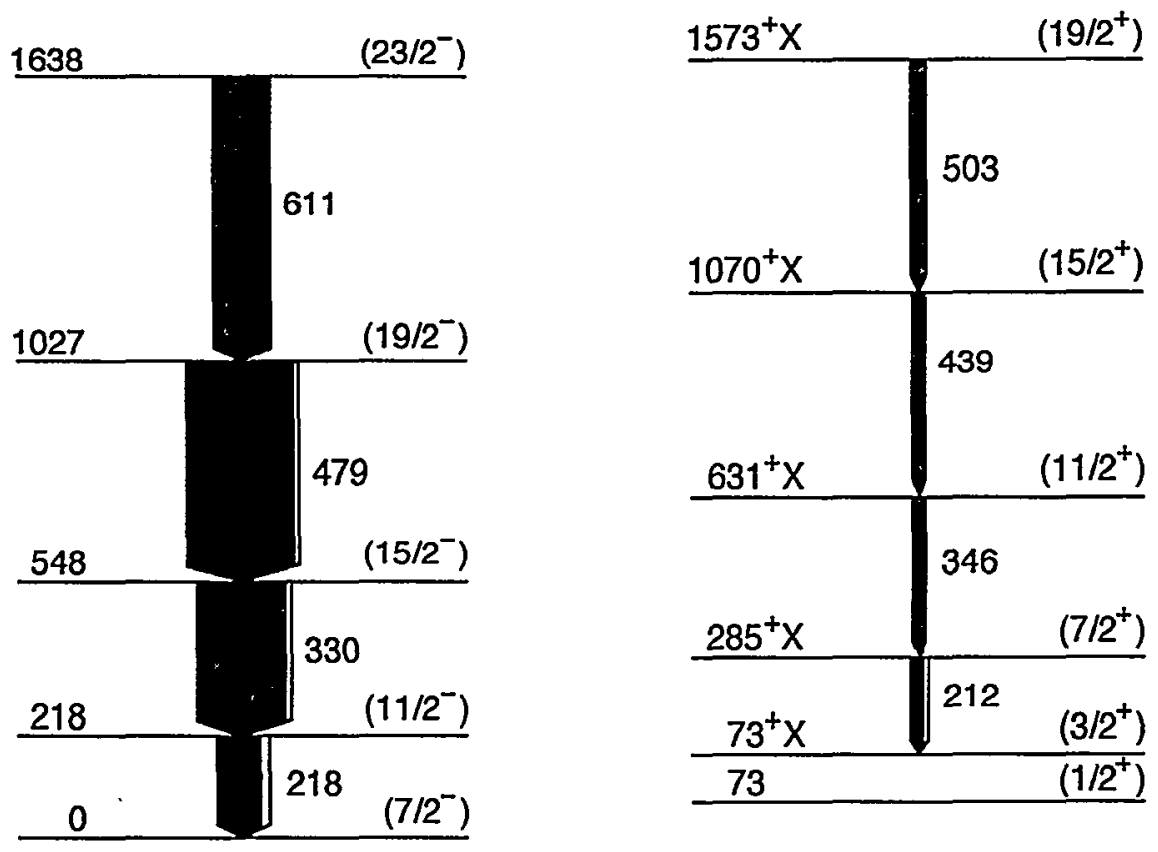

${ }^{141} \mathrm{HO}$

Fig. l-13. The preliminary level scheme proposed for ${ }^{141}$ Ho.

\section{b.9. Spectroscopy of $\mathbf{N}=84$ Nuclei at the Proton Drip Line (D. Seweryniak, J. Uusitalo, M. P. Carpenter, C. N. Davids, R. V. F. Janssens, T. Lauritsen, C. J. Lister, D. Nisius, P. Reiter, P. Bhattacharyya, $\uparrow$ K. Y. Ding, $\ddagger$ J. A. Cizewski, $\ddagger$ N. Fotiades,$\ddagger$ and A. O. Macchiavelli§)}

In-beam spectroscopic study of $\mathrm{N}=84$ nuclei located at the proton drip line was performed employing the Recoil Decay Tagging (RDT) method. The fusionevaporation reaction $58^{\mathrm{Ni}}+{ }^{102} \mathrm{Pd} \rightarrow 160 \mathrm{~W} *$ was used at the beam energy of $270 \mathrm{MeV}$. Gamma rays were detected using Gammasphere, coupled to the Fragment Mass Analyzer (FMA). This experiment was a continuation of the previous studies performed using the AYE-ball array of $\mathrm{Ge}$ detectors in which gamma rays belonging to several proton rich $\mathrm{N}=84$ isotones were identified for the first time. ${ }^{1}$ Thanks to much higher gamma-ray detection efficiency gamma-gamma coincidences were measured for the $\mathrm{N}=84$ isotones ${ }^{155} \mathrm{Lu},{ }^{156} \mathrm{Hf}$ and ${ }^{157} \mathrm{Ta}$, allowing us to establish their decay level schemes more firmly. In addition, gamma rays were assigned, for the first time, to the even more neutron deficient $N=84$ isotone ${ }^{158} \mathrm{~W}$. The following paragraphs summarize results obtained for the individual $\mathrm{N}=84$ isotones.
For ${ }^{155} \mathrm{Lu}$ gamma-gamma coincidences were measured tagging with alpha-decays from isomeric states $11 / 2^{-}$ and $25 / 2^{-}$. The ground state $1 / 2^{+}$alpha decay was too weakly populated to be used for tagging. This work is going to be a part of $K$. Y. Ding's thesis work.

For ${ }^{156} \mathrm{Hf}$ gamma-ray cascades built upon the alphadecaying $0^{+}$ground state and alpha-decaying $8^{+}$state were studied. When tagged with the ground state alpha decay a cascade consisting of three gamma rays can be seen. These transitions were proposed to form a $6^{+} \rightarrow$ $4^{+} \rightarrow 2^{+} \rightarrow 0^{+}$sequence (Fig I-14a). In addition, gamma rays feeding the $2^{+}, 4^{+}$and $6^{+}$states from the sides were obtained. When tagged with the alpha decay from the high-spin $8^{+}$state, a level scheme can be built at least up to spin 18 (Fig -I-14b).

For ${ }^{157} \mathrm{Ta}$ a proton decaying ground state $1 / 2^{+}$was recently reported by $R$. J. Irvine et al. ${ }^{2}$ In the work by R. D. Page et al. ${ }^{3}$ the observation of the alpha decay from the high-spin isomeric state $25 / 2^{-}$in ${ }^{157} \mathrm{Ta}$ was 
reported. In this work, recoil-alpha-alpha correlations did not show any evidence of such alpha decaying isomeric high spin state. Instead the gamma-spectrum tagged with alpha-decay from the isomeric state $11 / 2^{-}$ suggests that if such $25 / 2^{-}$state exists it is not populated or that there exists a state below it to which it can gamma decay (Fig I-14c). In fact the measured gamma-gamma coincidences suggests that close to a proposed $25 / 2^{-}$state there exists a state with a spin and parity of $23 / 2^{-}$. The alpha-decay (or proton decay) from the ground state $1 / 2^{+}$was too weak to be used for tagging.
Gamma-rays tagged with alpha-decay from the isomeric $8^{+}$state in $158 \mathrm{~W}$ is shown in (Fig. I-14d). The alphadecay from the ground state $0^{+}$was too weak for tagging purposes.

The measured energies were compared with the parameter-free shell model calculations using experimental one- and two-body matrix elements as described in the work C. T. Zhang et al. ${ }^{4}$ Relatively good agreement with the experimental level energies was obtained.

*ANL and University of Maryland, †Purdue University, $\ddagger$ Rutgers University, §Lawrence Berkeley National Laboratory

${ }^{1}$ D. Seweryniak et al., Proceedings of the Conference on Nuclear Structure at the Limits, July 22-26, 1996, Argonne, IL, ANL/PHY-97/1, p. 247 (1997).

${ }_{2}^{2}$. J. Irvine et al., Phys. Rev. C 55, R1621 (1997).

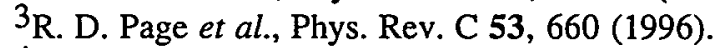

${ }^{4}$ C. T. Zhang et al., Phys. Rev. C 54, R1 (1996).

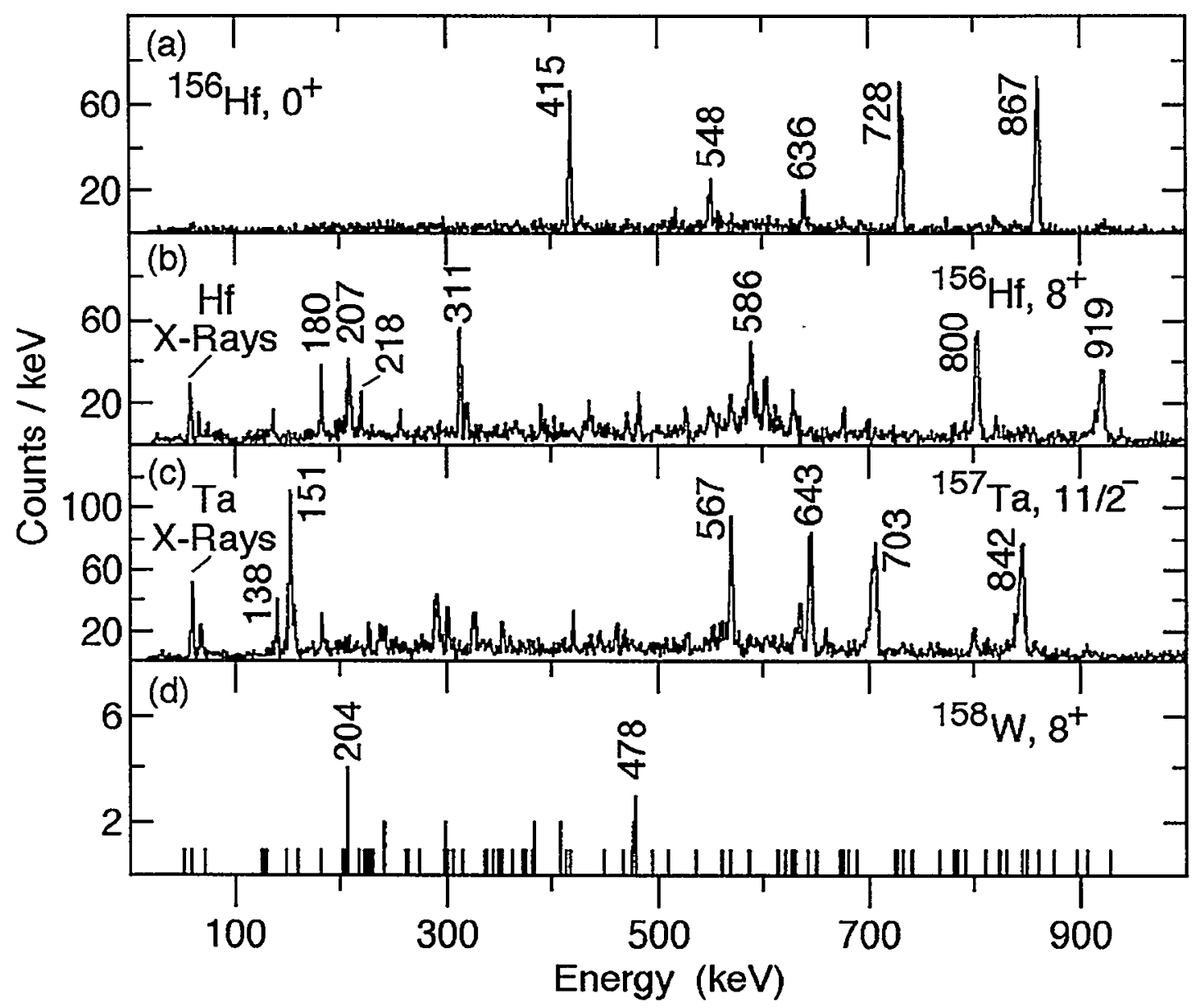

Fig. I-14. Alpha decay tagged gamma-ray spectra (see text). (a) ${ }^{156} \mathrm{Hf}, \mathrm{O}^{+},\left(\right.$b) ${ }^{156} \mathrm{Hf}, 8^{+},\left(\right.$c) ${ }^{157} \mathrm{Ta}, 11 / 2^{-}$and (d) $158 \mathrm{~W}, 8^{+}$. 


\section{b.10. Study of Excited States in the Proton-Emitter ${ }^{167}$ Ir and its Neighbors}

(M. P. Carpenter, R. V. F. Janssens, T. L. Khoo, D. Seweryniak, A. Sonzogni, I. Ahmad, L. T. Brown, C. N. Davids, G. Hackman, T. Lauritsen, C. J. Lister, P. Reiter, S. Siem, J. Uusitalo, I. Wiedenhoever, P. J. Woods,* J. A. Cizewski, $\uparrow$ W. Reviol, $\ddagger$ L. L. Riedinger, $\ddagger$ S. M. Fischer,§ J. J. Ressler,II W. B. Walters, II and P. Bhattacharyyall)

In the past several years, a number of new proton emitters were identified using current generation recoil mass spectrometers. ${ }^{1}$ These results have greatly enhanced our knowledge of the ground state properties of nuclei which lie beyond the proton drip line. For example, the decay rates for the majority of these proton emitters are well understood using a spherical shell model basis of $h_{11 / 2}, s_{1 / 2}$, and $d_{3 / 2}$ states. ${ }^{1}$ In the few cases where the spherical WKB model fails, the decay rates can be satisfactorily reproduced if one assumes that the proton is emitted from a deformed state. ${ }^{2}$

While a substantial amount of nuclear structure information was deduced about the ground-state properties of these nuclei which lie beyond the proton drip line, very little if anything is known about their excited states due to the fact that the cross-sections to form them via heavy-ion fusion-evaporation reactions is small $(<100 \mu b)$. In fact, the only data on nonisomeric excited states in proton emitters with $Z>50$ were obtained using the Recoil Decay Tagging (RDT) method, a technique which correlates the charged particle radioactivity of an ion implanted in a position sensitive silicon detector with a previously-implanted residue. ${ }^{3}$
With the coupling of Gammasphere to the Fragment Mass Analyzer (FMA) at Argonne, this lack of coincidence efficiency for small cross-section reactions was overcome. In an attempt to probe excited states in nuclei at the edge of stability, we measured excited states in the proton-emitter ${ }^{167} 7_{\mathrm{Ir}}{ }^{4}$ populated using the ${ }^{92} \mathrm{Mo}\left({ }^{78} \mathrm{Kr}, \mathrm{p} 2 \mathrm{n}\right)$ reaction at a beam energy of 360 $\mathrm{MeV}$. Gamma-rays in coincidence with residues were measured with Gammasphere and the FMA. The assignment of gamma-ray transitions to ${ }^{167}$ Ir was made using the RDT method. Figure I-15a shows a spectrum in coincidence with $A=167$ residues, and this spectrum is dominated by $\gamma$-rays associated with ${ }^{167} \operatorname{Re}(3 p)$ and ${ }^{167} \mathrm{Os}(2 \mathrm{pn})$. Figure I-15b is the $\gamma$-ray spectrum correlated with the alpha/proton decay of the $11 / 2$ level in ${ }^{167}$ Ir.

The data are currently being analyzed and a preliminary decay scheme suggests that the states built on the $11 / 2$ isomer are associated with a weakly deformed or spherical shape. This is in agreement with the conclusions of Ref. 4. In addition, we have obtained rather complete level structures on ${ }^{166-169}$ Os and $167,169 \mathrm{Re}$ which allowed us to more fully study the evolution from deformed to spherical shapes in this mass region.

*University of Edinburgh, United Kingdom, †Rutgers University, $\ddagger$ University of Tennessee, §DePaul University, qUniversity of Maryland, IIPurdue University

1P. J. Woods. and C. N. Davids, Ann. Rev. of Nucl. and Part. Sci. 47, 541 (1997) and ref. therein.

${ }^{2}$ C. N. Davids et al., Phys. Rev. Lett. 80, 1849 (1998).

${ }^{3}$ E. S. Paul et al., Phys. Rev. C 51, 78 (1995).

${ }^{4}$ C. N. Davids et al., Phys. Rev. C 55, 2255 (1997). 


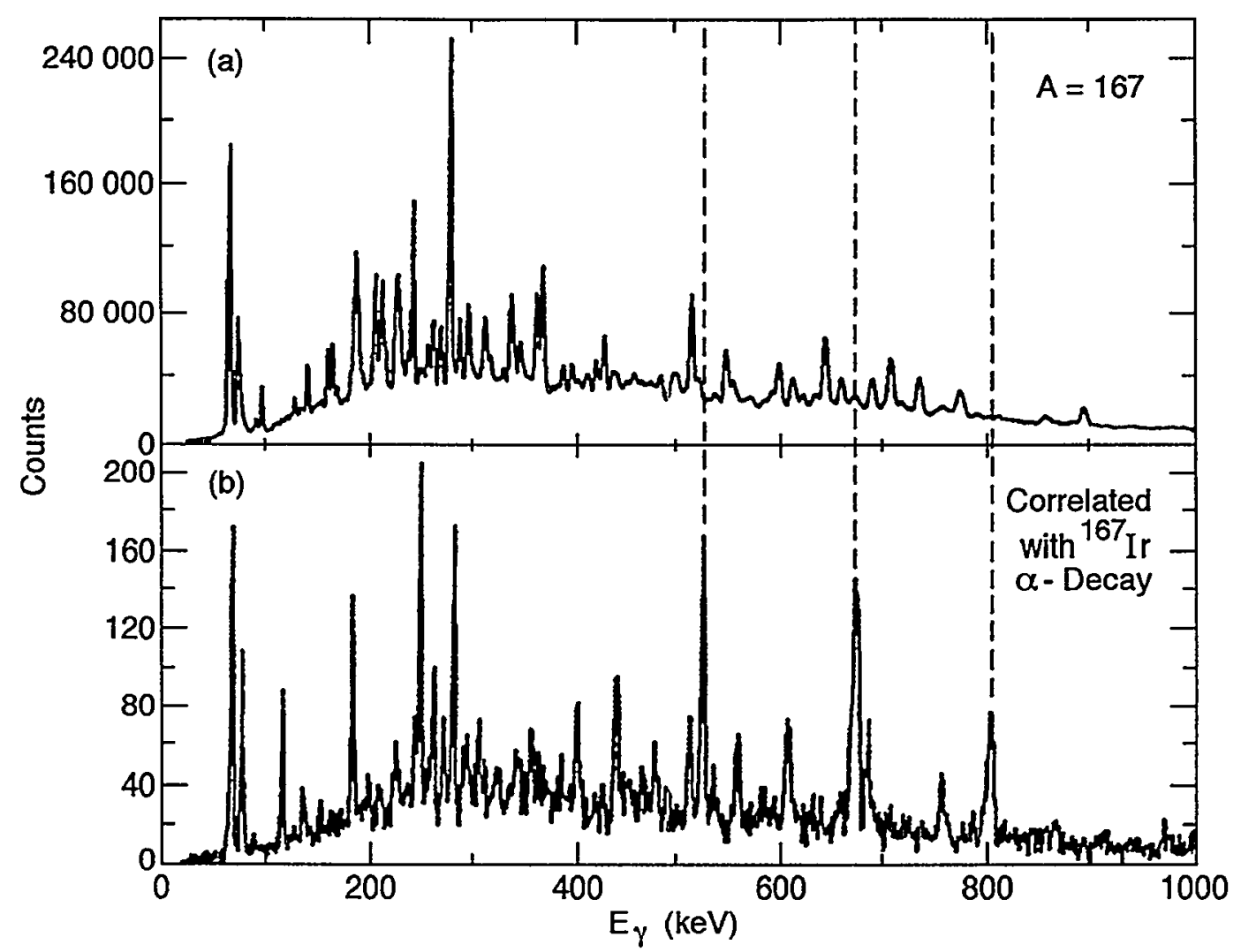

Fig. I-15. (a) Gamma-ray spectrum in coincidence with $A=167$ residues measured at the FMA focal plane. (b) Gamma-ray spectrum correlated with the alpha-decay of the $11 / 2^{-}$isomeric state in $167^{1 r}$.

b.11. Search for Enhanced Deformation in Light Hg Isotopes (F. G. Kondev, M. P. Carpenter, R. V. F. Janssens, M. Alcorta, L. T. Brown, C. N. Davids, T. L. Khoo, T. Lauritsen, C. J. Lister, D. Seweryniak, A. Sonzogni, J. Uusitalo, I. Wiedenhoever, P. Bhattacharyya,* S. M. Fischer, $\dagger$ W. Reviol, $\ddagger$ and L. Riedinger $\ddagger$ )

We recently published the first gamma spectroscopy data on the lightest $\mathrm{Hg}$ isotopes known so far, $176,178 \mathrm{Hg}$, from an experiment performed at ATLAS with the AYE-ball array and the Fragment Mass Analyzer (FMA). ${ }^{1}$ The interest in extending our knowledge to these very neutron-deficient $\mathrm{Hg}$ isotopes comes mainly from the fact that the entire chain of $\mathrm{Hg}$ isotopes displays a broad variety of interesting nuclear structure phenomena. For $N \geq 110$, the yrast structure at low spin is dominated by weakly deformed oblate rotational bands $\left(\beta_{2} \sim-0.15\right){ }^{2}$ At moderate spins $(I \sim$ $20 \hbar)$, these bands coexist with non-collective prolate $(\gamma$ $\left.=-120^{\circ}\right)$ excitations, ${ }^{3}$ and at the highest spins $(I-40 \hbar)$, superdeformed states are populated as well. ${ }^{4}$ The lighter $\mathrm{Hg}$ isotopes $(100 \leq \mathrm{N} \leq 110)$ exhibit shape coexistence: sequences of states associated with the small oblate deformation $\left(\beta_{2} \sim-0.15\right)$ characteristic of the ground state coexist with well developed rotational bands associated with a prolate shape $\left(\beta_{2} \sim-0.25\right)^{5}$.

Recent Nilsson-Strutinsky calculations ${ }^{6}$ using a Woods-Saxon potential suggested that for neutron number $\mathrm{N}<100$ the oblate ground state evolves steadily as a function of decreasing $\mathrm{N}$ towards a spherical shape, while the prolate minimum disappears and gives way to a new minimum at large deformation $\left(\beta_{2}=0.5-0.56\right)$ which is located between 3.5 and 5 $\mathrm{MeV}$ above the ground state. This prediction was borne out, at least to some degree, by our results. The new data indicate that the level structure associated with prolate deformation coexists with the oblate (or nearly spherical) ground state from $\mathrm{N}=110$ all the way down 
to at least $\mathrm{N}=98\left({ }^{178} \mathrm{Hg}\right)$ and that the prolate minimum comes closest in energy to the ground state at $N=102$. Furthermore, the behavior of this minimum with $\mathrm{N}$ appears to be rather symmetric around $\mathrm{N}=102$.

We re-examined ${ }^{178} \mathrm{Hg}$ using Gammasphere coupled to the FMA, utilizing the same reaction as we did with AYE-ball, namely, ${ }^{78} \mathrm{Kr}+{ }^{103} \mathrm{Rh}$. At a beam energy of $350 \mathrm{MeV}$, the two dominating mass channels measured at the FMA focal plane are $\mathrm{A}=177$ and 178 . In addition to the spectroscopic examination of $178 \mathrm{Hg}$, first level structures for $177,178 \mathrm{Au}$ were constructed and the level structure for ${ }^{178} \mathrm{Pt}$ was modestly extended. The data were analyzed using both mass-gated and recoil decay tagged gamma-gamma coincidence matrices.
In ${ }^{178} \mathrm{Hg}$, a second rotational band consisting of seven levels was observed. The states in this band were tentatively assigned odd-spins and negative parity. Initially, the band lies $\sim 1 \mathrm{MeV}$ above the yrast band. However, due to its larger moment of inertia, it rapidly approaches the yrast band with increasing spin, and we estimate that it will become yrast around I 23 $\hbar$. Based on the moment of inertia, this band appears to have significantly larger deformation than the prolate-yrast band. A different character for this band is also suggested by its decay properties, i.e. the band decays to the ground state through a number of states some of which may be spherical. Investigations into the parentage of this band are currently underway.

*University of Purdue, $\uparrow$ DePaul University, $\ddagger$ University of Tennessee

${ }_{1}$ M. P. Carpenter et al., Phys. Rev. Lett. 78, 3650 (1997).

${ }^{2}$ H. Hübel et al., Nucl. Phys. A453, 313 (1986).

${ }^{3} \mathrm{D}$. Ye et al., Phys. Lett. B236, 7 (1990).

${ }^{4}$ R. V. F. Janssens and T. L. Khoo, Ann. Rev. Nucl. Part. Sci. 41, 321 (1991).

5J. L. Wood, K. Heyde, W. Nazarewicz, M. Huyse and P. Van Duppen, Phys. Rep. 251, 101 (1992) and references therein.

6W. Nazarewicz, Phys. Lett. B305, 195 (1993).

\section{b.12. Spectroscopy of ${ }^{183} \mathrm{Tl}$ with Recoil-Mass and $Z$ Identification}

(M. P. Carpenter, R. V. F. Janssens, D. Seweryniak, J. Uusitalo, I. Wiedenhover,*

W. Reviol,* L. L. Riedinger,* C. R. Bingham,* W. Weintraub, $\dagger$ D. Jenkins, $\uparrow$

R. Wadsworth, $\dagger$ A. N. Wilson, $\ddagger$ S. Juutinen, $\ddagger$ K. Helariutta, $\S$ C. J. Gross,§

K. S. Toth, II J. C. Batchelder, II and J. A. Cizewskill)

The spectroscopy of ${ }^{183} \mathrm{Tl}$ was the focus of a recent experiment with Gammasphere at the FMA. The $182,183 \mathrm{Tl}$ nuclei were also studied as byproducts of $\mathbf{a}$. search for ${ }^{182} \mathrm{~Pb}$ at RITU in Jyväskylä. ${ }^{1}$ In these two experiments, the yrast sequence in ${ }^{183} \mathrm{Tl}$ was observed for the first time. The results reported here and in Ref. 2 are mainly based on the spectroscopy of ${ }^{183} \mathrm{Tl}$ with mass identification. The identified yrast sequence in ${ }^{183} \mathrm{TI}$ resembles the well-deformed (prolate) excited bands in adjacent nuclei of $\mathrm{Hg}, \mathrm{Tl}$, and $\mathrm{Pb}$, but its decay-out properties are different from those cases in two respects. (i) The rotational-like sequence is observed from medium spin to the $13 / 2^{+}$state, i.e. the population intensity stays within the band down to the bandhead. (ii) A strong $\gamma$-decay branch from the prolate band to a slightly-oblate structure, like in heavier Tl nuclei, is not observed. These features indicate that the prolate energy minimum in ${ }^{183} \mathrm{Tl}$ dropped significantly compared to ${ }^{185} \mathrm{Tl}^{3}$. In the present level scheme it is not clear how the ${ }^{183} \mathrm{Tl}$ band decays and, therefore, upper and lower-limit estimates for the energy of the $13 / 2^{+}$bandhead relative to the $9 / 2^{-}$isomeric state (oblate) were made $\left(95 \mathrm{keV} \leq E_{\text {rel }} \leq 424 \mathrm{keV}^{2}\right)$. However, with the estimated upper limit, the basic conclusions for the $\mathrm{i}_{13 / 2}$ band in ${ }^{183} \mathrm{Tl}$ are not affected by the uncertainty for the decay out of the band.

*University of Tennessee, $\nmid$ Univerisity of York, United Kingdom $\ddagger$ University of Jyväskylä, Finland, §Oak Ridge National Laboratory, TOak Ridge Associated Universities, \|University of Rutgers

${ }^{1} \mathrm{D}$. Jenkins, et al., to be published.

${ }^{2}$ W. Reviol et al., Proceedings of the Conference "Nuclear Structure '98", Gatlinburg, TN, AIP-Conference Proceedings, in print.

${ }^{3}$ G. Lane et al., Nucl. Phys. A586, 316 (1995). 


\section{b.13. High Spin Spectroscopy of $\mathbf{2 3 8 - 2 4 4}^{\mathrm{Pu}}$ (I. Wiedenhoever, R. V. F. Janssens, G.} Hackman, I. Ahmad, J. P. Greene, H. Amro, M. P. Carpenter, D. T. Nisius, P. Reiter, T. Lauritsen, C. J. Lister, T. L. Khoo, D. Seweryniak, J. Uusitalo, S. Siem,* J. Cizewsk, $†$ A. O. Macchiavelli, P. Chowdhury,§ E. H. Seabury,§ D. Cline,Il and C. Y. WuIl)

In two experiments performed with Gammasphere at ATLAS, the high spin states of the isotopes $238-244 \mathrm{Pu}$ were investigated by bombarding $240 \mathrm{Pu}$ and ${ }^{244} \mathrm{Pu}$ targets with a $208 \mathrm{~Pb}$ beam, and ${ }^{239} \mathrm{Pu}$, and ${ }^{242} \mathrm{Pu}$ targets with a ${ }^{207} \mathrm{~Pb}$ beam at energies above the Coulomb barrier. The data include Coulomb excitation of the target isotope as well as one- and two neutron transfer channels. In the cases of ${ }^{244} \mathrm{Pu}$ and ${ }^{242} \mathrm{Pu}$, the yrast band was extended to $34 \hbar$, revealing the completed $\pi i_{13 / 2}$ alignment, a "first" for actinide nuclei. The ${ }^{238} \mathrm{Pu}$ yrast sequence exhibits the onset of the quasiparticle alignment as well. The ground state band of $240 \mathrm{Pu}$ was measured up to the highest rotational frequencies ever reported in the actinide region $(\sim 300 \mathrm{keV})$, and shows no sign of particle alignment, in contrast to its heavier and lighter neighbors (see Fig. I-16).
One interesting phenomenon of nuclear structure in the actinide region is the coexistence between a quadrupole deformation with vibrational octupole (pear shaped) excitations. Our study revealed the relative position of the rotational bands associated with both states for all nuclei investigated. In the case of ${ }^{240} \mathrm{Pu}$, several observables such as the large E1 decay rates between octupole- and ground state band and the vanishing energy difference between both bands may suggest that both become one at high spin. This hypothesis would also explain the absence of particle alignment in this isotope. The observation that the nuclear structure of $240 \mathrm{Pu}$ differs significantly from its neighbors is at present not understood and places a challenge for theoretical models.

\footnotetext{
*Argonne National Laboratory and University of Oslo, Norway, †Argonne National Laboratory and Rutgers University, 立awrence Berkeley National Laboratory, §University of Massachusetts at Lowell, qUniversity of Rochester
} 


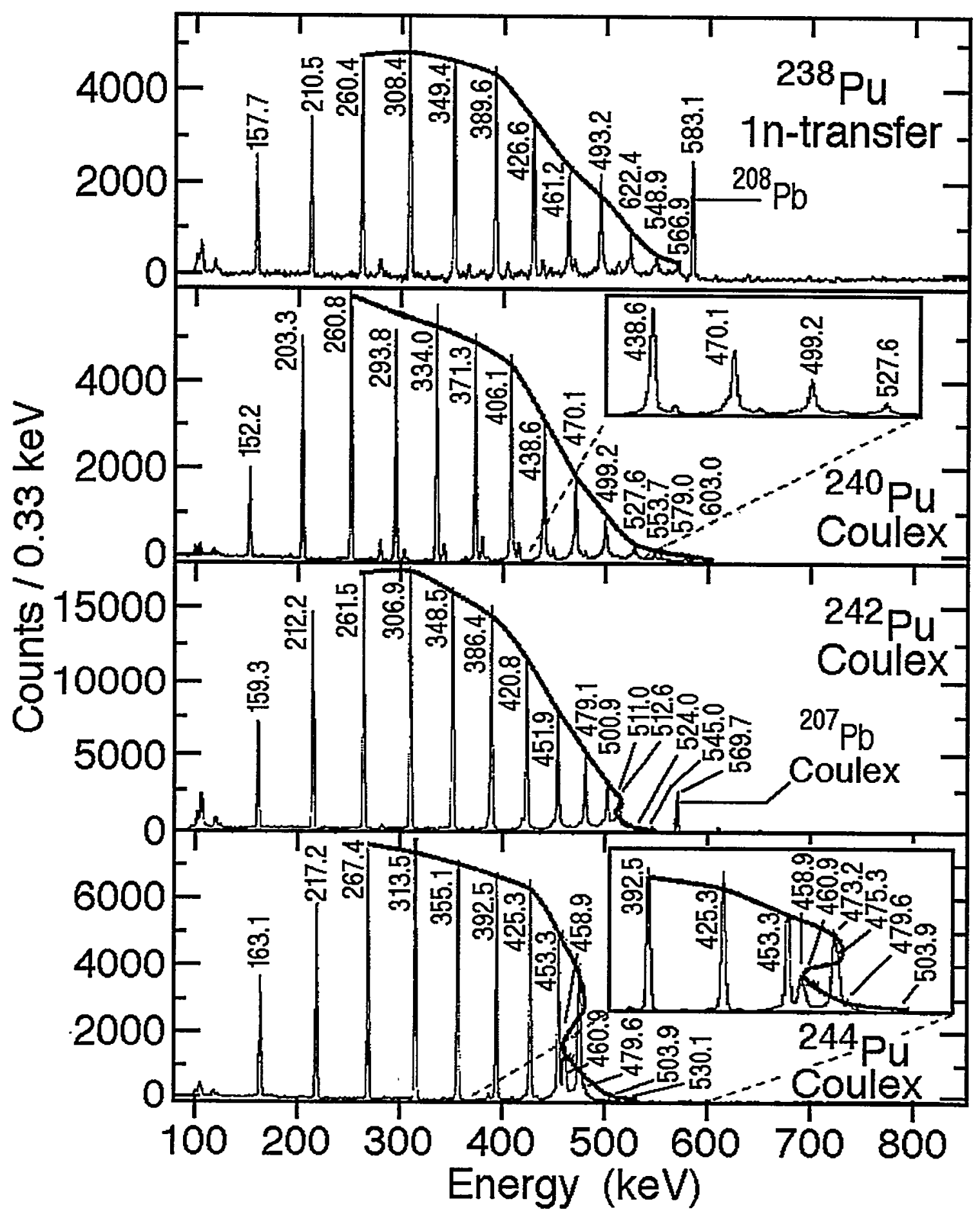

Fig. I-16. $\gamma$ spectra of the ground state rotational bands of $238-244$ Pu, measured with Gammasphere at ATLAS. The spectra are triple or quadruple gamma-coincidence events gated on transitions in the respective band. In the

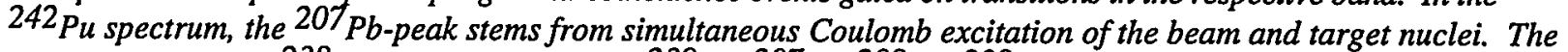
gamma-spectrum of $238 \mathrm{Pu}$ was observed in the ${ }^{239} \mathrm{Pu}\left({ }^{207} \mathrm{~Pb},{ }^{208} \mathrm{~Pb}\right) 238 \mathrm{Pu}$ neutron-transfer reactions. The crosscoincidences between the reaction products were used to separate reaction channels. 


\section{b.14. Study of ${ }^{248} \mathrm{Cm}$ Fission Products with Gammasphere (I. Ahmad,} M. P. Carpenter, R. V. F. Janssens, T. L. Khoo, T. Lauritsen, C. J. Lister, P. Reiter, D. Seweryniak, I. Wiedenhöver, P. Bhattacharyya, * C. T. Zhang,* P. J. Daly,* B. Grobowski,* B. Fornal, $\uparrow$ and R. Broda $\dagger$ )

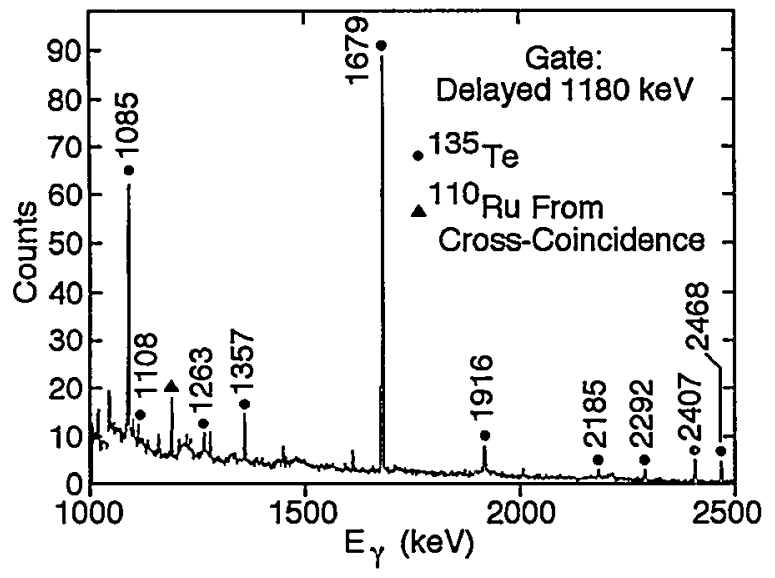

Fig. 1-17. Gamma ray spectrum measured with the Gammasphere and gated by the delayed 1080-keV gamma ray peak in 135 Te. The peaks in the spectrum are placed above the $0.5 \mu$ isomer.

Structures of many neutron-rich nuclei produced in the spontaneous fission of ${ }^{248} \mathrm{Cm}$ were deduced from the

*Purdue University, $\uparrow$ IFJ, Cracow, Poland

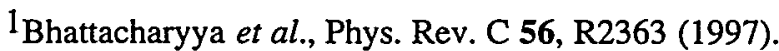

data accumulated in the experiment with the Eurogam2 array and a ${ }^{248} \mathrm{Cm}$ source, performed in 1994 . The experiment provided a large number of events of high quality but it did not have the required time information. This hampered the analysis of the data in the ${ }^{132} \mathrm{Sn}$ region where most of the nuclei have isomeric states. For this reason, we measured gamma-gamma coincidences with the Gammasphere using the same ${ }^{248} \mathrm{Cm}$ source we used in the Eurogam2 experiment. The experiment was carried out for 8 days around Labor Day last year and Gammasphere at that time contained $99 \mathrm{Ge}$ detectors. The data were collected with $\sim 1 \mu \mathrm{s}$ time range and $\sim 3 \times 10^{9}$ four or higher-fold events were recorded. Preliminary analysis of the data shows that the data are of high quality. A gamma-ray spectrum showing the quality of data is displayed in Fig. 1-17. The spectrum was obtained by placing a delayed gate on the $1180-\mathrm{keV}{ }^{135} \mathrm{Te}$ gamma-ray peak. Several gamma rays above the $0.5 \mu \mathrm{s}, 19 / 2^{-}$state appear in the spectrum. In the data from the Eurogam2 experiment, ${ }^{1}$ we were able to identify only 1085 -, 1357-, 1679- and $1407-\mathrm{keV}$ gamma rays. Analysis of the data is in progress.

\section{b.15. The $1 / 2^{+}[880]$ Single-Particle State in ${ }^{249} \mathrm{Cm}$ (I. Ahmad, B. B. Back, R. R. Chasman, J. P. Greene, T. Ishii, L. R. Morss* G. P. A. Berg, $\dagger$ A. D. Bacher, $\dagger$ C. C. Foster, $\uparrow$ W. R. Lozowski, $\uparrow$ W. Schmitt, $\uparrow$ E. J. Stephenson, $\uparrow$ and T. Yamanaka $\dagger)$}

The position of the $k_{17 / 2}$ shell orbital plays a significant role in determining the shell correction energy and hence the half-lives of the isotopes of superheavy elements. The $\Omega=1 / 2$ deformed component of this orbital, $1 / 2^{+}[880]$, comes down in energy with increasing prolate deformation and is expected to have an excitation energy of less than 2 $\mathrm{MeV}$ in nuclei with $\mathrm{N}=153$. Since this is a high- $\ell$ orbital, it is very weakly populated in (d,p) reactions and hence it was not observed in the ${ }^{248} \mathrm{Cm}(\mathrm{d}, \mathrm{p})$ reaction. High- $\ell$ states are strongly populated in $\left({ }^{4} \mathrm{He},{ }^{3} \mathrm{He}\right)$ reactions because of the angular momentum mismatch. We, therefore, performed the
${ }^{248} \mathrm{Cm}\left({ }^{4} \mathrm{He},{ }^{3} \mathrm{He}\right)$ reaction at the Indiana University Cyclotron Facility with $98.5-\mathrm{MeV}$ alpha beam. A 100 $\mu \mathrm{g} / \mathrm{cm}^{2}{ }^{248} \mathrm{Cm}$ target was prepared by molecular plating on a $75-\mu \mathrm{g} / \mathrm{cm}^{2}$ carbon foil and was bombarded with the alpha beam. The emerging ${ }^{3} \mathrm{He}$ ions were momentum analyzed with the K600 magnetic spectrometer and spectra were measured at angles of $4.1^{\circ}, 6.1^{\circ}, 10.2^{\circ}, 12.2^{\circ}$ and $16.2^{\circ}$. The spectrometer was calibrated with the ${ }^{3} \mathrm{He}$ ions produced in the ${ }^{208} \mathrm{~Pb}\left({ }^{4} \mathrm{He},{ }^{3} \mathrm{He}\right)$ and ${ }^{232} \mathrm{Th}\left({ }^{4} \mathrm{He},{ }^{3} \mathrm{He}\right)$ reactions at the same energy and angle. Figure I-18 shows the ${ }^{232} \mathrm{Th}\left({ }^{4} \mathrm{He},{ }^{3} \mathrm{He}\right)$ spectrum and clearly demonstrates the preferential population of high- $\ell$ states. Three strong 


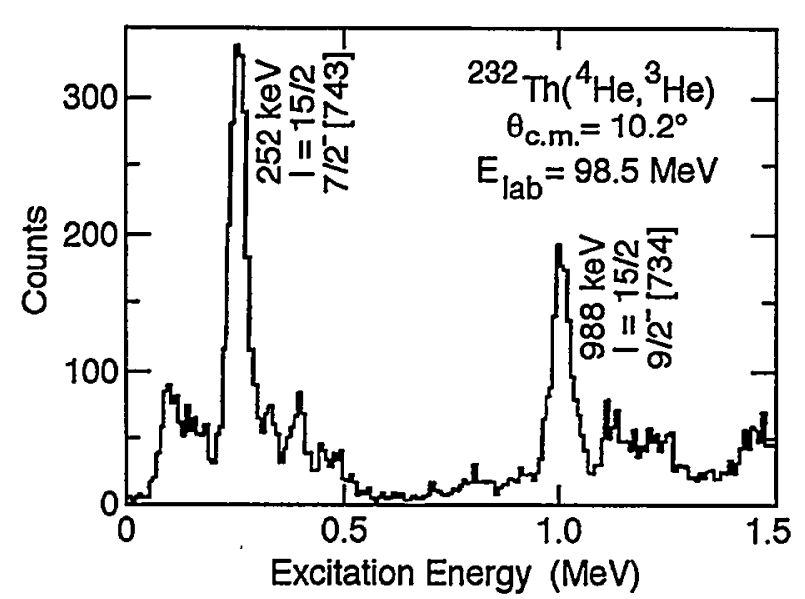

peaks were identified in the ${ }^{248} \mathrm{Cm}\left({ }^{4} \mathrm{He},{ }^{3} \mathrm{He}\right){ }^{249} \mathrm{Cm}$ spectrum. The 573-keV level was assigned to the $\mathrm{I}=$ $15 / 2$ member of the 11/2-[725] band. The 1560- and $1898-\mathrm{keV}$ levels are assigned to the $17 / 2,1 / 2+[880]$ and $15 / 2,13 / 2-[716]$ states. The cross sections for these two states calculated with the DWUCK code are found to be very similar and hence from the present data we cannot decide which level is the $1 / 2^{+}[880]$ state. The data, however, restrict the energy of this state between 1560 and $1898 \mathrm{keV}$. The results of this study were published. 1

*Chemistry Division, ANL, $†$ Indiana University

1. Ahmad et al., Nucl. Phys. A646, 175 (1999).

Fig. I-18. A portion of the ${ }^{3}$ He spectrum from the ${ }^{232} \mathrm{Th}\left({ }^{4} \mathrm{He},{ }^{3} \mathrm{He}\right)$ reaction measured with the K600 magnetic spectrometer at $10.2^{\circ}$ and $98.5 \mathrm{MeV}$ A-particle energy. The beam current was $25 \mathrm{pnA}$ and the accumulation time was 3.5 hours.

\section{b.16. Nuclear Structure of ${ }^{251} \mathrm{Cf}$ from the Decay of the $20 \mathrm{~h}$ 255Fm (I. Ahmad, M. P. Carpenter, R. V. F. Janssens, T. L. Khoo, T. Lauritsen, C. J. Lister, D. Seweryniak, I. Wiedenhöver, A. Sonzogni, J. Uusitalo, and P. Bhattacharyya*)}

Energies of the single-particle states in ${ }^{251} \mathrm{Cf}$, which are populated in the alpha decay of $255 \mathrm{Fm}\left(\mathrm{t}_{1 / 2}=20 \mathrm{~h}\right)$ and lie above the $N=152$ neutrons, are important for the calculation of the half-lives of superheavy elements. The last experiment on the decay of ${ }^{255} \mathrm{Fm}$ was performed 1 with a $25-\mu \mathrm{Ci}$ source and a $25-\mathrm{cm}^{3} \mathrm{Ge}(\mathrm{Li})$ detector in 1971. In that study all levels below 600 $\mathrm{keV}$ were identified. Since large $\mathrm{Ge}$ detectors and large arrays are now available and also larger amounts of source material can be obtained, we undertook the present measurement.

The nuclide ${ }^{255} \mathrm{Fm}$, which is the daughter product of $255 \mathrm{Es}$, was milked from the total amount of Es produced in the high flux isotope reactor (HFIR) at Oak Ridge. Three chemically purified samples of ${ }^{255} \mathrm{Fm}$, $\sim 1 \mathrm{mCi}$ each, were shipped to Argonne in three different weeks. The first two samples were used to measure gamma-singles spectra with a low-energy photon spectrometer (LEPS) and a $25 \%$ Ge spectrometer placed in a very-low background room. The decay of the spectra was followed for several days. We were able to collect a hundred times more data with higher resolution than the data in Ref. 1. All gamma rays previously reported were identified. In addition, many new gamma rays above $500 \mathrm{keV}$ were identified. Gamma rays with intensities as low as $1.0 \times 10^{-6} \%$ per ${ }^{255} \mathrm{Fm} \alpha$ decay were observed. The third sample was used to measure gamma-gamma coincidences with the Gammasphere. The data are being analyzed but we already have identified new vibrational bands in ${ }^{251} \mathrm{Cf}$. A sample of the $255 \mathrm{Fm}$ gamma ray spectrum is shown in Fig. I-19.

*Purdue University

${ }^{1}$ I. Ahmad et al., Phys. Rev. C 3, 390 (1971). 


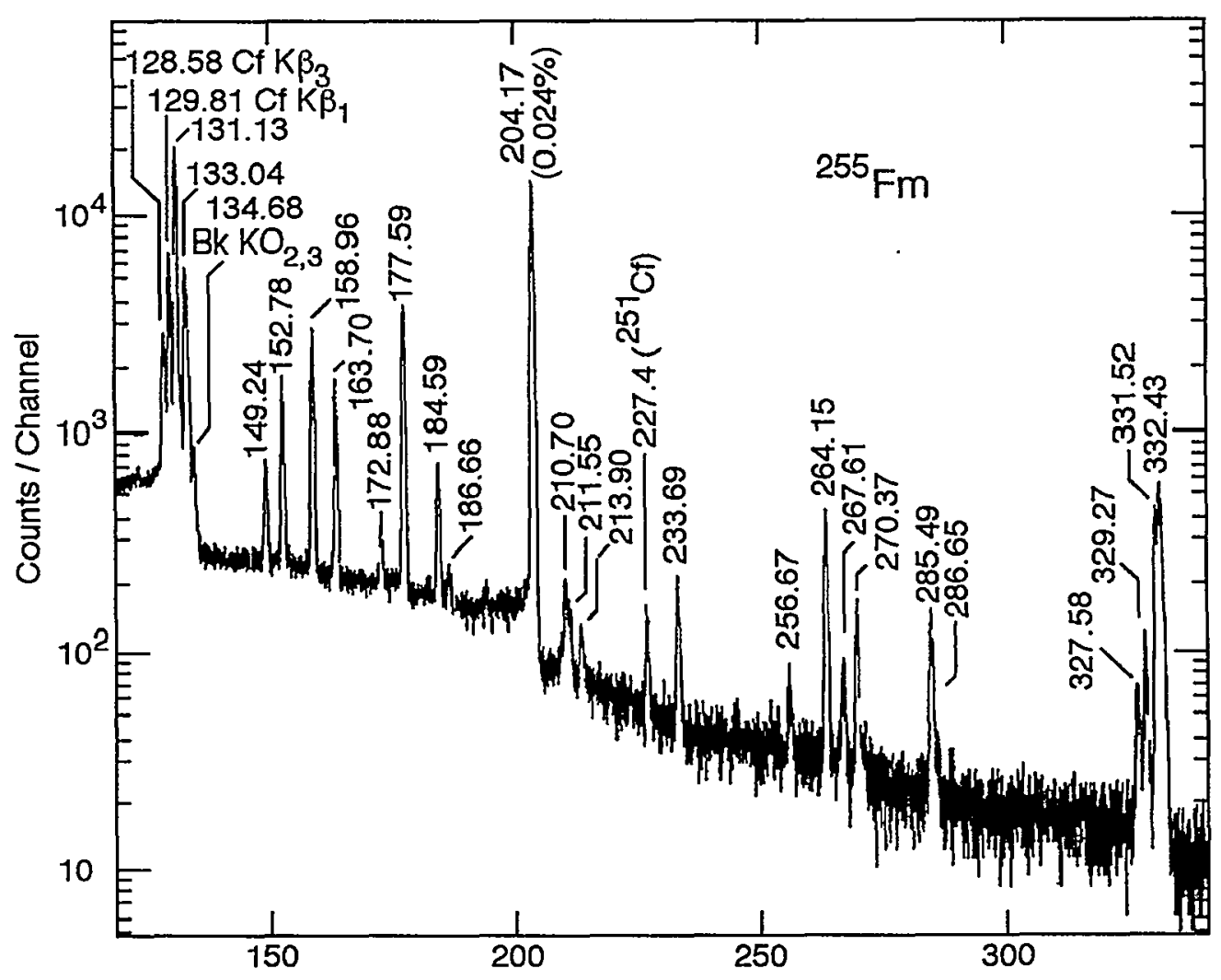

Fig. I-19. A portion of the 255 Fm gamma singles spectrum measured with a high-resolution LEPS spectrometer.

\section{b.17. Ground-State Band and Deformation of the $Z=102$ Isotope $254 \mathrm{No}$ (P. Reiter, T. L. Khoo, C. J. Lister, D. Seweryniak, I. Ahmad, M. Alcorta, M. P. Carpenter, C. N. Davids, G. Gervais, J. P. Greene, W. F. Henning, R. V. F. Janssens, T. Lauritsen, A. A. Sonzogni, D. Sullivan, J. Uusitalo, I. Wiedenhöver, J. A. Cizewski, ${ }^{*}$ S. Siem, $\uparrow$ N. Amzal $\ddagger$ P. A. Butler, $\ddagger$ A. J. Chewter, K. Y. Ding,§ N. Fotiades,§ J. D. Fox,II P. T. Greenlees, G. D. Jones, $;$ W. Korten,ll M. Leino,** and K. Vetter $\dagger \dagger)$}

The classical fission barriers of the heaviest elements with $Z>100$ approach zero because of the large Coulomb energy. However, a series of measurements 1 has established that elements with $\mathrm{Z}$ up to 112 are sufficiently bound against fission to preferentially decay by $\alpha$ emission. A large shell-correction energy leads to additional binding and, hence, creates sizable fission barriers of up to $8 \mathrm{MeV} .{ }^{2,3}$ The relative stability of these very heavy elements is a striking manifestation of shell structure in nuclei, and arises from the same mechanism responsible for the proposed ${ }^{4}$ stability of an "island" of superheavy elements around $Z=114, N=$ 184. Hence, nuclei with large $Z(>100)$, which are stable only because of the shell-correction energy, may be characterized as belonging to the family of superheavy nuclei, since their structures and formation mechanism.are governed by the same physics. We shall refer to these as transfermium nuclei (TFN).

The proposal of an "island" of superheavy elements was based on doubly-closed neutron and proton shells, associated with a spherical shape. ${ }^{4}$ Subsequent theoretical work (see, e.g., recent reviews 2,5 ) explained the observed stability of TFN with $Z$ up to 112; however, in these cases, the shell-correction energy is derived from the ability of the nucleus to deform, with not only quadrupole, but also higher multipole moments. Confidence in these calculations comes from their agreement with experimental $\alpha$-decay energies, $\alpha$ decay lifetimes and long fission lifetimes. $1,2,5$ A direct 
demonstration that these nuclei are deformed and a determination of the quadrupole deformation parameter constitute further important tests of these calculations.

The spin-dependent properties of TFN also provide another test of shell-correction energies, since rotational frequency and deformation play similar roles in determining the energies of orbitals (which represent the essence of the shell-correction). In addition, observation of high-spin $(I \geq 10)$ states in TFN gives information on the fission barrier at high angular momentum. This information is important for understanding the mechanism for producing the heaviest elements since the fission barrier governs their survival probability. The barrier at zero spin was calculated, but there are no predictions at finite spin, which would require calculations of the spin dependence of the shellcorrection energy. In fact, it is a priori not obvious that high-spin states of shell-stabilized nuclei will even survive against fission.

The heaviest element studied using (heavy ion,xn) reactions is uranium, with $Z=92$. In this work, we report on the ground-state band in the $Z=102$ isotope ${ }^{254} \mathrm{No}$, the heaviest element for which high-spin data now exist.

The ${ }^{208} \mathrm{~Pb}\left({ }^{48} \mathrm{Ca}, 2 \mathrm{n}\right)$ reaction was used to produce ${ }^{254} \mathrm{No}$, with a beam energy of $215 \mathrm{MeV}$, where the production cross section peaks ${ }^{6}$. Beams of up to 9 pnA of ${ }^{48} \mathrm{Ca}$ were provided by ATLAS. To enable the $208 \mathrm{~Pb}$ targets to survive such beams, they were mounted on a rotating wheel, which controlled pulsing of the beam, so that only the targets were irradiated. In addition, the beam was dispersed vertically by \pm 2.5 $\mathrm{mm}$ through wobbling (at $5 \mathrm{~Hz}$ ) with a magnetic steerer.

Gamma rays were detected using Gammasphere. In order to identify $\gamma$ rays from ${ }^{254}$ No in a background of $>10^{4}$ times more intense fission $\gamma$ rays, it was essential to require coincidences with evaporation residues. These were separated from the beam by the fragment mass analyzer (FMA). At the focal plane of the FMA, particles were detected in a position-sensitive detector, either a multichannel-plate detector in one experiment or a parallel-plate avalanche counter in a second experiment. After transmission through the focal-plane detector, the residues were implanted in a double-sided Si strip detector (DSSD), which had 1600 $1 \times 1-\mathrm{mm}$ pixels. To isolate the rare nobelium residues from a more copious background of scattered beam and some fission fragments, coincidence gates were set on a two-dimensional histogram of the flight time from the focal plane detector to the DSSD us. the DSSD implant energy. Further coincidence gates were placed on: (i) the time of flight of the evaporation residues from the target to the focal plane, and (ii) the focal-plane detector positions corresponding to two charge states $(q=20$ and 21 ) of ions with a mass of 254. The resulting $\gamma$ spectrum from ${ }^{254}$ No is shown in Fig. I-20a.

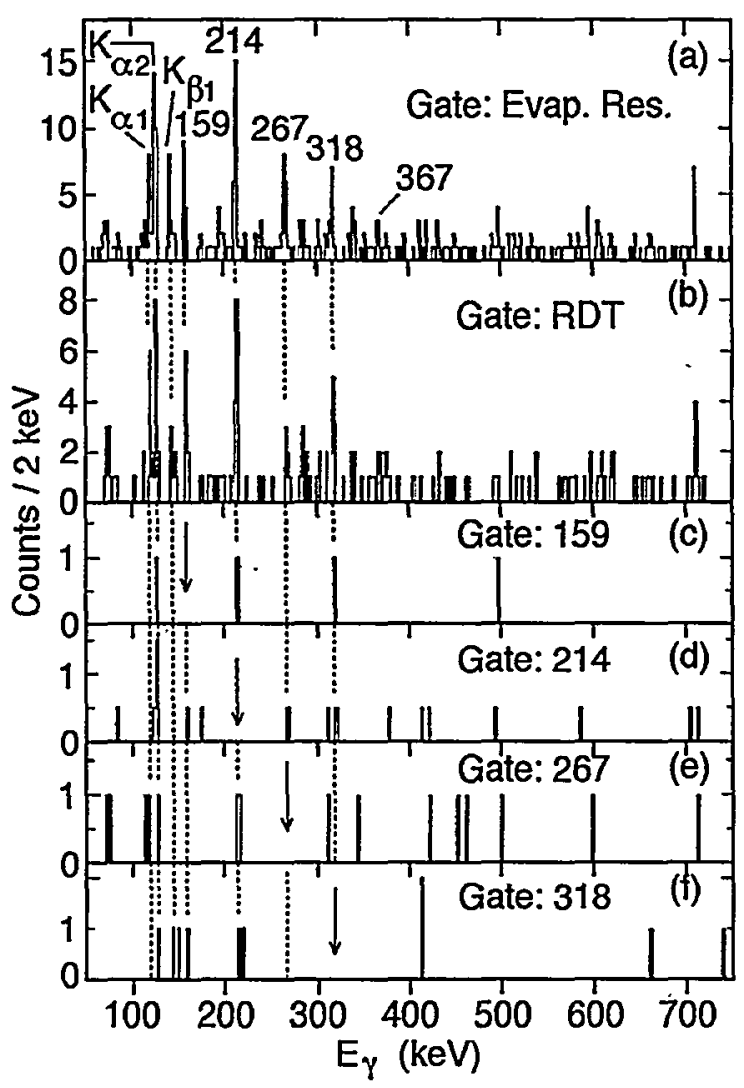

Fig. 1-20. (a) Gamma spectrum obtained using coincidence gates described in the text. The background level is 0.4 counts/channel. Peaks labelled by energy are assigned as transitions within the ground-state band of ${ }^{254} \mathrm{No}$. The peak at $73 \mathrm{keV}$ from $\mathrm{Pb} x$-rays appears from incomplete subtraction of random coincidences. (b) Spectrum with an additional requirement on the 254 No $\alpha$ peak. Coincidence spectra from gates set on the transitions indicated by arrows. Vertical dashed lines help visual alignment of peaks in the different panels.

The production of ${ }^{254} \mathrm{No}$ was unambiguously demonstrated by the observed $\alpha$ decay chain. After implantation of a ${ }^{254}$ No evaporation residue in a specific DSSD pixel, subsequent $\alpha$ decay in that pixel was used to identify $254 \mathrm{No}$, with additional 
requirements on energy $(8.09 \mathrm{MeV})$ and decay time (1$200 \mathrm{~s}$, commensurate with its $55 \mathrm{~s}$ half-life). Correlations of $\gamma$ rays with focal-plane signals corresponding to uniquely-identified No residues constitute the recoil decay tagging (RDT) technique, whereby unambiguous identification of the $\gamma$-ray parentage is achieved in the spectrum shown in Fig. I20b. This spectrum confirms that the spectrum in Fig. I-20a, which was generated without the $\alpha$-decay requirements, contains only ${ }^{254}$ No $\gamma$ rays. Hence, the latter spectrum, which has approximately twice as many counts, was used for further analysis. This spectrum shows the No $K_{\alpha}$ and $K_{\beta} x$-rays, a sequence of transitions with spacings that are characteristic of transitions from a rotational band, and $\gamma$ rays above $\sim 500 \mathrm{keV}$, which are presumably from excited bands. The transitions that exhibit rotational characteristics are assigned to the ground-state band of ${ }^{254}$ No.

Spectra from coincidence gates on individual transitions of this rotational band are given in Fig. I-20c-f. The high efficiency of Gammasphere enables coincidence relationships to be established even with the low statistics associated with a small production crosssection. Although the ground-band peaks contain only 1-2 counts, the background is $<0.01 /$ channel. Although not all pairwise coincidences are detected (as expected from the low statistics), a sufficient number are observed to support the assignment of a cascade within the ground-state band.

The identification of a rotational band in ${ }^{254} \mathrm{No}$ immediately establishes that the nucleus is deformed and constitutes an important confirmation of the predictions of theories that calculate the shell-correction energies of TFN. Fig. I-21 shows the moments of inertia, $\mathfrak{T}(1)$ and $\mathfrak{I}^{(2)}$, for the ground state band of ${ }^{254} \mathrm{No}$. $\left(\mathfrak{I}^{(1)}=\right.$ $\hbar^{2}(2 I-1) / \mathrm{E}_{\gamma}(I) ; \mathfrak{I}(2)=4 \hbar^{2} /\left(\mathrm{E}_{\gamma}(I)-\mathrm{E}_{\gamma}(I-2)\right) ; \hbar \omega=$ $E_{\gamma} / 2$.) The Harris parameterization,

$\mathfrak{I}^{(2)}=\mathfrak{I}_{0}+3 \mathfrak{I}_{1} \omega^{2} ; \mathfrak{I}^{(1)}=\mathfrak{I}_{0}+\mathfrak{I}_{1} \omega^{2}$

provides excellent fits of $\mathfrak{I}^{(2)}(\omega)$ and $\mathfrak{I}^{(1)}(\omega)$. From the parameters $\mathfrak{I}_{0}$ and $\mathfrak{I}_{1}$, we deduced the spins of the emitting states, using a procedure described in Ref. 7 and the expression

$I=\mathfrak{I}_{0} \omega+\mathfrak{S}_{1} \omega^{3}+1 / 2$.

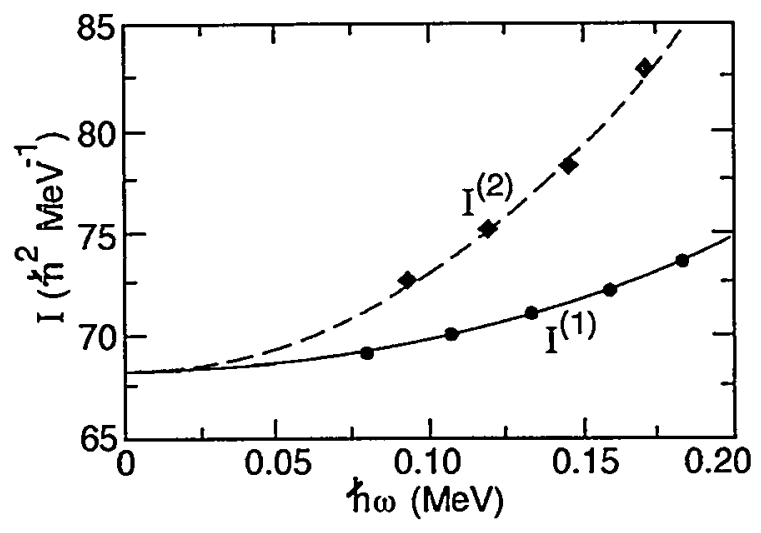

Fig. I-21. Moments of inertia, $\mathfrak{S}^{(1)}$ and $\mathfrak{S}^{(2)}$, for the ground-state band of ${ }^{254} \mathrm{No}$. The lines are fits to the data with Eqs. I, using $\mathfrak{I}_{0}=68.2 \hbar^{2} \mathrm{MeV}^{-1}, \mathfrak{I}_{1}=$ $164.9 \hbar^{2} \mathrm{MeV}^{-3}$.

The spins have even integer values between 6 and 14 (within .01), providing support for assigning the transitions to the ground-state band.

The proposed level scheme of ${ }^{254}$ No is shown in Fig. I-22. We estimate the energies of transitions from the $2^{+}$and $4^{+}$states as 44(1) and 102(1) keV, respectively, using Eq. (2). These $\gamma$ rays were not detected because the states decay almost entirely by internal electron conversion. The deduced transition energies also conform to those extrapolated from neighboring lighter nuclei, providing additional support for the assigned spins.

Lifetimes were not measured. However, the $\mathrm{B}(\mathrm{E} 2)$ values of rotors are related to the $2^{+}$level energies by empirical formulae. ${ }^{8,9}$ By relating the $B(E 2)$, quadrupole moment and deformation, we deduce a quadrupole deformation parameter of $\beta=0.27$ (2) for 254 No. (The uncertainty is given by the systematic deviations between measured $\mathrm{B}(\mathrm{E} 2)$ values in heavy nuclei and those deduced from the empirical relationship of Ref. 9) This value is in agreement with a value of 0.25 given by different macroscopic-microscopic model calculations $5,10,11$, and with respective values of 0.27 and 0.26 from a Hartree-Fock-Bogoliubov (HFB) calculation with the SLy4 force ${ }^{12}$ and from a relativistic Hartree-Bogoliubov calculation with the NL3 Lagrangian parameterization. ${ }^{13}$ Other HFB and relativistic mean-field calculations with other force parameterizations ${ }^{14}$ predict values as large as $\beta=0.3$. 
The moment of inertia is an important quantity for theory to describe, since it is sensitive to the singleparticle energies and pairing. The ground-state moments of inertia were recently calculated for some TFN, 15 and a preliminary value of $42.4 \mathrm{keV}$ has been obtained for the $2^{+}$energy of ${ }^{254} \mathrm{No}$, close to our deduced value of $44 \mathrm{keV}$. The measured moments of inertia of ${ }^{254}$ No increase with spin, as seen in Fig. I21.

The observation of states with spin up to 14 implies that neutron evaporation can compete against fission up to at least that spin. Hence, a fission barrier must still exist up to that angular momentum in 254 No. In fact, preliminary analysis of the multiplicity distribution from our experiment suggests that residues are formed with spin up to $\sim 18$. Our data further imply that the shell-correction energy, which creates the barrier, is reasonably robust with spin. From a pragmatic point of view, they also demonstrate that high-spin states of TFN can be studied by means of (heavy ion, xn) reactions.

In summary, the ground-state band was identified up to spin $12^{+}$(perhaps $14^{+}$) in ${ }^{254} \mathrm{No}$, an isotope of the heaviest element for which high-spin data now exist. This confirms predictions that the TFN gain stability against fission by exploiting the deformation degree of freedom. The deformation parameter, $\beta=0.27$, deduced from the $2^{+} \rightarrow 0^{+}$energy is close to values predicted by several calculations. An increase with frequency in in the moment of inertia is observed. The observation of states with spin up to 14 means that the fission barrier of ${ }^{254}$ No must still exist at least up to that spin. This work was published in Physical Review Letters. ${ }^{16}$

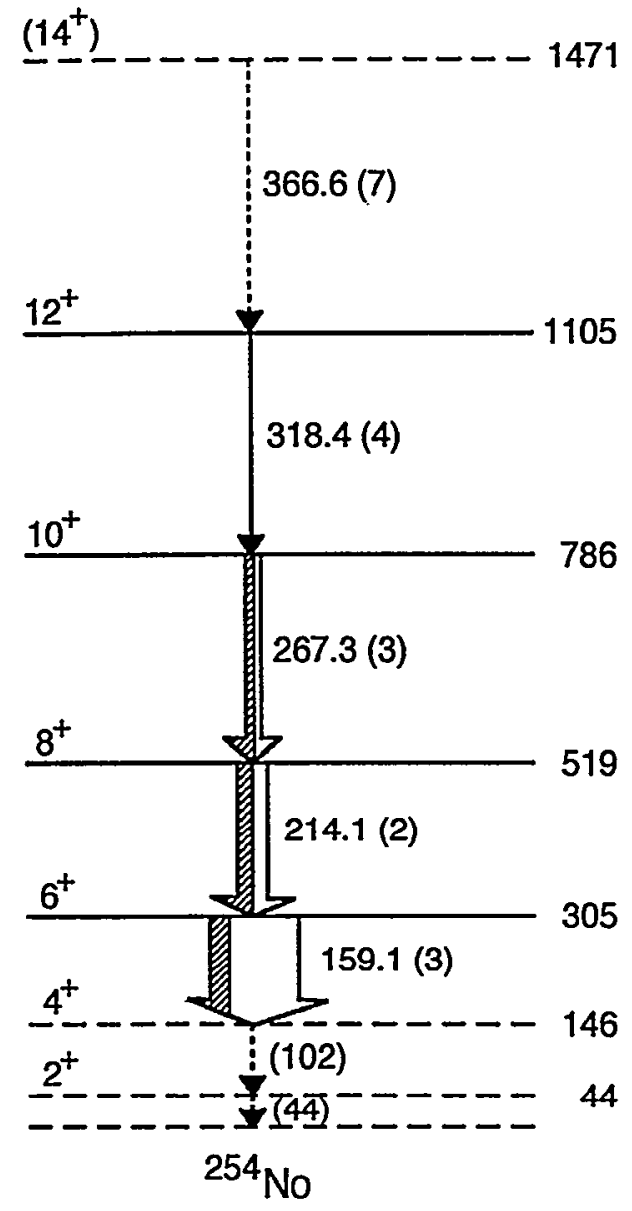

Fig. I-22. Proposed level scheme for the ground-state band of ${ }^{254}$ No. Spins are deduced using Eq. 3; the parity is assumed to be positive. The energies of the lowest two transitions, which were not detected because they decay by internal conversion electrons, were deduced by extrapolation. The $14^{+} \rightarrow 12^{+}$assignment is tentative. The widths of the filled and open arrows are proportional to the $\gamma$ and electron intensities, respectively; the latter were computed by assuming that the transitions have E2 multipolarity. The transition intensities decrease as spin grows, as expected for a (heavy ion, xn) reaction. 
*Argonne National Laboratory and Rutgers University, $\uparrow$ Argonne National Laboratory and University of Oslo, Norway, $\ddagger$ University of Liverpool, England, §Rutgers University, IFlorida State University, IIDAPNIA/SPhN, CEA Saclay, France, ${ }^{* *}$ University of Jyväskylä, Finland, ††Lawrence Berkeley National Laboratory

${ }^{1}$ S. Hofmann, Rep. Prog. Phys. 61, 639 (1998).

2P. Möller and J. R. Nix, J. Phys. G20, 1681 (1994).

${ }^{3}$ R. Smolanczuk et al., Phys. Rev. C 52, 1871 (1995).

${ }^{4}$ S. G. Nilsson et al., Nucl. Phys. A115, 545 (1968).

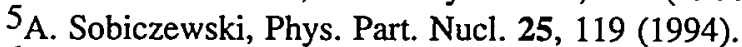

6 H. W. Gäggeler et al., Nucl. Phys. A502, 561c (1989).

7J. E. Draper et al., Phys. Rev. C 42, R1791 (1990); J. Becker et al., ibid. C 46, 889 (1992); G. Hackman et al., Phys. Rev. Lett. 79, 4100 (1997).

${ }^{8}$ L. Grodzins, Phys. Lett. 2, 88 (1962).

${ }^{9}$ S. Raman et al., At. Data Nucl. Data Tables 42, 1 (1989).

${ }_{10}$ S. Cwiok et al., Nucl. Phys. A573, 356 (1994).

11 P. Möller et al., At. Data Nucl. Data Tables 59, 185 (1995).

12 S. Cwiok et al., Nucl. Phys. A611, 211 (1996); W. Nazarewicz and P-H. Heenen, priv. comm. (1998).

${ }^{13}$ G. Lalazissis et al., priv. comm. (1998); Nucl. Phys. A608, 202 (1996).

${ }^{14}$ T. Bürvenich et al., Eur. Phys., in press; J. K. Rutz et al., Phys. Rev. C 56, 238 (1997).

15. Muntian, Z. Patyk and A. Sobiczewski, priv. comm. (1998).

16P. Reiter et al., Phys. Rev. Lett. 82, 509 (1999).

\section{b.18. Formation Mechanism of the $Z=102$ Isotope ${ }^{254}$ No and the Fission Barrier} at High Spin (P. Reiter, T. L. Khoo, C. J. Lister, D. Seweryniak, I. Ahmad, M. Alcorta, M. P. Carpenter, C. N. Davids, G. Gervais, J. P. Greene, W. F. Henning, R. V. F. Janssens, T. Lauritsen, A. A. Sonzogni, D. Sullivan, J. Uusitalo, I. Wiedenhöver, J. A. Cizewski, * S. Siem, $\dagger$ N. Amzal, $\ddagger$ P. A. Butler, $\ddagger$ A. J. Chewter, K. Y. Ding, $\S$ N. Fotiades, $\S$ J. D. Fox, I P. T. Greenlees, $\ddagger$ R.-D. Herzberg, $\ddagger$ G. D. Jones, $\ddagger$ W. Korten,II M. Leino, ${ }^{* *}$ and K. Vetter $\dagger$ )

The nucleus ${ }^{254}$ No derives its stability from a large shell-correction energy, which lowers the ground state, thereby creating a fission barrier. It belongs to the family of heavier deformed superheavy nuclei, which also owe their stability to the same mechanism. Hence, knowledge of the formation mechanism of ${ }^{254}$ No is pertinent to the production of superheavy nuclei, and may lead to. insights for increasing their production cross sections. Whereas the previous section discusses the structure of ${ }^{254} \mathrm{No}$, the present one addresses the production process. Surprisingly, much is still unknown about this process, which may be viewed in two steps: (a) the formation of a compound nucleus and (b) the survival of the latter against fission as it evaporates neutrons. It is not clear if the process is a straightforward fusion reaction, with neutron evaporation from an equilibrated compound nucleus, or if it involves preequilibrium neutron emission, which leaves a colder compound nucleus that is better able to survive against fission. The role of coupled-channel effects in determining the initial angular momentum of the compound system also has to be explored. In step (b), the fission barrier at high spin, which depends sensitively on the shell-correction energy at high angular momentum, determines whether the residue survives against fission. Hence, information on the barrier is not only important for understanding the formation of deformed superheavy nuclei, but also provides a measure of the robustness of the shell structure against rotation.

Data on the fission barrier and on the reaction mechanism come from the intensities of high-spin transitions, as well as the one-dimensional spin and sum-energy distributions and the two-dimensional entry distribution (in spin and energy) of ${ }^{254}$ No evaporation residues. We observed rotational members of the ground band up to spin 14 (see previous section), which immediately establishes that there must be a significant fission barrier up to at least that spin. We also measured the fold (number of firing detectors) vs. total energy associated with the formation of ${ }^{254}$ No residues 

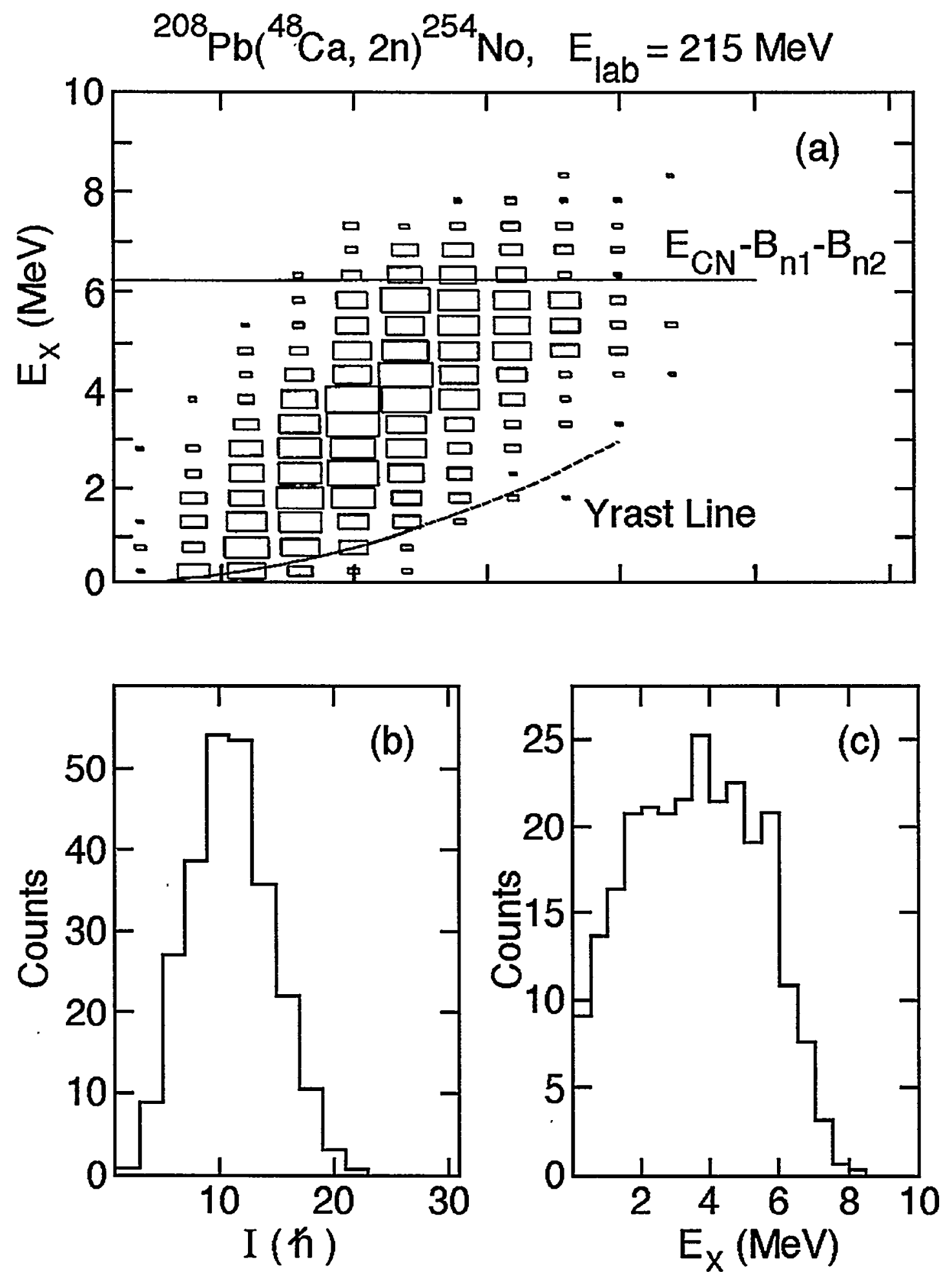

Fig. I-23. (a) Fold versus total energy associated with ${ }^{254}$ No residues (b\&c) Fold and energy distributions obtained from Fig. I-23 (a). These results are preliminary. 
(Fig. I-23a). The projections of this distribution give the fold and sum energy distributions (Fig. I-23b, c). To convert these distributions into entry, spin and sum energy distributions, a calibration of the fold and totalenergy responses of Gammasphere is required. Preliminary analysis suggests that ${ }^{254}$ No residues have, A first comparison points towards agreement with the ${ }^{254}$ No experimental spin distribution. This comparison and inspection of the measured quantities tentatively suggest that a straightforward fusion/evaporation process is involved. The survival of residues up to spin 18 indicates the preservation of a fission barrier up to at least that spin, contradicting the usual assumption that the barriers of superheavy nuclei damp away by spin $15 .{ }^{1}$ These results suggest that the shell structure is robust.
Our approach is to calclate, using a cascade evaporation code, the spin, sum energy and entry distributions for the evaporation residues, and to compare these with the measured quantities. Calculations using the code EVAP have begun. In our first measurements, the low excitation energy of the compound nucleus (19.3 MeV) resulted in a phase space limitation, such that there was insufficient energy to populate states with spin $>22$ after neutron evaporation. Therefore, a follow-up experiment with a larger excitation energy will be conducted, to ascertain if evaporation residues can survive to still higher spin, thereby providing information both on the fission barrier at higher spin and on the formation mechanism.

*Argonne National Laboratory and Rutgers University, † Argonne National Laboratory and University of Oslo, Norway, $\ddagger$ University of Liverpool, England, §Rutgers University, qFlorida State University, IIDAPNIA/SPhN, CEA Saclay, France, **University of Jyväskylä, Finland, ††Lawrence Berkeley National Laboratory

${ }^{1}$ P. Armbruster, Proc. Welch Symposium on Transactinide Nuclei, Houston, 1998.

b.19. Jyväskylä Experiment on Excited States in ${ }^{254}$ No (T. L. Khoo, M. Leino,* F. P. Hessberger, $\dagger$ R.-D. Herzberg, $\ddagger$ Y. Le Coz, $\S$ F. Becker, $\S$ P.A. Butler

A. J. Chewter, $\ddagger$ J. F. C. Cocks, * O. Dorvaux,* K. Eskola, IT J. Gerl, $\uparrow$ P. T. Greenlees $\ddagger$ K. Helariutta, * M. Houry,§ G. D. Jones, $\ddagger$ P. M. Jones, * R. Julin, ${ }^{*}$ S. Juutinen*, H. Kankaanpää,* H. Kettunen,* W. Korten, $§$ P. Kuusiniemi, * R. Lucas,§ M. Muikku,* P. Nieminen, * R. D. Page, $\neq$ P. Rahkila,* P. Reiter,ll A. Savelius, $*$ Ch. Schlegel, $\dagger$ Ch. Theisen, $\S$ W. H. Trzaska, ${ }^{*}$ and H.-J. Wollersheim $\dagger$ )

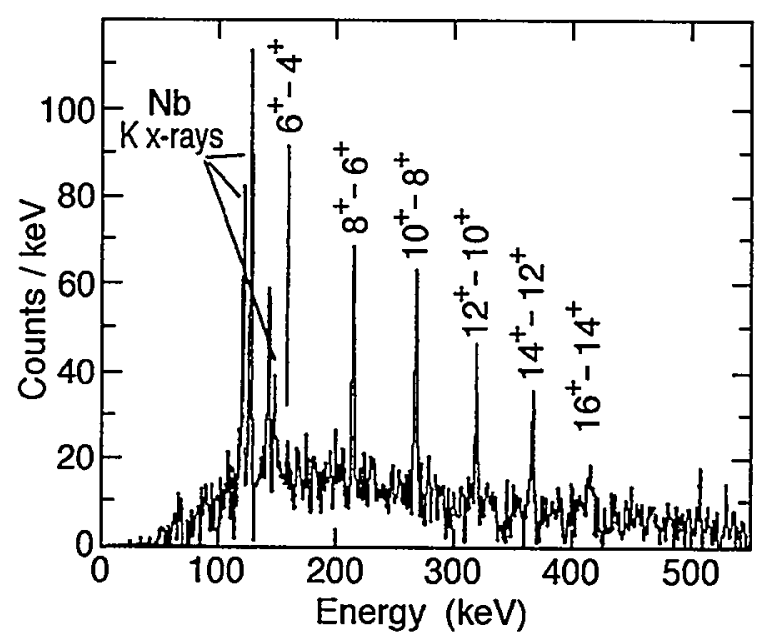

Fig. 1-24. Prompt gamma rays observed in the SARI array in coincidence with fusion products detected in the RITU focal plane particle detector. The assignment of transitions to 254 No is based on their presence in the recoil-decay tagged gamma-ray spectrum.

The nucleus ${ }^{254}$ No is one of the heaviest for which there is a possibility of investigating the excited states. The motivation for studying it was discussed above (see Reiter et al). An experiment was conducted at Jyväskylä, which employed the SARI detector array together with the gas-filled recoil separator RITU to study the structure of 254 No. The production reaction was ${ }^{48} \mathrm{Ca}+{ }^{208} \mathrm{~Pb}$. SARI consisted of four unshielded, segmented clover detectors placed at 50 degrees relative to the beam direction. Stationary $208 \mathrm{~Pb}$ targets of 250 $70-\mu \mathrm{g} / \mathrm{cm}^{2}$ thickness were used. The beam current was $10 \mathrm{pnA}$. An excitation function measurement found the maximum cross section of $\sim 2 \mu \mathrm{b}$ at a beam energy of 
$216 \mathrm{MeV}$ (two-thirds into the target), corresponding to 21-MeV excitation in the compound system. The method of recoil decay tagging (RDT) was used to identify in-beam gamma rays observed in the SARI detectors, on the basis of the $8.09-\mathrm{MeV}$ alpha particles emitted by ${ }^{254}$ No.

The gamma-ray spectrum of ${ }^{254}$ No, tagged by the time of flight and implant energy of the residue, is shown in Fig. I-24. The same transitions are observed in the RDT spectrum but with lower intensity due to the loss of escaping alpha particles. Ground state band transitions are observed up to $414 \mathrm{keV}$. The results confirm the transitions observed in the Argonne experiment and extend the ground band by one transition $\left(16^{+}-14^{+}\right)$.
It is of interest to compare the experiments at Argonne and at Jyväskylä. Compared to SARI, Gammasphere offered larger efficiency ( $-16 \%$ vs. $\sim 10 \%$ at $400 \mathrm{keV})$, significantly larger coincidence efficiency and lower background through Compton suppression. (The figure of merit is given by $[(\mathrm{P} / \mathrm{T})]^{\mathrm{n}}$, where $(\mathrm{P} / \mathrm{T})$ is the peak/total ratio and $n$ the coincidence fold.) On the other hand, compared to RITU, the efficiency of the FMA was 4-6 times smaller for transporting 254 No residues. The mass resolution of the FMA did not provide an advantage in this experiment since essentially only mass 254 was produced. For experiments on very heavy nuclei, the ideal apparatus would be the combination of Gammasphere and a highly efficient fragment separator, such as RITU.

*University Jyväskylä, Finland, †GSI, Darmstadt, Germany, $\ddagger$ University Liverpool, United Kingdom, §DAPNIA/SPhN CEA-Saclay, France, TUniversity of Helsinki, Finland, IILudwig-Maximillians University, Munich, Germany

b.20. New Actinide Signature Measurements (R. V. F. Janssens, I. Wiedenhöver, J. D. Cole,* M. W. Drigert,* R. Aryaeinejad,* E. L. Reber,* J. K. Jewell,* J. H. Hamilton, $\uparrow$ A. V. Ramayya, $\dagger$ C. J. Beyer, $\dagger$ G. M. Ter-Akopian, $\ddagger$ Ts. Yu. Oganessian, $\ddagger$ G. S. Popeko, $\ddagger$ and A. V. Daniel $)$

A set of experiments is underway at the ANL Intense Pulsed Neutron Source to study the radiation emitted from neutron-induced fission of ${ }^{235} \mathrm{U},{ }^{239} \mathrm{Pu}$, and other fissile isotopes important for characterization of $\mathrm{DOE}$ spent nuclear fuel. The first studies of fission fragments using arrays of Compton-suppressed $\mathrm{Ge}$ detectors were carried out in the mid-1980s. These studies showed that there was a great deal of new physics information (new gamma-rays, isotopes, decay modes, neutron and gamma-ray multiplicity) over what was previously studied using singles techniques. Of particular importance was the observation of previously unknown high energy (above $1 \mathrm{MeV}$ ) gamma-ray transitions in the prompt fission fragments. The results of the spontaneous fission measurements were applied to the problem of determining fissile isotopic ratios for arms control issues and are also forming the basis for techniques addressing concerns about the disposal and storage of spent nuclear fuel.

The current set of induced fission data will complement the data on spontaneous fission taken previously. For these measurements, 10 Compton Suppressed $\mathrm{Ge}$ detectors from the Argonne-Notre Dame BGO gammaray facility were moved to the IPNS and installed at the end of a beam line, along with an array of eight liquid scintillator detectors. The first measurement with a target of 2 gram of $93 \%$ enriched $235 \mathrm{U}$ was completed in December 1998 after an accumulated beam time of 10 weeks resulting in a data set of $1.5 \times 10^{9}$ events. A cursory analysis of the data indicates that at least 10 to 15 new isotopes may have been identified. The second measurement with a $96 \%$ enriched, 5 gram ${ }^{239} \mathrm{Pu}$ target will commence in mid February 1999.

*Idaho National Engineering Laboratory, $\uparrow$ Vanderbilt University, $\ddagger$ Joint Institute for Nuclear Research, Dubna, Russia 


\section{b.21. Decay of the Odd-Odd $\mathbf{N}=\mathbf{Z}$ Nuclide ${ }^{78} \mathbf{Y}$ (J. Uusitalo, $C$. $N$. Davids, D. Seweryniak, C. J. Lister, J. Greene, M. Huhta, ${ }^{*}$ P. Mantica, ${ }^{*}$ D. S. Brenner, $\dagger$ B. Tomlin, $\dagger$ J. J. Ressler, $\ddagger$ J. Rikovska, $\ddagger$ and W. B. Walters $\ddagger$ )}

The odd-odd $\mathrm{N}=\mathrm{Z}$ nuclide ${ }^{78} \mathrm{Y}$ was produced ${ }^{1}$ in the ${ }^{40} \mathrm{Ca}\left({ }^{40} \mathrm{Ca}, p n\right)$ reaction at $125 \mathrm{MeV}$. . Recoiling fragments separated by their $\mathrm{A} / \mathrm{Q}$ values were implanted into the tape of a moving tape collector and transported to a shielded position between two plastic $\beta$ detectors and two $G e \gamma$-ray detectors where $\beta-\gamma$ coincidences were recorded as a function of time. Gamma rays with energies of 279 (100\%), 504 (90\%), and $713(40 \%)$ $\mathrm{keV}$, previously identified as yrast transitions in the daughter nucleus ${ }^{78} \mathrm{Sr}$, were observed and found to decay with a half-life of 5.8(6) s. From the relative intensities of the $\gamma$-rays, a spin and parity of $5^{+}$and $T=$
0 are assigned to the parent state in ${ }^{78} Y$ undergoing $\beta$ decay. A production cross section of $4(1) \mu b$ was determined for ${ }^{78} \mathrm{Y}$ by comparision of the yield with those of other reaction products with known cross sections. An upper limit of $500 \mathrm{keV}$ can be set for the energy of this level relative to a possible highly deformed $\mathrm{T}=1,0^{+}$ground state. From this limit, it can be inferred that $\mathrm{T}=1 p_{\mathrm{n}}$ pairing is considerably quenched relative to such pairing in adjacent odd-odd $\mathrm{N}=$ $\mathrm{Z}{ }^{74} \mathrm{Rb}$. Two-quasiparticle rotor model calculations were used to account for the structure of ${ }^{78} \mathrm{Y}$ and adjacent nuclides.

*Michigan State University, $†$ Clark University, $\ddagger$ University of Maryland

1J. Uusitalo et al., Phys. Rev. C 57, 2259 (1998).

\section{b.22. Radioactive Decay of ${ }^{80} \mathrm{Y}$ and Low-Lying States in ${ }^{80} \mathrm{Sr}$ (C. N. Davids, D. Seweryniak, C. J. Lister, L. T. Brown, J. Döring, * H. Schatz,* A. Aprahamian,* R. C. de Haan,* J. Görres,* M. Wiescher,* and W. B. Walters $\dagger$ )}

The $\beta^{+} / \mathrm{EC}$ decay of a mass-separated ${ }^{80} \mathrm{Y}$ source was studied by $\beta$ - and $\gamma$-ray spectroscopy following the bombardment of a thin ${ }^{24} \mathrm{Mg}$ target with ${ }^{58} \mathrm{Ni}$ ions at $190 \mathrm{MeV}$. Figure I-25 shows the resulting extended decay scheme, in which 14 new states in ${ }^{80} \mathrm{Sr}$ were identified including the first observation of negativeparity states. The low-lying positive-parity states were found to be clustered indicating a pattern of one-, two-, and possibly three-phonon multiplets characteristic of an anharmonic vibrator. Some states in ${ }^{80} \mathrm{Sr}$ can also be grouped into a $\gamma$-vibrational band. In general the low-lying states in light strontium isotopes show vibration like collectivity which evolves to rotational behavior with increasing spin and decreasing neutron number. The observed ${ }^{80} \mathrm{Y} \beta$-decay fragmentation and $\log f t$ values were also used to assign spin and parity of $4^{-}$and $1^{-}$to the parent ground state and the $228.5 \mathrm{keV}$ isomeric state, respectively.

*University of Notre Dame, $\uparrow$ University of Maryland 


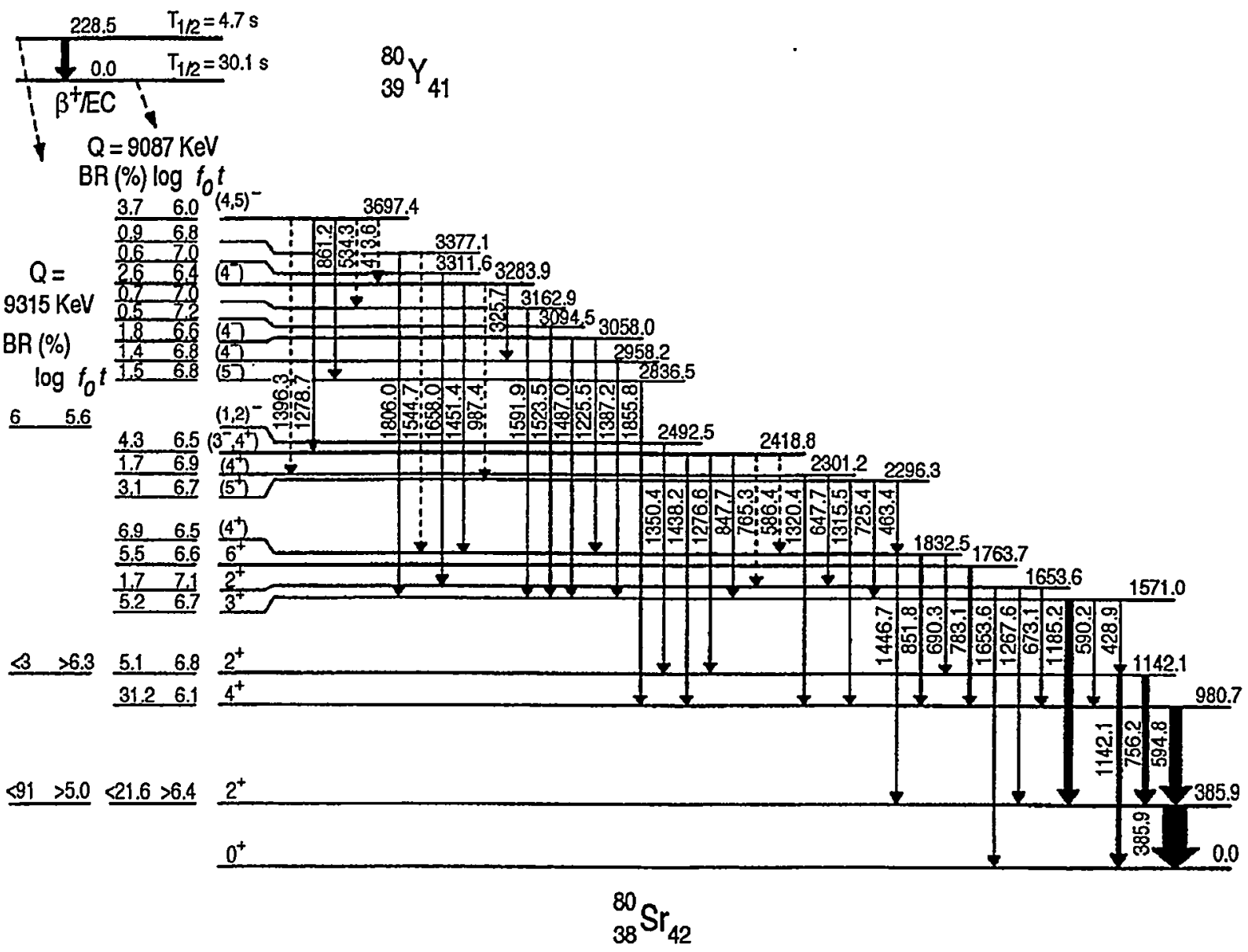

Fig. I-25. Level scheme deduced from the present decay study of $80_{Y} \rightarrow{ }^{80}$ Sr.

\section{b.23. ${ }^{80}$ Y Isomers and Decay (C. J. Lister, D. Seweryniak, C. N. Davids, J. Doering, A. Aprahamian, ${ }^{*}$ R. de Haan, ${ }^{*}$ and W. Walters $\dagger$ )}

An experiment to investigate the $\beta$-decay of $A=80$ isobars was analyzed carefully and published. 1 The original goal, to measure the half-life and decay energy of ${ }^{80} \mathrm{Zr}$ was not achieved in this first study, due to lack of information on states in the daughter ${ }^{80} \mathrm{Y}$. This data set provided the missing information. A $2 \mathrm{~s}, \mathrm{~J} \pi=1^{+}$ isomer was observed for the first time. A second isomer, of 10 microseconds, found in a GANIL experiment, ${ }^{2}$ was placed through our analysis and its most probable spin established. These isomers removed ambiguities from a recent detailed "in-beam" experiment, ${ }^{3}$ and the structure of ${ }^{80} \mathrm{Y}$ is now quite well established. Based on this new information, a new ${ }^{80} \mathrm{Zr}$ experiment has been successfully completed using the Oak Ridge Recoil Mass Spectrometer. ${ }^{4}$ A further paper, reporting new $\beta$-decay branches to states in ${ }^{80} \mathrm{Sr}$ was also published. 5

*University of Notre Dame, †University of Maryland

1J. Doering et. al., Phys Rev. C 57, 1159 (1998).

2P. H. Regan et al., Acta. Physica Polonica B 28, 431 (1997).

${ }^{3}$ D. Bucurescu et. al., Z. Phys. A352, 361 (1995).

${ }^{4}$ W. Walters et al, Private Communication (1999).

5J. Doering et al., Phys. Rev. C 59, 59 (1999). 


\section{b.24. Fine Structure in the Proton Decay of the Highly Deformed Nuclei ${ }^{131} \mathrm{Eu}$ and ${ }^{141 H o}$ (A. A. Sonzogni, C. N. Davids, M. P. Carpenter, J. Uusitalo, D. Seweryniak,* J. Schwartz, † J. Woods, $\ddagger$ J. J. Ressler,§ and W. B. Walters§)}

We have previously observed the proton radioactive decay of ${ }^{131} \mathrm{Eu}$ and ${ }^{141} \mathrm{Ho}^{1}$, and now report the observation of fine structure in the proton decays of these highiy deformed nuclei. Because the daughter nuclei ${ }^{130} \mathrm{Sm}$ and ${ }^{140} \mathrm{Dy}$ are also expected to be highly deformed, their first $2^{+}$states will lie at low excitation energies. Proton decay can proceed to the excited state as well as to the ground state, because the energy available is only slightly less than that for the ground state branch. In addition, the fact that the daughter state has spin-parity $2^{+}$means that lower angular momentum components of the decay can play a role, helping to offset the decrease in decay rate caused by the reduced available energy.

Figure I-26 shows the proton decay spectrum from ${ }^{131} \mathrm{Eu}$, formed in the inverse ${ }^{78} \mathrm{Kr}(402 \mathrm{MeV})+58 \mathrm{Ni}$ reaction. The lower energy peak represents a $\sim 20 \%$ branch feeding the first $2^{+}$state in ${ }^{130} \mathrm{Sm}$ lying at an excitation energy of $120(14) \mathrm{keV}$. This excitation energy fits in well with the extrapolated systematics for $2^{+}$energies in this region, and indicates a deformation of $\beta=0.34$.

In Ref. 1 the ground state decay rates were calculated using the theory of proton decay of deformed nuclei developed by Bugrov and Kadmenskii. ${ }^{2}$ The ${ }^{141} \mathrm{Ho}$ ground state was assigned a Nilsson configuration of $7 / 2-[523]$, while the ${ }^{131} \mathrm{Eu}$ decay rate was consistent with either a $3 / 2^{+}[411]$ or $5 / 2^{+}[413]$ configuration. In both cases the observed decay rates implied quadrupole deformations of 0.3 or greater. The same formalism was used to analyze the fine structure data, producing excellent agreement with the observed ${ }^{141} \mathrm{Ho}$ branching ratio and absolute rate. In the case of ${ }^{131} \mathrm{Eu}$ the calculations indicate that the observed decay rate and fine structure branching ratio can only be explained if the ${ }^{131} \mathrm{Eu}$ ground state configuration has a $3 / 2+[411]$ Nilsson configuration, in agreement with the prediction of Möller et al. ${ }^{3}$

*Argonne National Laboratory and University of Maryland, †Argonne National Laboratory and Yale University, łUniversity of Edinburgh, United Kingdom, §University of Maryland

${ }^{1}$ C. N. Davids et al., Phys. Rev. Lett. 80, 1849 (1998).

2 V. P. Bugrov and S. G. Kadmenskii, Sov. J. Nucl. Phys. 49, 967 (1989).

${ }^{3}$ P. Möller, J. R. Nix, and K.-L. Kratz, At. Data Nucl. Data Tables 66, 131 (1997).

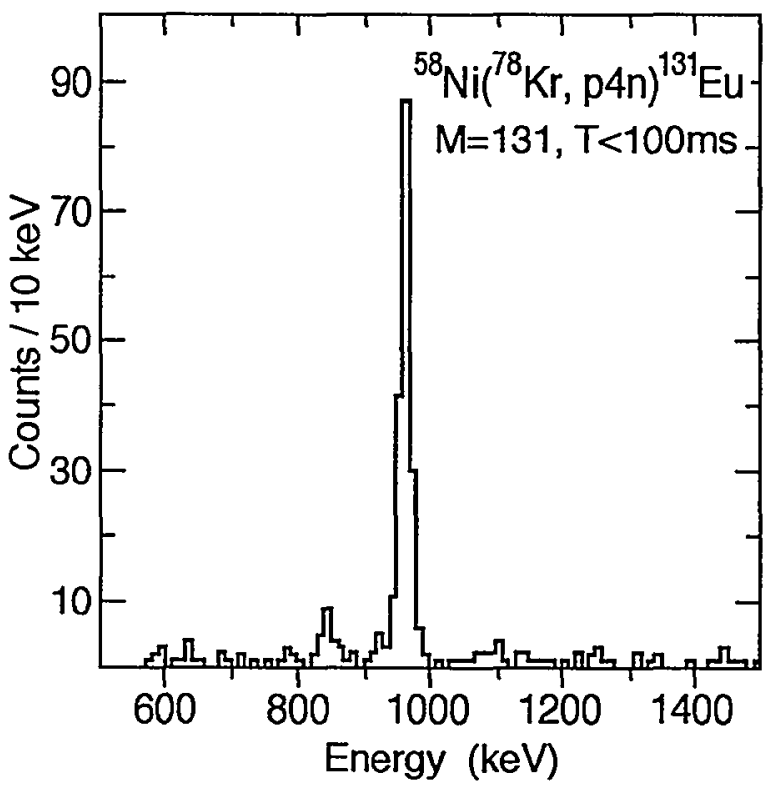

Fig. I-26. Energy spectrum of protons from the decay of ${ }^{131} \mathrm{Eu}$. 


\section{b.25. Decay Properties of New Isotopes ${ }^{172} \mathrm{Hg}$ and ${ }^{173} \mathrm{Hg}$ (D. Seweryniak, J. Uusitalo, M. P. Carpenter, D. Nisius, C. N. Davids, C. R. Bingham, $\dagger$ L. T. Brown, L. Conticchio, D. J. Henderson, R. V. F. Janssens, W. B. Walters,* J. Wauters, $\dagger$ and P. J. Woods $\ddagger$ )}

Within the last several years, a wealth of new information on nuclei lying at and beyond the proton drip line became available in the region of the nucleonic chart between the closed proton shells at $Z=50$ and $Z=$ 82. Much of this work was directed at the identification of new proton emitters in odd- $Z$ nuclei ranging from ${ }^{131} \mathrm{Eu}(\mathrm{Z}=63)$ to ${ }^{185} \mathrm{Bi}(\mathrm{Z}=83)^{1}$. In addition, studies of the alpha-decay properties of proton-rich even- $Z$ nuclei yielded complementary information on nuclei lying at the edges of stability. For example, measurements of alpha-decay energies and lifetimes far from the line of stability is one of the means to discriminate between different mass formulae. Growing uncertainty of different models when moving towards the proton-drip line is reflected in an increasing spread of predicted masses and alpha-decay $Q$ values.

In a recent experiment, the $\alpha$ decays of two neutrondeficient nuclei ${ }^{172} \mathrm{Hg}$ and ${ }^{173} \mathrm{Hg}$ were observed for the first time using the ${ }^{78} \mathrm{Kr}\left({ }^{96} \mathrm{Ru}, 2 \mathrm{n}\right)$ and ${ }^{80} \mathrm{Kr}\left({ }^{96} \mathrm{Ru}, 3 \mathrm{n}\right)$ reactions at the ATLAS accelerator of the Argonne National Laboratory. The reaction products were dispersed according to their mass over charge state ratios in the Fragment Mass Analyzer and implanted in a Double-Sided Si Strip detector where their subsequent decays were studied using the spatial and time correlation between implants and decays. A half-life of $250(-90+350) \mu \mathrm{s}$ and an energy of $7350(12) \mathrm{keV}$ was deduced for the alpha decay of $172 \mathrm{Hg}$. In $173 \mathrm{Hg}$ the half-life was measured to be $0.93(-0.26+0.57) \mathrm{ms}$ and the energy is $7211(11) \mathrm{keV}$. In addition, the half-life and energy of the $\alpha$ decay of ${ }^{174} \mathrm{Hg}^{2}$ was measured with higher precision. The reduced widths deduced for these $\mathrm{Hg}$ isotopes indicate that the observed decays are $\Delta \mathrm{l}=0$ transitions. The $\alpha$-decay $\mathrm{Q}$-values are compared with the calculated values by Möller and $\mathrm{Nix}^{3}$ and Liran and Zeldes. ${ }^{4}$ The latter calculations are in better agreement with the experimental data.

*University of Maryland, †University of Tennessee, $¥$ University of Edinburgh, United Kingdom

1P. J. Woods and C. N. Davids, Ann. Rev. Nucl. Part. Sci. 47, 541 (1997).

2 J. Uusitalo et al., Z. Phys. A358, 337 (1997).

3P. Möller, J. R. Nix, W. D. Myers, and W. J. Swiatecki, At. Data Nucl. Data Tables 59, 185 (1995).

${ }^{4}$ S. Liran and N. Zeldes, At. Data Nucl. Data Tables 17, 431 (1976).

\section{b.26. Identification of ${ }^{180} \mathrm{TI} \alpha$ Decay (C. N. Davids, D. Seweryniak, L. F. Conticchio, L. T. Brown, K. S. Toth, * J. C. Batchelder,$\dagger$ E. F. Zganjar, $\dagger$ C. R. Bingham, $\ddagger$ X.-J. Xu, $\ddagger$ J. Wauters, $\ddagger$ R. J. Irvine, $\S$ and W. B. Walters $\mathbb{D})$}

Using the FMA, the $\alpha$ decay of ${ }^{180} \mathrm{Tl}$ was identified following $92 \mathrm{Mo}$ bombardments of $90 \mathrm{Zr}$. At least three $\alpha$ transitions were observed, correlated with subsequent decays of ${ }^{176} \mathrm{Au}$. They have energies of 6281,6362 , and $6560 \mathrm{keV}$, and half-lives $\sim 1.5 \mathrm{~s}$. In addition, two weak $\alpha$ groups at 6208 and $6470 \mathrm{keV}$ were also correlated with ${ }^{176} \mathrm{Au}$ decay. It was not possible to determine how many decaying levels in ${ }^{180} \mathrm{Tl}$ are responsible for the $\alpha$ decays. In the same experiment, three $\alpha$ transitions were identified with the decay of ${ }^{179} \mathrm{Tl}$, two of which were assigned to originate from the decay of an $\mathrm{h}_{1} 1 / 2$ isomeric state.

*Oak Ridge National Laboratory, †Louisiana State University, $\ddagger$ University of Tennessee, §University of Edinburgh, United Kingdom, TUniversity of Maryland 


\section{SUPERDEFORMATION AND OTHER SPECTROSCOPY TOPICS}

Studies of the properties of nuclei under extreme conditions, and in particular, of superdeformed nuclei remain a topic of intense investigation within the Division. Research over the last few years emphasizes aspects related to the decay out of superdeformed bands as well as those related to the characterization of properties other than transition energies such as quadrupole moments. Other programs utilizing gamma-ray spectroscopy techniques are also summarized here.

\section{c.1. Decay Out of the Doubly Magic Superdeformed Band in the $\mathbf{N}=\mathbf{Z}$ Nucleus}

${ }^{60} \mathrm{Zn}$ (M. P. Carpenter, G. Hackman, R. V. F. Janssens, P. Reiter, D. Seweryniak, C.

E. Svensson,* D. Rudolph, $†$ C. Baktash, $\ddagger$ M. A. Bentley, $\S$ J. A. Cameron,* M. Devlin, $\mathbb{I}$ J. Eberth, | S. Flibotte,* A. Galindo-Uribarri, D. S. Haslip,* D. R. LaFosse,II T. J.

Laman,* I. Y. Lee,** F. Lerma,I A. O. Macchiavelli,** J. M. Nieminen,* S. D. Paul, D. C. Radford, $\ddagger$ L. L. Riedinger, $\dagger \dagger$ D. G. Sarantites, II B. Schaly,* O. Thelen, $\|$ H. G. Thomas, \| J. C. Waddington,* D. Ward,** W. Weintraub,†† J. N. Wilson,Il C. H. Yu, A. V. Afanasjev, $\dagger+\ddagger$ and I. Ragnarsson $\dagger$ )

Investigations throughout the chart of the nuclides led to the observation of many superdeformed (SD) rotational bands in the $A \sim 190,150,80$ and 60 nuclei. Although it was relatively straightforward to observe the long cascades of rotational transitions in these bands with modern gamma-ray arrays, discrete transitions connecting SD bands to states in the first well were more difficult to identify. As a result, the excitation energy, spin, and parity of levels in these superdeformed bands are unknown. Recently, significant progress was made in studying the decay out of SD bands in the A 190 region where the observation of linking transitions in ${ }^{194} \mathrm{Hg}^{1}$ and ${ }^{194} \mathrm{~Pb}^{2}$ led to definite quantum number assignments for $\mathrm{SD}$ states in these nuclei. In addition, a consistent description of the decay out process in A 190 SD bands in terms of a statistical process governed by the weak mixing of SD states with a "sea" of hot ND states was developed. ${ }^{3}$ The nature of the decay out process for SD bands in other mass regions remains an open question.

Recently, an SD band in ${ }^{60} \mathrm{Zn}$ built on the SD shell gaps at $N, Z=30$ was identified for the first time from data taken with the Gammasphere array. Two different experiments were conducted. In the first experiment, a $125-\mathrm{MeV}{ }^{28} \mathrm{Si}$ beam from the 88-Inch Cyclotron at Lawrence Berkeley National Laboratory accelerated onto a ${ }^{40} \mathrm{Ca}$ produced ${ }^{60} \mathrm{Zn}$ via the $2 \alpha$ channel. In the second experiment, a $134-\mathrm{MeV}^{32} \mathrm{~S}$ beam provided by the ATLAS facility at Argonne impinging onto a ${ }^{40} \mathrm{Ca}$ target produced ${ }^{60} \mathrm{Zn}$ via the $3 \alpha$ channel. In both experiments, evaporated charged particles were detected with the Microball, a $4 \pi$ array of CsI scintillators, in order to isolate weak reaction channels.

From the analysis of these data, a previously unknown rotational band was found consisting of 11 transitions. The quadrupole moment, $\mathrm{Q}_{t}$ of the band was measured to be $2.75 \pm 0.45$ eb (see Fig. I-27). The corresponding quadrupole deformation of $0.47 \pm 0.07$ confirm the SD character of the band. Of equal interest is the observation of linking transitions connecting the SD band to the yrast line, thus establishing the spins, parities and excitation energies of the SD states (Fig. I27). Unlike the $A=190$ region where linking transitions are found to have dipole character, these transitions in $60 \mathrm{Zn}$ are dominated by $\Delta I=2 \mathrm{E} 2$ transitions, indicating that the decay-out process in ${ }^{60} \mathrm{Zn}$ differs substantially from that observed in the A 190 region.

A paper reporting the results from these two experiments was published in Physical Review Letters. 4

*McMaster University, Ontario, Canada, $\nmid$ Lund University, Sweden, $\ddagger$ Oak Ridge National Laboratory, §Staffordshire University, Stafford, United Kingdom, qWashington University, |University of Cologne, Germany, **Lawrence Berkeley National Laboratory, ††University of Tennessee, \$Technical University of Munich, Germany

${ }^{1}$ T. L. Khoo et al., Phys. Rev. Lett. 79, 1233 (1996); G. Hackman et al., Phys. Rev. Lett. 79, 4100 (1997).

2 A. Lopez-Martens et al., Phys. Lett. B 380 (1996) 18.

${ }^{3}$ R. G. Henry et al., Phys. Rev. Lett. 73 (1994) 777.

${ }^{4}$ C. E. Svensson et al., Phys. Lett. 82 (1999) 3400. 

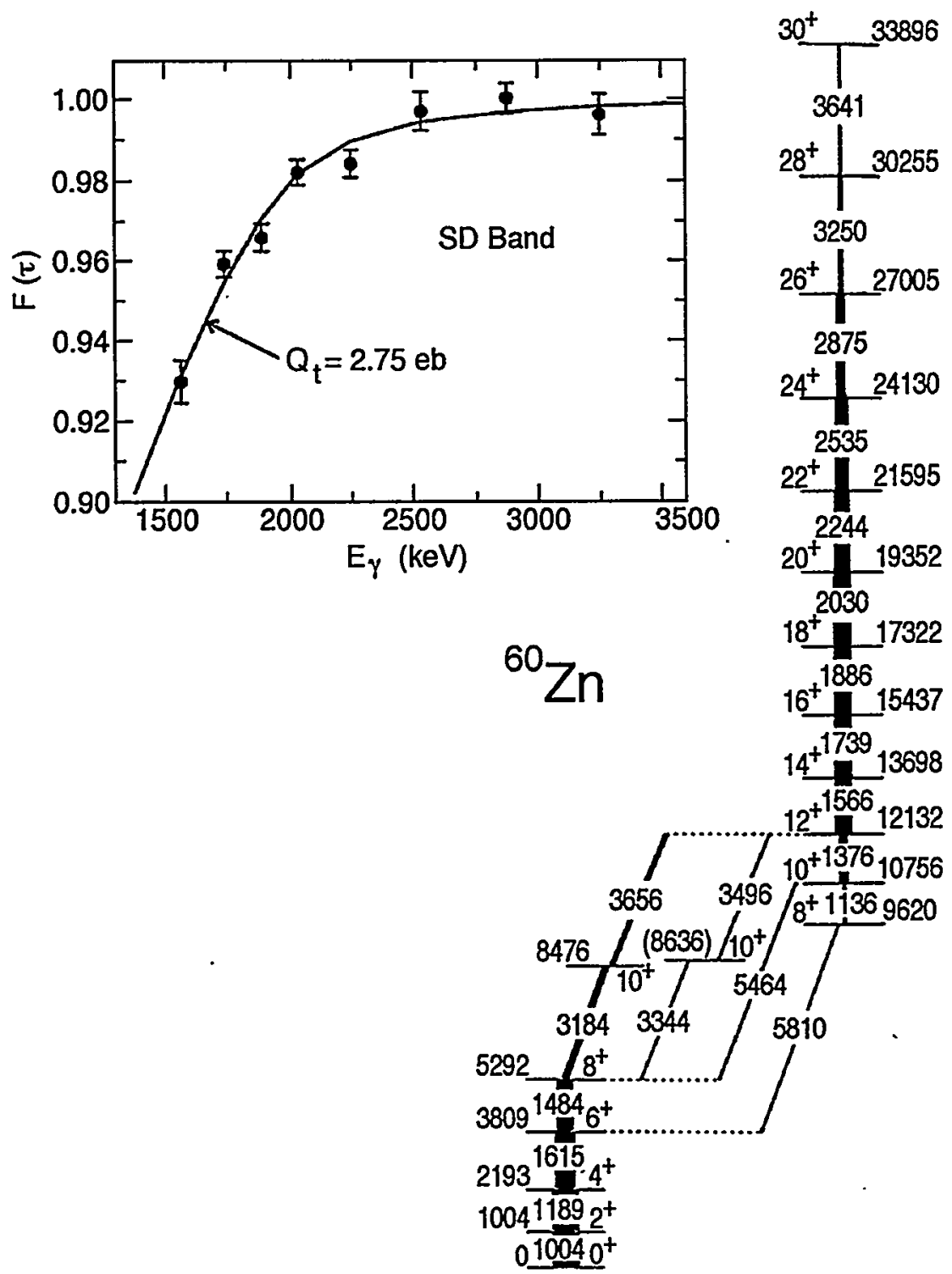

Fig. I-27. Partial decay scheme for ${ }^{60} \mathrm{Zn}$ showing the newly discovered superdeformed band and its decay. The inset shows the quadrupole moment determination.

c.2. Level Structures at High Spins in Nuclei near and at $\mathbf{N}=52$ (M. P. Carpenter, B. Crowell, R. V. F. Janssens, T. L. Khoo, T. Lauritsen, S. S. Ghugre,* U. Garg,*

B. Kharraja,* G. Smith,* W. Mueller, $\uparrow$ W. Reviol, $\uparrow$ L. L. Riedinger, $\uparrow$ and

R. Kaczarowski $\ddagger$ )

The level sequences in the $\mathrm{N}=50$ isotones of $\mathrm{Mo}, \mathrm{Tc}$, $\mathrm{Ru}$ and $\mathrm{Rh}$ nuclei exhibit single particle nature even at high spins $(I \sim 20 \hbar)$ and excitation energies $\left(E_{X} \sim 12\right.$ $\mathrm{MeV})$. On the other hand, rotational excitations are observed in nuclei with $\mathrm{N}>54$. Thus, the study of high spin states of the $\mathrm{N}=52,53,54$ isotones of $\mathrm{Mo}$, $\mathrm{Tc}, \mathrm{Ru}$ and $\mathrm{Rh}$ would greatly help in understanding the mechanism for the generation of the higher angular momentum states in this region, and to observe the transition from the spherical single-particle mode to the collective degrees of freedom. High-spin states in $94,95_{\mathrm{Mo}}, 94,95,96 \mathrm{Tc}, 96,97,98_{\mathrm{Ru}}$ and $97,98_{\mathrm{Rh}}$ were populated using a $142 \mathrm{MeV}{ }^{36} \mathrm{~S}$ beam on a ${ }^{65} \mathrm{Cu}$ 
target. Triple- $\gamma$ coincidences were measured using the early implementation phase of Gammasphere.

Several new transitions were identified in these nuclei, and the level sequences were substantially extended (up to a spin of $I \sim 20 \hbar$ and an excitation energy of $E_{X} \sim$ $12-14 \mathrm{MeV}$ ). Shell model calculations, using different model spaces and effective interactions, were carried out for several of these nuclei. The $\mathrm{N}=51,52$ isotones exhibit single particle nature and their higher angular momentum states are dominated by the excitation of a single g $_{9 / 2}$ neutron across the $\mathrm{N}=50$ magic shell. The "breaking" of the $\mathrm{N}=50$ core is evidenced by a number of transitions of $-2 \mathrm{MeV}$ followed by a fragmentation of intensity into several parallel cascades. The level sequences of the $N=53,54$ isotones, on the other hand, begin to indicate an onset of collectivity. The weakcoupling model has been quite successful in interpreting the level sequences of these nuclei. Vibrational model calculations as well as lifetime measurements would greatly facilitate a more complete understanding of the level sequences of these nuclei.

Three papers describing the results on the Mo, Ru and $\mathrm{Rh}$ nuclei studied were written and published in 1998. $1-3$

*University of Notre Dame, †University of Tennessee, $¥$ The Andrzej Soltan Institute for Nuclear Studies, Swierk, Poland

${ }^{1}$ B. Kharraja et al., Phys. Rev. C 57, 83 (1998).

${ }^{2}$ B. Kharraja et al., Phys. Rev. C 57, 2903 (1998).

${ }^{3}$ S. S. Ghugre et al., Phys. Rev. C 58, 3243 (1998).

\section{c.3. Quadrupole Moments and Identical Superdeformed Bands in ${ }^{149} \mathrm{~Tb}$ (R. V. F Janssens, M. P. Carpenter, S. M. Fischer, T. L. Khoo, T. Lauritsen, D. Nisius, B. Kharraja,* U. Garg,* H. Jin, * R. J. Ernst, * S. S. Ghugre,* E. F. Moore, $\dagger$ Th. Byrski, R. Kruecken,§ A. O. Macchiavelli,§ R. MacLeod,§ R. Kaczarowski,Il and I. M. Govilli)}

Five superdeformed (SD) bands were observed in ${ }^{149} \mathrm{~Tb}$, using the Gammasphere spectrometer. The measurement was performed with a backed target to obtain lifetime information from a DSAM analysis. It is proposed that the yrast $\mathrm{SD}$ band corresponds to the ${ }^{148} \mathrm{Gd}$ (yrast) $\times \pi 6^{3}$ intruder configuration. The first excited band (band 2) is found to be identical to the ${ }^{150} \mathrm{~Tb}$ yrast $\mathrm{SD}$ band. Band 3 is proposed to correspond to the $\pi[301] 1 / 2(\alpha=-1 / 2) \rightarrow[651] 3 / 2(\alpha=-1 / 2)$ proton-hole excitation coupled to the $\pi 6^{4} \vee([651] 1 / 2)^{-1} \vee 7^{1}$ intruder configuration. Bands 4 and 5, the weakest SD bands seen in the present work, show similarities with band 3 and are most likely associated with a similar intruder configuration. These results were published. 1

*University of Notre Dame, $\uparrow$ North Carolina State University, $\ddagger$ Institut de Recherches Subatomiques, Strasbourg, France, §Lawrence Berkeley National Laboratory, ISoltan Institute for Nuclear Studies, Swierk, Poland, IIPanjab University, Chandigarh, India

${ }^{1}$ B. Kharraja et al., Phys. Rev. C 58, R2640 (1998).

\section{c.4. Differential Quadrupole Moment Determinations in Superdeformed 151,152,153Dy (D. T. Nisius, R. V. F. Janssens, I. Ahmad, H. Amro, M. P. Carpenter, S. M. Fischer, G. Hackman, T. L. Khoo, T. Lauritsen, P. Reiter, E. F. Moore, ${ }^{*}$ B. Haas, † P. Fallon, $\ddagger$ R. M. Clark, $\ddagger$ and R. Krücken $\ddagger)$}

Some time ago, this collaboration published results of differential lifetime measurements in the superdeformed (SD) bands of $151,152 \mathrm{Dy}$ and ${ }^{151} \mathrm{~Tb} .{ }^{1}$ The main results can be summarized as follows: (1) differences in the quadrupole moments $\mathrm{Q}_{0}$ of the yrast $\mathrm{SD}$ bands in the three nuclei can be understood in terms of shape polarization effects by high- $\mathrm{N}$ intruder orbitals, in agreement with theoretical expectations, ${ }^{2,3}(2)$ the $Q_{0}$ 
moments of the excited SD bands in ${ }^{151}$ Dy proved important in proposing associated configurations and, surprisingly, (3) a slow sidefeeding component was found in the case of the ${ }^{152}$ Dy SD band, but not in the other cases.

In a new DSAM experiment, the ${ }^{120} \mathrm{Sn}\left({ }^{34} \mathrm{~S}, 4-\right.$ $5 n)^{152,151}$ Dy and ${ }^{122} \mathrm{Sn}\left({ }^{34} \mathrm{~S}, 5-6 \mathrm{n}\right){ }^{153,152}$ Dy reactions were studied at Gammasphere. The targets consisted of $1-\mathrm{mg} / \mathrm{cm}^{2}$ layers of isotopic $\mathrm{Sn}$ material evaporated onto a backing consisting of $70 \mu \mathrm{g} / \mathrm{cm}^{2}$ of $\mathrm{Al}$ and $15 \mathrm{mg} / \mathrm{cm}^{2}$ of Au. Fractions of full Doppler shift $F(\tau)$ were obtained for the yrast SD bands in $151,152 \mathrm{Dy}$ in the first reaction and for the $152 \mathrm{Dy}$. yrast SD band and the five SD bands in ${ }^{153}$ Dy in the second reaction. The analysis was performed with the code FITFTAU. ${ }^{1}$

Because of the difference in the reaction Q-values, the 152 Dy nucleus is produced at high excitation energy in the reaction on the ${ }^{120} \mathrm{Sn}$ target and at much lower excitation with the ${ }^{124} \mathrm{Sn}$ target. The results for the yrast SD bands in 151,152 Dy from the ${ }^{120}$ Sn target are in excellent agreement with the previous measurement, 1 including the presence of a time delay in the SD feeding for ${ }^{152} \mathrm{Dy}$. In contrast, while the $\mathrm{Q}_{0}$ moments obtained for ${ }^{152} \mathrm{Dy}$ in both reactions are within errors, no time delay was found in the side feeding for the $\left({ }^{34} \mathrm{~S}, 6 \mathrm{n}\right)$ reaction.

The ${ }^{153}$ Dy yrast SD band is interpreted as corresponding to the occupation of an additional $j_{15 / 2}$ neutron intruder, with respect to the ${ }^{152} \mathrm{Dy}$ core. Rather than the expected increase in the $Q_{0}$ moment $^{2}$ from the addition of a shape driving orbital, a decrease is observed: $\mathrm{Q}_{0}$ (band1) $=16.8 \pm 0.6 \mathrm{eb}$ vs $\mathrm{Q}_{0}\left({ }^{152} \mathrm{Dy}\right)=$ $18.2 \pm 0.3$ eb. Signature partner bands 2 and 3 are known to have identical dynamic moments of inertia to the ${ }^{152}$ Dy yrast SD band. The measured moments $\mathrm{Q}_{0}($ band2 $)=16.6 \pm 0.5 \mathrm{eb}$ and $\mathrm{Q}_{0}($ band 3$)=16.9 \pm 0.6$ eb are equal within errors, but are smaller than the moment of the identical ${ }^{152}$ Dy SD band. Finally, signature partner bands 4 and 5 exhibit a smaller moment $\mathrm{Q}_{0}$ (band4) $=13.9 \pm 1.0$ eb and $\mathrm{Q}_{0}$ (band5) $=$ $14.0 \pm 1.0 \mathrm{eb}$, much reminiscent of the values found in the Gd SD bands. Thus, the available experimental evidence points towards a "shrinking" of the mean field above the doubly magic 152 Dy SD nucleus. Calculations attempting to understand these results are underway.

*North Carolina State University and Triangle Universities Nuclear Laboratory, $\dagger$ Institut de Recherches

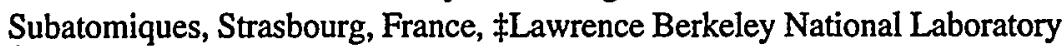

${ }_{1}$ D. Nisius et al., Phys. Lett. B392, 18 (1997).

2 W. Satula et al., Phys. Rev. Lett. 77, 5182 (1996).

${ }^{3}$ W. Savajols et al., Phys. Rev. Lett. 76, 4480 (1996).

\section{c.5. Search for Hyper-Deformation in ${ }^{151,152} \mathrm{Dy}$ (T. Lauritsen, T. L. Khoo, I. Ahmad, M. P. Carpenter, R. V. F. Janssens, C. J. Lister, A. Lopez-Martens, * H. Amro, $* \$$ S. M. Fischer, $§$ G. Hackman, F. Hannachi,* A. Korichi,* and E. F. Moore $\dagger$ )}

Several attempts were made to experimentally verify the existence of a hyper-deformed (HD) minimum in the nuclear potential energy surface. Such a minimum, created by shell effects and stabilized by rapid rotation, has been predicted by a number of theorists, 1,2 and is calculated to become yrast at very high spins ( $>70 \hbar$ ). None of the attempts has been successful so far.

Using the combination of Gammasphere and the FMA, a new search for HD was carried out in the fall of 1998.
Using the reaction ${ }^{76} \mathrm{Ge}\left({ }^{34} \mathrm{Se}, \mathrm{xn}\right)$ several Dy isotopes were populated at a much higher than usual energy and angular momentum (in the so called "over-shoot" mode). For example, ${ }^{152} \mathrm{Dy}$ was created at a spin of more than $75 \hbar$. Fission dominates at such high spins - but only at such high spins might the $\mathrm{HD}$ minimum be populated. The FMA was used to cleanly select the few nuclei that against all odds did not fission, but instead formed Dy residues at these high spins.

*CSNSM, Orsay, France, $\uparrow$ North Carolina State University, $\ddagger$ IReS, Strasbourg, France, §University of Pennsylvania ${ }^{1}$ Dudeck et al., Phys. Rev. B 211, 252 (1988).

${ }^{2}$ Chasman, Phys. Lett. B302, 134 (1993). 
It is hoped that with the residue mass gating of the FMA, as well as on events that also recorded a high fold and summed energy in the Gammasphere array, signatures of $\mathrm{HD}$ can be found. A search for HD ridges and planes in gamma-gamma matrices and gammagamma-gamma cubes is ongoing. The quasi-continuum of gamma rays in coincidence with the superdeformed bands in 152,151 Dy were extracted as well and should allow for a determination of the spin and energy of these bands using the quasi-continuum method. This analysis is in progress.

\section{c.6. Second Proton and Neutron Crossings in the Doubly-Odd 154,156 Tb Nuclei, High-Spin Structure of ${ }^{155}$ Tb (S. M. Fischer, R. V. F. Janssens, D. T. Nisius, D. J. Hartley,* J. L. Allen,* T. B. Brown,* F. G. Kondev,* J. Pfohl,* M. A. Riley,* P. Fallon, $\uparrow$ W. C. Ma, $\ddagger$ and J. Simpson§)}

High-spin states in the doubly-odd nuclei $154,156_{\mathrm{Tb}}$ were populated in two separate experiments at Gammasphere using the same ${ }^{36} \mathrm{~S}+{ }^{124} \mathrm{Sn}$ reaction at different beam energies. Yrast structures of both nuclei were extended to much higher spin $(1 \sim 48 \hbar)$ and several quasiparticle alignments were identified including the second neutron alignment and a clear delineation of the second proton crossing in ${ }^{156} \mathrm{~Tb}$. These results add to the systematics of these crossings for odd- $Z$ nuclei in the beginning of the rare earth region. The results can be understood in cranked shell model calculations. A manuscript was submitted for publication.

The reactions ${ }^{152} \mathrm{Sm}\left({ }^{7} \mathrm{Li}, 4 \mathrm{n}\right)$ at $45 \mathrm{MeV}$ and ${ }^{124} \mathrm{Sn}\left({ }^{36} \mathrm{~S}, \mathrm{p} 4 \mathrm{n}\right)$ at $165 \mathrm{MeV}$ were used to produce high-spin states of the $\mathrm{N}=90$ nucleus ${ }^{155} \mathrm{~Tb}$.
Previously known bands have been greatly extended in spin $(I-45 \hbar)$ and a new decoupled sequence was identified. Several band crossings or quasiparticle alignments were observed in each of the structures and allowed a configuration assignment to be given to the new band. $B(M 1) / B(E 2)$ transition strength ratios were extracted from the data and comparisons were made to theoretical predictions. A comprehensive analysis of the signature splitting in the energy levels and $B(M 1) / B(E 2)$ ratios from the proton $h_{11 / 2}$ bands of the A $\sim 160$ region has been made. The latter splitting apparently has (among other possible quantities) a large dependence on the quadrupole deformation and on the placement of the proton Fermi surface. A manuscript reporting these results was recently published. ${ }^{1}$

*Florida State University, †Lawrence Berkeley National Laboratory, $\$$ Mississippi State University, §Daresbury Laboratory, United Kingdom

${ }^{1}$ D. J. Hartley et al., Phys. Rev. C 58, 2720 (1998). 


\section{c.7. Search for Hyper-Deformation and Triaxial Superdeformed Bands in Hf Isotopes (T. Lauritsen, M. P. Carpenter, B. Herskind,* M. Bergstrom,* S. Bonneau,* G. B. Hageman,* G. Sletten,* S. Tormanen,* P. G. Varmette,* S. Odegaard,* M. A. Deleplanque-Stephens, $\uparrow$ I. Y. Lee $\uparrow$ F. Stephens, $\uparrow$ L. L. Riedinger, $\uparrow$ H. Hubel, $\S$ W. C. Ma,I A. Maj,ll A. Bracco,** F. Camera, ${ }^{* *}$ S. Frattini, $* *$ S. Leoni, ${ }^{* *}$ R. Bengtsson, $\dagger \dagger$ C. Fahlander, $\dagger \dagger$ and H. Ryde $\dagger \dagger$ )}

A search was performed for hyper-deformation (HD) in the region where the deepest $\mathrm{HD}$ well was calculated. 4,5 The particular reaction used was selected to be semisymmetric as well as 'cold' to enhance the probability for observing hyper-deformation. ${ }^{76} \mathrm{Ge}$ at $310 \mathrm{MeV}$ from ATLAS impinged on ${ }^{96} \mathrm{Zr}$ foils mounted on the Gammasphere target wheel and prompt gamma rays were observed with Gammasphere's 100 detectors. $\mathrm{Zr}$ targets are not very sturdy. Thus, the ATLAS beam was 'wobbled' in both vertical and horizontal direction to give a square beam spot of approximately $1 \mathrm{~cm} \times 1$ $\mathrm{cm}$. Using this technique 5 pna of beam could be deposited on the target. The $168-170 \mathrm{Hf}$ nuclei produced in the reaction should, apart from being populated hyper-deformed, also be populated in triaxial superdeformed pockets in the nuclear energy surface. Thus, triaxial superdeformed bands should be populated in these nuclei and a search for those is going on as well. Such bands have been seen in neighboring lutetium isotopes. $1-3$

*Niels Bohr Institute, Copenhagen, Denmark, †Lawrence Berkeley National Laboratory, $\ddagger$ University of Tennessee, §University of Bonn, Germany, TMississippi State University

INiewodniczanski Institute of Nuclear Physics, Krakow, Poland, **Universita di Milano, Italy, ††University of

Lund, Sweden

IW. Schmitz et al., Phys. Lett, B203, 397 (1993).

${ }^{2}$ H. Schnack-Petersen et al., Nucl. Phys. A594, 175 (1995).

${ }^{3}$ W. Schmitz et al., Nucl. Phys. A539, 112 (1992).

${ }^{4}$ Dudeck et al., Phys. Rev. B 211, 252 (1988).

${ }^{5}$ Chasman, Phys. Lett. B302, 134 (1993).

c.8. High-K Isomers in Neutron-Rich Hafnium Nuclei (I. Ahmad, M. P. Carpenter, G. Hackman, R. V. F. Janssens, T. L. Khoo, C. J. Lister, D. Nisius, P. Reiter, D. Seweryniak, I. Wiedenhöver, R. D'Alarcao,* P. Chowdhury,* E. Seabury,* P. M. Walker, $†$ and C. Wheldon $\dagger$ )

The conditions under which $\mathrm{K}$, the projection of the aligned nucleonic spins on the symmetry axis in deformed nuclei, is a good quantum number remain a topic of much current interest. In a previous publication, we reported on our success in populating new multi-qp isomers in the heaviest stable isotopes of $\mathrm{Lu}, \mathrm{Ta}$ and $\mathrm{W}$ using inelastic exeitation with ${ }^{238} \mathrm{U}$ beams. ${ }^{1}$ Here we report the results of our follow-up experiments with $\mathrm{Hf}$ targets. High- $\mathrm{K}$ isomers have long been predicted ${ }^{2}$ in isotopes heavier than ${ }^{180} \mathrm{Hf}$, the heaviest stable Hf isotope.

In our primary experiment, thick targets of isotopically enriched $180 \mathrm{Hf}$ and natural $\mathrm{Hf}$ were bombarded with pulsed $1.6-\mathrm{GeV}{ }^{238} \mathrm{U}$ beams from ATLAS, swept on and off in three different time periods in the microsecond-to-millisecond range, with a $20 \%$ duty cycle. Only delayed gamma rays were recorded in the beam-off periods by the ANL-Notre Dame BGO array. The results were clarified and extended by the more recent data obtained in conjunction with tests of the Gammasphere array operated with the beam sweeper at ATLAS, where a $1.3-\mathrm{GeV}{ }^{208} \mathrm{~Pb}$ beam was incident on the ${ }^{180} \mathrm{Hf}$ target for a few hours. The level schemes of

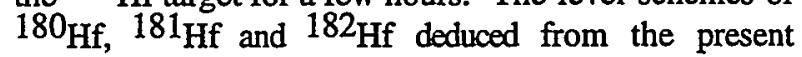
experiment are shown in Fig. I-28.

*University of Massachusetts, †University of Surrey, United Kingdom

${ }^{1}$ C. Wheldon et al., Phys. Lett. B425, 239 (1998).

${ }^{2}$ S. Aberg, Nucl. Phys. A306, 89 (1978). 


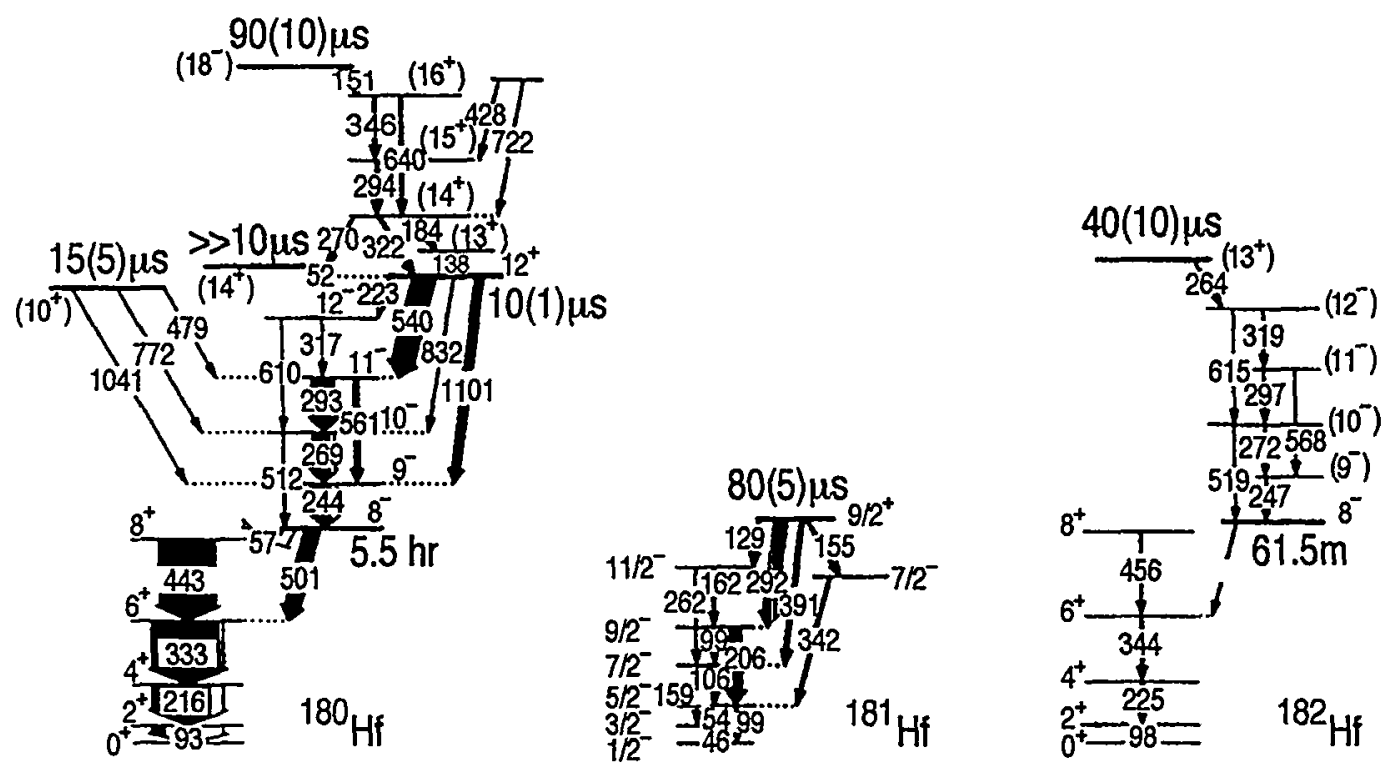

Fig. I-28. Decay scheme of new high-K isomers in $180,181,182 \mathrm{Hf}$. New data from the present work include all states above the $8^{-}$isomers in $180,182 \mathrm{Hf}$, and above $I=7 / 2$ in $181 \mathrm{Hf}$. The energy scale for ${ }^{181} \mathrm{Hf}$ is expanded by $a$ factor of 2.

A total of six new isomers were observed in the three nuclei, of which four involve four quasiparticles. Three of the four are assigned to $180 \mathrm{Hf}$ and one to $182 \mathrm{Hf}$. The decay pattern of the most strongly populated isomer in $180 \mathrm{Hf}$ ( 10 microsecond half-life) provides a classic example of $\mathrm{K}$-hindrances via the competition between an E3 and an E1 transition, and highlights the robustness of the $\mathrm{K}$ quantum number in this case.
Measured branching ratios of new rotational bands populated in this study allow configuration assignments to the isomers on which the bands are built. New isomers were also observed in the neutron-rich $\mathrm{Hf}$ nuclei via neutron transfer (Fig. I-28). The new data explore the role of residual spin-spin interactions for multi-qp configurations at the neutron-rich edge of the $\beta$ stability line. The results of this investigation were published. ${ }^{3}$

\section{c.9. Superdeformed Bands in ${ }^{189} \mathrm{Tl}$ (M. P. Carpenter, R. V. F. Janssens, D. Nisius, W. Reviol, * L. L. Riedinger,* E. D. Ellis, ${ }^{*}$ P. Fallon, $\dagger$ and S. M. Fischer $\left.\ddagger\right)$}

On several occasions (e.g. Ref. 1), we reported about a tentatively assigned superdeformed (SD) band in ${ }^{189} \mathrm{Tl}$ that seemed to be present in data taken with Early Implementation Gammasphere. However, the statistical accuracy of these data was not sufficient to be sure that the seven reported transitions were in cascade. Very recently, we could confirm the presence of this band and observe its signature partner during a 1-day run with the full Gammasphere (Fig. I-29). For this experiment, Gammasphere comprised 102 Compton-suppressed $\mathrm{Ge}$ detectors. A $172-\mathrm{MeV}{ }^{37} \mathrm{Cl}$ ion beam from the 88 -in. cyclotron was used to populate high-spin states in ${ }^{189} \mathrm{Tl}$ via the $4 \mathrm{n}$ evaporation channel. The target consisted of two self-supporting ${ }^{156} \mathrm{Gd}$ foils of thickness $0.6 \mathrm{mg} / \mathrm{cm}^{2}$ each. A total of 800 million triple and higher fold $\gamma$-ray coincidences were collected.

*University of Tennessee, †Lawrence Berkeley National Laboratory, $\ddagger$ University of Pennsylvania

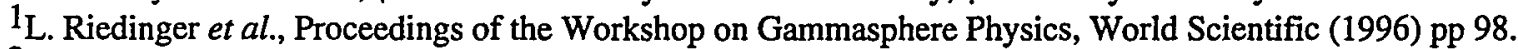

${ }^{2}$ W. Satula et al., Nucl. Phys. A529, 289 (1991).

${ }^{3}$ W. Reviol et al., Phys. Rev. C 58, R2644 (1998). 


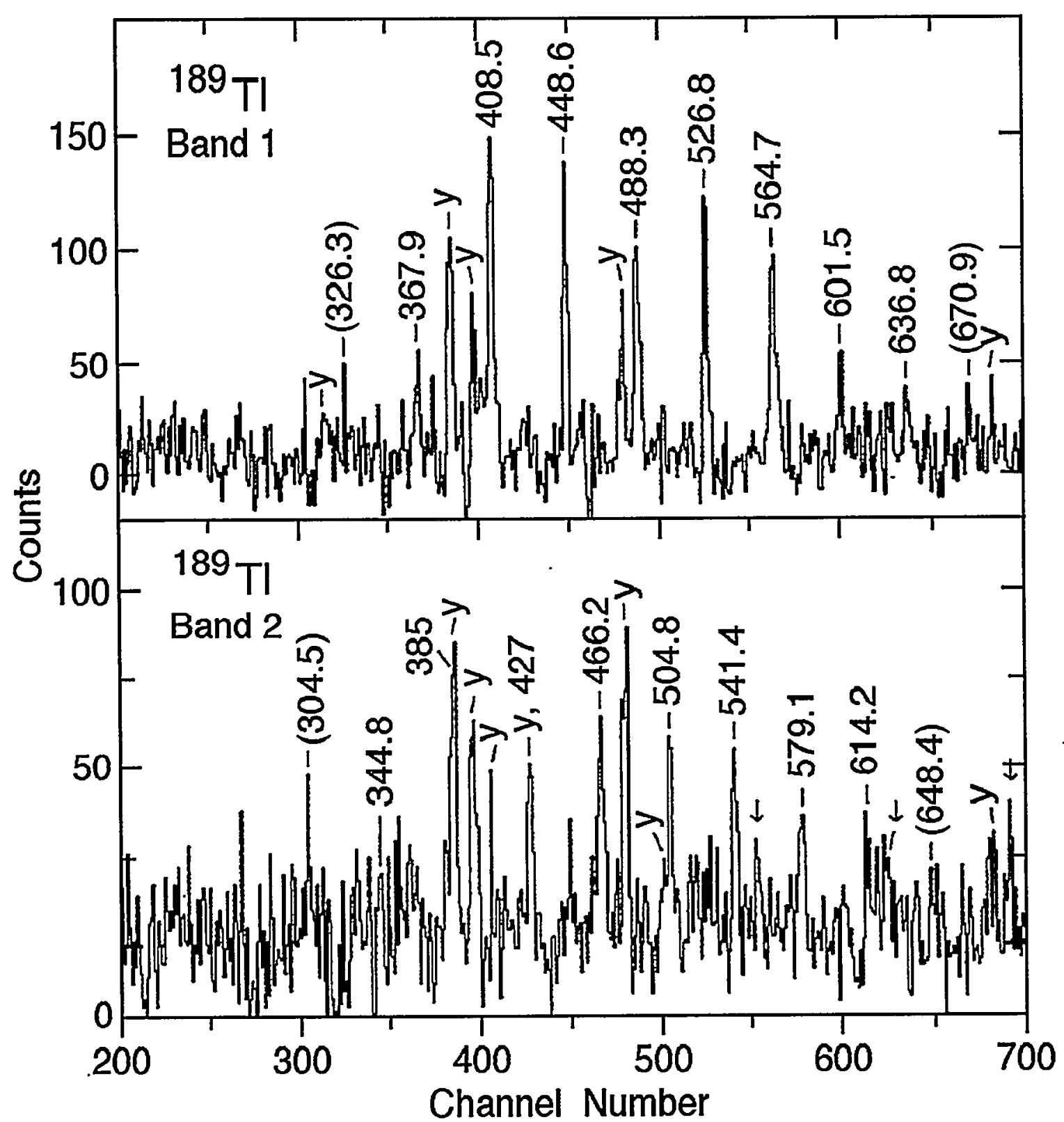

Fig. I-29. The two superdeformed bands of ${ }^{189} \mathrm{Tl}$. Yrast transitions are labeled " $Y$ ", while newly observed nearyrast transitions are indicated by an arrow.

The observed signature partner bands in ${ }^{189} \mathrm{Tl}$ are proposed to be based on the 5/2[642] $\left(i_{13 / 2}\right)$ proton configuration and extends this superdeformed region down to $N=108$. Interestingly, at this edge of the $\mathrm{A}$ $190 \mathrm{SD}$ region a gradual decrease of the quadrupole deformation $\left(\beta_{2}\right)$ compared to the central nuclei $(N=$ 112 ) is predicted (e.g. Rref. 2). A possible indication for this deformation trend could be differences in the
$\mathfrak{I}(2)$ moments of inertia between different isotopes which are now most significant for the series of the $189-195 \mathrm{Tl}$ nuclei. A fit of the $\mathfrak{S}^{(2)}$ values for ${ }^{193} \mathrm{Tl}$ gives a Harris parameter $\mathfrak{I}_{0}=95 \hbar^{2} / \mathrm{MeV}$ (proportional to $\beta_{2}$ ), while for ${ }^{189} \mathrm{Tl}$ a value of $90 \hbar^{2} \mathrm{MeV}$ is obtained. This decrease in $\mathfrak{I}_{0}$ of about $5 \%$ is in line with a similar drop in $\beta_{2}$. A paper reporting these results was recently published in Physical Review C. $^{3}$ 


\section{c.10. Fluctuations in the Strengths of Primary Transitions from Decay Out of a Superdeformed Band (T. L. Khoo, T. Lauritsen, I. Calderin, M. P. Carpenter, G. Hackman, I. Ahmad, S. Fischer, R. V. F. Janssens, D. Nisius, A. Lopez-Martens, $†$ T. Døssing,* A. Korichi, † F. Hannachi, $\nmid$ and E. F. Moore§)}

The decay from SD states is precipitated by sufficient mixing with one or two of the nearest normal-deformed (ND) states among which they are embedded. The decay spectrum is then governed by the admixed highlyexcited ND state. In analogy with neutron-capture gamma rays, we call the transitions that directly deexcite the state primary gamma rays. In the decay spectrum of ${ }^{194} \mathrm{Hg}$ (band 1), 34 primary transitions above $2.6 \mathrm{MeV}$ were detected above a $3 \sigma$ level. These transitions have a distribution in strength.

The fluctuations in the strength distribution can provide an indicator of the chaoticity of excited ND states around $4.3 \mathrm{MeV}$. In the fully chaotic limit, where the wavefunction is complex, the distribution is expected to be a $\chi^{2}$ distribution, with the number of degrees of freedom $v$ equal to 1 , giving the so-called PorterThomas distribution.
A technique based on the maximum likelihood method was developed for analyzing the distribution of the transition strengths. The analysis shows that the observed distribution is consistent with a $\chi^{2}$ distribution with $v=1$ (Porter-Thomas distribution). However, the uncertainties in $v$ and in the average strength $\theta$ are very large because the observed primary transitions represent the high-intensity tail of the maximum-likelihood distribution. In fact, some of the 1-step lines exhibit strengths up to $9 \theta$, raising the possibility that there may be some perseverance of selection rules for decay from states at $\sim 4.3 \mathrm{MeV}$. Since it is difficult to explain why this happens in only $194 \mathrm{Hg}$, a more likely explanation is that chance (and fortune) is responsible for the large 1-step strengths. (In ${ }^{194} \mathrm{~Pb}$, where the excitation energy of the SD band is significantly lower, the primary transitions are expected to be stronger.) In summary, the distribution of the strength of primary decay lines in ${ }^{194} \mathrm{Hg}$ is consistent with decay from a chaotic state.

*Niels Bohr Institute, Copenhagen, Denmark, †Centre de Spectrometrie Nucleaire et de Spectrometrie de Masse, Orsay, France, łInstitut de Physique Nucleaire, IN2P3-CNRS, Orsay, France, §North Carolina State University

\section{c.11. Spins and Excitation Energy of the Yrast Superdeformed Band in $191 \mathrm{Hg}$ (S. Siem,* P. Reiter, T. L. Khoo, M. P. Carpenter, T. Lauritsen, D. Gassmann, I. Ahmad, I. Calderin, S. M. Fischer, G. Hackman, R. V. F. Janssens, D. Nisius,

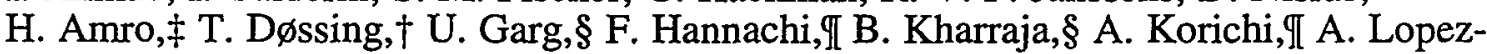 Martens, Il E. F.Moore, $\ddagger$ and C. Schuck $\mathbb{D}$}

The total gamma-spectrum following the decay out of the yrast $S D$ band in ${ }^{191} \mathrm{Hg}$ was extracted using the methods described in. 1 The gamma-rays following the ${ }^{174} \mathrm{Yb}\left({ }^{22} \mathrm{Ne}, 5 \mathrm{n}\right){ }^{191} \mathrm{Hg}$ reaction were collected with Gammasphere and the beam was provided by the $88^{\prime \prime}$ Cyclotron at LBNL. From the total gamma-spectrum the excitation energies and spins of the yrast SD band members were determined. The level fed by the 351 $\mathrm{keV}$ transition (the decay-out level) has an excitation energy of $5.73 \pm 1.0 \mathrm{MeV}$ and spin $17.0 \pm 0.7 \hbar$, leading to two possible spins: $33 / 2 \hbar$ or $35 / 2 \hbar$.

The result from the quasicontinuum analysis is in good agreement with the previous result from a one-step linking transition. ${ }^{2}$ A second one-step transition of $3310 \mathrm{keV}$, with $3 \sigma$ statistical significance, was tentatively assigned to feed a $33 / 2^{-}$known normaldeformed level. There are now two one-step transitions; both place the decay-out level at $6000 \mathrm{keV}$ (see Fig. I30). The angular distribution of the stronger one-step line $(2778 \mathrm{keV})$ shows a big positive value for the $\mathrm{A} 2$ coefficient $(0.57 \pm 0.48)$, which is consistent with $\Delta \mathrm{I}=$ 0 , suggesting a $35 / 2 \hbar$ spin assignment for the decayout level. We rule out the possibility of it being an E2 transition, because that would make the other one-step transition an M3 transition competing with an M1 transition that is not observed. The spin is consistent with a $j_{15 / 2}$ particle configuration assignment. ${ }^{3}$ However, the experimental data do not allow for a parity assignment. For the parity of the SD band to be consistent with a $j_{15 / 2}$ particle configuration assignment, the one-step lines must be M1 transitions. 
From neutron capture experiments it is known that the $\sim 8 \mathrm{MeV}$ E1 transitions are about 5 times more likely than $\mathrm{Ml}$ transitions. However, in ${ }^{191} \mathrm{Hg}$ the 1-step lines have significantly lower energy $(-3 \mathrm{MeV})$. In fact, M1 transitions around this energy are observed both in neutron capture and also from the decay of the SD band in ${ }^{194} \mathrm{~Pb}$.

*ANL and University of Oslo, Norway, $\uparrow$ The Niels Bohr Institute, Copenhagen, Denmark, $¥$ North Carolina State University, §University of Notre Dame, TCentre de Spectrometrie Nucleaire et de Spectrometrie de Masse, Orsay, France

1R. G. Henry et al., Phys. Rev. Lett. 73, 777 (1994); T. Lauritsen et al., Heavy Ion Physics 6, 229 (1997), Proc.

Symp. on Nucl. Structure at the Limits, ANL (1996).

2P. Reiter et al., Physics Division Annual Report for 1997, ANL-98/24, p. 38.

3M. P. Carpenter et al., Phys. Rev. C (1995).

4T. Døssing et al., Phys. Rev. Lett. 75 (1995) 1276.

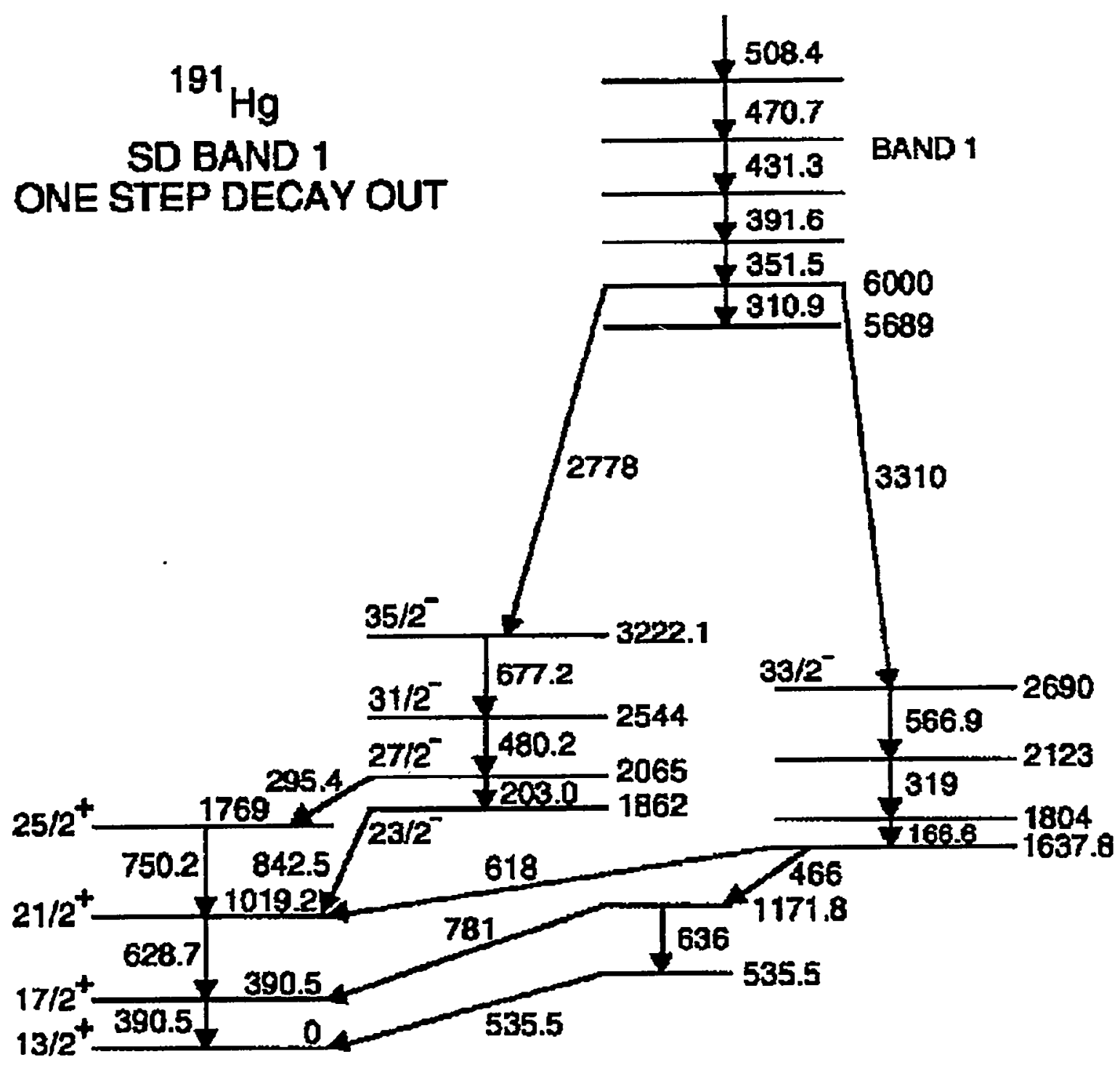

Fig. 1-30. Partial level scheme showing the one-step decay pathways connecting SD and ND levels. 
In conclusion, the excitation energy and spin of the yrast SD band in ${ }^{191} \mathrm{Hg}$ was determined to be $6000 \mathrm{keV}$ and $35 / 2 \hbar$ for the decay-out level. The energy above yrast of the SD band at the point of decay out is 2.778 $\mathrm{MeV}$. The excitation energy of the yrast SD band extrapolated to $13 / 2 \hbar$, the spin of the ground state, is $4.74 \mathrm{MeV}$. It is important to obtain these quantities in an odd-even nucleus since that gives information on the relative pair correlation energies in ND and SD states, thereby providing a stringent test for theory. Information on pair quenching in excited states will be obtained by comparing the quasicontinuum spectra following the decay of the SD bands in $191,192,194 \mathrm{Hg}$. In addition, these spectra will be compared with theoretical statistical decay spectra ${ }^{4}$ from excited states in even-even and odd-even nuclei.

\section{c.12. Determination of Spin and Excitation Energy of Superdeformed Bands in} ${ }^{192,194} \mathrm{Hg}$ (T. Lauritsen, T. L. Khoo, I. Ahmad, M. P. Carpenter, R. V. F. Janssens, C. J. Lister, A. Lopez-Martens, ${ }^{*}$ H. Amro, ${ }^{*} \ddagger$ S. Berger, L. Calderin, T. Dossing, $\mathbb{I}$ S. M. Fischer,§ G. Hackman, D. T. Nisius, F. Hannachi,* A. Korichi,* and E. F. Moore)

Superdeformed (SD) bands in the mass 190 region decay suddenly, over a few states, at relatively low spin and high excitation energy. The decay path is very fragmented and only in a few cases have one-step or two-step decays been seen. Presently, in this mass region, only three superdeformed bands have been linked to the normal states they decay to. Most of the gamma rays from the decay of the SD bands form a quasicontinuum. Thus, for most SD bands in this mass region, the excitation energy and spins of the bands are not known experimentally.

However, by extracting and analyzing the decay quasicontinuum spectrum in coincidence with the SD bands it is possible to determine the excitation energy and spin of the bands. This "quasi-continuum analysis method" was tested in ${ }^{194} \mathrm{Hg}$, which is one of the few cases where the excitation energy and spin of the first superdeformed band is known, and was then applied to the first superdeformed band in ${ }^{192} \mathrm{Hg}$, where no onestep decays were seen. The derived excitation energy and spin in ${ }^{194} \mathrm{Hg}$, using the quasi-continuum analysis method, were found to be in excellent agreement with the exact result determined from one-step and two-step decay lines in the nucleus. For ${ }^{192} \mathrm{Hg}$, extrapolated to zero angular momentum, the excitation energy of the $\mathrm{SD}$ band was found to be $5.7(6) \mathrm{MeV}$.

*CSNSM, Orsay, France, $\uparrow$ North Carolina State University, $\ddagger \mathbb{I R e S}$, Strasbourg, France, §University of Pennsylvania IINiels Bohr Institute, Copenhagen, Denmark

\section{c.13. Quasicontinuum Spectrum of Gamma Rays Which Depopulate SD States in ${ }^{194} \mathrm{~Pb}$ (T. L. Khoo, T. Lauritsen, D. P. McNabb, ${ }^{*}$ J. A. Cizewski, * K.-Y. Ding, * W. Younes, ${ }^{*}$ D. Archer, $\uparrow$ R. W. Bauer, $\dagger$ J. A. Becker, $\uparrow$ L. A. Bernstein, $\dagger$

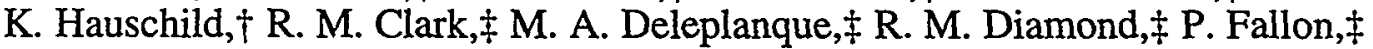 I. Y. Lee, $\ddagger$ A. O. Macchiavelli, $\ddagger$ F. S. Stephens $\ddagger$ and W. H. Kelley $\S)$}

Primary gamma rays from the decay out of the SD band in $194 \mathrm{~Pb}$ were identified and the spins, likely parity and excitation energies of the band were determined ${ }^{1}$ from experiments performed with both Eurogam and Gammasphere. The focus of this work is the analysis of Gammasphere data for the total decay spectrum. As for $192,194 \mathrm{Hg}$, this spectrum consists of both sharp primary transitions and an unresolved quasicontinuum component. In ${ }^{194} \mathrm{~Pb}$, however, the primary lines have a significantly larger fraction of the total decay yield, due largely to the smaller excitation energy of the SD band, which reduces the phase space for the unresolved component. The total decay spectrum out of SD states was extracted. The energy of the decaying SD states that is derived from the total decay spectrum agrees with that determined from the discrete lines. Inspection of the 
decay spectrum reveals a region below the end point, which has low counts. About $0.9 \mathrm{MeV}$ below the end point, the spectrum rises rapidly. This feature, which was predicted by Døssing et al., 2 reflects a region of low-level density immediately above the normal- deformed yrast line, to which decay is naturally reduced.

This work, which was submitted to Physical Review Letters, constituted part of the $\mathrm{Ph}$. $\mathrm{D}$. thesis of $\mathrm{D}$. McNabb from Rutgers.

*Rutgers University, †Lawrence Livermore National Laboratory, ¥Lawrence Berkeley National Laboratory, §Iowa State University

1A. Lopez-Martens et al., Phys. Lett. B380, 18 (1996); K. Hauschild et al., Phys. Rev. C 55, 2819 (1997).

${ }^{2}$ T. Døssing et al., Phys. Rev. Lett. 75, 1276 (1995).

\section{c.14. Intensity Distribution of Primary Transitions in Decay from the Superdeformed Band of ${ }^{194} \mathrm{~Pb}$ (T. L. Khoo, I. Ahmad, M. Carpenter, R. V. F. Janssens, T. Lauritsen, P. Reiter, A. Lopez-Martens, * F. Hannachi,* A. Korichi, $*$ E. Gueorguieva,* C. Schück, ${ }^{*}$ Ch. Vieu,* T. Døssing, $\dagger$ B. Herskind, $\dagger$ P. Varnelle $\uparrow \uparrow$, M. Meyer $\ddagger$ A. Astier, $\ddagger$ L. Ducroux $\ddagger$ S. Perries $\ddagger$ N. Redon,$\ddagger$ H. Hübel,$\S$ A. Goergen,§ S. Chmel,§ E. Mergel,§ A. Wilson, $\mathbb{~ C . ~ W . ~ B e a u s a n g , ~} \mathbb{I}$ and $\mathrm{K}$ Hauschild,II)}

In a preceding section, it is suggested that the fluctuations in the strengths of primary transitions from a SD band in ${ }^{194} \mathrm{Hg}$ are consistent with a PorterThomas form. This suggests that the admixed normaldeformed state, which mediates decay out of the SD band, has chaotic character. A limitation in studying the strengths of primary transitions in ${ }^{194} \mathrm{Hg}$ is that they are expected to be weak, on average. In fact, the weakest observable transition has a strength larger than the average strength of the Porter-Thomas distribution that best fits the data. A more favorable situation is expected for the SD band in ${ }^{194} \mathrm{~Pb}$, which lies at lower energy (2.7 MeV, vs. $4.3 \mathrm{MeV}$ in ${ }^{194} \mathrm{Hg}$ ), so that the phase space for quasicontinuum decay is smaller. As a consequence, primary transitions in ${ }^{194} \mathrm{~Pb}$ are expected to be about 35 times stronger, and the already observed primary transitions ${ }^{1}$ have about the estimated average strength. The strength of the weakest observable primary transitions should be well below the average, giving a better data set for testing if strength fluctuations are governed by a Porter-Thomas distribution. The maximum likelihood method will be used for this test.

An experiment was performed with Euroball at Legnaro to measure the sharp primary decay lines connecting SD and ND states in ${ }^{194} \mathrm{~Pb}$. The ${ }^{30} \mathrm{Si}\left({ }^{168} \mathrm{Er}, 4 \mathrm{n}\right)$ reaction at $142 \mathrm{MeV}$ was used to populate states in ${ }^{194} \mathrm{~Pb}$. At the time of the experiment, Euroball had 28 tapered-, 26 clover- and 15 cluster- Ge detectors. A large data set was obtained in a smooth run and analysis of the data is in progress.

*Centre de Spectrometrie Nucleaire et de Spectrometrie de Masse, Orsay, France, $\uparrow$ Niels Bohr Institute, Copenhagen, Denmark, $\ddagger$ Université Lyon, Villeurbanne, France, §University Bonn, Germany, TYale University, IlLawrence Livermore National Laboratory

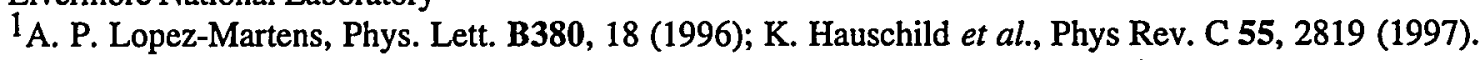




\section{c.15. Search for the Two-Phonon Octupole Vibrational States in 208Pb (H. Amro, R. V. F. Janssens, W. Henning, T. L. Khoo, I. Ahmad, M. P. Carpenter, T. Lauritsen, C. J. Lister, D. J. Blumenthal, G. Hackman, S. Fischer, E. F. Moore,* S. I. Sanders, $\dagger$ and S. Yates $\ddagger$ )}

The search for the two-phonon octupole excitations in the doubly-magic nucleus ${ }^{208} \mathrm{~Pb}$ remains a fascinating and unresolved problem in contemporary nuclear structure physics. A set of three experiments was carried out over the past few years to address the issue. The first two measurements were carried out using thick targets and beam energies roughly $20 \%$ above the Coulomb barrier. Only lifetime-dependent upper limits on the probability of populating members of the 2 phonon multiplet were extracted. The third mesurement was performed with a thin target and a beam energy about $7 \%$ below the Coulomb barrier. As a result of this measurement, upper limits on the excitation of $6^{+}$ states in ${ }^{208} \mathrm{~Pb}$ were deduced and the fragmentation of the $6^{+}$member of the multiplet is suggested. These results lead one to question the purity of the twophonon octupole vibrations. Only recently, theoretical calculations ${ }^{1}$ suggested that members of the 2-phonon multiplet (except for the $0^{+}$which is unambiguously identified $^{2}$ ) are indeed fragmented.

*North Carolina State University and the Triangle Universities Nuclear Laboratory, fUniversity of Kansas, $\ddagger$ University of Kentucky

$I_{V}$. Ponomarev et. al., Phys. Rev. Lett. 82, 501 (1999).

${ }^{2}$ M. Yeh et. al., Phys. Rev. Lett. 76, 1208 (1977). 


\section{REACTION DYNAMICS}

Over the last few years studies of various aspects of reaction dynamics using heavy-ion beams within the Division have steadily moved from measurements performed with ATLAS beams in the vicinity of the Coulomb Barrier towards higher beam energies. At present, the measurements at ATLAS focus mainly on reactions with exotic beams and on studies involving gamma-ray detection with Gammasphere: these are discussed elsewhere in this report. This section presents work being performed at relativistic energies; i.e. the analysis of the AGS E917 experiment and the preparations for the PHOBOS experiment at RHIC.

\section{d.1. Studies of $\mathrm{Au}+\mathrm{Au}$ Collisions at 6, 8, $10.8 \mathrm{GeV} /$ nucleon at the AGS}

(The E917 collaboration: B. B. Back, R. R. Betts, * A. Gillitzer, W. F. Henning, D. J. Hofman, V. Nanal, A. Wuosmaa, H. C. Britt,** W. C. Chang, C. Y. Chi, $\ddagger$

Y. Y. Chu, † J. B. Cumming, $\dagger$ J. C. Dunlop,II W. Eldredge, $\ddagger$ S. Y. Fung, $\ddagger$ R. Ganz, $\S$

E. J. Garcia-Solis, ** G. Heintzelman,Il B. Holzman,§ J. H. Kang,Il E. J. Kim,\|

S. Y. Kim,\| Y. Kwon,\| D. McLeod,§ A. C. Mignerey,** M. Moulson, †† C. A. Ogilvie,II

R. Pak, †† A. Ruangma, ** D. E. Russ,** R. Seto, + P. J. Stankas, ** G. S. F. Stephans, $\mathbb{1}$

H. Q. Wang, $\ddagger$ F. Wolfs, $\uparrow \dagger$ H. Xiang, $\ddagger$ G. Xu, $\ddagger$ H. B. Yao,Il and C. Zou $\ddagger)$

It was thought that high densities of up to 5-10 times those of normal nuclear matter may be achieved in central collisions of heavy nuclei at AGS energies, provided that the relative motion is stopped. There is possibly a new state of matter - the quark-gluon plasma (QGP) - which may emerge at these high densities. The question of the degree of stopping in head-on collisions is thus of central importance at these energies. Direct information pertaining to this issue may be obtained by studying the transverse mass spectra of protons, the majority of which are primordial, over a wide rapidity range to assess whether the observed rapidity distribution is consistent with the initial momentum of the projectile being converted to isotropic emission from a source at rest in the center-ofmass system.

Additional relevant information about the state of matter formed in these collisions may be obtained from measurements of the production of fundamental particles such as kaons, $\Phi$-meson, $\Lambda, \bar{\Lambda}$ and $\overline{\mathrm{p}}$ containing strange quarks. Both the production rate and their distribution in rapidity and transverse mass gives important clues about the state of matter produced in the heavy-ion collision, which may also manifest itself in subtle mass shifts or broadening of e.g. the short-lived $\Phi$-meson.

The purpose of the E917 experiment is therefore to study particle production, in-medium effects on particle masses, and collective flow phenomena at the high matter densities which may be achieved in $\mathrm{Au}+\mathrm{Au}$ collisions at relativistic energies.

\section{Experimental Arrangement}

The experimental arrangement is illustrated in Fig. I-31. Beams of ${ }^{197} \mathrm{Au}$ with momenta of $6.84,8.86$ and $11.69 \mathrm{GeV} / \mathrm{c}$ per nucleon corresponding to kinetic energies of $6.0,8.0$ and $10.8 \mathrm{GeV}$ per nucleon were obtained from the AGS at Brookhaven National Laboratory and focused onto a Au-target of $11 \mathrm{~mm}$ thickness, which corresponds to $\sim 3 \%$ interaction probability in the target. The trajectory of each beam particle was determined by a beam vertex detector - built at Argonne, ${ }^{1}$ - consisting of four planes of scintillating fibers read out by position-sensitive photo-multiplier tubes arranged in orthogonal pairs and located at $5.84 \mathrm{~m}$ and $1.72 \mathrm{~m}$ upstream from the target position. Further beam characterization was performed using beam-timezero and halo counters also placed upstream from the target. Triggers for beam interactions with the target were obtained by requiring that a signal of less than $75 \%$ of that expected for the full energy loss of a Aubeam nucleus was registered in a circular "bulls-eye" Čerenkov detector placed $11 \mathrm{~m}$ downstream from the target.

The centrality of each beam-target interaction was derived from the multiplicity of particles (mostly pions) registered in a multiplicity detector array subtending a solid angle of about $6.85 \mathrm{sr}$ around the target and/or by the total energy of the projectile remnant measured in the zero degree calorimeter.

In order to determine the reaction plane orientation in peripheral collisions, a hodoscope consisting of two orthogonal planes of $1 \mathrm{~cm}$ wide plastic scintillator slats 


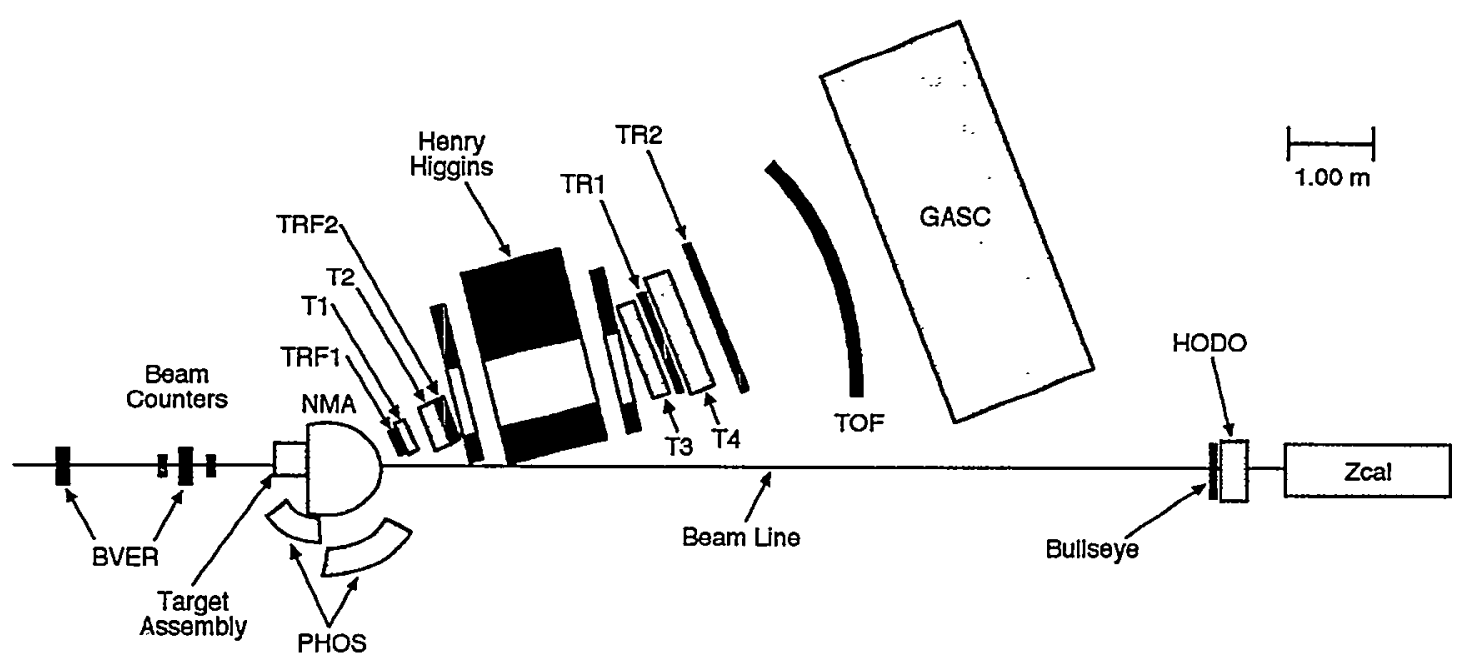

Fig. 1-31. Experimental arrangement.

was placed in front of the zero degree calorimeter. The azimuthal angle of the reaction plane determined from the average position of the charged projectile remnants in the hodoscope, relative to the beam axis will be used to study collective flow characteristics of peripheral collisions and the reaction plane dependence of the apparent source size obtained in a Hanbury-Brown Twiss analysis of pion pairs.

Particle spectra were obtained by momentum analysis in a movable magnetic spectrometer consisting of a $0.4-$ Tesla magnet (Henry Higgins) and a number of multiwire ionization chambers used to determine the straight line trajectories of particles entering and exiting the magnetic field region. In addition, a plastic scintillator wall located behind the spectrometer provided particle identification by time-of-flight measurements relative to the Cerenkov start detector located in front of the target.

This arrangement is capable of identifying charged pions, kaons, protons, anti-protons and heavier nuclei. The separation of pions and kaons is effective up to an energy of $\sim 1.75 \mathrm{GeV}$. Protons are separated from pions up to $\sim 3.4 \mathrm{GeV}$, although there is a negligible kaon contamination above $\sim 2.9 \mathrm{GeV}$.

The acceptance of the spectrometer is also sufficient to detect correlated decay products of

$$
\Phi \rightarrow \mathrm{K}^{+} \mathrm{K}^{-}, \Lambda \rightarrow \mathrm{p} \pi^{-} \text {, and } \bar{\Lambda} \rightarrow \overline{\mathrm{p}} \pi^{+} \text {. }
$$

\section{Stopping - Proton Spectra}

In Fig. I-32, spectra of the invariant probability for proton emission per trigger event are plotted for the $10.8-\mathrm{GeV} /$ nucleon beam energy as a function of the transverse mass $\mathrm{m}_{\mathrm{t}}-\mathrm{m}_{0}$ for the $5 \%$ most central collisions as determined from the energy deposition in the zero-degree calorimeter. The spectra are shown for different rapidity bins as indicated. Only statistical enror bars are shown. Note that adjacent spectra are offset by factors of ten to avoid overlapping. The range in $m_{t}$ reflects the acceptance of the spectrometer in the different rapidity bins ranging from backwards to near mid-rapidity at $y_{\mathrm{cm}}=1.613$. The curves represent the best fits to the spectra using a Boltzmann distribution, i.e. 
$\frac{1}{2 \pi m_{t}} \frac{d^{2} N}{d y d m_{t}}=C m_{t} e^{-m_{t} T}$

where $C$ is a normalization constant and $T$ is the inverse slope of the spectrum, both of which are determined from the fit to the data. We observe that the experimental $\mathrm{m}_{\mathrm{t}}$ spectra are in excellent agreement with this shape although they could also be described almost equally well by a pure exponential function.

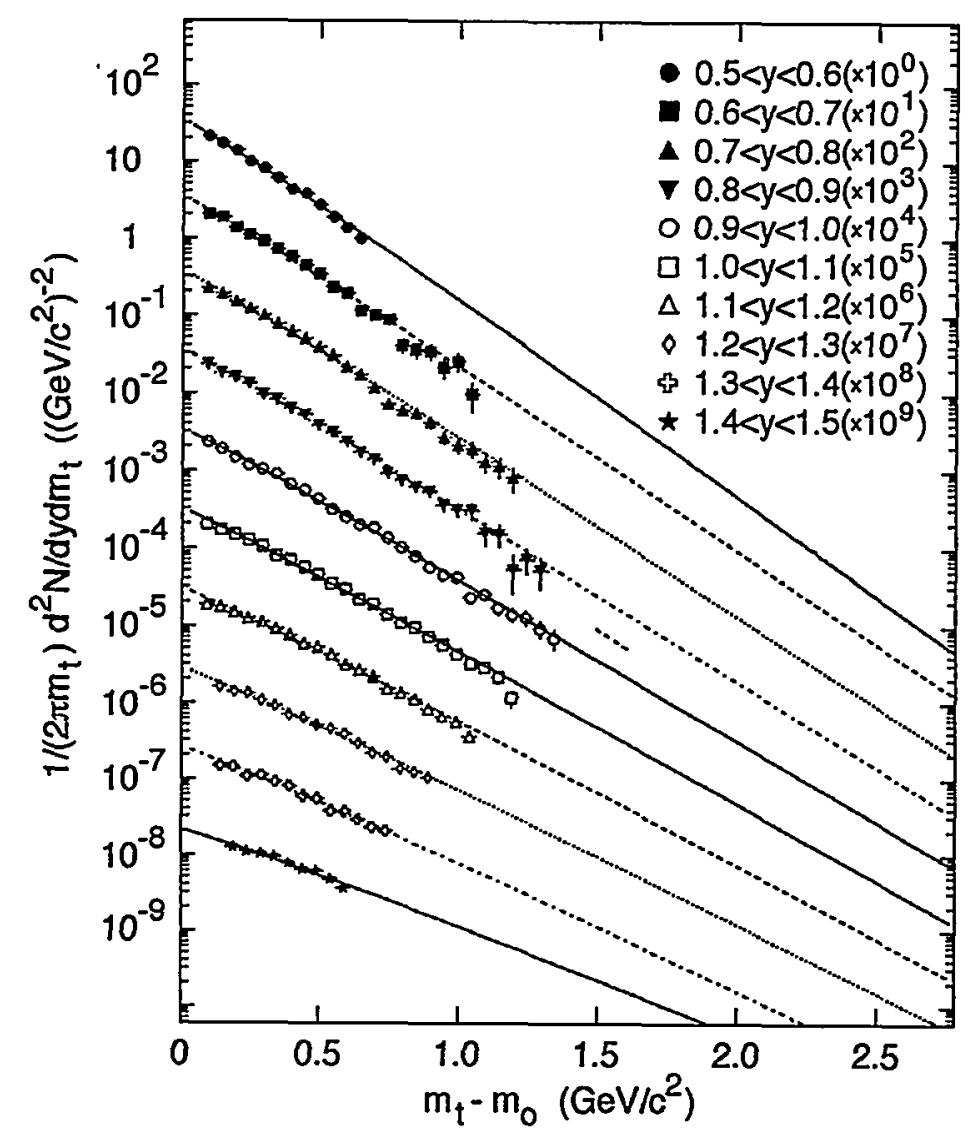

Fig. I-32. Proton $m_{t}$ spectra for central $(<5 \%) A u+$ Au collisions at a beam kinetic energy of $10.8 \mathrm{GeV} / \mathrm{nucleon}$ for different rapidity bins (laboratory system). Curves are Boltzmann distributions fitted to the data. Spectra for adjacent rapidity bins are offset by factors of ten to avoid overlapping.

From these fits we derive the total probability for proton emission per unit of rapidity, $\mathrm{dN} / \mathrm{dy}$, which is plotted as a function of rapidity in the center-of-mass frame, $y-y_{\mathrm{cm}}$, in the left panels of Fig. I-33. The derived inverse slopes $T$ are shown in the right-hand panels. Data are shown for $5 \%$ central collisions at all three beam energies, where the centrality for the 6- and 8-GeV/nucleon data are obtained from the multiplicity array at the target position. The measured points are represented by solid circles, whereas reflection around mid-rapidity results in the open points. Error bars on the fit parameters are purely statistical and do not include possible systematic errors.

We note that all three $\mathrm{dN} / \mathrm{dy}$ distributions are quite flat over the measured rapidity range. The inverse slopes, $\mathrm{T}$, show, however, a distinct peaking at mid-rapidity. The solid curves in Fig. I-33 represent the expectation for isotropic emission from a thermal source with temperature $\mathrm{T}_{0}$ at rest in the center-of-mass system ${ }^{2}$. This naive model gives a rather good 


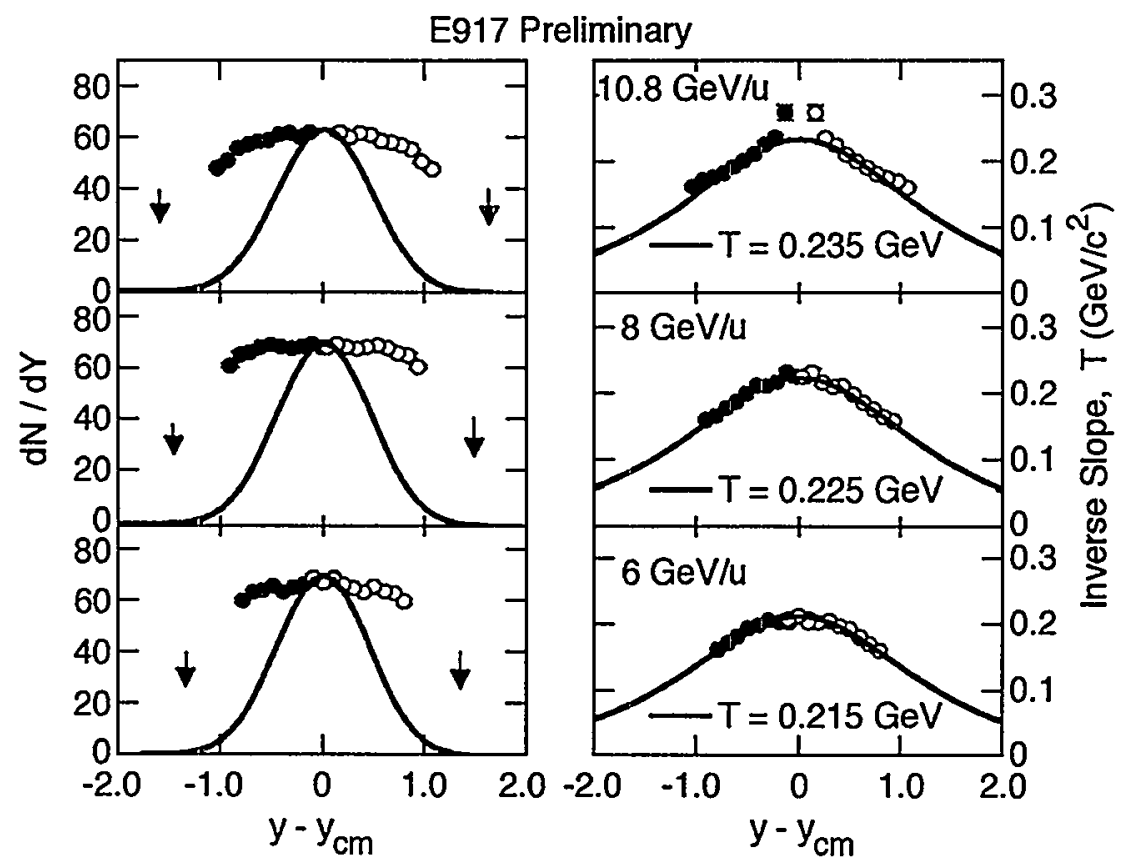

Fig. 1-33. Proton rapidity distributions in the center-of-mass system (left panels) and inverse slopes (right panels) are compared to a simple thermal source prediction for the three beam energies. The arrows indicate target and beam rapidities.

representation of the observed inverse slopes, but fails to reproduce the flat rapidity distributions. We conclude that there is not complete stopping at these energies. A manuscript describing the proton data is being prepared for publication in Physical Review C. A preliminary analysis was presented at the $15^{\prime}$ th Winter Workshop on Nuclear Dynamics, Park City, Utah, January 10-15, 1999.2

\section{Pion and Kaon Spectra}

There is significant theoretical interest in the study of kaon properties in dense nuclear matter. Qualitatively, the models suggest that $\mathrm{K}^{+}$mesons experience a weak repulsive potential inside the nuclear medium resulting in a slight increase in the effective mass with baryon density, whereas a strong attractive potential for $\mathrm{K}^{-}$mesons leads to a significantly reduced effective mass in the high baryon density environment. ${ }^{3}$ Because the $\Lambda \mathrm{K}^{+}$ production channel is expected to be larger than $\mathrm{K}^{+}$ $\mathrm{K}^{-}$pair production at AGS energies, this scenario results in a larger $\mathrm{K}^{-} / \mathrm{K}^{+}$yield ratio for central events near mid-rapidity, where high baryon density is expected and the $\Lambda \mathrm{K}^{+}$channel is suppressed.

The rapidity distributions of kaons were obtained from exponential fits to the transverse momentum, $\mathrm{m}_{\mathrm{t}}$, spectra at each rapidity bin. From these fits we obtain the integrated production probability, dN/dy, per unit of rapidity and the inverse slope, $T_{i n v}$, of these spectra. We emphasize that the inverse slopes, $\mathrm{T}_{\text {inv }}$, should not be interpreted as the temperature of the emitting source as it is well known that collective effects, such as radial expansion, can mimic high source temperatures.

The rapidity distributions, $\mathrm{dN} / \mathrm{dy}$, for $\mathrm{K}^{+}$and $\mathrm{K}^{-}$ emission for $0-5 \%$ central collisions are shown in Fig. I-34 for beam energies of 6, 8 and $10.8 \mathrm{AGeV}$. The rapidity distributions are observed to be peaked at mid-rapidity and the yields increase with beam energy without substantial change in the shape of the rapidity distributions. 

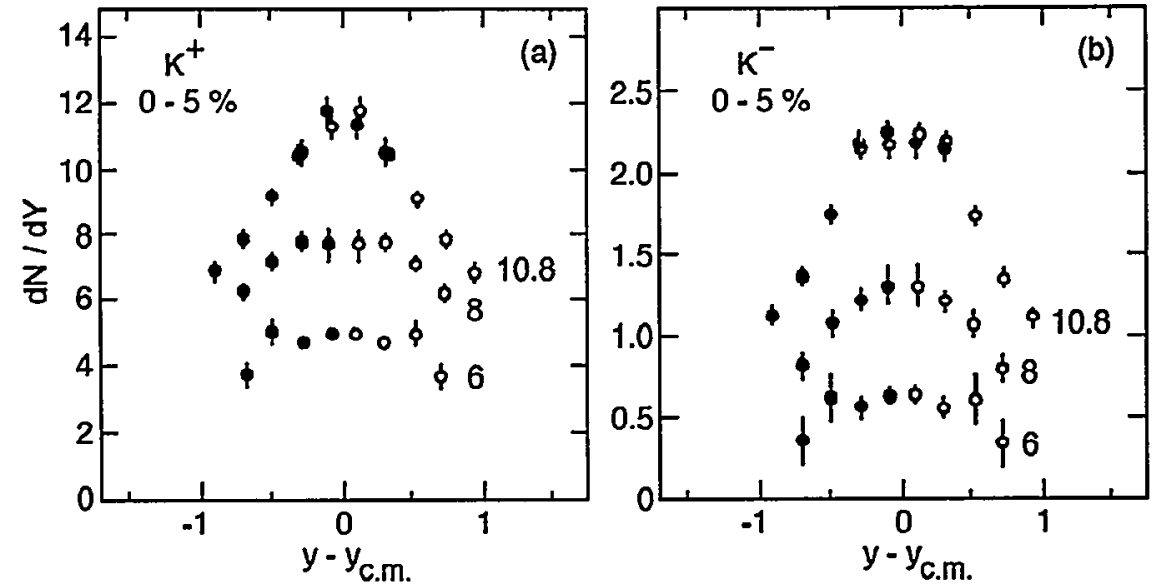

Fig. I-34. Rapidity distributions for $\mathrm{K}^{+}$(left panel) and $\mathrm{K}^{-}$(right panel) for $0-5 \%$ central events are shown for beam energies of 6,8 and $10.8(E 866) \mathrm{A} \mathrm{GeV}$.

Since the effects of the nuclear medium have opposite sign for $\mathrm{K}^{+}$and $\mathrm{K}^{-}$, the ratio of $\mathrm{K}^{-} / \mathrm{K}^{+}$production might be a very sensitive probe for studying such effects. In Fig. I-35a we show the $\mathrm{K}^{-} / \mathrm{K}^{+}$ratio as a function of rapidity for the three beam energies. The fact that this ratio peaks at mid-rapidity shows that the $\mathrm{K}^{-}$rapidity distribution is somewhat narrower than the $\mathrm{K}^{+}$distribution, which may be attributable to the higher threshold for $\mathrm{K}^{-}$production in nucleon-nucleon collisions.

E917 Preliminary
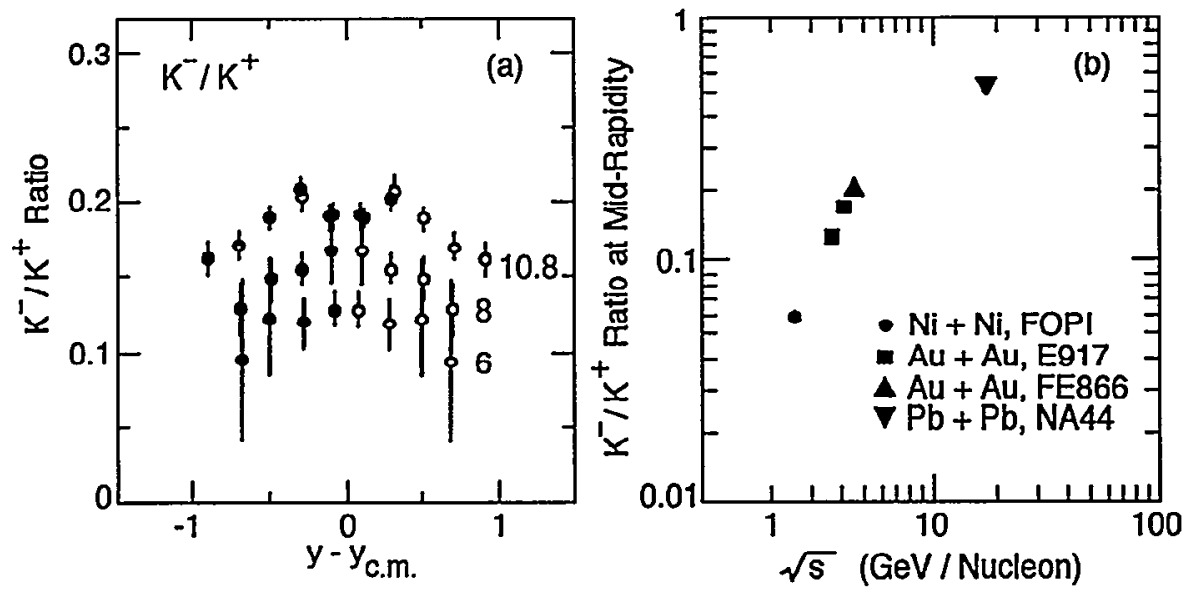

Fig. I-35. a: the rapidity distribution of $\mathrm{K}^{-} / \mathrm{K}^{+}$ratio for the $0-5 \%$ central events at various beam energies and $b$ : the $K^{-} / K^{+}$ratio at mid-rapidity at SIS, ${ }^{11}$ AGS and SPS ${ }^{12}$ energies.

The $\mathrm{K}^{-} / \mathrm{K}^{+}$ratio at mid-rapidity is shown in Fig. $35 \mathrm{~b}$ as a function of beam energy from SIS through AGS to SPS energies. The fact that this ratio asymptotically approaches unity with beam energy may be attributed to the reduced importance of the higher production threshold for $\mathrm{K}^{-}$. At the SPS beam energy of 160
$\mathrm{GeV} /$ nucleon we observe a near saturation in the population of the available phase space for both $\mathrm{K}^{+}$and $\mathrm{K}^{-}$. The analysis of these data is completed and will be published jointly with results obtained by the E866 collaboration at lower energies. A preliminary analysis was presented at the 15 'th Winter Workshop on Nuclear 
Dynamics, Park City, Utah, January 10-15, 1999.4 This work also constitutes the Ph.D. thesis for James Dunlop, MIT. ${ }^{5}$

4. $\bar{\Lambda}$ and $\overline{\mathrm{p}}$ Measurements

Experiment E917 measured anti-protons in the rapidity range $1.0<y<1.4$ and $\bar{\Lambda}$ were reconstructed from $\bar{p} \pi^{+}$pairs. The signal of $\bar{\Lambda}$ is clearly seen in the invariant mass distribution shown in Fig. I-36. The transverse mass spectra of $\overline{\mathrm{p}}$ and $\bar{\Lambda}$ in the rapidity range $1.0<y<1.4$ for the central $0-23 \%$ events are shown in Fig. I-37. The efficiency of detecting anti-

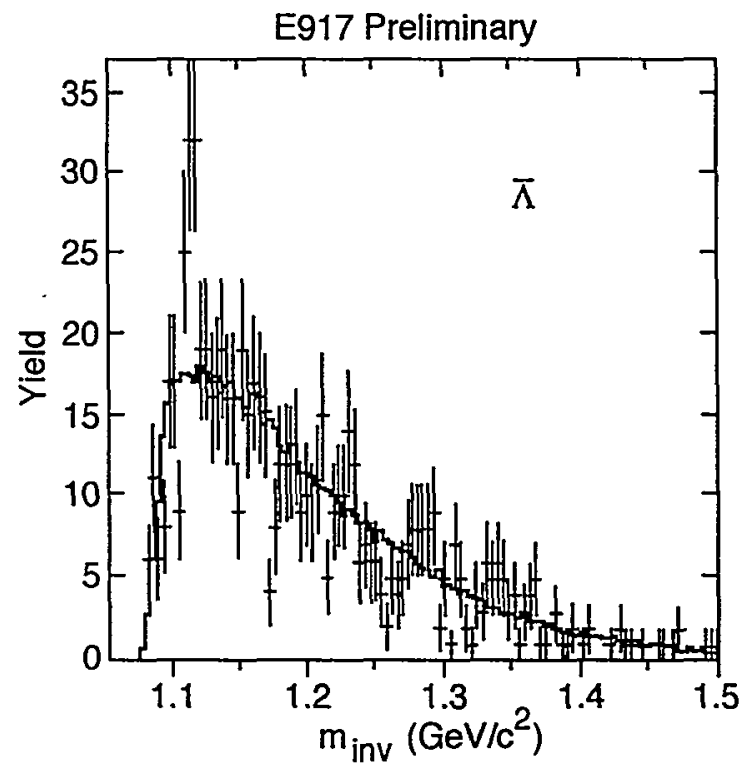

Fig. 1-36. Invariant mass spectrum of $\bar{\Lambda}$ reconstructed from $\overline{\mathrm{p}} \pi^{+}$pairs. The curve represent the fitted mixed-event background.

The E917 measurement of the $\bar{\Lambda} / \overline{\mathrm{p}}$-ratio is greater than unity for the $0-23 \%$ central collisions and consistent with $\mathrm{E} 859$ measurement in the $\mathrm{Si}+\mathrm{Au}$ system ${ }^{6}$. The ratio derived from the difference between the $\mathrm{E} 864^{7}$ and $\mathrm{E} 878^{8} \overline{\mathrm{p}}$ measurements lies within the upper bound of E917 results. It should be noted, however, that there exist several differences in the experimental measurements presented here and those of E864, E878, and E859. Most important are the ranges in $m_{t}$, rapidity and centrality measured by the different experiments. More data will be analyzed in the future to compare the results from the other experiments under similar centrality and rapidity cuts. The analysis of these data is completed and will be published soon. A preliminary analysis was presented at the $15^{\prime}$ th Winter protons from $\bar{\Lambda}$ decays is close to unity in our experiment. Assuming that the decay of $\bar{\Lambda}$ is the only source of hyperon feed-down into $\bar{p}$, the yield of $\bar{p}$ directly produced in the collision is:

$\mathrm{dN}\left(\overline{\mathrm{p}}_{\text {direct }}\right) / \mathrm{dy}=\mathrm{dN}\left(\overline{\mathrm{p}}_{\text {measured }}\right) / \mathrm{dy}-0.64 \mathrm{dN}(\bar{\Lambda}) / \mathrm{dy}$ thereby correcting for the $64 \%$ branching ratio of the $\bar{\Lambda}$ decay into the $\overline{\mathrm{p}} \pi^{+}$channel.

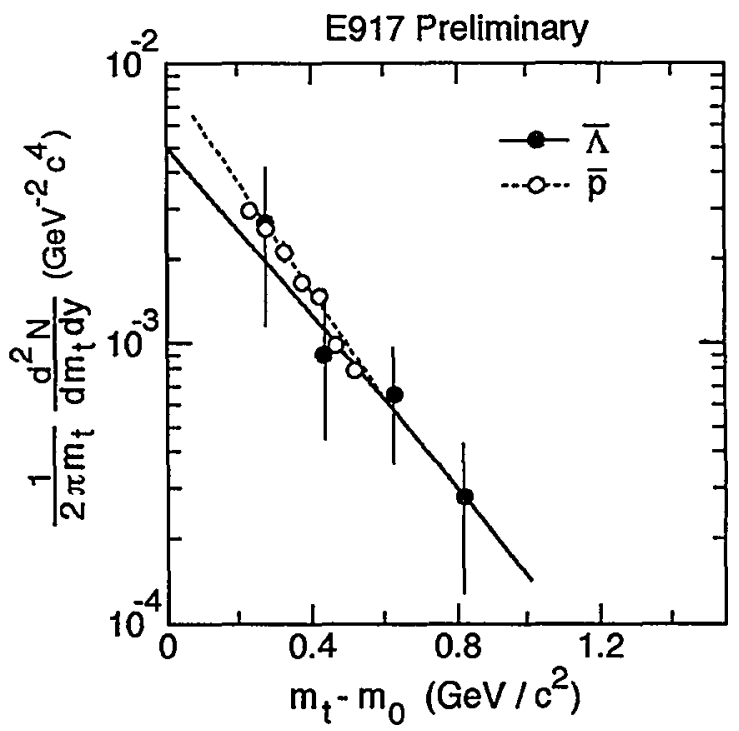

Fig. 1-37. Transverse mass $\left(m_{t}\right)$ spectra of $\overline{\mathrm{p}}$ (open circles) and $\bar{\Lambda}$ (solid circles) for $0-23 \%$ central events.

Workshop on Nuclear Dynamics, Park City, Utah, January 10-15, 1999. ${ }^{4}$ This work also constitutes the Ph.D. thesis for George Heintzelman, ${ }^{9}$ MIT.

\section{Phi-mesons}

One of the initial motivations for the E917 experiment was to search for any in-medium modifications of the mass centroid and/or width of the $\Phi$-meson in central Au collisions where very high nuclear densities are believed to exist. Experimentally, $\Phi$-mesons are identified via their $49 \%$ decay branch to a $\mathrm{K}^{+} \mathrm{K}^{-}$pair detected in the spectrometer. The $\Phi$ resonance in the invariant mass spectrum constructed from same-event $\mathrm{K}^{+} \mathrm{K}^{-}$pairs is clearly identifiable as illustrated in Fig. I- 
38. At the present stage of the analysis we have identified $430 \Phi$-mesons within the $0-77 \%$ centrality bin, and find no evidence of either a mass shift or broadening of the resonance. The analysis is still ongoing but it appears unlikely that any in-medium effect on the $\Phi$-meson will seen in this experiment. The analysis of the $\Phi$-meson data obtained at the $19^{\circ}$ spectrometer angle is completed and will be published soon. Additional data measured at $14^{\circ}$ are being analyzed. A preliminary analysis was presented at the 15 'th Winter Workshop on Nuclear Dynamics, Park City, Utah, January 10-15, 1999.4 This work will also constitutes the Ph.D. thesis for Hong Xiang, University of California at Riverside. ${ }^{10}$

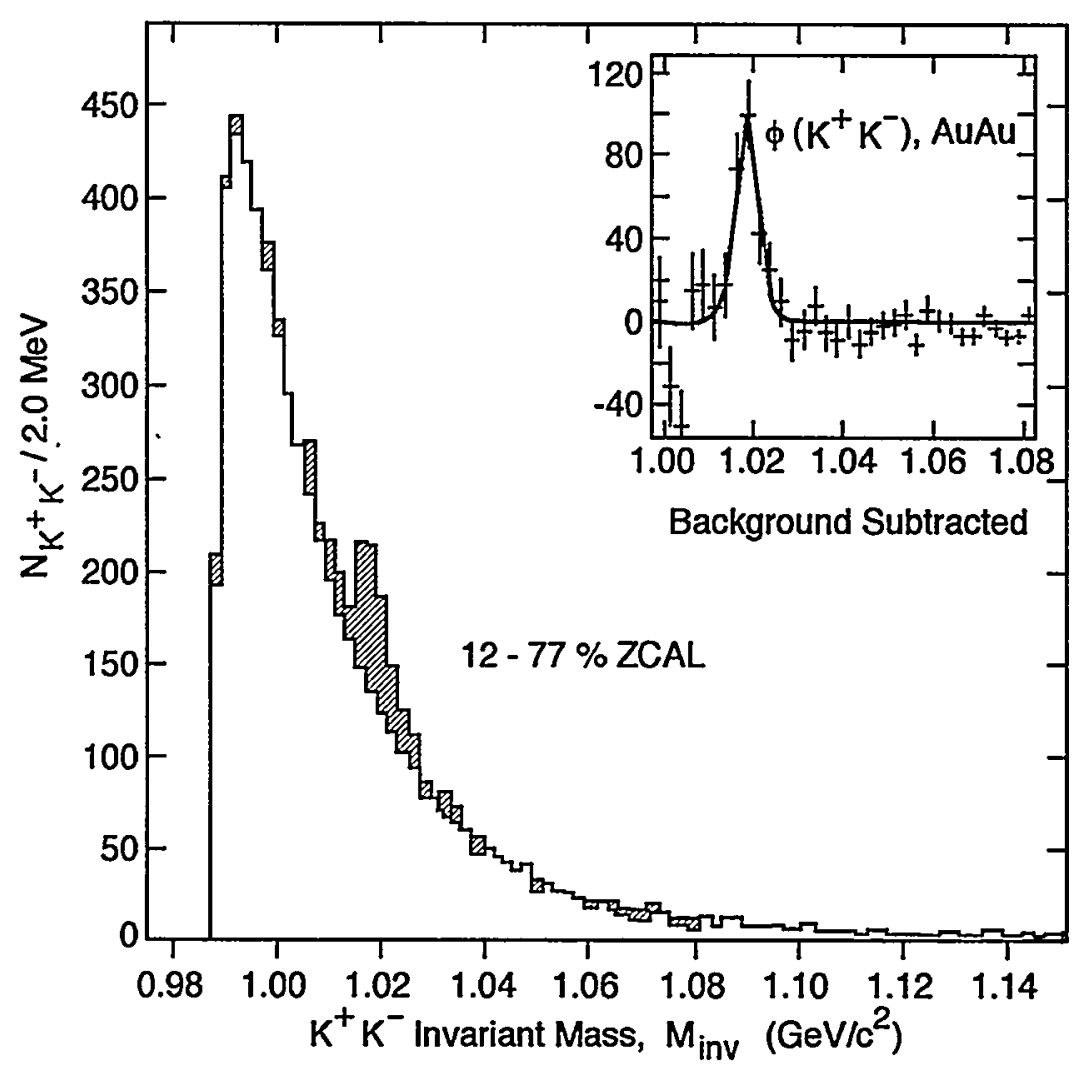

Fig. I-38. Invariant mass spectrum for $K^{+} K^{-}$pairs obtained in the $12-77 \%$ centrality bin.

\section{Reaction-plane and Directed Flow}

The reaction plane in non-central $\mathrm{Au}-\mathrm{Au}$ collisions were in the E917 experiment determined by measuring the centroid of the charged particle remnants of the beam hitting the $0^{\circ}$ hodoscope. It consists of 38 horizontal and 38 vertical plastic scintillator slats, each with a 0.8 $x 1.0 \mathrm{~cm}^{2}$ cross section and equipped with small Photomultiplier tubes on each end for optimum light collection. The azimuthal angle of the reaction plane is defined by this centroid and the beam axis. However, the transverse emittance of the AGS Au-beam is marginal for this study, and the E917 experiment was therefore augmented by a beam vertex detector, ${ }^{1}$ built at Argonne, which determined the intersection between the beam particle and the hodoscope to an accuracy of about $0.5 \mathrm{~mm}$. The accuracy of the reaction plane determination can be assessed by splitting the hits from single events into two sub-events in a random fashion and histogramming the difference in azimuthal angle as shown in Fig. I-39. It is seen that the reaction plane definition becomes more accurate for more peripheral collisions, i.e. larger impact parameters as expected on the basis of symmetry arguments. For central collisions the concept of a reaction plane becomes meaningless. 

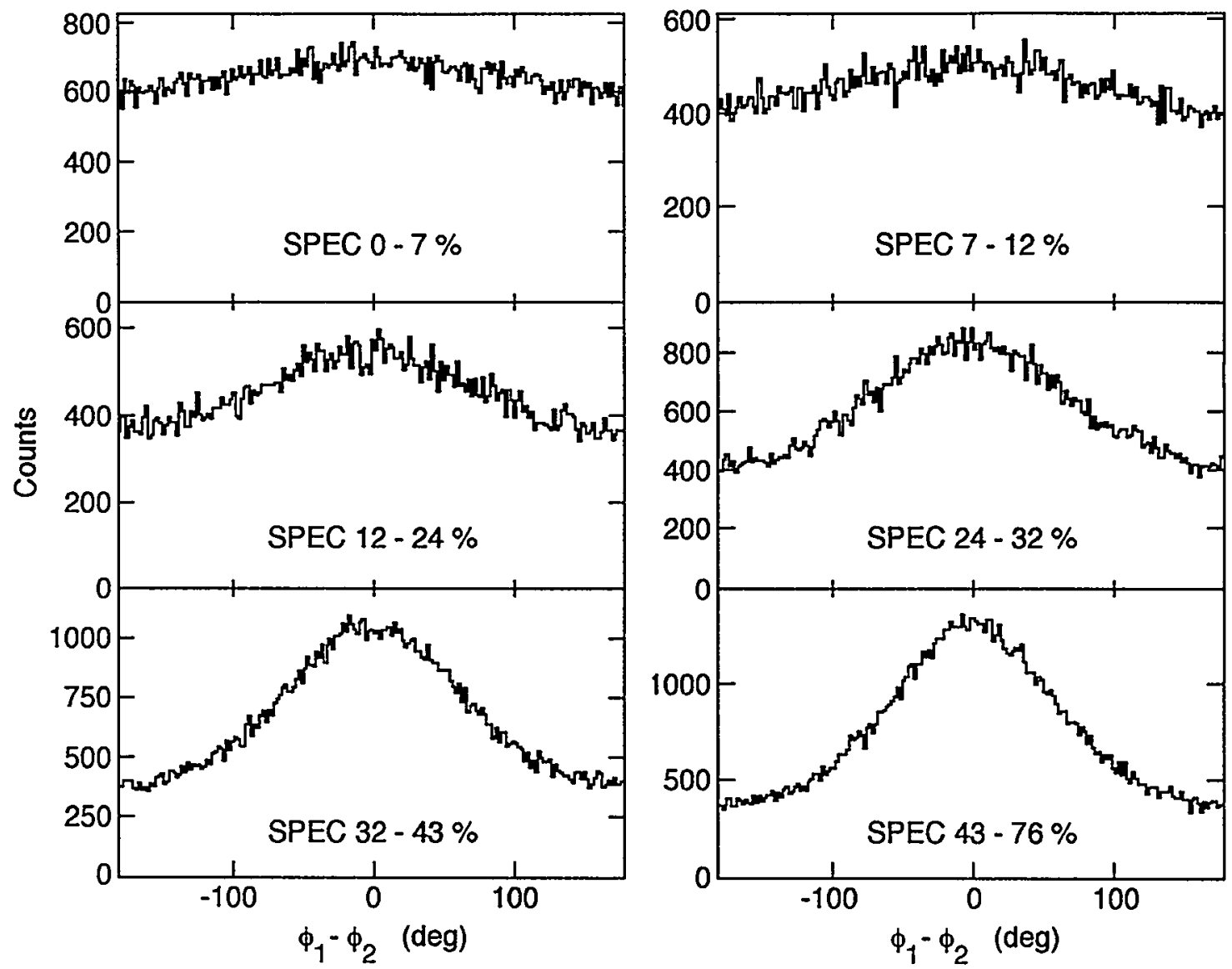

Fig. I-39. Reaction plane resolution.

The average total projection of transverse momentum onto the reaction plane, $\left\langle p_{x}\right\rangle$, was derived for protons measured in the spectrometer and is plotted as a function of rapidity in Fig. I-40. The open points are obtained by reflection and inversion of the measured solid points in order to show the dependence over a wide range of rapidity. This procedure is justified by symmetry arguments. The fact that $\left\langle\mathrm{p}_{\mathrm{X}}\right\rangle$ exhibits a strong dependence on rapidity shows that positive proton flow is indeed observed at this energy. The analysis of the flow signal from other measured particle species is presently underway. A preliminary analysis of this work was presented by David Hofman at the Relativistic Heavy-Ion Symposium at the APS Centennial Meeting in Atlanta, March, 1999, and will be published in Proceedings for the Relativistic Heavy-Ion Mini Symposium, Atlanta, GA, March 20-26, 1999, World Scientific (1999).

\section{Two-particle Correlations}

The volume of the nuclear fireball from which various particle species originate can be estimated by applying the Hanbury-Brown-Twiss analysis to pairs of identical particles. We have performed such an analysis for $\pi^{+} \pi^{+}$and $\pi^{-} \pi^{-}$pairs in Au-Au collisions at 6,8 , and $10.8 \mathrm{GeV} /$ nucleon with a minimum bias trigger. The results are illustrated in Fig. I-41 where the onedimensional correlation functions

$$
Q_{\text {inv }}=\sqrt{\left(\bar{p}_{1}-\bar{p}_{2}\right)^{2}-\left(E_{1}-E_{2}\right)^{2} / c^{2}}
$$


where $p_{i}$ and $E_{i}$ denote the momentum and energy of the particle, respectively for $\pi^{+} \pi^{+}$and $\pi^{-} \pi^{-}$pairs are plotted (solid points). The source radius, $R$, and the correlation strength, $\lambda$, are derived from fits to the data using the fitting function:

$$
C(Q)=1+\lambda e^{-Q_{\text {inv }}^{2} R_{\text {inv }}^{2} / \hbar^{2}}
$$

In Fig. I-42 we compare the source radii, $R_{\text {inv }}$, derived from these data with similar results obtained at both lower ${ }^{11}$ and higher energies. ${ }^{12}$ We observe a distinct trend that the source size appears to increase with beam energy, and that the present data fall nicely within this trend.

Future analyses will concentrate on HBT analysis as a function of the azimuthal angle relative to the reaction plane in order to study the shape of the source. In addition, we will attempt to carry out HBT analysis for other less abundant, particle species, such as protons and kaons. A preliminary analysis was presented at the 15 'th Winter Workshop on Nuclear Dynamics, Park City, Utah, January 10-15, 1999. 13 This work will also constitute the Ph.D. thesis for Burt Holzman, University of Illinois at Chicago.

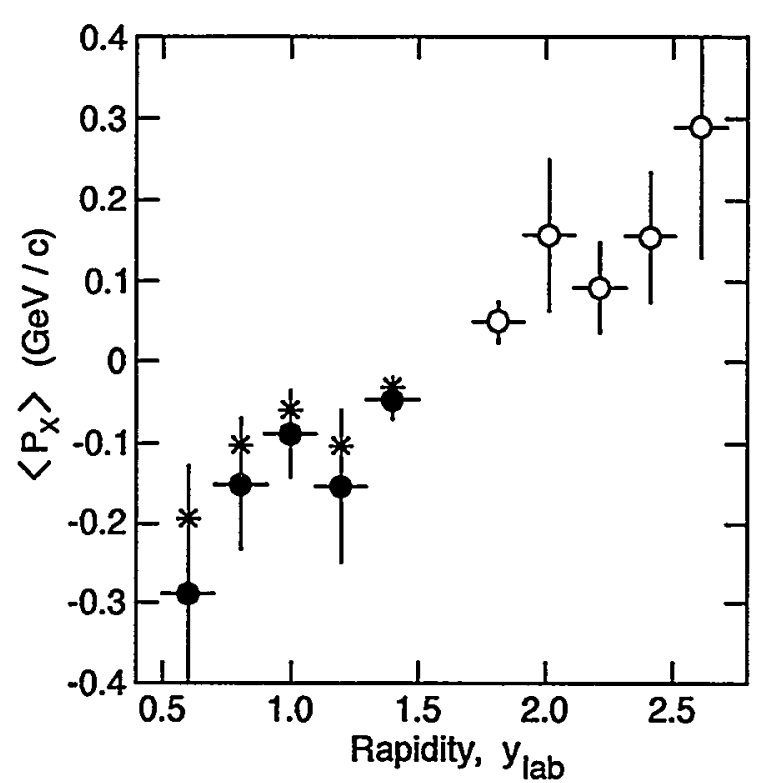

Fig. 1-40. Proton average transverse momentum in the reaction plane. The collective flow signal is obtained as the slope of this curve at $y=0$.

*Argonne National Laboratory and University of Illinois at Chicago, $†$ Brookhaven National Laboratory, $\ddagger$ University of California, §University of Illinois at Chicago, QMassachusetts Institute of Technology, llYonsei University, Seoul, Korea, ${ }^{* *}$ University of Maryland, $\uparrow \dagger$ University of Rochester, $\ddagger$ Columbia University

1B. B. Back, R. R. Betts, A. Gillitzer, W. F. Henning, D. J. Hofman, V. Nanal, A. H. Wuosmaa, R. Ganz, B. Holzman, D. McLeod, W. R. Binns, and J. W. Epstein, Nucl. Instrum. Methods A412, 191 (1998).

${ }^{2}$ B. B. Back for the E917 Collaboration, Proceedings of the 15th Winter Workshop on Nuclear Dynamics, Park City, Utah, 1999.

${ }^{3}$ W. Weise, Nucl. Phys. A610, 35c. (1996).

${ }^{4}$ W.-C. Chang for the E917 Collaboration, Proceedings of the 15th Winter Workshop on Nuclear Dynamics, Park City, Utah, 1999.

5J. C. Dunlop, Ph.D. Thesis, MIT, (1999).

${ }^{6}$ G. S. Stephans and Y. Wu, J. Phys. G 23, 1895 (1997).

7T. A. Armstrong et al., Phys. Rev. Lett. 79, 3351 (1997); Los Alamos Preprint Archive nucl-ex/9811002; G. J. Wang et al., Los Alamos Preprint Archive nucl-th/9807036 and nucl-th/9806006 (1998).

${ }^{8}$ D. Beavis et al., Phys. Rev. Lett. 75, 3633 (1995); Phys. Rev. C 56, 1521 (1997).

${ }^{9} \mathrm{G}$. Heintzelman, Ph.D. thesis, MT (1999).

${ }^{10} \mathrm{H}$. Xiang, Ph.D. Thesis, University of California at Riverside (1999).

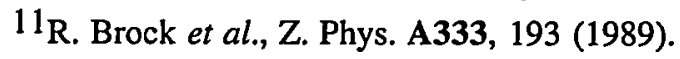

12I. G. Bearden et al., Phys. Rev. C 58, 1656 (1998).

13B. B. Holzman for the E917 Collaboration, Proceedings of the 15th Winter Workshop on Nuclear Dynamics, Park City, Utah, 1999. 


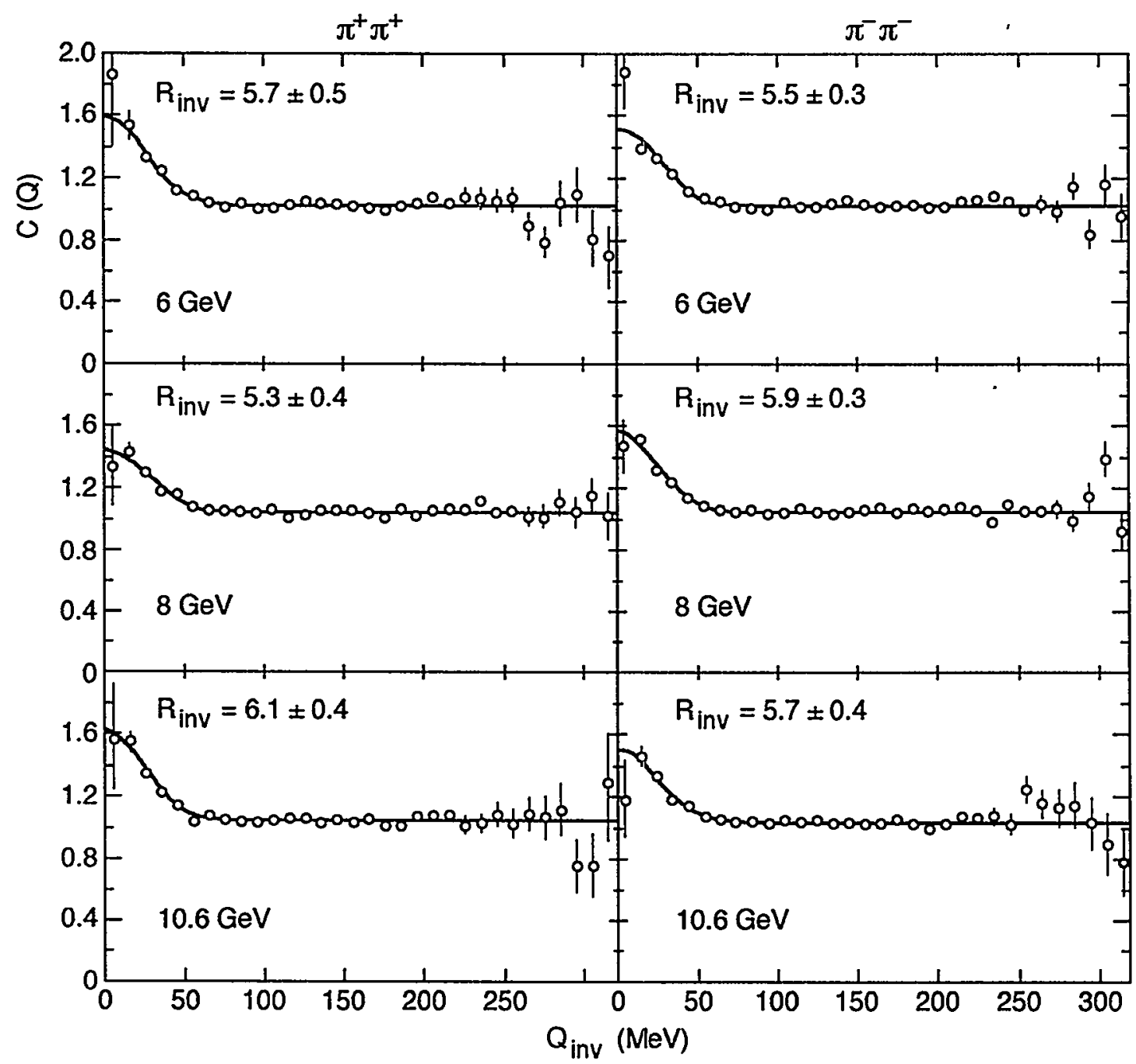

Fig. I-41. Pion HBT analysis of $\pi^{+} \pi^{+}$and $\pi \pi$ pairs at the three beam energies.

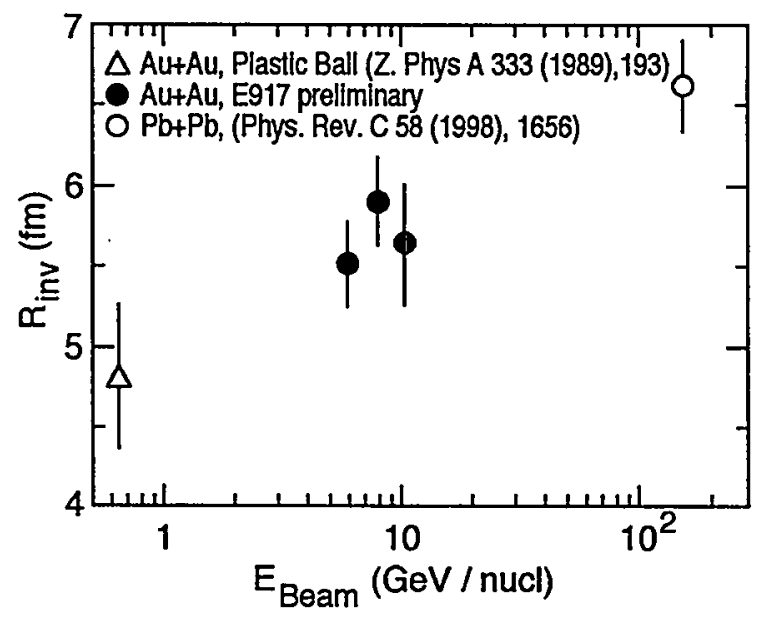

Fig. 1-42. Energy dependence of the source radius obtained from Hanbury-Brown Twiss analysis of $\pi^{-} \pi^{-}$pairs. 


\section{d.2. Reaction Plane Determination in Au + Au Collisions - Experiment E917 (D. J. Hofman, B. B. Back, A. H. Wuosmaa, R. R. Betts, * and B. Holzman*; for the E917 Collaboration)}

The study of collective flow in relativistic nuclear collisions provides unique insight into the complicated dynamics of these reactions. Most simply, collective flow can be understood as the global response of the combined system to the pressure gradients that develop in the high-baryon density region of the collision. These pressure gradients manifest themselves in many different forms of collective flow signals, such as directed flow and elliptic flow to mention two of the most often discussed. As the theoretical understanding of the various flow phenomena has progressed, it has become increasingly apparent that study of flow observables can yield information on such processes as thermalization, mean field effects, phase transitions or even the possible creation of the quark-gluon plasma. The formation of the quark-gluon plasma, just to give one example, may cause the pressure gradient from the baryon dense region to be reduced, or even absent entirely. Thus it is important to study the evolution of the many flow signals as a function of energy and particle type.

Flow signatures from experiment E917 will be extracted for Au + Au collisions at AGS energies of 8 and 10.7 $\mathrm{GeV} /$ nucleon. The magnitude of the observed flow signals will likely be quite small, falling somewhere in the range of a $2-10 \%$ effect, depending on the specific particle being examined. For experiment E917, the collective flow signal will be observed by making an accurate measure of the reaction plane on an event by event basis. It was the need for this accurate measure of the reaction plane which lead to the development of a beam vertexing detector system ${ }^{1}$ by Argonne National Laboratory for use in experiment E917. Once the reaction plane is determined, the azimuthal distribution of various particles relative to this plane can be deduced for any of the particles detected in the Henry Higgins spectrometer. A detailed analysis of these distributions will yield the flow signals of interest.

The reaction plane is determined on an event-by-event basis by combining beam trajectory data from the beam vertexing detector (BVER) with data from a Hodoscope (HODO) detector located 11.4 meters downstream of the target. The Hodoscope is a zero degree global detector kinematically sensitive to beam rapidity emission. It consists of two perpendicular planes of $1-\mathrm{cm}$-wide plastic scintillator slats that are individually viewed by two photomultiplier tubes attached to each end. There are a total of 38 slats for each of the $X$ and $Y$ planes. The purpose of the slat scintillators is to provide a charge weighted mean $\langle\mathrm{X}\rangle$ and $\langle\mathrm{Y}\rangle$ position for particles from the beam fragment. The reaction plane is thus determined from the deviation of the post-collision mean $\langle\mathrm{X}\rangle$ and $\langle\mathrm{Y}\rangle$ of the beam fragment $s$ as determined by the Hodoscope (XHODO, YHODO) from the initial beam trajectory beam position as deduced by the BVER detector $\left(\mathrm{X}_{\mathrm{BVER}}, \mathrm{Y}_{\mathrm{BVER}}\right)$. The reaction plane angle, $\phi$, is thus given by $\tan (\phi)=\left(\right.$ YHODO $_{\text {HO }}$ $\left.\mathrm{Y}_{\mathrm{BVER}}\right) /\left(\mathrm{X}_{\mathrm{HODO}}-\mathrm{X}_{\mathrm{BVER}}\right)$.

To better extract $\mathrm{XHODO}_{\mathrm{H}}$ and $\mathrm{YHODO}$, a response function was developed for the individual Hodoscope slat energy signals. Figure I-43 shows the response function fits to a central slat of the Hodoscope. This response function was parameterized as a function of slat number and reaction centrality for use in all

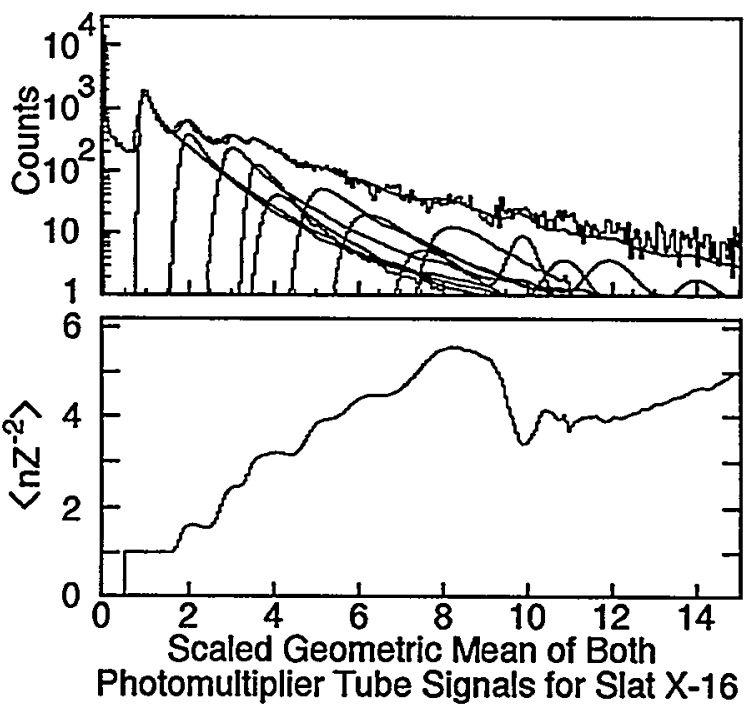

Fig. I-43. Top panel shows the geometric mean energy loss signal for one Hodoscope slat and the response function fits from the minimum ionizing proton, alpha and Li-like fragments emitted by the beam fragment. The bottom panel shows the resulting average charge value extracted from the response function which is used in the calculation for the mean $\langle X>$ and $\langle Y\rangle$ projectile position for each event. 
subsequent analysis. Additional work was necessary to correct for small shifts of relative geometry between the

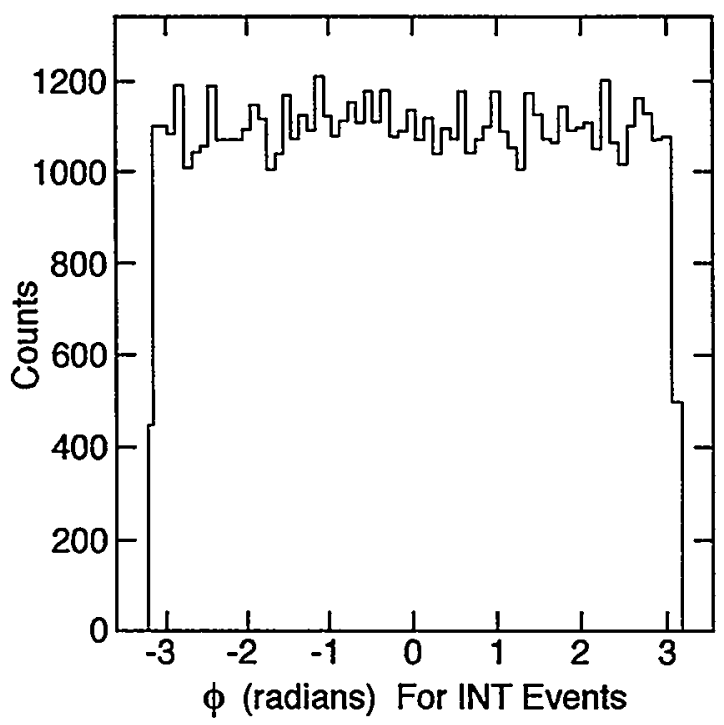

Fig.l-44. The resulting calculated reaction plane angle for interaction triggered (INT) events. A flat spectrum is expected, and this result gives confidence that the reaction plane extraction method is correct.

*Argonne National Laboratory and University of Illinois

${ }^{1}$ B. B. Back et al., Nucl. Instrum. Methods A412, 191 (1998).
BVER detector and the Hodoscope during the threemonth long period the experiment ran.

In order to verify the accuracy and validity of the reaction plane determination, the reaction plane for interaction triggered events was examined. The interaction trigger represents a global, un-biased, "symmetric" trigger that a reaction occurred, and thus the reaction plane should be flat (i.e. isotropic) in $\phi$ for these events. Figure I-44 shows the results of this procedure for a small portion of the data. The deduced reaction plane is flat with a mean near $0^{\circ}$. This result is very significant, and represents a great improvement over data from other experiments which have had variations of factors of two or larger in this spectrum.

The next stage of the analysis will be to determine the reaction plane resolution and then to use this deduced reaction plane to begin the extraction of flow signals. Flow signatures for protons and pions will be among the first results examined.

\section{d.3. The PHOBOS Experiment at RHIC (B. B. Back, R. R. Betts, A. H. Wuosmaa and the PHOBOS Collaboration - ANL, BNL, Case-Western Reserve, Krakow, MIT, NCU Taiwan, ORNL, University of Rochester, UIC, and University of Maryland)}

Qverview

At RHIC, heavy ions will collide at center-of-mass energies an order of magnitude higher than has previously been available anywhere. At this energy, it is expected that a baryon-free region of extremely high energy density will remain in the central region after the two colliding nuclei pass through each other. It is believed that this situation will be more than sufficient to create a system of deconfined quarks and gluons- the "Quark Gluon Plasma." After formation, the plasma should expand and cool before passing into the normal hadronic phase which in turn further expands until the hadrons cease to interact with each other ("freezeout"). The questions then are what are the direct probes and signatures of the plasma phase, and what identifiable traces of the quark-gluon phase remain in the observed hadronic final state? The PHOBOS experiment seeks to address these questions.

The PHOBOS experiment focuses on measurements of hadronic observables for a very large sample of events. The PHOBOS apparatus consists of a $4 \pi$ multiplicity detector and two multi-particle spectrometers capable of measuring and identifying particles with very low transverse momenta. The multiplicity detector will provide event-by-event charged particle multiplicity distributions, which can be used to find interesting events for study in more detail using the spectrometers. The multiplicity distributions are interesting on their own, and contain information on fluctuations and 
correlations, which relate to some of the proposed signatures of the plasma.

An overall view of the PHOBOS apparatus is shown in Fig. I-45; several different components are identified.
The fabrication of the multiplicity and Vertex detectors is the responsibility of the groups at Argonne and UIC. Together, these systems contain over 22000 channels of silicon pad detector.

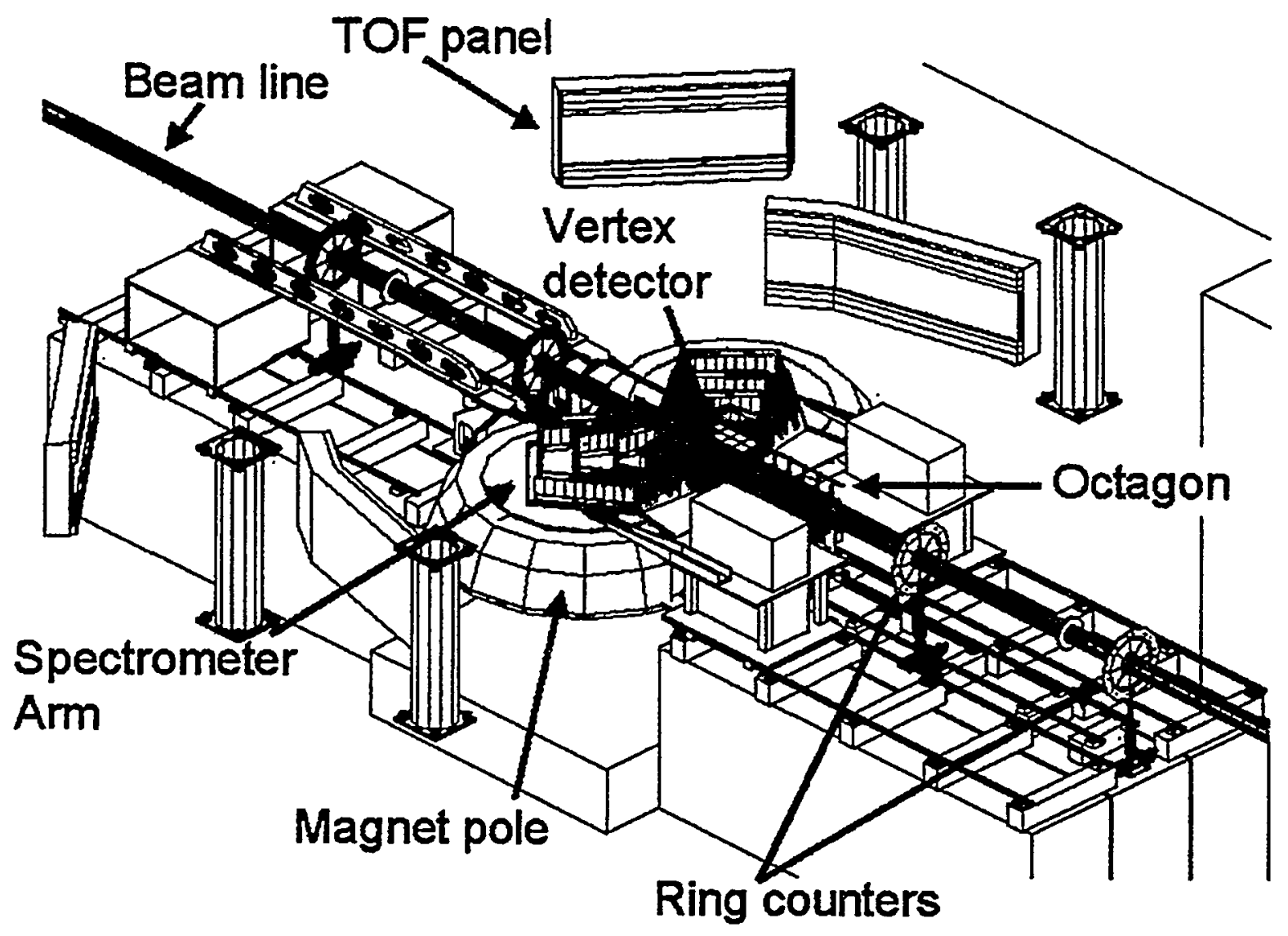

Fig. I-45. Overview of the PHOBOS experimental setup. The top half of the spectrometer magnet has been removed for clarity.

The Multiplicity detector will provide event-by-event information on the charged particle multiplicity distributions over \pm 5 units of pseudo-rapidity, with complete azimuthal coverage. For central $\mathrm{Au}+\mathrm{Au}$ collisions at RHIC, the total charged particle multiplicity in this pseudo-rapidity range is expected to be of order 6000 or greater. The multiplicity measurement is made using a centrally located, 11000 channel "Octagon" array of silicon pads supplemented with six 512 channel "Ring" counters placed up and down the beam pipe. These detectors will measure the energy deposited as a function of angle with respect to the collision vertex. Multiple hits can be handled in an average way by dividing the measured energy by the average expectation for a single charged particle at that point in the detector. Monte Carlo studies show that the charged particle multiplicity distributions for central $\mathrm{Au}+\mathrm{Au}$ collisions can be reconstructed with systematic errors small compared to normal Poisson fluctuations.

The Vertex detector is designed to locate the position of the interaction vertex on an event-by-event basis. It is composed of two pairs of planes of silicon pad detectors, each plane segmented into 2048 channels so that the occupancy is low. Particles emitted from the collision vertex can be tracked by combining pairs of hits on the inner and outer Vertex planes such that the 
position of the reconstructed interaction point can be determined to a precision of better than $1 \mathrm{~mm}$. Vertices may be reconstructed over the entire range of the RHIC collision diamond, corresponding to $\pm 20 \mathrm{~cm}$ about the center of the intersection region.

\section{PHOBOS Detector Assembly and Testing}

There exist a large number of tasks associated with the assembly and testing of the PHOBOS Multiplicity and Vertex array. These include fabrication of the mounting frames for the silicon detector modules, the assembly, and testing of the modules themselves, and the final mounting of modules on the support frames. The mechanical frames for the Octagon, Ring, and Vertex modules were fabricated in Krakow at the Institute for Nuclear Physics, and are now complete. Also complete is the carriage and support structure, which holds the Octagon frame. The responsibility for fabrication and testing of the detector modules, which are mounted on these structures, lies with the groups at Argonne and UIC.

\section{Module Assembly}

The assembly of the detector modules for the Multiplicity and Vertex arrays involves several steps.
The modules are comprised of silicon sensors designed jointly at ANL, ERSO/Miracle Technologies and the National Central University in Taiwan. These sensors each have different geometries. The Octagon sensors are rectangular with four rows of 30 pads, each with an area of approximately $23 \mathrm{~mm}^{2}$. The Ring sensors are larger, but more coarsely segmented, and have a trapezoidal shape with pad areas ranging from approximately 25 to $105 \mathrm{~mm}^{2}$. Finally, the Vertex detectors are rectangular with the outer and inner Vertex modules consisting of two, and four rows of 128 pads, respectively. Each Octagon and Ring module contains a single silicon sensor, mated to a printed circuit board hybrid that holds the front-end readout electronics chip. The front-end preamplifier-shaper-multiplexing electronics are the VA-HDR1 chips made by IDEAS in Norway. The Vertex modules are more complicated each with two sensors read out with eight preamplifier chips. Due to the increased power dissipation, the hybrids for the Vertex detectors are made from ceramic, instead of printed-circuit board, substrates. The assembly of these modules is carried out jointly by the University of Illinois at Chicago, the ANL Physics Division, and the Silicon Detector Laboratory at FNAL. Figures I-46 and I-47 are photographs of completed Octagon and Ring multiplicity modules.

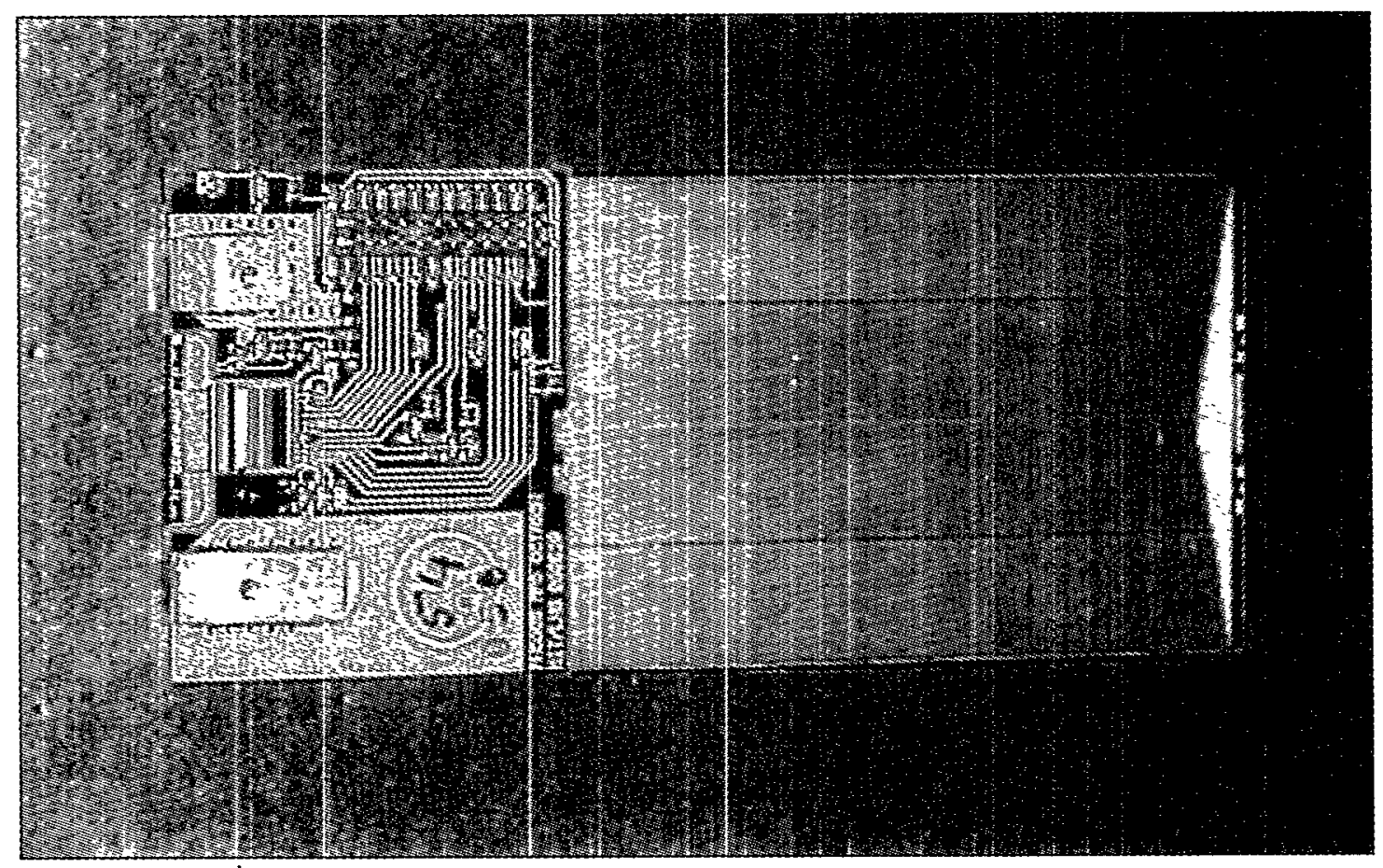

Fig. I-46. Octagon module. 


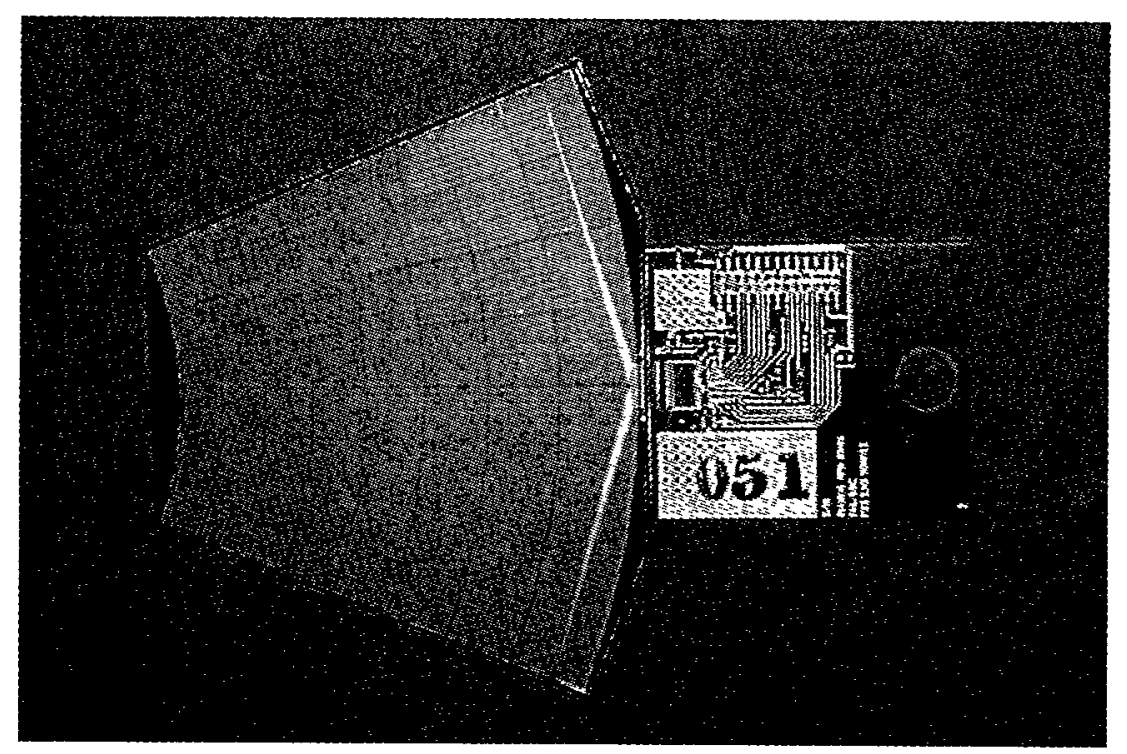

Fig. I-47. Ring module.

The assembly of the modules starts with the delivery of chips from Norway, and hybrids with surface-mount components attached from the Electronics Laboratory at MIT. The readout chips are precision aligned and glued to the hybrids at UIC, and then the readout controls for the chips are wire bonded to the hybrid at the Silicon Detector Laboratory at FNAL. The assembled hybrids are then electrically tested at ANL, and acceptable hybrids are returned to UIC. There, they are mated with silicon sensors, which are glued directly to the hybrids. The module is then returned to FNAL, where the connections between the sensor pads and the front-end electronics chip are made with wire bonding. The fully assembled module is again returned to ANL, where its electrical performance is again evaluated, and its response to particles from radioactive sources is measured. Only modules that pass all tests are accepted for installation in the experiment.

The modules are finally attached to their respective mounting frames, and connected to the readout framework. The readout for the Octagon and Ring detectors was also designed and fabricated at ANL. Figures I-48 and I-49 show completed Octagon and Ring modules mounted on their respective support frames. 


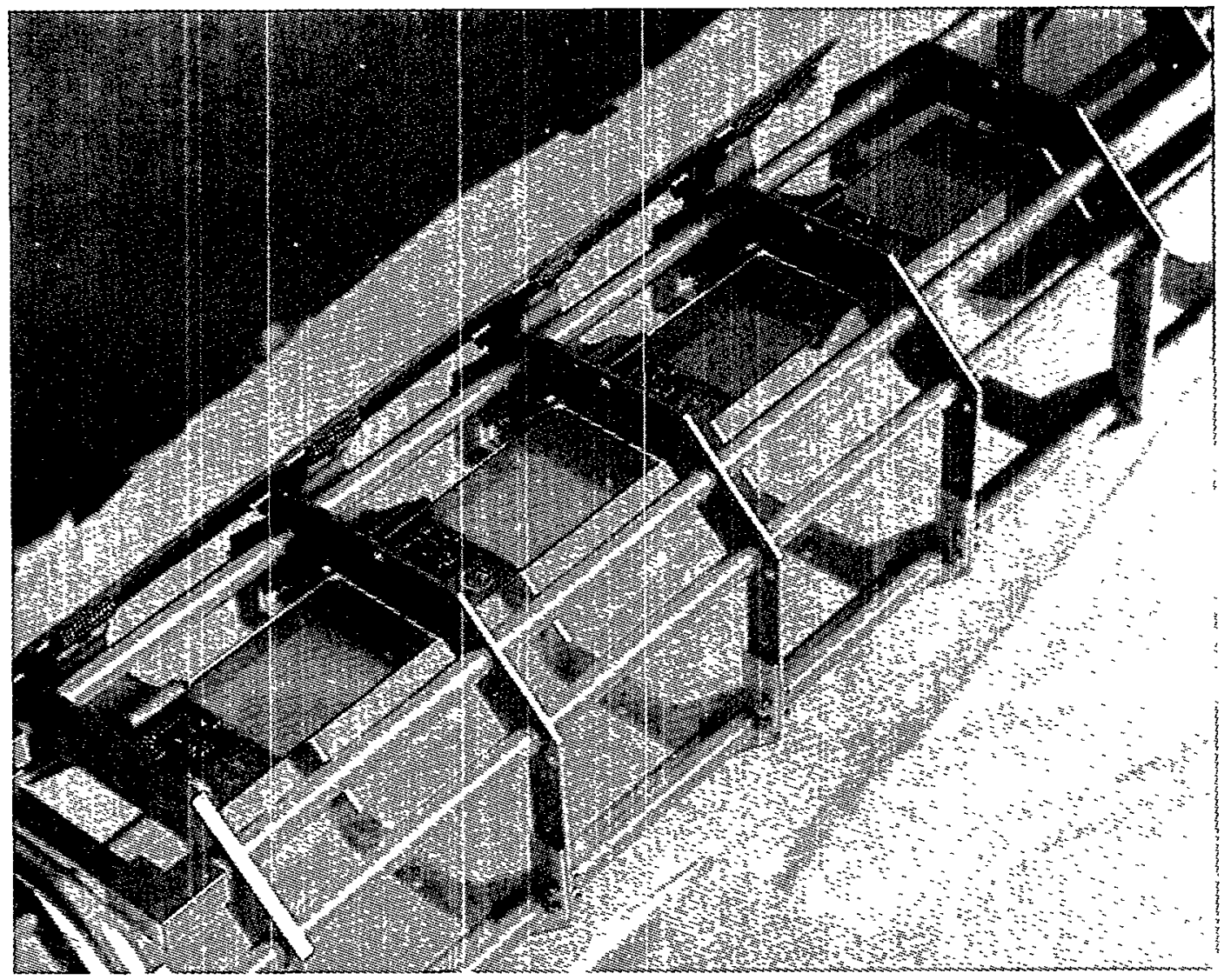

Fig. I-48. Completed Octagon modules mounted on the aluminum cooling and support frame. The mass-terminated cables connect the hybrids to the readout boards.

\section{Module Testing and Performance}

In PHOBOS, the detectors must measure the energy deposited by minimum-ionizing particles. This energy deposition is between approximately $100 \mathrm{keV}$ and 1 $\mathrm{MeV}$, depending on the particle's angle of incidence upon the sensor. In the multiplicity detectors, the number of particles striking a pad in an event is determined primarily from a measure of this deposited energy. The ability of the sensor to measure these small energies with good resolution is thus crucial.

The evaluation of the performance of completed modules is carried out in several steps. First, the operational full-depletion voltage is verified for each module. This measurement entails illumination of each sensor with conversion electrons from a ${ }^{113}$ Sn source $\left(E\left(e^{-}\right)=363,387 \mathrm{keV}\right)$. For $300-\mu \mathrm{m}$ thick silicon, approximately $45 \%$ of these electrons deposit their full energy in the sensor. The bias voltage of the sensor is increased until the peak-to-total ratio for the electron spectrum reaches a plateau. At typical electron-energy spectrum obtained with the ${ }^{113} \mathrm{Sn}$ source appears in Fig. I-50.

With the operational bias voltage established, the sensors are then illuminated with high-energy $\beta$ electrons from a ${ }^{106} \mathrm{Ru}$ source. For this measurement, the electrons travel through a plastic scintillator paddle that serves as a trigger for the data-acquisition system that reads out the chip. The signals for each pad are read out and histogrammed in a PC running LabView software. The parameters that characterize the performance of the module, including the signal-tonoise ratio for minimum ionizing particles with the most probably energy loss, the gain of each pad, and the number of non-working channels for each module, are determined. An energy spectrum for minimum-ionizing electrons from the ${ }^{106} \mathrm{Ru}$ source is shown in Fig. I-51. After this step, the modules are ready for installation in the experiment. 


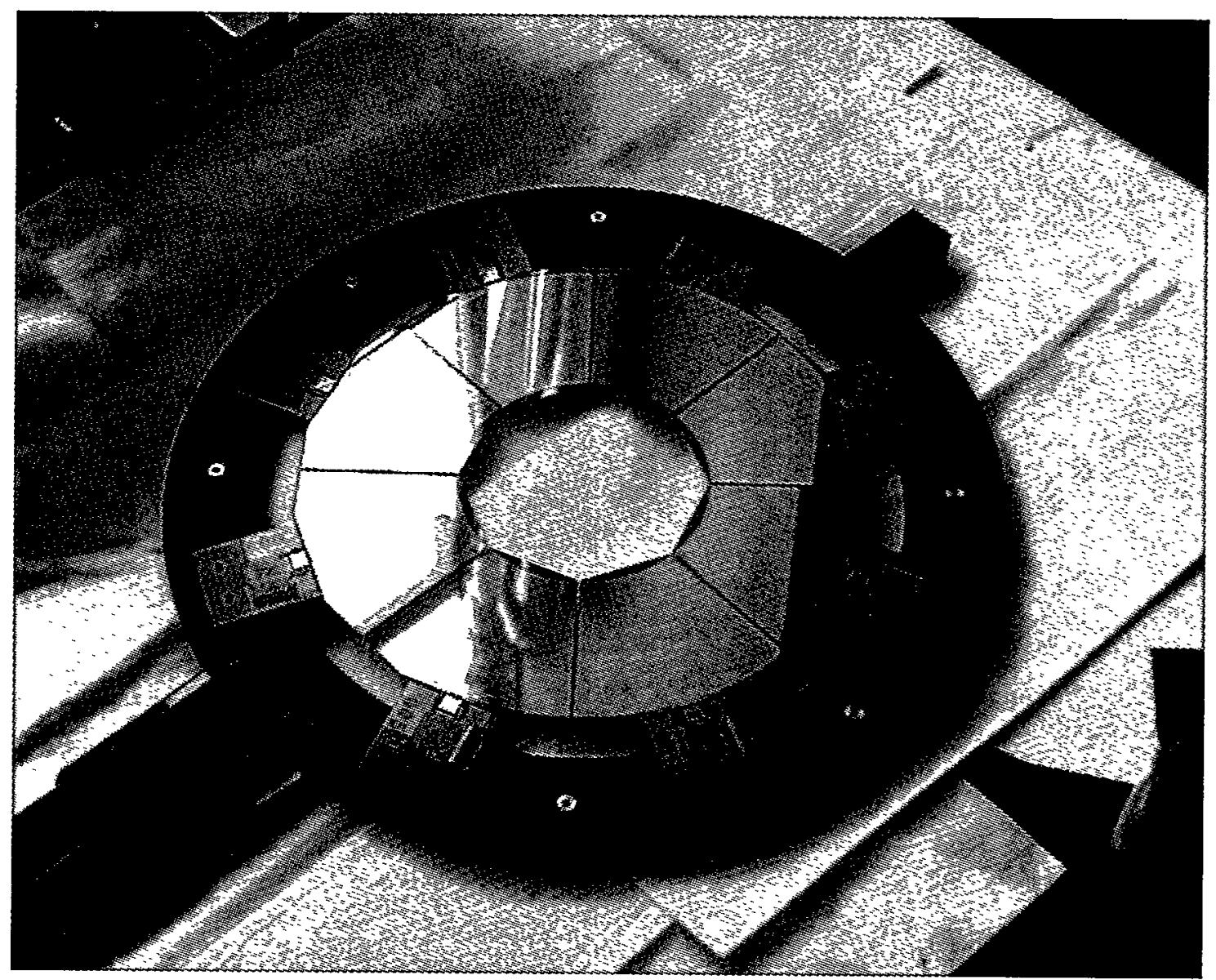

Fig. 1-49. One assembled multiplicity-ring counter, with eight completed ring detector modules attached.

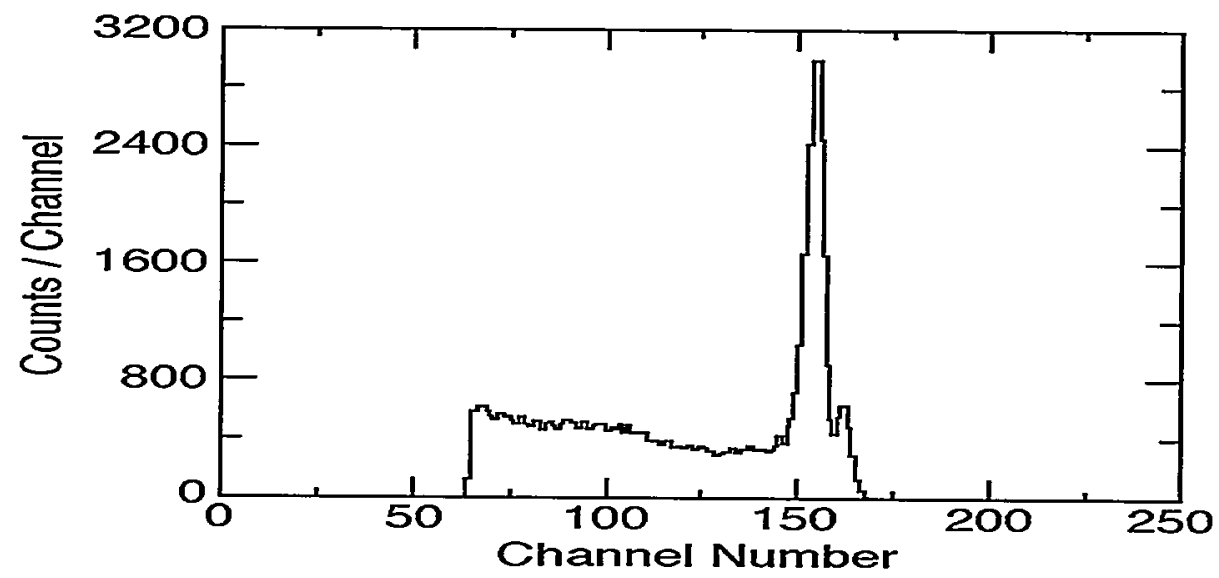

Fig. I-50. Conversion-electron spectrum obtained from one pad of one ring sensor. The $363 \mathrm{keV}$ and $387 \mathrm{keV} \mathrm{K}$ and $L$ conversion lines are clearly resolved. 


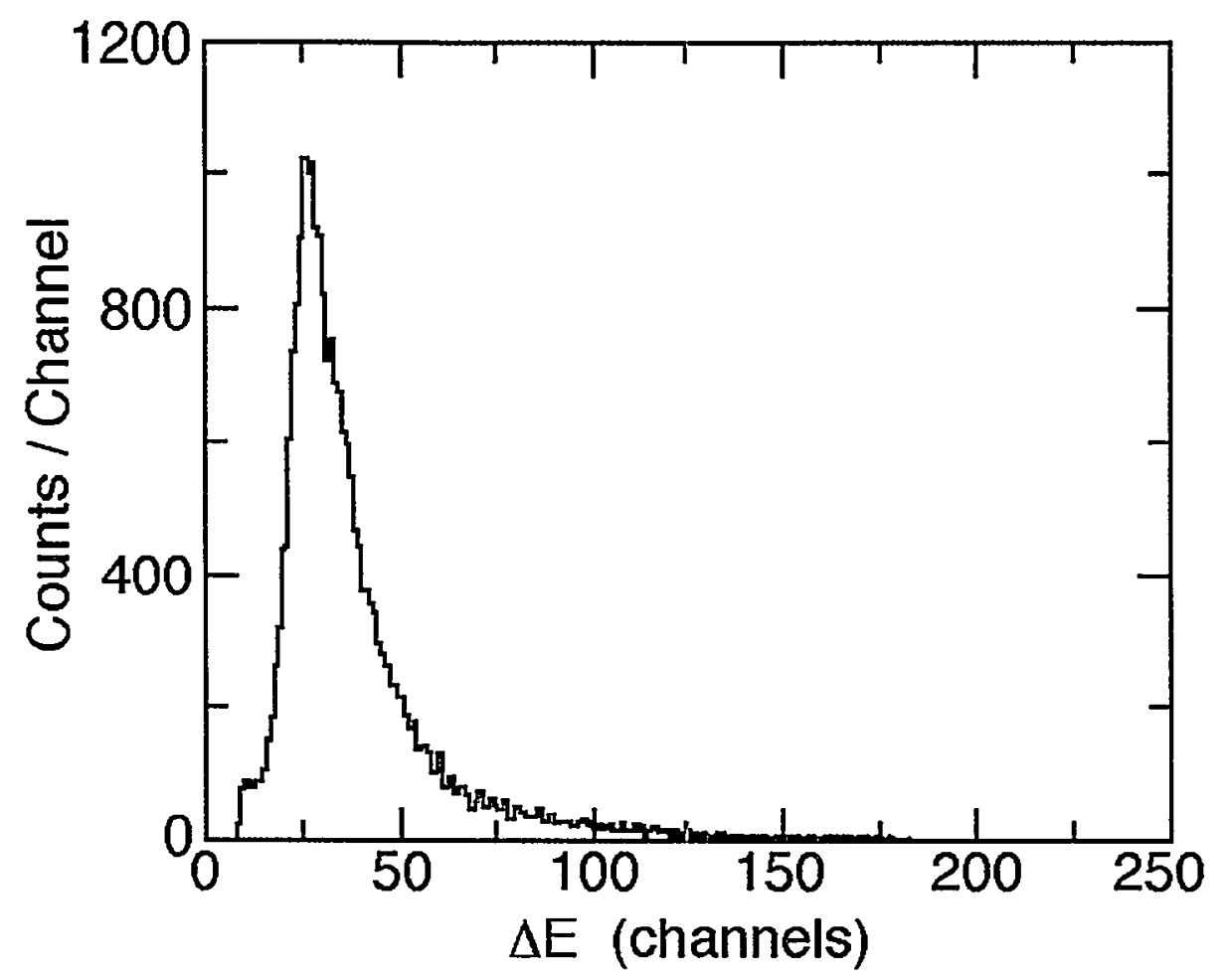

Fig. I-51. Energy spectrum of minimum ionizing electrons from a PHOBOS Octagon sensor.

\section{Multiplicity Reconstruction and Physics Analysis}

In central $\mathrm{Au}+\mathrm{Au}$ collisions at $100 \mathrm{GeV} / \mathrm{u}$, from 5000 to 10000 charged particles are expected to be produced in the pseudo-rapidity range $-5<\eta<5$. The pseudorapidity density is expected to range from 1000 at central rapidity to near zero at $\eta=5$. The techniques which are available for extracting the signal from the multiplicity detector therefore range from simple counting of hits in the regions of low occupancy in each sensor pad to more complex algorithms in the regions where there are expected to be multiple charged particles passing through each pad. In addition to the energy deposition in the sensor pads due to the primary flux of particles originating from the collision vertex, there are also significant contributions from background particles produced by secondary interactions in the beam pipe, detector structure, magnet and the detectors themselves. The magnitudes of these backgrounds clearly need to be understood before any meaningful measurement of the primary multiplicities can be made. The procedure for dealing with the issues of multiple occupancy and backgrounds is inextricably related to our ability to correctly simulate the response of the multiplicity detector. To date, this process has involved the simulation of central $\mathrm{Au}+\mathrm{Au}$ events generated using HIING together with a GEANT simulation of the PHOBOS detector. The results of this simulation were used to generate the fraction of the deposited energy in each pad from the primary particles and the average energy deposited per primary particle. These values will then be used to derive the multiplicities in the real data by first correcting the measured energy per pad for geometric effects and then determining the energy due to primaries and the number of such primaries. The efficacy of this procedure is shown in Fig. I-52 where input and output multiplicity distributions are compared. This procedure is adjusted to the characteristics of the events from HIING and the details of the GEANT simulation of PHOBOS, which, in all likelihood, will not reflect precisely in all respects the situation relevant to the real data. It will therefore be necessary to carefully investigate the validity of the assumptions in the multiplicity reconstruction package. This can be done in a number of ways. The detector is constructed in as symmetric a way as possible, however, some of the sources of background are asymmetric with respect to the nominal collision vertex - for example, the spectrometer magnet. Any asymmetry in the reconstructed events will therefore reflect a problem in the background estimations. In the central rapidity region, where the multiplicity detector occupancy is highest, there are other detectors with low occupancy covering the same region of pseudo-rapidity - 
the vertex detector and the front planes of the spectrometers. For these low-occupancy detectors, the multiplicity can be derived solely from the number of hits and thus used to check the values from the high occupancy sensors. Finally, the spectrometer data on the ratio of particle species and their energy spectra will provide important constraints on the event generators used as input for the correction factor determinations.

The PHOBOS detector is designed to take data with minimum bias trigger at essentially the full rate. In the first month of running, at $1 \%$ of the nominal RHIC luminosity, we expect to obtain some $10^{7}$ multiplicity distributions with over $10^{5}$ for the $5 \%$ most central collisions. Obviously, the first priority will be to assign sensitivities for signals of varying characteristics. Examples of such interesting events could be those with excess multiplicity in regions of pseudo-rapidity which could arise from Disoriented Chiral Condensate formation which can lead to anomalous charged pion fractions. Events with unusual patterns of fluctuations could signal the self-similar behavior characteristics of systems close to the critical point of a phase transition. Correlations in the pion. multiplicity arising from the Hanbury-Brown Twiss effect results in enhancements of pion pairs close in $\Delta-\eta$ and should be observable in our data.

It is clear that a first step in the search for these types of events must be a detailed understanding of the kind of fluctuations present in the data arising from pure statistics. Rather exotic looking events will obviously occur at some level and the question then is the observed fraction of events of any given type larger than that expected on a purely statistical basis. We have started to investigate this using events with the general characteristics expected for central collisions generated both with purely statistical fluctuations and with examples of the expected "signals" added in. Various analysis tools were studied such as deviation functions, correlation functions both of which are closely related to factorial moments, which have previously been used in such analyses. We have also started to look into "Wavelet" transforms of the data that are powerful tools in identifying structures localized in pseudo-rapidity with different widths in $h$. We derived the expectations for the frequency distributions of these quantities for pure Poisson statistics and will therefore be able to make a careful determination of the absolute multiplicities and the overall features of the distributions. This data set and the data to follow will allow for extremely sensitive searches for events with interesting characteristics.

Status and Schedule of Multiplicity and Vertex Detectors

A large fraction of the detector modules needed for the Multiplicity and Vertex arrays are now complete. All modules needed for the six Ring detectors, and more than $70 \%$ of the modules needed for the Octagon and Vertex detectors are assembled, tested, and ready for installation. The remainder will be assembled and tested during the May-June 1999 period.

RHIC will become operational for physics data taking on 1 November 1999. At this time, the entire multiplicity and Vertex detectors, the time-of-flight wall, and one of the two spectrometer arms will be complete and installed in the RHIC tunnel. Prior to the first physics run, a period of machine commissioning will take place beginning 1-June 1999. For this commissioning period, PHOBOS will install the Octagon frame with 13 detector modules, as well as two Vertex detector modules. We will use this preliminary setup to evaluate the performance of the modules and electronics. In addition, during this period we will attempt to reconstruct the first collision vertices, and do preliminary studies of interesting quantities such as the multiplicity at mid-rapidity, from early collisions at RHIC. 


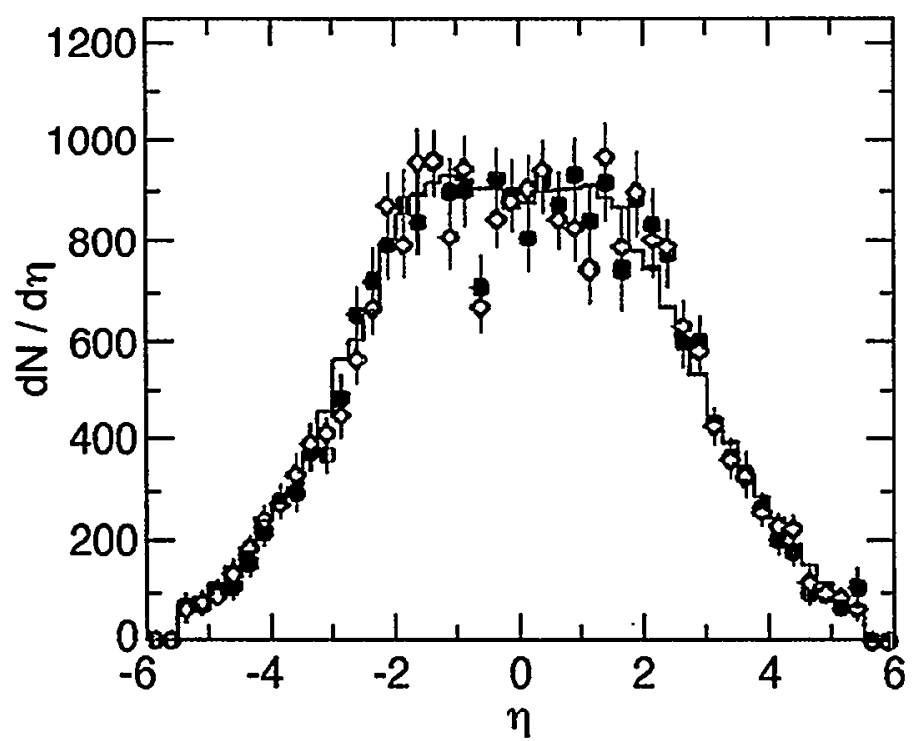

Fig. I-52. Multiplicity distribution from one HIJET event (solid symbols), the same event reconstructed (open symbols) and the average of 200 HIJET events (histogram). 


\section{E. FUNDAMENTAL INTERACTIONS AND OTHER TOPICS}

Over a year ago, the Canadian Penning Trap (CPT) was moved to ATLAS, where it was installed and made operational by a collaboration of Canadian and American scientists. The device aims at high-precision determination of atomic masses of short-lived isotopes. One of the main directions of research is high precision measurements of the mass of super-allowed $0^{+}$to $0^{+}$beta emitters $(50<\mathrm{A}<100)$. These mass determinations have a direct impact on the determination of the fundamental weak vector coupling constant and the unitarity test of the top row of the CabbiboKobayashi-Maskawa matrix.

This section also describes efforts devoted to other topics such as (1) the development of a new method to measure the electric dipole moment of the neutron, (2) the determination of the half-life of ${ }^{44} \mathrm{Ti}$, an isotope of importance in astrophysics, and (3) the physics of cooled beams of charged particles confined in storage rings and traps.

\section{e.1. Progress at the Canadian Penning Trap Mass Spectrometer (G. Savard, D. Seweryniak, J. Uusitalo, H. Fukutani, * K. S. Sharma,* R. C. Barber,* F. Buchinger, $\uparrow$ J. E. Crawford,$\uparrow$ S. Gulick, $\uparrow$ J. C. Hardy, $\ddagger$ J. K. P. Lee, $\dagger$ and R. B. Moore, $\dagger$ )}

The Canadian Penning Trap (CPT) Mass Spectrometer installed at ATLAS is a device aiming at the precise determination of the atomic masses of short-lived isotopes. It uses a combination of two ion traps, a radio-frequency quadrupole (RFQ) trap and a precision Penning trap, to capture and accumulate short-lived isotopes and confine them at rest in vacuum. This then enables properties of these isotopes to be precisely. determined using sample sizes as small as a few ions injected in the measurement trap.

The work performed this year on the CPT spectrometer centered around systematic mass measurements on stable isotopes to study possible sources of errors when performing high-precision $\left(\Delta \mathrm{M} / \mathrm{M} \sim 10^{-8}-10^{-9}\right)$ measurements. Measurements on heavy isotopes, particularly sensitive to electrostatic field imperfections in the Penning trap, were used to tune the device (see Fig. I-53), to check the short term and long term stability (see Fig. I-54) of the device, compare results with accurately known mass ratios, study the effect of the number of ions inside the trap, of the amplitude and time duration of the various excitations performed on the ions during a measurement (see Fig. I-55), of cleaning mechanisms to selectively remove contaminants from the trap, of evaporative cooling inside the trap to better center the ion cloud, etc. Properties of the ions ejected from the RFQ trap as a function of the ejection phase (see Fig. I-56), RF amplitude and pulse duration were also studied and their possible influence on the measured cyclotron frequencies investigated. The results were compared to a complex computer simulation that has been developed to mimic the CPT and adds to our quantitative understanding of the various sources of possible systematic errors. It is found that the resonance frequencies, and hence the magnetic field, decay steadily at a rate of about $2 \times 10^{-8}$ per day (well within the specifications of the superconducting magnet), with no noticeable short-term variations related to day/night, temperature or external field effects. The effect of the various parameters mentioned above on the resonance frequencies depend on the trap tuning and the excitation parameters and, for an

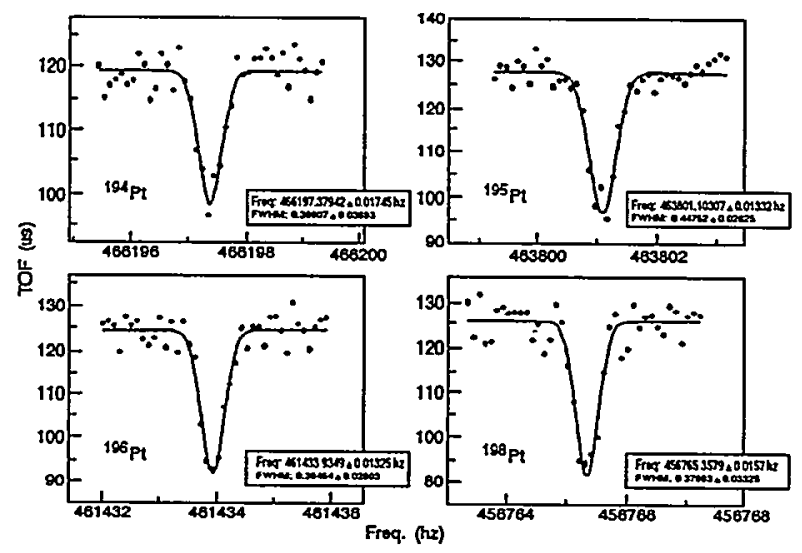

Fig. I-53. Cyclotron resonances obtained for 4 stable isotopes of Platinum produced by the laser ion source used to inject stable ions into the CPT. Each figure corresponds to about 15 minutes of data taking.

improperly tuned trap, can be very significant. For example, systematic shifts as large as $10^{-7}$ were observed when increasing the number of ions inside a "not-tuned" trap to more than 100 . It is, however, 
found that with the precision Penning trap properly tuned, the excitation parameters corresponding to a full conversion from initial magnetron motion to cyclotron motion and careful control of the injection parameters, the systematic shifts in mass ratios are kept below the $10^{-8}$ level for the range of parameters we have explored.

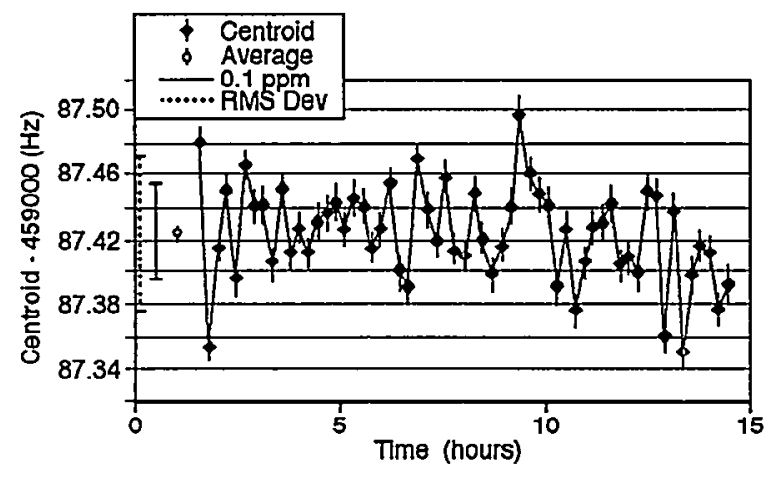

Fig. I-54. Stability of the cyclotron frequency measured at constant interval during a 15 hour run. Individual runs scatter at the $\pm 60 \mathrm{ppb}$ level, with no discernable systematic daily shifts, and the centroid is determined with a precision of $\pm 8 \mathrm{ppb}$.

The CPT is injected with radioactive ions collected at the back of the area II split-pole magnet in an RF gas cooler designed to stop, cool and accumulate these unstable isotopes and transfer them as a pulsed lowenergy beam to the CPT. On-line and off-line studies of the gas cell/gas cooler assembly were performed to determine optimum parameters for slowing down, cooling, bunching and transfer to the CPT. Test with a ${ }^{252} \mathrm{Cf}$ source and detection of the activity transported at different points along the system reproduced the $20 \%$ efficiency observed last year out of the gas cell and a redesign of the nozzle allowed the capture and transport efficiency of the cooler to be increased from roughly $50 \%$ to close to unity when operating the cooler as a transmission device. Operating the last section of the cooler as a linear trap to bunch the transfer of the radioactive ions can maintain this excellent efficiency as long as the trap is not saturated by space charge from other ions coming out of the gas cell. A number of measures to improve the purity of the helium used in the gas cell have resulted in this unity transmission after the gas cell to be maintained when ejecting the fission fragments out of the gas cooler at the $20 \mathrm{~Hz}$ rate we plan to operate it to inject radioactive ions into the CPT. Tests performed on-line with ${ }^{38} \mathrm{~K}$ activity produced with a ${ }^{28} \mathrm{Si}$ beam from ATLAS impinging on a ${ }^{12} \mathrm{C}$ target gave similar results. It was, however, found that transmission of heavier ions obtained directly from ATLAS showed very poor transmission. It is now believed that this was caused by the technique used to decelerate the beam before injection into the gas cell which involved running the gas-filled magnet at a high enough pressure to remove most of the energy of the ions by the time they reached the entrance window to the gas cell so that only a small residual energy needed to be removed by the high purity gas. The energy lost in the gas-filled magnet was sufficient for the rigidity of the ions to depart from the slowly varying with energy regime resulting in the ions reaching the focal plane at a large angle with respect to the axis of the gas cell. A tunable degrader is being constructed to be installed in front of the gas cell and allow the gas-filled magnet to be operated in a lower pressure regime where the charge focusing is obtained without distorting the orbits or introducing excessive multiple scattering.

\footnotetext{
*University of Manitoba, Winnipeg, Ontario, †McGill University, Montreal, Quebec, †Texas A\&M University
} 


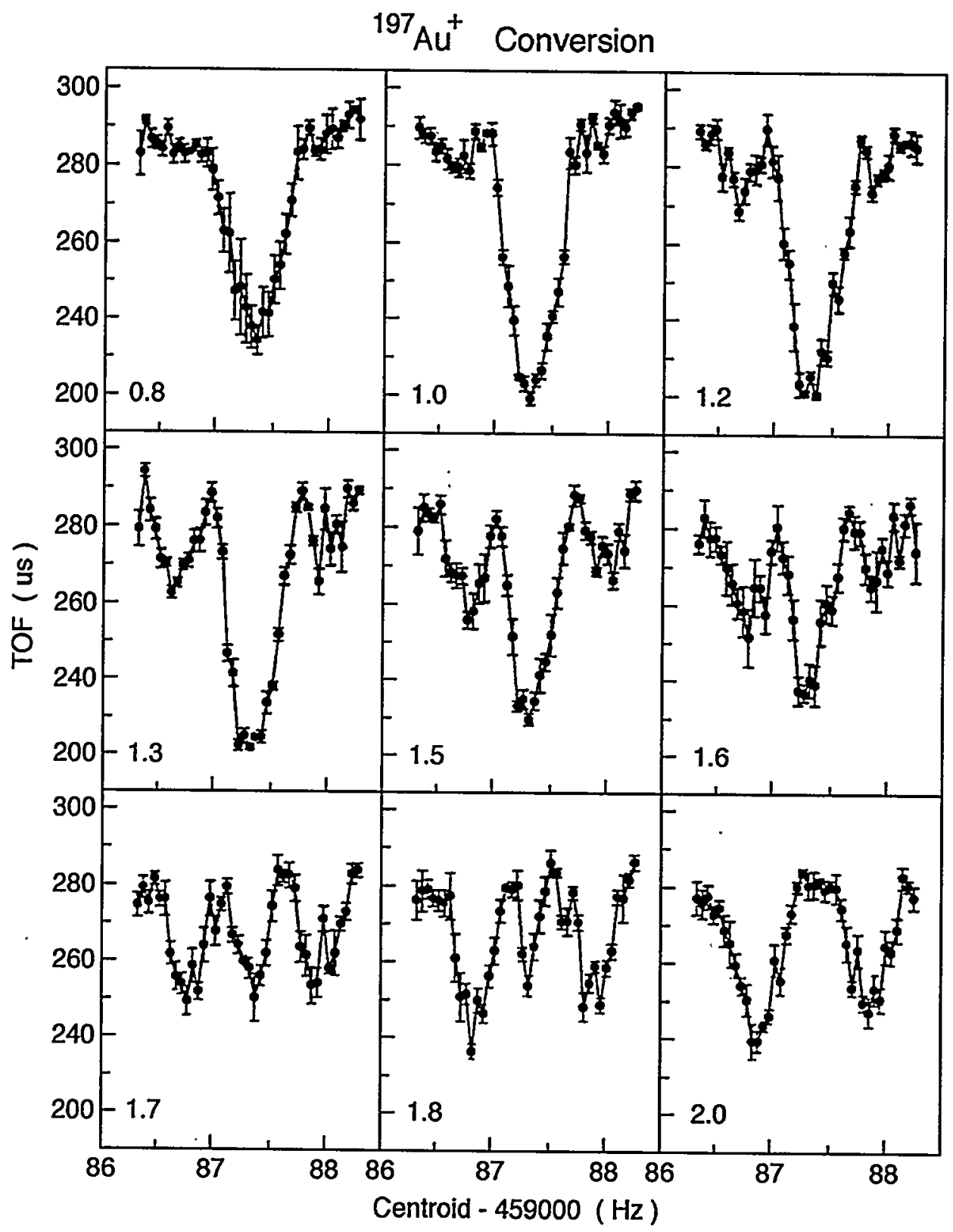

Fig. 1-55. Study of the time-of-flight (TOF) effect observed as a function of the cyclotron frequency excitation amplitude. An excitation amplitude resulting in a full conversion from the initial magnetron motion to cyclotron motion gives maximum signal amplitude and lowest sensitivity to a number of possible sources of systematic effects. It corresponds to the spectrum labeled 1 . As the amplitude is further increased conversion back to magnetron motion occurs up to the spectrum labeled 2 where the central peak has disappeared and we are left only with the sidebands of the excitation. 

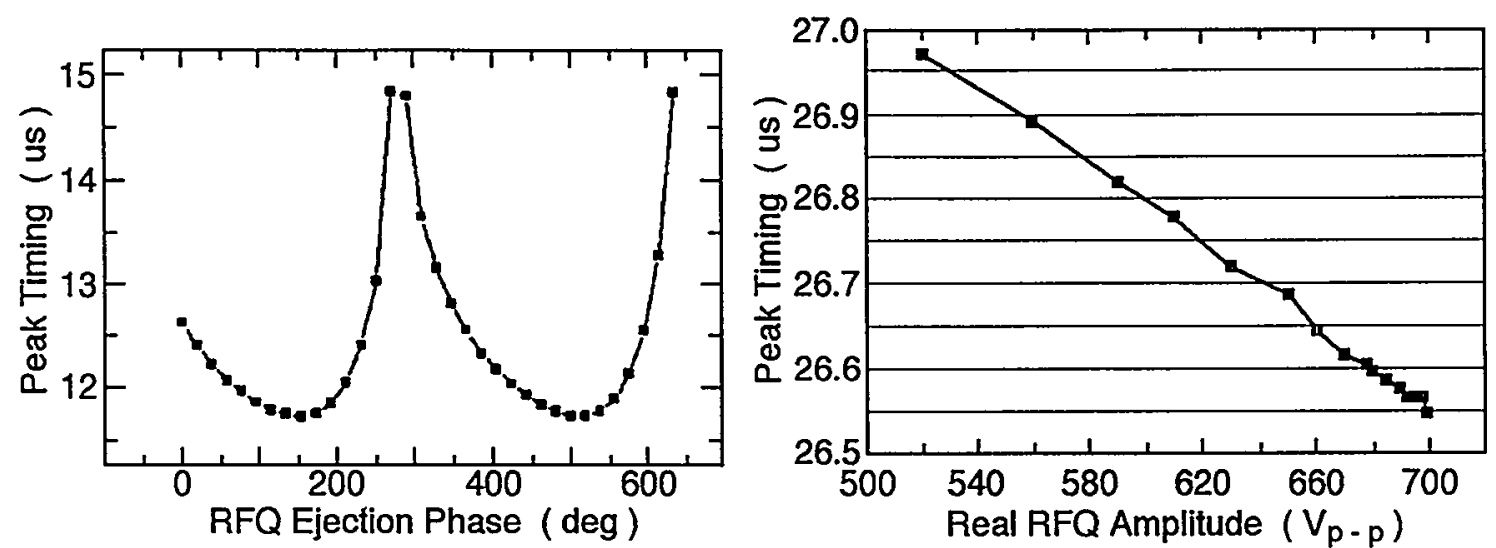

Fig. 1-56. Studies of the arrival time of the ions ejected from the $R F Q$ trap at an ion detector located below the Penning trap as a function of the ejection phase out of the $R F Q$ trap and the $R F$ amplitude.

\section{e.2. Development of a Large Accelerated Gas Cell System for the Collection of Fast Recoiling Radioactive Ions (G. Savard, D. Seweryniak, J. Uusitalo, B. Zabransky, H. Fukutani,* V. Pelletier, $\dagger$ and K.S. Sharma*)}

As part of the development of the injection system for the CPT mass spectrometer at ATLAS, a large gas cell (about 12-cm long running at 150 Torr) loaded with high-purity helium gas was constructed and used to stop reaction products at the focal plane of the area II Enge split-pole magnet. Gas flow out of the gas cell transported the thermalized ions to a radio-frequency gas cooler which cooled and accumulated them in a linear trap before transfer to the CPT. About $20 \%$ of the reaction products thermalized in the gas cell were extracted from the cell by the fast flow and half of those were accumulated in the linear trap. This demonstrated that large efficiencies can be achieved with large gas cells when the ionizing primary beam does not enter the stopping gas volume.

While the stopping volume used is much larger than in a standard IGISOL-type system, other possible applications requiring rapid thermalization of fast ions could use even larger effective stopping ranges. In particular, as part of the Argonne ISOL concept, it is proposed to use intense energetic heavy-ion beams to produce short-lived nuclei by projectile fragmentation, perform in-flight fragment analysis and separation on the fast moving recoils and stop the fragments in an IGISOL-type helium gas cell from which they would be quickly extracted as singly-charged ions and reaccelerated. This scheme combines the intrinsic advantages of in-flight fragmentation - short delay times - with those of the ISOL concept - high-quality beams of precise energy as required by experiments. It does, however, require much larger stopping volumes than even what was achieved with the original cell at the CPT. For this reason, development of a gas cell which would provide a larger stopping range while maintaining the fast extraction time was initiated.

The gas cell catcher necessary to stop reaction products produced by fragmentation requires a stopping length, after energy degradation and compensation of the recoils, of between 0.5 to 5 atmosphere-meter of helium, depending on the mass range of interest. Using solely gas flow to pull the ions out of such a large gas volume would be prohibitively slow with available pumping systems. It is, therefore, necessary to guide the ions towards the exit of the gas cell. This can be done with a combination of electrostatic and RF fields applied inside the cell. The cell being studied to stop fragmentation products would have a length of about 50 $\mathrm{cm}$ and be operated at pressures, depending on the fragments to be observed, varying from 1 to 10 atmospheres. An electric gradient along the length of the cell will drag the radioactive ions stopped in the gas towards the cell exit where a set of concentric electrodes will help focusing them on the exit hole. An RF field applied along the length of the cell prevents them from diffusing to the walls of the cell. At the exit hole, the gas velocity grows rapidly and pulls the ions out. The voltage gradients required to extract the ions in ms timescales is determined from ion mobility data. 
To demonstrate the feasibility of the concept, a scaled down $15-\mathrm{cm}$ prototype of the gas cell operating at up to 0.5 atmosphere was designed using an ion trajectory program in which the effect of the gas is added by a Monte Carlo approach simulating the gas collisions, fitted to reproduce the ion mobility data. The scaled down version of the gas cell has been constructed at Argonne (Fig. I-57) and preliminary measurements performed with reaction products validate the simulations (Fig. I-58). An efficiency of $20 \%$ has been achieved so far and the decrease in extraction time when an electric field is present has been demonstrated to be in good agreement with the predictions (Fig. I-59). Scaling to higher pressures should not present significant technical challenges.
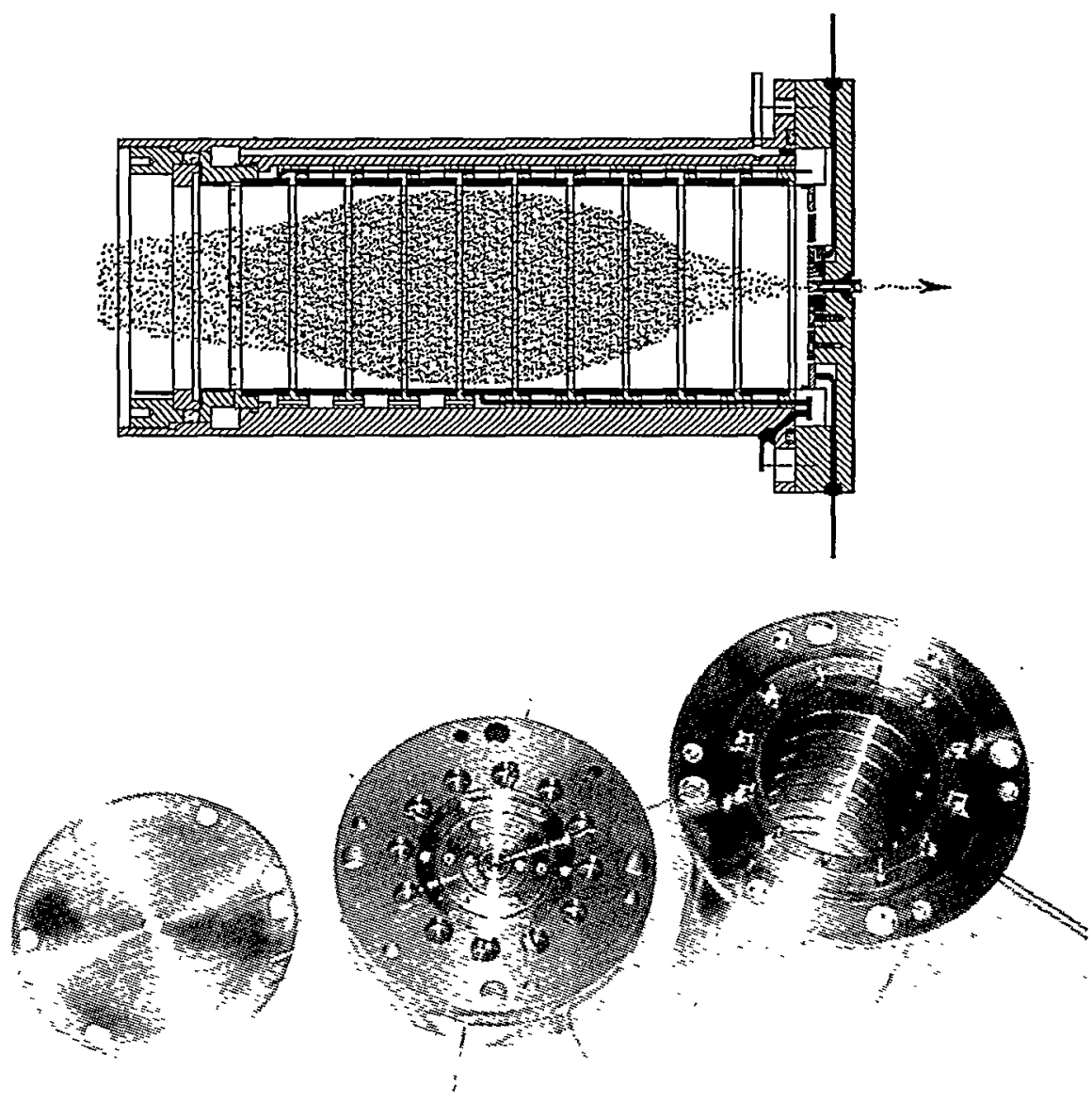

Fig. I-57. Top: Layout of the prototype accelerated gas cell with ions coming in through a thin window on the left, being thermalized in the cell and extracted as a thermal ion beam on the right. Bottom: Components of the gas cell tested with the CPT gas cooler setup. Shown at the right is the cylindrical stack of electrodes guiding the ions towards the exit plate, in the center is the set of concentric extraction electrodes which pull the ions towards the extraction hole, and at the left is the guard electrode covering the cell exit.

One issue of importance in IGISOL type systems is the amount of ionization created in the gas by the primary beam. Studies show that the efficiency of IGISOL systems start to decrease when the amount of energy loss in the gas exceeds a value between $10^{16}$ and $10^{19}$ $\mathrm{eV} / \mathrm{cm}^{3}$ depending on the exact geometry. We therefore looked at the ionization densities we could expect with such a gas cell located after a fragment separator. The ionization densities we will be dealing with, even at $100 \mathrm{~kW}$ of primary beam power, are far below this. For example, with $100 \mathrm{~kW}$ of $100-\mathrm{MeV} / \mathrm{u}{ }^{18} \mathrm{O}$ beam to produce ${ }^{14} \mathrm{Be}$ we would have a total of about $7.2 \times 10^{6}$ pps entering the cell from the magnetic separator system, a large fraction of them ${ }^{14} \mathrm{Be}$. Each particle would deposit about $70 \mathrm{MeV}$ of energy in the cell resulting in a total energy deposition of $5 \times 10^{14} \mathrm{eV}$ over a volume of about $1200 \mathrm{~cm}^{3}$ for an energy deposition density of about $4 \times 10^{11} \mathrm{eV} / \mathrm{cm}^{3}$. This is orders of magnitude below the levels where IGISOL systems show limitations. 

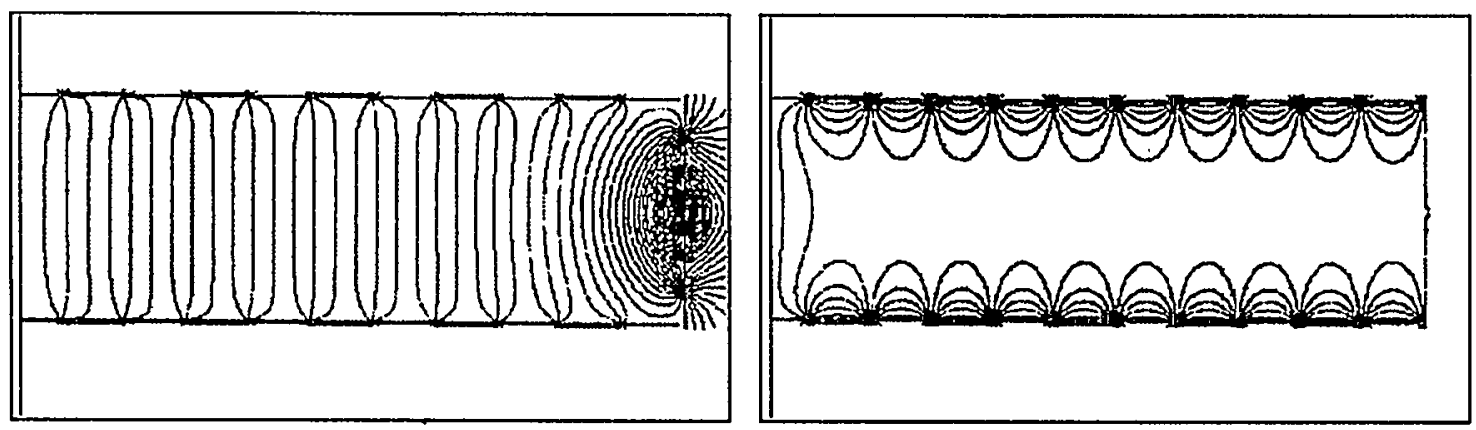

Fig. 1-58. Electrode geometry and resulting $D C$ and $R F$ electric field used in the simulation of the ion motion inside the gas cell (extraction hole is on the right). RF field is added on top of this DC field and a Monte-Carlo subroutine called by the Runga-Kutta program reproduces the effect of realistic ion-neutral collisions.

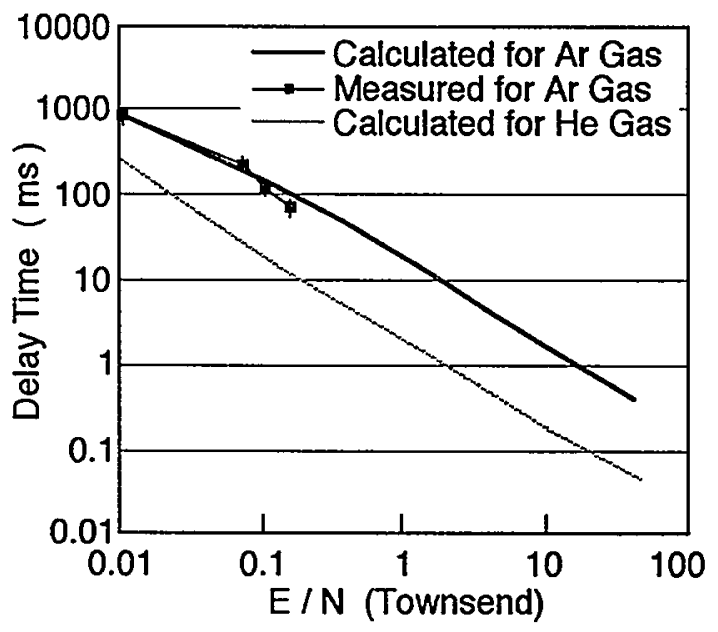

Fig. 1-59. Mean delay time between injection of a pulsed beam in the gas cell and extraction of the ions out of the. cell as a function of the electric field gradient applied inside the cell. A Townsend is $10^{-17}$ volts- $\mathrm{cm}^{2}$.

The ions extracted from the gas cell are still within a considerable gas flow and direct acceleration of these ions would result in very poor beam quality due to straggling in the gas during acceleration. The ions extracted from the gas cell will therefore first be channeled by an if quadrupole structure through a section of differential pumping to an acceleration region where the acceleration can take place unhampered by the residual pressure from the gas cell. Preliminary tests with the ions extracted from the accelerated gas cell injected and cooled in the CPT's RF gas cooler indicate a transmission of about $80 \%$ and a beam emittance well below the standard emittances observed at existing ISOL facilities (in the 10-30 $\pi \mathrm{mm}$ mrad range at $60 \mathrm{keV}$ ).

\footnotetext{
*University of Manitoba, Winnipeg, Ontario, $†$ McGill University, Montreal, Quebec
} 


\section{e.3. Temperature, Ordering and Equilibrium in an RF Ion Trap (J. P. Schiffer)}

The ordering of cold, laser-cooled, ions confined in an ion trap or storage ring has been studied both experimentally and through simulations. In earlier work systems under a constant focusing field were studied. While alternating quadrupole fields can be used in ion traps and are used in storage rings, the general opinion has been that some of the time-dependence of the focusing field will be transformed into "heat" and pose an ultimate limit to the size of systems that can be cooled.

In the past year, experimental results at Aarhus showed that considerably larger systems can form ordered systems in traps than was previously thought feasible [Phys. Rev. Lett. 81, 2878-2881 (1998)]. An example of this is shown in Figure I-60. Numerical simulations using molecular dynamics and following the motion in the oscillating confining field have been able to reproduce this - though since the motion is much faster than the characteristic times of an ion in an average trap field, the numerical integration of the equations of motion has to be carried out in much finer time steps and thus requires more computer time.

More generally, the limits of this coupling between the If motion and the "temperature" of ions was investigated. Figure I-61 shows the results of a simulation for 1000 ions, the ordered structures on the surface, the interior, and the amplitude of the rf motion of ions. These systems were "cooled" by gradually reducing the kinetic energy spread in the third dimension, perpendicular to the quadrupole motion, as it is done in laser cooling. A measure of an effective temperature in the other direction may be obtained by looking at the displacement of ions at the same point in the rf cycle and thus obtaining a measure of the aperiodic component of motion.

Once a system is cooled, it is allowed to propagate in the simulation, and the amount of energy transferred from the rf motion into random heat can be monitored. No such observable coupling was seen - suggesting that the time constant for such heating is at least hours.

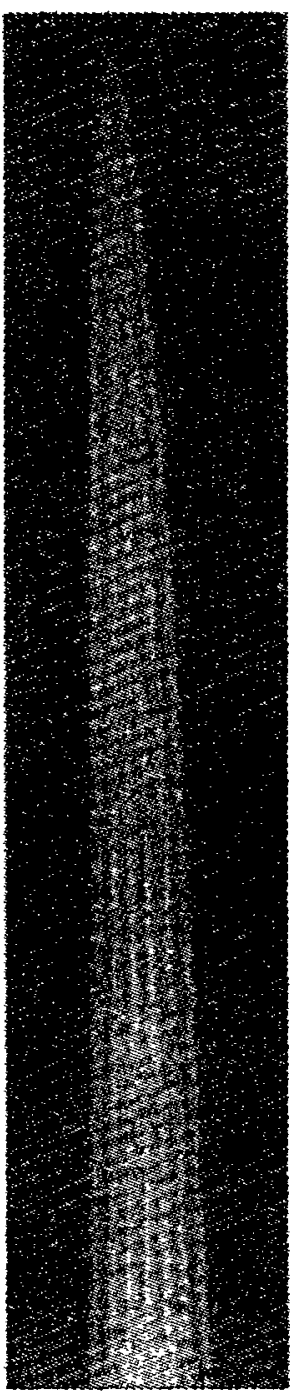

(a)

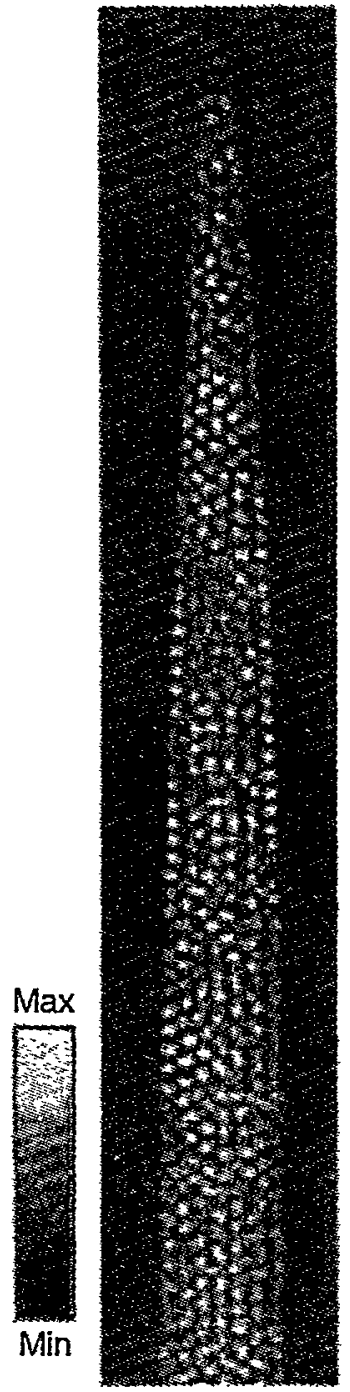

(b)
Fig. I-60. (a) CCD picture integrated over 2 sec of a very prolate crystal of about 3500 ions. (b) Molecular dynamic simulation with 3500 ions in a crystal with roughly the same aspect ratio as the one in (a).

This process was then repeated at systems prepared to different effective temperatures, whose density profiles are shown in Figure I-62. The corresponding coupling of rf energy into random heat begins to be noticeable at about the temperature where the ordering in the system dissolves. The coupling increases as the effective temperature squared, as is seen in Figure I-63. 


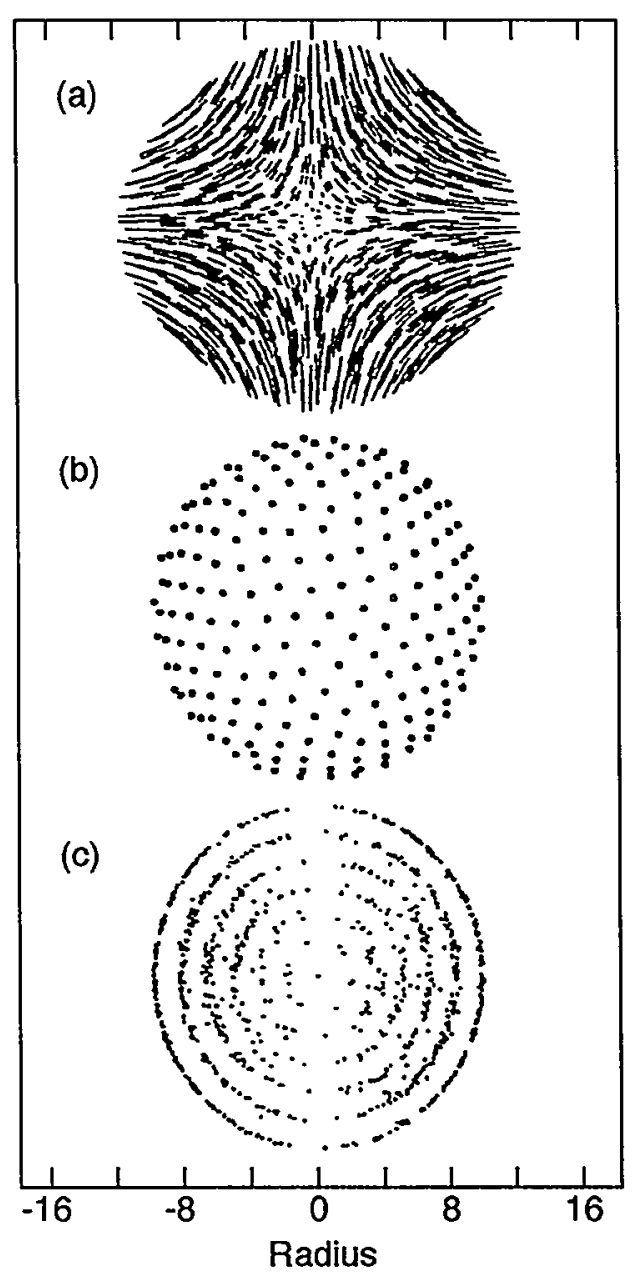

Fig. 1-61. The amplitude of the motion of particles in the applied rf field is shown in (a) on top. The arrangement of ions in the outermnost shell for the cooled 1000-ion system is shown in (b) in the middle, and the projection of the particles in the entire spherical system indicating the shell structure is in (c) on the bottom. The distance scale shown is in units of $a_{s w}$ of Seitz-Wigner radius for the cold system.

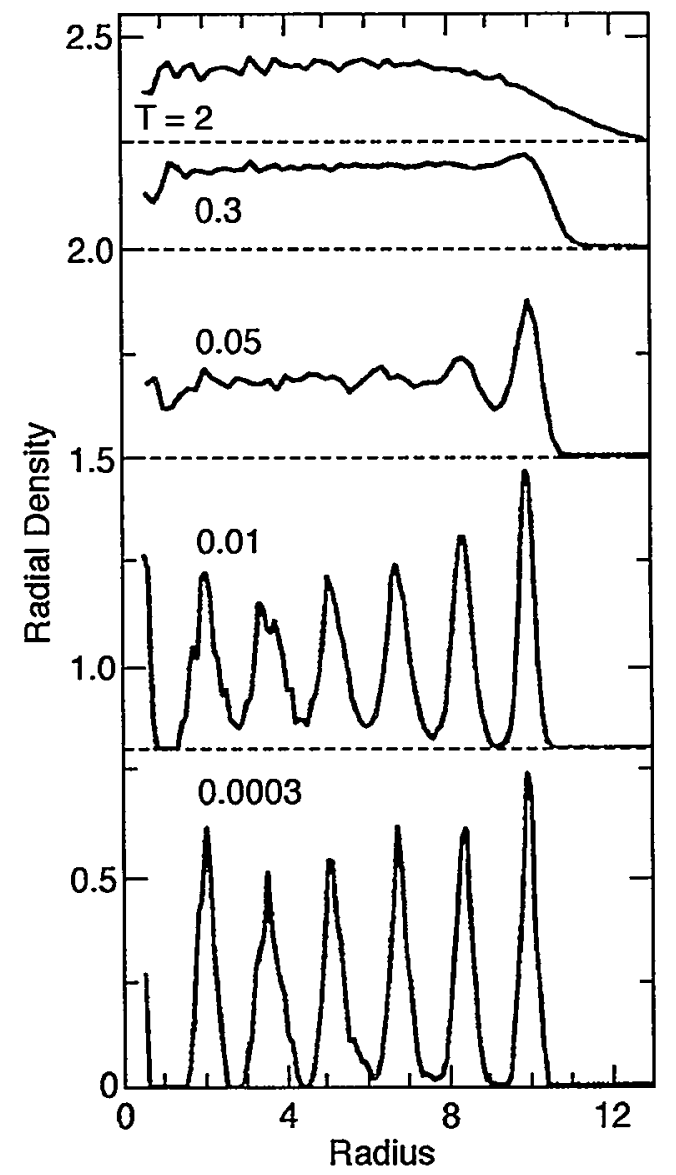

Fig. I-62. The average radial densities of the spherical 1000-ion system at various temperatures, derived from the aperiodic part of the kinetic energies. The radius is in units of $a_{s w}$, the density in terms of ions/ $a_{s w}{ }^{3}$ and the temperatures in units of $q^{2} / a_{s w}$. 


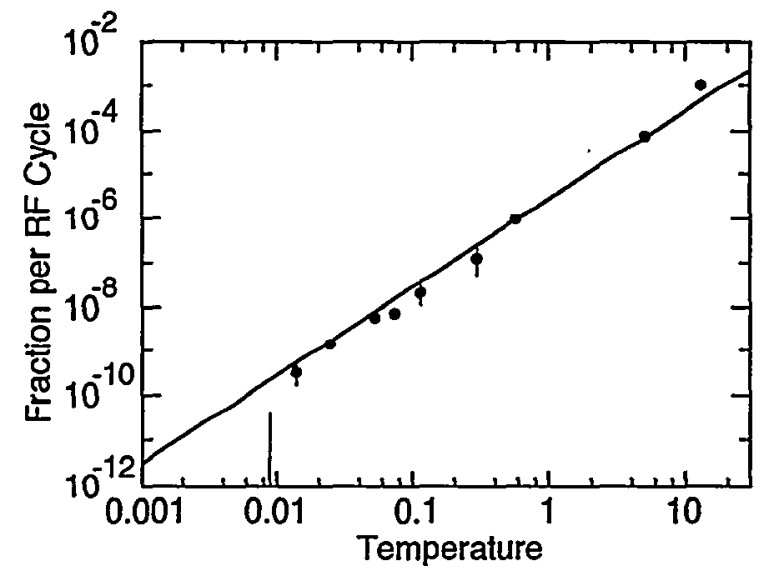

Fig. I-63. The coupling between the kinetic energy in the periodic motion of the quadrupole trap, and aperiodic thermal motion. The fraction of the kinetic energy in the periodic motion that is mixed into heat per rf period is plotted as a function of temperature in units of $q^{2} / a_{s w}$. The line represents a quadratic dependence of this coupling on temperature. The error bars represent an estimate in the uncertainty in determining this coupling from the simulations, due to the fluctuations in a finite system.

\section{e.4. A Long Measurement of the ${ }^{44}$ Ti Half-Life (I. Ahmad, J. P. Greene, W. Kutschera,* and M. Paul $\dagger$ )}

Theoretical models of supernova explosions were developed over the years which predict the amount of ${ }^{44} \mathrm{Ti}$ produced in these explosions. Recently the characteristic gamma line of $1157.0 \mathrm{keV}$ in ${ }^{44} \mathrm{Ti}$ decay was observed from the supernova explosion CAS A by the space-borne COMPTEL gamma-ray spectrometer. The COMPTEL spectrometer also detected a previously unobserved supernova explosion ${ }^{1}$ which is estimated to have occurred about 700 years ago. These observations, along with the half-life of ${ }^{44} \mathrm{Ti}$, provide the mass of the ${ }^{44} \mathrm{Ti}$ excreted in these explosions. Such masses can be used to test the theoretical models of supernova explosions and the formation of heavy elements.

Because of the importance of the ${ }^{44} \mathrm{Ti}$ half-life we undertook its measurement several years ago. We followed the decay of ${ }^{44} \mathrm{Ti}$ relative to ${ }^{60} \mathrm{Co}$ decay for 5 years and determined the ${ }^{44} \mathrm{Ti}$ half-life. We obtained a value of $59.0 \pm 0.6 \mathrm{y}$ and the results were published last year. ${ }^{2}$ We continued our measurements at Argonne and Jerusalem and followed the decay for an additional two years. At Argonne, we measured spectra at two sourceto-detector distances of 5.2 and $10.2 \mathrm{~cm}$. Also, spectra of a mixed ${ }^{44} \mathrm{Ti}+{ }^{60} \mathrm{Co}$ source and pure ${ }^{44} \mathrm{Ti}$ and ${ }^{60} \mathrm{Co}$ sources were measured. The half-life was determined from the ratio of counts in the 1157 and $1332 \mathrm{keV}$ peaks and from the ratio of the 1157 and $1173 \mathrm{keV}$ peaks. At Jerusalem, spectra of only the mixed source were measured and at one source-to-detector distance. The analyses of all these data sets gave a value of the half-life consistent with our published value of $59.0 \pm$ $0.6 \mathrm{y}$. A decay curve with the additional data points is displayed in Fig. I-64.

*University of Vienna, Austria, $†$ Hebrew University, Jerusalem, Israel

${ }^{1}$ A. F. Iyudin et al., Nature 396, 142 (1998). 2Phys. Rev. Lett. 80, 2550 (1998). 


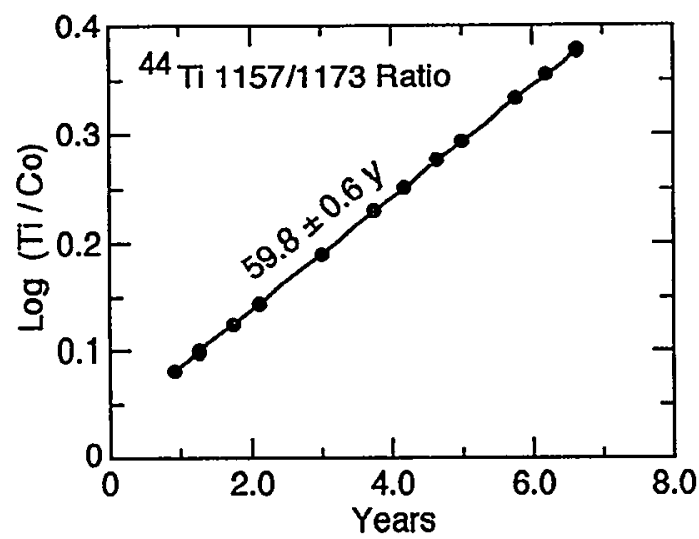

Fig. I-64. Semilogarithmic plot of the ratios of the counts in the $1157 \mathrm{keV}$ peak of ${ }^{44} \mathrm{Ti}$ to the counts in the 1173 $\mathrm{keV}$ peak of ${ }^{60}$ Co against time. The last three points were obtained after the publication of our paper.

\section{e.5. A Proposed Method for Measuring the Electric Dipole Moment of the Neutron by a Large Improvement of the Shull Method (G. R. Ringo and T. W. Dombeck*)}

The Shull Method of measuring the electric dipole moment (EDM) of the neutron is very different in systematic errors from the standard NMR method and our interferometric method. ${ }^{1}$ Where Shull and Nathans used a single reflection of neutrons in their experiment, we propose to use roughly 5000 reflections in a slot milled in a perfect silicon crystal. We are preparing to do a test of reflectivity of these crystals at the University of Missouri Reactor.

This method should be simple and less expensive than the one we proposed in 1997, but it may not be as sensitive.

*Fermi National Accelerator Laboratory

1M. S. Freedman, G. R. Ringo and T. W. Dombeck, Nucl. Instrum. Methods A396, 181 (1997). 


\section{F. EQUIPMENT DEVELOPMENT AT THE ATLAS FACILITY}

This section describes the various technical developments linked with the experimental program associated with Heavy-Ion research within the Division.

With Gammasphere completing a first full year of operation at ATLAS, it should come as no surprise that much effort was devoted to the effective operation of the device.

This section also contains a description of new developments associated with other programs such as the LEPPEX array, the production of radioactive beams, the FMA, target making and computing.

f.1. Installation and First Tests of Gammasphere (M. P. Carpenter, D. Nisius, R. V. F Janssens, J. Joswick, T. Lauritsen, C. J. Lister, P. Reiter, I Wiedenhöver, P. Wilt, B. Nardi, B. Chyna, J. Falout, J. Rohrer, M. Randolph, J. Weizeorick,* P. Fallon, $\dagger$ A. O. Macchiavelli, $\uparrow$ D. Landis, $\uparrow$ and I. Y. Lee $\dagger$ )

The reinstallation and re-commissioning of Gammasphere at ANL proceeded smoothly and rapidly due to extensive planning. Starting in the second week in November, when the support frame arrived and was unpacked, an intense five-week effort saw the entire reassembly and realignment accomplished. In the first three weeks the support frame was mounted on the rails, the gray cables unspooled and tested, the cable trays installed, the gray cables installed and the frame was aligned. The entire LN2 system was installed and tested. During this time the detector inventory was being readied for installation. The remaining BGO shield problems were fixed and the second half of the germanium ( $\mathrm{HpGe}$ ) inventory was annealed and tested. In the next two weeks the BGO shields were installed and then populated with $\mathrm{HpGe}$ detectors which were cooled. By Christmas the array was essentially rebuilt.

The post-Christmas period was dedicated entirely to improving the array performance. Many minor failures were identified as the array was turned on counter-bycounter. Each problem was solved as it arose. Consequently, by the second week in January, when detailed calibrations were performed, the system was found to operate better than it had ever done before, with an overall array resolution of $<2.4 \mathrm{keV}$ at $1332 \mathrm{keV}$ and a Peak/Total ratio of $>62 \%$ using ${ }^{60} \mathrm{Co}$.

The early test runs also saw many minor issues resolved and experience gained in beam tuning. In the data shack, the actual Gammasphere VXI system ran almost flawlessly, though considerable effort was needed to keep the auxiliary, detector ECL bus operational and free from hang-ups. With experience and replacement of faulty components the problems became steadily less severe. Similarly, in the data-room, in the VME crates, many hang-ups were initially experienced, with the EFF microproccessors, with memories, with tape drives and with data senders. Many of the problems were very difficult to trace and identify, as bad data from the shack can put unplanned strains on the front-end system. However, many modifications to the acquisition system were made in order to try to identify bad data and provide more preventative checking of the data stream. Consequently, by February the system was running reliably and by early summer crashes of the acquisition system had become rare. Most of the second half of the year saw runs with no crashes.

As the running reliability improved, attention was turned to making real improvements to the system. Many of these involved reductions of acquisition deadtime. A very fast trigger was built by externally collecting the individual VXI HpGe signals, a "fast clear" input for aborting events was installed, and the "pipeline" readout mode was made operational for the first time. Most of these features are now in regular use.

*Electronics Division, ANL, †Lawrence Berkeley National Laboratory 


\section{f.2. Gammasphere Operation (C. J. Lister, M. P. Carpenter, T. Lauritsen, R. V. F. Janssens, P. Reiter, and D. Seweryniak)}

Gammasphere started operating on January 15, 1998 and, after some short commissioning tests, started the PAC approved program on January 21. It has run rather smoothly since then, with 185.5 days of research data acquisition during the first full year of operation. During the entire time the array had a near-full compliment of detectors (100 or 101).
Despite almost non-stop experimentation and set-up, several problems with electronics and data acquisition were located and improvements were made, including the implementation of "pipelining" and "fast clear" facilities (to reduce deadtime) and a simplification of the EFF processor codes which seem to have reduced VME front-end crashes. We present some statistical data on operations

Total time $\quad$ 15th January -15 th January $=52$ weeks $=365$ days

$\begin{array}{llc}\text { Data Collecting “In-Beam" } & 155.5 \text { days } & 43 \% \\ & 30 \text { days } & 8 \% \\ \text { "Source Expts." } & 38 \text { days } & 10 \% \\ \text { "Gammasphere time" } & 61 \% & \\ \text { Data Collection } & 4452 \mathrm{hrs}\end{array}$

Experiments $36 \quad$ PAC Approved

Equipment Development Runs $\quad 6$

\section{f.3. Maintenance of Gammasphere's Germanium Detectors (I. Wiedenhöver, R. V. F. Janssens, P. Wilt, C. Lister, and D. Nisius)}

The germanium detectors of Gammasphere performed outstandingly in their first year of operation at ATLAS. In the standard configuration at the FMA, Gammasphere can hold 101 gamma detectors with their respective escape-suppression systems. The Gammasphere detectors were purchased between 1994 and 1996 and were constantly used in experiments at Lawrence Berkeley National Laboratory before Gammasphere was moved to ANL. As a result all the detectors had to be annealed before the start of operations at ATLAS. The vast majority of the experiments with Gammasphere at ATLAS ran with the maximum number of possible germanium detectors, a few with one "open" position.

During normal operation, two detectors developed electronic problems which could be solved in house by replacing the faulty components. Two detectors showed problems during the high humidity of the summer period. These problems were solved by placing desiccant material around the preamplifier electronics and sealing the housing. A total of eight detectors, of which five had reached Argonne in faulty condition, were sent back to the manufacturer for repair.

While the detectors in Gammasphere are always kept cold at liquid nitrogen temperature to maintain their electronic properties and the internal vacuum, they are warmed up to room temperature when they are taken out for operational reasons or repair. We adopted a procedure where the detectors warm up while the vacuum is maintained by an annealing station. Furthermore, the detector crystal and the molecular siff are heated for a short period to outgas possible vacuum contaminants. This procedure was applied on seventeen occasions. The Gammasphere detector lab possesses ten annealing stations which allows for an efficient operation.

For two series of experiments in June and NovemberDecember, thirteen detector systems, including the escape-suppression system, in the downstream direction 
of Gammasphere were removed to make room for neutron detectors. The $\mathrm{Ge}$ detectors in these most forward positions are usually exposed to an increased flux of high-energy neutrons, which degrades their performance over time. Although the detectors had not yet developed significant problems after almost one year of experiments, in November the opportunity was used to anneal all thirteen detectors. The procedure involves heating of the germanium crystal to $105^{\circ} \mathrm{C}$ for three to five days while maintaining high vacuum with a pumping station. Ten of the detectors came back to their original specifications, two were substantially improved. During all 32 operations with the Gammasphere annealing stations, only three detectors developed more serious problems and had to be sent back to the manufacturer.

The frequency of necessary maintenance was at a lower level than we had anticipated from experience with other multi-detector systems. The overall performance of the Gammasphere detector systems remains excellent.

\section{f.4. Gammasphere Software Developments (T. Lauritsen and M. P. Carpenter)}

One of the distinctions of the Gammasphere array, compared to other gamma ray detector arrays, is that the data stored on tape is highly processed. For example, all high-resolution germanium signals, as well as times, are aligned online before being written to data tapes. This makes the analysis of data from Gammasphere easy and straightforward. A number of new signals are now also aligned: 1) The BGO times, 2) the offset of BGO energies and 3) the low resolution signal from the germaniums. These improvements were made to allow for a better summed energy determination in the array. The Gammasphere array, without the heavy-mets on the BGO suppressor shields, is a good calorimeter. With the summed energy, $\mathrm{H}$, and modules that fired, $\mathrm{K}$, distributions it is possible to make distinctions between different reaction channels and gamma bands. These signals are now often used to enhance weak signals from more dominant (but less interesting) reaction channels and gamma bands. The HK distributions also allow for the extraction of the entry points for different reactions, i.e., the point in the spin and energy plane where the last particle has evaporated and the nucleus starts to cool by emitting gamma rays.
For certain experiments it is necessary to use a rotation target in order to prevent thin targets from melting. A target wheel from APEX was modified, moved to Gammasphere and a new control program was developed. This program runs in a 68030 processor residing in a VXI crate in the Gammasphere shack and interfaces the CAMAC crate's target wheel control module via a CES branch-driver. The target wheel can be controlled remotely from any of Gammasphere's workstations.

To monitor external data, as well as, for example, dataacquisition dead time, scalers are needed. When Gammasphere came to ANL the scaler system had a tendency to crash the acquisition system. Moreover, the setup of the scalers was very inflexible. A new scaler system was therefore developed. The new system is only called on demand (greatly reducing the chance of crashes) and has a flexible setup that users can modify themselves without help from Gammasphere software support. The scaler display is available from any of the Gammasphere workstations and is now also served on the web for convenient viewing. In the future, it will be possible to add the scaler data to the data stream going to tape as well.

\section{f.5. Calibration of Gammasphere as a Gamma-Ray Calorimeter and Multiplicity Counter (T. Lauritsen, I. Ahmad, M. P. Carpenter, R. V. F. Janssens, C. J. Lister, and T. L. Khoo)}

The Gammasphere array can function as a gamma-ray calorimeter and multiplicity detector - especially when the heavy mets are removed from the BGO suppresser shields. Both the summed energy of all gamma rays in coincidence with the primary beam as well as the number of modules that fired can be recorded with the fusion or fission events observed in the detector array.
From this information one can construct the entry distribution, i.e., the point in the spin and energy plane where the last particle has evaporated and the nucleus starts to cool by emitting gamma rays. However, in order to extract this entry distribution it is necessary to correct for the response of the Gammasphere array. By 
placing a ${ }^{88} \mathrm{Y}$ source in the center of the array and observe the gamma rays detected (in consecutive events) in the array and detector modules that fired, both the response of the array in terms of the absorbed energy as well as multiplicity was measured. Thus, the true summed energy and multiplicity, $\mathrm{H}$ and $\mathrm{K}$, can be found. This is done using a Monte Carlo technique to unfold the observed HK 2D distributions after they are properly background subtracted.

The measured HK response of the Gammasphere array is shown in Figure I-65. It is seen that, with 100 detectors in the array, on the average $70 \%$ of the energy is captured in the germanium and BGO detectors of the array.

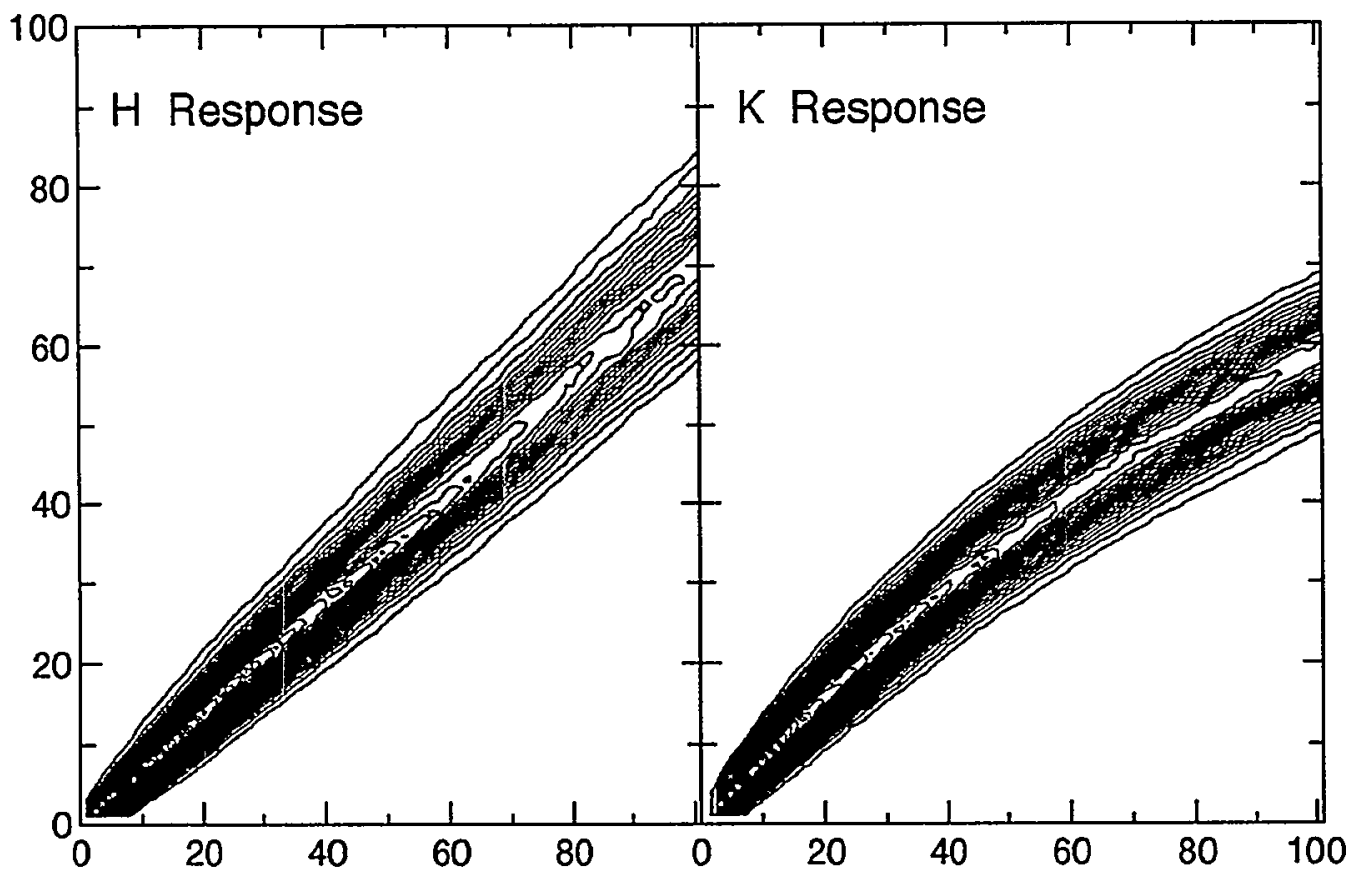

Fig. 1-65. Measured summed energy and multiplicity (HK) responses of Gammasphere with 100 detectors in the array. About $70 \%$ of the energy emitted at the center of the array is captured (for $898-k e V$ gamma rays).

\section{f.6. New Improved and Generalized Background Subtraction Procedure (T. Lauritsen, T. L. Khoo, M. P. Carpenter, and R. V. F. Janssens)}

In order to extract the quasi-continuum of gamma rays in coincidence with a superdeformed (SD) band it is paramount to do a proper background subtraction. It was known for some time that it was significantly more difficult to properly background subtract SD bands in the $A=150$ mass region than in the $A=190$ mass region. The problem is that in the $A=150$ region the SD gates are set in energy regions much higher than the mean energy of the un-gated gamma spectra. Thus, the usual (total) background spectra are not appropriate to use in the background subtraction.
A new, improved version of the angular sorting program, rebel, was developed which is using local backgrounds near the elliptical SD double gates. Furthermore, the program avoids using regions in the local background areas where strong peaks are present. The latter requirement is necessary in order to prevent individual strong normal coincidences from dominating in the statistically smaller sampling of the background spectra. With this new software it is, for the first time, possible to reliably extract the quasi-continuum of gamma rays for the yrast SD bands in $151,152 \mathrm{Dy}$. The background subtraction procedure works on summed 
energy and multiplicity (HK) distributions and gamma ray gated mass spectra as well. The HK distributions measure the points in the spin and energy plane where the last particle has evaporated and the nucleus starts to cool by emitting gamma rays.

Figure I-66 shows SD band-1 in ${ }^{151}$ Dy after the new background subtraction has been applied. The spectrum is also unfolded, corrected for summing effects, efficiency corrected and normalized to 10000 gamma cascades. Figure I-67 shows the summed energy and multiplicity distribution observed in Gammasphere when gates are placed on the lines in this SD band. The HK distribution is background subtracted using the new generalized background subtraction method.

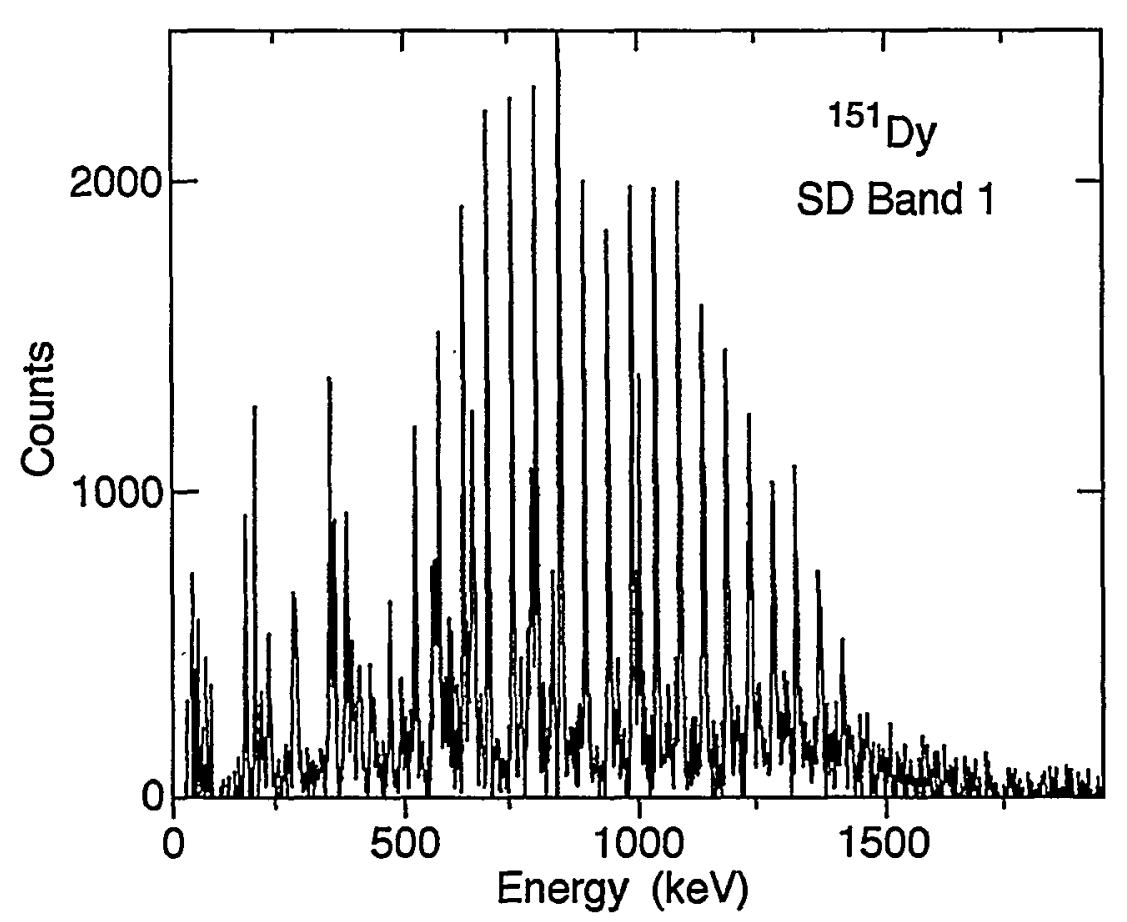

Fig. I-66. The yrast superdeformed band in 151 Dy background subtracted with a new procedure using local . background-background and peak-background spectra. The spectra are used to extract the quasi-continuum of gamma rays in coincidence with the yrast superdeformed in the nucleus.

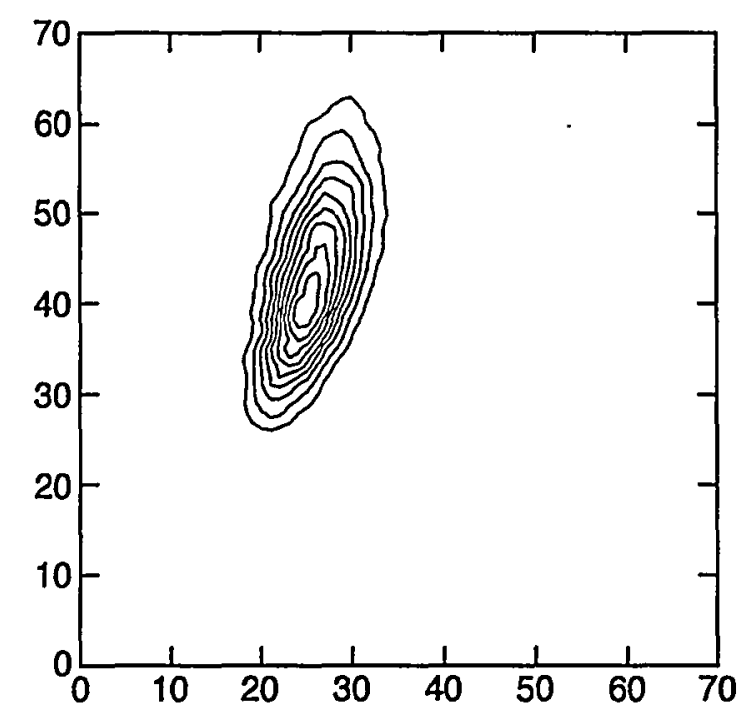

Fig. 1-67. The summed energy and multiplicity distribution (HK) observed in Gammasphere when gates are placed on the lines in the yrast $S D$ band in 151 Dy. The HK distribution is background subtracted using the new background subtraction method. 


\section{f.7. Neutron Detectors (C. J. Lister, J. Schwartz, D. Seweryniak, D. P. Balamuth, * P. Hausladen,* S. J. Freeman, $†$ B. J. Varley, $\dagger$ M. Leddy, $\uparrow$ S. J. Fischer, $\ddagger$ and D. Sarantites§)}

The development of a neutron detector array for Gammasphere continued in its evolution during 1998. Starting with a $7 \%$ efficient, 15 detector array of NE213 scintillators from existing University of Pennsylvania, University of Manchester and ANL equipment, first installed at Berkeley in 1996, the array has evolved into a $12 \%, 20$ detector array in 1998 at ANL, and will be further upgraded to a purpose-built array of $>20 \%$ efficiency with $>30$ elements in 1999. The detectors for the Gammasphere-specific array were designed and are being fabricated at Washington University.
During 1998, the Penn-Manchester array was installed for two blocks of experiments, in July and November. The installation involved removing 13 Gammasphere modules. Five PAC approved experiments involving the detection of neutrons were performed. They are listed in Table I-III. At the end of the runs, Gammasphere was returned to it's normal configuration and recalibrated. No loss of $\mathrm{HpGe}$ inventory occurred during these transitions. About one week of Gammasphere "down-time" was involved in setting up these experiments.

Table I-III

$\begin{array}{lll}\text { PAC697 } & \text { Balamuth et al. } & \mathrm{N}=\mathrm{Z} \text { nuclei, especially }{ }^{72} \mathrm{Kr} \\ \text { PAC722 } & \text { Rudolph et al. } & \text { Proton emission from excited bands } \\ \text { PAC689-2 } & \text { Freeman et al. } & 103 \text { Sn spectroscopy } \\ \text { PAC746 } & \text { Cederwall et al. } & \text { High Spins in } \mathrm{A}=90 \text { region } \\ \text { PAC716 } & \text { Clark et al. } & 96 \mathrm{Cd} \text { Isomers }\end{array}$

*University of Pennsylvania, $\nmid$ University of Manchester, United Kingdom, $\ddagger D e P a u l$ University, §Washington University

\section{f.8. Status of Channel-Plate-Based Heavy-Ion Detectors (C. J. Lister, B. Nardi, J. Greene, D. Henderson, P. Reiter, and J. Schwartz)}

For experiments where high count rates are anticipated, or energy-loss and multiple scattering are important considerations, a new focal-plane detection system for the FMA was built and run in many experiments. The system employs an image-making foil at the focal plane which generates electrons whenever heavy-ions pass, and a focusing and amplification system using electric fields and channel-plate detectors with a resistive readout. With careful calibration, the position resolution was measured to be about $2.2 \mathrm{~mm}$ FWHM, only slightly inferior to the normal gas detector. However, the sustained countrate capability was demonstrated to be in excess of $10^{5}$ ions/sec, and the imaging foils are much thinner than gas detector windows.
During 1998, these detectors passed from their testphase to reliable and regular use. They were demonstrated to work at both very low (PAC approved experiment 693) and very high (PAC approved experiment 673-2) countrates and with very low energy ions (PAC approved experiment 671LI $45 \mathrm{keV} / \mathrm{u}$ and $68120 \mathrm{keV} / \mathrm{u})$. For very low count rate work, operating two systems in coincidence lowers background count rates. Two systems are also good for correcting aberrations and rejecting scattered beam. For detection of the lowest energy heavy ions, forwardgoing electrons must be detected, as the yield of backward-going electrons falls too low for efficient research. 
Several areas of research and development were followed to allow consistent performance, and further work is needed. Two dedicated power supply mainframes with interlocks for countrate, vacuum status, and all voltages and currents were designed, commissioned and built by the Physics Division electronics group. These devices were found to be essential after several channel plates were damaged during early test experiments. The Fragment Mass Analyzer (FMA) is a hostile environment for low-level electron detection, and FMA sparks or vacuum excersions can cause intense, shortterm electron fluxes in the beamline. A rapid shutdown of the channel-plate acceleration voltages needs to be automatic to prevent rapid burnout. Since the new supplies were installed in August, no loss of channel plate performance was recorded.

Two related development areas are still under investigation. First, enclosing the channel plates and their readout in a well shielded environment lowers their noise and thus improves performance. Stray beamline electrons can be intercepted and "false" triggers can be substantially reduced. Second, reduction of background electrons, mainly from pressure gauges and light leaks, leads to the further enhancement of channel-plate performance, as detection thresholds can be lowered and efficiency increased.

In the image-foil area many projects were started and can be more carefully investigated now that the basic operating parameters are established. The Formvar base foils are in the $50-\mathrm{mg} / \mathrm{cm}^{2}$ region, but we hope to go to foils of $10 \mathrm{mg} / \mathrm{cm}^{2}$. The electrode material during the development phase was $20 \mathrm{mg} / \mathrm{cm}^{2}$ gold as it was found to be reliable to make and sturdy to use. However, lower- $Z$ and more electronegative materials were reported in the literature to provide superior performance and need to be evaluated. This will now be possible in "off-line" tests using a fission fragment source.

With the information we gained, a "second-generation" design study was started, mainly to make the mechanical operation more flexible and simple. A larger system, to match the $150 \mathrm{~mm}$ FMA focal plane, should now also be feasible.

\section{f.9. Prolonging the Useful Life of Gammasphere/FMA Targets under Bombardment with Intense Beams (T. L. Khoo, C. J. Lister, T. Lauritsen, P. Reiter, J. Schwartz, D. Seweryniak, S. Siem, G. Clifft, J. Falout, B. Nardi, and J. Fox*)}

In experiments with the Gammasphere/FMA combination, cross-sections are often small and large beam currents are required. In order to extend the useful lifetime of targets in such experiments, several developments were implemented. The most important one was the construction of a rotating target wheel. In addition, the beam spot can be enlarged by wobbling it vertically and/or horizontally.

The spot size for a well-tuned beam at the Gammasphere/FMA target position is usually very small. Measurements of spot sizes for beams of ${ }^{48} \mathrm{Ca}$ and ${ }^{76} \mathrm{Ge}$ reveal spot sizes of $<0.8 \times 0.5 \mathrm{~mm}$. These measurements were made by controlled paper burns, augmented by visual inspection, through the upstream transit telescope, of the glow on a ruby quartz, where the resolution $(\sim 2 \mathrm{~mm})$ was not quite adequate. With such a small spot size, fragile targets, such as lead, would be destroyed by intense heavy-ion beams. Furthermore, the target may be compromised (e.g. by beading, without actually being destroyed,) for experiments with the FMA, where evaporation residues must recoil out of the target into the FMA.
A target wheel was constructed for use with the Gammasphere target chamber. The design, adapted from that of the APEX target wheel, allows the wheel holder to be introduced into Gammasphere using a pentagon position, where there are no detectors. Targets are mounted on the wheel in four quadrants. In this manner, the beam is spread over a circumference of 10.8 $\mathrm{cm}$. Application of 4.5 volts results in a angular frequency of $\sim 710 \mathrm{rpm}$. Rotation of the wheel synchronizes pulsing of the beam, so that only the targets were irradiated but not the spokes of the target mounts. A CAMAC module provides signals for controlling the ATLAS beam sweeper and for monitoring the wheel orientation. Initialization and control of this CAMAC module can be done via software commands.

To further reduce the power density of the beam, it can be dispersed vertically (along a radius of the target wheel) by wobbling. This is achieved by modulating, at a low frequency $(\sim 5-10 \mathrm{~Hz})$, the current on the magnetic $y$-steerer in the FMA beam line. To wobble a 
$215-\mathrm{MeV} 10^{+48} \mathrm{Ca}$ beam by $\pm 1 \mathrm{~mm}$, requires an input current of $0.33 \mathrm{amp}$, achieved by applying \pm 0.83 volts. To disperse other beams, one should scale the applied voltage with the magnetic rigidity - this works only in the vertical direction.

By a combination of the target wheel and beam wobbling, a $0.5-\mathrm{mg} / \mathrm{cm}^{2} \mathrm{~Pb}$ target could withstand a 10 pnA beam of ${ }^{48} \mathrm{Ca}$ without noticeable deterioration, allowing nobelium residues to reliably recoil into and through the FMA. (New targets had to be conditioned by gradually increasing the beam current over a period of 4-8 hours in order to introduce wrinkles.) Inspection of the target under a microscope revealed none of the usual signs of damage (such as beading, cratering or breaks in crystalline structure).

In targets that are not so fragile as to require the use of the wheel, the beam power density can be reduced in a controlled manner (e.g. to give a $4 \times 1 \mathrm{~mm}$ spot for the FMA) by wobbling the beam in both the $x$ and $y$ directions. (The horizontal response to an external current is non-linear and must be visually checked for each beam.)

*Florida State University

\section{f.10. Performance of Planar Germanium Detectors for Low-Energy Gamma-Rays (I. Wiedenhöver, R. V. F. Janssens, C. Lister, M. Carpenter, M. Alcorta, P. Chowdhury,* and E. H. Seabury*)}

Gammasphere was designed to measure medium and high energy gamma-rays, which are of central interest for the majority of spectroscopy experiments. This objective led to the design of the Gammasphere detector type, which contains one ultra-pure large volume Germanium crystal in a cylindrical shape. Charge collection occurs with a radial electric field. For the measurement of low-energy gamma-rays under $100 \mathrm{keV}$, however, smaller crystals exhibit a better signal-tonoise ratio and better energy resolution. In addition, smaller crystals can be realized with a planar design for the electric field, which allows improvement in their timing characteristics and the count rate capability over coaxial detectors.

For some experiments, the energy resolution and timing characteristics for low-energy photons, such as X-rays or low-energy gamma-rays, are very important. To improve Gammasphere's performance in this respect, ANL purchased five low-energy photon spectrometer
(LEPS) detectors, which consist of relatively smallvolume planar germanium crystals. The performance of these detectors was measured and exceeded expectations. The resolution at $60 \mathrm{keV}$ is typically $800 \mathrm{eV}$, where the large volume detectors typically shows only $1600 \mathrm{eV}$ resolution. The time resolution at low energies was measured to be better than $20 \mathrm{~ns}$, whereas large volume detectors show values not better than 40 ns.

For a series of experiments, four LEPS detectors were mounted in Gammasphere replacing four standard detector systems. The detectors were brought as close to the target as mechanically possible. One important application of these low-energy detector systems lies in the measurement of X-ray energies, which are a characteristic function of the periodic number of the chemical elements. The coincidence events between Xrays and gamma-rays, thus, allows the assignment of unknown gamma-transitions to a reaction product of a certain charge number $Z$.

*University of Massachusetts at Lowell 


\section{f.11. Development of HpGeDSSD Planar Germanium Wafer Technology (C. J. Lister, M. P. Carpenter, R. V. F. Janssens, T. L. Khoo and T. Lauritsen, P. Wilt, and I. Wiedenhöver)}

The development of future gamma-ray arrays is undergoing deep consideration, both with respect to the design of a detector which can surpass Gammasphere and with consideration of what gamma-ray equipment is optimum for a future radioactive beam facility. One new technology which shows promise is the development of large-area planar germanium doublesided strip detectors (HpGeDSSD's). At ATLAS we are working on this technology to explore its potential. Beyond basic research, these detectors are being considered or used in space science, ${ }^{1}$ in environmental cleanup, ${ }^{2}$ and in medical physics through mini-PET scanners for medical imaging. 3

We are following several paths:

(a) Procurement and evaluation of the largest possible HpGeDSSD. We issued an RFQ for a spectroscopy-grade planar detector with size of about $90 \mathrm{~mm} \times 90 \mathrm{~mm} \times 20 \mathrm{~mm}$. The detector will have conventional B- and Li-implanted electrodes in p-type material with 16 strips on each side. One set of strips will have cold-FETS and have timing, energy resolution and countrate capability similar to our recently-acquired Gammasphere-compatible LEPS detectors.

(b) Design of a ultra-compact cryostat to hold the wafers in a minimum-mass environment. This design development will be essential if many wafers are to be stacked into a compact array. (c) Monte-Carlo simulations of the performance of stacks of wafers to evaluate the possible performance of an array, which we call the Gamma Ray Box (GARBO).

(d) Evaluation and costing of high-density electronics to use with HpGeDSSD's. With conventional electronics the cost of signal processing would far outweigh the cost of the actual detector. In the GARBO design, about 1000 channels of electronics are required, a five-fold increase over Gammasphere. It is not clear if digital pulse processing is desirable, or cost effective.

(e) Consideration of possible readout schemes and fast front-end processing of data, both for a single planar detector (both for interpolating between strips and for event reconstruction).

Even with 2 of the prototype detectors, many interesting cutting-edge research projects can be pursued including studies of linear polarization, spectroscopy of very heavy nuclei, source reconstruction for PET imaging, high-precision Doppler correction of gammarays emitted from very fast moving ions, and developing the "recoil-beta-tagging" method at the focal plane of recoil separators like the FMA.

We hope to have our first detector delivered in the fall of 1999 with an element that could be a building-block for GARBO in early 2000 .

\section{f.12. Electronics Development of General-Purpose, High-Speed Readout Module (P. Wilt, B. Harss, and J. Nolen)}

The realtime display module developed for the Fiber Optic Scanner (see Annual Report 1998, page 114) to be used with radioactive beams was modified into a general purpose high-speed buffer/readout and display module with a wide range of applications. The analogand the digital sections of the circuit, initially planned as one entity, were separated into two circuits. The digital buffer, read-out and display portion is being designed into Field Programmable Gate Array (FPGA) logic device, making it a generic base for a variety of analog front-end circuits. This flexibility allows the easy production of modules that perform, for example. as digital oscilloscopes, multi-channel analyzers or, serving its original purpose, display units for a fiber optic scanner. In every application, the device is equipped with capabilities for remote data read-out and full remote control.

Applications other than the fiber optic scanner display are remote monitoring of parameters of the ATLAS accelerator system, remote-controlled data taking in radiation areas of an ISOL type facility, setting up experiments, and the high-speed readout of large detector arrays. To achieve this flexibility, the device uses a 
sequencer circuit to read the analog information, processes it through a look-up memory table and stores the data in a histogram memory that can be read out by CAMAC or a high speed bus. The histogram memory is $4 \mathrm{k}$ words at 24 bits, while the processing, look-up memory, is a $4 \mathrm{k}$ by $4 \mathrm{k}$ by 10 bit high-speed EPROM memory array. The power of this logic circuit is that the FPGA and memory(s) can be reprogrammed into any data-acquisition/post-processing configuration required for an experiment with respect to the limitations of the FPGA size and speed. As originally planned, the final module will be equipped with CAMAC control for all its functions. However, the original digital read-out was expanded to support not only the CAMAC bus, but also a fast readout bus, allowing burst data transfer rates in excess of $100 \mathrm{MB} / \mathrm{s}$. The event rate limit for the present version of the digital module was demonstrated to exceed $10 \mathrm{MHz}$.

The analog circuit presently used has two self-triggering analog inputs equipped with track and hold circuits that will track pulses up to $5 \mathrm{~ns}$ risetime. The digital circuit can be configured to sample and display either channel or the ratio of the two channels. The setting to display one channel approximates a very fast MCA with remote readout capabilities, and was used this year extensively in the tuning process of radioactive beams from the gas cell setup described elsewhere in this Report. The application as controller for the fiber optic scanner will be demonstrated.

\section{f.13. Large Efficiency Heavy Ion Detector - LEPPEX (D. J. Hofman, B. B. Back, J. Falout, D. Henderson, P. Wilt, A. Sonzogni, and I. Dioszegi*)}

The LEPPEX (Large Efficiency Photon Particle Experiment) project involves the construction of two 30-element $\mathrm{BaF}_{2}$ detector arrays and a large solid angle coverage heavy-ion detection system. One 30 element $\mathrm{BaF}_{2}$ detector array was completed and used in experiments in 1997. This year the effort was turned to testing and construction of the heavy-ion detector system.

The heavy-ion detector system consists of eight compact, large area $\left(14 \times 49 \mathrm{~cm}^{2}\right)$, multi-wire avalanche detectors integrated into a vacuum chassis. The eight detectors are arranged in a barrel-shaped geometry covering $2 \pi$ in the azimuthal plane. The driving force behind the integrated detector and chamber design is to minimize detector dead space. Use of a meander-line readout design for the X-position plane was decided in 1997 following the successful tests of a prototype board.

Machining of the eight detector housings was completed at SUNY Stony Brook, and the first two detectors were outfitted with wire planes, meander-lines and front foils. These detectors were tested in two separate ATLAS experiments. Figure I-68 shows one detector mounted in the 36 " scattering chamber for testing with a 140 -
$\mathrm{MeV}{ }^{16} \mathrm{O}+{ }^{232} \mathrm{Th}$ reaction. Both detectors performed identically, with $\mathrm{X}$ and $\mathrm{Y}$ position resolutions of $<2$ $\mathrm{mm}$ and efficiencies of $100 \%$ at a counting rate of 2 $\mathrm{kHz}$. The detector efficiency fell to $80 \%$ at $5 \mathrm{kHz}$, illustrating the need to suppress target electrons even further above the $2 \mathrm{kV}$ bias voltage used in the ATSCAT tests. As a result of this test, a design goal of $15 \mathrm{kV}$ bias voltage on the target ladder assembly for the new chamber was set. The detectors were also mounted and tested in coincidence mode using a 434$\mathrm{MeV}{ }^{58} \mathrm{Ni}+{ }^{197} \mathrm{Au}$ reaction.

Machining of the vacuum chamber that will hold these eight detectors also proceeded throughout the year. Figure I-69 shows the vacuum frame after completion. Design of the back-plate for the frame is completed and construction is currently underway. To save construction costs, an existing detector platform currently not in use will be modified to work as a stand for the entire detector assembly.

If all goes well, the LEPPEX heavy-ion detection system should be assembled and ready for initial vacuum and source tests by the fall of 1999 . Experiments are planned in early 2000.

*SUNY at Stony Brook 


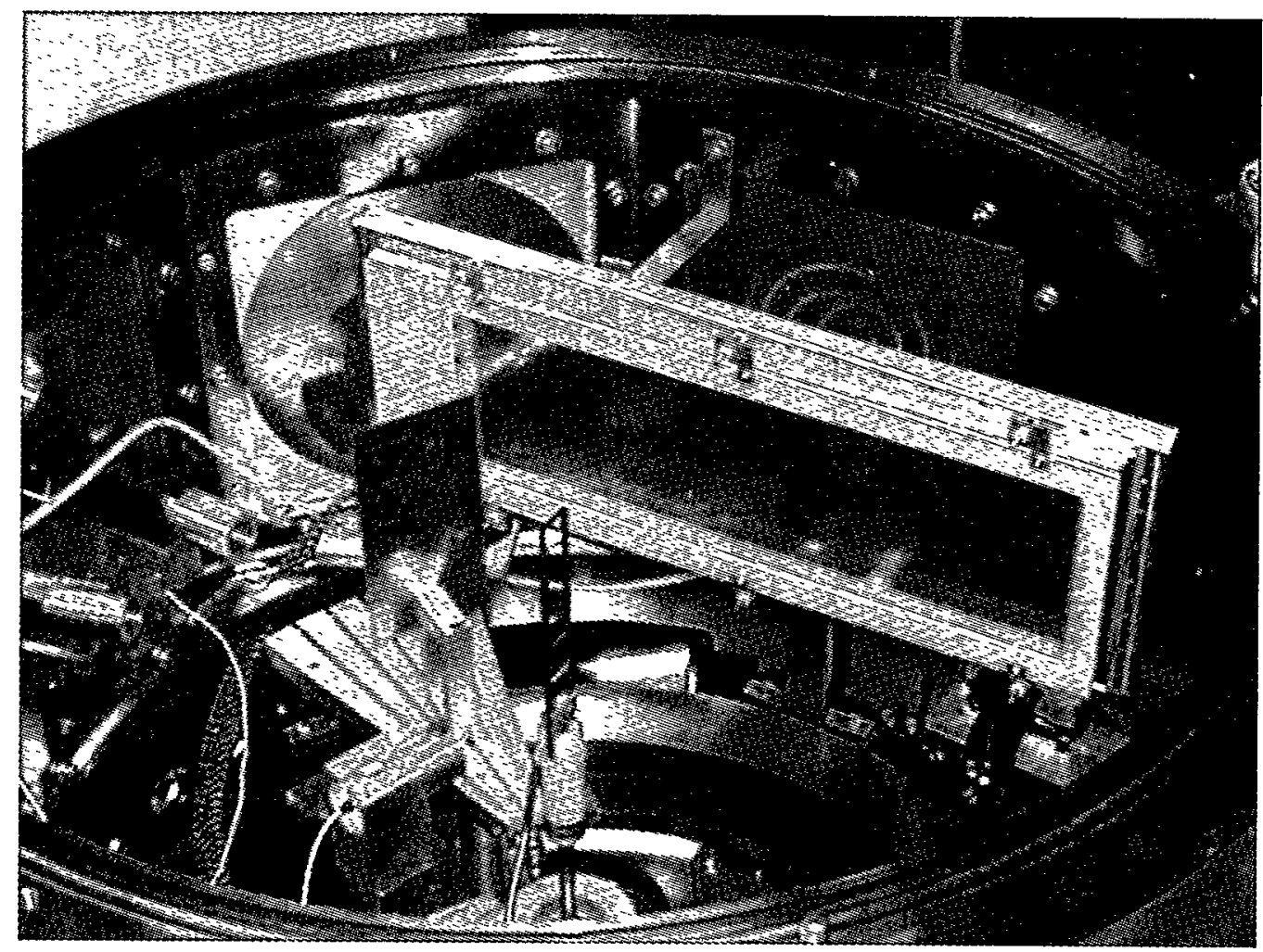

Fig. I-68. A picture of one completed detector mounted in the ATSCAT 36" vacuum chamber. The rectangular thin sheet mounted on the platform to the left side had a pattern of small holes drilled in it used for the resolution test. The detector on the platform opposite the detector was a silicon detector used for the coincidence efficiency test.

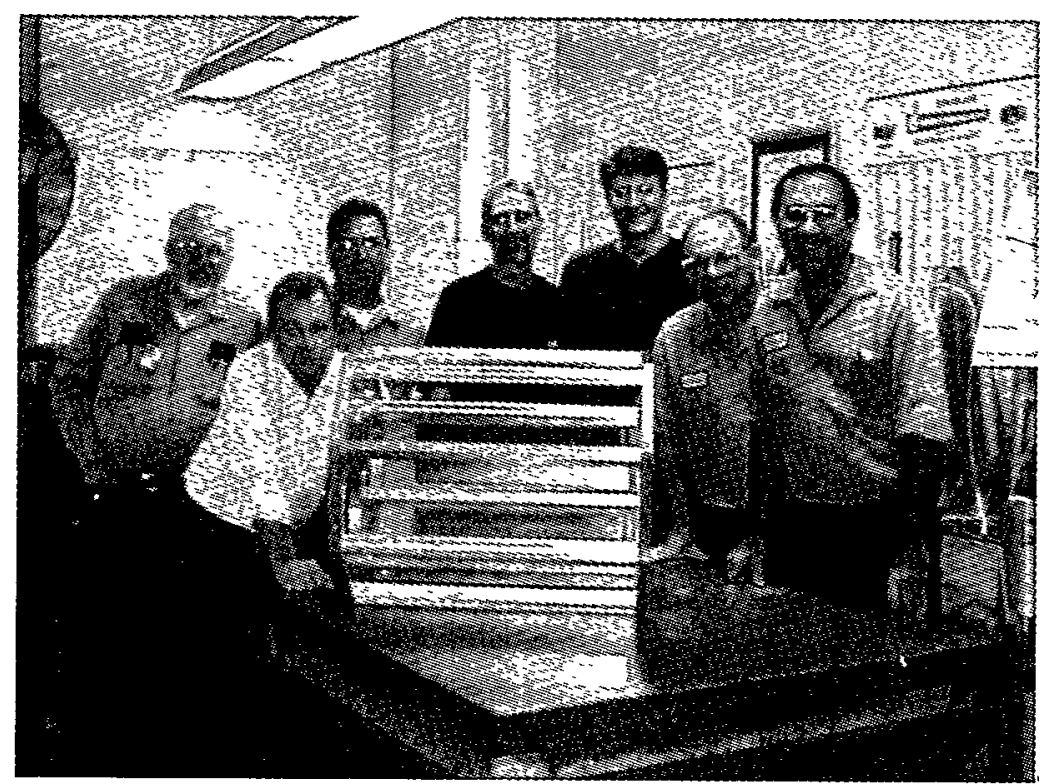

Fig. I-69. A picture of the completed vacuum chamber frame for the heavy-ion counters together with everyone involved in its design and construction. From left to right, we have Dave Compton, Joe Falout, Dan Nestor, Birger Back, Dave Hofman, Ray Rucinski, and Don Sutter. 


\section{f.14. Design of a Temperature Sensing System-LEPPEX (D. J. Hofman and L. DeRemer)}

The design and construction of a temperature sensing system that will be used in the LEPPEX (Large Efficiency Photon Particle Experiment) project was completed by L. DeRemer, a participant in the Summer 1998 Pre-College Research Program. The temperature sensor system was designed for use with the 30 -element $\mathrm{BaF}_{2}$ high-energy $\gamma$-ray detector. The light intensity output of $\mathrm{BaF}_{2}$ crystals is highly temperature dependent $\left(\sim 4 \% /{ }^{\circ} \mathrm{C}\right)$ and the line-shape of the slow component also varies dramatically as the crystals are cooled. ${ }^{1}$ A liquid cooled system is used to help stabilize the temperature of the $\mathrm{BaF}_{2}$ crystals. An active temperature monitoring system will allow us to make gain corrections for any variations in temperature during the course of an experiment.

The temperature sensing system consists of 20 temperature readout units, each of which is connected to individual thin thermocouple wires which are then threaded in between the $\mathrm{BaF}_{2}$ crystal modules. The temperature readout units were mounted on a custombuilt panel (see Figure I-70). Each unit was also connected to a multi-pin ribbon cable which fed into a single CAMAC module designed to read out up to 32 channels of individual voltage levels. The system was installed and successfully tested, and will be available for use in future experiments utilizing the $\mathrm{BaF}_{2}$ array.

lV. Nanal, B. B. Back, and D. J. Hofman, Nucl. Instrum. Methods A389, 430 (1997).

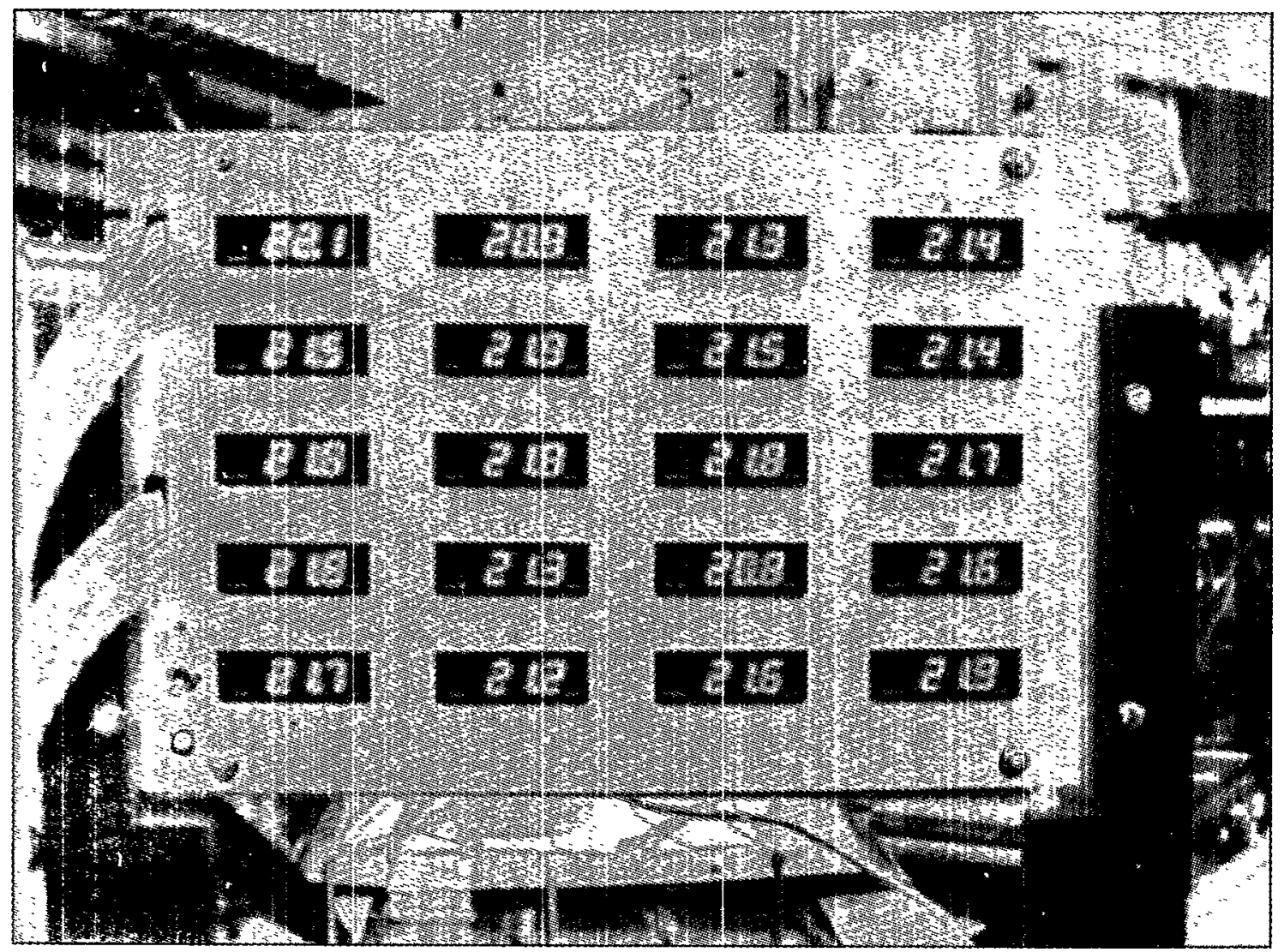

Fig. 1-70. View of the readout panel for the temperature sensing system which is mounted directly on the back of the LEPPEX BaF 2 detector array. Readout cables for CAMAC active monitoring during the experiment are visible on the left-hand side of the panel. 


\section{f.15. Nuclear Target Development (J. P. Greene and G. E. Thomas)}

The Physics Division operates a target development laboratory that produces targets and foils of various thicknesses and substrates, depending on the requirements, for experiments performed at the ATLAS and Dynamitron accelerators. The targets are prepared from both naturally occurring materials and stable isotopes which are supplied either in pure, elemental form or as stable compounds. Targets are made not only for the Physics Division but also for other divisions at the laboratory and occasionally for other laboratories and universities. In this regard, there has been a noticeable increase in requests for targets from individuals and groups outside of ANL. In particular, in support of new experimental facilities as they come online, examples include the $\mathrm{S} 800$ spectrometer at MSU and the $8 \pi$ Spectrometer and Gas-filled Separator at Berkeley.

In the past year, numerous targets were fabricated either as self-supporting foils, on various substrates or as "sandwich" targets. Targets produced included $\mathrm{Al}$,

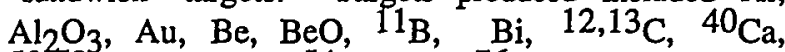
$50,52 \mathrm{Cr}, \mathrm{Cu}, \mathrm{CH}_{2},{ }^{54} \mathrm{Fe}$, FeS, ${ }^{76} \mathrm{Ge}$, Havar, Kapton, ${ }^{6} \mathrm{LiF},{ }^{24} \mathrm{Mg},{ }^{9} \mathrm{Mo}$, mylar, ${ }^{58} \mathrm{Ni}, 208 \mathrm{~Pb},{ }^{208} \mathrm{PbO}$, $\mathrm{PbS},{ }^{108} \mathrm{Pd}$, polyethylene, polypropylene, $\mathrm{Pt}$, ${ }^{96,104} \mathrm{Ru}, \mathrm{Se}, \mathrm{Si},{ }^{149} \mathrm{Sm},{ }^{120} \mathrm{Sn}, \mathrm{Ta}, \mathrm{Th},{ }^{46} \mathrm{Ti}, \mathrm{W}$, $176 \mathrm{Yb}$ and $94,96 \mathrm{Zr}$. Many of these target foils were fabricated using our small rolling mill. Rolling has become the method of choice for most of the thick targets used in Gammasphere experiments where the required surface densities range from 0.5 up to several $\mathrm{mg} / \mathrm{cm}^{2}$. There were also a few instances of thin film deposition applications involving coatings of $\mathrm{Ge}, \mathrm{SiO}_{2}$ and $\mathrm{Au}$ onto various parts and apparatus. Approximately 2318 targets were prepared for these various experiments, including accelerator support during this calendar year. With the relocation of Gammasphere to ATLAS, the support for research targets, absorbers, reset foils, etc. increased dramatically. By the end of February 1998, the number of targets produced for Gammasphere eclipsed the total number of targets previously made up to this time. An increase in Gammasphere experimental research was realized since the move, due in part to the availability of target production facilities at ANL. There were 567 targets prepared for Gammasphere over the year.

A major research effort has gone into fabrication by rolling of various window foils and the subsequent mounting of these foil windows in gas cells used both as targets and for the production of secondary beams.
These included for the most part, Havar and titanium, but also $\mathrm{Ni}, \mathrm{Cu}, \mathrm{Ta}$, polypropylene and Kapton. $\mathrm{A}$ large effort went into developing a soldering method for Havar windows on the production cell target and exploring various low-temperature epoxy compounds for $\mathrm{Ti}$ windows. In addition, much support was provided for the development of foils for use in various new detector set-ups and their experimental demands, including passive absorbers, reset foils, degrader foils and catcher foils. Several variations of metallized plastic foils were prepared for use in the channel plate detector at the FMA, and for a variety of windows employed in CPT experiments at the SPS in Area II including windows of $\mathrm{Ti}, \mathrm{Ni}$, Kapton and Havar.

As part of ATLAS support, carbon stripper foils of 2 $\mathrm{ug} / \mathrm{cm}^{2}$ for use in the Tandem as well as other thickness for additional stripping are being routinely produced by the target lab. A total of 886 carbon stripper and gold foils of various types were prepared for calendar year 1998. Also, there continues to be an increase in the preparation of various forms of isotopic source material for the production of enriched beams at ATLAS. This includes reducing separated isotopes into a form and shape suitable for introduction into PII ECR and for the SNICS source. Some examples include ${ }^{76} \mathrm{Ge},{ }^{54} \mathrm{Fe}$, $58 \mathrm{Ni}, \mathrm{TiO}_{2}$ and ${ }^{6} \mathrm{LiH}$. Procurement of stable and enriched material for ATLAS consumption continues to be provided by the target laboratory staff.

The target development laboratory includes state-of-theart equipment used for thin-film fabrication. The available techniques consist of multiple resistive heating, focussed ion beam sputtering, glow-discharge plasma deposition, electron beam and electron bombardment evaporation, electrodeposition and mechanical rolling. The evaporators are maintained under high vacuum and each vessel contains a quartzcrystal film-thickness monitor with deposition rate indicators. Also included are movable shutters, quartzlamp substrate heaters and thermocouple temperature sensors, allowing for complete process monitoring during target deposition.

Other auxiliary equipment used for target development includes equipment for electrodeposition, a small rolling mill, an alpha particle counting chamber, inert atmosphere glove box, laminar flow clean bench, pellet press, a reduction furnace, and a variety of precision balances. A turbo-pumped target storage facility is in operation for maintaining, under high vacuum, those 
targets which can readily oxidize in air. This system utilizes computer-controlled circuitry to prevent targets from exposure to atmosphere during power interruptions. A second storage system employs a bank of vacuum desiccators connected to a mechanicallypumped manifold for use by individual experimenters. Duplicates of both these systems are in operation within Target Area II at ATLAS. A new system consisting of two large glass desiccators and small turbo-pump system was constructed for material storage. This allows a separation of this material from the target storage desiccators, hence eliminating their exposure when transferring and retrieving targets.

A low-level radioactive source and target preparation laboratory was established at a separate location within the Division dedicated to the production of these sources and targets. Available preparation techniques include multiple resistive heating, electrodeposition and mechanical rolling. A diffusion-pumped vacuum evaporator is presently installed with plans for the addition of an electron beam evaporator system in the near future. A second much smaller evaporator system was constructed for close proximity evaporations of higher activity materials, to be used not only as targets, but for radioactive source development as well. The size of this system allows for minimal contamination and is presently installed within a hood. New developments in this area include the preparation of actinide targets of $\mathrm{Cm}$ and $\mathrm{Pu}$ for Coulomb excitation studies using the BGO array and Gammasphere at ATLAS. Also noted is the production of natural uranium and thorium foils by mechanical rolling.

\section{f.16. Physics Computing Facilities (K. Teh, D. R. Cyborski, and T. Lauritsen)}

The Physics Division maintains several computer systems for data analysis, computation, and general computing. These systems are conveniently grouped into various clusters and are described briefly below.

The Division's Unix cluster consists of Sun Sparcstations, DEC 3000/300 Alphas, and Linux PC workstations. Its primary function is data analysis and general computing. The VMS Analysis cluster consists of 3 Alphastations hosted by an AlphaServer. It is used primarily for sorting data obtained from ATLAS-based experiments. The primary analysis software package used is DAPHNE, although some users have adopted the PAW system. In addition, the Division maintains a 4-processor Silicon Graphics Origin 200 for numerically intensive computations.

Because of its older technology, the original Vax cluster is no longer used for data analysis. However, it continues to provide general computing services and serves as disk storage to Windows PCs and Macintoshes. It continues to serve as the central mail hub for the Division. The Computer Support Group obtained a dual-processor Linux PC and is now in the process of studying its viability as replacement for the Vax cluster.

In addition to the Divisional mail clusters, the Division also operates four additional clusters. The Theory group maintains a pair of IBM RS/6000 workstations which serve several $\mathrm{X}$-window terminals. The group also utilizes the Argonne IBM SP2 and the SGI Origin 2000 machines, both massively parallel machines, for large numerical computations. The Medium Energy group has 2 clusters: a Digital Unix cluster and a VMS cluster which are used for data analysis and general computing. Finally, the ATLAS accelerator group maintains its own cluster for controlling the ATLAS accelerator.

\section{f.17. Data-Acquisition Systems (K. Teh, D. R. Cyborski, and T. Lauritsen)}

The Division operates three MSU/DAPHNE dataacquisition systems. Each consists of an MSU VME front-end and a DAPHNE back-end for online monitoring which is hosted by an Alpha VMS workstation. No changes were made to these systems.

After much discussion, the Division has initiated an effort to replace the MSU/DAPHNE system with a new data-acquisition system. This new system, called SCARLET, involves both hardware and software development. A custom CAMAC controller called the PICA is currently being developed which will form the basis of the SCARLET data-acquisition system. A major goal of this system is to provide comparable acquisition performance at a fraction of the cost of the current MSU/DAPHNE systems. To achieve this goal, the system is based on off-the-shelf PC hardware and the Linux operating system. The projected end-user cost of a base SCARLET system is about $\$ 3 \mathrm{k}$. The aim is to have the system ready by the Spring of 2000 . 


\section{G. ASSISTANCE TO OUTSIDE USERS OF ATLAS}

\section{J. Hofman}

This year saw a large increase in the number of outside Users of ATLAS due to the start of Gammasphere experiments at ATLAS in January 1998. Outside Users were involved in about 95\% of all experiments performed in FY 1998. David J. Hofman is available in a user liaison capacity to handle the scheduling of ATLAS experiments, provide assistance in experiment proposal submission matters, and help facilitate the effective performance of research at ATLAS by outside scientists. In addition, a large portion of the Heavy-Ion in-house scientific staff spent time in experiment setup and preparation for the many different Gammasphere experiments.

The Program Advisory Committee, nominally consisting of six members from other institutions and two from Argonne, met twice during FY 1998. Program Advisory Committee meetings were held on October 31-November 1, 1997 and May 8-9, 1998 to recommend experiments for running time at ATLAS. In FY 1998 the Program Advisory Committee members were:

October 31 - November 1, 1997 Meeting

$\begin{array}{ll}\text { Peter Paul (chair) } & \text { SUNY at Stony Brook } \\ \text { David Fossan } & \text { SUNY at Stony Brook }\end{array}$

May 8-9, 1998 Meeting

$\begin{array}{ll}\text { Lee Riedinger } & \text { University of Tennessee } \\ \text { Robert Tribble } & \text { Texas A\&M University }\end{array}$

Both Meetings

David Balamuth
Russell Betts
Bernard Haas
I.-Y. Lee (chair for $2^{\text {nd }}$ meeting)
Witek Nazarewicz
Bradley Sherrill

David Balamuth

Russell Betts

Bernard Haas Witek Nazarewicz Bradley Sherrill

The PAC reviewed 42 proposals for 200 days of running time and 55 proposals for 273 days of running time at the two meetings, respectively. Of the submitted proposals, the Program Advisory Committee recommended acceptance of 50 proposals for a total of 214 days of running time.

The ATLAS User Executive Committee organized a joint User Group meeting chaired by David Fossan in conjunction with the Gammasphere, Berkeley 88" Cyclotron and HRIBF User Groups during the October 1998 Division of Nuclear Physics APS meeting held at Santa Fe, NM. Approximately 120 scientists attended the meeting. The main topics of discussion concerning Argonne were the status of ATLAS, updates on various experimental programs and issues surrounding Gammasphere. In FY 1998 the ATLAS Executive Committee consisted of David Fossan (SUNY Stony Brook) as Chairperson, Michael Wiescher (University of Notre Dame), and Frank Wolfs (University of Rochester).

A wide variety of experiments were carried out at ATLAS during the last year, with emphasis placed on utilization of Gammasphere during its tenure at ATLAS. The strength and diversity of the research program at ATLAS continues to rely heavily on active involvement by universities and other institutions. 


\section{a. Experiments Involving Outside Users}

All experiments in which outside users directly participated during FY 1998 are listed below. The spokesperson for each experiment is given in square brackets after the title, and the collaborators who were present for the experiment are given with their home institution below each entry.

(1) Precision Spectroscopy of the $1 \mathrm{~s} 2 \mathrm{~s}{ }^{3} \mathrm{~S}_{1}-1 \mathrm{~s} 2 \mathrm{p}{ }^{3} \mathrm{P}_{0}$ Fine Structure Transition in Helium-Like Ni ${ }^{26+}$ [Livingston]

S. Cheng, L. Curtis, University of Toledo; H. Berry, E. Jasper, K. Kukla, A. Livingston,

A. Vasilyev, University of Notre Dame; R. Ali, University of Nevada, Reno; R. Dunford,

E. Kanter, C. Kurtz, B. Zabransky, Argonne National Laboratory

(2) Study of 2- $\alpha$ Decaying High-Spin States in ${ }^{24} \mathrm{Mg}$ [Wuosmaa]

J. Cizewski, Rutgers University; B. Back, R. Betts, D. Hofman, R. Janssens, K. Lister,

V. Nanal, K. Rehm, I. Wiedenhoever, A. Wuosmaa, Argonne National Laboratory

E1-M1 Damping Interference in the Electric Field Quenching of Metastable $\mathrm{Ar}^{17+}$ Ions - II [Dunford]

S. Cheng, L. Curtis, University of Toledo; H. Berry, A. Livingston, A. Vasilyev, University of Notre Dame; R. Dunford, E. Kanter, Argonne National Laboratory

(4) Study of the Isometric Transitions in ${ }^{98} \mathrm{Cd}$ and ${ }^{102} \mathrm{Sn}$ [Seweryniak]

E. Seabury, University of Massachusetts, Lowell; J. Ressler, W. Walters, University of

Maryland; G. Hackman, University of Kansas; M. Huhta, Michigan State University;

C. Fahlander, Lund University; P. Reiter, Ludwig Maximilans-Universität München;

M. Lipoglavsek, Jozef Stefan Institute; C. Davids, S. Fischer, D. Henderson, R. Janssens,

K. Lister, J. Uusitalo, Argonne National Laboratory

(5) Half-Lives and Branching Ratios of Superallowed $0^{+} \rightarrow 0^{+} \beta$-emitters [Savard]

H. Fukutani, University of Manitoba; G. Gervais, Northwestern University; X. Feng, McGill

University; D. Hofman, G. Savard, D. Seweryniak, J. Uusitalo, Argonne National Laboratory

(6) Tests of the Area II Enge Split-Pole Upgrades for the CPT Mass Spectrometer Project [Savard]

H. Fukutani, K. Sharma, University of Manitoba; G. Hackman, University of Kansas; J. Hardy, Texas A \& M University; F. Buchinger, J. Crawford, X. Feng, S. Gulick, J. Lee, P. Martinez, McGill University; D. Hofman, G. Savard, J. Uusitalo, Argonne National Laboratory

(7) Preparation of the FMA and Auxiliary Detectors for Operation with Gammasphere [Lister] J. Schwartz, Yale University; P. Hausladen, University of Pennsylvania; P. Chowdhury,

E. Seabury, University of Massachusetts, Lowell; W. Walters, University of Maryland;

R. Macleod, Thomas Jefferson National Accelerator Facility; J. Cizewski, K. Ding, N. Fotiadis, Rutgers University; P. Reiter, Ludwig Maximilans-Universität München; P. Fallon,

A. Macchiavelli, M. Maier, Lawrence Berkeley National Laboratory; M. Carpenter, C. Davids,

D. Henderson, R. Janssens, T. Khoo, T. Lauritsen, K. Lister, D. Nisius, D. Seweryniak,

J. Uusitalo, Argonne National Laboratory

(8) Accelerator Mass Spectrometry of Actinide Elements with the ECR-ATLAS System [Paul]

B. Harss, TU Munich; D. Berkovits, Soreq Nuclear Research Center; F. Borasi, Northwestern

University; M. Paul, Hebrew University of Jerusalem; I. Ahmad, P. Billquist, C. Davids,

D. Henderson, W. Henning, C. Jiang, R. Pardo, K. Rehm, D. Seweryniak, R. Vondrasek,

Argonne National Laboratory

(9) Coulomb Excitation of Radioactive Beams Prepared by the Fragment Mass Analyzer [Schwartz] J. Schwartz, Yale University; P. Reiter, Ludwig Maximilans-Universität München; C. Davids

S. Fischer, D. Henderson, R. Janssens, K. Lister, D. Seweryniak, Argonne National Laboratory 
(10) Measurement of the ${ }^{3} \mathrm{He}\left({ }^{25} \mathrm{Al}, \mathrm{d}\right){ }^{26} \mathrm{Si}$ Reaction: Production of a ${ }^{25} \mathrm{Al}$ Beam [Champagne]

P. Parker, Yale University; A. Champagne, University of North Carolina-Chapel Hill; B. Harss, Tu Munich; R. Janssens, C. Jiang, R. Pardo, K. Rehm, A. Sonzogni, J. Uusitalo, Argonne National Laboratory

(11) Patterning of Vortex Channels and Square Arrays in High Temperature Superconductors with Heavy Ion Irridiation [Kwok]

L. Paulius, A. Petrean, Western Michigan University; D. Hofman, G. Karapetrov, W. Kwok,

D. Lopez, R. Olsson, Argonne National Laboratory

(12) Measurement of an Excitation Function of the ${ }^{17} \mathrm{~F}(\mathrm{p}, \alpha){ }^{14} \mathrm{O}$ Reaction [Rehm]

P. Parker, Yale University; X. Chen, University of Tennessee; B. Harss, Tu Munich;

J. Blackmon, Oak Ridge National Laboratory; R. Segel, Northwestern University; T. Wang,

Lawrence Livermore National Laboratory; M. Paul, Hebrew University of Jerusalem;

R. Janssens, C. Jiang, R. Pardo, K. Rehm, J. Schiffer, A. Sonzogni, J. Uusitalo,

I. Wiedenhoever, Argonne National Laboratory

(13) Development of a New Method for Measuring the Excitation Function for $(\alpha, \gamma)$ Reactions Induced by Radioactive Ion Beams [Jiang]

B. Harss, Tu Munich; D. Berkovits, Soreq Nuclear Research Center; F. Borasi, R. Segel, Northwestern University; M. Paul, Hebrew University of Jerusalem; W. Henning, R. Janssens, C. Jiang, R. Pardo, K. Rehm, G. Savard, J. Schiffer, A. Sonzogni, J. Uusitalo, Argonne National Laboratory

(14) Measurement of the Spins of States Observed in the Reactions ${ }^{12} \mathrm{C}\left({ }^{20} \mathrm{Ne},{ }^{12} \mathrm{C}^{12} \mathrm{C}\right){ }^{8} \mathrm{Be}$, ${ }^{12} \mathrm{C}\left({ }^{20} \mathrm{Ne},{ }^{16} \mathrm{O},{ }^{8} \mathrm{Be}\right){ }^{8} \mathrm{Be}$ and ${ }^{12} \mathrm{C}\left({ }^{20} \mathrm{Ne},{ }^{16} \mathrm{O}^{12} \mathrm{C}\right){ }^{4} \mathrm{He}$ [Murgatroyd]

N. Curtis, University of Surrey; B. Fulton, J. Murgatroyd, S. Singer, University of Birmingham;

D. Henderson, D. Hofman, I. Wiedenhoever, A. Wuosmaa, Argonne National Laboratory

(15) Study of the ${ }^{56} \mathrm{Ni}\left({ }^{3} \mathrm{He}, \mathrm{d}\right){ }^{57} \mathrm{Cu}$ Reaction [Schiffer]

B. Harss, Tu Munich; R. Segel, Northwestern University; P. Reiter, Ludwig Maximilans-

Universität München; D. Ackerman, Lab. Nazionali di Legnaro; M. Paul, Hebrew University of Jerusalem; I. Ahmad, J. Greene, D. Henderson, W. Henning, R. Janssens, C. Jiang, K. Rehm, J. Schiffer, D. Seweryniak, J. Uusitalo, I. Wiedenhoever, A. Wuosmaa, Argonne National

Laboratory

(16) Search for a Proton 2p-3h State in Light Odd Tl Isotopes [Bingham]

C. Bingham, W. Reviol, J. Wauters, X. Xu, University of Tennessee; J. Batchelder, K. Toth, Oak Ridge National Laboratory; J. Wood, Georgia Institute of Technology; C. Davids,

D. Seweryniak, J. Uusitalo, Argonne National Laboratory

(17) Study of ${ }^{8} \mathrm{~B}$ Neutrino Spectrum through the Decay Chain ${ }^{8} \mathrm{~B}\left(\mathrm{P}^{+}\right)^{8} \mathrm{Be}(2 \alpha)$ [Freedman]

S. Freedman, University of California; F. Borasi, Northwestern University; B. Fujikawa,

Lawrence Berkeley National Laboratory; M. Paul, Hebrew University of Jerusalem; R. Janssens,

C. Jiang, R. Pardo, K. Rehm, G. Savard, J. Schiffer, J. Uusitalo, Argonne National Laboratory

(18) Near Scission Emission of Intermediate Mass Fragments in ${ }^{12} \mathrm{C}+{ }^{232} \mathrm{Th}$ at ELAB $=150$ and $230 \mathrm{MeV}$ [de Souza]

R. Charity, L. Sobotka, Washington University; T. Bredeweg, B. Davin, R. Yanez, R. de Souza, Indiana University Cyclotron Facility; D. Hofman, Argonne National Laboratory

(19) Heavy Ion Beam Irradiation of Samples [Dando]

J. Dando, eMed Corporation; D. Hofman, S. Fischer, Argonne National Laboratory 
(20) Magnetic Rotation in ${ }^{104}$ Sn [Clark]

M. Devlin, D. Sarantites, J. Wilson, Washington University; D. Jenkins, C. Parry,

R. Wadsworth, A. Wilson, University of York; C. Chiara, D. Fossan, D. La Fosse, K. Starosta, S.U.N.Y. at Stony Brook; R. Clark, P. Fallon, A. Macchiavelli, D. Ward, Lawrence Berkeley National Laboratory

(21) Unsafe COULEX of the ${ }^{240} \mathrm{Pu}$ Nucleus [Janssens]

D. Cline, C. Wu, University of Rochester; S. Siem, University of Oslo; P. Chowdhury, E. Seabury, University of Massachusetts, Lowell; G. Hackman, University of Kansas; H. Amro North Carolina State University; I. Ahmad, M. Carpenter, S. Fischer, R. Janssens, T. Khoo, D. Nisius, I. Wiedenhoever, Argonne National Laboratory

(22) Exotic Structures in very Neutron-Deficient $55 \leq \mathrm{Z} \leq 59$, A 120 Nuclei [Smith]

M. Devlin, F. Lerma, D. Sarantites, J. Wilson, Washington University; R. Wadsworth,

A. Wilson, University of York; S. Freeman, M. Leddy, J. Smith, University of Manchester;

C. Chiara, D. Fossan, D. La Fosse, K. Starosta, S.U.N.Y. at Stony Brook; J. Mortara,

Lawrence Berkeley National Laboratory; R. Janssens, D. Seweryniak, Argonne National

Laboratory

(23) The Feasibility of Studying Octupole Correlations in $224,226 \mathrm{U}$ using Gammasphere and the FMA [Butler]

J. Schwartz, Yale University; N. Amzal, P. Butler, A. Chewter, P. Greenlees, R. Herzberg, University of Liverpool; P. Reiter, Ludwig Maximilans-Universität München; M. Carpenter, R. Janssens, T. Khoo, K. Lister, D. Seweryniak, Argonne National Laboratory

(24) Superdeformation in $30^{60} \mathrm{Zn}_{30}$ and Proton-Decay from Excited States in $33{ }^{66}$ As 33 [Svensson] M. Devlin, F. Lerma, D. Sarantites, J. Wilson, Washington University; O. Thelen, Universität zu Köln; M. Bentley, Staffordshire University; C. Baktash, A. Galindo-Uribarri, S. Paul, D. Radford, C. Yu, Oak Ridge National Laboratory; S. Flibotte, D. Haslip, T. Lampman, B. Schaly, J. Waddington, McMaster University; C. Svensson, D. Ward, Lawrence Berkeley National Laboratory; R. Janssens, M. Carpenter, Argonne National Laboratory

(25) Structure of Deformed Ho Isotopes Beyond the Proton Drip-Line [Woods] C. Bingham, R. Grzywacz, University of Tennessee; J. Ressler, W. Walters, University of Maryland; T. Davinson, R. Slinger, P. Woods, University of Edinburgh; P. Reiter, Ludwig Maximilans-Universität München; M. Carpenter, C. Davids, R. Janssens, D. Seweryniak, J. Uusitalo, I. Wiedenhoever, Argonne National Laboratory

(26) Proton Radioactivity of Deformed Tb and Pm Isotopes [Davids]

J. Ressler, W. Walters, University of Maryland; P. Woods, University of Edinburgh; C. Davids, D. Seweryniak, A. Sonzogni, J. Uusitalo, Argonne National Laboratory

(27) Mirror Symmetry in $26^{51} \mathrm{Fe}_{25}$ and $25^{51} \mathrm{Mn}_{26}$ [Bentley] W. Gelletly, P. Regan, University of Surrey; S. Vincent, University of Notre Dame; A. Bruce, L. Frankland, University of Brighton; M. Bentley, C. O'Leary, Staffordshire University; J. Cameron, T. Lampman, McMaster University; P. Reiter, Ludwig Maximilans-Universität München; C. Svensson, Lawrence Berkeley National Laboratory; D. Warner, Daresbury Laboratory; B. Rubio, CSIC-University of Valencia; M. Carpenter, C. Davids, R. Janssens T. Khoo, K. Lister, D. Nisius, D. Seweryniak, J. Uusitalo, Argonne National Laboratory

(28) High-Spin Collective Structures in $\mathrm{N}=\mathrm{Z}$ Nuclei in the Mass 40-46 Region [Sarantites] M. Devlin, F. Lerma, D. Sarantites, J. Wilson, Washington University; A. Axelsson, M. Weiszflog, Uppsala University; C. Baktash, A. Galindo-Uribarri, Oak Ridge National Laboratory; D. Rudolph, Lund University; M. Carpenter, Argonne National Laboratory 
(29) Hyperfine-Quenched Transition Rates in Helium-Like ${ }^{59}$ Co [Berry]

S. Cheng, L. Curtis, University of Toledo; H. Berry, E. Jasper, A. Livingston, A. Vasilyev, University of Notre Dame; R. Dunford, Argonne National Laboratory

(30) Study of the Breakout from the Hot CNO Cycle to the (rp) Process via the ${ }^{18} \mathrm{Ne}(\alpha, \mathrm{p})^{21} \mathrm{Na}$ Reaction [Rehm]

B. Harss, Tu Munich; F. Borasi, R. Segel, Northwestern University; P. Crotty, R. Janssens, C. Jiang, J. Nolen, R. Pardo, K. Rehm, J. Schiffer, A. Sonzogni, J. Uusitalo, I. Wiedenhoever, Argonne National Laboratory

(31) Structure and Formation Mechanisms of Heavy Elements [Reiter]

M. Leino, University of Jyväskylä; J. Cizewski, K. Ding, N. Fotiadis, Rutgers University; P. Reiter, Ludwig Maximilans-Universität München; P. Fallon, A. Macchiavelli, K. Vetter, Lawrence Berkeley National Laboratory; W. Korten, CEA Saclay; I. Ahmad, B. Back, C. Davids, R. Janssens, T. Khoo, T. Lauritsen, K. Lister, D. Seweryniak, A. Sonzogni, J. Uusitalo, I. Wiedenhoever, Argonne National Laboratory

(32) Study of Excited States in 167,169 Ir: Probing States Beyond the Proton Drip Line [Carpenter]

L. Riedinger, University of Tennessee; J. Ressler, W. Walters, University of Maryland;

P. Woods, University of Edinburgh; P. Bhattacharyya, Purdue University; P. Reiter, Ludwig

Maximilans-Universität München; I. Ahmad, M. Carpenter, C. Davids, S. Fischer, R. Janssens,

T. Khoo, T. Lauritsen, K. Lister, D. Nisius, D. Seweryniak, A. Sonzogni, J. Uusitalo,

I. Wiedenhoever, Argonne National Laboratory

Spectroscopy of $\mathrm{N}=\mathrm{Z}$ Nuclei Populated in the ${ }^{40} \mathrm{Ca}+{ }^{40} \mathrm{Ca}$ Reaction [Balamuth]

J. Schwartz, Yale University; D. Sarantites, Washington University; D. Balamuth, P. Hausladen, University of Pennsylvania; J. Durell, M. Leddy, B. Varley, University of Manchester;

S. Fischer, J. Greene, D. Henderson, K. Lister, D. Seweryniak, Argonne National Laboratory

(34) Search for Excitations of the ${ }^{100}$ Sn Core in ${ }^{102}$ Sn [Seweryniak]

E. Melby, A. Schiller, University of Oslo; J. Ressler, W. Walters, University of Maryland;

P. Reiter, Ludwig Maximilans-Universität München; M. Carpenter, C. Davids, R. Janssens,

D. Seweryniak, A. Sonzogni, J. Uusitalo, I. Wiedenhoever, Argonne National Laboratory

Shape Coexistence in Proton Rich Lead and Thallium Isotopes [Wadsworth]

D. Jenkins, R. Wadsworth, A. Wilson, University of York; C. Bingham, E. Ellis, W. Reviol,

L. Riedinger, W. Weintraub, University of Tennessee; K. Helariutta, S. Juutinen, University of

Jyväskylä; K. Toth, Oak Ridge National Laboratory; M. Carpenter, R. Janssens, T. Lauritsen,

D. Nisius, D. Seweryniak, J. Uusitalo, Argonne National Laboratory

Contrasting Causes of Enhanced Deformation in Light Pr, Nd, and Ce Nuclei [Riedinger]

D. Sarantites, J. Wilson, Washington University; D. Hartley, W. Reviol, L. Riedinger,

W. Weintraub, O. Zeidan, University of Tennessee; A. Galindo-Uribarri, S. Paul, Oak Ridge

National Laboratory

(37) Identification of Excited States in the Proton Emitter ${ }^{113} \mathrm{Cs}$ [Gross]

T. Ginter, Vanderbilt University; C. Bingham, R. Grzywacz, W. Reviol, University of

Tennessee; J. Batchelder, C. Gross, K. Toth, C. Yu, Oak Ridge National Laboratory;

A. Piechaczek, Louisiana State University; M. Carpenter, C. Davids, R. Janssens, K. Lister,

D. Seweryniak, Argonne National Laboratory 
(38) Alpha and Proton Calibration of Microball [Sarantites]

M. Devlin, F. Lerma, D. Sarantites, J. Wilson, Washington University; A. Axelsson,

M. Weiszflog, Uppsala University; R. Clark, Lawrence Berkeley National Laboratory;

M. Carpenter, S. Fischer, Argonne National Laboratory

(39) Ion Irradiations of Anisotropic High-Tc Superconductors: Probing Dynamics of Magnetic Vortices [Miller]

K. Gray, D. Hofman, D. Miller, Argonne National Laboratory

(40) Excited States Associated with Different Shapes in ${ }^{178} \mathrm{Hg}$ and Neighboring Odd-A Nuclei [Carpenter] T. Brown, Vanderbilt University; L. Riedinger, University of Tennessee; R. Nouicer, University of Illinois-Chicago; I. Ahmad, M. Carpenter, C. Davids, S. Fischer, R. Janssens, T. Khoo, T. Lauritsen, K. Lister, D. Seweryniak, A. Sonzogni, J. Uusitalo, I. Wiedenhoever, Argonne National Laboratory

(41) Structure and Formation Mechanism of Heavy Elements - Request for additional beam time for Experiment 693 - [Reiter]

S. Siem, University of Oslo; A. Chewter, G. Jones, University of Liverpool; J. Cizewski, K. Ding, Rutgers University; P. Reiter, Ludwig Maximilans-Universität München;

M. Carpenter, C. Davids, R. Janssens, T. Khoo, T. Lauritsen, K. Lister, D. Seweryniak, A. Sonzogni, J. Uusitalo, I. Wiedenhoever, Argonne National Laboratory

In-Beam Spectroscopy Study of the Proton Emitter ${ }^{109}$ I with Recoil-Decay Tagging Technique [Yu] A. Galindo-Uribarri, S. Paul, C. Yu, Oak Ridge National Laboratory; B. MacDonald, Georgia Institute of Technology

(43) Spectroscopy of the $\mathrm{N}=\mathrm{Z}$ Nucleus ${ }^{68}$ Se [Fischer]

J. Schwartz, Yale University; D. Balamuth, P. Hausladen, University of Pennsylvania;

S. Fischer, K. Lister, D. Seweryniak, Argonne National Laboratory

(44) Measurement of ${ }^{46} \mathrm{Ti}\left(\right.$ alpha, $\mathrm{n}{ }^{49} \mathrm{Cr}$ and ${ }^{46} \mathrm{Ti}$ (alpha,p) Cross Sections as the First Step to Study the ${ }^{44} \mathrm{Ti}($ alpha, $\mathrm{p}){ }^{47} \mathrm{~V}$ Reaction [Sonzogni]

B. Harss, Tu Munich; F. Borasi, R. Segel, Northwestern University; P. Lang, Fachhochschule Munchen; P. Crotty, R. Janssens, C. Jiang, K. Rehm, J. Schiffer, D. Seweryniak, A. Sonzogni, J. Uusitalo, I. Wiedenhoever, Argonne National Laboratory

(45) Search for Hyperdeformation and Triaxial-Superdeformation in $168-170 \mathrm{Hf}$ by use of the very Cold Semi-Symmetric Reactions, ${ }^{76} \mathrm{Ge}+{ }^{96} \mathrm{Zr} \rightarrow{ }^{172} \mathrm{Hf}$ [Herskind]

J. Domscheit, H. Hubel, Universität Bonn; D. Hartley, L. Riedinger, University of Tennessee;

S. Ødegård, University of Oslo; A. Bracco, S. Frattini, University of Milano; M. Bergström,

G. Hagemann, B. Herskind, K. Schmidt, G. Sletten, P. Varmette, University of Copenhagen;

W. Ma, Mississippi State University; M. Carpenter, R. Janssens, T. Khoo, T. Lauritsen, Argonne National Laboratory

(46) Measurement of the ${ }^{17} \mathrm{~F}(\mathrm{p}, \alpha){ }^{14} \mathrm{O}$ Reaction [Harss]

P. Parker, Yale University; B. Harss, Tu Munich; J. Blackmon, M. Smith, Oak Ridge National Laboratory; R. Janssens, C. Jiang, R. Pardo, K. Rehm, J. Schiffer, A. Sonzogni, J. Uusitalo, I. Wiedenhoever, Argonne National Laboratory

(47) Mirror Symmetry in ${ }^{65}$ As and ${ }^{65}$ Ge [Rubio]

J. Garces-Narro, W. Gelletly, Z. Podolya'k, University of Surrey; J. Espino, Universidad de

Sevilla; G. De Angelis, Lab. Nazionali di Legnaro; D. Cano-Ott, T. Martinez, B. Rubio, J. Tain, CSIC-University of Valencia; R. Janssens, K. Lister, D. Seweryniak, J. Uusitalo,

I. Wiedenhoever, Argonne National Laboratory 
(48) High-Accuracy Mass Determination of Neutron-Deficient Hg Isotopes: On-Line Commissioning of the CPT Spectrometer [Savard]

H. Fukutani, K. Sharma, University of Manitoba; F. Buchinger, J. Lee, McGill University;

D. Hofman, G. Savard, D. Seweryniak, J. Uusitalo, Argonne National Laboratory

(49) A New Search for Hyper-Deformation and the Determination of the SD Excitation Energy in the A $=150$ Region [Lauritsen]

F. Hannachi, A. Korichi, CSNSM; R. Janssens, T. Khoo, T. Lauritsen, K. Lister,

D. Seweryniak, J. Uusitalo, Argonne National Laboratory

(50) Spectroscopy of Low-Lying States in ${ }^{66}$ As [Lister]

J. Cizewski, Rutgers University; P. Reiter, Ludwig Maximilans-Universität München;

M. Carpenter, S. Fischer, W. Henning, T. Lauritsen, K. Lister, K. Rehm, D. Seweryniak,

I. Wiedenhoever, Argonne National Laboratory

\section{b. Outside Users of ATLAS During the Period 10/1/97 - 9/30/98}

This list includes all outside Users who were an experiment spokesperson (a), alternate spokesperson (b), student (*) or collaborator actually present at ATLAS for an experiment. An additional 68 collaborators on the various experiment proposals were not at ATLAS in person and are not represented in the list below.

(1) CEA Saclay

W. Korten

(2) CSIC-University of Valencia

* D. Cano-Ott

* T. Martinez

a B. Rubio

$\mathrm{J}$. Tain

(3) CSNSM

F. Hannachi

A. Korichi

(4) Daresbury Laboratory

b J. Simpson

D. Warner

(5) Fachhochschule Munchen

* P. Lang

(6) Georgia Institute of Technology

* B. MacDonald

J. Wood

(7) Hebrew University of Jerusalem

a M. Paul
(8) Indiana University Cyclotron Facility

* T. Bredeweg

* B. Davin

a R. de Souza

R. Yanez

(9) Jozef Stefan Institute

* M. Lipoglavsek

(10) Lab. Nazionali di Legnaro

D. Ackerman

G. De Angelis

(11) Lawrence Berkeley National

Laboratory

a R. Clark

b P. Fallon

B. Fujikawa

b I. Lee

A. Macchiavelli

M. Maier

* J. Mortara

a C. Svensson

K. Vetter

D. Ward

(12) Lawrence Livermore National Lab.

T. Wang 
(13) Louisiana State University

A. Piechaczek

(14) Ludwig Maximilans-Universität München

a P. Reiter

(15) Lund University

C. Fahlander

D. Rudolph

(16) Material Sciences Division, ANL

b G. Crabtree

b K. Gray

G. Karapetrov

a W. Kwok

D. Lopez

a D. Miller

* R. Olsson

(17) McGill University

F. Buchinger

J. Crawford

X. Feng

S. Gulick

J. Lee

* P. Martinez

(18) McMaster University

J. Cameron

S. Flibotte

* D. Haslip

* T. Lampman

* B. Schaly

J. Waddington

(19) Michigan State University

M. Huhta

(20) Mississippi State University W. Ma

(21) North Carolina State Univ.

* H. Amro

(22) Northwestern University

* F. Borasi

* G. Gervais

R. Segel
(23) Oak Ridge National Laboratory

b C. Baktash

J. Batchelder

J. Blackmon

b A. Galindo-Uribarri

a C. Gross

S. Paul

b D. Radford

b K. Rykaczewski

M. Smith

K. Toth

a C. Yu

(24) Purdue University

P. Bhattacharyya

(25) Rutgers University

J. Cizewski

* K. Ding

N. Fotiadis

(26) S.U.N.Y. at Stony Brook

* C. Chiara

b D. Fossan

D. La Fosse

K. Starosta

(27) Soreq Nuclear Research Center

D. Berkovits

(28) Staffordshire University

a M. Bentley

* C. O'Leary

(29) Texas A \& M University

J. Hardy

(30) Thomas Jefferson National Accelerator Facility

R. Macleod

(31) Tu Munich $a b^{*}$ B. Harss

(32) Universidad de Sevilla

J. Espino

(33) University of Birmingham

b B. Fulton

a J. Murgatroyd

S. Singer 
(34) University of Brighton

$$
\text { A. Bruce }
$$

* L. Frankland

(35) University of California

a S. Freedman

(36) University of Copenhagen

$$
\text { M. Bergström }
$$

G. Hagemann

a B. Herskind

* K. Schmidt

G. Sletten

P. Varmette

(37) University of Edinburgh

T. Davinson

* R. Slinger

ab P. Woods

(38) University of Illinois-Chicago

R. Nouicer

(39) University of Jyväskylä

* K. Helariutta

S. Juutinen

M. Leino

(40) University of Kansas

b G. Hackman

(41) University of Liverpool

* N. Amzal

a P. Butler

* A. Chewter

* P. Greenlees

R. Herzberg

G. Jones

(42) University of Manchester

J. Durell

S. Freeman

b M. Leddy

a J. Smith

B. Varley

(43) University of Manitoba

* H. Fukutani

b K. Sharma
(44) University of Maryland

* J. Ressler

b W. Walters

(45) University of Massachusetts, Lowell
P. Chowdhury

E. Seabury

(46) University of Milano
A. Bracco
* S. Frattini

(47) University of Nevada, Reno R. Ali

(48) University of North Carolina-Chapel Hill
a A. Champagne

(49) University of Notre Dame

ab H. Berry

* E. Jasper

* K. Kukla

ab A. Livingston

* A. Vasilyev

S. Vincent

(50) University of Oslo

* E. Melby

* S. Ødegård

* A. Schiller

* S. Siem

(51) University of Pennsylvania

a D. Balamuth

* P. Hausladen

(52) University of Rochester

D. Cline

C. $\mathrm{Wu}$

(53) University of Surrey

N. Curtis

* J. Garces-Narro

b W. Gelletly

Z. Podolya'k

P. Regan 
(54) University of Tennessee

a C. Bingham

$\mathrm{X}$. Chen

E. Ellis

R. Grzywacz

D. Hartley

b W. Reviol

a L. Riedinger

b J. Wauters

* W. Weintraub

$\mathrm{X} . \mathrm{Xu}$

* O. Zeidan

(55) University of Toledo
S. Cheng
L. Curtis

(56) University of York

* D. Jenkins

* C. Parry

a R. Wadsworth

A. Wilson

(57) Universität Bonn

* J. Domscheit

H. Hubel
(58) Universität zu Köln

* O. Thelen

(59)

Uppsala University

* A. Axelsson

M. Weiszflog

(60) Vanderbilt University

* T. Brown

* T. Ginter

(61)

Washington University

R. Charity

M. Devlin

* F. Lerma

a D. Sarantites

L. Sobotka

b J. Wilson

(62) Western Michigan University

L. Paulius

* A. Petrean

(63) Yale University

P. Parker

a* J. Schwartz

(64) eMed Corporation

a J. Dando

\section{Summary of the Continuing User Programs for FY 1998}

The experimental program at ATLAS changed and expanded quite dramatically since the arrival of Gammasphere and the Canadian Penning Trap. As a direct consequence, a wider base of Users from many different institutions has become actively involved in ATLAS research.

The character of each individual experiment also changed somewhat when compared to typical experiments performed prior to the arrival of these new detectors. Many experiments in FY1998 involved a relatively large number of collaborators from many different institutions working together on the same experiment to insure the success of the increasingly complicated detector configurations and subsequent data analysis.

Due to the large number of individuals and institutions involved in many different experiments, we have discontinued individual institution-based summaries of ongoing research. Instead, we refer the interested reader to the many reports on heavy-ion nuclear physics research in this annual report for both an overview of the many experiments and results, as well as for the detailed lists of collaborators and institutions which contributed. 


\section{OPERATION AND DEVELOPMENT OF ATLAS}

\section{OVERVIEW}

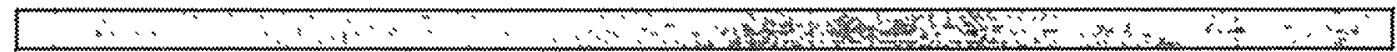

This section reports on the operation of the Argonne Tandem Linear Accelerator System (ATLAS) as a national user facility and related accelerator physics R\&D projects. ATLAS is used for basic research in nuclear and atomic physics, and occasionally for other areas of research and development, such as material science. Recent ATLAS operating performance and related development projects are described in the next section. ATLAS personnel are also involved in developing technology in support of a future advanced facility for beams of short-lived nuclei based on ATLAS.

ATLAS operates on a seven-day-per-week schedule. The installation of Gammasphere was completed in December 1997 and the experimental program with Gammasphere began in January 1998. For the 1998 calendar year, ATLAS provided 6176 hours of beam time for research, surpassing the 6000 -hour goal announced last year. Beams were provided from twenty-nine different isotopes at intensities up to $250 \mathrm{pnA}$. Statistics about beam hours and users are given in Table I-I.

ATLAS continued to provide a range of radioactive species with intensities generally in the range of $10^{5}$ to $10^{6}$ particles per second. This year $14.7 \%$ of all beam-time went to radioactive beams. Beams of long-lived ( $T_{1 / 2}>2$ hours) species produced at other facilities and placed in the ATLAS tandem ion source and beams of short-lived species produced in-flight by inverse-kinematics reactions have been developed at ATLAS. See the Heavy-Ion Research section for a summary of recent physics results from experiments using radioactive beams. 
Table I-I. SUMMARY OF ATLAS EXPERIMENTS AND USER STATISTICS

\begin{tabular}{|c|c|c|c|}
\hline & $\frac{\text { FY } 1998}{\text { (actual) }}$ & $\frac{\text { FY } 1999}{\text { (extrap.) }}$ & $\frac{\text { FY 2000 }}{\text { (pred.) }}$ \\
\hline \multicolumn{4}{|l|}{ Beam Use for Research (hr) } \\
\hline Nuclear Physics & 5136 & 5350 & 5400 \\
\hline Atomic Physics & 300 & 175 & 175 \\
\hline Accelerator Physics & 121 & 100 & 100 \\
\hline Other & 192 & 100 & 100 \\
\hline Total & 5749 & 5725 & 5775 \\
\hline $\begin{array}{l}\text { Number of Experiments } \\
\text { Receiving Beam }\end{array}$ & 53 & 55 & 50 \\
\hline $\begin{array}{l}\text { Number of Scientists } \\
\text { Participating in Research }\end{array}$ & 283 & 240 & 200 \\
\hline \multicolumn{4}{|l|}{ Institutions Represented } \\
\hline Universities (U.S.A.) & 28 & 28 & 25 \\
\hline DOE National Laboratories & 7 & 7 & 4 \\
\hline Other & 42 & 35 & 20 \\
\hline \multicolumn{4}{|l|}{ Usage of Beam Time (\%) } \\
\hline In-House Staff & 35 & 35 & 40 \\
\hline Universities (U.S.A.) & 30 & 30 & 45 \\
\hline Other DOE National Laboratories & 15 & 15 & 10 \\
\hline Other Institutions & $\underline{20}$ & 20 & -5 \\
\hline Total & $100 \%$ & $100 \%$ & $100 \%$ \\
\hline
\end{tabular}

\section{A. OPERATION OF THE ACCELERATOR}

(R. C. Pardo, B. Batzka, J. Bogaty, K. M. Borawski, B. E. Clifft, S. Daley, B. Millar, F. H. Munson, Jr., D. R. Phillips, D. Quock, A. Reuter, C. Roehrig, A. Ruthenberg, R. H. Scott, J. R. Specht, P. Strickhorn, J. Wheeler, R. C. Vondrasek, G. P. Zinkann)

Gammasphere arrived at ATLAS in the fall of 1997 and accepted first beams for tests in late December, 1997. The research program for Gammasphere began on January 17, 1998. With the arrival of Gammasphere at ATLAS, several projections were made regarding total beam time which would be provided for the research program as well as the percentage of that time for Gammasphere. For
ATLAS Operations, a goal of 6000 hours was set for beam-on-target during a twelve-month period.

The final statistics for FY1998 are now available as well as the statistics for the first quarter of FY1999 and the 6000-hour goal has been achieved. The detailed statistics are as follows:

\begin{tabular}{|l|l|l|}
\hline Time Period & Research Beam Hours & Reliability* \\
\hline FY1998 & 5749 & $93.4 \%$ \\
\hline Calendar 1998 & 6176 & 92.7 \\
\hline Jan. 1998 - Jan. 1999 & 6055 & $92.2 \%$ \\
\hline
\end{tabular}

$*$ Reliability $=100 *($ Total Research Hours $) /($ Total Research Hours+Unscheduled Maintenance $)$ 
For FY1998, $58 \mathrm{Ni}$ continued to be the most popular of the 29 isotopic species provided to the research program. The distribution of beam time shifted to lower mass species in FY1998. Only 20\% of beam time went to isotopes heavier that mass 58 , compared to $31 \%$ of all beam time for the same mass range in FY1997. The distribution of beams provided is shown in Figure II-1.

Since FY1995 ATLAS has had radioactive beams available for nuclear physics research. The radioactive beams developed to date include ${ }^{8} \mathrm{~B},{ }^{18} \mathrm{~F},{ }^{17} \mathrm{~F},{ }^{21} \mathrm{Na}$, ${ }^{25} \mathrm{Al},{ }^{56} \mathrm{Co}$, and ${ }^{56} \mathrm{Ni}$. During FY1998 $14.7 \%$ of the total beam time was with radioactive beams. Further development of radioactive beams is planned as required by the nuclear physics and nuclear astrophysics programs at ATLAS.
ATLAS operates on a seven-day per week schedule. An increase in funding as part of the DOE Scientific Facilities Initiative, that began in FY1996, increased the staff level and materials budget providing improved staffing for seven-day operation. This enhanced our ability to serve the high level of demand for beam time from the ATLAS user community. An unusually large level of staff turnover occurred in our operator pool during the last half of 1998 and strained our ability to continue to operate continuously. New operators have been hired and are now in our six-month long training program. Therefore it is expected that the staffing crisis will ease in mid-1999

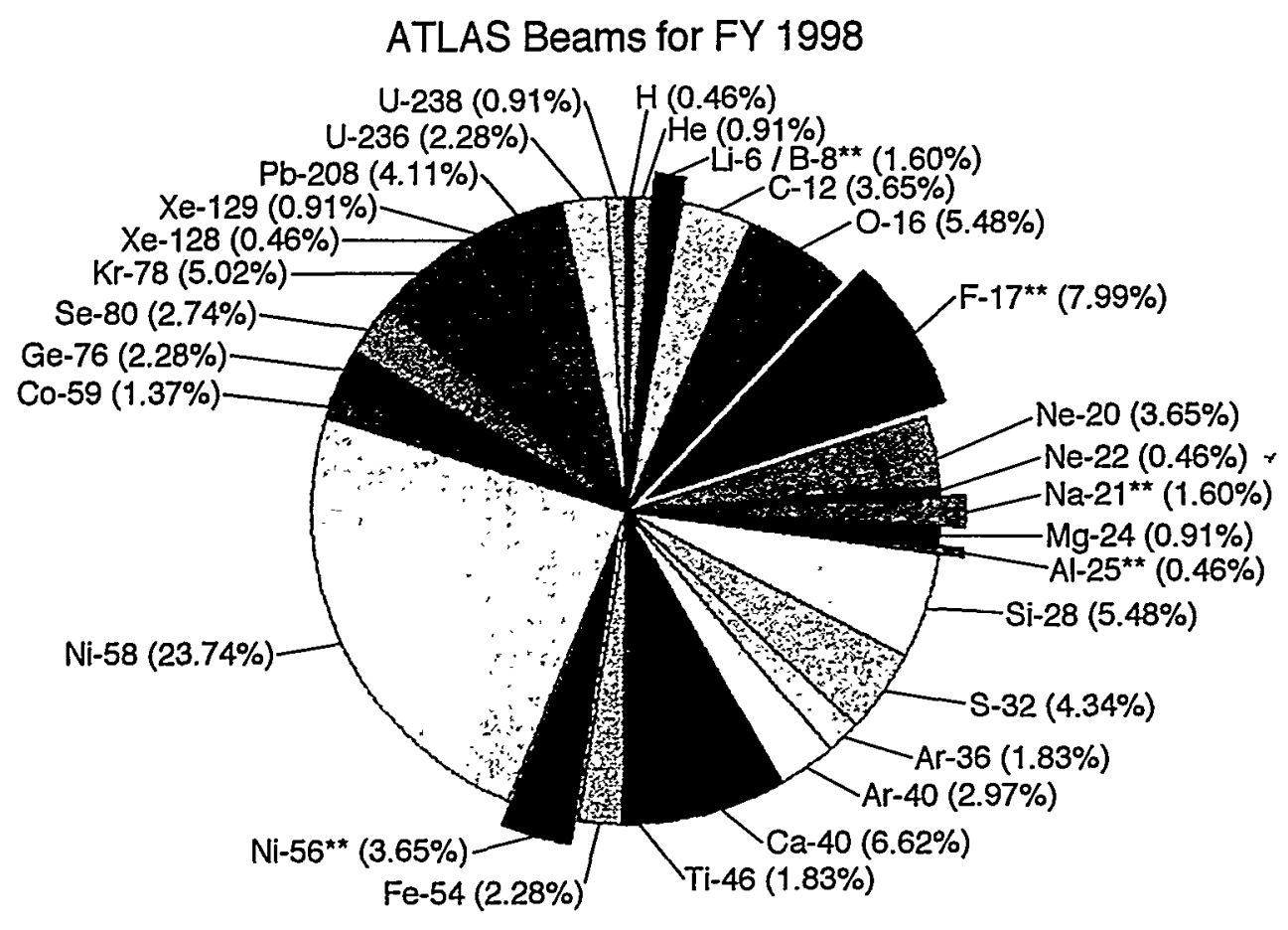

Figure II-1. Distribution of beam time, by isotope, provided by ATLAS in FY1998. A total of 29 different isotopes were provided to the research program. Radioactive beams of $17 \mathrm{~F}, 2 I_{\mathrm{Na}},{ }^{25} \mathrm{Al}$, and $56_{\mathrm{Ni}}$ comprised $14.7 \%$ of all beam time in FY1998. 


\section{a. Status of the New 14-GHz ECR Ion Source (ECR-II) (R. C. Pardo and R. C. Vondrasek)}

The new ATLAS ECR ion source and injection beamline system is complete and provided the first beam for the research program on September 7, 1998. This ion source design and construction was done in collaboration with C. Lyness and D. Xie of LBN and is similar to the LBNL AECR-U source. A beam of $16 \mathrm{O}^{5+}$ ions with an intensity on-target of $100 \mathrm{pnA}$ was used for the in-flight production of $17_{\mathrm{F}}$ for six days. This milestone had been delayed approximately 3 months due to shield problems in the high-voltage isolation transformers which required they be returned to the vendor for a design change. Then upon receiving and reinstalling the modified transformers, we discovered a noise problem with the unfiltered SCR high-current power supplies for the main ECR solenoid coils. Although they performed adequately when connected directly to the building power grid, when connected to the low impedance of the isolation transformers they create so much high-frequency switching noise on the power lines that other devices for the source will not operate correctly. We have implemented a short-term fix, but replacing both supplies is necessary.

An important area of development for ECR-II is the use of solid material for feed stock. Solid material is introduced into the plasma using either ovens or plasma sputtering. The oven is composed of an alumina tube with a thread machined in its outer diameter so that a $75 \% \mathrm{~W} / 25 \% \mathrm{Re}$ wire can be wrapped around the alumina. The W/Re wire is more flexible and has a longer lifetime than a pure $\mathrm{W}$ wire.

\begin{abstract}
A larger alumina tube is then placed over the heating element to ensure good electrical isolation and to protect the heating element. Multiple layers of 0.002 " dimpled tantalum foil encase the oven and the entire assembly is enclosed in a titanium tube with titanium end caps. Alumina crucibles are utilized for most materials barring undesired chemical reactions. The oven is heated using a 20V/10A DC power supply. The oven lifetime is approximately 375 hours with a maximum operating temperature of $1400{ }^{\circ} \mathrm{C}$ at an input power of $60 \mathrm{~W}$. The vapor pressure required for good beam production is in the $10^{-3}$ to $10^{-4}$ Torr range.
\end{abstract}

The oven has been used for beams of ${ }^{40} \mathrm{Ca},{ }^{48} \mathrm{Ca}$, ${ }^{76} \mathrm{Ge},{ }^{133} \mathrm{Cs},{ }^{197} \mathrm{Au}$, and ${ }^{209} \mathrm{Bi}$. Detailed parameter information is provided in Table II- $\Pi$. The initial usage rates were typically $\geq 1.0 \mathrm{mg} / \mathrm{hr}$. This relatively high consumption rate was due to the poor geometry for the radial introduction of material vapor. The majority of the material condenses within the radial slot and is lost to the plasma. A dimpled tantalum insert was fitted into the slot to create a hot surface and discourage sticking of the evaporate. This configuration reduced the consumption to $<0.2$ $\mathrm{mg} / \mathrm{hr}$. The overall source efficiency has been measured to be $0.20 \%$ into the peak charge state with the insert in use. This compares to a measured gas efficiency with a calibrated neon leak of $3.3 \%$ into the $6^{+}$charge state which was the peak of the distribution.

Table II-II. Beam intensity and material consumption of high-temperature oven-produced beams for

ECR2.

\begin{tabular}{ccccc}
\hline Beam & Charge State & Intensity $(\mathrm{e} \mu \mathrm{A})$ & Usage Rate $(\mathrm{mg} / \mathrm{hr})$ & $\begin{array}{c}\text { Approx. } \\
\text { Oven Temp }\left({ }^{\circ} \mathrm{C}\right)\end{array}$ \\
\hline${ }^{40} \mathrm{Ca}$ & $11+$ & 64.0 & 1.469 & 500 \\
${ }^{48} \mathrm{Ca} *$ & $13+$ & 0.43 & 0.089 & 500 \\
${ }^{76} \mathrm{Ge}$ & $17+$ & 2.5 & 0.188 & 1100 \\
${ }^{133} \mathrm{Cs}$ & $20+$ & 3.2 & 1.674 & 760 \\
${ }^{197} \mathrm{Au}$ & $31+$ & 5.8 & 1.196 & 1275 \\
${ }^{209} \mathrm{Bi}$ & $31+$ & 6.5 & 1.008 & 550 \\
\hline
\end{tabular}

\footnotetext{
*Using natural abundance material.
} 


\section{b. Project to Upgrade the ATLAS PII-ECR Ion Source (D. Moehs, R. C. Vondrasek, and R. C. Pardo)}

A major upgrade of the ATLAS $10 \mathrm{GHz}$ PII-ECR ion source, which began operation in 1987 , is in the design and procurement stages. The present source has served ATLAS well, but a major redesign of the magnetic confining fields and plasma chamber should yield significantly improved performance in both charge-state distribution and total beam current.

This old, two-stage source will be converted to a single-stage design including a high-gradient magnetic field, electron donor disk, large radial ports, and flexible modular design. The individual coils will be encased in an iron yoke that optimizes the magnetic field. Computer modeling of the magnetic field profile yields a minimum field along the axis of 3.0 $\mathrm{kG}$ with mirror ratios of 4.4 and 2.9 . An open hexapole configuration consisting of Nd-Fe-B bars enclosed in an austenitic stainless steel housing will be placed in an aluminum plasma chamber that will be water cooled along the poles of the hexapole. The hexapole field at the chamber wall, $4-\mathrm{cm}$ radius, is calculated to be $9.2 \mathrm{kG}$ along the magnet poles and $5.7 \mathrm{kG}$ between the poles, which are $2.4-\mathrm{cm}$ wide. A 3D model produced from individual 2D field profiles was used to check the end effects of the hexapole. Based on the models, this new field configuration is capable of supporting a second ECR resonance zone at $14 \mathrm{GHz}$, which may be attempted at a later date.

Retirement of the present ECR source is anticipated at the end of 1999. Disassembly and construction of the new source will then commence with first operation anticipated in May 2000.

\section{c. First Tests of a Traveling-Wave Chopper for ATLAS (R. C. Pardo, J. M. Bogaty, B. E. Clifft)}

Last year a ten segment traveling-wave chopper was constructed and successfully tested at $5 \%$ of the design $12 \mathrm{MHz}$ repetition rate. The chopper must remove unbunched tails from a partially bunched heavy-ion beam in order to avoid undue emittance growth in the linac and eliminate undesirable satellite beam bunches. A new bunching system developed in conjunction with the new ECR ion source project provides beam to the chopper plates while the bunch width is still approximately 16 ns. When poorly bunched beams traverse the traditional sine-wave chopper, unacceptable transverse emittance growth and unnecessary beam loss results; whereas the flat waveform of the traveling-wave chopper induces very little emittance growth. The measured transverse emittance growth in the traveling-wave chopper was negligible for a well bunched (3ns FWHM) beam and only $25 \%$ for a beam with a time width of $18 \mathrm{~ns}$ FWHM at the chopper plates. For the sinewave chopper which has been in use up to know, the beam emittance nearly doubled for the 18 ns wide beam bunches.

Construction of a driver power supply capable of full $12 \mathrm{MHz} \mathrm{CW}$ operation was undertaken this year and is now undergoing final testing off-line. Installation of the completed chopper is expected by mid-summer 1999 and full operational tests will then be undertaken.

\section{d. ATLAS Cryogenic System (J. R. Specht, B. Millar, and S. W. MacDonald)}

Vacuum-insulated liquid-nitrogen heaters mounted on bayonets with new electronic temperature controllers were built and installed on the three PII linac cryostats. These heaters eliminate ice and water buildup on the cryostats where cold nitrogen gas exits the cryostats. This gas is from liquid nitrogen used for cooling heat shields, VCX electronics and other devices.
A development program to find a solution to water and ice problems associated with the liquid-nitrogen heaters and lines for the booster and ATLAS cryostats was started. A prototype heater with controller was built and tested.

The above-mentioned condensation problems, when cured, will improve operational reliability and eliminate related safety problems. This upgrade will take 1-2 years to complete. 


\section{e. ATLAS Control System (F. H. Munson, D. Quock, C. Roehrig, J. Figueroa, B. Chapin)}

This year marked the completion of the most recent control system upgrade. The upgrade consisted of four phases. During this fourth and final phase the primary control Ethernet segment was converted to 100 $\mathrm{mbit} / \mathrm{sec}$. All preparation for retrofitting the original ECR source has been completed. This included installing a CAMAC crate and all necessary modules, the fiber optic link to the main CAMAC serial highway, and initial interfacing electronics. Control of some devices has been provided, but a demanding operating schedule has delayed the completion of the retrofit.

Changes to the control system are much less frequent than in recent years. Consequently, future tasks will include more investigation than implementation. New knob control units are being evaluated, and if the design is accepted knob control will be expanded to ion source devices. An evaluation of the more complicated processes will be made, and if necessary an attempt will be made to make these processes more robust where possible. A new version of the resonator "auto-scanning" process is being developed that will auto-scan PII resonators.

One of the goals of the control system upgrade project was to bring the system in line with current technologies. Consequently, an effort will be made to investigate remote processing. The purpose of this will be to reduce the number of CPU intensive processes running on the primary server. This approach is comparable to present day control system designs. 


\section{B. ACCELERATOR PHYSICS AND LINAC DEVELOPMENT}

a. In-Flight Production of Radioactive Beams at ATLAS (R. C. Pardo, B. Harss, K. E. Rehm, F. Borasi, J. Greene, D. Henderson, R. V. F. Janssens, C. L. Jiang, M. Paul, J. P. Schiffer, R. E. Segel, J. R. Specht, T. F. Wang, B. J. Zabransky, and G. P. Zinkann)

Last year a new beam optics geometry was described for the in-flight production of radioactive species using the inverse $(\mathrm{p}, \mathrm{n})$ and $(\mathrm{d}, \mathrm{n})$ reactions. The first beam produced with this technique was ${ }^{17} \mathrm{~F}$ and production rates up to $2 \times 10^{6}$ pps have been achieved. The new beam optics geometry consists of a superconducting solenoid immediately after the production target followed by a superconducting resonator. The combined effects of these devices increased the transport efficiency to target at the spectrograph to $6 \%$, in agreement with model calculations. Initial research results with this configuration for the ${ }^{17} \mathrm{~F}(\mathrm{p}, \alpha){ }^{14} \mathrm{O}$ reaction have now been published.

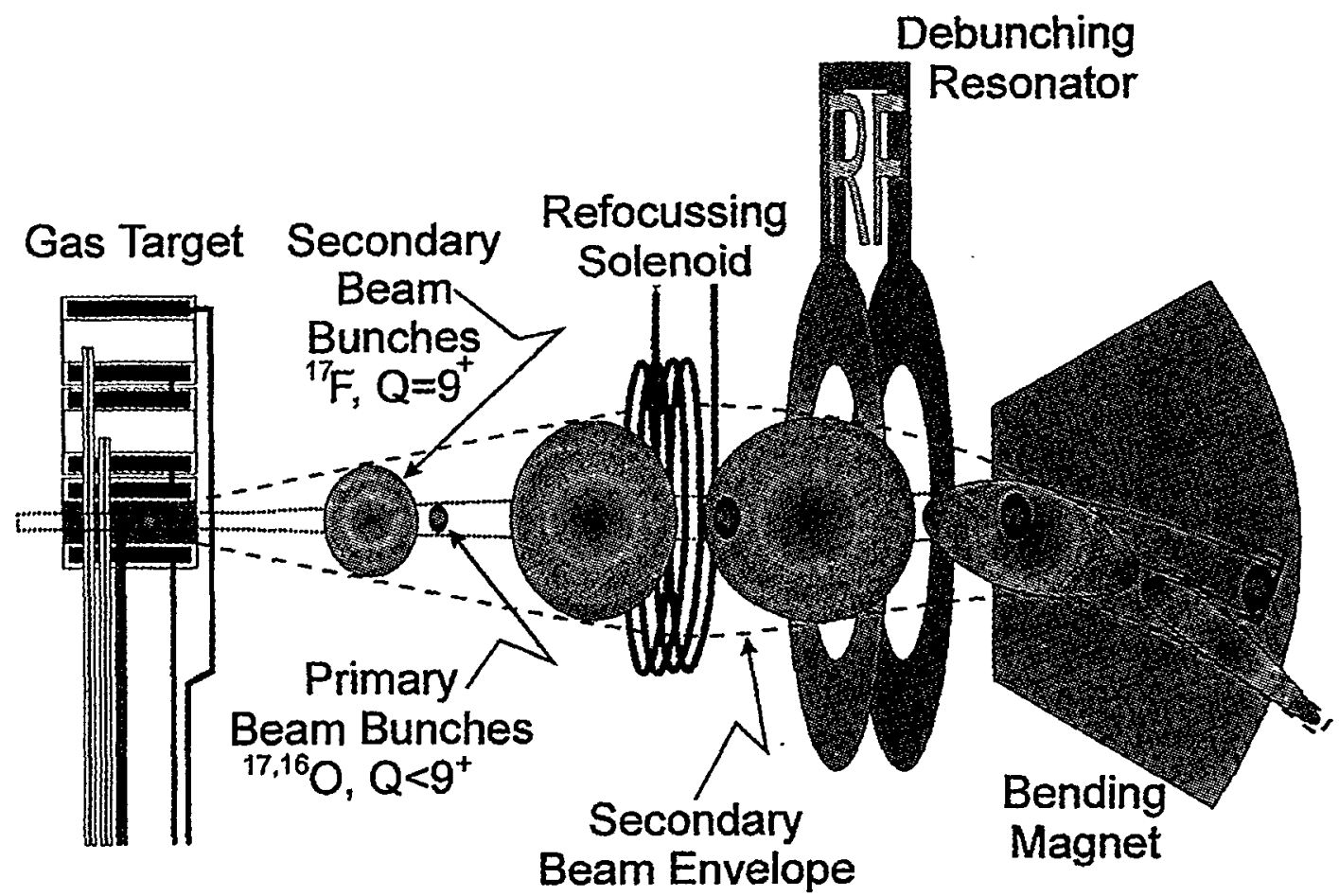

Figure II-2. Schematic floor plan of the radioactive-beam in-flight production facility at ATLAS. Following the production gas cell, a superconducting solenoid and rebunching resonator manipulate the beam phase space to improve energy resolution and transport efficiency. 
Table II-III. Data of beams produced at ATLAS with the in-flight technique.

\begin{tabular}{|c|c|c|c|c|}
\hline Beam & $\begin{array}{l}\text { Production } \\
\text { Reaction }\end{array}$ & $\begin{array}{l}\text { Secondary Energy } \\
\pm H W H M[\mathrm{MeV}]\end{array}$ & $\begin{array}{l}\text { Intensity (pps per pnA } \\
\text { primary beam)/ } \\
\text { (max. pps) }\end{array}$ & $\begin{array}{c}\text { Backgrounds } \\
\text { (fract. of total beam [\%]) }\end{array}$ \\
\hline $17_{\mathrm{F}}$ & ${ }^{17} \mathrm{O}(\mathrm{p}, \mathrm{n}){ }^{17_{\mathrm{F}}}$ & $\begin{array}{l}60-100 \\
\pm 0.3\end{array}$ & $1 \times 10^{4} / 2 \times 10^{6}$ & $\begin{array}{c}17 \mathrm{O}(10) \\
16 \mathrm{O}(<1) \\
14_{\mathrm{N}}(<0.1)\end{array}$ \\
\hline $17_{\mathrm{F}}$ & ${ }^{16} \mathrm{O}(\mathrm{d}, \mathrm{n})^{17} \mathrm{~F}$ & $\begin{array}{l}40-65 \\
\pm 0.2\end{array}$ & $\begin{array}{l}1 \times 10^{4} / 2 \times 10^{6}(65 \mathrm{MeV}) \\
3 \times 10^{3} / 3 \times 10^{5}(40 \mathrm{MeV})\end{array}$ & $\begin{array}{c}16 \mathrm{O}(10) \\
17 \mathrm{O}(10)\end{array}$ \\
\hline${ }^{25} \mathrm{Al}$ & ${ }^{24} \mathrm{Mg}(\mathrm{d}, \mathrm{n}){ }^{25} \mathrm{Al}$ & $\begin{array}{c}204 \\
\pm 0.8\end{array}$ & $1 \times 10^{3} / 1 \times 10^{5}$ & $\begin{array}{c}{ }^{24} \mathrm{Mg}(50) \\
{ }^{25} \mathrm{Mg}(<1)\end{array}$ \\
\hline${ }^{25} \mathrm{Al}$ & ${ }^{25} \mathrm{Mg}(\mathrm{p}, \mathrm{n}){ }^{25} \mathrm{Al}$ & $\begin{array}{c}180 \\
\pm 1.5\end{array}$ & $2 \times 10^{3} / 2 \times 10^{5}$ & $\begin{array}{c}{ }^{25} \mathrm{Mg}(20) \\
{ }^{24} \mathrm{Mg}(<1)\end{array}$ \\
\hline${ }^{21} \mathrm{Na}$ & ${ }^{20} \mathrm{Ne}(\mathrm{d}, \mathrm{n})^{21} \mathrm{Na}$ & $\begin{array}{l}113 \\
\pm 0.5\end{array}$ & $4 \times 10^{3} / 4 \times 10^{5}$ & $\begin{array}{c}{ }^{20} \mathrm{Ne}(50) \\
{ }^{21} \mathrm{Ne}(<0.1) \\
{ }^{18} \mathrm{~F}(<0.1)\end{array}$ \\
\hline${ }^{21} \mathrm{Na}$ & ${ }^{21} \mathrm{Ne}(\mathrm{p}, \mathrm{n})^{21} \mathrm{Na}$ & $\begin{array}{c}113 \\
\pm 0.3\end{array}$ & $8 \times 10^{3} / 8 \times 10^{5}$ & $\begin{array}{c}{ }^{21} \mathrm{Ne}(40) \\
20 \mathrm{Ne}(<0.1)\end{array}$ \\
\hline${ }^{8} \mathrm{~B}$ & ${ }^{6} \mathrm{Li}\left({ }^{3} \mathrm{He}, \mathrm{n}\right){ }^{8} \mathrm{~B}$ & $33 \mathrm{MeV}$ & $0.1 / 10$ & $\begin{array}{c}{ }^{6} \mathrm{Li}(99.9) \\
{ }^{7} \mathrm{Be}(<0.1)\end{array}$ \\
\hline
\end{tabular}

The technique for producing $17 \mathrm{~F}$ is generally applicable for a broad range of near-to-stability unstable species. Experiments with ${ }^{21} \mathrm{Na}$ and with $25 \mathrm{Al}$ beams are approved and scheduled for summer of 1999. For both beams, initial tests were performed in the fall of 1998 to determine the achievable beam intensity and quality. As in the case of $17 \mathrm{~F}$, both a $(d, n)$ and $a(p, n)$ reaction can be used to produce each of the desired isotopes. Another attempt was made to produce a ${ }^{8} \mathrm{~B}$ beam, which was only a partial success, since the production rate was lower than expected. However, a ${ }^{7} \mathrm{Be}$ beam of considerable intensity was produced in that experiment. A summary of radioactive beams developed with the in-flight technique at ATLAS is given in Table II-III.

The beam transport limitation at this point is the lack of a focusing element at the exit of the beamline switching magnet just after the superconducting buncher. We have requested funds in FY2000 for a new superconducting solenoid to replace the existing unit that will provide increased acceptance and focusing strength. The present solenoid can then be relocated to a point just after the beamline switching magnet and improve the beam transport to target in that section.

b . Timing Synchronization for Low-Intensity Beams (G. P. Zinkann, B. E. Clifft, J. A. Nolen, R. C. Pardo, K. E. Rehm, W. Q. Shen)

A variety of effects can cause transit time variations during acceleration through the tandem. Some examples of these are source platform voltage variations, stripper foil thickening, tandem voltage profile variations, or poor tandem voltage regulation due to low beam currents on the analyzing slits.. Such variations, if not corrected, would cause the ion bunch to arrive at the linac at the wrong time. Proper 
acceleration through the linac requires the time-ofarrival (TOA) at the entrance to the linac to be stable to a few tenths of a nanosecond. The room temperature helix resonant structure which is normally used to stabilize beam arrival time from the tandem is limited to beam intensity greater than 1 enA.

In order to measure the time-of-arrival of radioactive beams with intensities of as low as $10^{4} \mathrm{pps}$, a plastic scintillator detection system has been developed. The new low-beam-current detection system is located about 0.25 meters downstream from the tandem energy analyzing slits. The scintillator is mounted on a linear motion feedthrough assembly and installed in the vertical plane on the beam line. A vertical insertion, rather than horizontal, is desirable because in the horizontal plane tandem voltage fluctuations will cause the 90 degree analyzing magnet to sweep the full beam current onto the scintillator.

A phase-lock control circuit was constructed to use the pulses generated by intercepting a small fraction, approximately $1 \%$, of the beam current on the scintillator and convert it into an input control signal for the existing voltage-controlled phase shifter of the pre-tandem buncher. Low-energy events that are not within $10 \%$ of the beam energy are rejected. A TAC output from the good events is used to create a phase error signal to control the pre-tandem buncher by sending good events to a sample-and-hold circuit.

The system has now been successfully used for experiments with ${ }^{56} \mathrm{Ni}$ and ${ }^{44} \mathrm{Ti}$ and tested with beams of ${ }^{28} \mathrm{Si}$ at intensities as low as $2 \times 10^{4}$ pps. Intercepted beam event rates of 500 to 1000 particles per second are sufficient for stable control of the beam time-of arrival to approximately $0.3 \mathrm{~ns}$.

\section{c. A Vibration Damper for a Low Velocity Four Gap Accelerating Structure ( G. P. Zinkann, K. W. Shepard, and A. Facco*)}

The low velocity $(b=.008$ and .016$)$ accelerating structures in the PII (Positive Ion Injector) accelerator have the largest mechanical oscillation amplitudes out of all of the ATLAS resonators. These mechanical vibrations cause excursions in the resonant frequency of the cavity that, even with state-of-the-art phase control, result in irreducible variations of the if phase of the cavity. This phase noise, in turn, limits the time-energy resolution of ATLAS beams. A vibration damper for use in superconducting cavities has been demonstrated at the INFN Legnaro Laboratory. In a collaborative effort, their design has been adapted to the ATLAS PII resonators. Early tests on-line indicate a reduction of vibration effects by a factor of two, which can be exploited in the future to improve the limits on beam quality at ATLAS.

*INFN, Legnaro, Italy

\section{d. Superconducting Accelerating Structures for Low-Beta Heavy Ions (K. W. Shepard, M. Kedzie, P. Potukuchi* and S. Ghosh*)}

Construction of the first twelve niobium quarter-wave resonant cavity accelerating structures for the New Delhi booster linac is nearing completion. This project represents the first production of niobium cavities by commercial vendors within the United States for actual use in a linac. The contract for the project calls for fabricating ten resonators. However, because of increased productivity from the electronbeam welder, resulting in a cost reduction, we will produce twelve resonators. Figure II- 3 shows the first two completed resonators. In cold test the first resonator exceeded the nominal design goal performance of $3 \mathrm{MV} / \mathrm{m}$ with 4 watts of $\mathrm{rf}$ input power. The second resonator is being tested presently. The remaining ten resonators are 95 percent complete and are being prepared for the final electron-beam welds. Fabrication of all the resonators along with the slow tuners is expected to be complete in the next 2-3 months.

*Nuclear Science Centre, New Delhi, India 


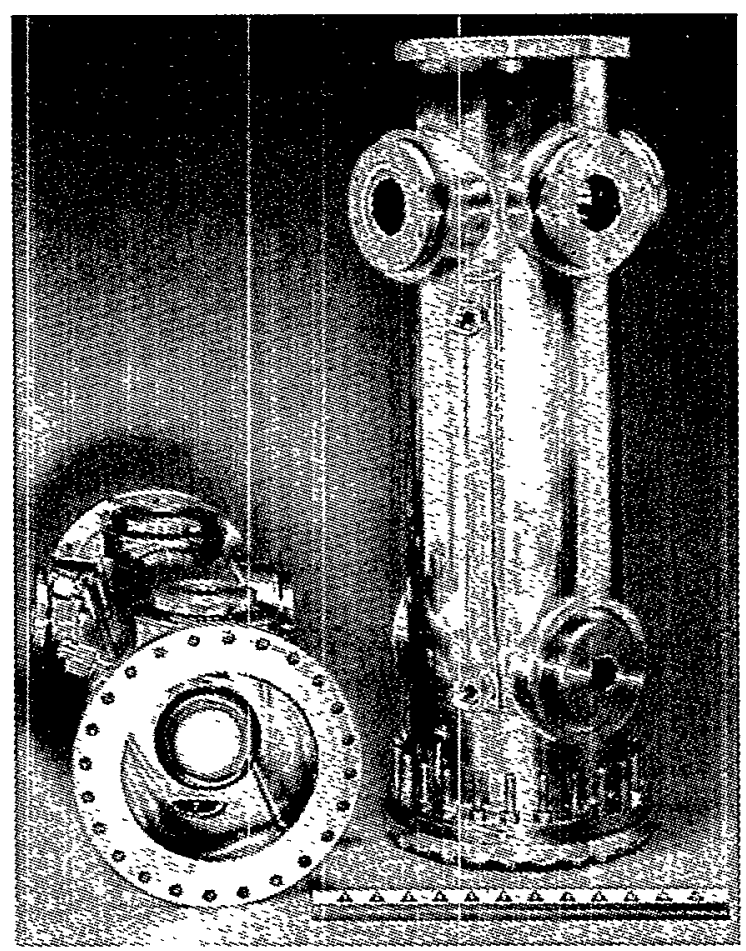

Figure II-3. Photograph of the first two resonators for the New Delhi booster linac complete with the stainless steel outer jacket. 


\section{R \& D RELATED TO A FUTURE ADVANCED EXOTIC BEAM FACILITY}

\section{a. Introduction}

Intense efforts over the past few years, supported in part by Argonne Laboratory Directed Research and Development (LDRD) funds, have led to an exciting concept for an advanced facility for beams of exotic short-lived nuclei of the ISOL (Isotope Separation On Line) type. Such a facility is based on an intense driver beam that produces short-lived nuclear species which are stopped, the desired isotope is selected, and accelerated to the appropriate energy in a second accelerator.

The recent Argonne work involves a number of technical innovations. Most importantly, it includes a novel approach that avoids the most serious drawbacks of an ISOL facility for a broad range of beams: delay between the production of the short-lived isotopes and their acceleration and the underlying sensitivity to the chemistry of extracted species. A scheme has been developed which provides for shorter delay times and insensitivity to the chemical properties. It is based on intense heavy-ion beams from the production linac, the use of the projectile fragmentation mechanism, and subsequent extraction of the desired fragment from a high-pressure helium-gas stopper cell.

The overall Argonne concept aims squarely at providing the intense beams of short-lived nuclei needed to carry out the research described in the community White Paper (1997) on the Science Opportunities with an ISOL Facility. Our initial plans, described in the 1995 Argonne Working Paper (ANL Yellow Book), have found widespread attention. The concept of using a high-power primary linac for light and heavy ions, including the generation of fast neutrons from a deuteron beam for a two-step production scheme, has triggered broad international interest.

The current concept builds and expands on these initial ideas, with several new developments: i) $a$ superconducting $c w$ production linac, providing intense beams of light ions (up to $10^{16} / \mathrm{s}$ ), light heavyions (up to $10^{15} / \mathrm{s}$ ), and heavy ions (up to $10^{14} / \mathrm{s}$ ); $i$ ) 2-step targets to handle beam powers in excess of $100 \mathrm{~kW}$; iii) utilization of a range of reaction mechanisms to optimize yields of radioactive ions
2-Step Fast Neutron Fission

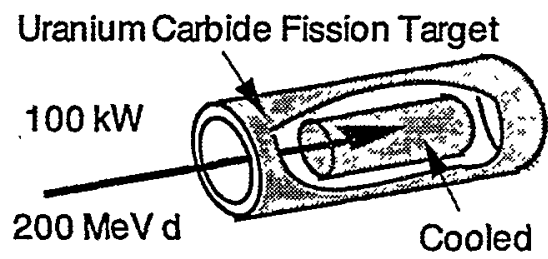

Tungsten Neutron

Production Target

\section{2-Step Projectile Fragmentation} (Solid Stopper)

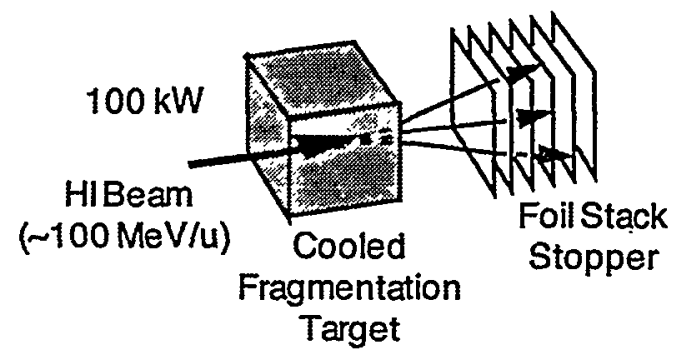

\section{2-Step Projectile Fragmentation}

(High Pressure He Gas Stopper Cell)

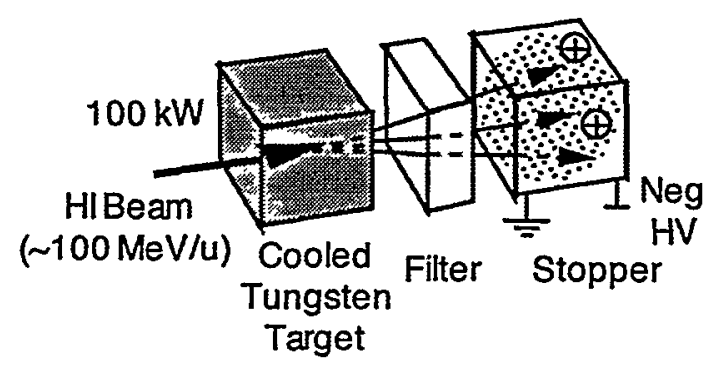

\section{One-Step Spallation Target}

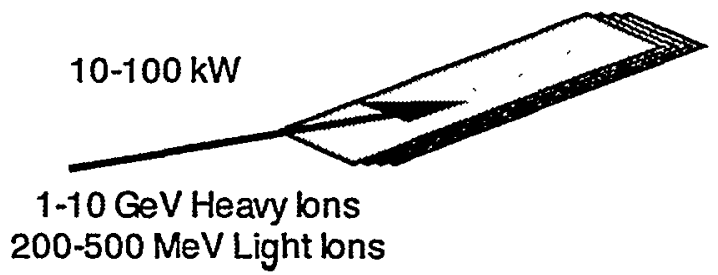

Fig. II-4. Production target concepts for the Argonne ISOL facility. 
and to match the high-power target concepts; iv) development of a cw injector into ATLAS, with a new $R F Q$ accelerator for $1^{+}$ions and non-equilibrium low-velocity stripping; and v) superconducting acceleration and focusing structures for efficient injection into ATLAS.
Figure II-4 illustrates schematically the principal approaches and solutions of the Argonne concept to the key issues of an ISOL facility: high-power production targetry and secondary (radioactive) ion-beam extraction.

\section{b. Radioisotope Production and High-Power Targetry}

The most critical issues for an advanced ISOL facility concern the generation of radioisotopes in the ISOL production target, and their subsequent extraction as an ion beam. The key technical challenges (target power density, chemical aspects of diffusion, fast effusion geometries, high ionization yields, and ion-source beam emittances) tie into aspects of production mechanisms and target geometry.

The ANL concept for the advanced ISOL facility is based on a high-power superconducting linac providing a broad range of beams, up to uranium. Projected intensities range from close to $10^{16}$ protons/s to $10^{13}$ uranium ions/s.

With these intense beams and the high target power densities expected from the Argonne driver linac, target cooling becomes a central issue. In conventional ISOL production schemes the target and ion source are integrally coupled so that cooling the primary target may interfere with the extraction of the radioactive nuclides. With the two-step schemes these functions are physically separated as described below. The ohmic heating by the beam is restricted to the primary target. Advantage is taken of recent developments in cooling with liquid lithium. Engineering designs of windowless flowing liquid lithium targets exist at Argonne in the Energy Technology Division and the Technology Development Division, which were developed for stopping 10-MW deuteron beams for the thermonuclear fusion program. Figure II-5 shows a photograph of an operating lithium pump system at Argonne National Laboratory.

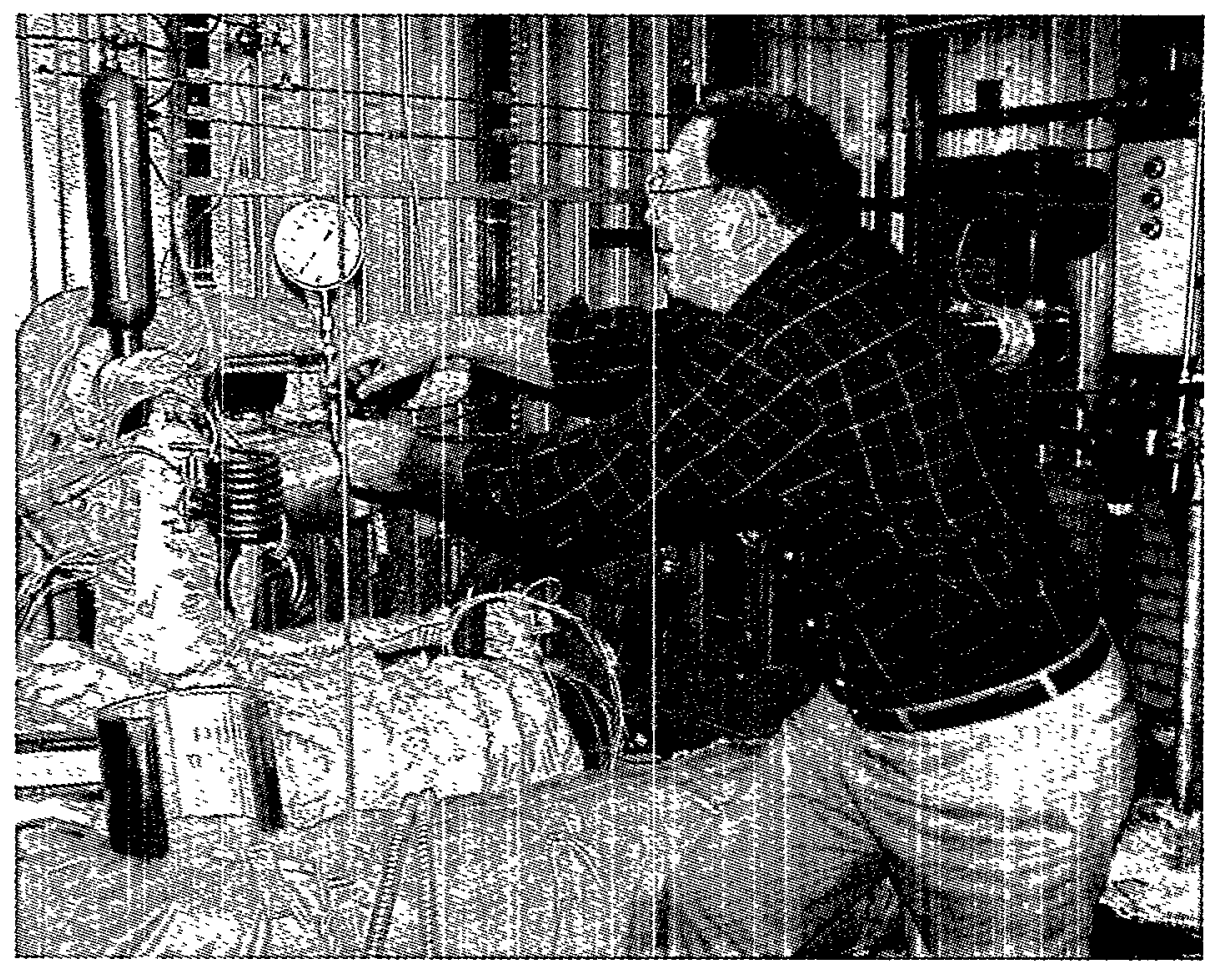

Fig. II-5. An operating liquid-lithium pump system circulating 200 gallons/min (100 times the flow needed for the ISOL target) in the Argonne Technology Development Division. 


\section{b.1. Fragmentation/high-pressure He gas stopper cell}

A new and very promising development based on the so-called IGISOL technique, that is currently being tested at Argonne in conjunction with ion accumulation for the Canadian Penning Trap, may provide a breakthrough in ISOL technology, particularly for very short-lived isotopes and elements not generally amenable to the ISOL technique (e.g. refractory elements). It uses projectile fragmentation of a highenergy heavy-ion beam, say uranium, a fragment separator to select a stabilized isotope of choice, and stops these in a high-pressure helium or argon gas cell where they come to rest as $1^{+}$ions and cannot neutralize in a medium with high ionization potential.
Using a combination of RF and static electric fields they are extracted in a few milliseconds from the gas volume with high efficiency, independent of their chemical properties. This advanced two-step fragmentation target concept (Fig. II-6) will make irrelevant the slow diffusion processes which have limited, to a great extent, the variety of radioactive beams available with ISOL based facilities.

Recent tests at Argonne with the CPT gas-catcher and ion-guide system (see Fig. II-7) have demonstrated $20 \%$ extraction efficiency of heavy-ion reaction products from the gas cell and, for a gas cell with voltage electrodes installed (see Fig. II-8), extraction times of ions injected in a pulsed mode reduced to about 60 milliseconds with

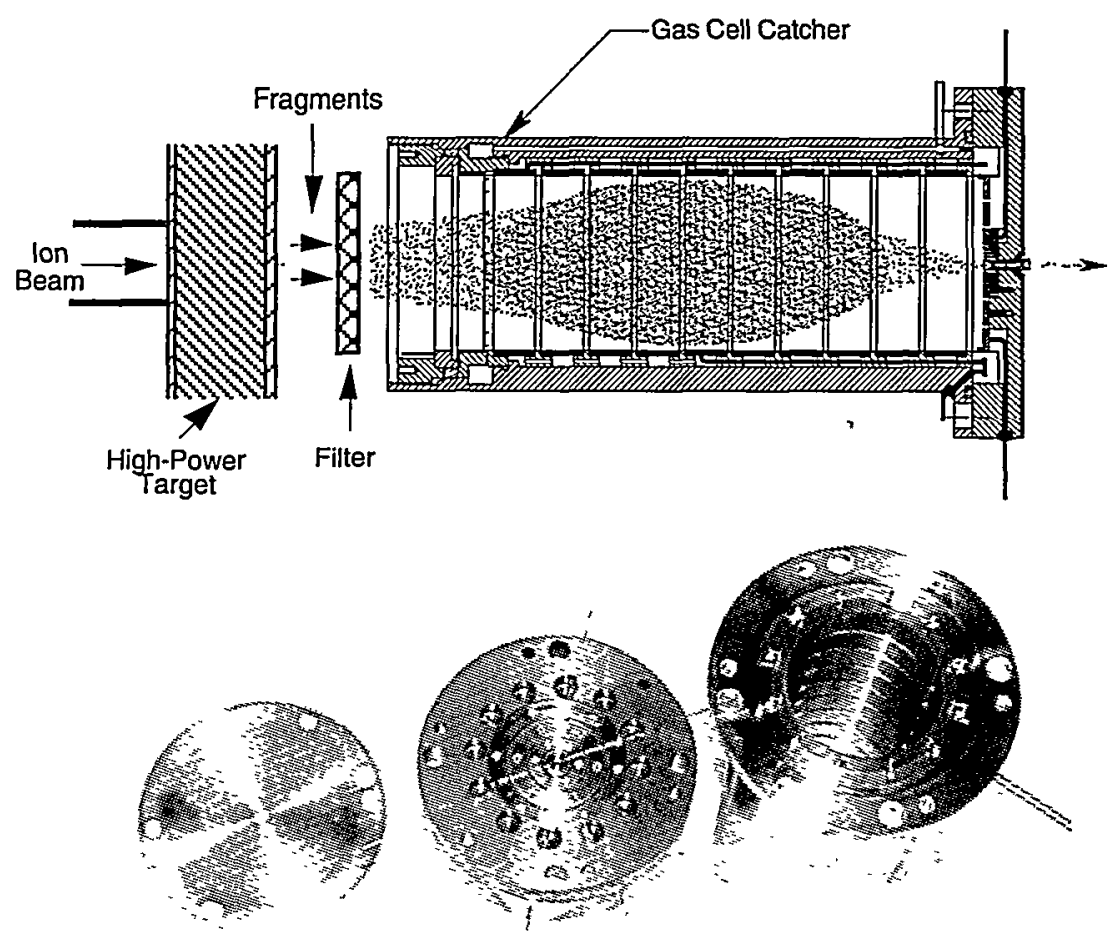

Fig. II-6. Top: Schematic layout of the advanced two-step fragmentation target. The driver beam impinges on a well-cooled primary target where the fragmentation products are formed. These products are stopped in the gas cell catcher as $\mathrm{I}^{+}$ions, and are guided by electric fields to the extraction hole. A filter, which could be a selective absorber or magnetic device, may be placed between the production target and the gas stopper cell. Bottom: Components of the gas cell catcher used in preliminary release studies at ATLAS. Shown are the cylindrical stack of electrodes which guide ions towards the exit plate (right), the concentric extraction electrodes which pull the ions towards the extraction hole (center), and the guard plate covering the cell exit (left). 
a very modest gradient of $8 \mathrm{~V} / \mathrm{cm}$ for argon gas in the cell. Figure $\Pi-8$ shows mean extraction times as a function of voltage to pressure ratio. This first version of the biased gas cell should be able to operate with a gradient in excess of $100 \mathrm{~V} / \mathrm{cm}$ which will provide extraction times below $1 \mathrm{~ms}$ for helium and a few $\mathrm{ms}$ for argon.

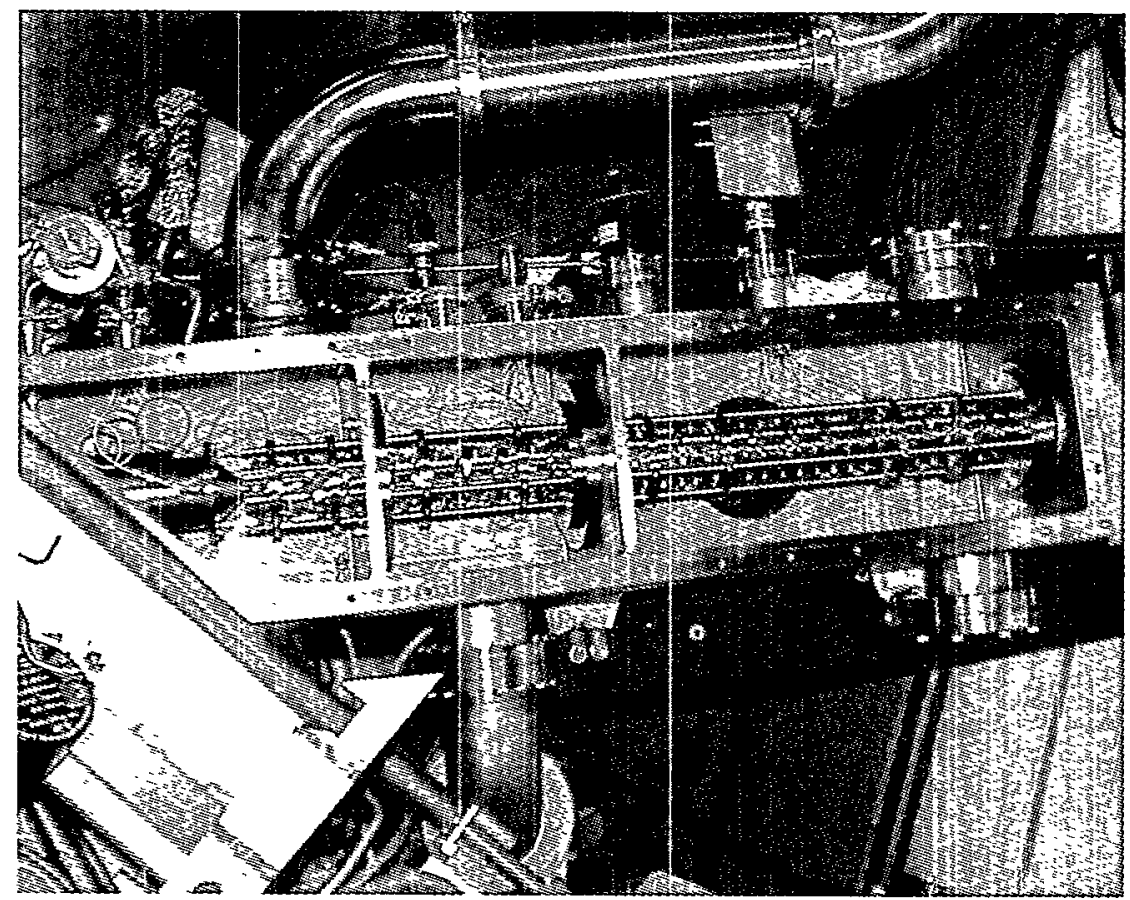

Fig. II-7. A photograph of the gas cell and ion guide system used in efficiency and delay time tests at Argonne.

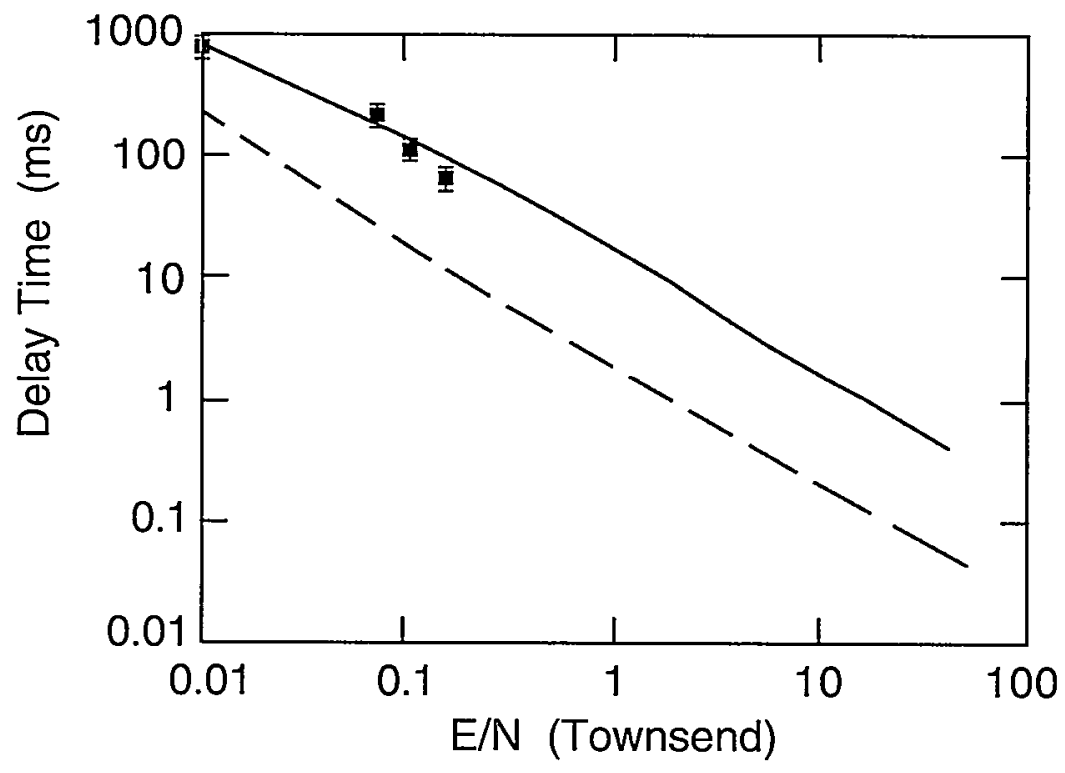

Fig. II-8. Mean delay time between injection of pulsed beam in a gas cell and extraction of the ions out of the cell as a function of electric field. The squares represent measured data points in an argon gas cell. The solid line denotes calculated delay times for Ar gas and the dashed line for He gas. A townsend is $10^{-17}$ volt- $\mathrm{cm}^{2}$. 


\section{b.2. Two-step, neutron-generator target}

In this scheme, a very intense flux of neutrons is produced by deuterons incident on a primary target, and the neutrons induce fission in the secondary uranium target. With the use of neutron-induced fission, mass distributions extending to the very neutron-rich isotopes are obtained. To optimize the solid angle of the secondary uranium target for the fast and the evaporation neutrons, a cylindrical geometry will be used as shown in Fig. II-9. This geometry is well suited to the production of radionuclides that have high yields in lowenergy neutron-induced fission. With the Los Alamos LAHET Code, the fission fragment production rates have been calculated for realistic geometries. The calculated production yield of the doubly magic nucleus ${ }^{132} \mathrm{Sn}$ (40 sec half-life) produced with a $400-\mu \mathrm{A}, 250-$
$\mathrm{MeV}$ deuteron beam in a compact secondary target ( $2 \mathrm{~cm}$ radial thickness by $10 \mathrm{~cm}$ axial length) is $2 \times 10^{11}$ per second.

This compact secondary target design is optimized for both diffusion from the porous UC target matrix and effusion from the cylindrical vacuum space surrounding the target material. The thickness of the effusion space is optimized via three-dimensional Monte Carlo modeling which includes both the temperature-dependent sticking times and the total path length effects. The length and diameter of the secondary target are kept to a minimum for optimal extraction of short-lived products. Use of thicker and longer secondary targets can increase the yields of longer-lived isotopes.

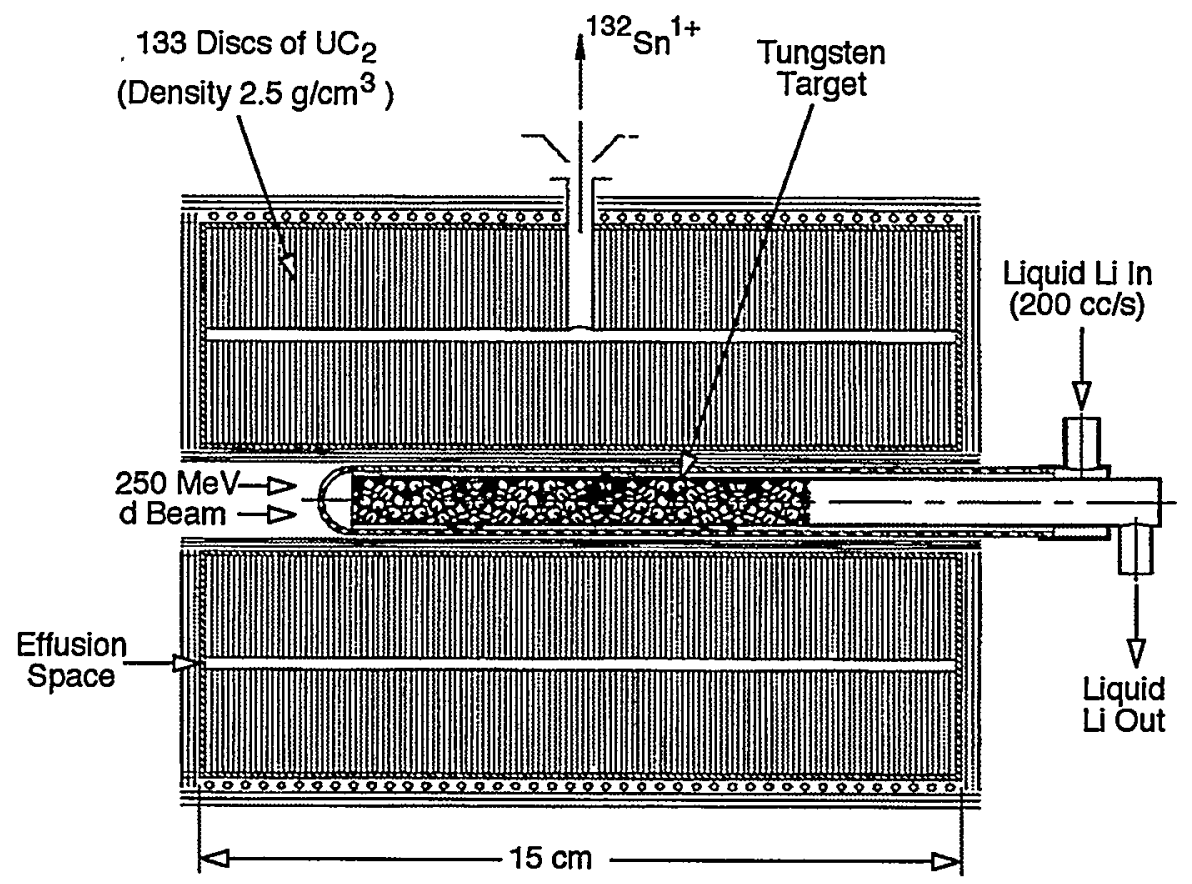

Fig. II-9. Schematic of a two-step, high-power target for the production of fission fragments by secondary neutrons. A deuteron beam on a tungsten primary target produces the neutrons. Circulating liquid lithium cools the primary target. The secondary uranium carbide target is heated to $2000^{\circ} \mathrm{C}$ by a combination of the fission power and supplemental electrical power to facilitate the release of fission products.

\section{b.3. Direct irradiation spallation target}

A third target design concept is being developed to utilize an important radionuclide production mechanism, heavy-ion-induced spallation of heavy targets such as uranium. Yields of very neutron-rich sodium isotopes, for example, were observed in the pioneering work with $86 \mathrm{MeV}$ per nucleon ( $1 \mathrm{GeV}$ total energy) carbon beams at the ISOLDE facility at the CERN Synchrocyclotron to be a factor of $\sim 50$ larger than those obtained with $600-\mathrm{MeV}$ protons. Based on these ISOLDE yield measurements, scaled to $100 \mathrm{~kW}$ of 
$1.4 \mathrm{GeV}$ carbon on a $\mathrm{UC}_{\mathrm{X}}$ target, mass separated beams of ${ }^{31} \mathrm{Na}$ (17 ms half-life) could be obtained with intensities of over $5 \times 10^{6}$ per second. With beams of $1.4 \mathrm{GeV}$ carbon, $2 \mathrm{GeV}$ neon and $4 \mathrm{GeV}$ argon that our driver linac allows, this reaction mechanism could prove to be very useful. However, this requires a direct irradiation of the $\mathrm{UC}_{\mathrm{X}}$ target with the intense heavy-ion beam as the two-step geometries are not applicable.

Direct irradiation of the standard low-density porous $\mathrm{UC}_{\mathrm{x}}$ used at $\mathrm{ISOLDE}^{9}$ cannot be scaled easily to high beam power due to the very low thermal conductivity of this form of the material. Hence, a geometry is being considered for irradiating a large area sheet of thin $(0.1$ to $1 \mathrm{~mm}$ thick) higher density $\mathrm{UC}_{\mathrm{X}}$, taking advantage of the short range of the heavy ions. Such a sheet, tilted almost parallel to the driver beam, can dissipate the large beam power by black body radiation, while thin sample dimensions prevent high internal temperatures within the sheet. This arrangement has a very open geometry that results in very short effusion times, as calculated by the three-dimensional modeling code.

\section{c. Superconducting Driver Linac Studies and Cavity Development (K. W. Shepard, M. Kedzie, J. R. Delayen, *, C. Piller,* and J. Mammosser*)}

A key requirement for a high-performance, multi-beam driver linac is the development of superconducting cavities to accelerate particles over the velocity range $0.2<\mathrm{v} / \mathrm{c}<1$. Two prototype niobium $350-\mathrm{Mhz}$ spoke-loaded cavities (Fig. II-10) were completed that are designed for particle velocities of 0.3 and $0.4 \mathrm{c}$. In initial cold tests, accelerating gradients of $5 \mathrm{MV} / \mathrm{m}$ were achieved at $4.5 \mathrm{~K}$ (see Fig. II-11). These early results are sufficient to establish the feasibility of the superconducting linac approach for building a multibeam driver. More conventional, lower frequency inter-digital and quarter-wave resonators can be used at the low velocity end of the linac.

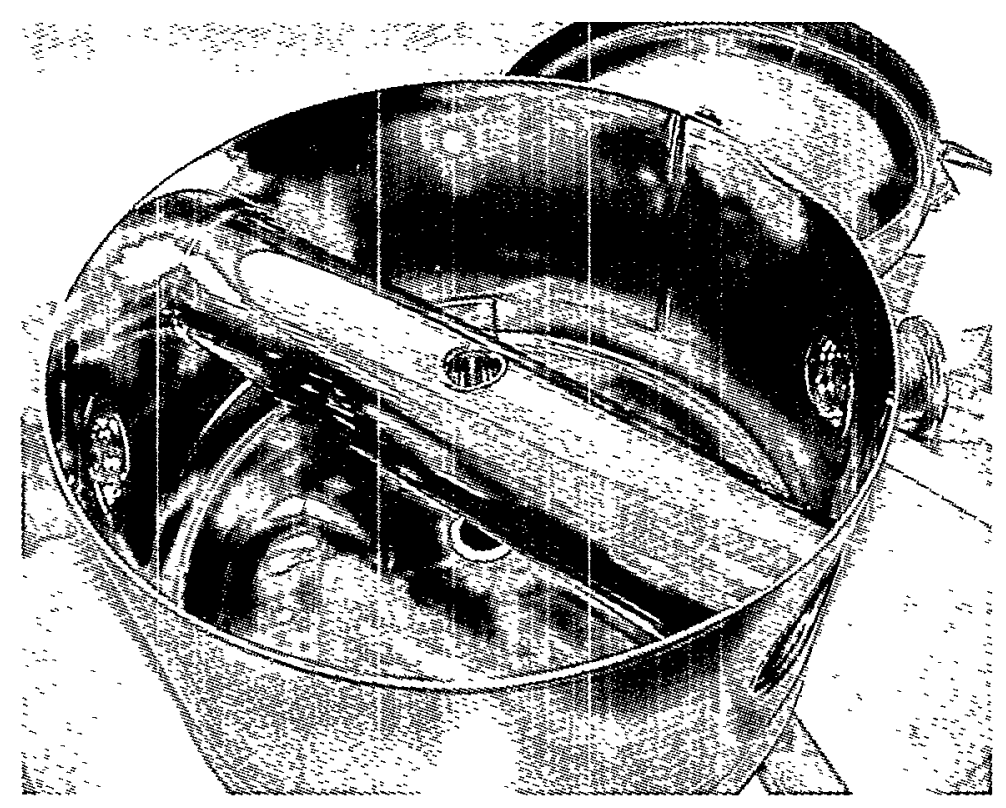

Fig. II-10. Niobium elements of the prototype $350 \mathrm{MHz}$ spoke cavity.

*Thomas Jefferson National Accelerator Facility 


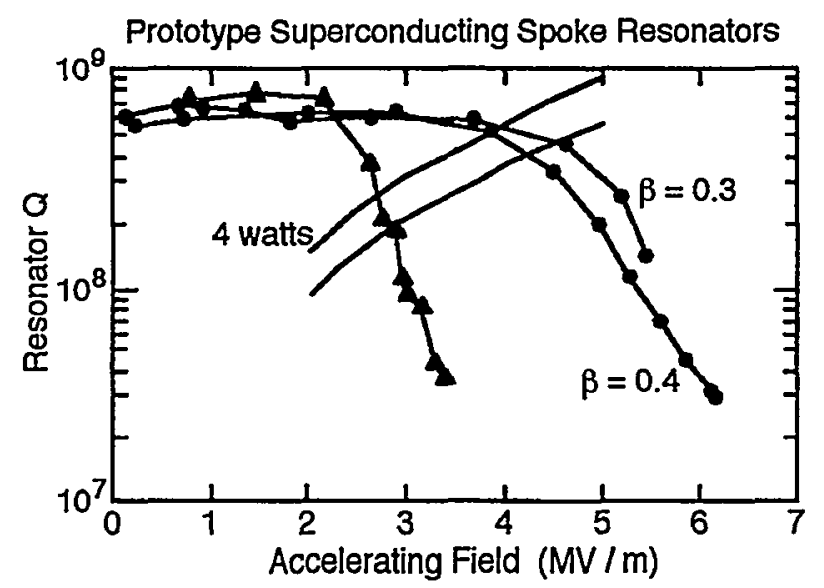

Fig. II-11. Accelerating gradients of $5 \mathrm{MV} / \mathrm{m}$ achieved at $4.5 \mathrm{~K}$.

In Fig. II-12, a schematic layout of the driver is shown with platforms at the low energy end. At both ends, RF switching can be used to simultaneously accelerate beams from alternate sources and irradiate multiple target modules, an important feature for the development of new beams while also permitting the simultaneous delivery of other beams to more than one experiment.

The Physics Division has in storage at ANL a complete 4-kW liquid helium refrigerator system. This refrigerator was received as surplus equipment from the Superconducting Super Collider (SSC) project. It was installed and tested at the SSC Magnet Test Laboratory. This refrigerator system is approximately three times more energy efficient than the existing ATLAS refrigerators and has the capacity to handle both the new ISOL and existing ATLAS superconducting linacs. Valued at about $\$ 10 \mathrm{M}$, it consists of compressors, coldboxes, and control systems (Fig. II-13).

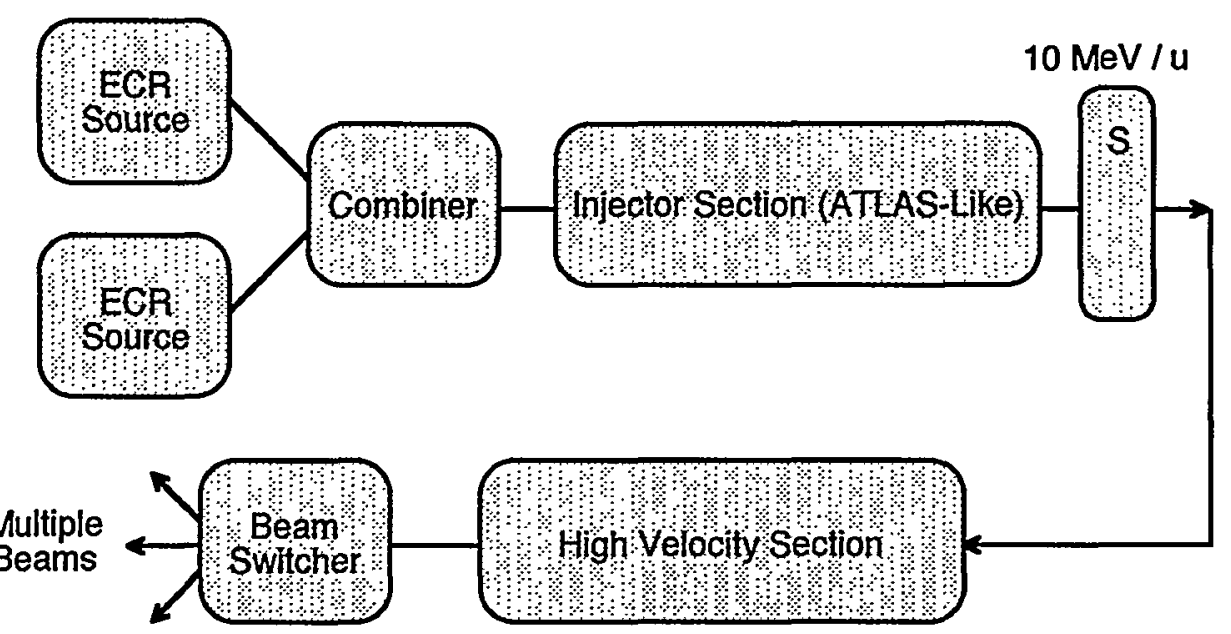

Fig. II-12. Schematic layout of the light-ion/heavy-ion superconducting production linac, including beam merging and separation sections that allow multiple-beam and multiple-target operation. 


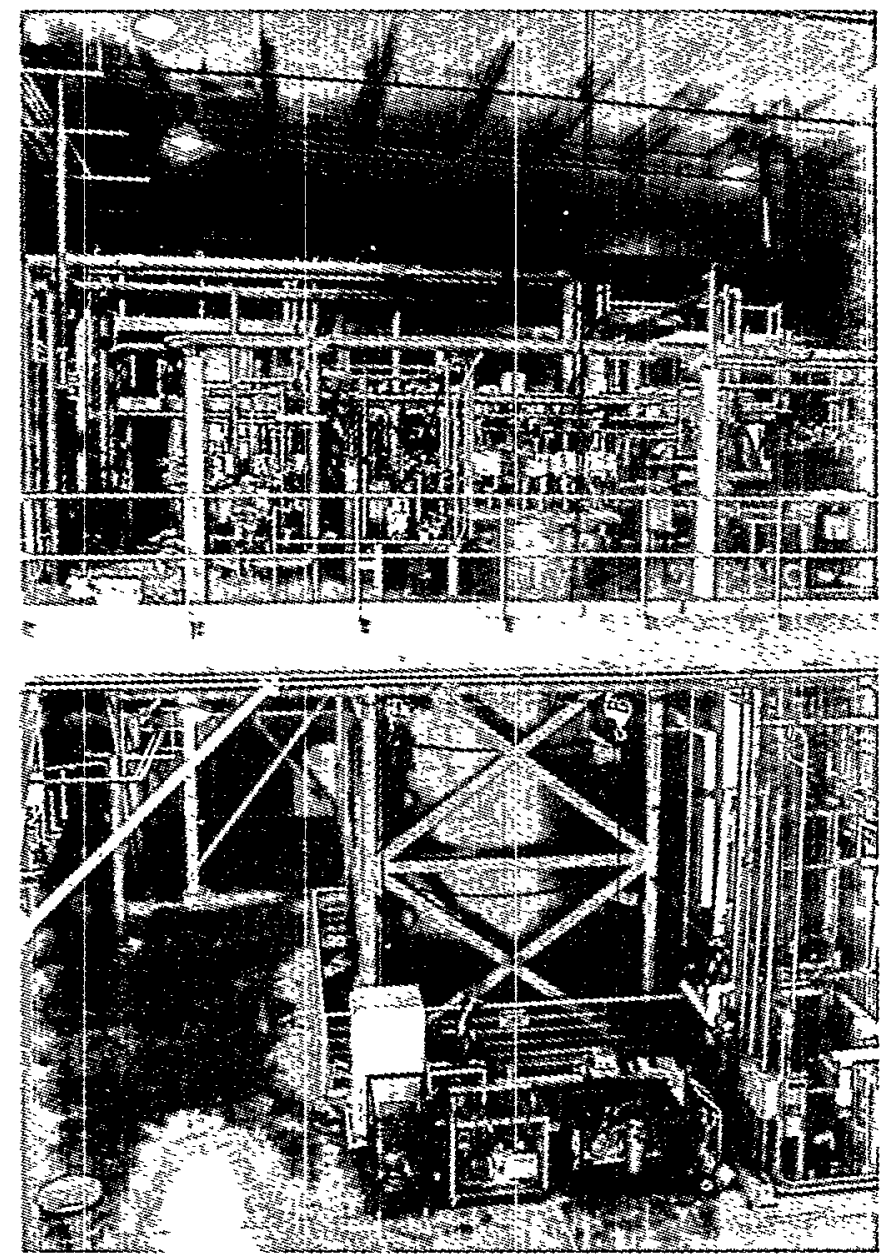

Fig. II-13. The $4 \mathrm{~kW}$ liquid helium refrigerator coldboxes from the SSC before dismantling in Texas. The system was removed in 1996 and placed in storage at Argonne.

\section{d. The Post-Accelerator}

The post-accelerator will utilize the present ATLAS facility. An essential feature of the post-accelerator is to preserve the excellent beam quality currently available at ATLAS for stable beams of any mass up to uranium in the energy range $6-15 \mathrm{MeV}$ per nucleon. The high overall efficiency of the acceleration process will be of the utmost importance. The post-acceleration will involve several steps: the extraction of $1^{+}$beams from the target/ion-source configuration, a high resolution mass separator, a low charge-state injector linac consisting of two $\mathrm{cw}$ RFQ's for $1+$ ions up to mass 240 , and a linac with interdigital structures based on those currently used in the ATLAS positive-ion injector. The schematic layout of the post accelerator is shown in Fig. II-14.

\section{d.1. Ion sources}

The present proposal is based on $1+$ ions, dominantly from the high-pressure helium gas cell and ion guide.

Designing the post-accelerator to work with $1^{+}$ions is therefore highly desirable as it assures maximum flexibility in the choice of ion sources. Furthermore, it enables the implementation of new concepts such as the gas fragment/catcher technique discussed above.

In addition, a test stand has been built at the Dynamitron facility (Fig. II-15) for the development of radioactive beam ion sources and targets based on the neutron-generator 2-step target concept and the 


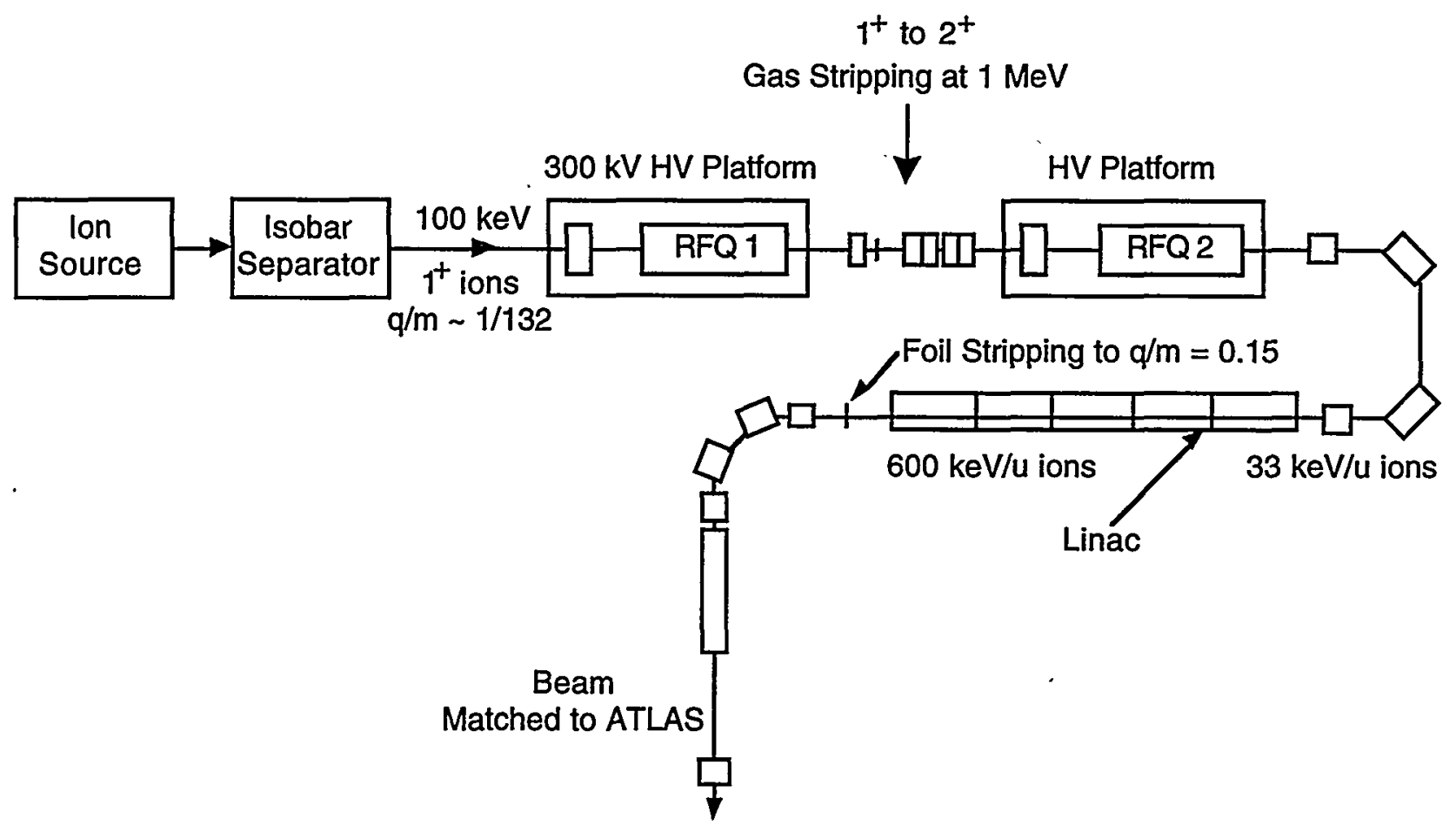

Fig. 14. Pre-acceleration and stripping of $1^{+}$ions for injection into ATLAS.

spallation target concept. Neutrons are produced by the bombardment of light targets with $3.0-5.0 \mathrm{MeV}$ deuteron beams which are then used to produce fission fragments on a ${ }^{238} \mathrm{U}$ target. A surface ionization source was built using some parts of the Brookhaven Tristan ion source. This ion source and the isotope separator built behind it permit us to study release efficiencies and delay times of various isotopes. The Dynamitron is capable of providing $100 \mu \mathrm{A}$ of deuteron beam with energies up to $5 \mathrm{MeV}$. Measurements are underway to address ISOL-related issues.

\section{d.2. Isobar separator}

To obtain beams of a single species, mass separation is essential. In unfavorable cases, the radionuclide of interest may have a much more intense neighboring isobar, requiring a separator with a resolution of 20,000 or higher. Important parameters of such an isobar separator are the emittance acceptance and energy-spread compensation. The present proposal includes one or two large isobar separators with emittance acceptances of $10 \pi \mathrm{mm}-\mathrm{mr}$ at $100 \mathrm{keV}$ and the ability to compensate for an ion source energy spread of at least $10-20 \mathrm{eV}$. The ion-optical scheme of using two magnetic separators with deceleration inbetween, as originally proposed by Wollnik, will be used. Ion optical simulations of such a system to fifth order have been performed using a modified version of the code COSY INFINITY. 


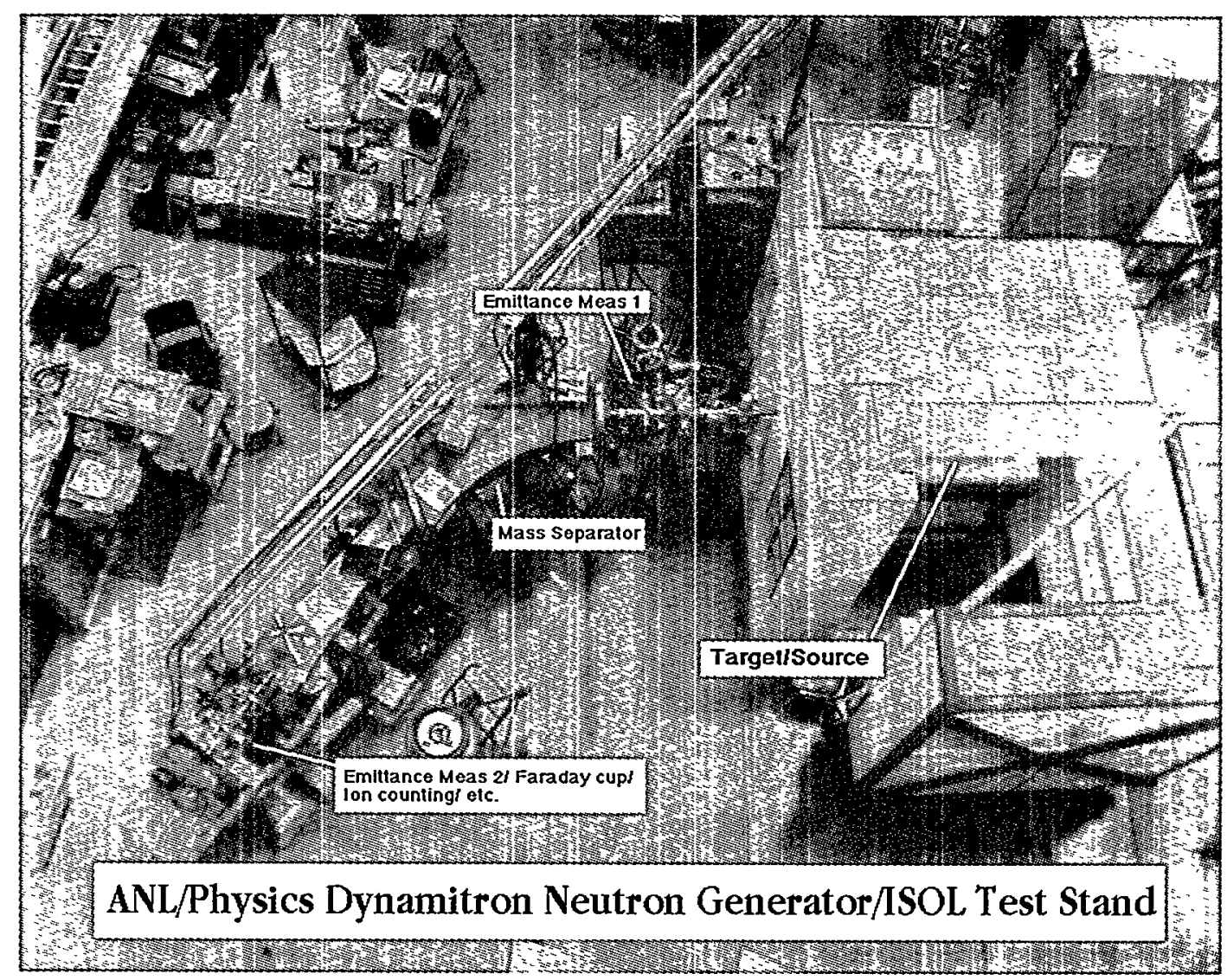

Fig. II-15. Bird's eye view of the experimental setup at the Dynamitron used to measure delay times and release efficiencies with the two-step, neutron generator target. The mass separator is an ISOLDE type general purpose separator built by Danfysik.

\section{d.3. Stripping scheme}

The science program of the Advanced ISOL Facility foresees mass-separated ion beams at three different energy regimes: unaccelerated beams at ion source energies, beams in the $1 \mathrm{MeV}$ per nucleon energy range, and beams accelerated to $6-15 \mathrm{MeV}$ per nucleon. For ions of the lower energies and lower atomic masses, it is possible to deliver the beams directly in the $1^{+}$charge state with very high efficiency. For heavy ions to be accelerated to higher energies, considerations of cost efficiency necessitate the use of one or two stages of ion stripping. The present proposal involves stripping to $2^{+}$or $3^{+}$in a thin helium gas cell for masses above 70 after the initial acceleration to $1 \mathrm{MeV}$ in the first RFQ. A series of measurements with the Dynamitron accelerator of the ANL Physics Division has shown the efficiency for this process to be as high as 40 $50 \%$ for a broad range of heavy ions (see Fig. II-16). For acceleration to energies above $600 \mathrm{keV}$ per nucleon, a foil stripper is used to increase the charge-to-mass ratio to $>0.15$, with a typical efficiency of about $20 \%$. Hence, the overall efficiency of the process varies with both mass and energy depending on the need for: no stripping $(100 \%)$, gas stripping only $(-50 \%)$, foil stripping only $(\sim 20 \%)$, or foil and gas stripping $(\sim 10 \%)$. Various groups around the world are developing alternatives to gas or foil strippers, the so-called charge breeders. A charge breeder concept could be readily incorporated into the present proposal. 


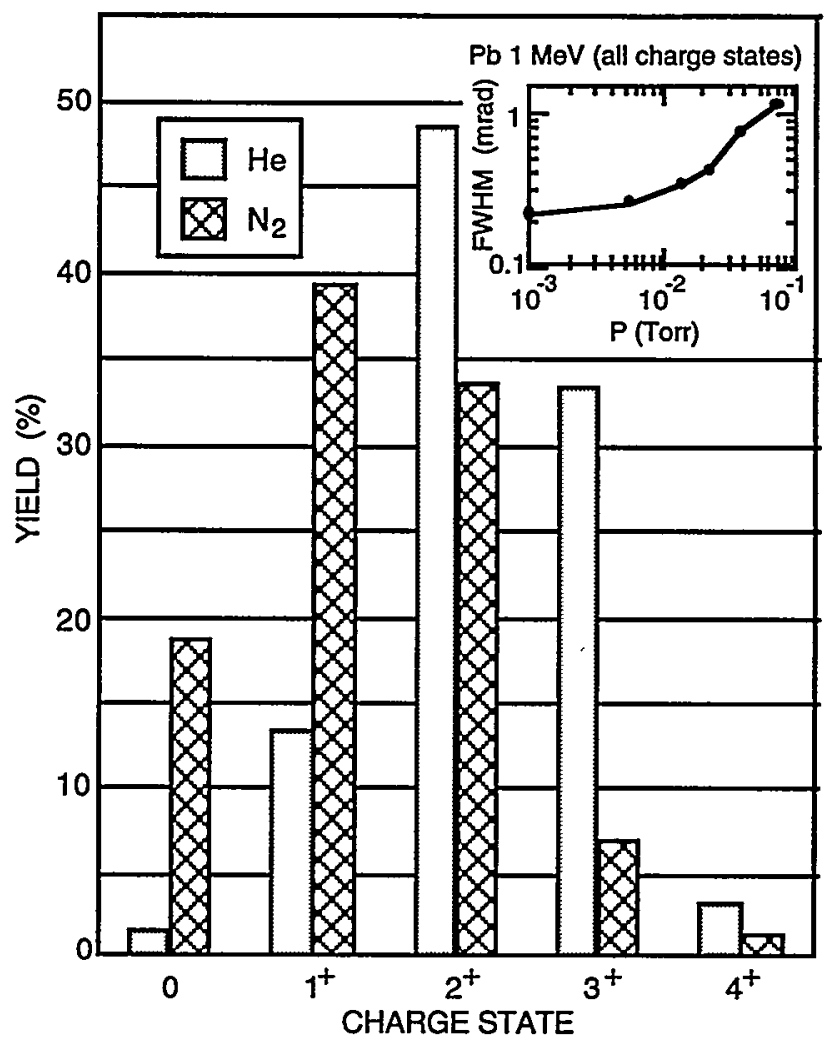

Fig. II-16. Charge state distribution of $1 \mathrm{MeV} I^{+}$Pb ions incident on gas strippers of nitrogen (left bars) and helium (right bars), respectively, measured at the Dynamitron. The figure indicates improvement in the yield of $2^{+}$

$\left(\right.$ or $\left.3^{+}\right)$ions in He gas. The inset shows multiple scattering as a function of the pressure of the helium gas.

\section{d.4. A 12-Mhz CW RFQ for Injecting Low-Charge-State Ions into ATLAS}

Beam tests of a prototype RFQ accelerator are being performed. The RFQ is a $16-\mathrm{mm}$ aperture, $\mathrm{cw}$ device operature at $12 \mathrm{Mhz}$ and is intended for use as the initial element of a preaccelerator system for injecting radioactive ions beams into the existing ATLAS accelerators. Early tests of the RFQ achieved stable operation at a cw intervane voltage as high as $108 \mathrm{keV}$, a voltage well above the design value of $100 \mathrm{kV}$ (see
Fig. II-17). The RFQ was injected with single-charged ions of mass up to 132 , the largest $A / q$ the $R F Q$ was designed to accelerate. Initial beam tests are being performed with $\mathrm{dc}$, unbunched beams of singley-charged ${ }^{132} \mathrm{Xe}$ and ${ }^{84} \mathrm{Kr}$. The output beam time-energy distribution was measured with a $\mathrm{Si}$ detector. The observed energies are in excellent agreement with numerical beam simulations. Tests are planned with a dual-detector system to measure the longitudinal emittance of accelerated beam. 


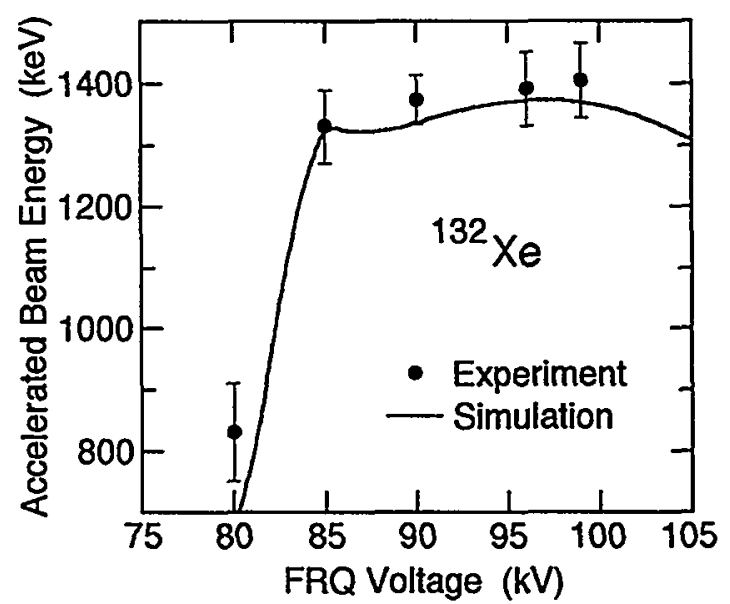

Fig. II-17. ${ }^{132}$ Xe accelerated beam energy as a function of $R F Q$ voltage.

\section{d.5. Superconducting linacs}

Following the room-temperature RFQ accelerator sections described above, all of the radioactive beam acceleration is done with superconducting linear accelerators. The present proposal is to add a high charge-to-mass ratio injector section $(\mathrm{m} / \mathrm{q}<70)$ for the initial acceleration up to $600 \mathrm{keV}$ per nucleon. The superconducting resonators of this section are of the type currently in use for the ATLAS low-velocity injector $(v \geq 0.008 \mathrm{c})$. However, due to the higher mass-to-charge ratio of the new injector, the transverse focusing requirements are more demanding. The present concept is to use high-gradient superconducting quadrupole triplets for focusing in the new injector as opposed to the superconducting solenoid focusing

\section{e. Experimental Areas and Systems}

The present experimental areas and equipment will be part of the planned facility. In addition, an expansion of the experimental area is foreseen that will double the floor space, thus making room for the advanced specialized equipment needed to best utilize the often low intensities of the radioactive beams far from the elements used in the present ATLAS injector. Beams from the high $\mathrm{m} / \mathrm{q}$ injector can be delivered, without further stripping, at energies up to $600 \mathrm{keV} / \mathrm{u}$ to either the intermediate energy experimental areas or transported without further acceleration to the apparatus in the highenergy experimental areas. For acceleration to energies above $600 \mathrm{keV} / \mathrm{u}$, the high $\mathrm{m} / \mathrm{q}$ injector is followed by the foil stripper and a short superconducting matching section. The matching section provides beams at up to $1.2 \mathrm{MeV} / \mathrm{u}$ in the intermediate energy experimental areas or delivers the beam for further acceleration by the present ATLAS linacs. Energies up to the $15 \mathrm{MeV}$ per nucleon range, depending on ion mass, are available.

valley of stability. The requirements for such equipment were discussed at the "Workshop on the Experimental Equipment for an Advanced ISOL Facility", held at Berkeley on July 22-25, 1998, and recommendations were made for the needed experimental equipment. 
f. Facility Layout

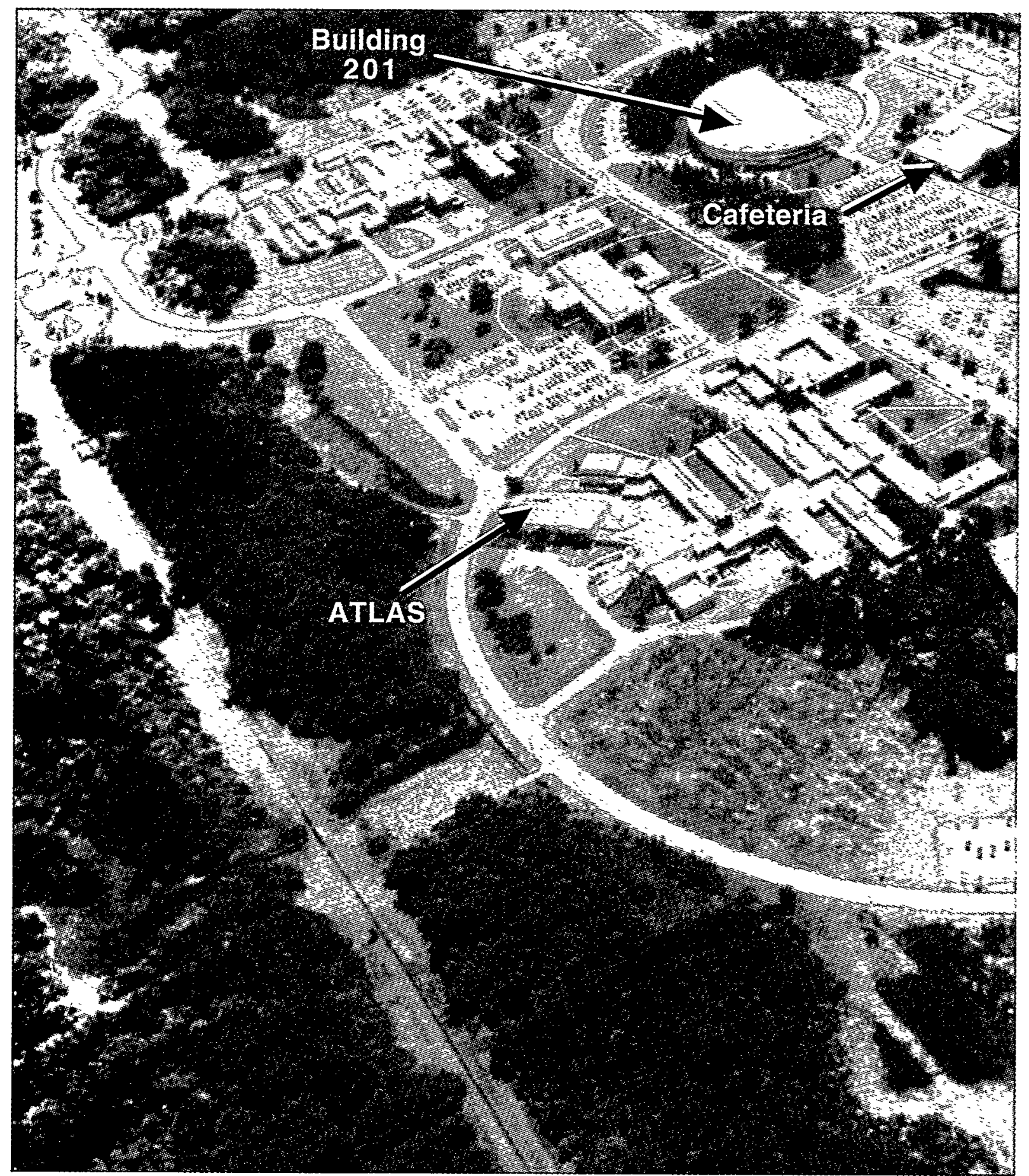

Figure II-18. Aerial view of the site for the proposed Argonne ISOL facility. 


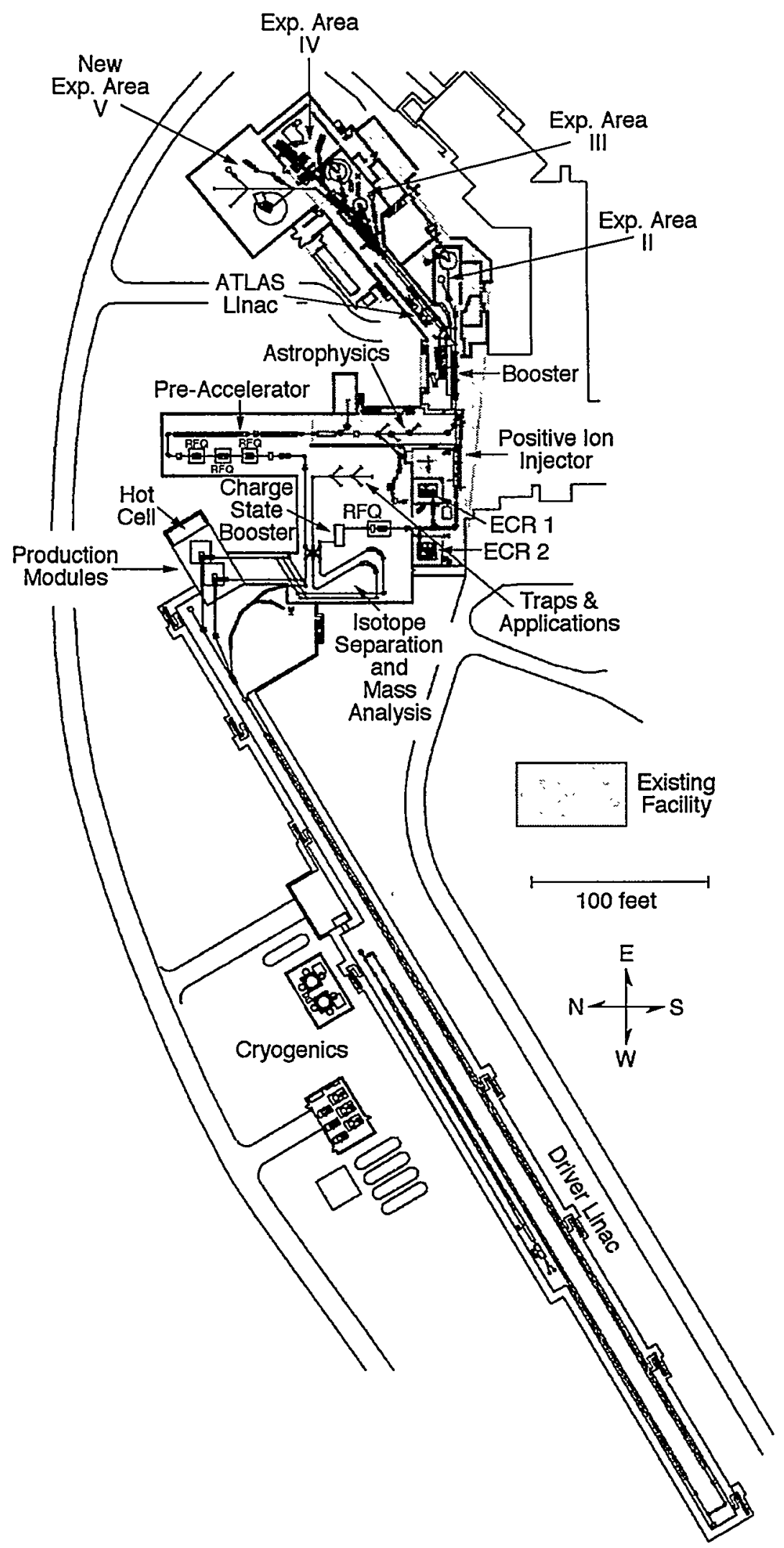

Figure II-19. The technical layout of the facility. 


\section{MEDIUM-ENERGY NUCLEAR PHYSICS RESEARCH}

\section{OVERVIEW}

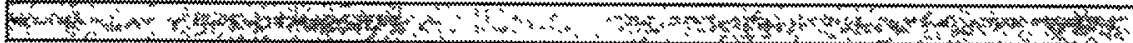

In order to understand how to incorporate the quark-gluon structure of the nucleon into a fundamental description of nuclear forces, the medium-energy research program in the Argonne Physics Division emphasizes the study of processes in nuclei in which interactions with the constituents of the nucleon describe the basic physics. Examining related physics topics at both the quark and hadronic levels in complementary experiments is most revealing of the low-energy structure of the strong interactions. Because energetic leptons provide an accurate, well-understood probe of these phenomena, primary emphasis is placed on the experiments involving electron and muon scattering.

The electron beams of the Thomas Jefferson National Accelerator Facility (TJNAF) are ideally suited for studies of nuclei at hadronic scales and represent one center of the experimental program. Staff members led in the construction of experimental facilities, serve as spokesmen for three experiments, and are actively involved in four others. The group completed construction of the broad-purpose Short Orbit Spectrometer which forms half of the coincidence spectrometer pair that is the base experimental equipment in Hall C. We continue to upgrade the SOS detector package and improve the understanding of the spectrometer optics and acceptance. Argonne led the first experiment to be carried out at TJNAF in FY1996 and have completed parts of four other experiments. In FY1998 a cryogenic helium target was installed in Hall $\mathrm{C}$ and used in measurements of the virtual pion field in light nuclei. We anticipate running an average of two TJNAF experiments a year for the next several years.

The first results from TJNAF build upon previous ANL experiments at Stanford Linear Accelerator Center (SLAC), MIT-Bates and Saclay (ALS). For the first time, forward-backward angle measurements of the (e,e'p) reaction were used to perform longitudinal-transverse separations of the proton spectral function at high momentum transfers. Exclusive deuteron photo-disintegration experiments identified that this reaction obeys quark-counting-rule scaling arguments at large transverse momenta. These measurements were pushed to photon energies of 4.0 GeV at TJNAF and will be extended to higher energies in FY1999. The simplest extension of this work, to coherent pion photo-production on the deuteron, did not see evidence of the quark counting rule behavior. Measurements of kaon production on hydrogen and deuterium provide important information on the basic strangeness production mechanisms, the poorly-known low-energy hyperon- 
nucleon interaction and the electromagnetic form factor of the $\mathrm{K}^{+}$. In the future these experiments will measure hypernuclear states on few-body nuclei as the first step in a hypernuclear physics program at TJNAF. They will also search for a bound $\Sigma$-hypernuclear state that was recently seen in hadronic strangeness exchange reactions. Pion production measurements in FY1998 on hydrogen, deuterium, ${ }^{3} \mathrm{He}$, and ${ }^{4} \mathrm{He}$ will determine the charge form factor of the pion and measure the change in the pion field in the nuclear medium. Since the pion contains valence antiquarks, these measurements complement our high-energy Drell-Yan measurements of the antiquark distributions in nucleons and nuclei.

HERMES, a broadly based North American-European collaboration is studying the spin structure of the nucleon using internal polarized targets in the HERA storage ring at DESY. Deep inelastic scattering was measured with polarized electrons on polarized hydrogen and ${ }^{3} \mathrm{He}$. Argonne concentrated on the hadron particle identification of HERMES, a unique capability compared to other spin structure experiments. In 1998, Argonne led a major upgrade of the spectrometer to replace threshold Cerenkov counters with a dual-radiator ring-imaging Cerenkov counter (RICH) to provide complete hadron identification in the experiment. This will allow HERMES to make decisive measurements of the flavor dependence of the spin distributions and to begin measurements of the spin dependence of the glue. The HERMES experiment is also making significant advances in unpolarized deepinelastic scattering physics with its coincident hadron detection. Argonne scientists extended the physics program in studies of exclusive vector meson production in polarized-beam-polarized-target measurements and also measurements on nuclear targets. Clear evidence is seen on the nuclear targets for the effect of the coherence scale in the production mechanism.

Measurements of high-mass virtual photon production in high-energy protoninduced reactions determined the flavor dependence of the sea of antiquarks in the nucleon. These measurements give insight into the origin of the nucleon sea. In the same experiment, the nuclear dependence of the Drell-Yan process and the nuclear dependence of the production of heavy quark resonances such as the $\mathrm{J} / \Psi$ and $Y$ were determined. These results provide constraints on the gluon distributions of nucleons and nuclei and a significant baseline for attempts to use heavy vector meson production as a signal of the formation of the quark-gluon plasma in relativistic heavy-ion experiments. A new initiative is underway to continue the Drell-Yan measurement with higher luminosity at the FNAL main injector.

The technology of laser atom traps provides a unique environment for the study of nuclear and atomic systems and represents a new thrust for the group. Initially the efforts focus on developing high-efficiency and high-sensitivity trapping techniques for the isotope analysis of noble gases. Single atom sensitivity in a noble atom trap was demonstrated in FY1998. This technique provides a new approach to such diverse problems as dating old ground water or mapping out the atmospheric concentration of fission products. A longer-term goal is to use trapped radon atoms to search for time-reversal symmetry violation in electric dipole moment measurements. Related atomic physics techniques are being used to measure the nuclear moments of unstable cesium isotopes in an ISOL source test facility. 


\section{A. SUBNUCLEONIC EFFECTS IN NUCLEI}

\section{a.1. HERMES, Measurements of Spin-Structure Functions and Semi-Inclusive Asymmetries for the Proton and Neutron at HERA (J. Arrington, H. E. Jackson, T. G. O'Neill, D. H. Potterveld, D. De Schepper, and collaborators at 33 other institutions)}

HERMES is an international collaboration that is carrying out a program of studies of the spin structure of the nucleon at the DESY Laboratory in Hamburg, Germany. The collaboration currently consists of approximately 30 institutions from North America and Europe including several institutions from the CIS. HERMES is located in the East Hall of the $6.4-\mathrm{km}$ circumference HERA positron-proton collider. The experiment is arranged so that the $27.5-\mathrm{GeV}$ longitudinally polarized positron beam interacts with polarized internal gas targets (the proton beam is not used). The HERMES spectrometer was designed to measure the dependence of the $\left(e, e^{\prime}\right)$ deep inelastic scattering cross section on the relative orientation of the beam and target spins. The beam is self-polarized transverse to the beam direction by the Sokolov-Ternov effect. Spin rotators located at the entrance and exit of the East straight section precess the spin direction from vertical to longitudinal at the target position and, following the target, back to the vertical direction. The polarization of the beam with the rotators in place was measured for both positrons and electrons. Polarizations of about $50 \%$ are stable and reproducible, and are measured using transverse and longitudinal Compton backscattering polarimeters with systematic uncertainties of better than $\pm 5 \%$. The beam polarization develops in about 25 minutes. As a result of the ease with which the direction of the target polarization can be changed, it will be possible to study parallel and perpendicular polarization asymmetries for all targets.

Data were taken on a polarized ${ }^{3} \mathrm{He}$ target in 1995 , on a polarized ${ }^{1} \mathrm{H}$ target in 1996 and 1997, and on a polarized $2_{\mathrm{H}}$ target in 1998. The HERMES target consists of an open-ended thin-walled storage cell through which the circulating positron beam of the HERA accelerator passes. A magnetic holding field provides a quantization axis for the target polarization. The target densities are about $7.5(5) \times 10^{13}$ atoms $/ \mathrm{cm}^{2}$ for hydrogen (deuterium) and $3.5 \times 10^{14}$ atoms $/ \mathrm{cm}^{2}$ for ${ }^{3} \mathrm{He}$. Typical polarizations of the target are $90 \%$ (50\%) for the hydrogen/deuterium $\left({ }^{3} \mathrm{He}\right)$ sources, and are measured to better than $\pm 5 \%$ with polarimeters. The target spin orientation was reversed frequently (every 10 minutes in 1995, every 2 minutes in 1996-1998) to minimize systematic errors from experimental timedependences. Luminosities are in the range of 4-30 $\times$ $10^{31}$ nucleons $\mathrm{cm}^{-2} \mathrm{sec}^{-1}$. The gases are pure so that there is no target dilution. The extremely small target thickness and absence of end windows minimize external radiative corrections. Unpolarized gases of hydrogen, deuterium, nitrogen, and krypton, with thicknesses of around $10^{15}$ atoms $/ \mathrm{cm}^{2}$, are also used for data taking. In these cases the maximum target thickness is determined by the impact on the stored positron lifetime.

The HERMES spectrometer, shown in Fig. III-1, was designed to detect scattered positrons and hadrons from deep inelastic scattering. The spectrometer consists of two identical halves above and below the positron ring plane. This provides two independent measurements of observables and thus a cross check on systematic uncertainties. A dipole magnet with a bending strength of $1.3 \mathrm{~T} \cdot \mathrm{m}$ provides background rejection and momentum analysis of charged particles. A fly's eye lead glass calorimeter and a system of segmented hodoscopes provide a trigger on deep inelastic scattered positrons. A transition radiation detector provides strong off-line discrimination against hadrons. Tracking chambers before the magnet, in the magnetic field, and behind the magnet provide charged particle tracking. A pair of threshold gas Cerenkov counters were used for identification of pions during the 1995-1997 runs. In 1998 , the Cerenkov counter was upgraded to a ring imaging configuration (see section a.3). Relative luminosity is monitored with $\approx 1 \%$ precision by the coincident detection, in symmetrically placed bismuth tungstenate calorimeters, of the positron-electron pair from symmetric Bhabha scattering off the target atomic electrons. The target empty luminosity rate is negligible. A collimator system prevents the large flux of synchrotron photons from impinging on the target

IJ. Ashman et al., Zeit. Phys. C 52, 1 (1991)

2L.S. Osborne et al., Phys. Rev. Lett. 40, 1624 (1978)

${ }^{3}$ A. Bialas and M. Gyulassy, Nucl. Phys. B 291, 793 (1987) 
cell. The angular acceptance of the experiment is $40<$ $\theta<220 \mathrm{mrad}$. The kinematic range accessible is 0.02 $<\mathrm{x}<0.8$ and $0.2<\mathrm{Q}^{2}<20(\mathrm{GeV} / \mathrm{c})^{2}$. Argonne provided the Cerenkov counter used for particle identification, led the RICH upgrade effort, and developed the drifilm coating technique for the ultrathin target cell required for this experiment.

Analysis of the inclusive (e,e') data taken with the polarized ${ }^{3} \mathrm{He}$ and $\mathrm{H}$ targets was performed to extract the neutron and proton spin structure functions $\mathrm{g}_{1}^{\mathrm{n}}(\mathrm{x})$ and $\mathrm{g}_{1}^{\mathrm{F}}(\mathrm{x})$. In the quark parton model $\mathrm{g}_{1}(\mathrm{x})$ is equal to the total contribution to the nucleon spin of quarks with momentum fraction $x$, weighted by the square of the quark charge. The results are in good agreement with those reported from SLAC experiments E-142, E-143, and E-154, and represent the only high-precision confirmation of the SLAC results. The confirmation was especially important because all previous spin structure measurements suffered from large corrections for target dilution by other atomic species and by target end caps. The HERMES measurement was performed with a pure atomic species and no end caps, and so was not subject to these systematic uncertainties.

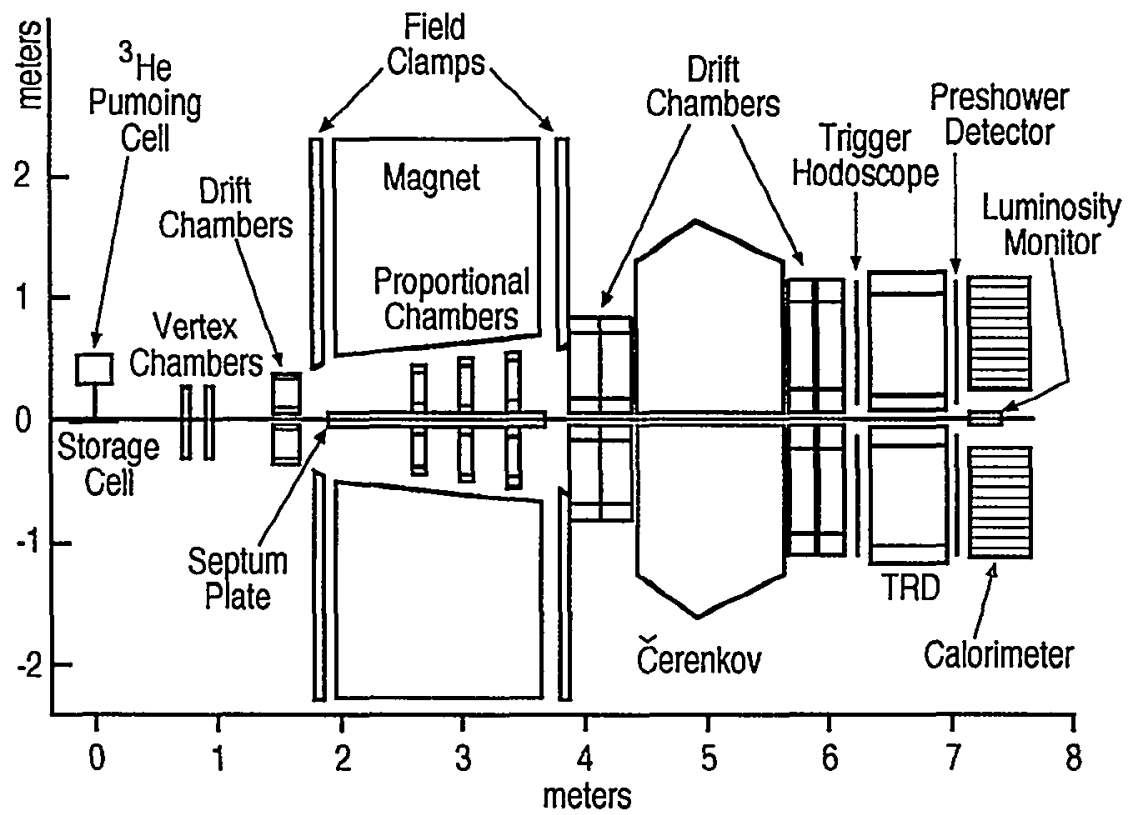

Fig. III-1. Schematic diagram of the experimental apparatus (side view).

Much more detailed information about the spin content of the nucleon can be obtained from the spin-dependence of $\left(e, e^{\prime} h^{\ddagger}\right)$ semi-inclusive deep inelastic scattering (SIDIS). (Here $\mathrm{h}^{ \pm}$refers to a positive or negative hadron detected in coincidence with the scattered positron.) Under the factorization approximation, which relates the detected hadron to the struck quark, the asymmetries for different hadron species can be combined to extract the contribution of each quark flavor (i.e., up, down, or strange) to the nucleon spin, and to distinguish between the contributions of valence and sea quarks. The HERMES data provide the most accurate measurement of the SIDIS (e, $\mathrm{e}^{\prime} \mathrm{h} \mathrm{f}^{\ddagger}$ ) asymmetry at $0.03<x<0.3$. Thanks to the Cerenkov counter provided by the ANL MEP group, HERMES is the first experiment to measure the SIDIS asymmetry on a specific hadron species, namely the pion. The ANLdirected upgrade of the Cerenkov to Ring Imaging Configuration will allow the first measurement of the asymmetry for SIDIS kaon production as well, providing sensitivity to the strange quark polarization.

HERMES is also breaking new ground in SIDIS physics on nuclear targets such as nitrogen. Attenuation by the nuclear medium provides sensitivity to the lifetimes and interactions of virtual quantum states mediating the SIDIS reaction. For example the nuclear transparency for exclusive $\rho^{\circ}$ electroproduction, 
shown in section a.2, is senstitive to the properties of virtual quark-antiquark fluctuations of a virtual photon. Similarly, the production of SIDIS pions can be suppressed by interactions of the struck quark and of the pion with the nuclear medium. This is tested by comparing, for different targets, the ratio of the SIDIS $\left(\mathrm{e}, \mathrm{e}^{\prime} \pi^{ \pm}\right)$events to the inclusive DIS (e,e') events. Figure III- 2 shows this ratio for nitrogen, divided by the ratio for deuterium as a function of the laboratory virtual photon energy $v$. At low $v$, the pion forms inside the nucleus and is strongly absorbed, leading to a ratio significantly less than unity. At high $v$, time dilation ensures that the struck quark fragments into the pion outside of the nucleus and the ratio is closer to one. This indicates that the struck quark interacts less strongly with the nuclear medium than does the final pion. In addition the $v$ value at which the transition between these two regimes occurs indicates the time scale necessary for the struck quark to form the pion. As the figure shows, the HERMES data have greatly improved our knowledge of this transition region.

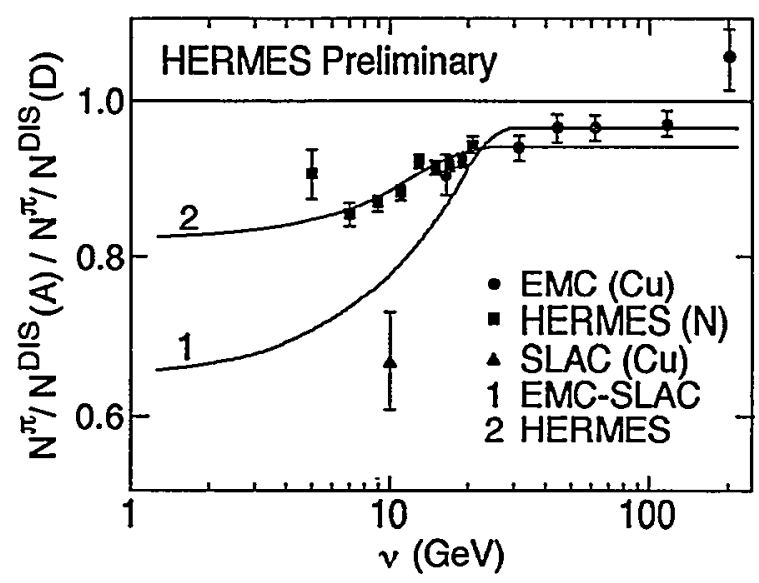

Fig. III-2. World data for nuclear to deuterium pion attenuation ratio as a function of virtual photon energy, v. The filled squares are the preliminary HERMES nitrogen data. The filled circles and triangle are the $E M C^{1}$ and $S L A C^{2}$ measurements on copper targets. The solid lines are fits to a LUND fragmentation model ${ }^{3}$ with similar parameters for nitrogen and copper.

\section{a.2. Nuclear- and Spin-dependent Cross Section for Exclusive $\rho^{0}$ Electroproduction (T. G. O'Neill, J. Arrington, D. De Schepper, H. E. Jackson, D. H. Potterveld, and collaborators at 33 other institutions)}

A signal for the exclusive electroproduction of the neutral $\rho$ meson was isolated as a subset of the standard HERMES deep inelastic scattering data. In this reaction, the positron emits a virtual photon which then fluctuates into a quark-antiquark pair of lifetime $1_{c}$. A diffractive interaction with the target converts the quarkantiquark pair into the detected $\rho^{0}$. The $\rho^{0}$ meson, which decays into 2 pions, manifests itself in a peak at $\mathrm{M}_{\rho}=0.77 \mathrm{GeV}$ in the invariant mass of the 2-pion system. Energy and momentum conservation can be used to ensure that there are no additional particles in the final state, resulting in a clean signal for the exclusive $\left(e, e^{\prime} \rho^{0}\right)$ reaction. Figure III-3 exhibits the HERMES measurement of the nuclear transparency for $\rho^{0}$ production from ${ }^{2} \mathrm{H},{ }^{3} \mathrm{He}$, and nitrogen targets. The transparency measures the reduction in the reaction cross section by interactions of the reaction participants with the nuclear medium. At small $l_{c}$ the transparency

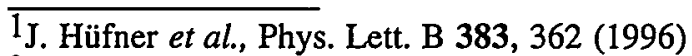

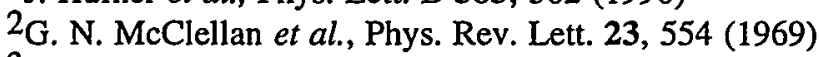

${ }^{3}$ M. R. Adams et al., Phys. Rev. Lett. 74, 1525 (1995) reflects the interactions of the outgoing $\rho^{0}$ meson with the nuclear medium. At large $1_{c}$, the transparency is further reduced by the interactions of the virtual photon's quark-antiquark fluctuations. The data agree with the prediction of the Glauber multiple scattering theory, ${ }^{1}$ confirming expectations for the lifetime of the quark-antiquark fluctuations and indicating that the interactions with the nuclear medium are approximately like those of a free $\rho^{0}$ meson. Publications are also being prepared on the unpolarized $\rho^{0}$ production cross section and on the $\rho^{\circ} \rightarrow \pi^{+} \pi^{-}$decay angular distributions. In addition, the HERMES data will be used to make the world's first determination of the dependence of $\rho^{\mathbf{o}}$ production on target and beam spin orientations. The HERMES $\rho^{\circ}$ production data should shed new light on the details of the $\rho^{0}$ production reaction mechanism. 

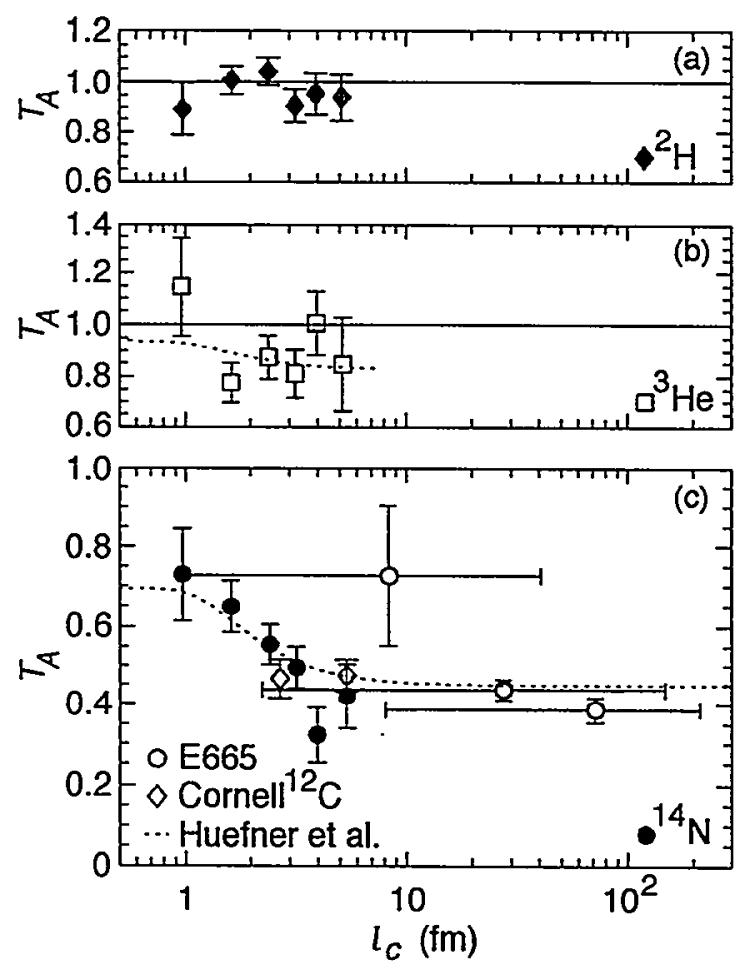

Fig. III-3. Nuclear transparency $T_{A}$ as a function of $l_{c}$ for a) ${ }^{2} \mathrm{H}$ (filled diamond), b) ${ }^{3} \mathrm{He}$ (open square), and c) ${ }^{14} \mathrm{~N}$ (filled circle) targets. The error bars include statistical and point-to-point systematic uncertainties added in quadrature. The respective $2.5 \%$, $5.5 \%$, and $5.9 \%$ systematic uncertainties in the overall normalizations of $T_{2_{H}}, T_{3_{H e}}$, and $T_{14_{N}}$ are not shown, since they do not influence the significance of the $l_{c}$ dependencies. Panel $(c)$ includes comparisons with previous experiments with photon (open diamonds) ${ }^{2}$ and muon (open circle) ${ }^{3}$ beams. The dashed curves are the Glauber calculation of Hüfner et al. for ${ }^{3} \mathrm{He}$ and ${ }^{14} \mathrm{~N} .{ }^{1}$

\section{a.3. A Dual Radiator Ring Imaging Cerenkov Counter for HERMES}

(H. E. Jackson, K. G. Bailey, D. M. De Schepper, T. G. O'Neill, D. H. Potterveld, J.-O. Hansen,* R. S. Kowalczyk, $\dagger$ and the HERMES collaboration)

The HERMES experiment emphasizes studies of the spin structure of the nucleon through the measurement of pion, kaon, and nucleon semi-inclusive spin asymmetries. If the hadrons can be unambiguously identified, the resulting data can be used to tag leading hadrons, thus providing a means of direct study of the flavor dependence of polarized structure functions and of the sea polarization. If hadrons from the strange particle decays can be identified, low background measurements of $\Lambda$ decay will provide a probe of polarization transfer in $\Lambda$ production. If kaons can be reliably identified, clean measurements of open charm production by means of detection of the charmed meson decay becomes an attractive option for probing processes involving gluons.

In order to provide hadron identification over the full acceptance of HERMES, the existing threshold Cerenkov counters in the HERMES spectrometer were replaced by a dual-radiator Ring Imaging Cerenkov Counter (RICH) which employs a new technology. The detector was designed and built by a collaboration of 8 institutions, led by Argonne (Argonne, Bari, Caltech, Frascati, Gent, Rome, Tokyo and Zeuthen), in 12 months from concept approval to installation. The installation of the RICH in the spectrometer of the HERMES experiment (DESY, Hamburg) was completed on May 22, 1998. It is the first use of recently developed clear, hydrophobic aerogel as a Cerenkov radiator in a RICH system. In order to identify almost all of the pions, kaons, and protons in the HERMES acceptance, the RICH is filled with a second radiator $\left(\mathrm{C}_{4} \mathrm{~F}_{10}\right.$ gas) which in combination with the aerogel, provides complete hadron identification from $2 \mathrm{GeV} / \mathrm{c}$ to about $16 \mathrm{GeV} / \mathrm{c}$. Rings from the aerogel $(n=1.03)$ provide identification up to about 9 $\mathrm{GeV} / \mathrm{c}$, while rings from the gas $(n=1.0015)$ are used for higher momenta (see Fig. III-4). The RICH consists of a pair of identical counters, one in the upper half and a second in the lower half of the HERMES spectrometer. Each photon detector is an array of 1934

*TJNAF, †Jet Propulsion Lab 


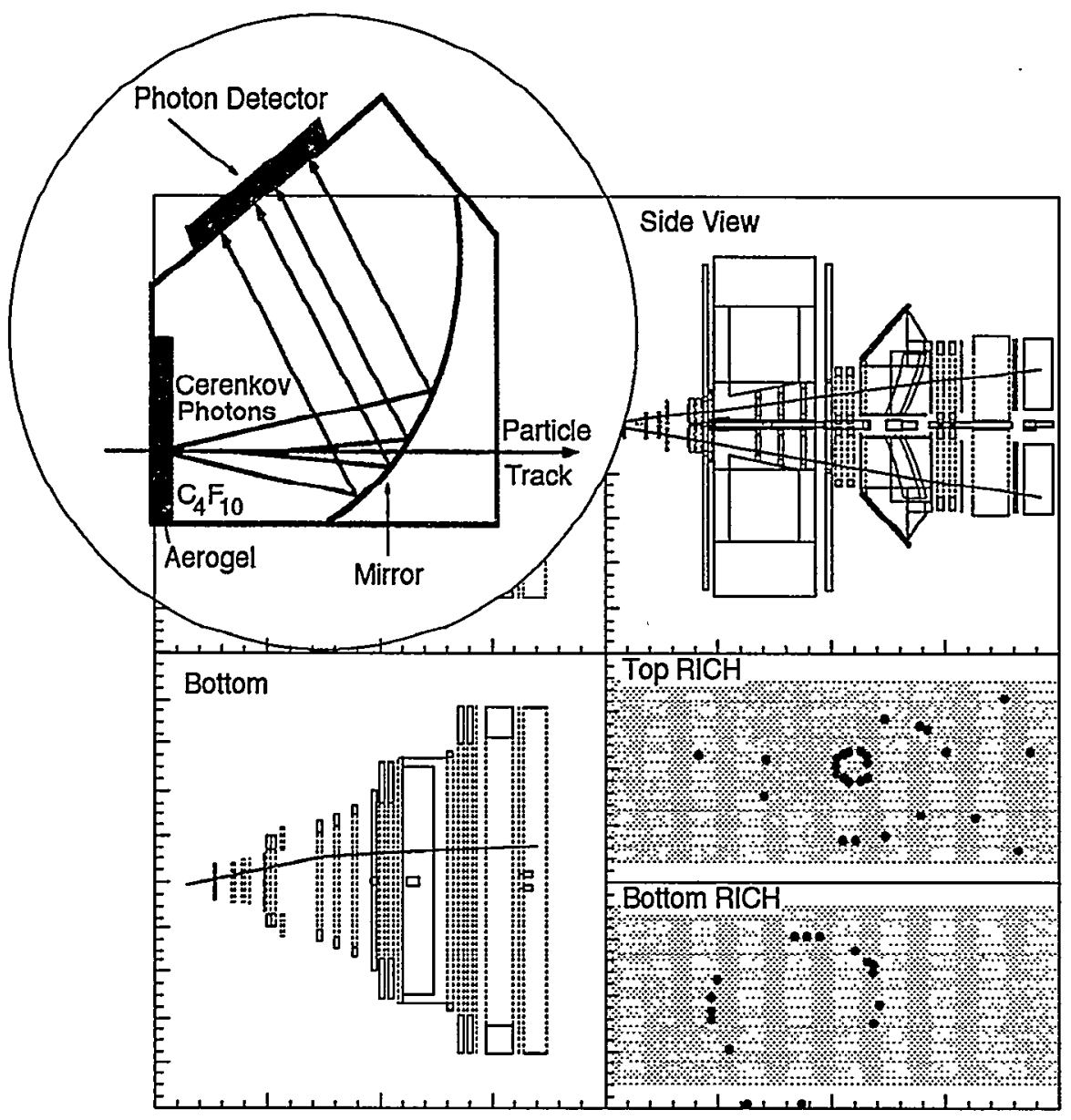

Fig. III-4. The hit pattern in the RICH photon detector as viewed in the HERMES event display. The hit pattern in the top RICH shows an inner ring of hits from photons generated by the gas as well as hits on a larger radius corresponding to photons from aerogel. The hits in the bottom RICH result from photons generated only in the aerogel by a particle whose momentum is below the threshold for Cerenkov light from the gas. A schematic view of the top RICH is shown in the insert.

3/4" photomultiplier tubes arranged in a planar array of honeycomb packing. For a fully relativistic particle, a typical ring pattern observed in the photon detector consists of a small inner ring generated by photons from the gas and a larger concentric ring of photon hits generated by photons from the aerogel. With this system, $99 \%$ of the hadrons can be unam- biguously identified, making HERMES unique among contemporary experimental studies of the spin structure of the nucleon. Now HERMES will be able to exploit fully, flavor tagging in studies of spin structure using deep-inelastic lepton scattering. In the initial operation, in terms of ring resolution, the system already performs. in reasonable proximity to the design values. The resolution appears to be limited by imperfections in the geometry of the aerogel tiles. 


\section{a.4. The Energy Dependence of Nucleon Propagation in Nuclei as Measured in} the (e,e'p) Reaction (D. F. Geesaman, J. Arrington, K. Bailey, W. J. Cummings, D. DeSchepper, H. E. Jackson, C. Jones, S. Kaufman, T, O'Neill, D. Potterveld, J. Reinhold, J. P. Schiffer, B. Zeidman, P. Bosted,* A. Lung, $\dagger$ D. Abbott, $\ddagger$ R. Carlini, $\ddagger$ J. Dunne, $\ddagger$ R. Ent,$\ddagger$ J.-O. Hansen $\ddagger$ D. Mack $\ddagger$ D. Meekins,$\ddagger$ J. Mitchell $\ddagger$ S. Wood $\ddagger$

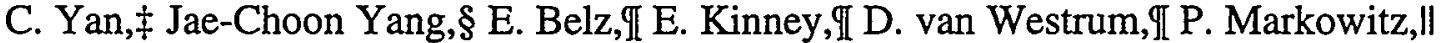
K. A. Assamagan, ** O. K. Baker,**l K. Beard,** J. Cha,** T. Eden,** P. Gueye,** W. Hinton, ${ }^{* *}$ C. Keppel, $* *$ R. Madey, ${ }^{* *}$ G. Niculescu, $* *$ I. Niculescu, ${ }^{* *}$ L. Tang, $* *$ Wooyong Kim, $\uparrow \dagger$ C. Bochna, $\ddagger \ddagger$ H. Gao, $\ddagger \ddagger$ R. Holt, $\neq \ddagger$ M. Miller, $\neq \ddagger$ A. Nathan, $\neq \ddagger$

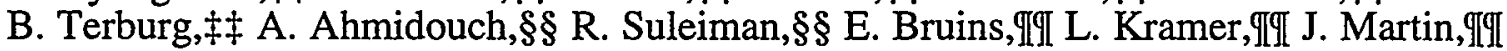
A. Mateos, IIT R. Milner,IIT W. Kurchinetz, ITI C. Williamson, IIT W. Zhao, ITI E. Beise,III H. Breuer, I| || N. Chant, || || F. Duncan, || || J. J. Kelly, || || R. Mohring, || || M. Khandaker,*** K. McFarlane, $* * *$ C. Salgado, *** S. Beedoe, $\dagger+\dagger$ S. Dangoulian, $\nmid \dagger \dagger$ C. Jackson, $\uparrow \dagger \dagger$

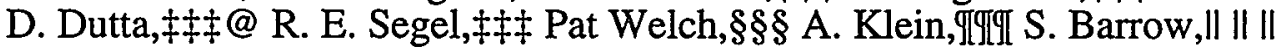
D. Beatty, || || || H. T. Fortune, || || || D. Koltenuk, || || || W. Lorenzon, || || || J. Yu, || || || V. Frolov, **** J. Pricel**** P. Stoler,**** R. Gilman, $\nmid \dagger \dagger \dagger$ J.-E.Ducret,

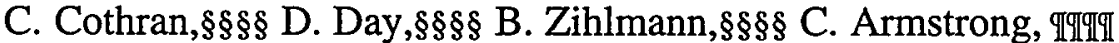

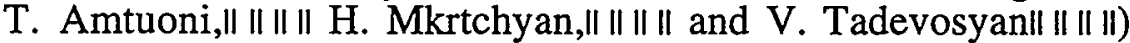

ANL led the first experiment to be carried out at TJNAF in 1995-1996. This experiment built upon earlier ANL work at MIT and SLAC using the (e,e'p) reaction to study the propagation of $0.35-3.3 \mathrm{GeV}$ protons through nuclear material and the reaction mechanism in the quasifree region. The Hall $\mathrm{C}$ collaboration selected this experiment as one of the two commissioning experiments. Electrons were detected in the High Momentum Spectrometer and protons were detected in the Short Orbit Spectrometer except at the highest $Q^{2}$ setting where the roles of the spectrometers were reversed. The experiment utilized TINAF beams of $0.845,1.645,2.445$, and $3.245 \mathrm{GeV}$ with up to $50 \mu \mathrm{A}$ intensity on targets of $\mathrm{C}, \mathrm{Fe}$ and $\mathrm{Au}$. Hydrogen targets with used to check the normalization at each kinematic setting. Full commissioning studies of each spectrometer were performed to calibrate this experiment and to serve as a baseline for future experiments.

Measurements were made at $\mathrm{Q}^{2}$ values of $0.64,1.3,1.8$ and $3.3(\mathrm{GeV} / \mathrm{c})^{2}$ corresponding to average proton kinetic energies of $0.35,0.70,0.97$ and $1.8 \mathrm{GeV}$ to span the threshold for pion production in $p$-p collisions where the nature of the p-p cross section changes from dominantly elastic to dominantly inelastic. At $\mathrm{Q}^{2}$ of 0.64 and 1.8 data were taken at two values of the virtual photon polarization to examine the separate contributions of longitudinal and transverse photon exchange. In all aspects the experimental apparatus performed as expected. The statistics, kinematic coverage and experimental precision significantly exceed those of previous measurements. This data set provides precise measurements of nuclear transparency as well as a broad survey of nuclear spectral functions from recoil momentum of 0 to $300 \mathrm{MeV}$ and missing energy of 0 to $150 \mathrm{MeV}$.

The measurements of the average nuclear transparency were published in 1998. Radiatively corrected spectral functions were extracted for each target and momentum transfer. These spectral functions suggest that the widths of deeply bound hole states in Fe and Au may be somewhat larger than were expected. Figure III-5 shows the results of the separation of the longitudinal and transverse spectral functions on carbon for missing momenta from 0 to $80 \mathrm{MeV}$ at $\mathrm{Q}^{2}=\left(\mathrm{GeV} / \mathrm{c}^{2}\right)$ and previous results 1 at lower momentum transfer. In all cases the longitudinal and transverse spectral

*American University; †Caltech; ¥TJNAF; §Chungnam National University, Taejon, Korea; qUniversity of

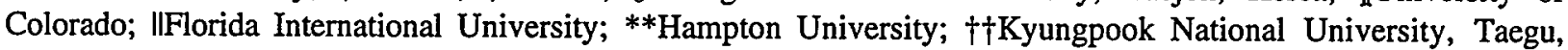
S. Korea; ł $\ddagger$ University of Illinois, Urbana; §§Kent State University; IIIMTT; II IIUniversity of Maryland; ***Norfolk State University; $\dagger \dagger \dagger$ North Carolina A\&T University; $\$$ Northwestern University; $\S \S$ Oregon State University; Tपप्OOld Dominion University; II II IIUniversity of Pennsylvania; ${ }^{* * *}$ Rensselaer Polytechnic Institute;

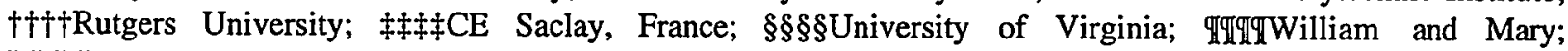
II II II IIYerevan Physics Institute, Armenia; @ This experiment will form the basis for the Ph.D. theses of these students

1P. E. Ulmer et al. Phys. Rev. Lett. 59, 2259 (1987) 
functions of the p-shell knockout strength are consistent. However, there is a substantial excess of transverse strength in the missing energy region from $30-60 \mathrm{MeV}$. At $\mathrm{Q}^{2}=1.8(\mathrm{GeV} / \mathrm{c})^{2}$, it is difficult to extract the longitudinal strength reliably, but the transverse spectral function is not consistent with the longitudinal spectral function measured at $Q^{2}=0.64$ $(\mathrm{GeV} / \mathrm{c})^{2}$ as would be expected from a single particle knockout mechanism. This suggests that the $(e, e$ 'p) reaction mechanism is more complicated than expected at the lower momentum transfers, and based on the large excess of transverse strength, it is likely that meson exchange contributions are quite important.

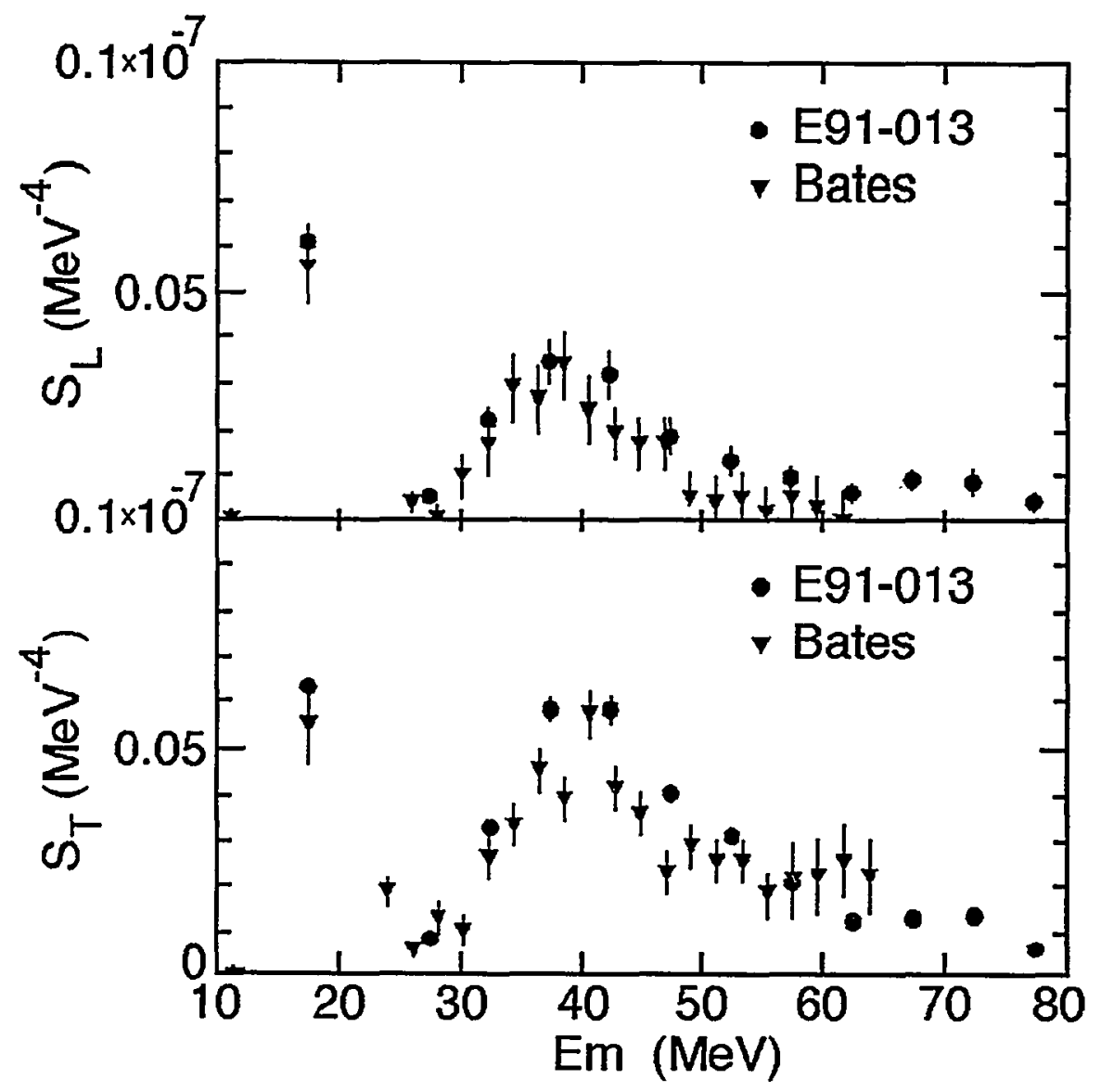

Fig. III-5. Longitudinal $\left(S_{L}\right)$ and transverse $\left(S_{T}\right)$ spectral functions for ${ }^{12} \mathrm{C}$ at $Q^{2}$ of 0.64 (circles) (GeV/c) ${ }^{2}$ integrated in missing momentum from 0 to $80 \mathrm{MeV} / \mathrm{c}$ along with results from a previous experiment ${ }^{1}$ at 0.14 $(G e V / c)^{2}$. The lowest $E_{m}$ point is an average over the $p$ shell region. 
a.5. Electroproduction of Kaons and Light Hypernuclei (J. Arrington, K. Bailey, H. Gao, D. F. Geesaman, H. E. Jackson, T. G. O'Neill, D. Potterveld, J. Reinhold, J. P. Schiffer, B. Zeidman, and E91-016 Collaboration)

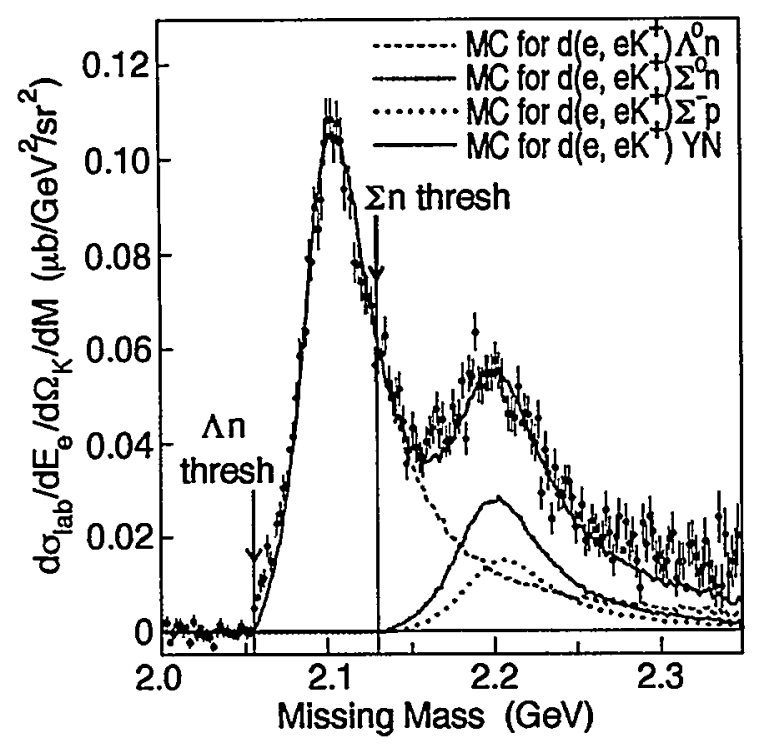

Fig. III-6. Missing mass spectrum for $D\left(e, e^{\prime} K^{+}\right) Y N$, where $Y$ is a hyperon and $N$ is the spectator nucleon. $E_{e}=3.245 \mathrm{GeV}$ and $1.5^{\circ}$ is the central angle of the SOS spectrometer relative to the virtual photon. The curves represent Monte-Carlo calculations that include spectrometer responses, production cross sections, and radiative corrections. The $\Lambda$ and $\Sigma^{\circ}$ peaks at 2.1 and $2.2 \mathrm{GeV}$ are normalized to proton data at the same kinematic conditions. The sum of the $\Sigma$ peak at $2.2 \mathrm{GeV}\left(\sim 1 / 2 \Sigma^{\circ}\right), \Sigma^{\circ}$, and $\Lambda$ peaks results in the line that matches most of the data.

In TJNAF experiment E91-016 "Electroproduction of Kaons and Light Hypernuclei" quasifree electroproduction of $\mathrm{K}^{+}$on deuterium and hydrogen was measured at incident electron energies of 3.245 and $2.445 \mathrm{GeV}$ with coincident detection of the emergent $\mathrm{e}$ and $\mathrm{K}^{+}$in the HMS and SOS magnetic spectrometers in
Hall C. Angular distributions for the reactions $\mathrm{D}\left(\mathrm{e}, \mathrm{e}^{\prime} \mathrm{K}^{+}\right) \mathrm{YN}$ and $\mathrm{H}\left(\mathrm{e}, \mathrm{e}^{\prime} \mathrm{K}^{+}\right) \mathrm{Y}$, where $\mathrm{Y}$ is either $\Lambda$ or $\Sigma$, were studied at forward angles with respect to the virtual photon for $\mathrm{Q}^{2}=0.376$ and $0.50(\mathrm{GeV})^{2}$. Particle identification utilizing time-of-flight techniques together with Aerogel Cerenkov detectors yield clean missing mass spectra, such as those shown in Fig. III-6, and allow subtraction of random backgrounds.

Roughly half of the approved beam time was used during the production runs late in CY1997; the balance of the experiment, (e,e' $\left.\mathrm{K}^{+}\right)$on ${ }^{3,4} \mathrm{He}$ is scheduled to run late in 1999.

Missing mass spectra show two broad peaks arising from quasifree $\Lambda$ production on the proton and $\Sigma$ production on both the proton and neutron; Fermi motion within the deuteron broadens these peaks. Subtraction of the proton yield, suitably broadened and normalized, from the deuteron data, allows extraction of the cross section for kaon production on the neutron as indicated in Fig. III-6.

The angular distributions of both $\Lambda$ and $\Sigma^{-}$are somewhat forward peaked, while that for the $\Sigma^{0}$ is flat over the range covered. Since the cross section ratio, $\Sigma^{-}$ $\Sigma^{\circ}$, is $<1$ at all angles, the previous expectation of isospin $1 / 2$ in the $\Sigma$-nucleon system is clearly incorrect. The present data will constrain theoretical models that currently predict $\Sigma^{-}$cross sections that vary by more than an order of magnitude. Cross sections for the production of $\omega$ mesons over much of the angular range studied were obtained from analysis of the recoil proton spectra gathered during the hydrogen runs.

E91-016 provides data for the Ph.D. theses of students at Hampton University, University of Pennsylvania, and Temple University. 


\section{a.6. Momentum Transfer Dependence of $H\left(e, e^{\prime} K^{+}\right) Y$ Reactions (J. Arrington,} K. Bailey, D. F. Geesaman, H. E. Jackson, T. G. O'Neill, D. Potterveld, J. Reinhold, J. P. Schiffer, B. Zeidman, and E93-18 Collaboration)

In TJNAF experiment E93-18, the momentum transfer dependence of the reaction $\mathrm{H}\left(\mathrm{e}, \mathrm{e}^{\prime} \mathrm{K}^{+}\right) \mathrm{Y}$, where $\mathrm{Y}$ is $\Lambda$ or $\Sigma^{0}$, was studied at $\mathrm{Q}^{2}=0.5,1.0,1.5$, and $2.0(\mathrm{GeV} / \mathrm{c})^{2}$ at incident electron energies ranging from 2.445 to $4.045 \mathrm{GeV}$. The HMS and SOS spectrometers in Hall $\mathrm{C}$ were used for detection of the emergent electrons and $\mathrm{K}^{+}$, respectively. For each $\mathrm{Q}^{2}$, data were obtained at three different values of the polarization of the virtual photon in order to separate the longitudinal and transverse parts of the cross section. Together with data on the neutron (E91-16), this study is vital for understanding processes that lead to production of bound hypernuclei. Students from Hampton University and the University of Maryland utilized data from E93-18 for doctoral theses.

For $\Lambda$ production, the ratio, $R=\sigma_{\mathrm{L}} / \sigma_{\mathrm{T}}$, was found to reach a maximum $\approx 1$ at $\mathrm{Q}^{2}=0.75(\mathrm{GeV} / \mathrm{c})^{2}$. The data were compared to calculations and provide significant constraints on theoretical models. These results were published; ${ }^{1}$ the $\Sigma^{0}$ data are still being analyzed.

${ }_{1}$ G. Niculescu et al., Phys. Rev. Lett. 81, 1805 (1998)

a.7. Measurements of Inclusive Cross Section and $R=\sigma_{L} / \sigma_{T}$ in the Nucleon

Resonance Region (J. Arrington, D. F. Geesaman, H. Gao, T. G. O'Neill,

D. Potterveld, J. Reinhold, C. Keppel,* K. Assamagan, * O. K. Baker,* W. W. Buck,*

A. Cochran,* L. Gan,* A. Gasparian,* R. Green,* P. Gueye,* I. Niculescu,*

E. Segbefia,* L. Tang,* C. Williams,* L. Yuan,* P. Bosted, $†$ S. Rock, $\uparrow$

B.D. Anderson, $\ddagger$ G. Petratos, $\ddagger$ J.W. Watson, $\ddagger$ W. M. Zhang, $\ddagger$ W. Lorenzon,,

J. Dunne, I S. Beedoe,ll S. Danagoulian,|l C. Jackson,ll R. Sawafta,II V. V. Frolov, **

J. Napolitano,** J. Price, ** P. Stoler, ** C. Armstrong, $\uparrow \dagger$ R. Carlini, $\uparrow \uparrow$ R. Ent, $\uparrow \dagger$

K. Garrow, $\dagger \dagger$ J. Gomez, $\uparrow \dagger$ A. Lung, $\uparrow \dagger$ D. Mack, $\uparrow \dagger$ J. H. Mitchell, $\uparrow \dagger$ A. Saha, $\uparrow \dagger$

W. Vulcan, $\uparrow \dagger$ S. Wood, $\uparrow \dagger$ C. Yan, $\uparrow \dagger$ C. Cothran, $+\ddagger$ D. Day, $\neq+$ M. Zeier, $+\ddagger$

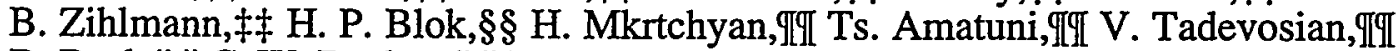

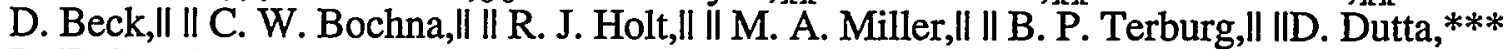

R. E. Segal,*** B. W. Filippone, $\dagger \dagger \dagger$ E. Kinney, $\neq+\ddagger$ D. van Westrum, $+\neq \ddagger$

D. M. Koltenuk, $\S \S \S$ and R. M. MohringIIIID

Reliable global descriptions of inclusive electroproduction data are necessary for electron-nucleon scattering model development, accurate radiative correction calculations, and the extraction of form factors, structure functions, and parton distribution functions from inclusive electron scattering experiments. High precision cross section measurements in the resonance region, combined with a separation of the longitudinal $\left(\sigma_{\mathrm{L}}\right)$ and transverse $\left(\sigma_{\mathrm{T}}\right)$ components, will substantially improve the global description of electroproduction at moderate to high $\mathrm{Q}^{2}$ and large Bjorken-x. In addition, an observed scaling relationship between resonance electroproduction and deep inelastic scattering, termed Bloom-Gilman duality, suggests a common origin for both kinematic regimes. A fundamental quark description for both properties of electroproduction may become possible by studying duality with new resonance electroproduction data and better measurements of $R=\sigma_{\mathrm{L}} / \sigma_{\mathrm{T}}$.

A series of measurements of inclusive electron scattering in the resonance region was taken in Hall C at TJNAF during 1995 and 1996 running. Data were obtained from both hydrogen and deuterium allowing extraction of the resonance production cross section from both the proton and the neutron. The cross sections were measured for momentum transfers between 0.5 and $5.0(\mathrm{GeV} / \mathrm{c})^{2}$. Bloom-Gilman duality was observed both for the entire region and locally in the vicinity of each prominent resonance.

Additional measurements will allow extraction of the ratio of longitudinal to transverse electron scattering off the proton. There exist few separation measurements of 
the ratio $\mathrm{R}$ in the resonance region at moderate or high momentum transfers. Theoretical models indicate that both the $\sigma_{\mathrm{L}}$ and the $\sigma_{\mathrm{T}}$ structure functions should manifest Bloom-Gilman duality. These models of duality can be tested for the first time with the proposed measurements of $R$. TJNAF experiment $94-110$ is scheduled to run in August of 1999 in Hall C and will measure inclusive electroproduction throughout the resonance region up to $\mathrm{Q}^{2}=7.5(\mathrm{GeV} / \mathrm{c})^{2}$. The experiment proposes to reduce the uncertainty in $\mathrm{R}$ from greater than $100 \%$ to approximately $10 \%$.

*Hampton University; †The American University; $\ddagger$ Kent State University; §University of Michigan; TMississippi State University; II North Carolina A\&T University; ${ }^{* *}$ Rensselaer Polytechnic Institute; ††Thomas Jefferson National Accelerator Facility; $\ddagger U$ University of Virginia; $\S \S$ Vrije Universiteit; GTYerevan Physics Institute; II IIUniversity of Illinois; ***Northwestern University; $\uparrow \dagger \uparrow$ California Institute of Technology; Colorado; §§§University of Pennsylvania; qGTUniversity of Maryland

\section{a.8. Measurements of Deuteron Photo-disintegration up to $4.0 \mathrm{GeV}$ (D. F. Geesaman, H. E. Jackson, T. G. O'Neill, D. H. Potterveld, B. Zeidman, J. Arrington, and the E89-012 and E96-003 collaborations)}

Constituent-counting-rule behavior was observed previously in high energy proton-proton scattering and photo-meson productions from the proton. Only one nuclear reaction thus far has exhibited this behavior. Argonne experiments NE8 and NE17 at SLAC showed that the deuteron photodisintegration $d(\gamma, p) n$ reaction at a proton center-of-mass angle of $90^{\circ}$ starts to show the scaling behavior at the unexpectedly low photon energy of around $1.0 \mathrm{GeV}$. Extending the SLAC measurements to higher photon energies and performing a detailed angular distribution study is essential to identify the limits of the kinematic regime of this behavior and to investigate the underlying mechanisms.

TJNAF experiment E89-012, one of the two hall-C commissioning experiments performed in 1996, measured for the first time the differential cross section for $d(\gamma, p) n$ up to $4.0 \mathrm{GeV}$ at center-of-mass angles $36^{\circ}$, $52^{\circ}, 69^{\circ}$, and $90^{\circ}$. The $\mathrm{d}(\gamma, \mathrm{d}) \pi^{\circ}$ reaction was also studied at $\mathrm{E}_{\gamma}=0.8-3.2 \mathrm{GeV}$. The $\mathrm{d}(\gamma, \mathrm{p}) \mathrm{n}$ results ${ }^{1}$, shown in Fig. III-7, are in good agreement with the previous measurements and the $90^{\circ}$ data continue to show the scaling behavior up to $4 \mathrm{GeV}$ and are also in fair agreement with an asymptotic meson-exchange model calculation. ${ }^{2}$ The data at $37^{\circ}$ and $53^{\circ}$ do not exhibit the counting-rule behavior, but also do not extend to as high a region of transverse momenta as the $90^{\circ}$ data. The preliminary $\mathrm{d}(\gamma, \mathrm{d}) \pi^{\circ}$ results are consistent with previous measurements at low photon energy, but do not show any signs of scaling at a center-of-mass angle of $90^{\circ}$. In contrast, the results at $136^{\circ}$ are in good agreement with the constituentcounting-rule behavior.

In the spring of 1999 we will extend the forward angle measurements to $5.5 \mathrm{GeV}$ to further study the energy and angular dependence of these reactions.

${ }_{1}^{1}$ C. Bochna et al., Phys. Rev. Lett. 81, 4576 (1998)

${ }^{2}$ S. I. Nagornyi, Yu. A. Kasatikin, and I. K. Kirchenko, Sov. J. Nucl. Phys. 55, 189 (1992)

${ }^{3}$ T.-S. H. Lee, Argonne National Laboratory Report No. PHY-5253-TH-88; T.-S. H. Lee, Proceedings of the International Conference on Medium and High Energy Nuclear Physics, Taipei, Taiwan, 1988 (World Scientific, Singapore 1988), p.563

${ }^{4}$ S. J. Brodsky and J. R. Hiller, Phys. Rev. C 28, 475 (1983)

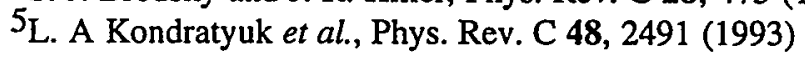




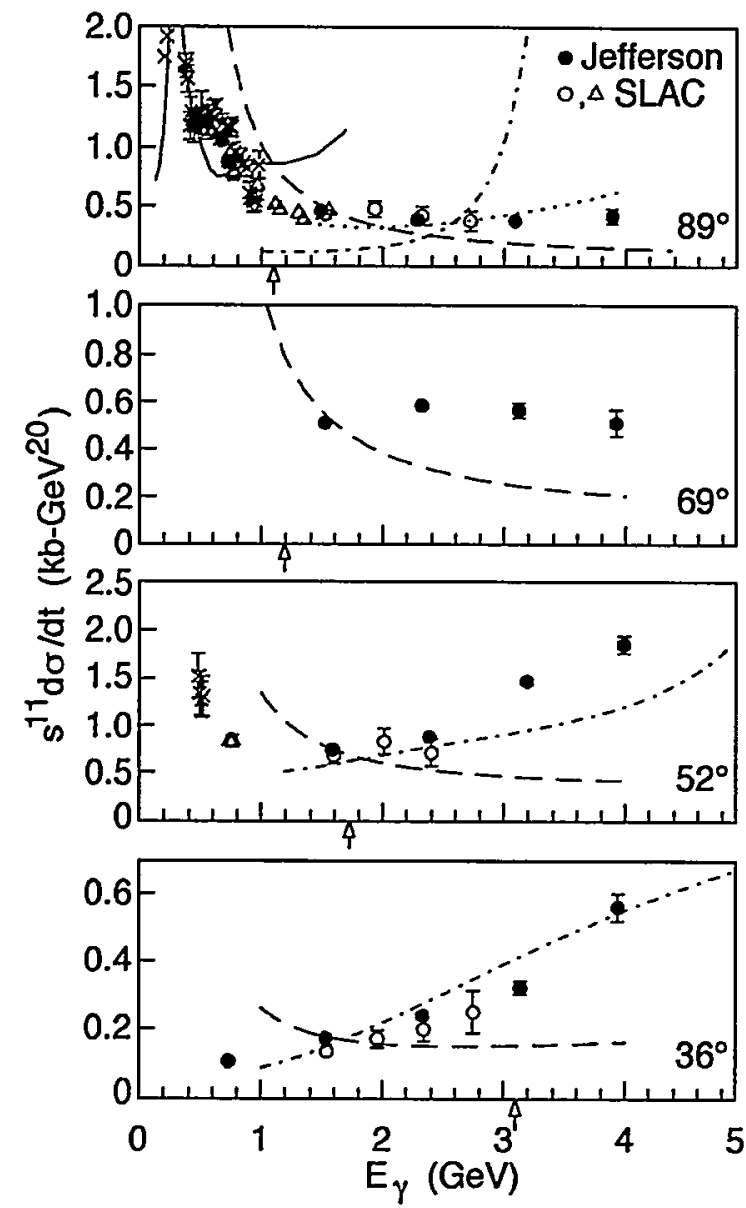

Fig. III-7. $s^{11} d \sigma d t$ vs. $E_{\gamma}$ for the $d(\gamma, p)$ in reaction. The present work is shown as solid circles with statistical uncertainties only, the SLAC NE17 data are shown as open circles, the SLAC NE8 data are shown as open triangles, and other existing data are shown as crosses. The solid line is the meson-exchange model calculation of Lee. ${ }^{3}$ The long-dashed line is the RNA analysis, ${ }^{4}$ and the dotted line is Nagornyi's ${ }^{2}$ asymptotic meson-exchange calculation. The dash-dotted line is the QGS calculation. 5 The arrows indicate the photon energies where $p_{T}^{2}=1.0(\mathrm{GeV} / \mathrm{c} \text {. })^{2}$. The previous data are shown above at nominal center of mass angles of $37^{\circ}, 53^{\circ}$, and $90^{\circ}$.

\section{a.9. A Study of Longitudinal Charged-Pion Electroproduction in $\mathrm{D},{ }^{3} \mathrm{He}$, and ${ }^{4} \mathrm{He}$ (H. E. Jackson, J. Arrington, K. Bailey, D. De Schepper, D. Gaskell, D. F. Geesaman, J. Reinhold, B. Mueller, D. H. Potterveld, T. G. O'Neill, B. Zeidman, and the E91-003 Collaboration)}

According to the simplest models of the nucleonnucleon force, pion-exchange currents in nuclei should give rise to mass-dependent enhancement of the nuclear pion charge distribution. Longitudinal pion electroproduction should be a clean direct probe of the nuclear pion currents because of the dominance of the pion-pole process for charged-pion emission in the direction of the virtual photon. If current conceptions of pion-exchange currents in nuclei are correct, longitudinal electroproduction will be suppressed at lower momentum transfers and enhanced at higher momentum transfers. These currents should also manifest themselves in the quark-antiquark distribution functions as observed in deep-inelastic scattering on nuclei. However, analysis of parton distribution functions show no evidence for any mass enhancements of the sea quarks. Recent data from Drell-Yan studies which probe directly the quark-antiquark sea, show no mass dependence. These results, suggest that a reformulation of pion-exchange models of the mediumand short-range properties of nuclear forces may be required. In an attempt to probe exchange currents directly, we carried out a series of measurements of single-charged-pion electroproduction on the proton, deuteron, and ${ }^{3} \mathrm{He}$ at the TJNAF. The goal is to measure the longitudinal cross section in parallel kinematics by means of a Rosenbluth separation, and to search for target-mass dependent effects. The results from these measurements should provide insight into the absence of any enhancement of sea quark distributions in nuclei as measured in deep-inelastic scattering. 
The measurements have been carried out in two kinematic configurations, one of which corresponds to a momentum transfer for which the electroproduction is quenched, and a second for which, according to the standard pion-exchange model of nuclear forces, one expects a substantial enhancement. Measurements at different photon polarizations for each configuration will be used to carry out a Rosenbluth separation of the longitudinal and transverse cross sections. The data analysis to date has been focused on obtaining accurate simulations of the spectrometer acceptances which will insure the precision required for reliable separations. By making a direct comparison of the cross sections for each target measured in the identical geometry, absolute cross section measurements will not be necessary. The experiment was carried out in Hall $\mathrm{C}$ at TJNAF. The scattered electrons were observed in the high momentum spectrometer and the short-orbit spectrometer, built by the Argonne group, served as the pion arm. An example of the data obtained is shown in Figure III-8, in which the missing mass spectra for $\pi^{+}$and $\pi^{-}$ production on ${ }^{3} \mathrm{He}$ are compared. The $\pi^{+}$yield was divided by 2 in order to make a direct comparison of the $\pi^{+}$production on the protons in ${ }^{3} \mathrm{He}$ with $\pi^{-}$ production on the neutron. For the $\pi^{+}$production, in addition to the quasifree component which appears to be the same as for $\pi^{-}$there is a sharp peak corresponding to coherent production leading to a triton in the final state. In addition, for the $\pi^{+}$, there is a component corresponding to a deuteron and neutron in the final state. The kinematic conditions for the experiment are summarized in Table III-I. Data analysis is in progress to extract the longitudinal cross sections for each target.

TABLE III-I. Kinematic conditions for TJNAF experiment E91-003.

\begin{tabular}{ccccccccc}
\hline \hline $\begin{array}{c}\mathrm{E} \\
\mathrm{GeV}\end{array}$ & $\begin{array}{c}\omega \\
\mathrm{GeV}\end{array}$ & $\begin{array}{c}\Theta_{\mathrm{e}} \\
\text { deg }\end{array}$ & $\begin{array}{c}\Theta_{\mathrm{q}} \\
\text { deg }\end{array}$ & $\begin{array}{c}\mathrm{W} \\
\mathrm{GeV}\end{array}$ & $\begin{array}{c}\mathrm{Q}^{2} \\
G e V / c^{2}\end{array}$ & $\begin{array}{c}\mathrm{p}_{\pi} \\
\mathrm{GeV} / \mathrm{c}\end{array}$ & $\varepsilon$ & $\begin{array}{c}P_{\text {recoil }} \\
G e V / c\end{array}$ \\
\hline 0.845 & 0.46 & 66.87 & 27.11 & 1.160 & 0.396 & 0.327 & 0.43 & 0.451 \\
1.645 & 0.46 & 26.04 & 41.94 & 1.160 & 0.396 & 0.327 & 0.86 & 0.451 \\
& & & & & & & & \\
1.645 & 1.108 & 39.33 & 15.46 & 1.600 & 0.400 & 1.079 & 0.49 & 0.197 \\
3.245 & 1.108 & 13.79 & 23.54 & 1.600 & 0.400 & 1.079 & 0.89 & 0.197 \\
\hline
\end{tabular}




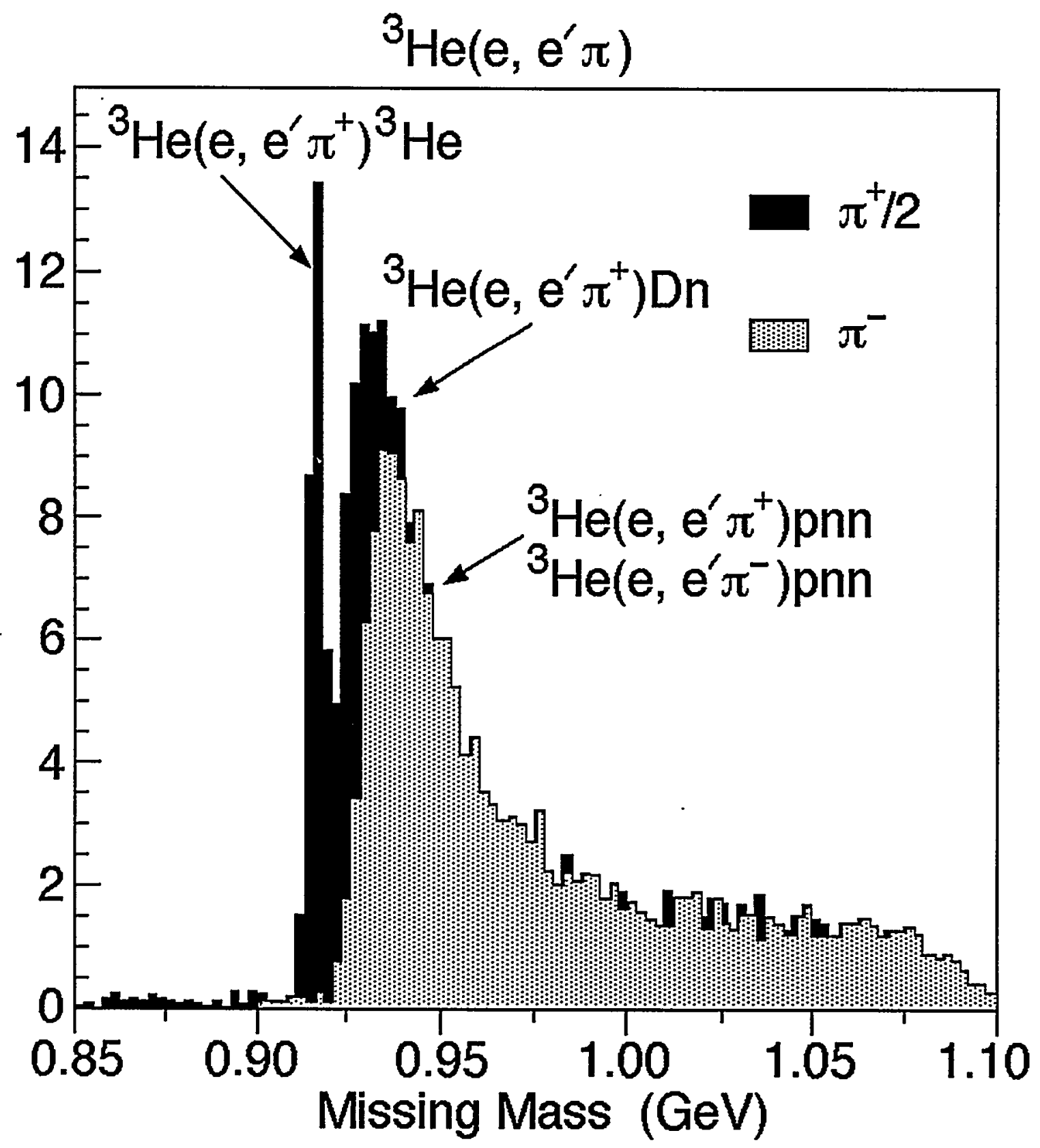

Fig. III-8. Comparison of missing mass spectra for charged pion electroproduction on ${ }^{3} \mathrm{He}$ at an incident electron energy of $1.645 \mathrm{GeV}$ and a proton-photon invariant mass of $1.160 \mathrm{GeV}$. 


\section{a.10. The Charged Pion Form Factor (H. E. Jackson, D. Gaskell, B. Mueller, and the E93-021 Collaboration)}

The pion, as the lightest meson, is an ideal laboratory for testing theoretical predictions at the border of nonperturbative and perturbative QCD regimes. At intermediate and high $\mathrm{Q}^{2}$, reliable experimental data for the charged pion form factor $\left(\mathrm{F}_{\pi}\right)$ are scarce. Experiment E93-021 took data at TJNAF for the ${ }^{1} \mathrm{H}\left(\mathrm{e}, \mathrm{e}^{\prime} \pi^{+}\right)$n reaction in Hall $\mathrm{C}$ late in 1997 for $\mathrm{Q}^{2}$ values of 0.6-1.6 (GeV/c) $)^{2}$. The forward longitudinal response in this process is dominated by knockout of virtual pions. The data analysis in progress is focused on obtaining longitudinal and transverse separated cross sections. $F_{\pi}$ will be extracted from these data. In addition, data were acquired on a deuterium target. The cross section ratio $\pi^{-1} / \pi^{+}$is sensitive to the reaction mechanism. Analysis of these data will yield ratios which can be compared to recent Regge model calculations.

\section{a.11. Short-Orbit Spectrometer for Hall C at TJNAF (H. E Jackson, K. Bailey, D. H Potterveld, and B. Zeidman)}

The Short-Orbit Spectrometer (SOS) built by the Argonne Medium Energy Physics group under contract to TJNAF is now used routinely in the experiments carried out at TJNAF. The SOS is a large acceptance, compact, general purpose magnetic spectrometer for charged particles. It is installed in Hall $\mathrm{C}$ where it is a resource available to all users. The optical design is point-to-point in both the dispersive (vertical) and transverse planes, with a $40 \%$ momentum bite, a solid angle of $7 \mathrm{msr}$, and a maximum central momentum of $1.75 \mathrm{GeV}$. For a $1-\mathrm{mm}$ target spot, the first-order resolving power is approximately 2200 , with an angular resolution of better than $5 \mathrm{mr}$. Because of the strong edge focusing, the optical length of the SOS is only $7.3 \mathrm{~m}$. The short length reduces the fraction of pions and kaons that decay in flight - an important feature for experiments involving the detection of these particles. Operation of the SOS in conjunction with the High Momentum Spectrometer in Hall $\mathrm{C}$ provides a coincidence capability for electron scattering experiments and serves as a general purpose second arm in a wide variety of experiments at TJNAF. The SOS was built with a spherical bearing at the target and a hydraulic lifting system that will allow measurements of scattered particles at angles up to 20 degrees out of the horizontal plane.

In 1998, we conducted a pion production experiment (E91-003) that used the SOS with a Cerenkov detector that was upgraded by ANL. In 1999 we will participate in 3 experiments that use the SOS. In support of these experiments we are working to improve the understanding of the SOS optics, through the analysis of fieldmap data measured by ANL, and through the analysis of optics data measured during these experiments. We are also working with the TJNAF staff to upgrade the control and monitoring software for the SOS magnets.

\section{a.12. ${ }^{3}$ He Target for Hall C at TJNAF (K. Bailey, H. E. Jackson, J. Reinhold, and B. Zeidman)}

Cryogenic targets of the lightest nuclei, $\mathrm{H}, \mathrm{D},{ }^{3,4} \mathrm{He}$, are of special interest for the physics program in Hall C. For this, TJNAF constructed a cryo-target system for liquid hydrogen and deuterium targets, but ANL experiments E91-03 and the balance of E91-16 require high-density helium targets. The existing hydrogen targets were replaced by $4-\mathrm{cm}$ - diameter vertical-flow aluminum cells, wall thickness of $0.013 \mathrm{~mm}$, that were tested to burst at approximately $400 \mathrm{psi}$. With an operating pressure of $150 \mathrm{psi}$ at around $5 \mathrm{~K}$, densities exceeding that of liquid ${ }^{3} \mathrm{He}$ at a pressure of 1 atmosphere are attained.

ANL constructed a gas-handling system to fill the target and also recover the expensive ${ }^{3} \mathrm{He}$ gas after an experiment is finished or in the event of a sudden cooling loss during operation. The gas-handling system is constructed of $1 / 4$ " stainless steel tubing with 
hermetically sealed diaphragm and bellows valves. An 80 -gal vessel is used as a room temperature storage and recovery tank for the approximately $300 \mathrm{~g}^{3} \mathrm{He}$ used in the target loop. A cryo compressor, consisting of a 1gal pressure vessel immersed in a liquid helium bath, surrounded by a liquid nitrogen shield, is used to transfer the gas into the target and pressurize it to $10 \mathrm{~atm}$. This system was successfully tested, installed in Hall $C$ and used during experiment E91-03, the first experiment at TJNAF to use a ${ }^{3} \mathrm{He}$ target as well as a similar ${ }^{4} \mathrm{He}$ target. It is now considered standard equipment and will be used for both ${ }^{3} \mathrm{He}$ and ${ }^{4} \mathrm{He}$ targets in experiment 91 016 now scheduled for late 1999.

\section{a.13. Nuclear Vector Polarization in the Laser Driven Polarized Target at IUCF and Measurement of $\overrightarrow{\mathbf{d}}(\overrightarrow{\mathbf{p}}, \mathbf{p p})$ n Reaction (W. J. Cummings, H. Gao, R. Kowalczyk, Z.- T. Lu, R. V. Cadman,*R. J. Holt,* M. A. Miller,* B. Owen,* E. Schulte, ${ }^{*}$ E. Thorsland, ${ }^{*}$ C. Grosshauser,$\uparrow$ W. Kilian, $\dagger$ W. Nagengast,$\dagger$ K. Rith, $\dagger$ F. Schmidt, $\uparrow$ J. Stenger $\dagger$ J . Sowinski, $\ddagger$ F. Sperisen, $\ddagger$ J. Brack,II B. Fox, II E. Kinney,II W. Kirwin,T C. E. Jones, § and D. K. Topopkovll)}

The laser-driven polarized deuterium target concept originally developed at ANL is being used in its first nuclear physics experiment as an internal target in the Indiana University Cyclotron Facility cooler ring to study polarized proton scattering from vector polarized deuterium. The target operation was improved considerably with the introduction of small amounts of $\mathrm{O}_{2}$ gas to improve the dissociator performance. Stable target operation was demonstrated over several-day intervals with polarizations at or above the design goal of $23 \%$. This performance was also reproducible after several-week down periods.

Tests continue with beam to study and optimize the target performance. First measurements on $d(\vec{p}, p p) n$ reactions have begun. Recent calculations indicate the polarization observables in this reaction are sensitive to the models of the three-nucleon forces. This physics will continue to be addressed in additional running in 1999.

*University of Illinois, Urbana; †University of Erlangen-Nürnberg; 拍diana University Cyclotron Facility; TUniversity of Colorado; §Caltech; llBudker Institute for Nuclear Physics, Novosibirsk

\section{a.14. An Ultra-Sensitive Trace Analyzer of Krypton-81 and Krypton-85 Atoms (K. Bailey, C.-Y. Chen, D. J Lin, Z.-T. Lu, T. P. O'Connor, and L. Young)}

We are developing laser trapping techniques for the purpose of building a practical ultra-sensitive trace analyzer of ${ }^{81} \mathrm{Kr}\left(\mathrm{t}_{1 / 2}=2 \times 10^{5} \mathrm{yr}\right)$ and ${ }^{85} \mathrm{Kr}\left(\mathrm{t}_{1 / 2}=\right.$ $10.8 \mathrm{yr}$ ) concentrations in gas samples. This new technology would help support the DOE missions in environmental technology and nuclear non-proliferation. For example, the ${ }^{81} \mathrm{Kr} / \mathrm{Kr}$ isotope abundance measurements can be applied to date old groundwater on the time scale of $10^{5}-10^{6}$ years, which will help characterize potential radioactive waste burial sites. It can also be applied to date deep ice core samples obtained from Greenland and Antarctica, which will extend our knowledge of prehistoric earth climates to a million years ago. The same technique can be applied to map the atmospheric concentration of $85 \mathrm{Kr}$, primarily produced by nuclear-fuel reprocessing facilities, in order to help assess the global nuclear non- proliferation effort. Moreover, the techniques developed here directly benefit our other projects on measuring the nuclear moments and radii of unstable isotopes and on testing fundamental symmetries.

In 1998 we realized laser trapping of metastable krypton atoms. Figure III-9 shows the fluorescence signal from the trapped atoms as the laser frequency is scanned over the resonance frequency of each isotope. It clearly shows that the laser trap is isotopically selective. A quantitative measurement on the selective power is underway. Since ${ }^{81} \mathrm{Kr}$ and ${ }^{85} \mathrm{Kr}$ are so rare in natural samples, they would only appear in our trap/detector one atom at a time, and our system has to be able to detect a single trapped atom. We have investigated and applied several techniques, including imaging, chopping the atomic beam, and using a special UHV-compatible a 


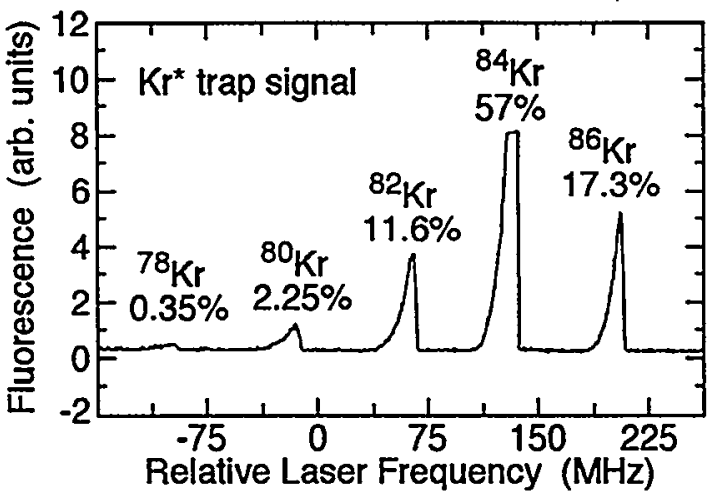

black paint and achieved the sensitivity necessary to see a single trapped atom (Fig. III-10). Having demonstrated these two capabilities, we moved on to build an atomic beam machine that is stable and reliable so that it can be used for counting the two rare isotopes. We recently finished building this new machine and are currently testing and characterizing its properties. Our near-term goal is to detect ${ }^{81} \mathrm{Kr}$ and $85 \mathrm{Kr}$ atoms, and demonstrate the principle of our new approach.

Fig. III-9. The fluorescence signal from laser trapped. metastable krypton atoms. Atoms of different isotopes are trapped at different laser frequencies due to isotope shifts. The ${ }^{84} \mathrm{Kr}$ signal is saturated.

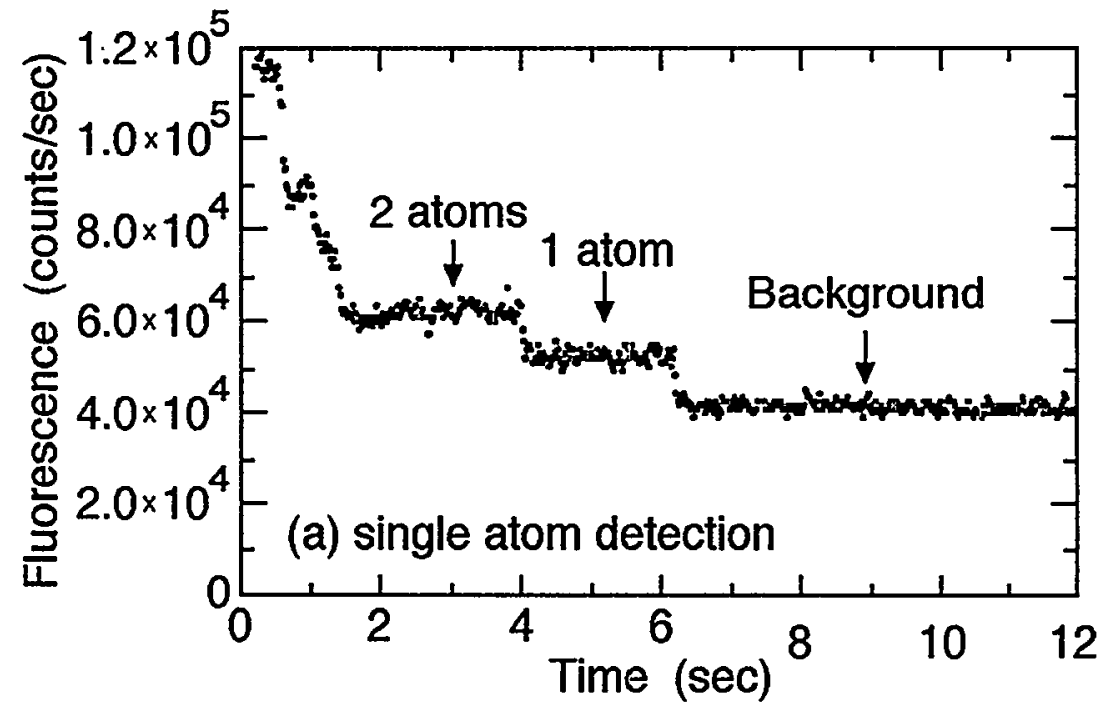

Fig. III-10. The decay of trap fluorescence over time. The discrete step in fluorescence is a result of a single atom leaving the trap.

\section{a.15. Nuclear Moments and Radii of Unstable Cesium Isotopes \\ (K. Bailey, Z.-T. Lu, J. Nolen, T. P. O'Connor, G. Savard, and L. Young)}

The recent development of an ISOL source and an online mass separator at the Dynamitron ${ }^{1}$ presents an opportunity for us to study nuclear properties of long chains of unstable isotopes. By measuring the hyperfine structures and isotope shifts of the atoms using precision laser spectroscopy, the nuclear spins, magnetic dipole moments, electric quadrupole moments and charge radii can be determined. Such information, especially when mapped over a long chain of isotopes, helps to understand the nuclear structure and force, and complements the mass measurements that are being carried out in our division. ${ }^{2}$ Moreover, isotopically selective laser spectroscopy methods can also be used to identify new isotopes that have yet to be discovered.

We are developing laser spectroscopy techniques to study unstable cesium isotopes. We plan to inject the mass-separated cesium ions onto a heated yttrium foil 
that serves as a neutralizer. The neutral cesium atoms boiled off the foil will enter a glass cell, in which the laser spectroscopy will be performed. In 1998 we built two diode lasers and a sensitive fluorescence detector.
We also investigated non-stick coatings that will be used to reduce loss rate due to atoms sticking to the glass walls.

\begin{abstract}
${ }_{1}^{1}$ Ion Sources and Targetry for Radioactive Beams, J. Nolen et al., Physics Division Annual Report, ANL-98/24, page 117 (1998)

2 The Canadian Penning Trap Mass Spectrometer, G. Savard et al., Physics Division Annual Report, ANL-98/24, page 75 (1998)
\end{abstract}

\title{
a.16. Testing Time-Reversal-Symmetry in Radon Atoms (K. Bailey, C.-Y. Chen, D. J. Lin, Z.-T. Lu, T. P. O'Connor, and L. Young)
}

Time-reversal-symmetry is known to be violated in neutral kaon decay. Many theoretical models that offer an explanation of this phenomenon also predict the existence of a small and permanent electron electricdipole-moment (EDM). By searching for the signature of such an EDM in atoms, high-precision spectroscopy experiments set an upper limit of "EDM electron $<4 \times$ $10^{-27} \mathrm{e} \mathrm{cm}$. This value is higher than, but within an order of magnitude to, the values predicted by some supersymmetry models. Our plan of using trapped and cooled metastable radon atoms presents an opportunity for large improvements from two sources: 1) the signature of an EDMM is enhanced in heavy atoms. For example, the enhancement in radon atoms is ten times what it is in cesium atoms; 2 ) cold atoms allow a much longer observation time and, consequently, result in more precise measurements.

Since radon atoms are radioactive and rare (among radon isotopes, ${ }^{222} \mathrm{Rn}$ has the longest half-life of only 3.8 days), a high efficiency in preparing and trapping radon atoms is essential in order to conduct an EDM search with radon atoms. In 1998 we continued developing techniques to improve the efficiency of producing metastable noble gas atoms, slowing and loading atoms into a magneto-optical trap, and detecting small numbers of trapped atoms.

\section{a.17. Di-lepton Production with 800-GeV Protons Measurement of $\bar{d} / \overline{\mathbf{u}}$ in the Nucleon (D. F. Geesaman, S. B. Kaufman, N. Makins, B. A. Mueller, J. D. Bush,* L. D. Isenhower,* M. E. Sadler,* R. S. Towell,* J. L. Willis,* D. K. Wise,* C. N. Brown, $\dagger$ W. E. Cooper, $\uparrow$ X. C. He $\ddagger$ W. M. Lee,$\ddagger$ G. Petitt $\ddagger$ D. M. Kaplan,$\S$ T. A. Cary, II G. T. Garvey, II D. M. Lee,II M. J. Leitch,II P. L. McGaughey,I J. M. Moss, II B. K. Park, I J. C. Peng,I P.E. Reimer,I W. E. Sondheim, II T. N. Thompson,II P. N. Kirk,\| Y. C. Wang,\| Z. F. Wang,ll M. E. Beddo,** T. H. Chang,** G. Kyle, ** V. Papavassiliou, ** J. Selden,** J. C. Webb, ** T. C. Awes, $\dagger \dagger$ P. W. Stankus, $\dagger \dagger$ G. R. Young,,$\dagger$ E. A. Hawker, C. A. Gagliardi, $\neq \ddagger$ R. E. Tribble, $\neq \ddagger$ M. A. Vasiliev, and P. M. Nord§§)}

No fundamental symmetry requires the $\overline{\mathbf{u}}$ quark distribution of the proton to be equal to the $\bar{d}$ distribution, but for many years, lacking evidence to the contrary, this assumption was used in parameterizing parton distribution functions of the nucleon. Following evidence from deep inelastic scattering of an integral difference in these distributions, E866 at FNAL measured the $x$ dependence of the ratio of $\bar{d} / \bar{u}$ from $0.04<x<.31$. In 1997 E866 completed running at FNAL on di-muon production on hydrogen, deuterium, beryllium, copper and tungsten targets. By focusing on high-mass Drell-Yan pairs and kinematics in which the target $\overline{\mathrm{u}}$ distributions dominate the cross section, it was possible to extract the $\bar{d} / \bar{u}$ ratio and the $\bar{d}-\bar{u}$ difference (see Fig III-11) from the ratio of deuterium to hydrogen cross sections. (See Fig. III-11). The flavor difference is a pure flavor non-singlet quantity: its integral is $Q^{2}$ independent and its $\mathrm{Q}^{2}$ evolution at leading order does not depend on the gluon distribution of the proton. 
The large differences seen in Fig. M-11 must be nonperturbative in nature. Three approaches which can accommodate such differences are illustrated by the curves: 1) hadronic models of the meson cloud of the nucleon (solid and dashed curves represent difference choices of the $\pi \mathrm{NN}$ and $\pi \mathrm{N} \Delta$ vertex functions). 2) Chiral quark models which couple mesons directly to the constituent quarks (dot-dashed). 3) Instanton models (dotted). One conclusion is that the chiral quark models lead to much too soft $\mathrm{a} \overline{\mathrm{d}}-\overline{\mathrm{u}}$ distribution.

Analysis of the E866 data continues focusing on the following issues: 1) Complete ratio analysis of the entire Drell-Yan deuterium and hydrogen data sets. 2) Extract absolute Drell-Yan cross sections on hydrogen and deuterium. 3) Extend measurements of the nuclear dependence of Drell-Yan, $J / \Psi$ and $Y$ production into new kinematic ranges compared to previous measurements. 4) Extract decay angular distributions of $\mathrm{J} / \Psi$ and $\Upsilon$ production to test the new models of these reactions which were developed to reproduce higher energy collider data. 5) Determine decay angular distributions of Drell-Yan production to test the next-to-leading-order QCD calculations of this process.

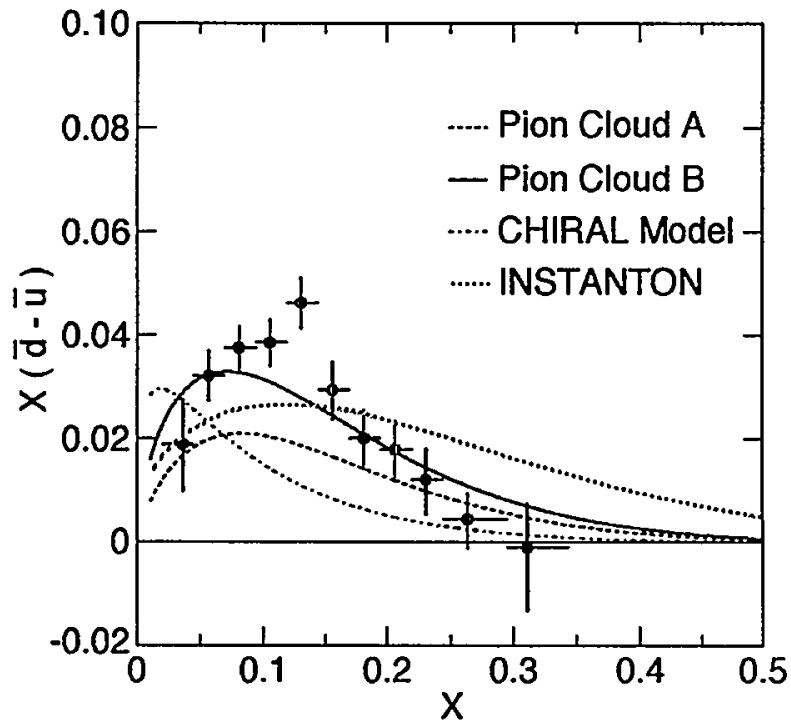

Fig. III-11. The $x$ dependence of $x(\overline{\mathrm{d}}-\overline{\mathrm{u}})$ of the proton at a mass scale of $7.35 \mathrm{GeV}$. The curves represent four model calculations of $x(\overline{\mathrm{d}}-\overline{\mathrm{u}}){ }^{I}{ }^{1}$ The solid and dashed curves include pions, nucleons and deltas with differing vertex cut-offs. The dot-dashed curve is a chiral quark model and the dotted curve is an instanton inspired prediction.

*Abilene Christian University; †Fermi National Accelerator Laboratory; $\ddagger$ Georgia State University; $\S$ Illinois Institute of Technology; qLos Alamos National Laboratory; ILouisiana State University; **New Mexico State University; ††Oak Ridge National Laboratory; $\ddagger \ddagger T e x a s$ A\&M University; §§Valparaiso University

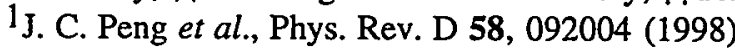

\section{a.18. $\Upsilon$ and $\mathrm{J} / \psi$ Production from $800-\mathrm{GeV}$ Protons Incident on $\mathrm{D}_{2}$ and $\mathrm{H}_{2}$ Targets}

(D. F. Geesaman, S. B. Kaufman, N. Makins, B. A. Mueller, J. D. Bush,* L. D. Isenhower,* M. E. Sadler,* R. S. Towell,* J. L. Willis, ${ }^{*}$ D. K. Wise, ${ }^{*}$ C. N. Brown, $\dagger$ W. E Cooper $\uparrow$ X. C. He $\ddagger$ W. M. Lee $\ddagger$ G. Petitt, $\ddagger$ D. M. Kaplan,$\S$ T. A. Cary,II G. T. Garvey,II D. M. Lee,II M. J. Leitch,II P. L. McGaughey,I J. M. Moss,I B. K. Park, II J. C. Peng,II P. E. Reimer,I W. E. Sondheim,II T. N. Thompson,II P. N, Kirk,II Y. C. Wang,\| Z. F. Wang,\| M. E. Beddo,** T. H. Chang, ** G. Kyle,** V. Papavassiliou,** J. Selden,** J. C. Webb,** T. C. Awes, $\nmid \dagger$ P. W. Stankus, $\nmid \dagger$ G. R. Young, $\uparrow \dagger$, E. A. Hawker, $+\ddagger$ C. A. Gagliardi, $\ddagger$ R. E. Tribble, $\ddagger$ M. A. Vasiliev, $\ddagger$ D. D. Koetke, $\S \S$ and P. M. Nord§§)

Fermilab experiment E866 is designed to detect pairs of oppositely charged muons produced in collisions between $800-\mathrm{GeV}$ protons and variety of fixed targets. The primary motivation for E866 was to detect dimuon events generated in Drell-Yan reactions, but the detector is sensitive to any process which produces dimuon pairs. One such process is heavy quark resonance production. $\Upsilon$ and $\mathrm{J} / \Psi$ particles are produced when partons from the beam and target annihilate and form a virtual gluon which then hadronizes into a heavy resonance state. These states have several percent branching ratios into dimuon pairs and are detected during normal data acquisition. The virtual gluon which produces the resonance can be generated by the 
annihilation of either a quark/antiquark pair or a pair of gluons. Resonance production is therefore sensitive to both the quark and gluon distributions within the beam and target.

The E866 hydrogen and deuterium data samples contain approximately $30000 \Upsilon$ and 1 million $J / \psi$ events. The resonance events are separated from the underlying Drell-Yan continuum by fitting the mass spectra to line shapes generated by a realistic simulation of the E866 detector and trigger, and the separation is performed for several bins in $x_{\text {Feynman. The absolute }} \mathrm{Y}$ and $\mathrm{J} / \Psi$ production cross sections for hydrogen and deuterium are obtained from the extracted results by normalizing the yields to the integrated luminosity and correcting the results by the acceptance of the detector. The cross sections values can then be compared to predictions based various models using the various parton distributions available in the literature. In practice, a more stringent test of the models is achieved by comparing the $\mathrm{D}_{2} / \mathrm{H}_{2}$ cross section ratio since the dominant systematic uncertainties, the acceptance correction, and charge normalization largely cancel in the ratio. In Fig. III- 12 the $\mathrm{D}_{2} / \mathrm{H}_{2}$ cross section ratio extracted from the data is compared to a color evaporation model calculation for the yield. The model calculation overpredicts the ratio for all $\mathrm{x}_{\mathrm{Feynman}}$.

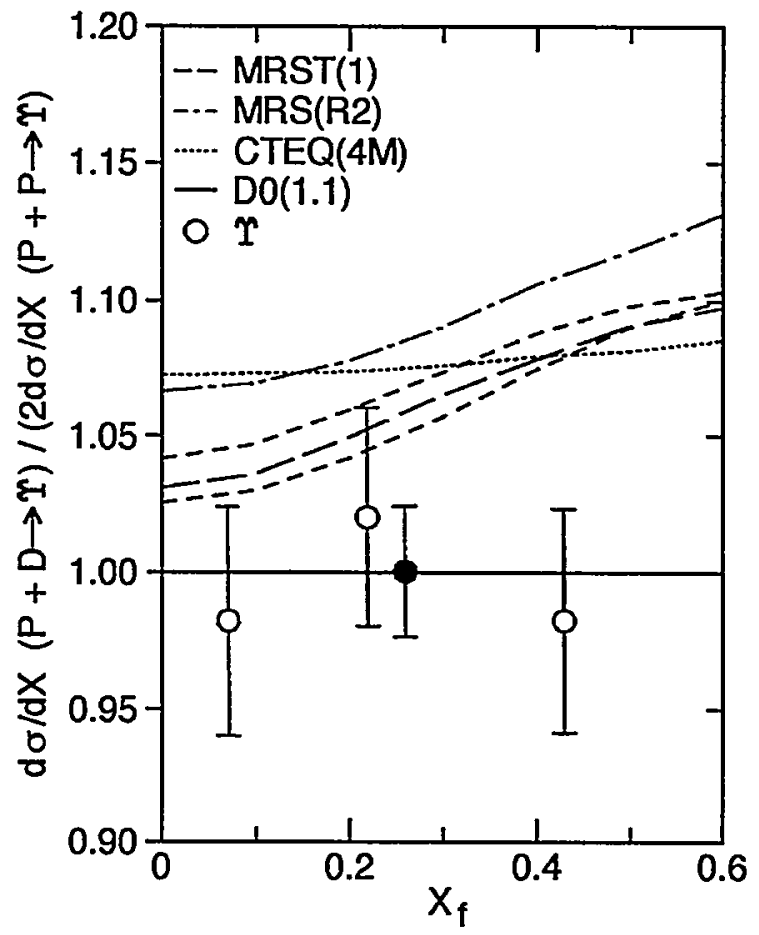

Fig III-12. The data points represent the ratio of cross sections for $r$ production from deuterium and hydrogen. The curves correspond to color evaporation model calculations with four parameterizations at the nucleon parton distributions.

\footnotetext{
*Abilene Christian University; †Fermi Na;tional Accelrator Laboratory; $\ddagger$ Georgia State University; §lllinois Institute of Technology; ILos Alamos National Laboratory; II Louisiana State University; **New Mexico State University; ††Oak Ridge National Laboratory;
} 


\section{a.19. Di-lepton Production With 120-GeV Protons to Extend the Measurement of} $\overline{\mathbf{d}} / \mathbf{u}$ in the Nucleon (D. De Schepper, D. F. Geesaman, B. A. Mueller, T. G. O'Neill, D. H. Potterveld, L. D. Isehower,* M.E. Sadler,* C. N. Brown, $\dagger$ G. T. Garvey, $\ddagger$ M. J. Leitch, $\ddagger$ P. L. McGaughey,$\ddagger$ J.-C. Peng $\ddagger$ P. E. Reimer $\ddagger$ C. A. Gagliardi,,

R. E. Tribble, $\S$ and D. D. KoetkeI)

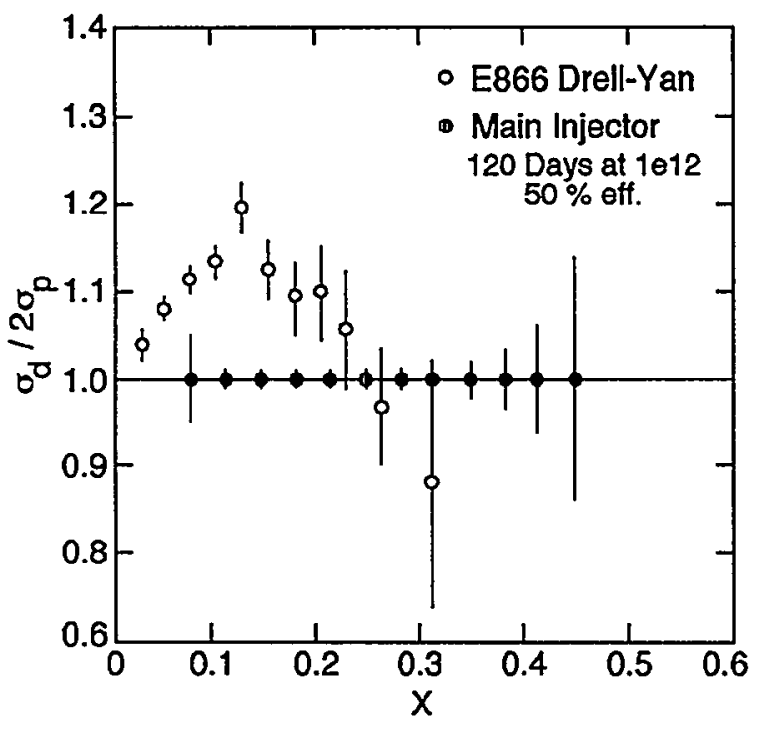

Fig. III-13. FNAL E866 results for the $x$ dependence of the ratio of the Drell-Yan production cross sections on deuterium and hydrogen. The statistical uncertainties expected for an experiment proposed for the FNAL main injector are shown as the error bars on the solid circles which are arbitrarily plotted at a ratio of 1.0. The systematic errors are estimated to be less than $1 \%$.
The FNAL E866 measurements of the ratio of $\bar{d} / \bar{u}$ distributions using the Drell-Yan reaction with 800 $\mathrm{GeV}$ protons on targets of hydrogen and deuterium reveal an unexpected $x$ dependence at the highest $x$ values that could be measured in that experiment. A significant extension of the $x$ range of the measurement requires high intensity proton beams of lower energy and are ideally suited for $120 \mathrm{GeV}$ beams of the new FNAL main injector. A letter of intent was submitted to FNAL to carry out high precision Drell-Yan measurements using the primary proton beam from the main injector. A representation of the statistical errors that could be obtained from such measurements is shown in Fig. III-13. Measurements would also be made on nuclear targets with similar precision to extend our knowledge of the nuclear dependence of the antiquark distributions to higher $\mathrm{x}$ values.

FNAL encouraged the collaboration to submit a full proposal for this experiment. The collaboration is developing this proposal for the spring of 1999 with an expected start of the experiment in 2001-2002.

*Abilene Christian University; †Fermi National Accelerator Laboratory; $\ddagger$ Los Alamos National Laboratory; §Texas A\&M University; TValparaiso University 


\section{THEORETICAL PHYSICS}

\section{OVERVIEW}

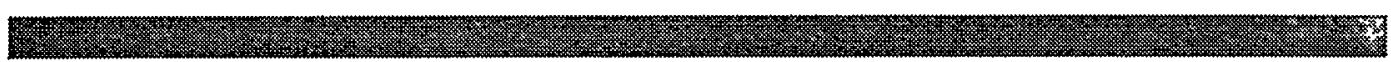

Theoretical research in the Physics Division addresses a broad range of problems involving the structure and dynamics of hadrons and nuclei. There is a strong emphasis on comparison to data provided by experimental groups at Argonne and at other facilities around the world. The principal areas of research include nuclear dynamics with subnucleonic degrees of freedom, nuclear forces and nuclear systems, and heavy-ion reaction and structure studies. Our work includes the modeling of quantum chromodynamics in meson and baryon structure, developing reaction theories for medium-energy baryon-baryon interactions and meson production, and studying electron scattering within the framework of relativistic Hamiltonian particle dynamics. We construct realistic two- and three-nucleon potentials that give very accurate fits to elastic scattering data, and then use these in detailed many-body calculations of the properties of light and closed-shell nuclei, hypernuclei, nuclear matter and neutron stars. Heavy-ion research includes the structure and reactions of nuclei far from stability produced at radioactive beam facilities, coupled-channels calculations of reactions near the Coulomb barrier, and studies of deformed and superdeformed nuclei observed at ATLAS. We also pursue some research in atomic physics, fundamental quantum mechanics, and quantum computing. Most of our projects involve collaborators at domestic and foreign universities and other national laboratories. Several require major numerical simulations using state-of-the-art computers, including Argonne's massively parallel IBM SP and SGI Onyx systems. 


\section{A. NUCLEAR DYNAMICS WITH SUBNUCLEONIC DEGREES OF FREEDOM}

The objective of this research program is to investigate the role of mesons, nucleon resonances, and quark-gluon degrees of freedom in nuclear dynamics.

The Dyson-Schwinger equations (DSEs) provide a nonperturbative approach to studying the continuum formulation of QCD, making accessible phenomena such as confinement, dynamical chiral symmetry breaking, and bound state structure and interactions. However, they also provide a generating tool for perturbation theory and hence their application is tightly constrained at highenergy. This is the particular feature of the phenomenological application of DSEs: their ability to furnish a unified description of high-and low-energy phenomena in QCD. This last year has seen many successful applications. For example, we used our exact pseudoscalar meson mass formula to prove that heavy-meson masses rise linearly with the mass of their heaviest constituent; applied the equation of state obtained in our simplest model of QCD at finite temperature and chemical potential to the calculation of the properties of quark stars; demonstrated that all gap-equation-based studies of QCD using the rainbow truncation will yield mean-field exponents for the chiral symmetry restoration transition; and achieved a unified description of light- and heavy-meson observables.

We are continuing our effort to develop reaction theories for relating the structure of nucleon resonances $\left(\mathrm{N}^{*}\right)$ as predicted by various QCD-based hadron models to the data of $\pi \mathrm{N}$ and $\gamma \mathrm{N}$ reactions. The model was applied to investigate the $d\left(e, e^{\prime} \pi\right)$ reactions which are being pursued at TJNAF and MIT-Bates. The amplitudes of vector meson photoproduction at a few $\mathrm{GeV}$ were analyzed based on the diffractive Pomeron-exchange and meson-exchange mechanisms. Models of hyperon-nucleon and hyperon-hyperon interactions were constructed by using SU(3) symmetry and the data on nucleon-nucleon and hyperon-nucleon reactions. The models were used to investigate the strange hadron matter that could be created in relativistic heavy-ion collisions, electroproduction of hyperons on the deuteron, $n p \rightarrow \Lambda p$ weak reactions, and the structure of the hypernucleus ${ }_{\Lambda}^{16} \mathrm{O}$.

We are continuing our investigation of quark models of hadrons and of electron scattering from few-nucleon systems, in the framework of relativistic Hamiltonian dynamics. Quark confinement is the source of important qualitative differences between relativistic quark and few-nucleon models. The structure of quark models that respect Poincaré covariance together with flavor and color symmetry need not be a quark-particle structure. Such models provide simple relations of the properties of nucleon resonances and nucleons. Conventional form factors, directly related to electromagnetic observables, are either defined by spin matrix elements evaluated in a special 'frame', or invariant factors appearing in covariant spinor representations of the currents. We found that the well-known Lorentz covariant representations of current kernels for spin $1 / 2$ and spin 1 can be generalized to arbitrary integral and half-odd integral spin by emphasizing the $O(1,2)$ subgroup that leaves the four-momentum transfer invariant. These relations have significant implications for the current structures of hadronic models. 


\section{a.1. Pion Mass and Decay Constant (P. Maris, C. D. Roberts, and P. C. Tandy*)}

The axial-vector Ward-Takahashi identity was exploited to derive the explicit relation between the pion BetheSalpeter amplitude, $\Gamma_{\pi}$, and the quark propagator in the chiral limit, independent of assumptions about the form of the quark-antiquark scattering kernel. $\Gamma_{\pi}$ necessarily involves a non-negligible $\gamma_{5} \gamma \bullet P$ term $(P$ is the pion four-momentum). Exact, gauge-invariant and renormalization-point-independent expressions were obtained for the pion decay constant, $f_{\pi}$, and mass, both of which depend on $\Gamma_{\pi}$. The equivalence between $f_{\pi}$ and the pion Bethe-Salpeter normalization constant in the chiral limit was demonstrated. Preserving the axialvector Ward-Takahashi identity is crucial in any study of the pion itself, and in any study whose goal is a unified understanding of the properties of the pion and other hadronic bound states. An article describing this work was published. ${ }^{1}$

*Kent State University

1P. Maris, C. D. Roberts, and P. C. Tandy, Phys. Lett. B 420, 267 (1998)

\section{a.2. Heavy- to Light-Meson Transition Form Factors (P. Maris, C. D. Roberts, M. A. Ivanov, ${ }^{*}$ and Yu. L. Kalinovsky*)}

Semileptonic heavy-heavy and heavy-light meson transitions were studied as a phenomenological application of a heavy-quark limit of Dyson-Schwinger equations. Employing two parameters: $\mathrm{E}$, the difference between the mass of the heavy meson and the effective mass of the heavy quark; and $\Lambda$, the width of the heavymeson Bethe-Salpeter amplitude, we calculated the transition form factor $f_{+}(t)$ for all such processes on their entire kinematically accessible t-domain. Our study favored a value of the leptonic decay constant of the $\mathrm{B}$-meson, $\mathrm{fB}$, in the range $0.135-0.17 \mathrm{GeV}$ and with $\mathrm{E}=0.44 \mathrm{GeV}$ and $1 / \mathrm{A}=0.14 \mathrm{fm}$ we obtained the prediction $\mathrm{f}_{+}^{\mathrm{B} \pi}(0)=0.46$. We estimated that, as a result of neglecting $1 / \mathrm{m}_{\mathrm{C}}$ corrections, our calculated values of the slope of the heavy-to-heavy meson transition form factor, $\rho^{2}=0.87$, and $\mathrm{f}_{+}^{\mathrm{DK}}(0)=0.62$ are too low by approximately $15 \%$. However, the bulk of these corrections should cancel in our calculated values of $\mathrm{Br}(\mathrm{D} \rightarrow \pi \ell v) / \mathrm{Br}(\mathrm{D} \rightarrow \mathrm{K} \ell v)=0.13$ and $\mathrm{f}_{+}^{\mathrm{B} \pi}(0) / \mathrm{f}_{+}^{\mathrm{DK}}(0)=$ 1.16 , which are then robust predictions that agree with recent experimental results. An article describing this work was published. ${ }^{1}$ These studies are particularly timely, with the construction of many B-factories in the next few years.

*Joint Institute for Nuclear Research, Dubna, Russia

${ }^{1}$ M. A. Ivanov, Yu. L. Kalinovsky, P. Maris, and C. D. Roberts, Phys. Rev. C 57, 1991 (1998)

\section{a.3. Thermodynamic Properties of a Simple, Confining Model (C. D. Roberts, D. Blaschke, ${ }^{*}$ and S. Schmidt*)}

We studied the equilibrium thermodynamics of a simple, confining, Dyson-Schwinger equation model of 2-flavor QCD at finite temperature and chemical potential. The model has two phases: one characterized by confinement and dynamical chiral symmetry breaking; and the other by their absence. The phase boundary is defined by the zero of the vacuum-pressure difference between the confined and deconfined phases.
Chiral symmetry restoration and deconfinement are coincident with the transition being of first order, except for $\mu=0$, where it is second order. We showed that nonperturbative modifications of the dressed-quark propagator persist into the deconfined domain and lead to a dispersion law modified by a dynamically-generated, momentum-dependent mass-scale, and hence that the

*University of Rostock, Germany 
Stefan-Boltzmann limit for the bulk thermodynamic quantities is attained only for large values of temperature and chemical potential. This behavior at finite temperature was observed in numerical simulations of lattice-QCD but our study is the first to demonstrate that it is mirrored at finite chemical potential. An article describing this work was published. 1

${ }^{1}$ D. Blaschke, C. D. Roberts, and S. Schmidt, Phys. Lett. B 425, 232 (1998)

\section{a.4. Chemical Potential Dependence of $\pi$ and $\rho$ properties (P. Maris, C. D. Roberts, and S. Schmidt*)}

Using a confining, Dyson-Schwinger equation model of QCD at finite temperature $T$ and chemical potential $\mu$, we studied the behavior of $\langle\bar{q} q\rangle, m_{\pi}, f_{\pi}$ and the masses of the $\rho$ - and $\phi$-mesons. For each of these quantities there is a necessary anticorrelation between its response to $\mathrm{T}$ and its response to $\mu$. ( $(\langle\overline{\mathrm{q}} \mathrm{q}\rangle)$ and $\mathrm{f}_{\pi}$ decrease with $T$ and increase with $\mu ; m_{\pi}$ is almost insensitive to $T$ and $\mu$ until very near the border of the confinement domain; and the mass of the longitudinal component of the vector mesons increases with $T$ and decreases with $\mu$. At $T=0$, the $\rho$-meson mass, $m_{\rho}$, is reduced by approximately $15 \%$ at nuclear matter density. These results are a consequence of the necessary momentumdependence of the dressed-quark self-energy, and provide counterexamples to the "universal scaling" law that has been conjectured. An article describing this work was published. 1

*University of Rostock, Germany

${ }_{1}^{1}$ P. Maris, C. D. Roberts, and S. Schmidt, Phys. Rev. C 57, R2821 (1998)

\section{a.5. Deconfinement at Finite Chemical Potential (C. D. Roberts, A. Bender,* G. Poulis, * A. W. Thomas, ${ }^{*}$ and S. Schmidt $\dagger$ )}

In a confining, renormalizable, Dyson-Schwinger equation model of two-flavor QCD we explored the chemical-potential dependence of the dressed-quark propagator, which provides a means of determining the behavior of the chiral and deconfinement order parameters, and low-energy pion observables. We found coincident, first-order deconfinement and chiral symmetry restoration transitions at $\mu_{c}=375 \mathrm{MeV}$. $\mathrm{f}_{\pi}$ is insensitive to $\mu$ until $\mu-\mu_{0}=0.7 \mu_{c}$ when it begins to increase rapidly. $\mathrm{m}_{\pi}$ is weakly dependent on $\mu$, decreasing slowly with $\mu$ and reaching a minimum $6 \%$ less than its $\mu=0$ value at $\mu=\mu_{0}$. In a two-flavor free-quark gas at $\mu=\mu_{c}$ the baryon number density would be approximately $3 \rho_{0}$, where $\rho_{0}=0.16 \mathrm{fm}^{-3}$ while in such a gas at $\mu_{0}$ the density is $\rho_{0}$. These results preclude the possibility of pion condensation and provide a counterexample to the "universal scaling" laws that have been conjectured. They also suggest that a quark gluon plasma exists in the core of neutron stars. An article describing this work was published. ${ }^{1}$

*University of Adelaide, Australia, tUniversity of Rostock, Germany

1 A. Bender, G. Poulis, C. D. Roberts, S. Schmidt, and A. W. Thomas, Phys. Lett. B 431, 263 (1998) 


\section{a.6. Asymptotic Behavior of $\mathbf{F} \pi\left(\mathbf{q}^{2}\right)$ (P. Maris and C. D. Roberts)}

The discovery of a necessary pseudovector component in the pion Bethe-Salpeter amplitude eliminates a longstanding misapprehension about its bound-state structure. It has important observable consequences. For example, at large pion energy this piece of the amplitude is dominant and hence controls the large- $q^{2}$ behavior of the electromagnetic pion form factor. In a model-independent analysis we showed that the momentum dependence of the pseudovector amplitude is the same as that of the quark mass function, i.e., that it behaves as $1 / \mathrm{k}^{2}$, up to logarithmic corrections. From this it follows that $\mathrm{q}^{2} \mathrm{~F}_{\pi}\left(\mathrm{q}^{2}\right)=$ constant at large spacelike $-q^{2}$, in agreement with perturbative analyses in QCD. If the pseudovector component is neglected one finds $q^{4} F_{\pi}\left(q^{2}\right)=$ constant. The Dyson-Schwinger equation approach that we employed in deriving these results also allows a calculation of low-energy pion observables, such as $m_{\pi}, f_{\pi}$ and $r_{\pi}$. Hence, we realized a framework that unifies both low- and high-energy scales in studying the strong interaction. An article describing this work was published. 1

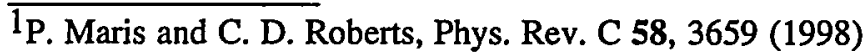

\section{a.7. Analysis of Chiral and Thermal Susceptibilities (C. D. Roberts, D. Blaschke,* A. Höll, * and S. Schmidt*)}

We calculated the chiral and thermal susceptibilities for two confining Dyson-Schwinger equation models of QCD with two light flavors, a quantitative analysis of which yields the critical exponents, $\beta$ and $\delta$, that characterize the seeond-order chiral symmetry restoration transition. The method itself is of interest, minimizing the influence of numerical noise in the calculation of the order parameter for chiral symmetry breaking near the critical temperature. For the more realistic of the two models we find: $T_{c} \sim 153 \mathrm{MeV}$, and the non-meanfield values: $\beta=0.46 \pm 0.04, \delta=4.3 \pm 0.3$ and $1 /(\beta \delta)=0.54 \pm 0.05$. We found that the critical temperature is not sensitive to the details of the model and discussed our results in comparison with those of other models and lattice simulations. An article describing this work was published. 1

*University of Rostock, Germany

${ }^{1}$ D. Blaschke, A. Höll, C. D. Roberts, and S. Schmidt, Phys. Rev. C 58, 1758 (1998)

\section{a.8. Infrared Behavior of Propagators and Vertices (C. D. Roberts, P. Maris, and F. T. Hawes*)}

We elucidated constraints imposed by confinement and dynamical chiral symmetry breaking on the infrared behavior of the dressed-quark and -gluon propagators, and dressed-quark-gluon vertex. In covariant gauges the dressing of the gluon propagator is completely specified by $P\left(\mathrm{k}^{2}\right):=1 /\left[1+\Pi\left(\mathrm{k}^{2}\right)\right]$, where $\Pi\left(\mathrm{k}^{2}\right)$ is the vacuum polarization. In the absence of particle-like singularities in the dressed-quark-gluon vertex, extant proposals for the dressed-gluon propagator that manifest $P\left(k^{2}=0\right)=0$ and $\max \left(\not\left(k^{2}\right)\right)-10$ neither confine quarks nor break chiral symmetry dynamically. This class includes all existing estimates of $P\left(k^{2}\right)$ via numerical simulations. An article describing this work was published. 1

*University of Adelaide, Australia

${ }^{1}$ F. T. Hawes, P. Maris, and C. D. Roberts, Phys. Lett. B 440, 353 (1998) 


\section{a.9. A Dynamical, Confining Model and Hot Quark Stars (C. D. Roberts, D. Blaschke,* S. Schmidt,* H. Grigorian, $\dagger$ and G. Poghosyan $\dagger$ )}

We explored the consequences of an equation of state (EOS) obtained in a confining Dyson-Schwinger equation model of QCD for the structure and stability of non-strange quark stars at finite-T, and compared the results with those obtained using a bag-model EOS. Both models support a temperature profile that varies over the star's volume and the consequences of this are model independent; e.g., the maximum mass of a quark star is reduced as the central temperature is increased and the maximum attainable radius of a pure quark star is $R$ $\sim 8-10 \mathrm{~km}$, as illustrated in Fig. IV-1. However, in our model the analogue of the bag pressure is $(T, \mu)$ dependent, which is not the case in the bag model. This is a significant qualitative difference and comparing the results effected a primary goal of elucidating the sensitivity of quark star properties to the form of the EOS. An article describing this work was accepted for publication.

*University of Rostock, Germany

†Yerevan State University, Armenia

Fig. IV-1. Upper figure - gravitational mass of quark stars as a function of the central energy density: our model (upper panel); bag-model (lower panel). At a central temperature of $T(0)=50 \mathrm{MeV}$ (dashed lines) the maximum mass is lowered by $\sim 20 \%$ when compared to the $T(0)=0$ case (solid lines). Lower figure - gravitational mass of quark stars as a function of the radius. At $T(0)=50 \mathrm{MeV}$, the objects are more compact than when $T(0)=0$.
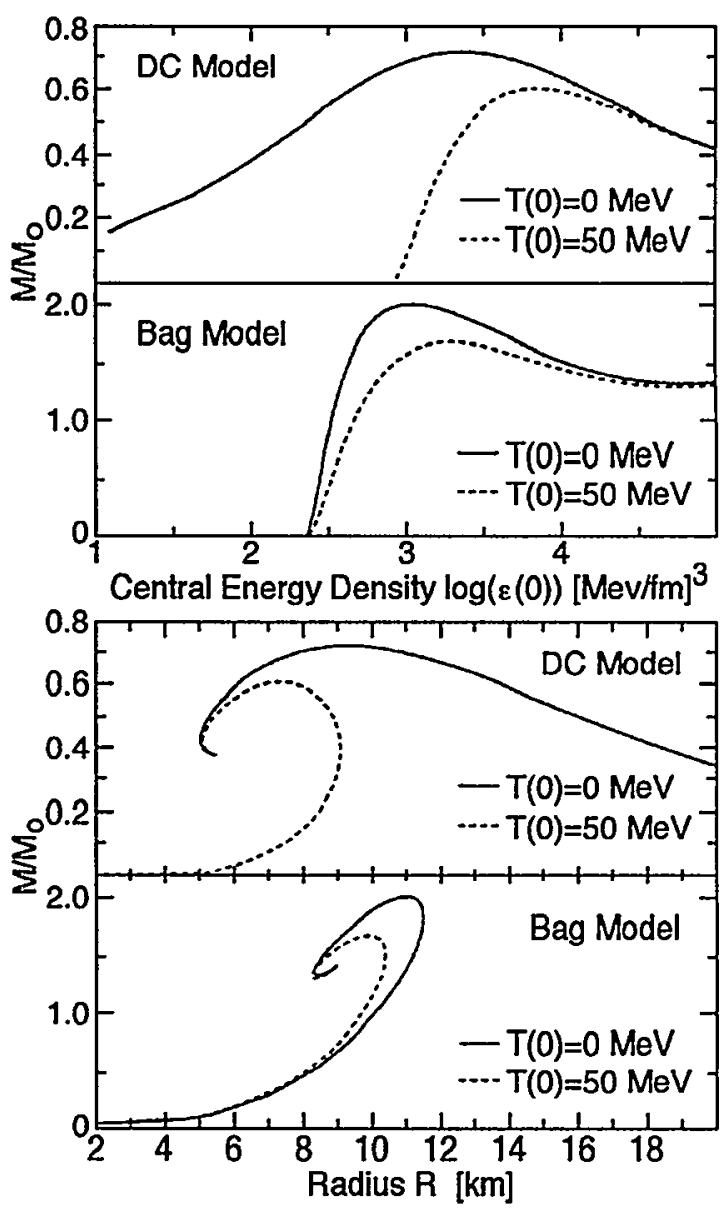

\section{a.10. Mean Field Exponents and Small Quark Masses (C. D. Roberts, P. Maris, and A. Höll*)}

We demonstrated that the restoration of chiral symmetry at finite-T in a class of confining Dyson-Schwinger equation (DSE) models of $\mathrm{QCD}$ is a mean field transition, and that an accurate determination of the critical exponents using the chiral. and thermal susceptibilities requires very small values of the currentquark mass: $\log _{10}\left(\mathrm{~m} / \mathrm{m}_{\mathrm{u}}\right) \leq-5$, making their determination via lattice-QCD simulations very difficult. Other classes of DSE models characterized by qualitatively different interactions also exhibit a mean field transition. Incipient in this observation is the suggestion that mean field exponents are a result of the gap equation's fermion substructure and not of the interaction. This conclusion can likely only be false if $1 / \mathrm{N}_{\mathrm{c}}$-corrections to the quark-gluon vertex are large in the vicinity of the transition. An article describing this work was accepted for publication.

*University of Rostock, Germany 


\section{a.11. Survey of Heavy-meson Observables (C. D. Roberts, M. A. Ivanov,* and Yu. L. Kalinovsky†)}

We employed a Dyson-Schwinger equation model to effect a unified and uniformly accurate description of light- and heavy-meson observables, which we characterized by heavy-meson leptonic decays, semileptonic heavy-to-heavy and heavy-to-light transitions: $\mathrm{B} \rightarrow \mathrm{D}^{*}, \mathrm{D}, \rho, \pi ; \mathrm{D} \rightarrow \mathrm{K}^{*}, \mathrm{~K}$; radiative and strong decays: $\mathrm{B}^{*} \rightarrow \mathrm{B} \gamma ; \mathrm{D}^{*} \rightarrow \mathrm{D} \gamma, \mathrm{D} \pi$; and the rare $B \rightarrow K^{*} \gamma$ flavor-changing neutral-current process. The results are illustrated in Table IV-I and Fig. IV-2. We elucidated the heavy-quark limit of these processes and, using a model-independent mass formula valid for all nonsinglet pseudoscalar mesons, demonstrated that their mass rises linearly with the mass of their heaviest constituent. In our numerical calculations we eschewed a heavy-quark expansion and relied instead on the observation that the dressed c,b-quark mass functions are well approximated by a constant, interpreted as their constituent-mass: we found $\hat{\mathrm{M}}_{\mathrm{c}}=1.29 \mathrm{GeV}$ and $\hat{\mathrm{M}}_{\mathrm{b}}=4.54 \mathrm{GeV}$. The calculated heavy-meson leptonic decay constants and transition form factors are a necessary element in the experimental determination of CKM matrix elements. The results also show that this framework, as employed hitherto, is well able to describe vector meson polarization observables. An article describing this work was submitted for publication.

*BLTP, JINR, Dubna, †LCTA, JINR, Dubna

Table IV-I. Calculated values of a range of light- and heavy-meson observables: leptonic decay constants, charge radii, strong coupling constants, decay widths and branching ratios.

\begin{tabular}{|c|c|c|c|c|c|}
\hline & Obs. & Calc. & & Obs. & Calc. \\
\hline $\mathrm{f}_{\mathrm{K}^{\mathrm{r}} \mathrm{K}}$ & $0.472 \pm 0.038$ & 0.47 & $-\mathrm{f}_{\mathrm{K}}^{2} \mathrm{r}_{\mathrm{K}}^{2} \mathrm{o}$ & $(019 \pm 0.05)^{2}$ & $(0.15)^{2}$ \\
\hline$g_{\rho \pi \pi}$ & $6.05 \pm 0.02$ & 5.88 & $\Gamma_{\mathrm{D}} * 0(\mathrm{MeV})$ & $<2.1$ & 0.019 \\
\hline $\mathrm{g}_{\mathrm{K}}^{*} \mathrm{~K} \pi^{\mathrm{o}}$ & $6.41 \pm 0.06$ & 5.96 & $\Gamma_{\mathrm{D}^{*+}}(\mathrm{keV})$ & $<131$ & 28.7 \\
\hline $\mathrm{g}_{\rho}$ & $5.03 \pm 0.012$ & 5.93 & $\Gamma_{\mathrm{B}^{*+} \mathrm{B}^{+} \gamma}(\mathrm{keV})$ & & 0.028 \\
\hline $\mathrm{f}_{\mathrm{Bs}}(\mathrm{GeV})$ & $0.195 \pm 0.035$ & 0.266 & $\mathrm{G}_{\mathrm{B}} * \mathrm{O}_{\mathrm{B}} \mathrm{O} \gamma(\mathrm{keV})$ & & 0.022 \\
\hline $\mathrm{f}_{\mathrm{D}}^{*}(\mathrm{GeV})$ & & 0.238 & $\mathrm{~B}\left(\mathrm{D}^{*+} \rightarrow \mathrm{D}^{+} \pi^{0}\right)$ & $0.306 \pm 0.025$ & 0.322 \\
\hline $\mathrm{f}_{\mathrm{B}} *(\mathrm{GeV})$ & & 0.225 & $\mathrm{~B}\left(\mathrm{D}^{*+} \rightarrow \mathrm{D}^{\mathrm{o}} \pi^{+}\right)$ & $0.683 \pm 0.014$ & 0.673 \\
\hline $\mathrm{f}_{\mathrm{Ds}} / \mathrm{f}_{\mathrm{D}}$ & $1.10 \pm 0.06$ & 1.23 & $\mathrm{~B}\left(\mathrm{D}^{*+} \rightarrow \mathrm{D}^{+} \gamma\right)$ & $0.011_{-0.007}^{+0.021}$ & 0.005 \\
\hline $\mathrm{f}_{\mathrm{Bs}} / \mathrm{f}_{\mathrm{B}}$ & $1.14 \pm 0.08$ & 1.47 & $\mathrm{~B}\left(\mathrm{D}^{* 0} \rightarrow \mathrm{D}^{0} \pi^{0}\right)$ & $0.619 \pm 0.029$ & 0.641 \\
\hline $\mathrm{f}_{\mathrm{D}} * / \mathrm{F}_{\mathrm{D}}$ & & 1.34 & $\mathrm{~B}\left(\mathrm{D}^{*} \mathrm{o} \rightarrow \mathrm{D}^{0} \gamma\right)$ & $0.381 \pm 0.029$ & 0.359 \\
\hline $\mathrm{f}_{\mathrm{B}} / / \mathrm{f}_{\mathrm{B}}$ & & 1.25 & $\mathrm{~B}\left(\mathrm{~B} \rightarrow \mathrm{K}^{*} \gamma\right)$ & $(5.7 \pm 3.3)_{10^{-5}}$ & 10.3 \\
\hline
\end{tabular}



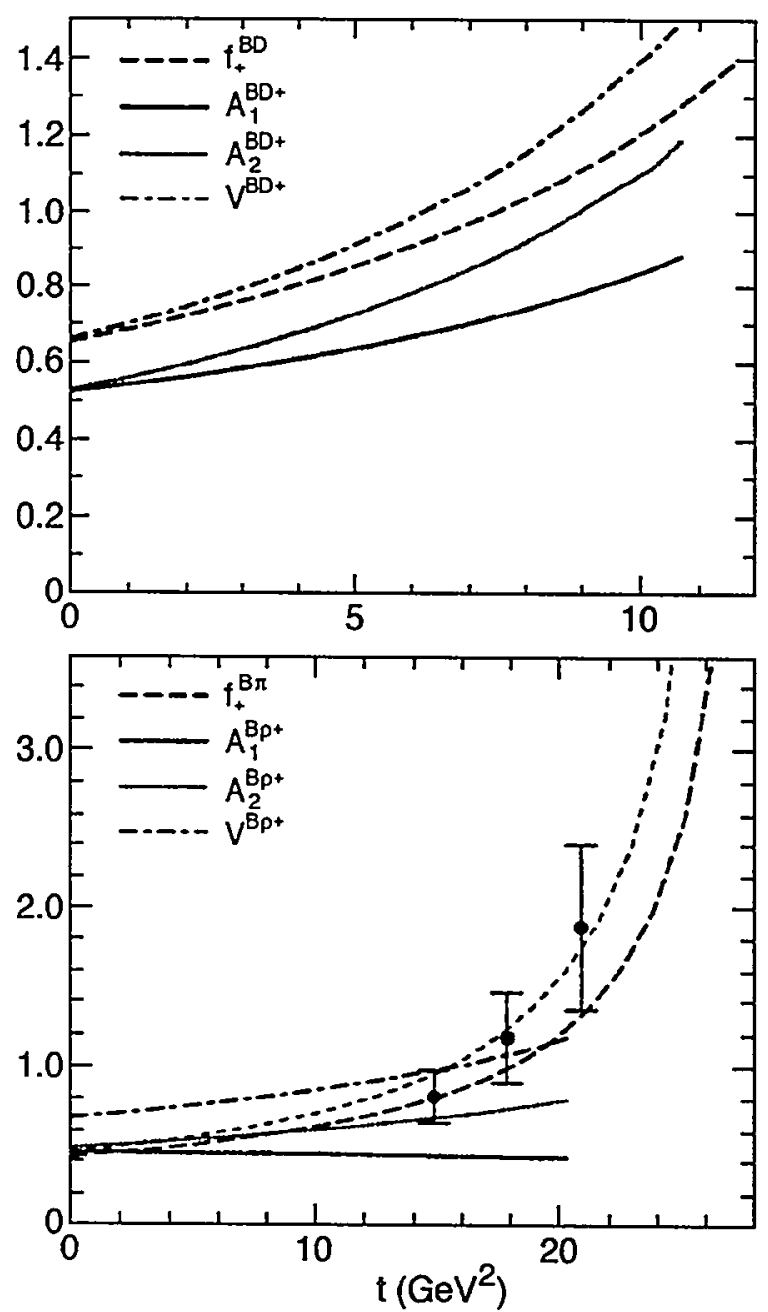

Fig. IV-2. Upper panel - calculated form of the semileptonic $B \rightarrow D$ and $B \rightarrow D^{*}$ form factors. Lower panel - the semileptonic $B \rightarrow \pi$ and $B \rightarrow \rho$ form factors with, for comparison, data from a lattice simulation and a vector dominance, monopole model: $f_{+}^{\mathrm{B} \rightarrow \pi}(t)=$ $0.46 /\left(1-t / m_{\mathrm{B}^{*}}^{2}\right)=5.325 \mathrm{GeV}$, the light, short-dashed line.

\section{a.12. Diquark Condensation at High Density (J. C. R. Bloch, C. D. Roberts, and S. Schmidt)}

Diquark correlations may play an important role in the strong interaction because ladder-like dressed-gluon exchange is attractive in the color anti-triplet scalar and pseudovector channels. This interaction, considered alone, yields a bound scalar $\left(m_{0^{+}}^{u d} \sim 740 \mathrm{MeV}\right)$ and vector $\left(\mathrm{m}_{1^{\mathrm{u}}}^{\text {ud }} \sim 950 \mathrm{MeV}\right)$ diquark, and predicts the formation of a diquark condensate at high baryon density. However, it cannot be a complete picture because real diquarks are not observed. The defect is the truncation. If one proceeds beyond the ladder-truncation one uncovers repulsive contributions that destroy diquark binding. We ane exploring how these contributions affect diquark condensation. 


\section{a.13. Electromagnetic Nucleon Form Factors. (J. C. R. Bloch, C. D. Roberts, and S. Schmidt)}

We are employing the Dyson-Schwinger equation framework to calculate the electromagnetic nucleon form factor. This is a first and important step in extending the successful meson phenomenology to the baryon sector, a long-held goal. The wealth of experimental nucleon form factor data, over a large range of $q^{2}$, means that this application will provide an excellent environment to test, improve and extend the approach. That will make possible calculations of the neutron electric form factor, the $\pi \mathrm{NN}$ coupling constant and many other quantities of contemporary experimental interest.

\section{a.14. Non-perturbative Gluon and Ghost Propagators in QCD (J. C. R. Bloch and D. Atkinson*)}

We solved a truncated set of coupled Dyson-Schwinger equations for the non-perturbative gluon and ghost propagators in QCD. Their asymptotic infrared behavior was calculated analytically: the ghost propagator is singular as $\left(\mathrm{p}^{2}\right)^{-\kappa-1}$ while the gluon propagator vanishes as $\left(\mathrm{p}^{2}\right)^{2 \mathrm{k}-1}$. The renormalization group invariant running coupling constructed from these results has an infrared fixed point. The value of $\kappa$ depends on the truncation scheme and seems to have a value between 0.7 and 1 . Using bare vertex Ansätze for the triple gluon vertex and for the ghost-ghost-gluon vertex and treating the angular integrals exactly yields the intriguing result $\mathrm{K}$ identical to 1 . The solution for all momenta was calculated numerically using a y-max angular averaging scheme. The complete numerical treatment with exact calculation of the angular integrals is currently underway.

*University of Groningen, The Netherlands

\section{a.15. Dynamical Chiral Symmetry Breaking in QCD (J. C. R. Bloch and D. Atkinson*)}

We are investigating the consequences of the nonperturbative behavior of an infrared vanishing gluon and infrared singular ghost propagator, determined from coupled Dyson-Schwinger equations, on the quark propagator and on the realization of dynamical chiral symmetry breaking. In a first naive attempt we constructed an effective running coupling with infrared fixed point from the gluon and ghost propagators and solved the Dyson-Schwinger equation for the quark propagator. We found that chiral symmetry breaking does occur, however the generated mass scale is too low to be successfully used in phenomenological hadronic

*University of Groningen, The Netherlands calculations. However, as the ghost fields do not appear explicitly in the quark equation, we are currently studying how they indirectly enter in the calculation of the quark propagator through the quark-gluon vertex as is easily noted from the Ward-Takahashi identity. First calculations using a modified Ball-Chiu vertex, incorporating the effects of the ghost propagator, do not seem to consistently yield dynamical chiral symmetry breaking without the introduction of some additional parametrization. 


\section{a.16. Electromagnetic Production of Mesons and Nucleon Resonances (T.-S. H. Lee, T. Sato,* and T. Yoshimoto*)}

A dynamical model of electromagnetic production of mesons on the nucleon was developed at energies accessible to TJNAF. Motivated by the existing QCDbased hadron models, we assume that the basic resonant interaction mechanisms of the model Hamiltonian are the absorption and emission of mesons by a bare quark core. The matrix elements of the nonresonant interactions which cannot be calculated from the considered hadron models are deduced from the effective

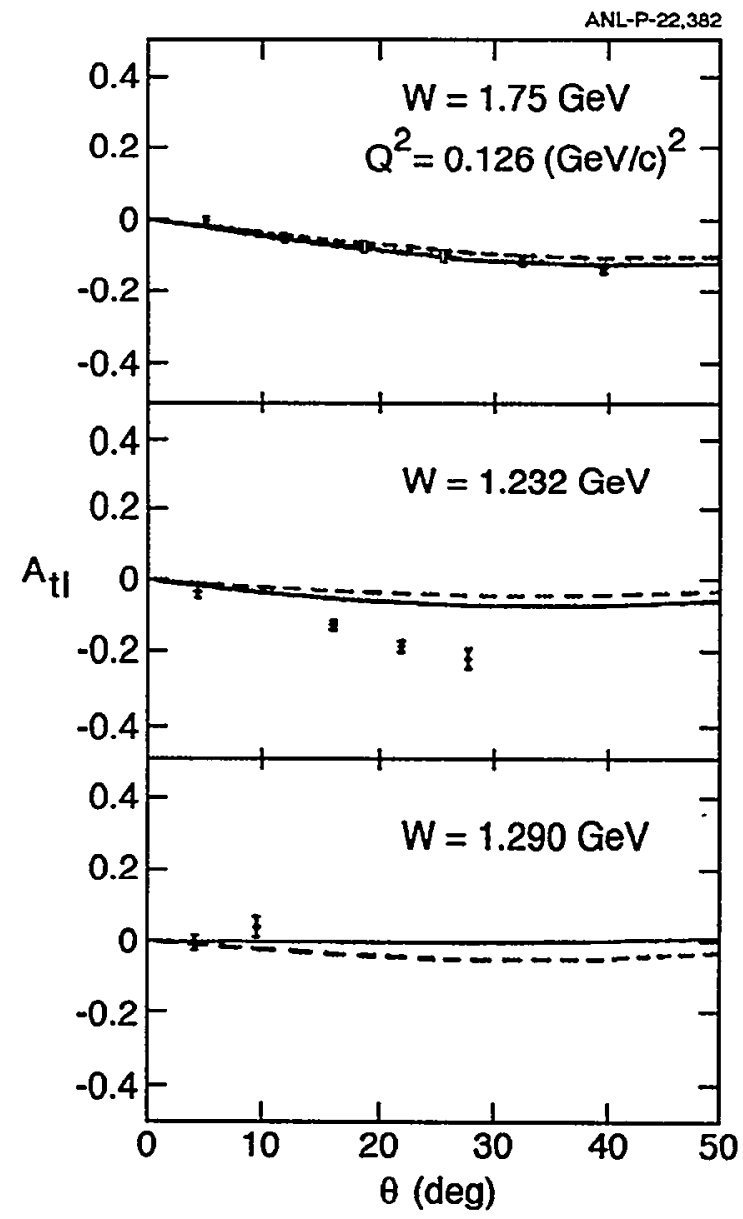

Fig. $N$-3. The predicted transverse-longitudinal asymmetry $A_{t l}$ are compared with the recent data from MIT-Bates. The dotted curves are from calculations with no $\Delta$ deformation.
Lagrangians with chiral symmetry. The standard projection operator technique was applied to obtain a set of unitary scattering equations for describing $\pi \mathrm{N}$ and $\gamma \mathrm{N}$ reactions up to the $\mathrm{GeV}$ region where the $\eta$ and $2 \pi$ channels are important. In FY 1998, we showed that our model in the $\Delta$ excitation region is consistent with the recent $\mathrm{p}\left(\mathrm{e}, \mathrm{e}^{\prime} \pi\right)$ data from TJNAF and MIT-Bates, as shown in Fig. IV-3. Our current focus is to apply our approach to examine how the quark-quark interactions within the constituent quark model can be directly determined by the $\pi \mathrm{N}$ and $\gamma \mathrm{N}$ reaction data.

The main task is to calculate the meson-baryon and photon-baryon form factors. Our current focus is on the $S_{11}$ resonances and their coupling with the $\gamma \mathrm{N}, \pi \mathrm{N}$, $\pi \Delta$ and $\eta \mathrm{N}$ channels. We found that the form factors predicted by the one-gluon-exchange (oge), one-meson-

*Osaka University, Japan

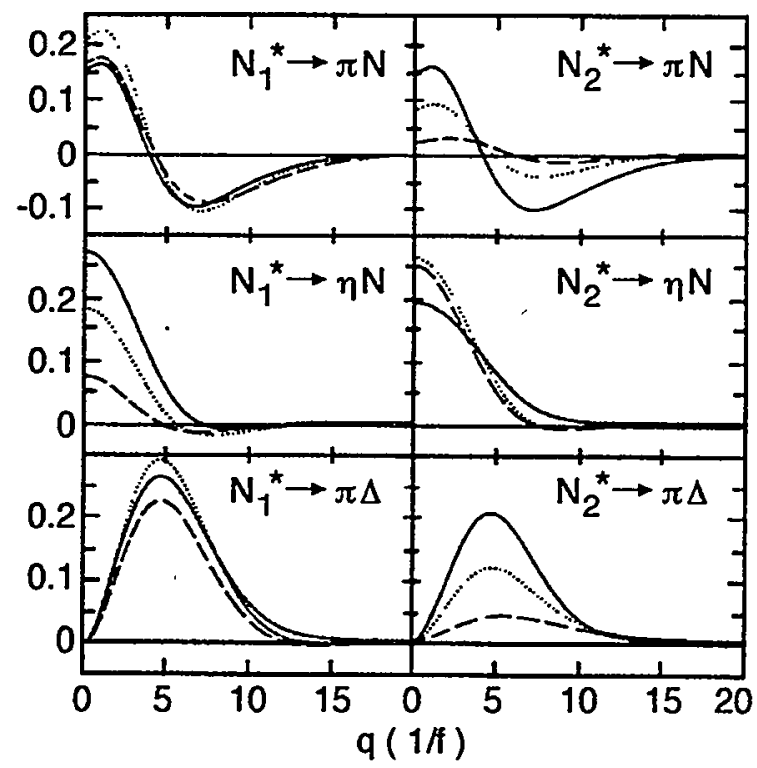

Fig. IV-4. The $N^{*} \rightarrow \pi N, \eta N, \pi \Delta$ form factors. The solid, dotted and dashed curves are respectively from the oge, ome, and ogme models. 
exchange (ome), and a phenomenological mixture (ogme) of oge and ome can be distinguished by the $\pi \mathrm{N}$ scattering data. Their differences are shown in Fig. IV-4. A phenomenological quark-quark potential was extracted from fitting the $\pi \mathrm{N}$ phase shifts in $S_{11}$ channel. 'A paper describing our results is being prepared. We are now extending this effort to investigate other $\mathrm{N}^{*}$ resonances.

\section{a.17. Study of the $\mathbf{N} \Delta$ Interactions With $\mathbf{d}\left(\mathrm{e}^{\prime} \mathrm{e}^{\prime} \pi\right)$ Reactions (T.-S. H. Lee)}

The dynamical model described in section a.16 was used to predict the $\mathrm{d}\left(\mathrm{e}, \mathrm{e}^{\prime} \pi\right)$ reaction in the $\Delta$ excitation region. The predictions are in agreement with the preliminary results from TJNAF. It is shown that the polarization observables of $\mathrm{d}\left(\mathrm{e}, \mathrm{e}^{\prime} \pi\right)$ reactions are useful for investigating the $N \Delta$ interactions which are crucial in exploring the $\Delta$ components in nuclei and the properties of $\Delta$-rich systems created in relativistic heavy-ion collisions. Our predictions for a typical kinematics are shown in Fig. IV-5.

Fig. IV-5. The predicted differential cross sections (in units of $\left.\mathrm{pb} / \mathrm{sr}^{2}-\mathrm{MeV}^{2}\right)$ and asymmetries for $d\left(e, e^{\prime} \pi\right)$ reaction at $E_{e}=880 \mathrm{MeV}, E_{e}=580 \mathrm{MeV}, \theta_{e, e}=20^{\circ}$ and $\theta_{y, \pi}=30^{\circ}$. The $x$-axis is the pion momentum in unit of $\mathrm{MeV} / \mathrm{c}$. The asymmetries are defined as $A(B, T)$ with $B$ and $T$ denoting respectively the beam and target polarization directions. The solid curves are the full calculations. The dotted curves are obtained when the $N \Delta$ interaction is turned off.
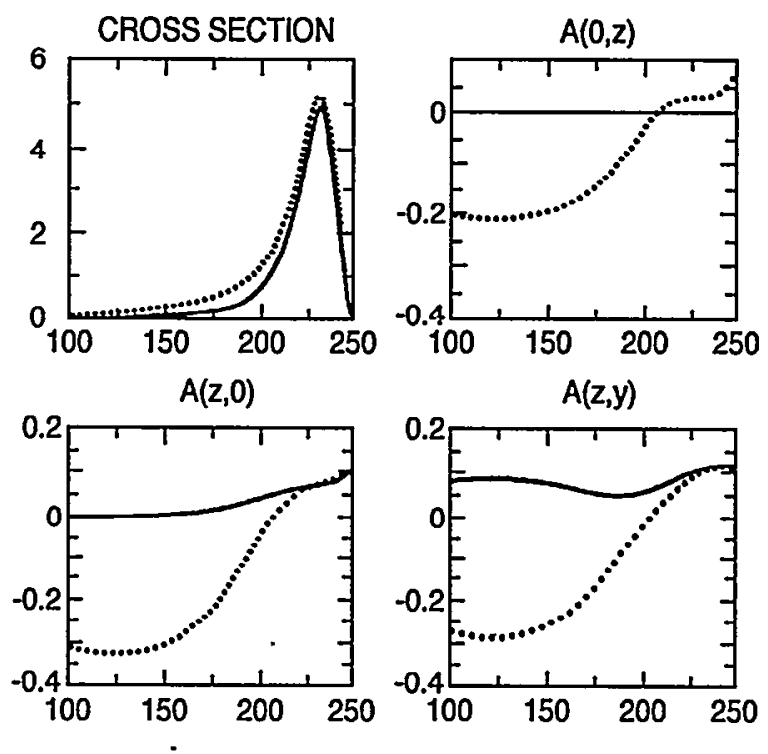

\section{a.18. Structure of the Vector Meson Photoproduction Amplitude at a Few GeV (T.-S. H. Lee, A. Titov, ${ }^{*}$ and H. Toki†)}

The structure of the $\phi$ photoproduction amplitude in the $\sqrt{s} \sim 2-5 \mathrm{GeV}$ region is analyzed based on Pomeronexchange and meson-exchange mechanisms. The SU(3) symmetry and the $\phi$ decay widths are exploited to determine the parameters that are needed to predict the amplitudes due to pseudoscalar meson $\left(\pi^{\mathbf{0}}, \eta\right)$ exchange, scalar meson $\left(\sigma, a_{0}, f_{o}\right)$ exchange, and the $\phi$-radiation from the nucleon. In addition to the universally accepted Pomeron exchange with an intercept $\alpha(0) \sim$ 1.08 , we investigate the role of a second Pomeron with $\alpha(0)<0$, as inspired by the glueball $\left(\mathrm{J}^{\pi}=0^{+}, \mathrm{M}_{\mathrm{b}}{ }^{2} \sim 3\right.$ $\mathrm{GeV}^{2}$ ) predicted by Lattice QCD calculation and Dual Landau-Ginsburg model. It is found that the existing limited data at low energies near threshold can accommodate either the second Pomeron or the scalar meson exchange. The differences between these two competing mechanisms are shown to have profound effects on various density matrices which can be used to calculate the cross sections as well as various single and double polarization observables. We predict a definite isotopic effect: polarization observables of $\phi$ photoproduction on the proton and neutron targets can have differences of a factor 2 and more. In Fig. IV- 6 we show how the second Pomeron with $\alpha(0)<0$ can account for the $\phi$ total cross section data. A paper describing our results is being prepared for publication. The extension of our approach to investigate $\rho$ production on the nucleon and nuclear targets is in progress, aiming at interpreting the recent data from DESY and TJNAF.

*Bogoluibov Laboratory of Theoretical Physics, JNR, Dubna, Russia

†Research Center for Nuclear Physics, Osaka University, Japan 

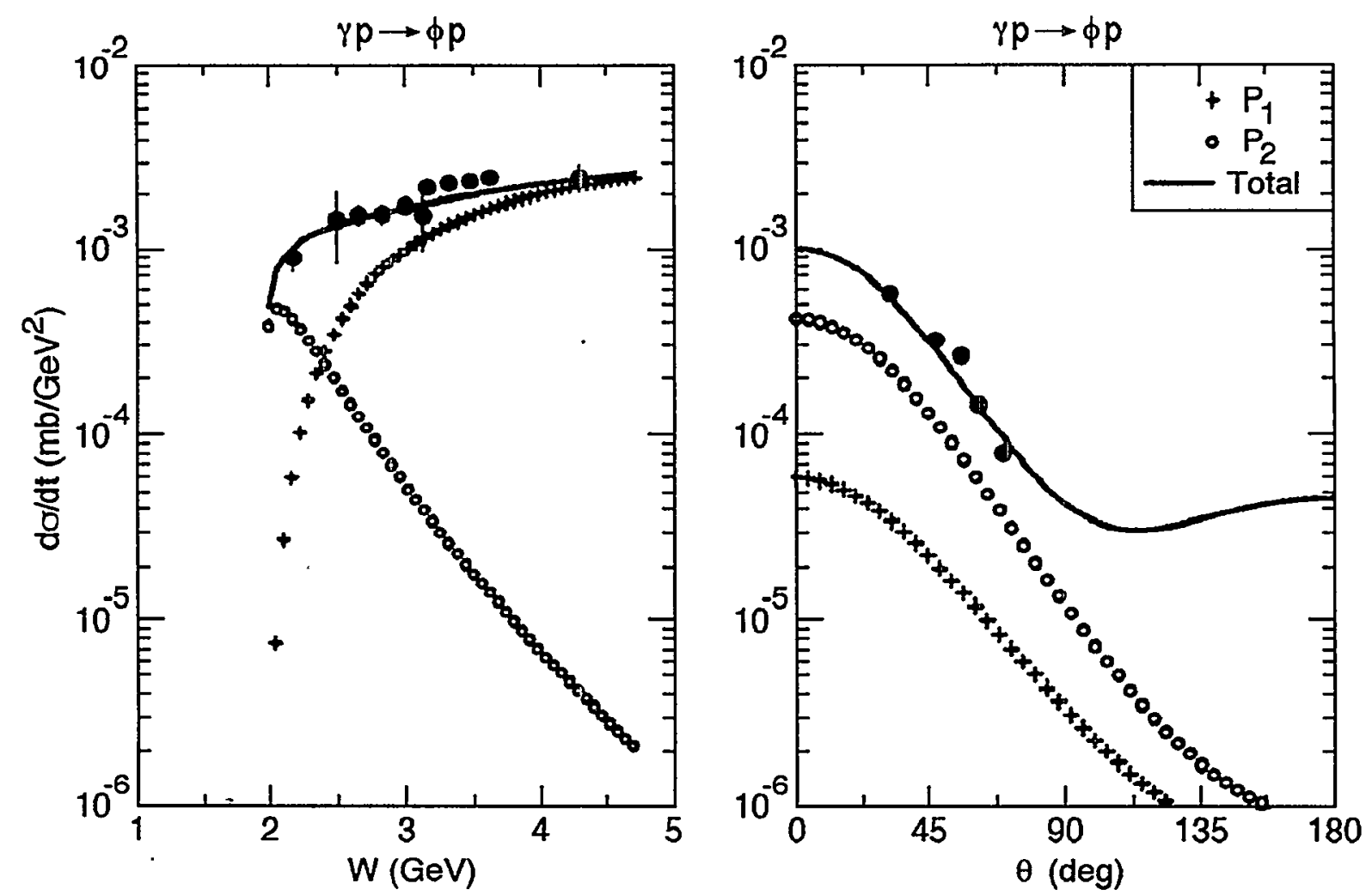

Fig. IV-6. The differential cross sections of $\not p \rightarrow \phi p$ reaction. $P_{i}$ denotes the contribution from the ith Pomeron.

\section{a.19. Non-perturbative Chiral Approach to $\mathbb{K}^{-p} \rightarrow \gamma \mathbf{Y}$ Reactions (T.-S. H. Lee, J. A Oller,* E. Oset,* and A. Ramos $\dagger$ )}

A recently developed nonperturbative chiral approach to describe the $S=-1$ meson-baryon reactions was extended to investigate the near threshold $\mathrm{K}^{-} \mathrm{p} \rightarrow \gamma \Lambda, \gamma \Sigma^{0}$ reactions. With the parameters governed by chiral SU(3) symmetry, we show that the predicted branching ratios $\Gamma_{\mathrm{K}^{-} \mathrm{p} \rightarrow \gamma \Lambda} \Gamma_{\mathrm{K}^{-} \mathrm{p}} \rightarrow$ all and $\Gamma_{\mathrm{K}^{-} \mathrm{p} \rightarrow \gamma \Sigma^{0} / \Gamma_{\mathrm{K}^{-} \mathrm{p}}}$ $\rightarrow$ all are close to the experimental values. The coupling with the $\eta$ channels, which was shown to be important in the $S=-1$ meson-baryon reactions, is also found to be significant here. Our results are consistent with the interpretation of the $\Lambda(1405)$ as a quasi-bound meson-baryon state as found in other similar chiral approaches. A paper describing our results was published. 1

*University of Valencia, Spain

†University of Barcelona, Spain

${ }^{1}$ T.-S. H. Lee, J. A. Oller, E. Oset, and A. Ramos, Nucl. Phys. A643, 402 (1998) 


\section{a.20. Soft-core Hyperon-nucleon and Hyperon-hyperon Potentials (V. G. J. Stoks* and Th. A. Rijken $\dagger$ )}

A new soft-core OBE potential model was constructed for the low-energy YN interactions. The potentials are generated by the exchange of nonets of pseudoscalar, vector, and scalar mesons. As standard in the Nijmegen soft-core models, we also include the $\mathrm{J}=0$ contributions from the tensor $f_{2}, f_{2}^{\prime}, a_{2}$, and Pomeron Regge trajectories, and use Gaussian form factors to guarantee that the potentials have a soft behavior near the origin. An important innovation with respect to the previous soft-core potential is the assignment of the cutoff masses for the baryon-baryon-meson (BBM) vertices in accordance with broken $\mathrm{SU}(3)_{\mathrm{F}}$, which serves to connect the NN and the YN channels. As a novel feature, we allow for medium strong breaking of the coupling constants, using the ${ }^{3} \mathrm{P}_{\mathrm{o}}$ model with a Gell-Mann-Okubo hypercharge breaking for the BBM coupling. Charge-symmetry breaking in the $\Lambda \mathrm{p}$ and $\Lambda \mathrm{n}$ channels is included as well. We constructed six hyperon-nucleon potentials which describe the available YN cross section data equally well, but which exhibit some differences on a more detailed level. The differences are constructed such that the constructed models encompass a range of scattering lengths in the
$\Sigma \mathrm{N}$ and $\Lambda \mathrm{N}$ channels. In all cases, we obtained $\chi^{2} / \mathrm{N}_{\text {data }} \sim 0.55$ for $35 \mathrm{YN}$ data. In particular, we were able to fit the precise experimental datum $\mathrm{r}_{\mathrm{R}}=0.468 \pm$ 0.010 for the inelastic capture ratio at rest. For the scalar-meson mixing angle we obtained values $\tau_{S}=37^{\circ}$ $40^{\circ}$, which points to almost ideal mixing angles for the scalar $\bar{q} q$ states. The G-matrix results indicate that the remarkably different spin-spin terms of the six potentials appear specifically in the energy spectra of $\Lambda$ hypernuclei. A paper describing our results was published. ${ }^{1}$

The model was extended to obtain baryon-baryon potentials for the complete baryon octet. By using the SU(3) symmetry, the resulting potentials for the $S=-2,-3$, and -4 sectors do not contain any additional free parameters, which makes them among the first models of this kind. Various properties of the potentials are illustrated by comparing scattering lengths, total cross sections, and searching for the possible existence of bound states. A paper describing our results is being prepared for publication.

*ANL and University of Adelaide, Australia, $\uparrow$ University of Nijmegen, Netherlands

1V. G. J. Stoks and Th. A. Rijken, Phys. Rev. C 59, 21 (1999)

\section{a.21. Strange Hadron Matter and SU(3) Symmetry (T.-S. H. Lee and V. G. J. Stoks*)}

We calculate saturation curves for strange hadron matter using the SU(3) baryon-baryon potentials described in section a.20. All possible interaction channels within the baryon octet (consisting of $N, \Lambda, \Sigma$, and $\Xi$ ) are considered. It is found that a small $\Lambda$ fraction in nuclear matter slightly increases binding, but that larger fractions $(>10 \%)$ rapidly cause a decrease. Our results are shown in Fig. IV-7. Charge-neutral $N, \Lambda, \Xi$ systems, with equal densities for nucleons and cascades, are only very weakly bound. The dependence of the

*ANL and University of Adelaide, Australia binding energies on the strangeness per baryon, $f_{s}$, is predicted for various $\mathrm{N}, \Lambda, \Xi$ and $\mathrm{N}, \Lambda, \Sigma, \Xi$ systems. These results are shown in Fig. IV-8. The implications of our results in relativistic heavy-ion collisions and the core of a dense star are discussed. We also discuss the differences between our results and previous hadron matter calculations. A paper describing our results was submitted for publication. 

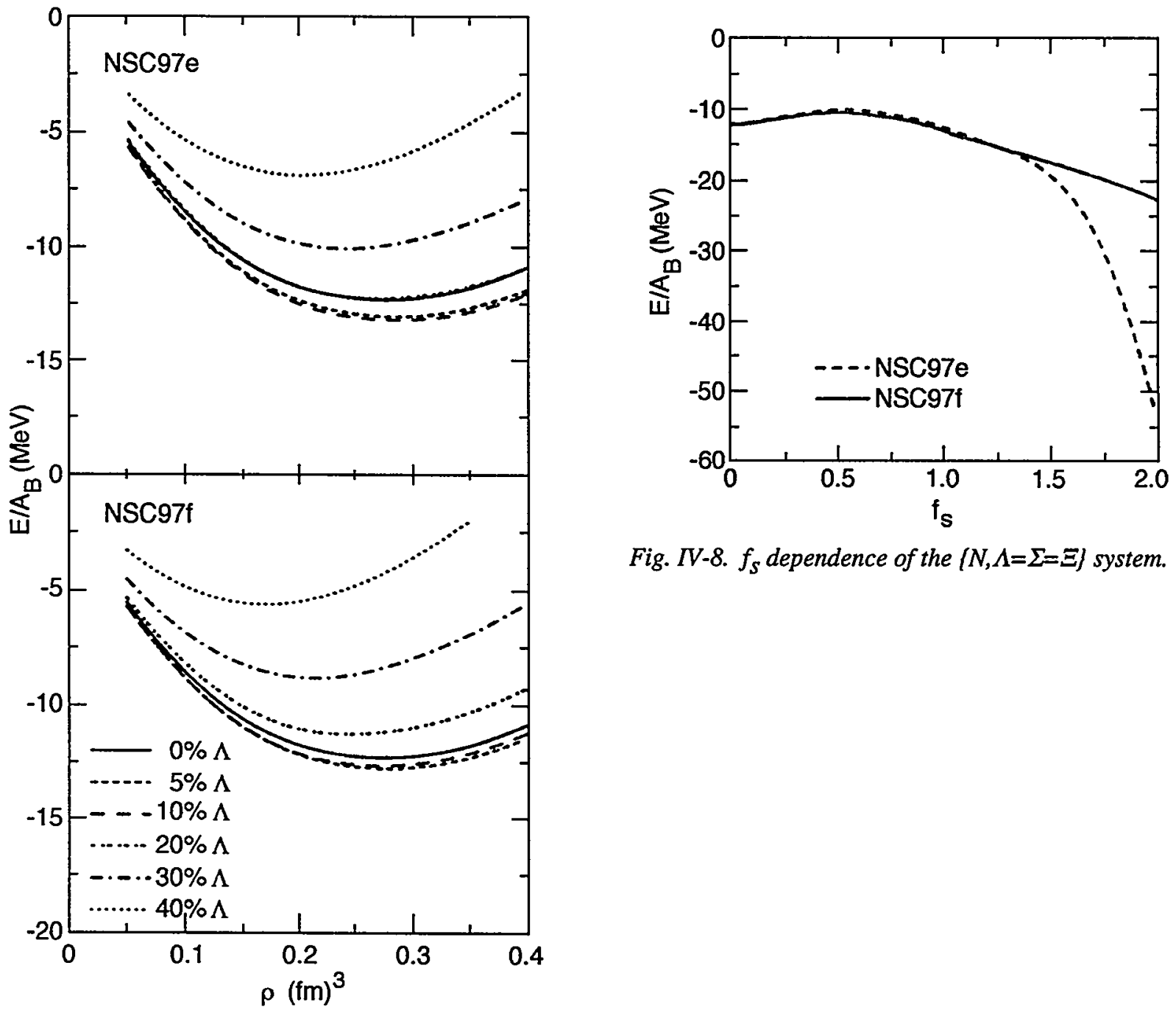

Fig. $I V-8 . f_{S}$ dependence of the $[N, \Lambda=\Sigma=\Xi]$ system.

Fig. IV-7. Saturation of $N+\Lambda$ systems for various fractions $\rho_{\Lambda} /\left(\rho_{N}+\rho_{\Lambda}\right)$.

\section{a.22. Study of Hyperon-Nucleon Interactions with d(e,e'K) Reactions (T.-S. H. Lee, V. G. J. Stoks, ${ }^{*}$ and B. Saghai $\dagger$ )}

The $d\left(e, e^{\prime} K^{+}\right)$reaction was investigated by using the kaon production amplitudes developed by the SaclayLyon collaboration. Our focus is on the dependence of the reaction cross sections on the final hyperon-nucleon interactions, aiming at testing the SU(3) models of baryon-baryon potentials described in the section a.20. The predicted results for $\Lambda$ production are in agreement with the preliminary data from TJNAF. To account for the $\Sigma$ production, we are refining the Saclay-Lyon amplitudes to fit the new data from TJNAF and extending the model to also account for the kaon production on the neutron.

*ANL and University of Adelaide, Australia, †CEA-Saclay, France 


\section{a.23. Study of the pn $\rightarrow$ p $\Lambda$ Reactions (T.-S. H. Lee, V. G. J. Stoks, ${ }^{*}$ T. Ogaito, $\uparrow$ H. Nabetani, $\ddagger$ and T. Sato $\ddagger$ )}

The $\mathrm{pn} \rightarrow \mathrm{p} \Lambda$ reaction was investigated within the oneboson-exchange model. Both the strong and weak meson-baryon-baryon vertices are fixed by the $\mathrm{SU}(6)_{\mathrm{W}}$ symmetry. The final hyperon-nucleon interaction is described by the SU(3) baryon-baryon model described in section a.20. The main feature of our investigation is to account for the nonlocal effects in the weak transition matrix elements which were neglected in all previous work. We made predictions of various polarization observables which can be used to determine the interference between the parity-conserving and parity-violating terms by using the data from the experiment being pursued at several laboratories. In Fig. IV-9 we show our preliminary results for the asymmetries $\bar{A}_{p}$ for the proton and $\bar{A}_{\Lambda}$ for the $\Lambda$. A paper describing our results will be prepared for publication.

*ANL and University of Adelaide, Australia, †Fukui Medical University, Japan, łOsaka University, Japan

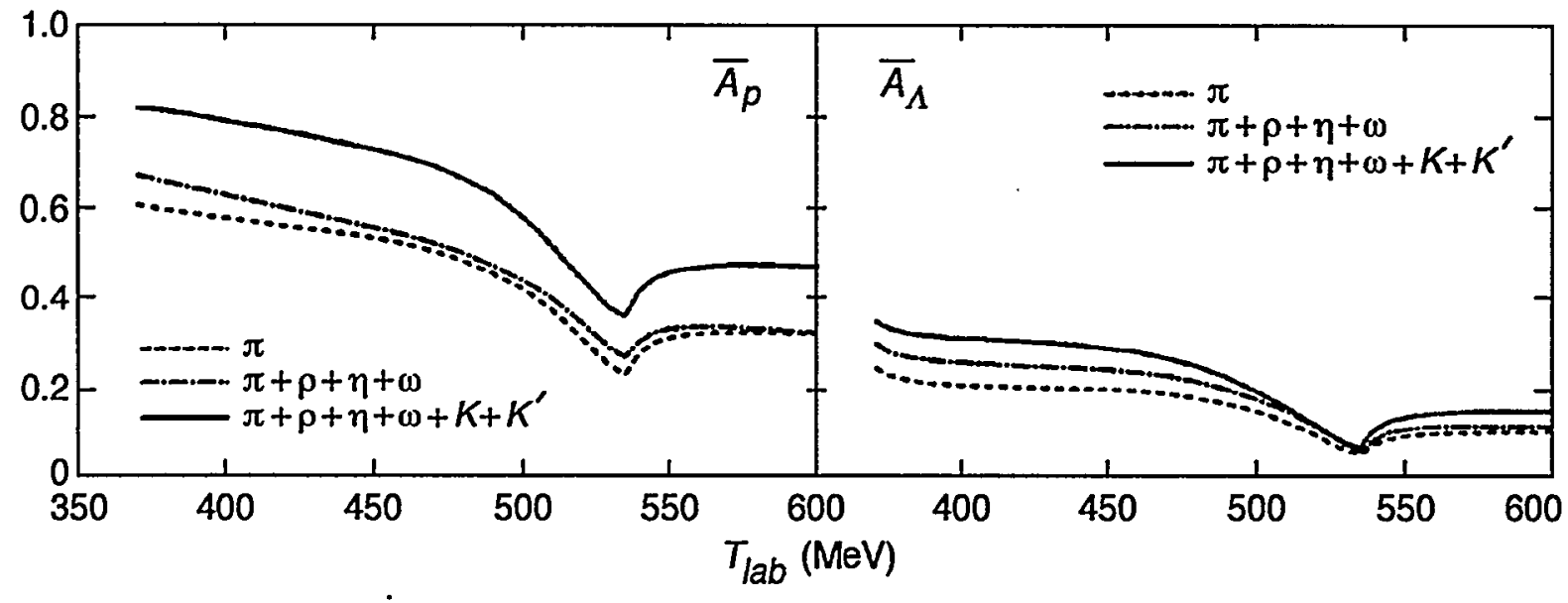

Fig. IV-9. Contribution of each meson exchange mechanism to the $\overline{\mathrm{A}}_{p}$ and $\overline{\mathrm{A}}_{\Lambda}$. All meson exchange mechanisms are included in the solid line while the dash-dotted line includes non-strangeness ones. The dashed line includes only the pion-exchange mechanism.

\section{a.24. Two-frequency Shell Model for Hypernuclei (T.-S. H. Lee, T. T. S. Kuo,* and Y. Tzeng $\dagger$ )}

A two-frequency shell model is proposed for investigating the structure of hypernuclei starting with a hyperon-nucleon potential in free space. In a calculation for ${ }_{\Lambda}^{16} \mathrm{O}$ using the folded-diagram method, the applicability of the model is demonstrated by showing that the predicted $\Lambda$ single-particle energies have saturation minima at an oscillator frequency $\hbar \omega_{\Lambda}$ $\sim 10 \mathrm{MeV}$, which is considerably smaller than $\hbar \omega_{\mathrm{N}}$

*SUNY at Stonybrook, $\uparrow$ Academia Sinica, Taiwan
$=14 \mathrm{MeV}$ for the nucleon orbits. A fairly strong dependence on $\hbar \omega_{\Lambda}$ was also observed for the calculated $\Lambda$ particle-nucleon hole interactions. The model was applied to demonstrate that the $\Lambda \mathrm{NN}$ threebody force induced by the $\Lambda \mathrm{N} \leftarrow \Sigma \mathrm{N}$ transitions can significantly change the predicted spectrum. A paper describing our results was submitted for publication. 


\section{a.25. Covariant Quark Model for the Baryons (F. Coester, K. Dannbom,* and D. O. Riska*)}

A family of simply solvable covariant quark models for the baryons is presented. With optimal parameter choices, the models reproduce the empirical spectra of the baryons in all flavor sectors to an accuracy of a few percent. Complete spectra are obtained for all states of the strange, charm and beauty hyperons with $\mathrm{L} \leq 2$. The magnetic moments and axial coupling constants of the ground state baryons correspond to those of conventional quark models. We construct currentdensity operators that are consistent with empirical nucleon form factors at low and medium energies.

A theoretically consistent description of the baryons based on the concept of constituent quarks should build in confinement and Poincare invariance from the beginning. Confinement is an obvious consequence of the unbounded discrete mass spectrum. The requirements of Poincare invariance for the mass operator are easily met by explicit construction: the Hilbert space of states $H$ is the tensor product of functions representing the center-of-mass motions with the representation space of the "little group". The eigenfunctions of the four-momentum are related to observables only in combination with a corresponding representation of the current density operators. The construction of a Poincare covariant quark model of the baryons involves both the construction of a mass operator with appropriate spectral properties and the construction of Poincaré covariant current density operators. The emphasis in the present work is consistency with general principles and simplicity while discarding the notion of constituent quarks as a system of free particles that incidentally are confined in an infinite potential well. The key to the satisfactory description of the spectrum is a mass operator which is the sum of two commuting terms: (i) a confining mass operator with the basic point spectrum, and (ii) a simple spin- and flavor-dependent hyperfine term large enough to affect the level order. Observable form factors and transition amplitudes depend on two distinct features of a quark model that are not separately observable: (i) the wave function, which differs for different baryons, and (ii) the quark-current structure that is the same for baryons. ${ }^{1}$

*University of Helsinki

${ }^{1}$ F. Coester, K. Dannbom, and D. O. Riska, Nucl. Phys. A 634, 335 (1998)

\section{a.26. Poincaré Covariant Quark Models of Baryon Form Factors (F. Coester and D. O. Riska*)}

Poincaré covariant quark models of the nucleon, the $\Delta$ resonance and their excitations are explored. The baryon states are represented by eigenfunctions of the fourvelocity and a confining mass operator, which reproduces the empirical baryon masses up to $\sim 1700$ $\mathrm{MeV}$ to an accuracy of $\sim 6 \%$. Models of constituent quark currents provide the relations between ground-state properties and transition amplitudes. ${ }^{1}$ To be published in "Few-Body Systems" (probably before the end of the century).

\footnotetext{
*University of Helsinki

${ }^{1}$ F. Coester and D. O. Riska, accepted for publication in Few Body Systems
} 


\section{a.27. Null-Plane Invariance of Hamiltonian Null-Plane Dynamics (F. Coester, W. H. Klink,* and W. N. Polyzou*)}

The mathematical formulation of Hamiltonian nullplane dynamics involves two unitary representations, $U_{0}(L, a)$ and $U(L, a)$, of the Poincare group on the Hilbert space of physical states which are identical for those Poincare transformations that leave the null-plane $\mathrm{x}^{0}+\mathrm{x}_{3}=0$ invariant. It follows that for every other null-plane, $n \cdot x=x^{0}+\hat{n} \cdot x=0$, there is a conjugate unitary Poincaré representation, $\mathrm{U}_{\mathrm{n}}(\mathrm{L}, \mathrm{a})$, that is identical to $U_{o}(L, a)$ for those Poincaré transformations which leave that null-plane invariant. The unit vectors $\hat{\mathbf{n}}$ label a set of left-coset representatives of $\operatorname{SO}(1,3)$ relative to the subgroup that leaves the null-plane $x^{0}+$ $\mathrm{x}_{3}=0$ invariant. There are irreducible unitary representations of the Poincaré group on functions over the unit sphere. Translations are represented by the identity. As suggested by Karmanov, it is useful to treat the orientation $\hat{\mathbf{n}}$ of the null-planes as additional unphysical degrees of freedom. The extended Hilbert space of states is the tensor product of the original Hilbert space with functions over the unit sphere. On this extended Hilbert space we have Poincaré representations with interactions appearing only in the four-momentum. "Null-plane invariance" is the requirement that observable quantities must be invariant under rotations of the null-planes. Impulse currents do not satisfy this null-plane independence requirement. The requirement is satisfied in practice by adding appropriate interaction currents. These procedures are inherently ambiguous requiring additional criteria to define "minimal" interaction currents. The construction of Poincaré covariant Hamiltonian dynamics from a Fock-space representation of a local Lagrangian requires additional considerations, since regularization destroys commutation relations of the Poincare generators. On the extended Fock space there are unitary Poincare representations with interactions occurring only in the four-momentum. Additional steps are needed to achieve the required null-plane independence of the observables. This framework is useful for comparison with other forms of dynamics. ${ }^{1}$

\footnotetext{
*University of Iowa

${ }^{1}$ F. Coester, W. H. Link, and W. N. Polyzou, Few Body Systems 24 (1998) in print.
}

\section{B. NUCLEAR FORCES AND NUCLEAR SYSTEMS}

The goal of this program is to achieve a description of nuclear systems ranging in size from the deuteron to nuclear matter and neutron stars using a single parametrization of the nuclear forces. Aspects of our program include both the construction of two- and three-nucleon potentials and the development of many-body techniques for computing nuclear properties with these interactions. Detailed quantitative, computationally-intensive studies are essential parts of this program.

Quantum Monte Carlo (QMC) calculations of light $(\mathrm{A} \leq 8)$ nuclei with realistic interactions have been the main focus of our recent efforts. Our nonrelativistic Hamiltonian contains the accurate Argonne $v_{18}$ two-nucleon (NN) potential, which includes charge-independence-breaking terms, and the Urbana IX three-nucleon (NNN) potential. The QMC calculations include both variational (VMC) and Green's function (GFMC) methods. We begin with the construction of variational trial functions based on products of correlated two- and three-body operators. Energy expectation values are evaluated with Metropolis Monte Carlo integration and parameters in the trial functions are varied to minimize the energy. These optimized variational wave functions can then be used to study other nuclear properties. They also serve as a starting point for the GFMC calculations, which systematically remove higher excited-state components from the trial wave functions by a propagation in imaginary time, $\tau$. 
We are currently studying all $\mathrm{A} \leq 8$ nuclei with experimentally-known bound-state or resonance energies, including some 38(15) excited states in VMC (GFMC). These are the first and only calculations treating these nuclei directly with realistic NN and NNN interactions. The groundstate energies tend to be somewhat underbound compared to experiment, with the disagreement increasing with both $\mathrm{A}$ and $\mid \mathrm{N}-\mathrm{Zl}$. However, there is broad agreement for the ordering and excitation energies of the higher states. To improve on the spectrum, we spent considerable effort this year investigating new NNN potential terms. In addition, VMC calculations including twobody charge and current operators were made for the elastic and transition electron scattering form factors in ${ }^{6} \mathrm{Li}$ and for the ${ }^{7} \mathrm{Li}\left(\mathrm{e}, \mathrm{e}^{\prime} \mathrm{p}\right)^{6} \mathrm{He}$ reaction; both calculations are in excellent agreement with experiment. The VMC wave functions are also being used to obtain astrophysically interesting cross sections, starting with the reaction ${ }^{4} \mathrm{He}(\mathrm{d}, \gamma)^{6} \mathrm{Li}$. Finally, work on the $\mathrm{A}=9$ nuclei was initiated during 1998.

The present QMC calculations of light nuclei grow exponentially as nucleons are added, and are unlikely to be usable past $A=12$. Work on a cluster GFMC method for larger nuclei has been in progress since late 1997. At present we can make only a short propagation in $\tau$ before statistical errors get too large, but we can already see an improvement on the older cluster VMC energies of $\sim 1 \mathrm{MeV} /$ nucleon. We are also studying the properties of neutron drops with the goal of providing additional constraints for the construction of Skyrme interactions for modeling neutron-rich nuclei in the crusts of neutron stars.

Studies of hypernuclei are continuing on several fronts. We made major revisions to our calculations of $\Lambda$ single-particle energies in matter, including derivation of density-dependent effective $\Lambda N$ and $\Lambda \mathrm{NN}$ interactions. We also continue to examine the effect of $\Lambda$-induced distortion of nuclear cores in light hypernuclei, and charge-symmetry breaking of the $\Lambda \mathrm{N}$ interaction.

\section{b.1. Variational Monte Carlo Calculations of Light p-shell Nuclei (R. B. Wiringa, S. C. Pieper, V. R. Pandharipande,* and J. Carlson†)}

We performed an extensive series of variational Monte Carlo (VMC) calculations for all nuclei up to $A=8$ using realistic two- and three-nucleon interactions. The variational wave functions, $\Psi_{\mathrm{v}}(\mathbf{R})$, obtained in these calculations are used as input to the more precise Green's function Monte Carlo (GFMC) calculations described below, and are also being used to study electron scattering and low-energy electroweak reactions of these nuclei. The Hamiltonian contains the recentlyconstructed Argonne $\mathrm{v}_{18} \mathrm{NN}$ interaction, which gives an excellent fit to two-nucleon data, and the Urbana IX NNN potential, which was adjusted to give the correct three-nucleon binding energy and reasonable saturation properties for nuclear matter. The variational wave functions include central, spin, isospin, tensor, and spin-orbit two-body correlations and three-body correlations for the NNN interaction. The $\Psi_{\mathrm{V}}(\mathbf{R})$ for sshell nuclei $(A=3,4)$ give upper bounds to the groundstate binding energy $\sim 2 \%$ above exact Faddeev or GFMC calculations.

The $\Psi_{\mathrm{V}}(\mathbf{R})$ for A=6-8 nuclei are considerably more complicated because of the need to place the additional nucleons in p-shell orbitals. For the one-body part of the trial function, we have used an LS coupling scheme to construct states with the desired $\mathrm{JM}$ values. At present we use a complete p-shell basis for $A=6,7$ nuclei, including states of [2] and [11] spatial symmetry in $A=6$ and of [3], [21], and [111] spatial symmetry in $A=7$, where $[\mathrm{n}]$ denotes the Young pattern.

*University of Illinois at Urbana-Champaign, $\nmid$ Los Alamos National Laboratory 


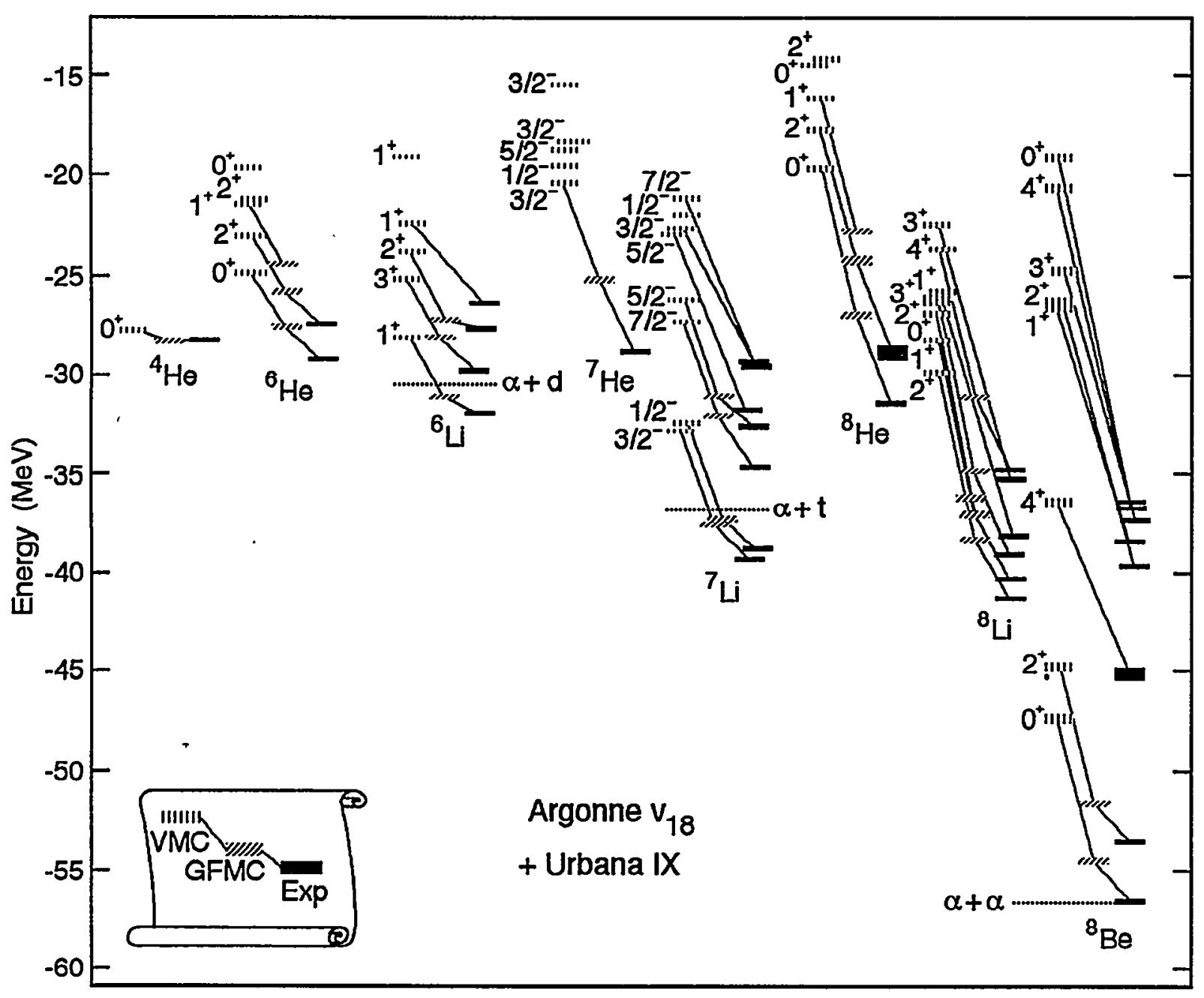

Fig. IV-10. VMC, GFMC, and experimental energies for $A=4-8$ nuclei. 


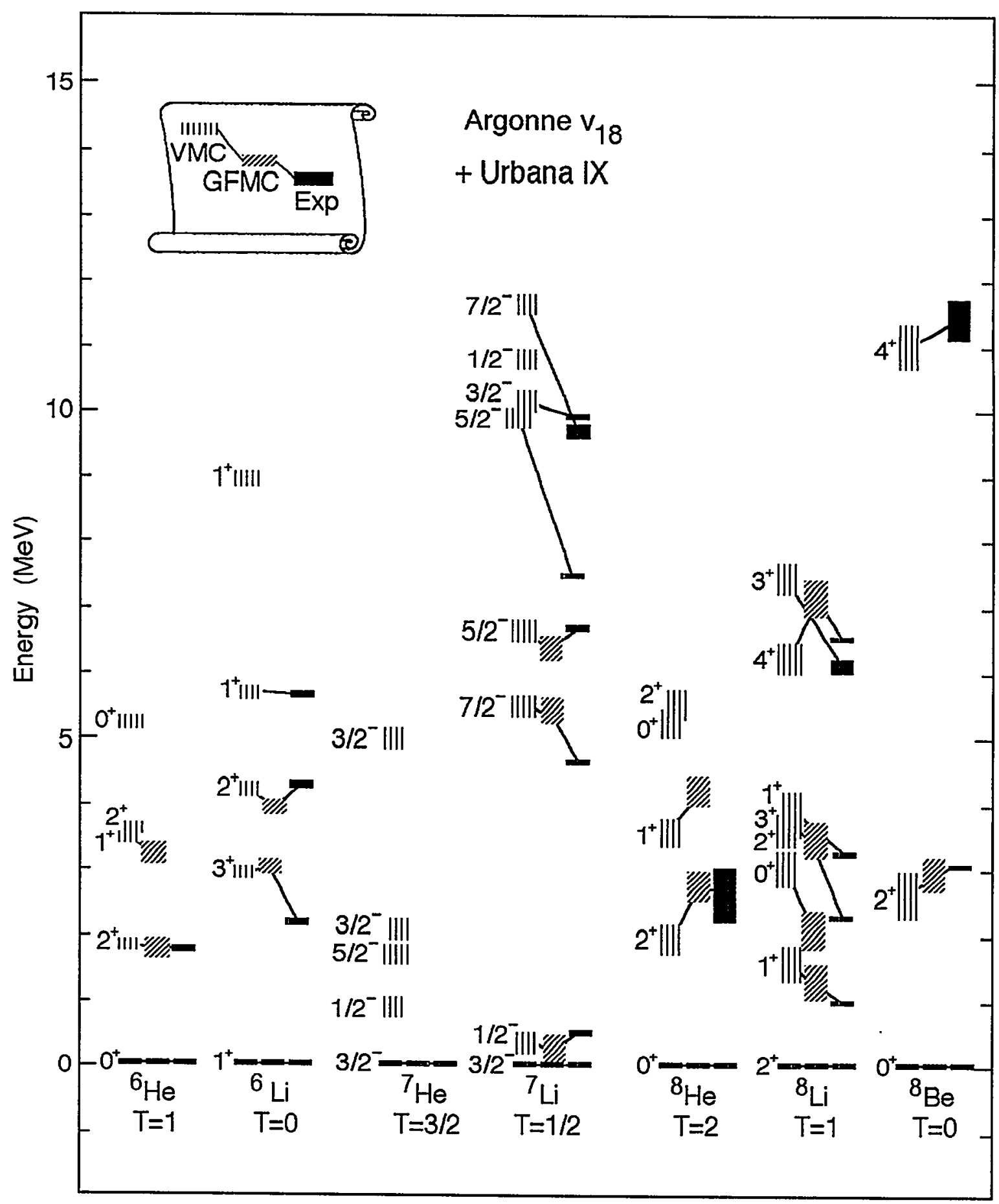

Fig. IV-11. VMC, GFMC, and experimental excitations for $A=6-8$ nuclei. 
In 1998 our $A=8$ calculations were expanded to include the two highest spatial symmetries for each nucleus, which are [4] and [31] in ${ }^{8} \mathrm{Be},[31]$ and [22] in ${ }^{8} \mathrm{Li}$, and [22] and [211] in ${ }^{8} \mathrm{He}$. Because of the tensor two-body correlations, many additional symmetry components are present in the full wave function. We diagonalize our wave functions in the different symmetry components so we can project out all higher excited states with the same quantum numbers as the ground or first excited states.

At present we have made calculations for the ground states of all the $A=6-8$ nuclei, and for 38 different excited states (not counting isobaric analogs); recent results are shown in Figs. IV-10 and IV-11. The binding energies of the ground states are 3-8 MeV high compared to the final GFMC results. However, the excited state energies are in generally good agreement with the GFMC excitations (compared to their respective ground states) in the 15 cases where GFMC was also used. Compared to experiment, these states generally occur in the correct order and with reasonable excitation energies. We also computed all the isobaric analog multiplets to extract the isovector and isotensor energy differences. A recent summary of this work was published. ${ }^{1}$

In 1998 we also did initial programming for $A=9$, sufficient to produce accurate time estimates. The nuclei ${ }^{9} \mathrm{Li}$ and ${ }^{9} \mathrm{Be}$ will require $4-5$ times the computational effort required for ${ }^{8} \mathrm{Li}$, the most difficult case we have completed so far. They also use about 4 times more memory, which will require moving the codes from Argonne's IBM SP, where they currently reside, to the new SGI Onyx machine with its shared memory structure. Ground-state calculations for these nuclei should be feasible in 1999.

1R. B. Wiringa, Nucl. Phys. A631, 70c (1998)

\section{b.2. Green's Function Monte Carlo Calculations of Light p-shell Nuclei} (S. C. Pieper, R. Roncaglia, R. B. Wiringa, J. Carlson,* and V. R. Pandharipande $\dagger$ )

In the past decade, the ground states of ${ }^{2} \mathrm{H},{ }^{3} \mathrm{H},{ }^{3} \mathrm{He}$, and ${ }^{4} \mathrm{He}$, and the low-energy scattering states of ${ }^{5} \mathrm{He}$ were studied with Hamiltonians of the form described in the previous section in an essentially exact fashion (limited only by statistical errors) by using the Green's function Monte Carlo (GFMC) method. Our work in the last few years has focused on extending these calculations to six-, seven-, and eight-body nuclei and their excited states. These are the first calculations of the structure of such nuclei with realistic many-nucleon Hamiltonians and thus they also test the range of applicability of such Hamiltonians.

In the GFMC calculations, we operate on a trial wave function with the imaginary time propagator, $\exp \left[-\left(\mathrm{H}^{\prime}-\right.\right.$ $\left.E_{0}\right) \tau$, where $H^{\prime}$ is a simplified Hamiltonian, $E_{0}$ is an estimate of the eigenvalue, and $\tau$ is the imaginary time. To save computer time, the trial wave function is a simplified form of the variational wave functions described in the previous section. The excited-state components of the trial wave function will then be damped out for large $\tau$, leaving the exact lowest eigenfunction with the quantum numbers of the input variational wave function. The expectation value of $\mathrm{H}$ is computed for a sequence of increasing values of $\tau$ to determine the convergence. Our $\mathrm{H}^{\prime}$ contains the reprojected v8 part of the NN potential and full $3 \mathrm{~N}$ potential. The small correction $\mathrm{H}-\mathrm{H}^{\prime}$ is computed perturbatively. The many-body propagator is written as a symmetrized product of exact two-body propagators, with the $3 \mathrm{~N}$ potential treated in lowest order.

GFMC calculations for fermion systems suffer from the well-known sign problem in which the statistical error grows exponentially with imaginary time; in our original calculations, this effectively limited $\tau$ to 0.06 $\mathrm{MeV}^{-1}$, which means that components of the trial wave function with excitation energies less than $\sim 10 \mathrm{MeV}$ were not appreciably damped out. We believe this problem was solved for nuclei by using "constrained path propagation". In this approach, configurations whose overlap with the trial wave function is small or negative are discarded with a probability such that the average overlap for the discarded configurations is zero. We know of no theorems demonstrating that this is a correct procedure, but we find that the energies and many other observables obtained in this manner are insignificantly different from those obtained with full

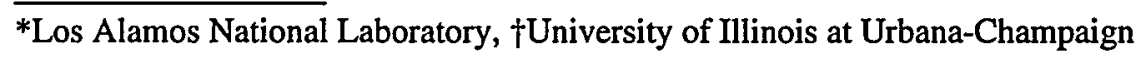


GFMC propagation. With this method there is no sign problem and we can propagate to arbitrarily large $\tau$. However, we recently encountered a problem with this method in neutron drops (systems of neutrons in an artificial external well). In this case, the constrained path can result in the wrong (by 1 to $2 \mathrm{MeV}$ out of $\sim 40$ $\mathrm{MeV}$ ) binding energy. We are presently devoting much effort to trying to determine the cause of this failure.

Figure IV-10 shows a comparison of our VMC and GFMC calculations with experiment for a variety of nuclear states. For $\mathrm{A}>4$, the Hamiltonian is not providing enough binding; this problem also gets worse with increasing $\mid \mathrm{N}-\mathrm{Zl}$. However, as is shown in Fig. IV-11, the Hamiltonian does a good job of predicting the spectrum of excitation energies for a given $A, Z$, and $\mathrm{T}$, except for some of the higher-lying states.

The computer resources (both CPU time and memory) required by our present GFMC method increase exponentially with the number of nucleons. At present calculations of up to $A=8$ nuclei can be routinely made with propagation to $\tau=.2 \mathrm{MeV}$ and we are beginning to explore $A=9$; in the next few years we might be able to reach $A=12$. However using the present method for larger nuclei will not be practical. To overcome this limit we are developing a cluster-expansion GFMC method. This method, and the computer program, is based on our cluster VMC calculation of $16 \mathrm{O}$ that was made a few years ago. In the cluster VMC, expectation values are expanded in terms corresponding to the number of nucleons whose spin and isospin degrees of freedom are active. The complete Jastrow wave function is used in every order of the expansion and thus all integrations are 3A-dimensional. To extend the method to GFMC, one writes the GFMC mixed estimate for a given $\tau$ as an $\mathrm{N} \times 3 \mathrm{~A}$ dimensional integral where $\mathrm{N}$ is the number of time slices used to reach $\tau$, and then makes the spin- and isospin-cluster expansion of this entire expectation value. Preliminary results obtained for ${ }^{16} \mathrm{O}$ and the Argonne $\mathrm{v}_{8}$ ' potential using four-body clusters and propagated to only $\tau=$ $0.004 \mathrm{MeV}^{-1}$ give an improvement of $\sim 1 \mathrm{MeV} /$ nucleon in the binding energy compared to the VMC result. However we are experiencing large statistical fluctuations; we are attempting to solve this by developing a different Metropolis sampling method.

\section{b.3. Studies of Three-Nucleon Interactions in Nuclear Systems (S. C. Pieper, R. B. Wiringa, V. R. Pandharipande,* A. Akmal,* J. Carlson, $\uparrow$ and J. L. Forest}

We made GFMC calculations of the energies of 25 states for nuclei with $3 \leq \mathrm{A} \leq 8$ using the Hamiltonian consisting of the Argonne $v_{18} \mathrm{NN}$ and Urbana IX NNN potentials. These calculations show that this Hamiltonian underbinds p-shell nuclei by $0.8 \mathrm{MeV}$ in ${ }^{6} \mathrm{Li}$ to $5 \mathrm{MeV}$ in ${ }^{8} \mathrm{He}$. The error increases with both $\mathrm{A}$ and $\mid \mathrm{N}-\mathrm{ZI}$. However, with the exception of some underpredicting of the spin-orbit splittings, the excitation spectra of the nuclei are reasonably well reproduced. The rms error for the 10 ground states is $2.3 \mathrm{MeV}$ and for the 15 excitation energies is $0.5 \mathrm{MeV}$; with no NNN potential at all these numbers are 7.3 and $0.5 \mathrm{MeV}$, respectively.

These results show that the simple Urbana NNN potentials, which have been used successfully for more than a decade in studies of s-shell nuclei and dense matter, need to be extended for work in the p-shell. Our approach is to use theoretical guidance to suggest the structure of contributions to the NNN potential, but to consider the coupling constants and short-range shapes of the potential to be adjustable. This is in the same spirit as the development of realistic NN potentials. We considered a number of new terms. We find that new potential terms are often not perturbative, i.e., an expectation value of the new term using the GFMC wave function from just Argonne $\mathrm{v}_{18}$ and Urbana IX may be misleading. Thus each new term must be added to the GFMC propagator and a new GFMC calculation made. Furthermore, as the strength of the new term is adjusted, the propagations must be repeated.

The dominant term of the Urbana potential is the Fujita-Miyazawa two-pion term with intermediate excitation of one nucleon to a $\Delta$. We have now studied three-pion ring terms containing one and two $\Delta$ excitations. These are repulsive in s-shell nuclei and attractive in p-shell nuclei and correct the overall loss of binding energy with respect to both $A$ and $|N-Z|$. The Tucson-Melbourne potential contains the FujitaMiyazawa term and a term arising from s-wave $\pi \mathrm{N}$ scattering. We find that the latter term somewhat increases spin-orbit splitting. 
We also studied NNN potentials arising from $\sigma$ exchange between one pair of nucleons and various mesons between one of those nucleons and a third nucleon. In particular, a NNN spin-orbit potential can be made in this manner. This can completely correct the small spin-orbit splittings obtained with just Urbana IX however the corresponding two- $\sigma$ NNN potential is also required to compensate for the large general attraction generated by the NNN spin-orbit potential.
At present we have several models of new NNN potentials, each containing four adjustable coupling constants. They result in rms errors of only 0.3 to 0.5 $\mathrm{MeV}$ for the ground states in $3 \leq \mathrm{A} \leq 8$. The spectra for $A \leq 7$ are also significantly better than with just Urbana IX, however there are one or two problems in $A=8$ nuclei. We are still studying new NNN potentials.

\section{b.4. Microscopic Calculation of ${ }^{6} \mathrm{Li}$ Elastic and Transition Form Factors (R. B. Wiringa and R. Schiavilla*)}

Variational Monte Carlo calculations were made for the elastic electron scattering and inelastic form factors of ${ }^{6} \mathrm{Li}$. The six-body wave functions were obtained for our standard Hamiltonian containing the Argonne v18 two-nucleon potential and the Urbana IX three-nucleon potential. Consistent charge and current operators including one- and two-body terms were also constructed and evaluated. Elastic results include the longitudinal and transverse form factors and tensor polarization. Transition form factors were calculated for the $(5 \pi ; T)=\left(3^{+} ; 0\right),\left(0^{+} ; 1\right),\left(2^{+} ; 0\right)$, and $\left(2^{+} ; 1\right)$ states.

Our calculations show that the elastic longitudinal form factors $F_{L}{ }^{2}(q)$ and $F_{T}{ }^{2}(q)$ are well reproduced in impulse approximation (IA) and that meson-exchange current (MEC) contributions are not large but do improve the agreement with data, particularly in the first minimum of $F_{L}$ near $3 \mathrm{fm}^{-1}$. The longitudinal transition form factor to the $\left(3^{+} ; 0\right)$ excited state is also in reasonable agreement with data at the IA level, and addition of the small MEC corrections improves the agreement in the same region near $3 \mathrm{fm}^{-1}$. The transverse transition form factors to the $\left(0^{+} ; 1\right)$ and $\left(2^{+} ; 1\right)$ states are also very well reproduced, with the MEC corrections helping significantly above $2 \mathrm{fm}^{-1}$.

We also extracted the $\mathrm{B}(\mathrm{E} 2)$ and $\mathrm{B}(\mathrm{M} 1)$ values, obtaining reasonable agreement with experiment. These calculations are the first $a b$ initio six-body calculations based on realistic interactions and they work beautifully without the need for effective charges used in traditional shell model studies. A colorful letter on this work was published. ${ }^{1}$

*TJNAF and Old Dominion University

${ }^{1}$ R. B. Wiringa and R. Schiavilla, Phys. Rev. Lett. 81, 4317 (1998) 


\section{b.5. Nuclear Structure Studies With the ${ }^{7} \mathrm{Li}\left(\mathbf{e}, \mathrm{e}^{\prime} \mathbf{p}\right)$ Reaction (R. B. Wiringa, L. Lapikás,* and J. Wesseling*)}

In previous years we calculated single-nucleon momentum distributions in many of the $A \leq 8$ nuclei and a variety of cluster-cluster overlap wave functions, such as $\left\langle\left.\mathrm{dp}\right|^{3} \mathrm{He}\right\rangle,\left\langle\left.\mathrm{ddl}\right|^{4} \mathrm{He}\right\rangle$, and $\left\langle\alpha \mathrm{dl}{ }^{6} \mathrm{Li}\right\rangle$. This past year we studied the overlaps $\left.\left\langle{ }^{6} \mathrm{He}\left(\mathrm{J}^{\pi}\right)+\mathrm{p}\left(\ell_{\mathrm{j}}\right)\right\rangle_{\mathrm{Li}}\right\rangle$, shown in Fig. IV-12, for $\mathrm{J}=0,2$, or higher, $\ell=\mathrm{p}$ or $\mathrm{f}$, and $\mathrm{j}=$ $1 / 2$ to $7 / 2$. In that figure the overlaps are compared to the difference of the single-proton momentum distributions in ${ }^{7} \mathrm{Li}$ and ${ }^{6} \mathrm{He}$, which should approximate the p-shell proton wave function. The spectroscopic factors obtained from these overlaps are given in the figure and compared to the predictions of the CohenKurath shell model.
There is a noticeable quenching of the strength compared to traditional shell-model calculations. A recent analysis of previously unpublished ${ }^{7} \mathrm{Li}\left(e, e^{\prime} p\right)^{6} \mathrm{He}$ experiments performed at NIKHEF obtained spectroscopic factors to the $0^{+}$ground state of ${ }^{6} \mathrm{He}$ of $0.42(4)$ and to the $2^{+}$first excited state of $0.16(2)$, in excellent agreement with our predictions of 0.41 and 0.18 , respectively. In addition to this absolute normalization, our overlap functions also result in an excellent description of the measured missing energy spectra. A letter reporting these results was submitted for publication.

*NIKHEF

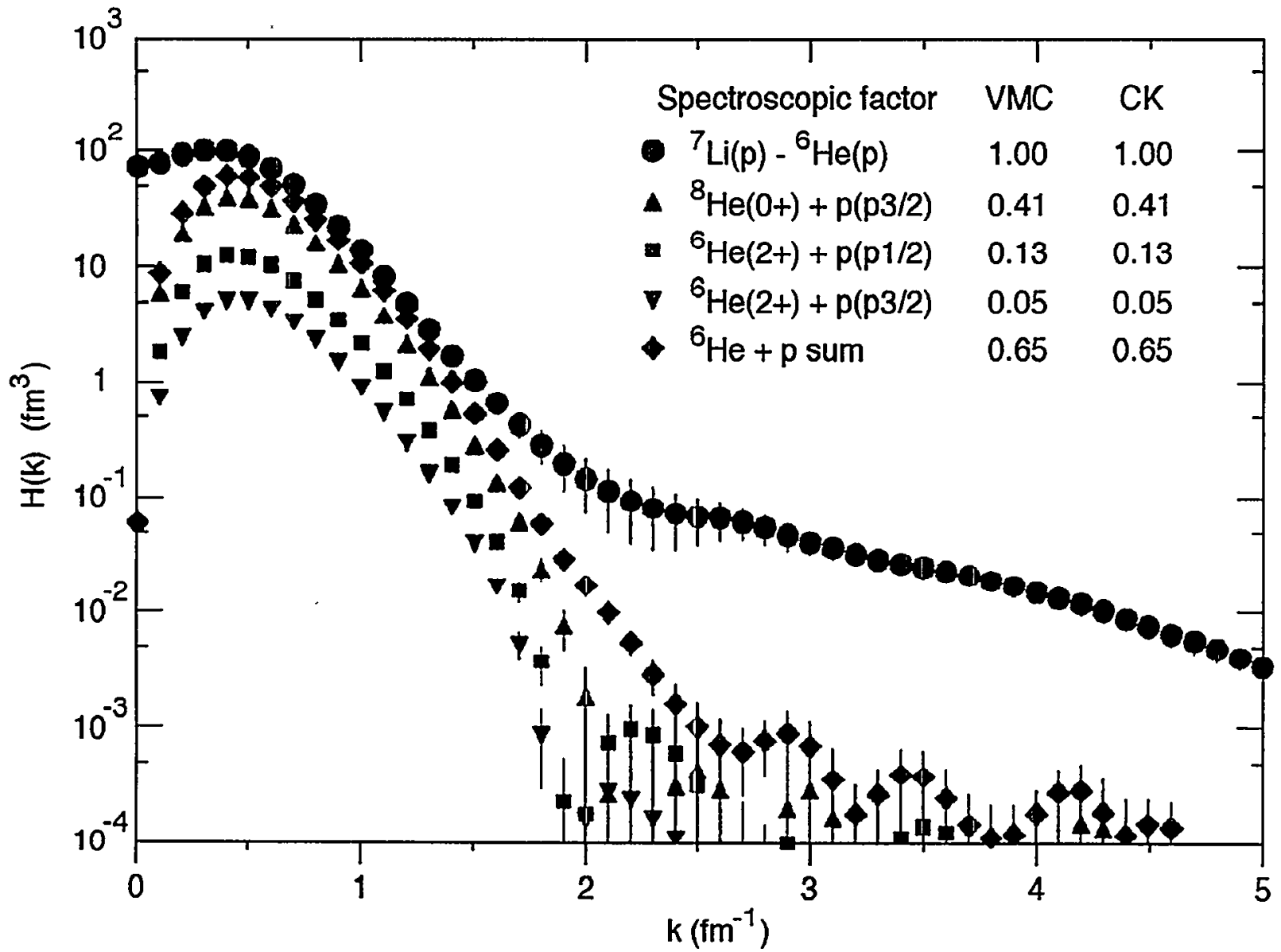

Fig. IV-12. Momentum distributions and cluster overlaps for $A=6,7$ nuclei. 
b.6. Weak Capture of Protons by Protons (V. G. J. Stoks, R. B. Wiringa, R. Schiavilla, * W. Glöckle $\nmid$ H. Kamada, $†$ A. Nogga, $\dagger$ J. Carlson, $\ddagger$ R. Machleidt,$\S$ V. R. Pandharipande,II A. Kievsky,II S. Rosati,Il and M. Viviani II)

The proton-proton weak capture reaction ${ }^{1} \mathrm{H}\left(\mathrm{p}, \mathrm{e}^{+} \mathrm{v}_{\mathrm{e}}\right)^{2} \mathrm{H}$ is the most fundamental process in stellar nucleosynthesis; it is the first reaction in the pp-chain reaction with wave functions obtained from a number of modern, phase-equivalent, realistic interactions. These include the Argonne $\mathrm{v}_{18}, \mathrm{CD}$ Bonn, and several Nijmegen NN potentials. The low-energy pp scattering solutions are treated very precisely, with long-range electromagnetic interactions including vacuum polarization and other smaller effects; these reduce the cross section by $\sim 1 \%$ from the result obtained with just a Coulomb and nuclear potential. Two-body axial currents are also evaluated and are found to boost the cross section by $\sim 1.5 \%$. This is the first calculation of weak pp capture to include both effects.

To minimize the uncertainty in the axial two-body current operator, its matrix element was adjusted to reproduce the measured Gamov-Teller matrix element of tritium $\beta$-decay in calculations using trinucleon wave functions from the same interactions. For the trinucleon calculations a NNN potential was added to the NN potential and adjusted to reproduce the correct trinucleon binding energy in each case. A thorough analysis of the ambiguities that this procedure introduces in evaluating the two-body current contribution to the pp capture was made. Its inherent model-dependence is in fact found to be very small. The overlap integral $\Lambda^{2}(E=0)$ for weak pp capture is predicted to be in the range 7.05-7.06, including the axial two-body current contribution, for all interactions considered. This is the most precise determination of the weak pp capture rate ever made. A paper on this work was published. ${ }^{1}$

*TJNAF and Old Dominion University, $\nmid$ Ruhr-Universität Bochum, $\neq$ Los Alamos National Laboratory, §University of Idaho, TUniversity of Illinois at Urbana-Champaign, II INFN Sezione di Pisa

$1_{R}$. Schiavilla et al., Phys. Rev. C 58, 1263 (1998)

\section{b.7. Radiative Capture Reactions for Astrophysical Applications (R. B. Wiringa and K. M. Nollett*)}

Radiative capture reactions play a major role in many astrophysical processes, including primordial nucleosynthesis and stellar evolution. We are attempting to use the many-body quantum Monte Carlo wave functions discussed above to study radiative capture reactions involving light p-shell nuclei. Our first project is to obtain the low-energy cross section for ${ }^{4} \mathrm{He}(\mathrm{d}, \gamma)^{6} \mathrm{Li}$. This reaction was the primary source for primordial ${ }^{6} \mathrm{Li}$ production in the big bang. The primary nuclei synthesized in the big bang were $2 \mathrm{H}$, ${ }^{3} \mathrm{He},{ }^{4} \mathrm{He}$, and ${ }^{7} \mathrm{Li}$. Trace amounts of ${ }^{6} \mathrm{Li}$ should also have been made, although a primordial abundance has not yet been unambiguously identified in astronomical observations. The cross section at $\mathrm{keV}$ energies is sufficiently small that laboratory experiments have only established an upper bound, which could be orders of magnitude too high.

Our QMC wave functions are good for bound states, but are not as well developed for scattering states. Hence, we are evaluating the appropriate electromagnetic matrix elements between a ${ }^{6} \mathrm{Li}$ ground state and a correlated $\alpha-\alpha$ pair, which will then be folded with a continuum $\alpha-d$ wave function obtained from a suitable optical potential. At present, we have explored some optical potentials and set up the matrix element evaluation. One of the interesting challenges is to find ways of Monte Carlo sampling in the tail of the ${ }^{6} \mathrm{Li}$ ground state where most of the capture reaction takes place. We also developed an improved variational wave function for ${ }^{6} \mathrm{Li}$ that at long range has the form of $\alpha$ and $d$ clusters with the correct empirical binding.

If this method is successful, we can expect to study a number of other interesting radiative capture reactions such as ${ }^{4} \mathrm{He}\left({ }^{3} \mathrm{H}, \gamma\right){ }^{7} \mathrm{Li}$ and ${ }^{4} \mathrm{He}\left({ }^{3} \mathrm{He}, \gamma\right){ }^{7} \mathrm{Be}$, which also play an important role in primordial nucleosynthesis, and for which the experimental uncertainties are $\sim 30 \%$. Also of interest is the reaction ${ }^{7} \mathrm{Be}(\mathrm{p}, \gamma){ }^{8} \mathrm{~B}$, which is important in the solar neutrino problem; the value of this reaction rate is currently in much dispute. A good warm-up exercise for these calculations will be the 
thermal neutron capture reactions ${ }^{6} \mathrm{Li}(\mathrm{n}, \gamma){ }^{7} \mathrm{Li}$ and ${ }^{7} \mathrm{Li}(\mathrm{n}, \gamma)^{8} \mathrm{Li}$, which should provide a good test of our many-body wave functions, and for which good experimental data is available.

*University of Chicago

\section{b.8. $\Lambda$ Single-Particle Energies (A. R. Bodmer and Q. N. Usmani*)}

We made revisions and further calculations to our work of the previous year. These changes have improved and refined our earlier calculations but have not made any major changes in our conclusions. In our work we calculate the $\Lambda$ single-particle energies $B_{\Lambda}$ of hypernuclei $(\mathrm{HN})$ in terms of $\Lambda$-nuclear interactions. The Fermi hypernetted chain (FHNC) method is used to obtain, in terms of the $\Lambda \mathrm{N}$ and $\Lambda \mathrm{NN}$ potentials, the binding $D(\rho)$ to nuclear matter and the effective mass $\mathrm{m}_{\Lambda}^{*}(\rho)$ at densities $\rho \leq \rho_{\mathrm{o}}\left(\rho_{\mathrm{o}}\right.$ is normal nuclear matter density). However we now also obtain the corresponding effective $\Lambda \mathrm{N}$ and $\Lambda \mathrm{NN}$ interactions. The $\Lambda$-core nucleus potential $U_{\Lambda}(r)$ is then obtained by suitably folding these interactions into the core density; the Schrödinger equation for $U_{\Lambda}(r)$ and $m_{\Lambda}^{*}(p(r))$ is then solved to obtain the $B_{\Lambda}$. We show that the fringing field due to the finite range of the effective interactions plays an important role. Another major change is that we now use only a dispersive type $\Lambda \mathrm{NN}$ potential for which we can make reliable calculations, but also include a phenomenological $\rho$ dependence allowing for less repulsion at $\rho<\rho_{0}$, i.e. in the surface. Our best fits give a large $\rho$ dependence which translates into an A-dependent $\Lambda N N$ strength nicely consistent with variational Monte Carlo calculations of ${ }_{\Lambda}^{5} \mathrm{He}$ indicating an effective $\Lambda N N$ potential which becomes more repulsive for larger $A$. The probable interpretation is in terms of a dispersive plus a two-pion-exchange $\Lambda N N$ potential since the latter is known to give an attractive contribution for light HN but is conjectured to give a repulsive contribution for larger $\rho$ and hence for heavier HN. For the well depth we obtain $D\left(\rho_{0}\right) \approx 29 \pm 1$ $\mathrm{MeV}$. The exchange fraction of the $\Lambda \mathrm{N}$ potential corresponds to $\mathrm{m}_{\Lambda}^{*}\left(\rho_{\mathrm{o}}\right) \approx 0.75 \mathrm{~m}_{\Lambda}$ and to a ratio of p- to s-state $\Lambda N$ potential strengths of $\approx 0.45$. An important new result is that charge symmetry breaking is quite significant for heavier HN having a large neutron excess, and has a strength very well consistent with the value we obtained in earlier work from the $\mathrm{A}=4 \mathrm{HN}$ (see below). This work was submitted for publication.

*University of Putra Malaysia

\section{b.9. Core-Nucleus Distortion in Hypernuclei (A. R. Bodmer and Q. N. Usmani*)}

We included the changes in our study of the $\Lambda$ singleparticle energies in a study of the effects of the spherical distortion of the core nucleus by the $\Lambda$ in a hypernucleus, and we also improved our calculations so as to obtain more complete results. The response of the core is determined by an appropriately chosen energydensity functional which depends on the nuclear compressibility and which gives a very good description of the nuclear binding energies. The forcing action of the $\Lambda$ is determined by the nuclear density dependence of the $\Lambda$ binding in nuclear matter which is obtained from our work on the $\Lambda$-single energies. Because of the strongly repulsive $\Lambda N N$ forces, this $\Lambda$ binding saturates at a density not very much less than the central density of nuclei, and results in a core-nucleus distortion which is much less than would be obtained with only $\Lambda \mathrm{N}$ forces. The effects of core distortion turn out to be small even for light hypernuclei. Our results justify the usual assumption that spherical core distortion effects are small and can mostly be neglected.

\footnotetext{
*University of Putra Malaysia
} 


\section{b.10. Charge-Symmetry Breaking $\Lambda$-Nucleon Interaction (A. R. Bodmer, Rita Sinha,* and Q. N. Usmani*)}

Some time ago we showed that the charge-symmetrybreaking interaction, as obtained from the $\mathrm{A}=4$ hypernuclei $\left({ }_{\Lambda}^{4} \mathrm{H}\right.$ and ${ }_{\Lambda}^{4} \mathrm{He}$ ) was spin-independent. This cannot be understood with conventional mesonexchange models, including the recently published latest version of the Nijmegen models of the $\Lambda \mathrm{N}$ potential.
The calculations of $\mathrm{A}=4$ hypernuclei are being extended to include more realistic nucleon-nucleon forces with noncentral and momentum-dependent components which could possibly modify this result. Preliminary indications are that our earlier conclusions are likely to remain unchanged.

*University of Putra Malaysia

\section{NUCLEAR STRUCTURE AND HEAVY-ION REACTIONS}

This research focuses on nuclear structure in unusual regimes: nuclei far from stability, and superdeformed nuclei at high spin. We also study heavy-ion reactions near the Coulomb barrier. Much of this work is closely tied to experiments performed at ATLAS and at radioactive-beam facilities.

Our studies of drip-line nuclei focused on breakup reactions induced by the Coulomb and nuclear fields from a target nucleus. A critical issue was to develop a realistic description of breakup mechanisms as a necessary tool for extracting or testing the nuclear structure properties of drip-line nuclei. An example of particular interest to solar neutrino physics is the low-lying E1 strength of ${ }^{8} \mathrm{~B}$ which determines the radiative proton capture on ${ }^{7} \mathrm{Be}$ in the sun. $\mathrm{E} 1$ transitions dominate the $\left({ }^{8} \mathrm{~B},{ }^{7} \mathrm{Be}+\mathrm{p}\right)$ breakup on a heavy target but they are masked by E2 transitions, and also by nuclear processes. We separated and extracted the E1 and E2 components by analyzing measurements of ${ }^{7} \mathrm{Be}$ fragment momentum distributions. This is possible because the distributions exhibit an asymmetry that is caused by the $\mathrm{E} 1+\mathrm{E} 2$ interference.

The studies of heavy-ion reactions at energies close to the Coulomb barrier are based on applications of the coupled-channels technique. The objective is to obtain a consistent, unified, quantum-mechanical explanation of fusion reactions, compound-nucleus spin distributions, elastic and inelastic scattering, and transfer reactions. The calculations are constrained by the structure of the interacting nuclei. We used this technique to investigate the fusion of nuclei far from stability, such as ${ }^{17} \mathrm{~F}$.

Our studies of superdeformed nuclei, at both low and high spins, address the issues of possible new regions of superdeformation and hyperdeformation. Special emphasis is being put on the study of fission barriers at high spin, and the relation between fission barriers and the possibility of producing very extended nuclear shapes. Other areas of interest are the structure of heavy elements and superheavy elements, density dependence of two-body interactions, and the phenomenon of proton radioactivity. The techniques we use to study these problems are a deformed central potential approach for surveying nuclear structure over a large region, self-consistent mean-field calculations for more detailed studies of particular nuclides, and many-body wave functions when residual interaction effects are small and a mean-field approach is inadequate. 
Much of our work is computer intensive and we are adapting our codes to exploit the massively parallel IBM SP supercomputer system at Argonne. The use of the SP system allows us to calculate energy surfaces as a function of angular momentum, using the Strutinsky method with cranking, in a four-dimensional deformation space consisting of quadrupole, octupole, hexadecapole, and necking degrees of freedom. We carried out studies of nuclear energy surfaces in nuclei with masses ranging from $\mathrm{A} \sim 80$ to $\mathrm{A} \sim 200$. We are analyzing these calculations to look for nuclei that are good candidates for experimental investigation of superdeformation. Our recent analysis of energy surfaces near $A=100$ suggests that very extended minima in several nuclides near ${ }^{108} \mathrm{Cd}$ are experimentally accessible. These studies are being complemented with studies of selected nuclei, in a collaborative effort with J. L. Egido and L. M. Robledo, using the HartreeFock method with the finite-range Gogny interaction. We are adapting our many-body code for parallel computer systems and at the same time substantially modifying this code to allow the use of general two-body matrix elements rather than multipole-multipole matrix elements. This will allow detailed nuclear structure studies with exact number projection and good parity. We are involved in heavy-element nuclear structure studies and are applying these results to investigations of the stability of superheavy elements.

\section{c.1. Momentum Distributions of ${ }^{7}$ Be Fragments in Breakup Reactions of ${ }^{{ }^{8}} \mathbf{B}$ (H. Esbensen, B. Davids, * S. M. Austin,* and others*)}

We analyzed our measurements of longitudinal momentum distributions of $7_{\mathrm{Be}}$ fragments that were produced in the breakup of ${ }^{8} \mathrm{~B}$ on a lead target. Our analysis is based on first-order Coulomb dissociation and a single-particle description of the valence proton in ${ }^{8} \mathrm{~B}$. This allows us to scale independently the strengths of the E1 and E2 transitions the model predicts. The best fits to the data are illustrated in Fig. IV-13. They were achieved by increasing the predicted E1 strength by $22 \%$ and reducing the E2 strength by $15 \%$. This implies an $\mathrm{S}$-factor of $22 \mathrm{eV}^{*}$ barn for the radiative capture of proton on ${ }^{7} \mathrm{Be}$, which is consistent with the currently adopted value.

More importantly, the analysis shows that E2 transitions do play a role by producing an asymmetry in the measured distributions. A measure of the E2 strength, which is sometimes used in the literature, is the ratio of the S-factors for E2 and E1 transitions at

*Michigan State University

${ }^{1}$ B. Davids et al., Phys. Rev. Lett. 81, 2209 (1998) about $600 \mathrm{keV}$; the best fit requires the ratio $\mathrm{S}(\mathrm{E} 2) / \mathrm{S}(\mathrm{E} 1)=(7 \pm 2) 10^{-4}$. This is most likely a lower limit because our analysis was based on first-order perturbation theory, and higher-order processes are expected to reduce the calculated asymmetry. This work was published. 1

It should be noted that these measurements are not particularly sensitive to $E$ transitions near threshold, where the S-factor is normally quoted, since they probe the $E 1$ (and E2) strength distribution over a wider range of excitation energies, with maximum weight near 600 $\mathrm{keV}$. The extracted $\mathrm{S}$-factor is therefore somewhat uncertain and model-dependent. A measurement of the ${ }^{8} \mathrm{~B}$ decay energy spectrum, obtained by measuring the momenta of the proton and the ${ }^{7} \mathrm{Be}$ fragment in coincidence, is now underway, and it will hopefully provide a more accurate determination of the S-factor near threshold. 


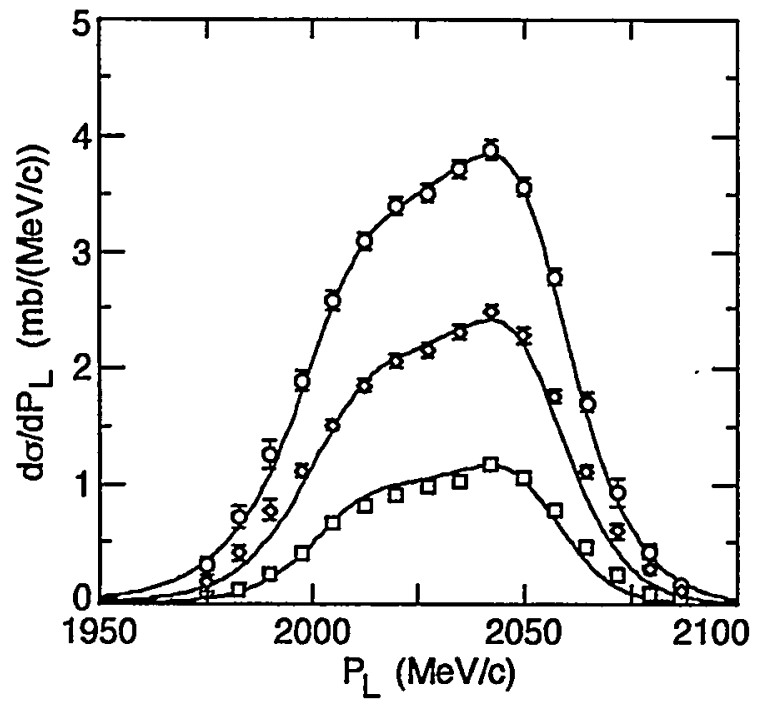

Fig. IV-13. Longitudinal momentum distributions of $7_{\text {Be fragments, produced in breakup reactions of }{ }^{8} B \text { on a }}$ $\mathrm{Pb}$ target at $44 \mathrm{MeV} / \mathrm{u}$. The calculated distributions have been adjusted, as explained in the text, to fit the measurements for the different acceptance angles of $7_{\mathrm{Be}}$ fragments, namely $\theta_{\max }=1.5,2.4$, and 3.5 degrees.

\section{c.2. Nuclear-Induced Breakup of Halo Nuclei (H. Esbensen and G. F. Bertsch*)}

Approximations that are commonly used in analyzing reaction data for ordinary, tightly-bound nuclei may become unreliable when applied to the breakup of weakly-bound halo nuclei. We have, in particular, investigated the validity of perturbation theory and a coupled-channels treatment, which includes couplings to continuum states but ignores the couplings between continuum states. We find that these two methods are unrealistic and that they exaggerate the influence of the nuclear-induced breakup.

We tested the two approximations in the sudden limit (relevant to experiments at intermediate and high energies) by comparing to the eikonal approximation which provides an exact description in the sudden limit. An example is illustrated in Fig. IV-14, where we show the nuclear induced ${ }^{11} \mathrm{Be} \rightarrow{ }^{10} \mathrm{Be}+\mathrm{n}$ breakup probabilities for a light target. The breakup is here induced by a real neutron-target interaction, whereas the core-target interaction was assumed complex. The exponential fall-off at large impact parameters is governed by the transverse halo density; the behavior at small impact parameters is determined by coreabsorption. The coupled-channels result (dashed curve) does not differ much from the first-order calculation (open circles) but they are both much larger (about $60 \%$ ) than the eikonal result (solid curve). The discrepancy is even larger when we adopt a complex neutron-target interaction that is realistic at 40-60 $\mathrm{MeV} / \mathrm{u}$.

The failure of the two approximations is caused by the fact that the nucleon-target interaction is much too strong to justify a perturbative or a truncated coupledchannels treatment. This work was submitted for publication.

*INT, University of Washington 


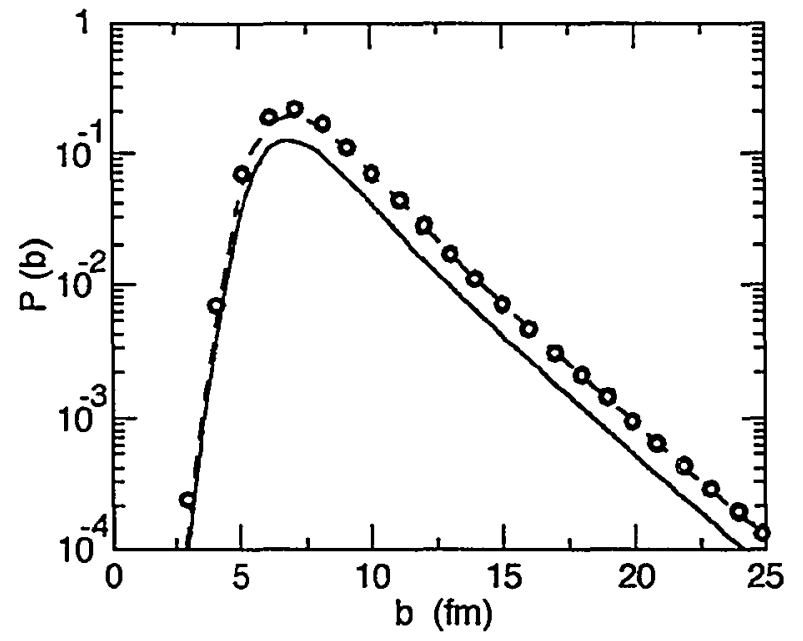

Fig. IV-14. Probabilities for the $11_{B e} \rightarrow 10_{B e+n}$ diffraction dissociation on a light target, as functions of the impact parameter. They were all calculated in the sudden limit. The results of first-order perturbation theory (open circles) and a coupled-channels calculation which ignores continuum-continuum couplings (dashed curve) are compared to the exact eikonal result (solid curve).

\section{c.3. Breakup Reactions of ${ }^{8}$ B at Low Energies (H. Esbensen and G. F. Bertsch*)}

The ${ }^{8} \mathrm{~B} \rightarrow{ }^{7} \mathrm{Be}$ breakup at $26 \mathrm{MeV}$ on a nickel target was recently measured at Notre Dame. ${ }^{1}$ A realistic calculation of the breakup is quite difficult at such a low energy, partly because higher-order processes are expected to be important, and partly because nuclear processes may also play a role, as indicated by DWBA calculations. ${ }^{2}$ We adopted a semiclassical description, where we follow the time-evolution of the wave function of the valence proton in ${ }^{8} \mathrm{~B}$, essentially to all orders in the Coulomb and nuclear fields from the target, and use Coulomb trajectories to describe the relative motion of projectile and target.

The results of various calculations are compared to the measurement in Fig. IV-15. The top thin curve is the result of first-order Coulomb dissociation. This calculation is based on calculated E1 and E2 strength distributions. It exceeds the measurement by almost a factor of 5 . The middle thicker curve is the result of a dynamical calculation, which includes the effects of the E0, E1 and E2 Coulomb fields from the target to all orders. The discrepancy with the measurement is now reduced to a factor of 2.4. This reduction is caused, partly by higher-order Coulomb processes, and partly by the fact that we here have used the correct multipole

*INT, University of Washington

$1_{\mathrm{J}}$. von Schwarzenberg et al., Phys. Rev. C $\underline{53}, 2598$ (1996)

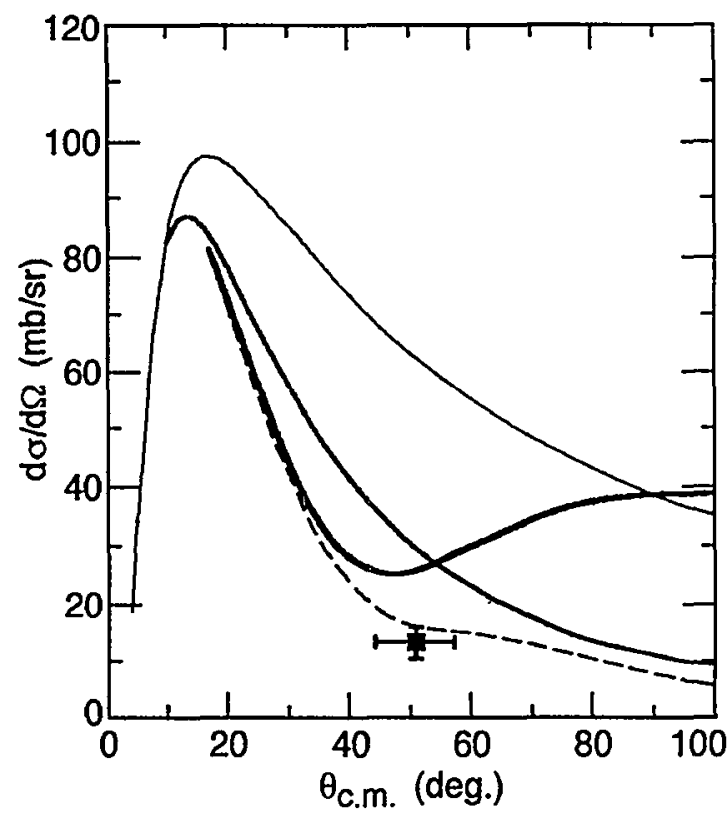

Fig. IV-15. Differential cross section for the ${ }^{8} B \rightarrow{ }^{7} B e$ breakup at $26 \mathrm{MeV}$ on a ${ }^{58} \mathrm{Ni}$ target, as a function of the $8_{B}$ center of mass scattering angle. The results of the different calculations (explained in the text) are compared to the measurement. ${ }^{l}$ 
expansion of the Coulomb field from the target, in particular, when the target penetrates the valence proton density.

The thickest solid curve in Fig. IV-15 includes, in addition to the E0, E1, and E2 Coulomb fields, the effect of the associated multipole fields of a complex proton-target nuclear interaction. The nuclear interaction of the ${ }^{10} \mathrm{Be}$ core with the target was ignored in these calculations. The result shows that the Coulomb-nuclear interference reduces the breakup probability at 20-55 degrees but not enough to reproduce the measurement. The dashed curve shows the separate contribution from diffraction dissociation, whereas proton stripping makes up the difference between the two curves.

It is noted that the effect of the nuclear field is weaker than obtained in the DWBA approach. ${ }^{2}$ This is most clearly seen at large angles where the peak of the DWBA calculation is about twice as high as obtained in our dynamical calculation, although the two calculations were based on the same proton-target interaction. This discrepancy confirms that the nuclear field is much too strong to justify a perturbative description of the nuclear induced breakup of weakly bound nuclei. This work was submitted for publication, together with the work reported in section c. 2 above.

2F. M. Nunes and I. J. Thompson, Phys. Rev. C 57, 2818 (1998)

\section{c.4. Coupled-Channels Calculations of $17 \mathrm{~F}+208 \mathrm{~Pb}$ Fusion Reactions}

(H. Esbensen, C. L. Jiang and K. E. Rehm)

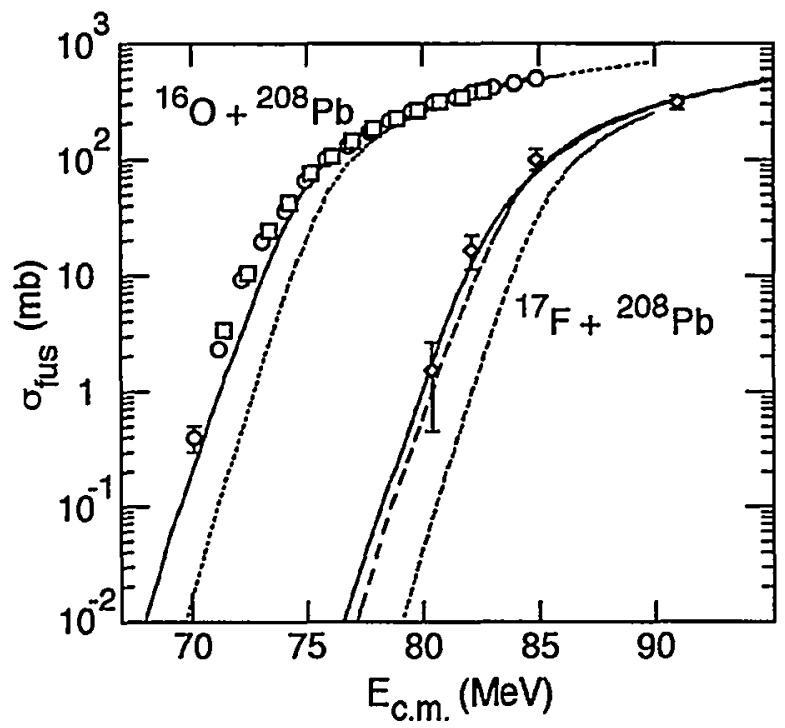

Fig. IV-16 Measured and calculated fusion cross section for $16 \mathrm{O}$ and $17_{\mathrm{F}}$ beams on a $208 \mathrm{~Pb}$ target. The solid curves are the results of the full calculations described in the text. The dotted curves are the results obtained without any coupled-channels effects. The dashed curve is the result we obtain when we ignore the singleparticle excitation of the valence proton in $17 \mathrm{~F}$.

\footnotetext{
${ }_{1}$. E. Rehm et al., Phys. Rev. Lett. 81, 3341 (1998)

${ }^{2}$ C. R. Morton et al., Phys. Rev. C 52, 243 (1995)
}

The fusion-fission cross section for $17 \mathrm{~F}+{ }^{208} \mathrm{~Pb}$ was recently measured at energies in the vicinity of the Coulomb barrier. ${ }^{1}$ The data are consistent with the measured fusion cross sections for ${ }^{16} \mathrm{O}+{ }^{208} \mathrm{~Pb}, 2$ shifted in energy by the ratio $9 / 8$ of the charges of the two projectiles. This suggests that the weakly-bound proton in ${ }^{17} \mathrm{~F}$ affects the fusion rate only by changing the charge of the projectile.

In order to test this view, we performed coupledchannels calculations for the fusion, both of ${ }^{16} \mathrm{O}+{ }^{208} \mathrm{~Pb}$ and of $17 \mathrm{~F}+208 \mathrm{~Pb}$, and made comparisons to the available data. The calculations for $16 \mathrm{O}+208 \mathrm{~Pb}$ included couplings to the lowest $2^{+}$and $3^{-}$states, in both projectile and target. In the calculations for $17 \mathrm{~F}+208 \mathrm{~Pb}$ we included the exact same states and couplings, assuming that excitations of the ${ }^{16} \mathrm{O}$ core were not affected by the valence proton. We included, in addition, the quadrupole Coulomb and nuclear couplings between the $5 / 2^{+}$ground state and the excited $1 / 2^{+}$states in $17 \mathrm{~F}$, at $0.495 \mathrm{MeV}$.

The results of our calculations are shown in Fig. IV-16. The calculations for the ${ }^{16} \mathrm{O}$ beam reproduce the data above the Coulomb barrier but there are some discrepancies below the barrier; this is a well-known and unsolved problem. The calculations for the $17 \mathrm{~F}$ beam are in reasonable agreement with the measurements. 
They show, in particular, that the couplings associated with the excitation of the valence proton do not enhance the subbarrier fusion cross section much, compared to the calculation which ignores these couplings (the dashed curve). This is consistent with the experimental findings. Other aspects of the ${ }^{17} \mathrm{~F}$ experiment, as for example the significance of Coulomb dissociation, are discussed in the publication. 1

\section{c.5. Many-Body Wave Functions (R. R. Chasman)}

In the past few years, we developed many-body variational wave functions that allow one to treat pairing and particle-hole two-body interactions on an equal footing. The complexity of these wave functions depends only on the number of levels included in the valence space, but does not depend on the number of nucleons in the system. In these wave functions, we conserve particle number and parity strictly; projecting states of good particle number and parity before carrying out the variational calculations. We added a cranking term to the many-body Hamiltonian and modified the projection procedure to get states of good signature before variation. This allows us to study pairing collapse as a function of angular momentum. We also extended this program to calculate spectroscopic factors involved in proton decay. This is useful for studies of nuclides near the proton drip line.

By using residual interaction strengths (e.g. the quadrupole interaction strength or pairing interaction strength) as generator coordinates, one gets many different wave functions; each having a different expectation value for the relevant interaction mode. These wave functions are particularly useful when one is dealing with a situation in which the mean-field approximation is inadequate and a configuration interaction treatment is needed. Because the same basis states are used in the construction of all of the manybody wave functions, it is possible to calculate overlaps and interaction matrix elements for the many-body wave functions obtained from different values of the generator coordinates (which are not in general orthogonal) easily. The valence space can contain a very large number of single-particle basis states, when there are constants of motion that can be used to break the levels up into groups.
Wave functions of this sort become more realistic as the size of each group is increased. To increase this size, it seems necessary to parallelize the code. Another issue involved in making the description of nuclei more realistic is the choice of matrix elements. Until now, we used a multipole-multipole interaction to calculate matrix elements which simplifies the many-body calculations substantially.

With these thoughts in mind, we parallelized our manybody code to run on the SP computer and at the same time we modified the code to handle arbitrary two-body matrix elements. To include the latter feature, we have rewritten the code almost completely. We find that parallelization speeds up the code in a reasonable way as we increase the number of processors, until we reach a critical number of processors. Beyond this number of processors, there is essentially no decrease in running time. This critical number of processors depends on the specific system being studied and is typically between 5 and 10 processors. With the parallelization of our code, we should be able to make fairly realistic studies of systems with large numbers of levels ( 9 or 10 doubly degenerate levels per group).

Together with J. L. Egido and L. M. Robledo, we adapted subroutines for calculating Gogny interaction matrix elements and Coulomb matrix elements to use with this many-body code. The inclusion of the repulsive Coulomb matrix elements in our code caused a breakdown of our iterative method for finding extrema. In many instances, the method gives maxima rather than minima. By introducing a suitable counter-term based on exact sum rules, we have overcome this difficulty. At present, we are looking into ways to speed up the convergence of our iterative procedure. The convergence gets slower as the number of levels in a group increases. 


\section{c.6. Very Extended Shapes in Nuclei (R. R. Chasman)}

In the past few years, large computer resources have become available on the massively parallel processor IBM SP system at Argonne, in addition to the resources provided by NERSC. We parallelized the code used to calculate single-particle spectra to exploit the SP system and devoted a large part of our efforts to calculating energy surfaces in a four-dimensional shape space that includes reflection-asymmetric shapes. We study the nuclear energy surfaces as a function of mass, charge, shape and angular momentum, using the Strutinsky method. In this approach, one makes quantum corrections to a smooth liquid drop behavior using the calculated single-particle energy levels. In earlier studies, we found that it is often not sufficient to use only quadrupole and hexadecapole deformations to describe very extended reflection-symmetric nuclear shapes. When we added a necking degree of freedom, we found previously unknown minima. These minima are characterized by very extended capsule shaped nuclei with axis ratios of $2.2: 1$ in the $\mathrm{A}=180$ mass region. We have now added octupole deformation to this shape space. The inclusion of these two degrees of freedom to our shape space substantially increases our ability to describe nuclear shapes compared to a typical shape space consisting only of quadrupole and hexadecapole deformations. As parity is no longer a good quantum number when octupole deformation is included, the size of the matrices we diagonalize is doubled. In a typical calculation, we diagonalize matrices that are $600 \times 600$. Several thousand such diagonalizations are needed to determine energy surfaces.

There remains a need to test calculated fission barriers and to generally understand nuclear properties at the highest spins. Using the four-dimensional deformation space described above, we analyzed the high-spin energy surfaces of the $N=86$ isotones going from $\mathrm{Sn}(Z=50)$ to Dy $(Z=66)$. There is a high-spin superdeformed minimum in all of these nuclides ( $\sim 1.85: 1$ axis ratio) that becomes yrast at high spin. These shapes are well known experimentally in the Dy region.

We find that as the proton number decreases from $\mathrm{Z}=$ 66 to $Z=50$, the fission barrier increases by roughly 10 $\mathrm{MeV}$ at a given angular momentum. The superdeformed minimum associated with $N=86$ is present for both $\mathrm{Sn}$ and Dy. This result suggests that we can extend the study of nuclear properties at extreme deformations to a new regime of angular momenta, with the availability of radioactive nuclear beam facilities. A preliminary version of this work was published. ${ }^{1}$

We extended our high-spin Strutinsky calculations ${ }^{2}$ to nuclei in the $A=100$ mass region. Many of the very extended minima we find will be accessible with projectiles produced at an exotic beam facility. However, our calculations show very extended minima in nuclides in the vicinity of ${ }^{108} \mathrm{Cd}$ that are accessible using existing facilities. In Fig. IV-17, we show the energy surface of ${ }^{108} \mathrm{Cd}$ at $\mathrm{I}=60 \hbar$; and in Figs. IV-18 and IV-19, we display the proton and neutron singleparticle energy level diagrams associated with the minimum at $\left(\eta_{2}=0.7, \eta_{\text {neck }}=-0.1\right)$, as a function of rotational frequency.

Several odd-parity excited states were found in the superdeformed minima of the $\mathrm{Hg}$ region. Because of their low excitation energies, it had been thought that

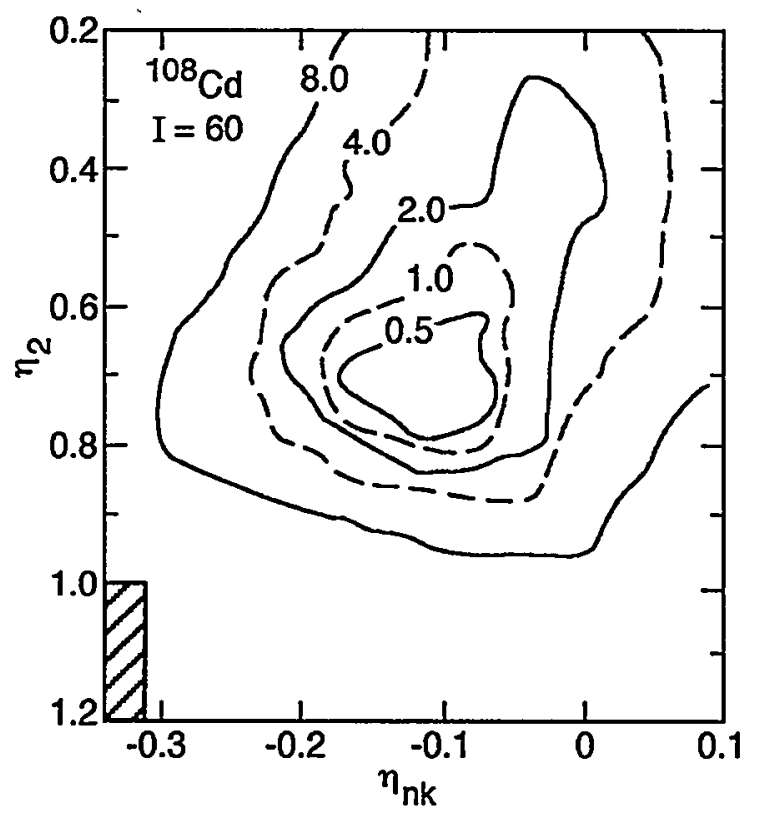

Figure IV-17. Contour diagram of the energy surface of ${ }^{108} \mathrm{Cd}$, as a function of $\eta_{2}$ and $\eta_{n k}$ at $I=60 \hbar$.

1R. R. Chasman, Workshop on the Science for an Advanced ISOL Facility (1997) p. 69

2R. R. Chasman, PHY-9018-TH-98

${ }^{3}$ R. R. Chasman, Phys. Rev. Lett. 80, 4610(1998)

${ }^{4}$ R. R. Chasman Phys. Lett. B 219, 227 (1989) 


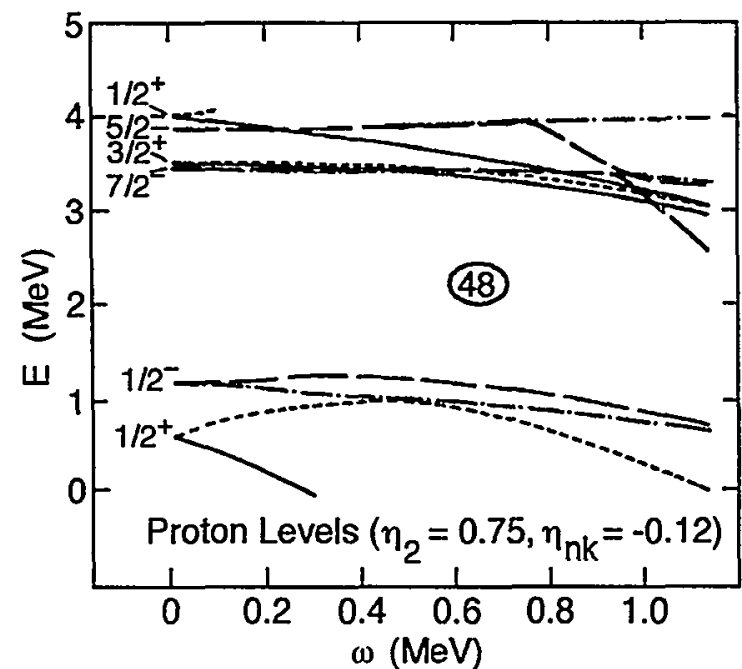

Figure IV-18. Proton single particle levels as a function of rotational frequency for the deformation $\eta_{2}$ $=0.75, \eta_{n k}=-0.12$. The levels are distinguished by parity and signature.

these states are very collective. Making use of a particle number conserving treatment of pairing, we found ${ }^{3}$ that one can calculate the excitation energies of these states quite well as simple broken pair excitations; using conventional pairing force strengths and the single-particle levels that were obtained when superdeformation in this mass region was first ${ }^{4}$

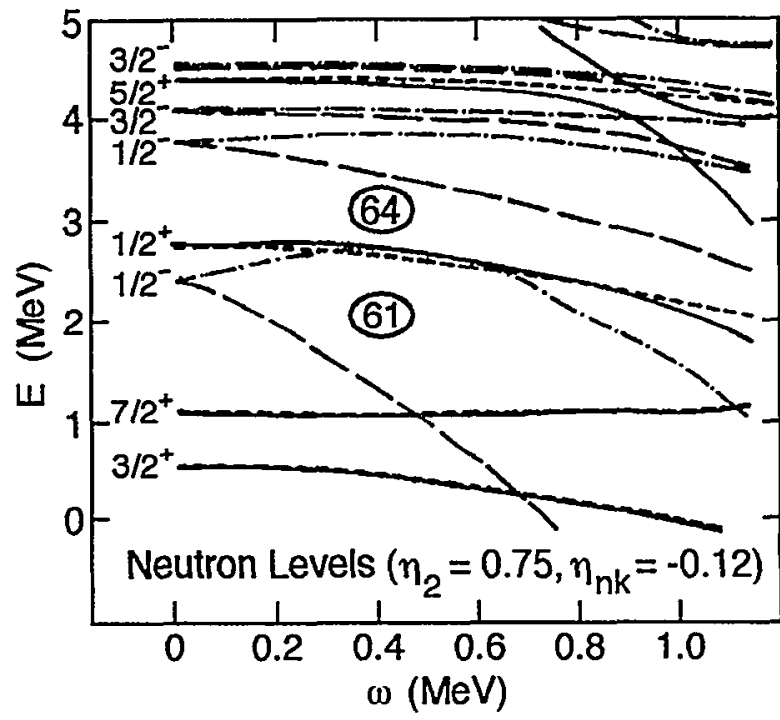

Figure IV-19. Neutron single particle levels as a function of rotational frequency for the deformation $\eta_{2}$ $0.75, \eta_{n k}=-0.12$

predicted. The transition matrix elements connecting these excited states to the superdeformed yrast band remain to be explained.

We are analyzing nuclides in the region $70<\mathrm{A}<100$, searching for very extended minima

\section{c.7. Single Particle States in the Heaviest Elements (I. Ahmad and R. R. Chasman)}

The search for superheavy elements has been a major theme of nuclear structure research for the past twenty years. Theoretical predictions of the stability of superheavy elements depend crucially on the singleparticle energy level spacings in the vicinity of 114 protons and 184 neutrons. Our approach is to learn as much as possible about these levels from spectroscopic studies of nuclides in the $A=250$ region. This is possible because there are members of the relevant spherical multiplets that drop rapidly in energy with increasing deformation, and are fairly close to the ground state in the strongly-deformed nuclides near $\mathrm{A}=$ 250. The orbitals that are important for fixing the shell corrections near $\mathrm{N}=184$ are the $h_{11 / 2}, j_{13 / 2}$ and $k_{17 / 2}$ spherical states. For each of these spherical orbitals, there is a corresponding deformed orbital whose energy in the $\mathrm{A}=250$ region is quite sensitive to one of these spherical states, e.g. the 1/2-[761] orbital that has already been identified in ${ }^{251} \mathrm{Cf}$ is quite sensitive to the spherical $j_{13 / 2}$ orbital. The position of the $1 / 2+[880]$ deformed orbital is very sensitive to the $k_{17 / 2}$ spherical state. According to our calculations, using a momentum-dependent potential, this state should be found at $\sim 1500 \mathrm{keV}$ in ${ }^{251} \mathrm{Cf}$ and should be populated in an $\left(\alpha,{ }^{3} \mathrm{He}\right)$ reaction. We calculated signatures for the low-lying states in ${ }^{251} \mathrm{Cf}$ and the calculated energies and signatures are in good agreement with the experimentally-observed (d,p) spectrum. We expect

1. Ahmad et al., Nucl. Phys. 646, 175 (1999)

2R. R. Chasman and I. Ahmad, Phys. Lett. B 392, 255 (1997) 
to see the high-J states in a $\left(\alpha,{ }^{3} \mathrm{He}\right)$ transfer reaction. As a ${ }^{250} \mathrm{Cf}$ target is not available, we studied the high-J states in $249 \mathrm{Cm}$, which is an isotone of ${ }^{251} \mathrm{Cf}$. The $\left(\alpha,{ }^{3} \mathrm{He}\right)$ experiment was carried out and two high-J peaks were observed at $\sim 1.6$ and $\sim 1.9 \mathrm{MeV}$. These peaks are candidates for the $1 / 2+$ [880] band. Additional coincidence studies will be needed to make a definitive assignment. This study ${ }^{1}$ is published.

Using the Stutinsky method, we found ${ }^{2}$ we could get very good agreement with the known low-lying levels in ${ }^{251} \mathrm{Cf}$ using a momentum-independent Woods-Saxon potential. This calculation gives the $1 / 2+[800]$ orbital at $\sim 2.0 \mathrm{MeV}$. Although it is not clear yet how well this corresponds to the experimental value in ${ }^{249} \mathrm{Cm}$, we used the potential parameters generated from this fit to study the stability of superheavy elements. To determine potential parameters for protons in the heavy elements, we utilized data from our spectroscopic studies of $255 \mathrm{Md}$. These studies show the 1/2-[521] orbital to lie above the 7/2-[514] orbital, but the magnitude of the energy difference is not known. Determining the potential in this way, we extrapolate directly to the superheavy element region. As the usual zero point correction to mass estimates is questionable in that it treats only the quadrupole deformation mode,

\section{c.8. Studies of Nuclear Energy Surfaces L. M. Robledo*)}

This collaborative research program is focused on the study of nuclear energy surfaces at high spin, with an emphasis on very deformed shapes using two complementary methods: 1) the Strutinsky method for making surveys of mass regions, and 2) Hartree-Fock calculations using a Gogny interaction to study specific nuclei that appear to be particularly interesting from the Strutinsky method calculations. The great advantage of the Strutinsky method is that one can study the energy surfaces of many nuclides $(-300)$ with a single set of calculations. Although, the Hartree-Fock calculations are quite time consuming relative to the Strutinsky calculations, they determine the shape at a minimum without being limited to a few deformation modes. We completed a study of ${ }^{182} \mathrm{Os}$ using both approaches. In our cranked Strutinsky calculations, that incorporate a we replaced this correction in our mass estimates by a term that depends quadratically on the sum of the proton and neutron shell corrections. This correction is easy to evaluate and gives quite reasonable corrections where masses are known.

Using the potential parameters derived from the heaviest elements for which there is detailed spectroscopic information, we find that our calculated lifetimes are sufficiently long so as to be able to observe elements at least through $\mathrm{Z}=120$.

In our analysis, we assumed that the $1 / 2$-[521] orbital lies just above the 7/2-[514] orbital in $255 \mathrm{Md}$. In fact, this level spacing is not yet known. Our estimates of superheavy element stability will be affected by the experimental value of this level spacing. We hope to determine this spacing through experimental studies, and thereby refine our estimates of superheavy element lifetimes. Another important feature in determining the binding energy of superheavy elements is the magnitude of the pairing interaction strength. We are analyzing excited states in even-even Fm isotopes, to improve our estimates of pairing interaction strengths in the heaviest known elements.

\section{(R. R. Chasman, J. L. Egido,* and}

necking mode deformation in addition to quadrupole and hexadecapole deformations, we found three well separated, deep, strongly deformed minima. The first is characterized by nuclear shapes with axis ratios of 1.5:1; the second by axis ratios of 2.2:1 and the third by axis ratios of 2.9:1. We also studied this nuclide with the density-dependent Gogny interaction at $\mathrm{I}=60$ using the Hartree-Fock method and found minima characterized by shapes with axis ratios of 1.5:1 and 2.2:1. A comparison of the shapes at these minima, generated in the two calculations, shows that the necking mode of deformation is extremely useful for generating nuclear shapes at large deformation that minimize the energy. The Hartree-Fock calculations are being extended to larger deformations in order to further explore the energy surface in the region of the 2.9:1 minimum.

\footnotetext{
*Universidad Autonoma de Madrid

${ }_{1}^{1}$ R. R. Chasman and L. M. Robledo, Phys. Lett. B 351, 18 (1995)

2J. L. Egido, L. M. Robledo and R. R. Chasman, Phys. Lett. B 393, 13 (1997)

${ }^{3}$ L. M. Robledo, J. L. Egido and R. R. Chasman Proceedings of the Conference on Nuclear Structure at the Limits, July 22-26 (1996) p. 124
} 
With the recent availability of large computer resources on the SP system at Argonne, together with the continued availability of computing resources at NERSC, it is feasible to carry out Strutinsky calculations on a large four-dimensional grid in a deformation space that includes octupole deformation in addition to quadrupole, hexadecapole and necking deformations. We are carrying out this study of the A 180 region concentrating on the questions: 1) how do the inclusion of necking and reflectionasymmetric degrees of freedom modify nuclear energy surfaces, and 2) how soft are the many, known, very deformed nuclear shapes in this region to octupole deformation.

We completed ${ }^{1}$ the first phase of these studies, using the Strutinsky method to calculate nuclear energy surfaces in the four-dimensional space discussed above. Comparing the results obtained with and without octupole deformation, we found major modifications of the energy surface in many nuclei in the region around $176 \mathrm{~W}$. These effects are strong at all values of the angular momentum. There are reductions of the total energy of $\sim 7 \mathrm{MeV}$ for the necked-in shapes at the largest deformations. This feature can be understood in terms of incipient fission fragments. The reflectionasymmetric shape is necked in such a way as to exploit the large shell corrections associated with a strongly deformed fragment in the vicinity of $100 \mathrm{Zr}$ and a spherical fragment in the vicinity of $80 \mathrm{Zr}$. The symmetric necked-in shape at these large deformations corresponds to two strongly deformed incipient fission fragments near $A=90$ and is not favored. The effects are huge here, compared to typical octupole deformation energy gains of less than $1 \mathrm{MeV}$.

As in the case for Strutinsky method calculations, the introduction of reflection asymmetry doubles the basis space that must be used in Hartree-Fock calculations. We extended the Hartree-Fock codes to allow us to calculate the properties of very deformed reflectionasymmetric shapes, using a one-center basis. We finished a calculation 2,3 of the energy surface of ${ }^{176} \mathrm{~W}$ at $I=0$, using the Gogny interaction. In these calculations, we also found a substantial lowering of the fission barrier when reflection-asymmetric shapes are allowed. At I $=0$, where the Hartree-Fock calculations were carried out, the fission barrier is lowered by 4.5 $\mathrm{MeV}$ when reflection-asymmetric shapes are included. At $\mathrm{I}=0$, the reduction is $6.2 \mathrm{MeV}$ in the Strutinsky calculations. The agreement between these two very different methods is impressive. In the Hartree-Fock calculations, the asymmetric division is into fission fragments of ${ }^{66} \mathrm{Ni}$ and ${ }^{110} \mathrm{Pd}$.

\section{c.9. Nuclear Structure Near the Proton Drip Line (R. R. Chasman)}

Recently, proton emission was observed in the light $\mathrm{Pb}$ nuclei. It seems that one might hope to use this phenomenon to extract some interesting spectroscopic data on nuclear shapes and orbitals in much the same way that one gets spectroscopic information through single-nucleon transfer reactions such as $(\mathrm{d}, \mathrm{p})$ and $(\mathrm{d}, \mathrm{t})$ reactions. In order to explore this possibility, we developed a treatment of proton emission spectroscopic factors using many-body wave functions. In the case we have studied, ${ }^{185} \mathrm{Bi} \rightarrow{ }^{184} \mathrm{~Pb}$, the energetics of the reaction are such that only the ground state is populated. However, we calculate a very small spectroscopic factor for the proton decay to the ground state $(0.1 \%)$ in

1C. N. Davids et al., Phys. Rev. Lett. 76, 592 (1996) lowest order. To understand the observed ${ }^{1}$ spectroscopic factor $(-3 \%)$, it is necessary to carry out configuration mixing of the many-body wave functions. We are carrying out such calculations and extending them to neighboring nuclides.

We expectt our calculations for this mass region will be improved by using more levels, as we can do with our parallelized code. Also, we can now handle Coulomb matrix elements, which should give more realistic results. This is quite important for treating the diverse shapes of this region; shapes that differ substantially in deformation. 


\section{ATOMIC THEORY AND FUNDAMENTAL QUANTUM MECHANICS}

\section{d.1. Interactions of High-Energy Photons with matter (M. Inokuti)}

In support of experimental work in atomic physics with the use of synchrotron radiation, theoretical studies are being conducted on the physics of Compton scattering by bound electrons, focusing on topics selected in view of basic importance, timeliness, and potential in applications.

When the photon energy greatly exceeds the binding energy of an electron, the effect of electron binding to an atom is largely understood in the framework of an impulse approximation, which accounts for the momentum of an electron at the instant of the Compton scattering. However, the physics remains to be explored for a more general case, where full details of atomic structure, described for instance in terms of the Green's function, need to be considered. ${ }^{1}$ For a

1 M. Jung et al., Phys. Rev. Lett. 81, 1596 (1998)

${ }^{2}$ M. Dingfelder et al., Radiat. Phys. Chem. 53, 1 (1998) hydrogen-like atom, or within a simple-electron approximation, full analysis of the binding effect is theoretically feasible. Efforts will be made to elucidate basic points of the physics involved and to suggest items for experimental exploration.

Work on the Compton scattering by liquid water was completed. Results of recent measurements by a group at Sendai were compared closely with an earlier semiempirical determination ${ }^{2}$ of the Bethe surface (the strength of the Compton scattering plotted as a function of both energy transfer and momentum transfer), within the impulse approximation and a reasonably good agreement was found. A paper on this topic will appear in Radiation and Environmental Biophysics.

\section{d.2. Interactions of Fast Charged Particles with Matter (M. Inokuti)}

Stopping power, the total yield of ionization, its fluctuation, ionization cross sections, and other quantities related to the energy loss of fast charged particles penetrating through matter, are important in many applications such as the detection of particles and the analysis of their charges and kinetic energies. A review of the current understanding of "W, F, and I: Three quantities basic to radiation physics" was written for publication in the Proceedings of a symposium, 29 March 1998, held in Tokyo in honor of Tadayoshi Doke on the occasion of his seventieth birthday.

Another review on "Electron collision cross sections of atoms" was produced for inclusion in a forthcoming volume on "Atomic Collisions". in the LandoltBörnstein Numerical Data and Functional Relationships in Science and Technology, Springer-Verlag. This article reports on various cross sections for electron collisions with free atoms, and presents values recommended as best in the light of current knowledge. 


\section{d.3. Electron Energy-loss Spectrum of Nanowires (H. Esbensen and G. F. Bertsch*)}

Recent experimental interest in electron energy-loss spectroscopy (EELS) of carbon nanotubes and silicon whiskers motivated us to calculate the energy-loss spectrum of fast electrons interacting with a thin dielectric wire. Assuming that the electron beam does not penetrate the wire but passes it on a trajectory perpendicular to the wire at a certain impact parameter, we were able to derive an analytic expression for the energy loss spectrum, with the dielectric function of the material as input.

*INT, University of Washington

1Phys. Rev. B 58, 14031 (1998)

\section{d.4. Quantum Robots (P. Benioff}

Work begun last year on quantum robots was continued. A quantum robot is a mobile system with an on board quantum computer and ancillary memory, output, and control systems. The quantum robot interacts with other quantum systems in its environment. The dynamics is described by tasks that are alternating sequences of computation and action phases. Each computation phase determines the action for the next phase. Input includes the local state of the environment and states of the memory and output systems. The following action phase carries out the action determined in the previous computation phase. Local environmental observations may be needed. The task is complete when the desired goal is reached.

The dynamical description in terms of a unitary discrete time-step operator $\mathrm{T}=\mathrm{T}_{\mathrm{a}}$ (action phase) $+\mathrm{T}_{\mathrm{c}}$ (computation phase), given previously for motionless noninteracting environmental systems, was expanded to

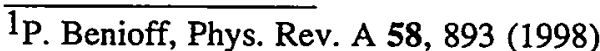

The formula we derived for the energy-loss spectrum can, in particular, be evaluated in closed form for a conducting wire. The spectrum displays in this case the characteristic peak of a surface plasmon excitation, and a low-energy peak, associated with excitations of electronic motion along the wire, which diverges as $1 / E$ as the energy loss $\mathrm{E}$ goes to zero. This work has been published. ${ }^{1}$

include moving interacting systems whose dynamics is described by a unitary step operator $\mathrm{T}_{\mathrm{E}}=\mathrm{e}^{-\mathrm{iH} \Delta}$. Here $\Delta$ and $\mathrm{H}_{\mathrm{E}}$ are the time interval and environment Hamiltonian. The time-step operator $\mathrm{T}$ is replaced by

$\Gamma=\mathrm{T}_{\mathrm{E}}^{1 / 2} \mathrm{~T}_{\mathrm{a}} \mathrm{T}_{\mathrm{E}}^{1 / 2}+\mathrm{T}_{\mathrm{E}}^{1 / 2} \mathrm{~T}_{\mathrm{c}} \mathrm{T}_{\mathrm{E}}^{1 / 2}=\Gamma_{\mathrm{a}}+\Gamma_{\mathrm{c}}$

which is exact in the limit $\Delta=0$.

Last year a simple example of a task, measuring the distance between the quantum robot and a motionless particle on a lattice by counting the number of lattice steps between the quantum robot and the particle, was described in terms of a decision tree. The dynamics was restricted to exclude dispersion in the result. This year the description was expanded to allow for dispersion in the action phase part of the task. A description of accuracy was included. 1 


\section{d.5. Definitions of Truth, Validity, Consistency and Completeness in Quantum Mechanics (P. Benioff}

This work is an outgrowth of foundational reasons why a study of quantum computers and quantum robots is interesting. This is based on the premise that, besides their use for efficient computation, these systems form a base for study of systems with some characteristics of intelligence. An essential activity of these systems is the construction of valid physical theories using mathematics and physics. If quantum mechanics is universally applicable, then quantum mechanics must describe its own validation by these systems. It is expected that such a theory will include a coherent combination of mathematical logical concepts with quantum mechanics. To this end it is necessary to bring mathematical logical concepts into quantum mechanics.

This was done for a simple example by defining truth, validity, consistency, and completeness for a quantum mechanical version of a simple classical expression enumeration machine described by Smullyan. ${ }^{1}$ Some of the expressions are chosen as sentences denoting the presence or absence of other expressions in the enumeration. Two of the sentences are self-referential. It is seen that for the quantum version, truth, consistency and completeness have different properties than in the classical version. This is based on the fact that, following Feynman, the evolving system state is a linear sum over many different expression sequence path states. Because of this the truth of a sentence $S$ is defined only on those paths containing $S$. It is undefined elsewhere. Also $S$ and its negation can both be true provided they appear on separate paths. This satisfies the definition of consistency. It was proved that validity implies consistency. It was shown that the requirements of validity and maximal completeness strongly restrict the allowable dynamics for the quantum system.

The question of the existence of a valid and maximally complete dynamics was discussed. It was shown that there exists a quantum computer that is valid and complete for the set of sentences considered. It seems likely that the quantum computer constructed is exponentially efficient in the sense that the proof that the quantum computer is valid and complete for all included expressions of length $n$ seems to be polynomial in $n$.

${ }_{1}$ R. Smullyan, Gödel's Incompleteness Theorems, Oxford University Press (1992)

\section{d.6. Force-Free Interactions and Nondispersive Phase Shifts in Interferometry (M. Peshkin)}

It was pointed out by Zeilinger that certain so-called topological interactions lead to energy-independent, or nondispersive, phase shifts in neutron interferometry. That result has been useful to experimenters in confirming the results of theories involving effects of the Aharonov-Bohm type. It has also been used, not always correctly, as an operational signature of topological effects. Zeilinger's result was obtained by calculating the phase shifts in each of what he called the generalized Aharonov-Bohm experiments with neutrons and observing that they were all nondispersive.

I have sharpened Zeilinger's observation by proving from the Schroedinger equation that the issue is absence of forces on the neutron. Force-free interactions imply nondispersive phase shifts. The converse proposition, that a nondispersive phase shift implies a force-free interaction, was shown to be false, but the counter examples appear to be physically rather contrived.

A paper that presents this result and applies it to several nondispersive phenomena of current interest, and that also seeks to clarify the distinction between force-free interactions and topological interactions, has been accepted for publication in Foundations of Physics. 


\section{d.7. A No-go Theorem for Matter-Wave Interferometry, with Application to Neutron Electric-Dipole Moment Experiments (M.Peshkin)}

There has for some years been interest in ideas for neutron-interferometry measurements of the neutron EDM that might have a chance to be significantly more sensitive than current experiments based on the Ramsey method. One such idea is to expose a neutron to a strong electric field gradient, separating the neutron's wave function into two slightl;y different wave packets with opposite neutron spin in the direction of the force exerted on the neutron through its EDM. The separation of the two wave packets by that force is minute, but amplification by ingenious interferometric tricks may make it measurable. Published discussions of some of those ideas suffer from the use of semiclassical phase shift approximations that suffice for ordinary interferometry but that become uncertain when applied to the very delicate EDM proposals.

I have been able to avoid the uncertainties of semiclassical approximations by proving a no-go theorem from the Schroedinger equation which shows that the desired amplification is impossible in some, but not all, of the proposed geometries.

\section{E. OTHER ACTIVITIES}

\section{e.1. Theory Institute on Nuclear Structure (R. R. Chasman and H. Esbensen)}

The Physics Division theory group hosted a workshop on "Nuclear Structure", August 3-7, 1998. The workshop brought together about 20 theorists from many countries, who conduct research in many areas of nuclear structure.

The purpose of the workshop was to examine the strengths of various methods to advance our understanding of the nuclear structure of moderately heavy nuclei. The methods discussed included HartreeFock and Strutinsky Method calculations and large-scale shell-model calculations. The methods were applied to the analysis of superdeformation at high spin, with emphasis on problems of angular momentum projection and particle number conservation and studies of nuclei far from stability. The subjects discussed also included studies of light nuclei, including halo nuclei, using large-space shell-model calculations and quantum Monte Carlo calculations. A confluence is developing in the problems that can be handled with the various methods and this was brought out at the workshop. The questions pertaining to nuclear structure and reaction studies of nuclei far from stability, discussed at the workshop, will be occupying the attention of both the experimental and theoretical communities for the next decade.

A schedule of four talks per day allowed ample time for discussions of critical issues and results by the participants. The workshop was funded by the Associate Laboratory Director for Physical Research. 


\section{e.2. 11th Annual Midwest Nuclear Theory Get-Together (R. B. Wiringa)}

The Theory Group hosted the 11th Annual Midwest Nuclear Theory Get-Together on September 25 and 26, 1998. Nuclear theorists from a number of midwest universities get together every fall to find out what different people and groups in the region are working on. The organizational duties rotate among the participants, but Argonne has become the regular meeting place by virtue of its facilities and central location. The organizer for 1998 was Paul Ellis of the University of Minnesota. The meeting provides a good chance for students to broaden their horizons and get some practical speaking experience in a friendly atmosphere. The format is very informal, with an agenda of talks being volunteered at the beginning of the meeting. This year we had the largest attendance ever: 30 faculty, postdocs, and students from ten different universities in Indiana, Iowa, Kentucky, Michigan, Minnesota, Ohio, and Wisconsin, along with the Argonne staff. Some 28 presentations were made over Friday afternoon and Saturday morning. Topics included low-energy theorems at finite temperature, light-front and effective field theories, QCD, neutron resonances, quantum Monte Carlo methods, pionic fusion, relativistic heavy-ion collisions, and many other topics. A good time was had by all. 


\title{
V. ATOMIC AND MOLECULAR PHYSICS RESEARCH
}

\section{OVERVIEW}

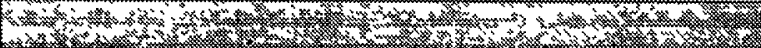

The major goal of the atomic physics program is to establish a refined and quantitative understanding of $\mathrm{x}$-ray interactions with atoms and molecules. These studies of photoprocesses provide a fundamental basis for the $\mathrm{X}$-ray methods used in applications, e.g. Compton scattering studies of electron momentum distributions, electronic and geometric structure determination using angle-resolved photoemission, core-hole shifts in complex chemical environments, EXAFS determination of local structure, and crystallographic determination of global structure. Research during the past year focuses on benchmark measurements of $\mathrm{x}$-ray photoionization and scattering on simple gas phase targets (rare gases) which serve as a testbed for the development of theoretical methods that can be extended to complex systems. A general and accurate theoretical method to describe x-ray scattering and photoionization has not yet been developed due to the need to include accurate descriptions of both wavefunctions and the x-ray atom interaction. A complementary effort tests relativistic many-body theory in few-electron systems in the intermediate- $\mathrm{Z}$ regime where electron correlation, relativistic, and $\mathrm{QED}$ effects are simultaneously important. Progress in understanding relativistic interactions in atoms should assist development of quantum chemistry codes for heavy elements currently of interest for nuclear waste remediation.
\end{abstract}

Studies of X-ray interactions with atoms were enabled by the Advanced Photon Source, APS. This year four experiments were aimed at challenging established theoretical concepts such as form-factor, dipole and independent particle approximations in high-energy photoionization and scattering. All experiments were conducted at the Basic Energy Sciences Synchrotron Radiation Center, BESSRC, located at the APS. Photoionization processes are typically calculated using the electric dipole approximation, E1, an extension of techniques used at ultraviolet energies. Higher-order terms, M1 and E2, are manifest in forwardbackward asymmetries of the ejected photoelectrons. In a continuation of our earlier work done at NSLS, these asymmetries were studied at higher photon energies in atomic rare gases using a rotatable photoelectron spectrometer. Formfactors are widely used to describe x-ray scattering from complex systems. However, there are substantial corrections to the form-factor construct which become increasingly dominant near thresholds. These corrections were mapped out at energies nearer threshold using a new system that measures, simultaneously, both reference and sample gas scattering at multiple angles. The ejection of two electrons by a single high-energy photon is an inherently many-body process, requiring theory beyond independent particle approximation. Double ionization 
processes, induced by Compton scattering as well as photoionization, were studied. The double ionization of helium provides a stringent test of scattering theories in a few-body system, whereas the study of double K-ionization provides an important probe of electron-electron correlation in a heavy system where relativistic effects are also important.

Studies of few-electron systems continued at the ATLAS and GSI facilities. Progress was made in testing relativistic-many-body theories by measuring the spectral shapes of two-photon decay at intermediate and high nuclear charge. The final analysis of the data for two-photon decay of He-like nickel $(Z=28)$ was completed and a model independent measurement of the two-photon shape was obtained. In addition, the first observation of two-photon decay at very high $\mathrm{Z}$ with a measurement of the decay of the $2{ }^{1} S_{0}$ level in He-like Au $(Z=79)$ was made at GSI in Germany.

Progress was made in development of a laser-cooled $\mathrm{Li}$ trap to be used both for studies of photon and electron-induced ionization processes. The study of the three-electron $\mathrm{Li}$ system extends the large body of work performed on He. Studies of multiple-ionization processes using intense, high-field lasers are of particular current interest, with the study of the electron correlation in such systems at the forefront of such research.

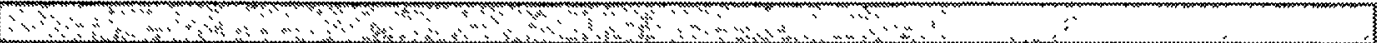


a . Forward-Backward Asymmetries of Atomic Photoelectrons (S. H. Southworth, B. Krässig, E. P. Kanter, J. C. Bilheux, R. W. Dunford, D. S. Gemmell, S. Hasegawa, and L. Young)

Linearly-polarized, narrow-band X-ray beams in the 4-19 $\mathrm{keV}$ energy range provided by the BESSRC-CAT undulator beamline at the APS were used to measure forward-backward asymmetries of atomic photoelectrons. A diagram of the experimental system is shown in Fig. V-1. A parallel-plate electron analyzer (PPA) is rotated about the polarization vector $\varepsilon$ with the polar angle fixed at the magic angle $\theta=\cos ^{-1}\left(3^{-1 / 2}\right) \approx$ $54.7^{\circ}$. The forward-backward asymmetry with respect to the $\mathrm{X}$-ray propagation vector $\mathbf{k}$ is determined from photoelectron intensities measured at four azimuthal angles $\varphi=45^{\circ}, 135^{\circ}, 225^{\circ}$, and $315^{\circ}$. Those angles are magic with respect to both $\varepsilon$ and $k$, and simple data analysis procedures account for $<100 \%$ polarization and small angular misalignments. ${ }^{1}$ The intensities of scattered and fluorescent X-rays recorded by an X-ray detector positioned opposite the PPA are used to normalize the electron intensities for variations in X-ray beam intensity and sample gas density.

Angle-dependent intensities of $\mathrm{Kr}$ 1s photoelectrons were measured at several kinetic energies from threshold to $5 \mathrm{keV}$. Selected results shown in Fig. V-2 demonstrate backward enhancement at $32 \mathrm{eV}$, no forward-backward asymmetry at $1217 \mathrm{eV}$, and forward enhancement at $5017 \mathrm{eV}$. The angular dependence of $\mathrm{Kr}$ $\mathrm{L}_{2,3}-\mathrm{MM}$ Auger electrons, which is expected to be forward-backward symmetric, was used to determine the small instrumental asymmetry. The measured $\mathrm{Kr} 1 \mathrm{~s}$ asymmetries are consistent with calculations which include interference between electric-dipole and electricquadrupole transitions. ${ }^{2}$ The data are being analyzed for comparison with the predicted threshold dependence ${ }^{2}$ and the significance of electric-octupole amplitudes at high energy. 3

1 M. Jung, B. Krässig, D. S. Gemmell, E. P. Kanter, T. LeBrun, S. H. Southworth, and L. Young, Phys. Rev. A54, 2127 (1996).

2 A. Bechler and R. H. Pratt, Phys. Rev. A39, 1774 (1989) and Phys. Rev. A42, 6400 (1990).

${ }^{3}$ L. A. LaJohn and R. H. Pratt, Phys. Rev. $\underline{A 58}, 4989$ (1998).

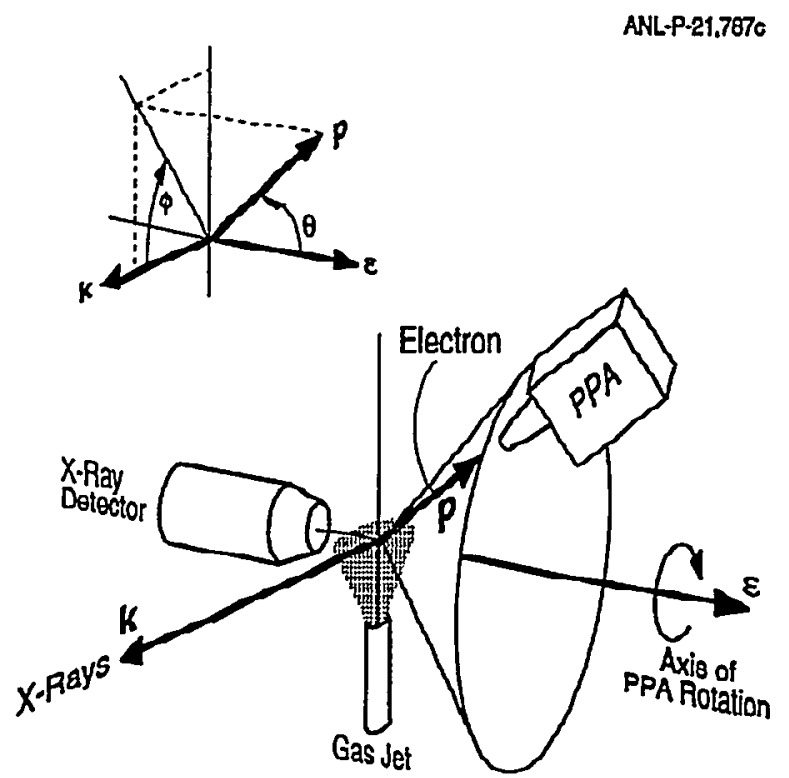

Fig. V-1. Schematic diagram of the experimental system used for measurements of forward-backward photoelectron asymmetries. The PPA is a parallel-plate electron energy analyzer. The photoelectron momentum $p$ is measured relative to the polarization vector $\varepsilon$ and propagation vector $k$ of the $X$-ray beam. 

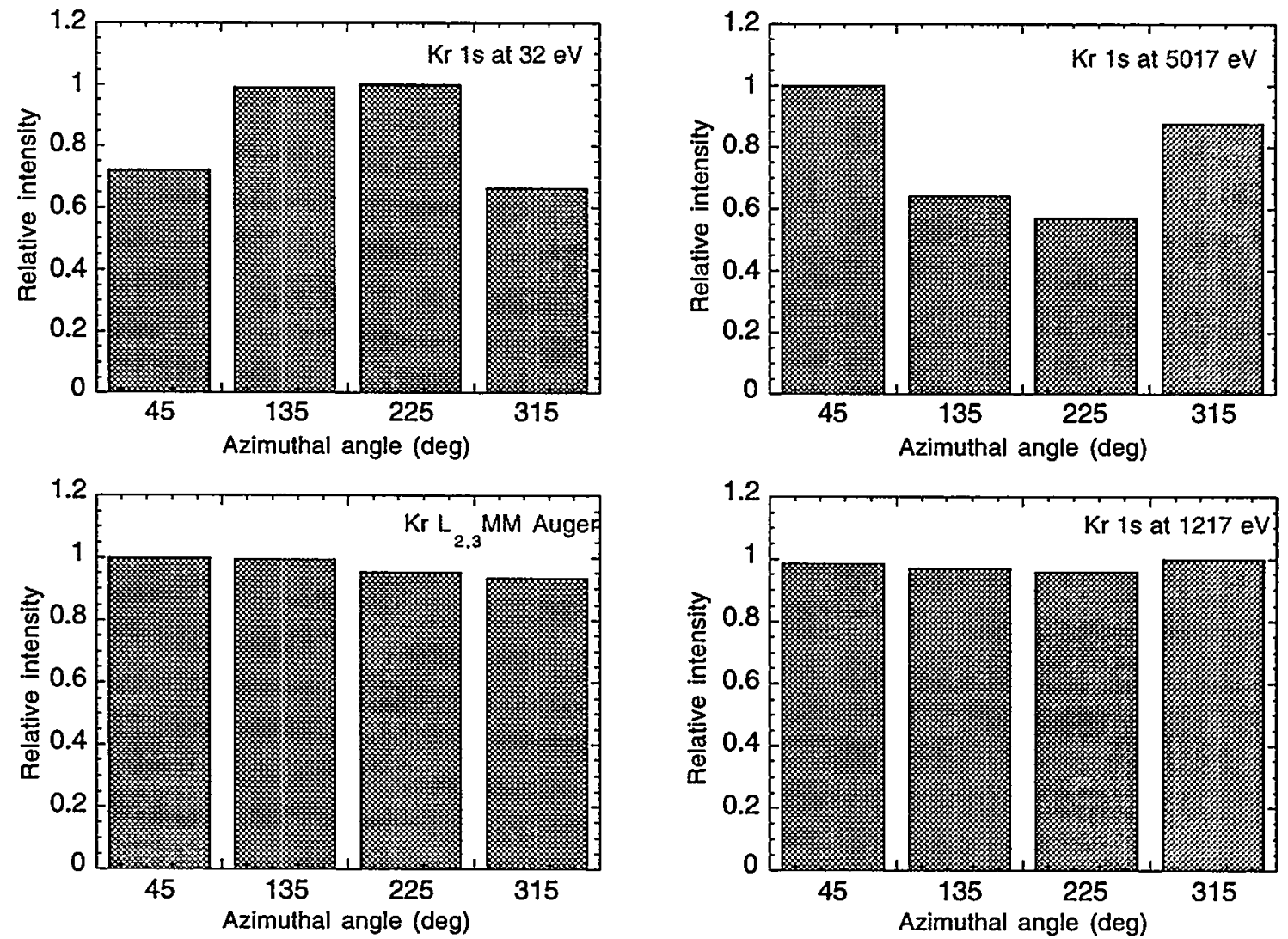

Fig. V-2. Angular dependence of the relative intensities of $\mathrm{Kr} 1 \mathrm{~s}$ photoelectrons measured at $32 \mathrm{eV}, 1217 \mathrm{eV}$, and $5017 \mathrm{eV}$ above threshold and for $\mathrm{Kr} L_{2,3} M M$ Auger electrons.

b . Electron Pair Correlation Functions from X-ray Scattering (L. Young, R. W. Dunford, D. S. Gemmell, E. P. Kanter, B. Krässig, S.H. Southworth, R. Bonham,* and P. Lykos*)

Within the validity of the first Born approximation measurements of the total (elastic + inelastic) scattering cross-sections yield information on the electron-electron pair correlation function and its various moments through a Fourier transform. However, it is necessary to go beyond the first Born approximation for measurements of $\approx 1 \%$ accuracy, i.e. at the level required to extract electron pair correlation functions. Our initial measurements of elastic and inelastic scattering cross sections in $\mathrm{Ne}$ and He revealed the need to include nonlocal exchange, electron correlation, relativity and dynamic effects (second order $\mathrm{p} \bullet \mathrm{A}$ terms) to obtain theoretical agreement with $1 \%$ accuracy. ${ }^{1}$ The experimental accuracy was obtained by measuring ratios: total scattering in $\mathrm{Ne}$ to $\mathrm{He}$ and the ratio of Compton to Rayleigh scattering in Ne. The theoretical agreement was obtained assuming that the corrections to the simplest independent-particle, non-relativistic, firstorder approach could be treated perturbatively.

After this initial experiment, a follow-up experiment was performed at lower momentum transfers, where the perturbative approach might not be adequate. The analysis of this scattering data revealed that systematic 
uncertainties were unacceptably large because of the long data acquisition times using the relatively low flux from an unfocussed X-ray beam from the BESSRC bending magnet beamline. Subsequently, we designed a new scattering chamber to permit simultaneous measurement of both sample and reference gases at scattering angles of $45^{\circ}$ and $90^{\circ}$. A set of data was taken using the high flux X-rays from the undulator beamline for the energy range 4.3 to $15 \mathrm{keV}$. Data analysis is underway.

*Illinois Institute of Technology

${ }^{1}$ M. Jung et al., Phys. Rev. Lett. 1, 1596 (1998).

\section{c. Double Ionization of Helium by X-Ray Compton Scattering (B. Krässig, R. W. Dunford, D. S. Gemmell, S. Hasegawa, E. P. Kanter, S. H. Southworth, L. Young, W. Schmitt*, H. Schmidt-Böckingt, and T. Weber $\left.{ }^{\dagger}\right)$}

The process of helium double ionization is of fundamental importance to the understanding and modeling of electron correlation effects. Theoretical treatments of this process require incorporation of electron correlation both in the intial bound state and the final three-body continuum state. Experimentally, this process is very difficult to determine because its cross section is very small. Published results in the energy range between the onset of Compton ionization at a few $\mathrm{keV}$ up to about $20 \mathrm{keV}$ display substantial discrepancies in the energy dependence of the ratio $\mathrm{R}_{\mathrm{C}}=\mathrm{He}^{++} / \mathrm{He}^{+}$in this process.

*GSI Darmstadt, Germany †Universität Frankfurt, Germany
We have measured the ratio $R_{C}$ to high precision at several X-ray energies between 8 and $20 \mathrm{keV}$. The experiments were performed at the BESSRC-CAT undulator beam line ID 12 at the APS. A specially designed recoil momentum-dispersive ion time-of-flight spectrometer was employed in the experiment to separate Compton scattering ionization events from photoabsorption events. The results are compared to published experimental and theoretical results up to 20 $\mathrm{keV}$ in Fig. V-3. Contrary to the previous experimental determinations, our results clearly delineate the energy dependence of $R_{C}$ in this regime and they strongly favor the theoretical prediction based on the MBPT method. 


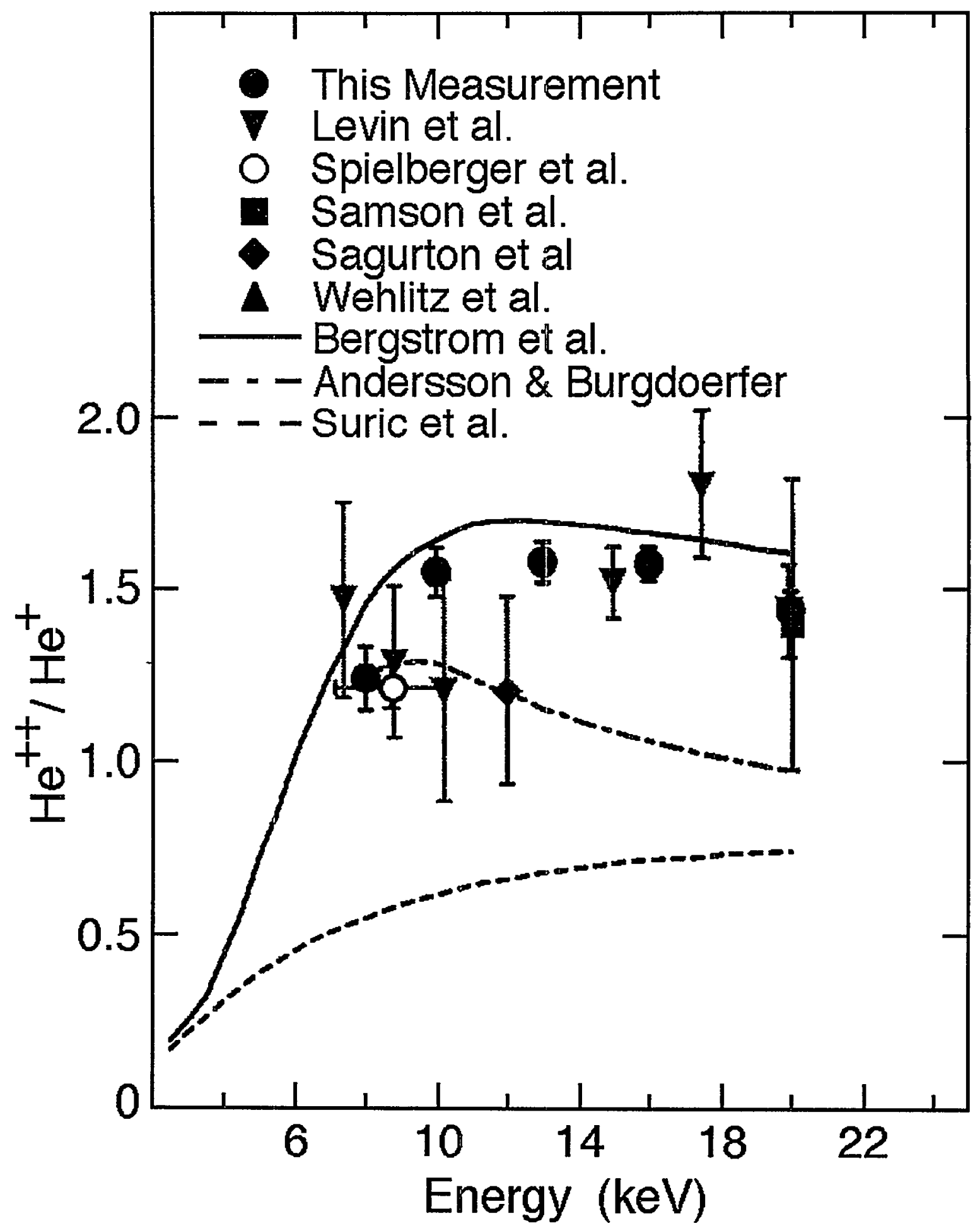

Fig. $V$-3. The ratio $R_{C}=H e^{++} / \mathrm{He}^{+}$for Compton scattering as a function of the $X$-ray energy. References to the data sets quoted in the legend can be found in Spielberger et al., Phys. Rev. Lett. 76,4685 (1996). 


\section{d. Observation of Double K-Ionization by X-Ray Photoionization of Molybdenum (R. W. Dunford, E. P. Kanter, B. Krässig, and S. H. Southworth)}

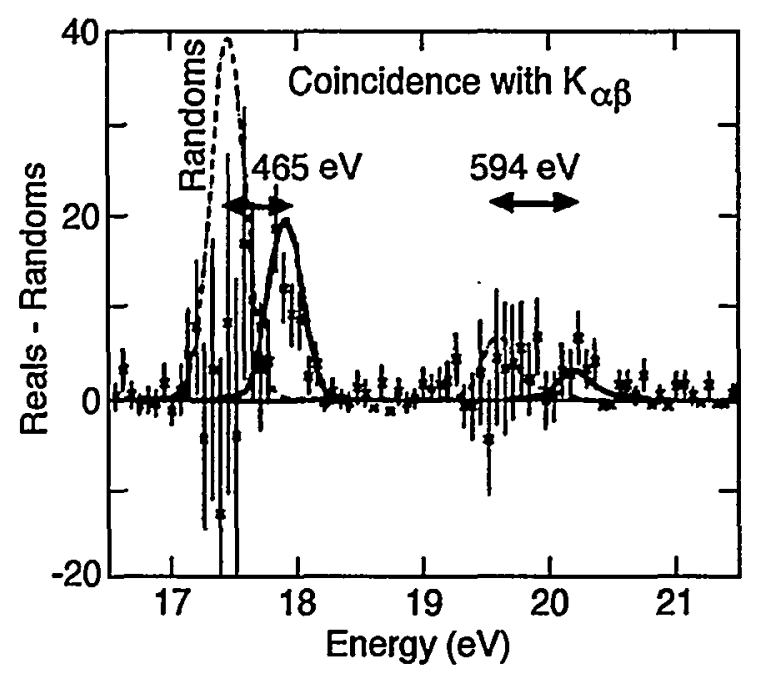

Fig. V-4. Energy in detector " $C$ " in coincidence with $K$ fluorescence detected in detector " $D$ " for true coincidence (i.e. reals-randoms). For comparison, the randoms are shown in the normal diagram line positions as measured (dashed lines) as well as shifted up in energy by the $M C D F$ theoretical predictions (solid curves).

The double ionization of helium was extensively studied for more than 30 years. 1 Because of the single-particle nature of the photon-electron dipole operator, ${ }^{2}$ double ionization would not occur in a two-electron atom in the absence of the $e-e$ interaction and thus this process serves as an extremely sensitive probe of electron correlations within atoms. With the advent of modern third-generation synchrotron radiation sources, there was considerable progress both experimentally ${ }^{3}$ and theoretically ${ }^{4}$ in recent years and attention turned to methods of probing such processes in other systems as a more extensive test of the new theories. 5

Most of that effort is directed toward light, few-electron systems such as $\mathrm{H}^{-}$and $\mathrm{Li}^{+}$because it is generally held that the effects of electron-electron correlations are most pronounced in such systems. This is a consequence of the $1 / Z$ falloff of the ratio of the electron-electron potential to the electron-nucleus interaction with increasing atomic number. While that argument is certainly valid with regard to energy eigenvalues, it is not necessarily pertinent to double ionization which is instead sensitive to eigenfunctions. Because double ionization proceeds through the Z-independent e-e interaction, the effect of electron-electron correlations $w$ was in fact found to be constant with increasing $\mathrm{Z}^{6}$ and consequently more challenging to treat in high- $Z$ systems where relativistic effects simultaneously become important. 7

We recently demonstrated a relatively simple method to isolate the effects of electron-electron correlations in heavy atoms by the creation of double-K-vacancy states in X-ray photoionization and subsequent detection through the observation of the K-hypersatellite transitions as those states relax. Using hard X-rays provided by the Advanced Photon Source, we unambiguously detected the double K-ionization molybdenum. The presence of these double-hole states is signaled by the observation of $K_{\alpha, \beta}$ hypersatellite fluorescence detected in coincidence with the subsequent satellite transition filling the second vacancy (see Fig. V-4). From these data we deduced the relative probabilities for production of double- and single- $K$ vacancies by photon impact at $50 \mathrm{keV}$ and find that ratio to be $2.96(50) \times 10^{-4}$. This is the most precise measure of that quantity in a heavy atom.

That ratio was shown to provide an important probe of electron-electron correlations in high-Z atoms where theoretical descriptions must consider those correlations simultaneously with relativity. In order to provide a more definitive test, however, it is important to separate the contributions of shake processes from the double ionization yield. We plan to do this in the near future by extending our measurements to Xe where previous experiments with radioactive $\mathrm{Cs}$ sources provide an independent measurement of the shakeoff/shakeup contributions.

\footnotetext{
${ }_{1}^{1}$ T. A. Carlson, Phys. Rev. 156, 142 (1967).

2 R. P. Madden and K. Codling, Phys. Rev. Lett. 10, 516 (1963).

3 J. Ullrich et al., J. Phys. B30, 2917 (1997), and references therein.

${ }^{4}$ A. S. Kheifets and I. Bray, Phys. Rev. A57, 2590 (1998), and references therein.

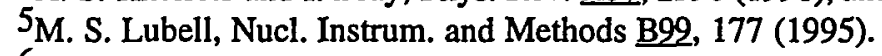

6J. P. Briand et al., Phys. Rev. A23, 39 (1981).

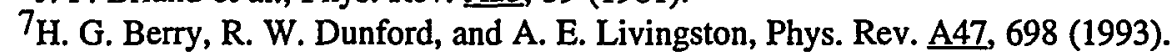




\section{e. Two-Photon Decay (R. W. Dunford, E. P. Kanter, H. W. Schäffer,* P. H. Mokler,* A. E. Livingston, $†$ S. Cheng, $\ddagger$ L. J. Curtis $\ddagger$ C. Kozhuharov,* A.Krämer,* T. Ludziejewski, * H.-T. Prinz, * P. Rymuza,§ Z. Stachura,I Th. Stöhlker,* P. Swiat,Il}

In the process of two-photon decay, two photons $\left(\omega_{1}\right.$, $\omega_{2}$ ) with a sum-energy, $\omega_{0}=\omega_{1}+\omega_{2}$, are emitted as an atom makes a transition to a lower-lying state. ${ }^{1} \mathrm{~A}$ summation over all possible virtual intermediate states (bound and continuum) determines the shape of the continuous two-photon emission spectrum. Hence, the complete structure of the atomic system is reflected in the spectral distribution of the photons, $A\left(\omega_{i}\right) d \omega_{i}$. A measurement of the spectral shape of two-photon decay determines more information than lifetime measurements which test the transition probability integrated over this distribution. We have recently completed measurements of two-photon decay in Helike $\mathrm{Kr}$ and $\mathrm{Ni}$ at ATLAS and established the spectral shape for intermediate $Z$ ions. 2,3 At very high- $Z$, lifetime measurements are not feasible for the study of two-photon decay because the relevant lifetimes are too short, and measurement of the spectral distribution provides the only means for studying three decays. Here, one can probe the influence of a strong central field on the total structure of an ion, and study the role of the negative energy continuum, which appears directly in the sum over virtual intermediate states in the second order matrix element. To explore these issues we have measured the photon energy distribution from decay of the $1 \mathrm{~s} 2 \mathrm{~s}{ }^{1} \mathrm{~S}_{0}$ level in He-like gold $(\mathrm{Z}=79$, $A=197$ ) having a total $X$-ray transition energy $\omega_{0}=68.3$ $\mathrm{keV}$. This isotope is ideal due to it's high- $Z$, simple nuclear shape, and non-zero nuclear spin $(I=3 / 2)$. The nuclear spin causes hyperfine quenching of the $2{ }^{3} \mathrm{P}_{0}$ state and this eliminates potential contributions from the E1M1 two-photon decay branch from this state. ${ }^{4}$ In the past year, we have made the first measurement of two-photon decay in a very high- $Z$ two-electron ion.

In the experiment, 5 the He-like $\mathrm{Au}^{77+}$ ions were provided by the heavy-ion accelerator facility SIS (GSIDarmstadt) at an energy of $106 \mathrm{MeV} / \mathrm{u}$ (44 percent of the velocity of light). After penetrating a $100-\mu \mathrm{g} / \mathrm{cm}^{2}$ thick $\mathrm{Al}$ target $1 \mathrm{~s} 2 \mathrm{~s}{ }^{1} \mathrm{~S}_{0}$ projectile states were excited and decayed directly behind the target (within a few $\mu \mathrm{m}$ ). Photons were detected with two $\mathrm{X}$-ray detectors placed at 60 degrees to the beam direction in the laboratory frame. In the emitter system the detection angles transform to 90 degrees with respect to the beam axis giving an angle $\theta$ of 180 degrees between the directions of the two-photons which corresponds to the maximum intensity as a function of opening angle.

Figure V-5 is a plot of the some of the data from the experiment. These are compared to Monte-Carlo simulations based on the results of a fully relativistic calculation by Derevianko and Johnson. ${ }^{6}$ The experimental results for gold show the first indication of the influence of the strong field on the continuum shape for two-photon decay. The effects are most pronounced for asymmetric photon energies in the highest $Z$ ions, so in future runs we plan to improve the experiment to provide a more extended photon energy range and carry out measurements in ions with even higher atomic numbers.

\footnotetext{
*GSI, Darmstadt, Germany, †University of Notre Dame, $\ddagger$ University of Toledo, §Soltan Institute, Swierk, Poland, IIInstitute of Nuclear Research, Cracow, Poland, IIJagiellonian University, Cracow

${ }^{1}$ M. Göppert-Mayer, Ann. Phys. (Leipzig) 2, 273 (1931).

${ }^{2}$ R. Ali, I. Ahmad, R. W. Dunford, D. S. Gemmell, M. Jung, E. P. Kanter, P. H. Mokler, H. G. Berry, A. E. Livingston, S. Cheng, and L. J. Curtis, Phys. Rev. A55, 994 (1997).

${ }^{3}$ H. W. Schäffer, R. W. Dunford, E. P. Kanter, S. Cheng, L. J. Curtis, A. E. Livingston, and P. H. Mokler, Phys. Rev. A.59, 245 (1999).

${ }^{4}$ G. W. F. Drake, Nucl. Instrum. and Methods B9, 465 (1985).

${ }^{5}$ H. W. Schäffer, R. W. Dunford, C. Kozhuharov, A. Krämer, T. Ludziejewski, P. H. Mokler, H.-T. Prinz, P. Rymuza, L. Sarkadi, T. Stöhlker, P. Swiat, and A. Warczak, Hyperfine Interactions 114, 49 (1998).

${ }^{6}$ A. Derevianko and W.R. Johnson, Phys.Rev. A56, 1288 (1997).
} 


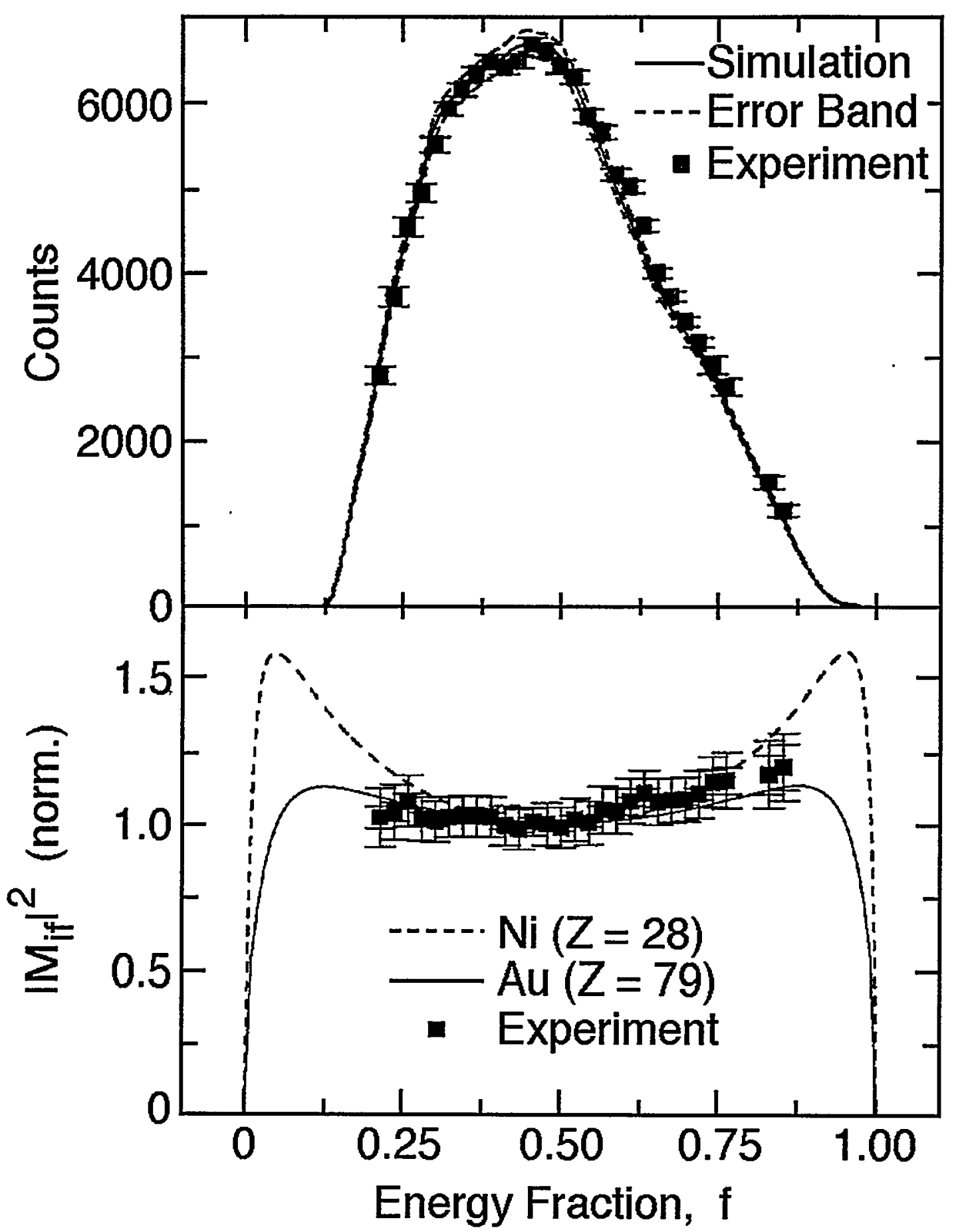

Fig. V-5. The X-ray continuum for the two-photon distribution of He-like Au $77+$ ions (top part) plotted as a function of the fraction of the transition energy $f$. The measured data points are compared with a Monte Carlo simulation (full curve; the dashed curves gives the error band). The square of the energy dependent matrix element (bottom part) extracted from the experiment is compared with the fully relativistic theory for $\mathrm{Au}^{77+}$ and $\mathrm{Ni}^{26+}$ (full and dashed lines, respectively 6 ). 
f . K-Shell Excitation in Ion-Atom Collisions (R. W. Dunford, E. P. Kanter, T. Stöhlker, * F. Bosch, * H. Geissel, * C. Kozhuharov,* T. Ludziejewski, * P. H. Mokler, $*$ C. Scheidenberger, ${ }^{*}$ D. C. Ionescu, $\dagger$ P. Rymuza, $\ddagger$ Z. Stachura,$\S$ and A. WarczakID

Apart from electron capture, projectile excitation is the most important mechanism for photon production in collisions of fast, highly charged ions with target atoms. Despite this, few data exist for excitation in this regime and we have begun a systematic study of this process. Recent results 1,2 from experiments done using the SIS heavy ion synchrotron at GSI include studies of the formation of excited states in hydrogenlike and heliumlike bismuth projectiles $(Z=83)$ in collisions with low- $Z$ targets. The excited states were observed by measuring their radiative decay to the ground state. An important feature of this work is that excitations to the various L-shell sublevels could be separately resolved. This was possible because the relatively large fine structure splitting of these ions allows emission from the $P_{1 / 2}$ and $P_{3 / 2}$ levels to be resolved in the germanium $\mathrm{X}$-ray detectors used. The results of this work were compared with recent fully relativistic calculations and showed that relativistic wavefunctions and magnetic interactions were both important in these collisions. Particularly significant was the demonstration that the magnetic part of the Lienard-Wiechert interaction must be added coherently with the electric part and leads in this case to a significant reduction of the $\mathrm{K}$-shell excitation cross section.

For lower $\mathrm{Z}$ projectiles, little information is available for state-specific excitation in the intermediate energy regime where the orbital velocities of the projectile electrons are similar to the velocity of the ion. We will study this regime at ATLAS by measuring cross sections for excitation from the ground state of He-like
$\mathrm{Ni}$ ions to each of the $\mathrm{n}=2$ excited states. These data would extend the state specific information ${ }^{3}$ available for $\operatorname{Ar}(Z=18)$ up to nickel $(Z=28)$ where relativistic corrections to the energy levels of the projectile ion are more important. Such data would complement the work done at GSI in the very high $Z$ regime.

In the planned experiment, $600-\mathrm{MeV}$ nickel ions prepared in the He-like $26^{+}$charge state will be directed to a gas target. X-ray photons from excitation of the $1 \mathrm{~s}$ electrons will be monitored by a pair of $\mathrm{Si}(\mathrm{Li}) \mathrm{X}$-ray detectors. One detector will view the center of the gas target while the other will be moved along the beam line with a translation stage. Identification of final states produced in the collisions will be done using a combination of low resolution X-ray spectroscopy using $\mathrm{Si}(\mathrm{Li})$ detectors and measurements of the lifetimes of the excited states. A foil target will also be used for comparison.

One of the specific issues we will address is that of direct excitation to the $1 \mathrm{~s} 2 \mathrm{~s}{ }^{3} \mathrm{~S}_{1}$ or $1 \mathrm{~s} 2 \mathrm{p}{ }^{3} \mathrm{P}_{2}$ He-like states in a single collision. Excitation to these levels requires a spin flip and is forbidden in the lowest order theories currently available. It can occur via higher order processes however. In earlier experiments at ATLAS, the $1 \mathrm{~s} 2 \mathrm{~s}{ }^{3} \mathrm{~S}_{1}$ and $1 \mathrm{~s} 2 \mathrm{p}{ }^{3} \mathrm{P}_{2}$ levels were significantly populated via excitation in thin carbon foils. $4-6$ In this case, excitation may involve multiple collisions. Comparison of data for gas targets and foil targets would allow a better understanding of the mechanism for population of these levels.

\footnotetext{
*GSI, Darmstadt, Germany, †LBNL, 抽S, Swierk, Poland, §INP, Cracow, Poland, qJagiellonian University, Cracow, Poland

${ }^{1}$ T. Stöhlker, et al., Phys. Rev. A57, 845 (1998).

2T. Stöhlker, et al., Phys. Lett. A238, 43 (1998).

${ }^{3}$ L. Adoui, et al., Nucl. Instrum. and Methods B98, 312 (1995).

${ }^{4}$ R. W. Dunford, H. G. Berry, K.-O. Groeneveld, M. Hass, E. Bakke, M. L. A. Raphaelian, A. E. Livingston, and L. J. Curtis, Phys. Rev. A38, 5423 (1988).

$5_{R}$. W. Dunford, M. Hass, E. Bakke, H. G. Berry, C. J. Liu, M. L. A. Raphaelian, and L. J. Curtis, Phys. Rev. Lett. $\underline{62}, 2809$ (1989).

${ }^{6}$ R. W. Dunford, C. J. Liu, J. Last, N. Berrah-Mansour, R. Vondrasek, D. A. Church, and L. J. Curtis, Phys. Rev. A44, 764 (1991).
} 


\section{g. Laser-Cooled Li Target for Investigation of Ionization Processes (S. Hasegawa, Z.-T. Lu, L. Young, M. Lindsay,* and S. J. Sibener†)}

The detailed understanding of the dynamics of multiple ionization processes remains a fundamental issue in. atomic physics. 1 Recent experimental and theoretical investigations have greatly enhanced the understanding of the nature of double photoionization processes, particularly in He [cf. 2 for a review]. However, direct triple photoionization of $\mathrm{Li}$ has only recently been observed ${ }^{3}$ and theoretical understanding of this process is at an early stage. The observed $\sigma^{3+} / \sigma^{+}$differs from the predicted ratio for the high-energy limit by a factor of 10. Investigation of multiple ionization processes using a sample of low momentum spread with large solid angle detection will allow angular distribution and polarization dependence to be determined and thus provide stringent tests of developing theories.
During this year, a magneto-optic trap of $\mathrm{Li}$ atoms for studies of ionization processes using recoil ion momentum spectroscopy has been realized. A new apparatus to contain a pulsed magneto-optic trap of $\mathrm{Li}$, ion time-of-flight spectrometer and electron gun has been constructed and is currently being tested. Initial tests demonstrated a trap of $>10^{9}$ atoms $/ \mathrm{cm}^{3}$ using a simplified loading scheme. A first experiment using this apparatus will measure absolute electron impact ionization cross sections in $\mathrm{Li}$ over the energy range $50-1000 \mathrm{eV}$. These cross sections have been the subject of recent theoretical investigations, ${ }^{4}$ and the existing experimental data is inconsistent. 5

*University of Louisville, $\dagger$ University of Chicago

${ }^{1}$ H. W. van der Hart and C. H. Greene, Phys. Rev. Lett. $\underline{81}, 4333$ (1998).

2J. H. McGuire et al., J. Phys. B28, 913 (1995).

${ }^{3}$ R. Wehlitz et al., Phys. Rev. Lett. $\underline{81}, 1813$ (1998).

${ }^{4}$ I. Bray, J. Phys. B28, L247 (1995); X.Z. Qian, S. F. Pan Phys. Lett. A211, 281 (1996); D.W. Chang and P. L. Altick, J. Phys. B28, 1049 (1995).

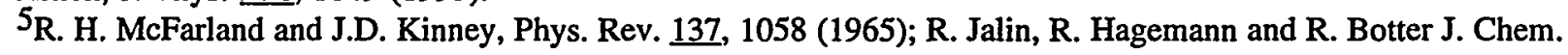
Phys. 59, 952 (1973). 


\section{OTHER EDUCATIONAL ACTIVITIES IN THE PHYSICS DIVISION}

\section{a. Enhancement of Minority Involvement in DOE Nuclear Physics Programs (B. Zeidman)}

The ANL Physics Division, through its Minority Program, continues to attract highly qualified students who apply for participation in the programs of the Physics Division and other ANL divisions. The program is directed toward identification of physics departments with relatively strong programs and faculty interested in stimulating their students to pursue research, particularly in summer programs. Several returning, as well as new, minority physics majors participated in the ANL Summer Program during 1998. Over a dozen former participants are currently enrolled in programs leading to doctorates in physics. A Michigan State University student who spent several summers working with J. Nolen at ATLAS as an undergraduate is currently engaged in thesis research in accelerator physics under Nolen's direction. Another student who recently passed his qualifiers at the University of Michigan will do his thesis research in the ANL Physics Division.

The program is an ongoing effort based upon personal interactions with a substantial number of qualified minority students and faculty. During visits to the Physics Departments of HBCU colleges (Historically Black Colleges and Universities) and other institutions with large minority populations, lectures are presented and there are discussions of activities in physics at ANL and other laboratories, graduate programs, etc. Other activities include attendance at meetings of minority organizations, appointment as Adjunct Professor at Hampton University including supervision of graduate thesis research, and serving on the Advisory Boards of the Centers of Excellence at both Hampton University and Morehouse College.

\section{b. Nuclear Physics Award for Faculty in Undergraduate Institutions (B. Zeidman)}

The goal of the 'Faculty Program', is to enhance undergraduate science education through faculty awards for minority and HBCU faculty that will allow them to directly participate in the ANL Physics Division research program and increase the number of undergraduates involved in research. A participant has obtained independent funding for continuing research collaboration with ANL that involves several undergraduate students. Several minority faculty members and students have been involved in research collaborations with the Physics Division for the past few years and will return this year. In order to maximize the scope of the program, existing educational programs are being utilized to supplement support for some of the participants and more formal collaborative arrangements are being discussed. 


\section{c. Scientific Support of SciTech Museum Exhibits and Outreach Programs (D. Henderson)}

SciTech (Science and Technology Interactive Center) is a hands-on science museum located in Aurora, Illinois, near Argonne. With the help of volunteers and institutional support from Argonne, Fermilab, and several technological companies, SciTech has become an acknowledged leader in developing exhibits to teach modern science in a museum context.
D. Henderson of the Physics Division serves voluntarily as an exhibit developer on a regular basis. Several other staff members volunteer from time to time. The Physics Division collaborates with SciTech in developing exhibits for the museum and for use in Argonne's public educational activities. These efforts involve no significant programmatic costs. 


\section{STAFF MEMBERS OF THE PHYSICS DIVISION}

Listed below are the staff of the Physics Division for the year ending December 31, 1998. The program headings indicate only the individual's current primary activity.

SCIENTIFIC STAFF

EXPERIMENTAL NUCLEAR PHYSICS STAFF

\section{Regular Staff}

Irshad Ahmad, Ph.D., University of California, 1966

Birger B. Back, Ph.D., University of Copenhagen, 1974

* R. Russell Betts, Ph.D., University of Pennsylvania, 1972 Michael P. Carpenter, Ph.D., University of Tennessee, 1987

$\dagger$ Cary N. Davids, Ph.D., California Institute of Technology, 1967

¥ Stuart J. Freedman, Ph.D., University of California, 1972 Donald F. Geesaman, Ph.D., State University of N.Y., Stony Brook, 1976

$\S$ Walter F. Henning, Ph.D., Technical University of Munich, 1968 David J. Hofman, Ph.D., State University of New York, Stonybrook, 1994

I Harold E. Jackson, Jr., Ph.D., Cornell University, 1959

II Robert V.F. Janssens, Ph.D. Univ. Catholique de Louvain, Belgium, 1978 Cheng-lie Jiang, Ph.D. China Institute of Atomic Energy, 1960 Teng Lek Khoo, Ph.D., McMaster University, 1972

** Walter Kutschera, Ph.D., University of Graz, Austria, 1965 Torben Lauritsen, Ph.D., State University of New York, 1990 Christopher J. Lister, Ph.D., University of Liverpool, 1977 Zheng-tian Lu, Ph.D., University of California, Berkeley, 1994

†† Jerry A. Nolen, Jr., Ph.D., Princeton University, 1965

Thomas P. O'Neill, Ph.D., California Institute of Technology, 1994

Richard C. Pardo, Ph.D., University of Texas, 1976

David H. Potterveld, Ph.D., Caltech, 1988

Karl Ernst Rehm, Ph.D., Technical University, Munich, 1973

Guy Savard, Ph.D., McGill University, 1988

¥ John P. Schiffer, Ph.D., Yale University, 1954

Dariusz Seweryniak, Ph.D., Uppsala University, 1994

Kenneth W. Shepard, Ph.D., Stanford University, 1970

Kenneth Teh, Ph.D., Vanderbilt University, 1988

Alan H. Wuosmaa, Ph.D., University of Pennsylvania, 1989

$\S \S$ Benjamin Zeidman, Ph.D., Washington University, 1957

* Joint appointment with the University of Illinois-Chicago.

$\dagger$ Adjunct Professor, Vanderbilt University.

\# On leave of absence at the University of California, Berkeley.

$\S$ Director of the Physics Division.

I Associate Director of the Physics Division. Joint appointment with the University of Chicago.

II Adjunct Professor, North Carolina State University and Adjoint Professor, Vanderbilt University.

** On leave of absence at the University of Vienna.

t† Director of the ATLAS Facility. Adjunct Professor, Michigan State University.

Associate Director of the Physics Division. Joint appointment with the University of Chicago.

$\S \S$ Adjunct Professor, Hampton University. Retired June 1998. 


\section{Special Appointments}

Lowell M. Bollinger, Ph.D., Cornell University, 1951

Sheldon B. Kaufman, Ph.D., University of Chicago, 1953

* Michael Paul, Ph.D., Hebrew University of Jerusalem, 1973

G. Roy Ringo, Ph.D., University of Chicago, 1940

Ralph E. Segel, Ph.D., Johns Hopkins University, 1955

George E. Thomas, B.A., Illinois Wesleyan, 1943

$\dagger$ Ben Zeidman, Ph.D. Washington University, 1957

\section{Term Appointments}

‡ William Cummings, Ph.D., Stanford University, 1992

\section{THEORETICAL NUCLEAR PHYSICS STAFF}

\section{Regular Staff}

Richard R. Chasman, Ph.D., University of California, 1959

Henning Esbensen, Ph.D., University of Aarhus, 1977

$\S$ Tsung-Shung Harry Lee, Ph.D., University of Pittsburgh, 1973

Steven C. Pieper, Ph.D., University of Illinois, 1970

Craig T. Roberts, Ph.D., Flinders University of South Australia, 1989

Robert B. Wiringa, Ph.D., University of Illinois, 1978

\section{Special Appointments}

Paul Benioff, Ph.D., University of California, 1959

Arnold R. Bodmer, Ph.D., Manchester University, 1953

Fritz Coester, Ph.D., University of Zurich, 1944

Mitio Inokuti, Ph.D., University of Tokyo, 1962

Dieter Kurath, Ph.D., University of Chicago, 1951

Robert D. Lawson, Ph.D. Stanford University, 1953

Harry J. Lipkin, Ph.D., Princeton University, 1950

II Vijay Pandharipande, Ph.D., University of Bombay, 1969

Murray Peshkin, Ph.D., Cornell University, 1951

* Special Term Appointee from the Hebrew University of Jerusalem, Israel.

$\dagger$ Special Term Appointee.

\$ Terminated June 1998 .

$\S$ Adjunct Professor, University of Pittsburgh.

II Special Term Appointee from the University of Illinois, Urbana. 


\section{ATOMIC AND MOLECULAR PHYSICS STAFF}

\section{Regular Staff}

* Robert Dunford, Ph.D., University of Michigan, 1978

Donald S. Gemmell, Ph.D., Australian National University, 1960

* Elliot P. Kanter, Ph.D., Rutgers University, 1977

* Bertold Kraessig, Ph.D., University of Freiburg, 1994

* Stephen H. Southworth, Ph.D., University of California, Berkeley, 1982

$\dagger$ Zeev Vager, Ph.D. Weizmann Institute of Science, 1962

* 'Linda Young, Ph.D., University of California, Berkeley, 1981

\section{Special Appointments}

William J. Childs, Ph.D., University of Michigan, 1956

F. Paul Mooring, Ph.D., University of Wisconsin, 1951

Gilbert J. Perlow, Ph.D., University of Chicago, 1940

\section{TEMPORARY APPOINTMENTS}

\section{Postdoctoral Appointees}

John Arrington (from California Institute of Technology, Pasadena, California): Medium-energy physics.

(Juny 1998--

)

Jacques Bloch (from University of Durham, N.C.):

Theoretical physics studies.

(September 1998--

Chun-Yen Chen (from Kansas State University, Manhattan, Kansas):

Medium-energy physics.

(March 1998--

Dirk De Schepper (from MTT, Cambridge, Massachusetts):

Medium-energy physics.

(May 1997--February 1998)

Patrick Decrock (from K. U., Leuven, Belgium):

Accelerator development.

(January 1996--February 1998)

Gregory Hackman (from McMaster University, Hamilton, Ontaro, Canada):

Heavy-ion research at ATLAS.

(September 1995-March 1998)

Shuichi Hasegawa (from University of Tokyo, Japan):

Atomic and molecular physics studies.

(September 1996--September 1998)

Robert Kaye (from Florida State University, Tallahassee, Florida):

Linac development.

(April 1998--

* Transferred to Chemistry Division October 1998.

$\dagger$ Joint Appointment with Weizmann Institute of Science, Rehovot, Israel. 
Nathalie Lecesne (from University of Caen, Caen, France):

Accelerator development.

(November 1997--February 1998)

Pieter Maris (from University of Groningen, The Netherlands):

Theoretical studies.

(September 1996--August 1998)

Douglas Moehs (from Texas A\&M University, College Station, Texas):

ATLAS operations and development.

(August 1998-- )

Bryon Mueller (from California Institute of Technology, Pasadena, California):

Medium-energy physics.

(June 1997--

Vandana Nanal (from Tata Institute, Bombay, India):

Heavy-ion research at ATLAS.

(August 1995--May 1998)

David Nisius (from Purdue University, W. Lafayettte, Indiana):

Heavy-ion research at ATLAS.

(November 1996-March 1998)

Maria Petra (from University of Illi8nois, Urbana, Illinois):

ATLAS operations and development.

(February 1998-- )

* Jorg Reinhold (from Technical University of Munich, Germany):

Medium-energy research.

(September 1995--December 1998)

Peter Reiter (from University of Heidelberg, Germany):

Heavy-ion research at ATLAS.

(August 1996--July 1998)

Riad Rejoub (from University of Nevada, Reno, Nevada): ATLAS Operations and development. (June 1998--

)

Renato Roncaglia (from Indiana University, Bloomington, Indiana):

Theoretical studies.

(September 1997-- )

$\dagger$ Dariusz Seweryniak (from University of Uppsala, Sweden):

Heavy-ion research at ATLAS.

(January 1995--January 1998)

Alejandro Sonzogni (from University of Washington, Seattle, Washington): Heavy-ion research at ATLAS.

(May 1997-- )

Juha Uusitalo (from University of Jyvaskyla, Jyvaskyla, Finland):

Heavy-ion research at ATLAS.

(April 1997-. ) )

* Feodor-Lynen Postdoctoral Fellowship from Alexander von Humboldt Foundation.

$\dagger$ Joint appointment with the University of Maryland. Joined the regular staff January 1998. 
Kalman Varga (from Atomki, Debracen, Hungary):

Theoretical studies.

(April 1998--

Ingo Wiedenhover (from University of Cologne, Cologne, France):

Heavy-ion research at ATLAS.

(September 1997-- )

\section{TECHNICAL AND ENGINEERING STAFF}

(and areas of activity)

Henning O. Back (B.S. Indiana University, 1997)

Technical assistance, heavy-ion physics.

Kevin G. Bailey (B.S. University of Nebraska, 1989).

SOS construction for CEBAF.

Brian T. Batzka (B.S. University of Houston, 1992).

ATLAS lead operator.

* Peter J. Billquist

ECR heavy-ion source, ATLAS operation.

John M. Bogaty (A.A.S. DeVry, 1961).

Electrical systems, ATLAS operation and development.

Benny E. Clifft (A.S.E.E., DeVry, 1959).

Electrical systems, ATLAS operation and development.

$\dagger$ Joseph Falout (B.S.M.E. University of Illinois, 1970).

Experimental equipment design.

John P. Greene (M.S. DePaul University, 1982).

Target preparation.

$\dagger$ Ray E. Harden (A.A.S. Milwaukee School of Engineering, 1957).

ATLAS operator

$\dagger$ Dale J. Henderson (B.S. Elmhurst College, 1951).

Detector development, technical assistance, heavy-ion physics.

James M. Joswick (A.A.S. Milwaukee School of Engineering, 1964).

ATLAS experimental equipment maintenance, technical assistance, heavy-ion physics.

$\dagger$ Raymond B. Kickert

ATLAS experimental equipment maintenance, technical assistance, heavy-ion physics.

\# Charles A. Kurtz (M.S. University of Arkansas, 1984).

Technical assistance, atomic physics.

$\dagger$ Paul Markovich (B.S. Purdue University, 1972).

Surface chemistry, ATLAS development and operation.

* In charge of Dynamitron operations.

$\dagger$ Special Term Appointee.

\#* Transferred to MSD in February 1998. 
Thomas P. Mullen (B.S. Marquette University, 1966).

Division ESH/QA engineer.

Floyd Munson, Jr. (A.A.S. DeVry, 1966, B.S. Lewis University, 1993). Control system for ATLAS.

Bruce G. Nardi (A.A.S. Morton Jr. College, 1967; A.A.S. DeVry, 1969). Electronics design and maintenance.

Tom O'Connor (M.S. DePaul University, 1995). Technical assistancet, medium-energy physics.

Tad Pennington (M.S. University of Missouri, 1989; M.S. University of Alabama, 1998) Detector development, technical assistance, heavy-ion physics.

Deborah Quock (M.S. Rice University, 1985) Technical assistance, ATLAS control system.

* Anne Reuter (B.S. University of Rochester, 1993).

Operate, upgrade and maintain ATLAS cryogenic system.

James R. Specht (A.A.S. DeVry, 1964).

Cryogenics engineer. ATLAS development and operation.

Philip Strickhorn (B.S. DeVry, 1990).

Electrical and technical assistance with ATLAS operations.

$\dagger$ Ian R. Tilbrook (B.S. Pennsylvania State University, 1987; MBA, Keller Graduate School of Management, 1994).

ATLAS operator.

Richard Vondrasek (B.S. University of Mlinois, 1990). ATLAS operator.

Philip R. Wilt (Johnstown Technical School 1973). Electronics design and maintenance.

Bruce J. Zabransky (M.S. University of Illinois, Chicago, 1973). Dynamitron operation.

Gary P. Zinkann (B.S. DeVry, 1975). ATLAS operations supervisor.

\section{ADMINISTRATIVE STAFF}

‡ Allan Bernstein, M.B.A., Rosary College, 1986

$\ddagger$ James E. Nelson, B.A., University of Illinois, 1975

$\S$ Karen J. Thayer

* Terminated December 1998.

$\dagger$ Transferred to Information and Publishing Division March 1998. 


\section{VISITORS AND STUDENTS}

Long-Term Visitors (at Argonne more than 4 months)

Jean-Chritophe Bilheux (from University of Orsay, France):

Atomic physics at accelerators.

(April--October 1998)

II Jolie Cizewski (from Rutgers University, Piscataway, New Jersey):

Heavy-ion research at ATLAS.

(August 1997--June 1998)

Xin Feng (from McGill University, Montreal, Quebec):

Heavy-ion research at ATLAS.

(January--July 1998)

Hiroshi Fukutani (from University of Manitoba, Canada):

Heavy-ion research at ATLAS.

(May 1998--

Rudolf Ganz (from University of Heidelberg):

Heavy-ion research at ATLAS.

(February 1996--January 1998)

Subhendu Ghosh (from Nuclear Science Centre, New Delhi, India): ATLAS development.

(August 1997--

)

Boris Harss (from Technical University of Munich, Germany):

Heavy-ion research at ATLAS.

(June 1997--

)

Peter Lang (from Fachschule Munich, Germany):

Heavy-ion research at ATLAS.

(April--August 1998)

* Donald McLeod (from University of Illinois, Chicago, Illinois):

Heavy-ion research at ATLAS.

(June 1994--

Daniel Martin (from McGill University, Montreal, Quebec):

Heavy-ion research at ATLAS.

(November 1998-- )

\footnotetext{
* In charge of Dynamitron operations.

$\dagger$ Terminated in January 1997.

‡ Assistant Director of the Physics Division.

$\S$ Staff Assistant.

I Maria Goeppert-Mayer Argonne Scholar.
} 
Prakash Potukuchi (from Nuclear Science Centre, New Delhi, India):

ATLAS development.

(March 1996--

Kumar Sharma (from University of Manitoba, Canada):

Heavy-ion research at ATLAS.

(July 1998--

Sunniva Siem (from University of Oslo, Norway):

Heavy-ion research at ATLAS.

(January 1998--

Rolf Siemssen (from KVI, Groningen, Netherlands):

Heavy ion rresearch at ATLAS.

(October 1998--

James Stewart (from DESY Laboratory, Hamburg, Germany)

Medium-energy physics.

(March 1998--

Vincent Stoks (from TRIUMF, Canada):

Theoretical studies.

(December 1997--June 1998)

Ralf Thee (from Technical University, Darmstadt, Germany):

Heavy-ion research at ATLAS.

(August-December 1998)

Hassina Zaim (from University Orsay, France):

Heavy-ion research at ATLAS.

(April--October 1998)

Short-Term Visitors (at ANL less than 4 months)

* Nour-eddi Berrah (Western Michigan University, Kalamazoo, Michigan):

Atomic physics research.

(October 1991--

Susan Fischer (DePaul University, Chicago, Mlinois):

Heavy-ion research at ATLAS.

(October 1998--

)

Clive Halliwell (University of Illinois, Chicago, Illinois):

Heavy-ion research at ATLAS.

(June 1998--

Jeffrey Hangst (University of Aarhus, Denmark):

Heavy-ion research at ATLAS.

(April 1995--

)

* Edward Hohman (York Township High School, Lyons, Illinois):

Summer student coordinator.

(June-August 1998)

* Guest Faculty Research Participant. 
Mikhail Ivanov (JINR, Dubna, Russia):

Nuclear theory studies.

(August-September 1998)

Judith Katzy (University of Illinois, Chicago, Illinois):

Heavy-ion research at ATLAS.

(February 1998-. )

* Wojciech Kucewicz (University of Illinois, Chicago, Illinois):

Heavy-ion research at ATLAS.

(April 1998-- )

Bruce McKellar (University of Melbourne, Australia):

Theoretical physics studies.

(November-December 1998)

Rachid Nouicer (University of Chicago, Chicago, Illinois):

Heavy-ion research at ATLAS.

(April 1998-- )

Anna Maria Porcellato (INFN, Legnaro, Italy):

Heavy-ion research at ATLAS.

(July--September 1998)

* Carol Tanner (University of Notre Dame, Notre Dame, Indiana):

Accelerator atomic physics.

(March 1992--April 1998)

Yau Wah (University of Chicago, Chicago, Illinois):

Heavy-ion research at ATLAS.

(January 1998--

)

\section{Resident Graduate Students}

Hanan Amro (North Carolina State University, Raleigh, North Carolina): Heavy-ion research at ATLAS.

(January 1995-- )

Larry Todd Brown (Vanderbilt University, Nashville, Tennessee):

FMA development at ATLAS

(January 1994-- )

Patrick Crotty (University of Chicago, Chicago, Illinois):

Heavy-ion research at ATLAS.

(January 1998-- )

David Gaskell (Oregon State University, Corvallis, Oregon):

Medium-energy physics.

(September 1998--

Burt Holzman (University of Illinois, Chicago, Illinois):

Heavy-ion research at ATLAS.

(June 1997--

Douglas Koltenuk (University of Pennsylvania, Philadelphia, Pennsylvania):

Medium-energy studies.

(August 1997--September 1998)

* Guest Faculty Research Participant. 
David Lin (Northern Illinois University, Dekalb, Illinois):

Medium energy physics.

(June 1997-August 1998)

Mauricio Portillo (Michigan State University, E. Lansing, Michigan):

Accelerator development.

(September 1995--

Jazmin Schwartz (Yale University, New Haven, Connecticut):

Heavy-ion research at ATLAS.

(May 1995--

Russell Strickland (University of Chicago, Chicago, Illinois):

Heavy-ion research at ATLAS.

(March 1997--February 1998)

\section{Guest Graduate Students}

Francesca Borasi (University of Milan, Milan, Italy):

Heavy-ion research at ATLAS.

(November 1997-- ）

Dipangkar Dutta (Northwestern University, Evanston, Illinois):

Medium-energy studies.

(June 1994--

Douglas Koltenuk (University of Pennsylvania, Philadelphia, Pennsylvania): Medium-energy studies.

(August 1997--September 1998)

Kenneth Nollett (University of Chicago, Chicago, Illinois):

Theoretical studies.

(May 1997--

)

Vincent Pelletier (McGill University, Montreal, Quebecv): Heavy-ion research at ATLAS.

(May-August 1998)

Michael Reuter (University of Chicago, Chicago, Illinois):

Heavy-ion research at ATLAS.

(September 1997-- ）

\section{Undergraduate Students}

Lamont Anderson (Illinois Institute of Technology)

Teresa Barlow (Illinois Benedictine College)

Chris Beltran (New Mexico State University)

Robert Blevins (Pennsylvania State University)

Daniel Braithwaite (University of Chicago)

George Chancellor (North Central College)

Scott Davis (University of Illinois-Urbana)

Juan Figueroa (Benedictine University)

Jeff Fingler (University of Manitoba)

Andrew Geraci (University of Chicago)

Guillaume Gervais (Northwestern Univrsity)

Jerome Gura (University of St. Francis)

Taft Hayden (Daley College)

Sarah Heany (U. S. Military Academy)

Edward Hill (University of Chicago) 
Brian Johnson (Northwestern Unviersity)

Kim Lewis (Dillard University)

Jaime Morales (University of Texas-El Paso)

Sultan Muhammad (Ohio State University)

Brian Napolitano (College of DuPage)

Jason Noeth (St. Norbert College)

John Orrell (Rensselaer Polytechnic Institute)

Eric Pahnke (College of DuPage)

Adam Peterson (Joliet Jr. College)

Joseph Snider (University of Chicago)

Devin Sullivan (Carnegie-Mellon University)

John Szezech (University of Illinois-Chicago)

Lisa Tung (Northern Illinois University)

Keri Unterzuber (North-Central College)

Michael Witt (North Central College)

Adam Zelko (Northern Illinois Univrsity)

Pre-College Program (Just Graduated from High School)

(June--August 1998)

Martin Alcorta (Downers Grove South High School)

Rudra Banerji (Oak Park-River Forest High School)

Terrell Bennett (Proviso West High Sschool)

Peter Coley (Benet Academy)

Lindsay Deremer (Oak Park-River Forest High School)

Leah Goodman (Wheaton-Warrenville High School)

Alice Lam (Downers Grove North High School)

Jennifer Nelson (Ressurection High School)

\section{High School Student Aide}

Monica Green (Amos Alonzo Stagg High School)

Silvia Palomino (Joliet Central High School) 
PUBLICATIONS DURING 1998

(The arrangement follows approximately the Table of Contents)

\section{HEAVY-ION NUCLEAR PHYSICS RESEARCH}

Level Structures of $96,97,98 \mathrm{Ru}$ at High Angular Momentum

B. Kharraja, S. S. Ghugre, U. Garg, R. V. F. Janssens, M. P. Carpenter,

B. Crowell, T. L. Khoo, T. Lauritsen, D. Nisius, W. Reviol, W. F. Mueller,

L. L. Riedinger, and R. Kaczarowski

Phys. Rev. C 57, 83-96 (1998)

Hartree-Fock-Bogoliubov Description of Quasiparticle Excitations in the Superdeformed Wells of $191,192 \mathrm{Hg}$ and $192,193 \mathrm{Tl}$

P.-H. Heenen and R. V. F. Janssens

Phys. Rev. C 57, 159-165 (1998)

Nuclear Structure of ${ }^{241} \mathrm{Pu}$ from Neutron-Capture, (d,p)-, and (d,t)-Reaction Measurements

D. H. White, R. W. Hoff, H. G. Borner, K. Schreckenbach, F. Hoyler,

G. Colvin, I. Ahmad, A. M. Friedman, and J. R. Erskine

Phys. Rev. C 57, 1112-1133 (1998)

New Isomer in ${ }^{80} \mathrm{Y}$

J. Doring, H. Schatz, A. Aprahamian, R. C. de Haan, J. Gorres, M. Wiescher,

W. B. Walters, J. Rikovska, L. T. Brown, C. N. Davids, C. J. Lister,

D. Seweryniak, and B. Foy

Phys. Rev. C 57, 1159-1166 (1998)

Shell Effects in Superdeformed Minima

P.-H. Heenen, J. Dobaczewski, W. Nazarewicz, P. Bonche, and T. L. Khoo

Phys. Rev. C 57, 1719-1726 (1998)

Decay of the Odd-Odd $\mathrm{N}=\mathrm{Z}$ Nuclide ${ }^{78} \mathrm{Y}$

J. Uusitalo, D. Seweryniak, P. F. Mantica, J. Rikovska, D. S. Brenner, M. Huhta, J. Greene, J. J. Ressler, B. Tomlin, C. N. Davids, C. J. Lister, and W. B. Walters

Phys. Rev. C 57, 2259-2263 (1998)

Multineutron Transfer in ${ }^{58} \mathrm{Ni}+{ }^{124} \mathrm{Sn}$ Collisions at Sub-Barrier Energies

C. L. Jiang, K. E. Rehm, H. Esbensen, D. J. Blumenthal, B. Crowell,

J. Gehring, B. Glagola, J. P. Schiffer, and A. H. Wuosmaa

Phys. Rev. C 57, 2393-2400 (1998)

Nuclear Structure of ${ }^{94,95}$ Mo at High Spins

B. Kharraja, S. S. Ghugre, U. Garg, R. V. F. Janssens, M. P. Carpenter,

B. Crowell, T. L. Khoo, T. Lauritsen, D. Nisius, W. Reviol, W. F. Mueller,

L. L. Riedinger, and R. Kaczarowski

Phys. Rev. C 57, 2903-2911 (1998) 
Lifetime Measurements of Yrast and Excited Superdeformed Bands in ${ }^{192,193} \mathrm{Hg}$

B. C. Busse, P. Fallon, R. Kruecken, D. Ackermann, I. Ahmad, S. J. Asztalos,

D. J. Blumenthal, M. P. Carpenter, R. M. Clark, M. A. Deleplanque,

R. M. Diamond, S. M. Fischer, F. Hannachi, R. V. F. Janssens, T. L. Khoo,

A. Korichi, T. Lauritsen, I. Y. Lee, A. Lopez-Martens, A. O. Macchiavelli,

R. W. MacLeod, E. F. Moore, D. Nisius, G. Schmid, D. Seweryniak,

F. S. Stephens, and K. Vetter

Phys. Rev. C 57, R1017-R1021 (1998)

First Observation of Excited States in ${ }^{118} \mathrm{Ba}$ : Possible Evidence for Octupole Correlations in

Neutron-Deficient Barium Isotopes

J. F. Smith, C. J. Chiara, D. B. Fossan, G. J. Lane, J. M. Sears,

I. Thorslund, H. Amro, C. N. Davids, R. V. F. Janssens, D. Seweryniak,

I. M. Hibbert, R. Wadsworth, I. Y. Lee, and A. O. Macchiavelli

Phys. Rev. C 57, R1037-R1041 (1998)

Observation of the Exotic Nucleus ${ }^{145} \mathrm{Tm}$ via Its Direct Proton Decay

J. C. Batchelder, C. R. Bingham, K. Rykaczewski, K. S. Toth, T. Davinson,

J. A. McKenzie, P. J. Woods, T. N. Ginter, C. J. Gross, J. W. McConnell,

E. F. Zganjar, J. H. Hamilton, W. B. Walters, C. Baktash, J. Greene, J. F. Mas, W. T. Milner,

S. D. Paul, D. Shapira, X. J. Xu, and C. H. Yu

Phys. Rev. C 57, R1042-R1046 (1998)

High-Spin Properties of Octupole Bands in ${ }^{240} \mathrm{Pu}$ and ${ }^{248} \mathrm{Cm}$

G. Hackman, R. V. F. Janssens, T. L. Khoo, I. Ahmad, J. P. Greene, H. Amro,

D. Ackermann, M. P. Carpenter, S. M. Fischer, T. Lauritsen, L. R. Morss,

P. Reiter, D. Seweryniak, D. Cline, C. Y. Wu, E. F. Moore, and T. Nakatsukasa

Phys. Rev. C 57, R1056-R1059 (1998)

Positron-Electron Angular Correlations in Internal Pair Conversion

A. H. Wuosmaa, C. M. Conner, I. Ahmad, B. B. Back, R. R. Betts,

R. W. Dunford, S. M. Fischer, R. Ganz, J. P. Greene, N. I. Kaloskamis, L. Lamm,

C. J. Lister, V. Nanal, D. E. Roa, and J. P. Schiffer

Phy. Rev. C 57, R2794-R2798 (1998)

Detailed Angular Correlation Analysis with $4 \pi$ Spectrometers; Spin Determinations and

Multipolarity Mixing Measurements in ${ }^{128} \mathrm{Ba}$

I. Wiedenhoever, O. Vogel, H. Klein, A. Dewald, P. von Brentano,

J. Gableske, R. Kruecken, N. Nicolay, A. Gelberg, P. Petkov, A. Gizon, J. Gizon, D. Bazzaco,

C. Rossi Alvarez, G. de Angelis, S. Lunardi, P. Pavan, D. R. Napoli, S. Frauendorf, F. Donau,

R. V. F. Janssens, and M. P. Carpenter

Phys. Rev. C 58, 721-728 (1998)

Mass Measurements of Proton-Rich Medium Mass Nuclides

B. D. Foy, D. S. Brenner, C. N. Davids, D. Seweryniak, D. Blumenthal,

R. L. Gill, N. V. Zamfir, D. D. Warner, and C. J. Barton

Phys. Rev. C 58, 749-753 (1998) 
Electron Capture Decay of ${ }^{116}$ In and Nuclear Structure of Double $\beta$ Decays

M. Bhattacharya, A. Carcia, M. M. Hindi, E. B. Norman, C. E. Ortiz,

N. I. Kaloskamis, C. N. Davids, O. Civitarese, and J. Suhonen

Phys. Rev. C 58, 1247-1255 (1998)

Identification of ${ }^{180} \mathrm{Tl} \alpha$ Decay

K. S. Toth, X.-J. Xu, C. R. Bingham, J. C. Batchelder, L. F. Conticchio,

W. B. Walters, L. T. Brown, C. N. Davids, R. J. Irvine, D. Seweryniak, J. Wauters,

and E. F. Zganjar

Phy. Rev. C 58, 1310-1313 (1998)

Quadrupole Moments and Identical Superdeformed Bands in ${ }^{149} \mathrm{~Tb}$

B. Kharraja, U. Garg, H. Jin, R. J. Ernst, S. S. Ghugre, R. V. F. Janssens,

M. P. Carpenter, S. M. Fischer, T. L. Khoo, T. Lauritsen, D. Nisius,

E. F. Moore, Th. Byrski, R. Krucken, A. O. Macchiavelli, R. MacLeod, R. Kaczarowski, and I. M. Govil

Phys. Rev. C 58, 1422-1429 (1998)

Identification of Yrast States in ${ }^{187 \mathrm{~Pb}}$

A. M. Baxter, A. P. Byrne, G. D. Dracoulis, P. M. Davidson, T. R. McGoram,

P. H. Regan, C. Chandler, W. Gelletly, C. Wheldon, R. Julin, J. F. C. Cocks,

K. Helariutta, P. Jones, S. Juutinen, H. Kankaanpaa, P. Kuusiniemi, M. Leino, M. Muikku,

A. Savelius, R. V. F. Janssens, T. Brown, M. P. Carpenter, C. N. Davids, T. Lauritsen,

D. Nisius, and P. T. Greenlees

Phys. Rev. C 58, 2671-2676 (1998)

In-Beam $\gamma$-Ray,Spectroscopy of Pt Isotopes Located at the Proton Drip Line

D. Seweryniak, D. Ackermann, H. Amro, L. T. Brown, M. P. Carpenter, L. Conticchio,

C. N. Davids, S. M. Fischer, G. Hackman, S. Hamada, D. J. Henderson, R. V. F. Janssens,

D. Nisius, P. Reiter, W. B. Walters, and P. J. Woods

Phys. Rev. C 58, 2710-2714 (1998)

High-Spin Structures in ${ }^{155} \mathrm{~Tb}$ and Signature Splitting Systematics of the $\pi \mathrm{h}_{11 / 2}$ Bands in Odd-A 160 Nuclei

D. J. Hartley, T. B. Brown, F. G. Kondev, J. Pfohl, M. A. Riley, S. M. Fischer,

R. V. F. Janssens, D. T. Nisius, P. Fallon, W. C. Ma, and J. Simpson

Phys. Rev. C 58, 2720-2735 (1998)

High-Spin States in $97,98 \mathrm{Rh}$

S. S. Ghugre, B. Kharraja, U. Garg, R. V. F. Janssens, M. P. Carpenter, B. Crowell, T. L. Khoo, T. Lauritsen, D. Nisius, W. Mueller, W. Reviol, L. L. Riedinger, and R. Kaczarowski

Phys. Rev. C 58, 3243-3251 (1998)

Fragmentation of the Two-Phonon Octupole Vibrational States in $208 \mathrm{~Pb}$ K. Vetter, A. O. Macchiavelli, D. Cline, H. Amro, S. J. Asztalos, B. C. Busse, R. M. Clark, M. A. Deleplanque, R. M. Diamond, P. Fallon, R. Gray, R. V. F. Janssens, R. Krucken, I. Y. Lee, R. W. MacLeod, E. F. Moore, G. J. Schmid, M. W. Simon, F. S. Stephens, and C. Y. Wu

Phys. Rev. C 58, R2631-R2635 (1998) 
First Direct Measurements of $\mathrm{g}$ Factors of the Three Superdeformed Bands of ${ }^{194} \mathrm{Hg}$

R. H. Mayer, G. Kumbartzki, L. Weissman, N. Benczer-Koller, C. Broude,

J. A. Cizewski, M. Hass, J. Holden, R. V. F. Janssens, T. Lauritsen, I. Y. Lee,

A. O. Macchiavelli, D. P. McNabb, and M. Satteson

Phys. Rev. C 58, R2640-R2643 (1998)

Superdeformed Bands in ${ }^{189} \mathrm{Tl}$

W. Reviol, D. E. Ellis, L. L. Riedinger, M. P. Carpenter, S. M. Fischer,

R. V. F. Janssens, D. Nisius, and P. Fallon

Phys. Rev. C 58, R2644-R2648 (1998)

Radioactive Decay of ${ }^{80} \mathrm{Y}$ and Low-Lying States in ${ }^{80} \mathrm{Sr}$

J. Doring, A. Aprahamian, R. C. de Haan, J. Gorres, H. Schatz, M. Wiescher,

W. B. Walters, L. T. Brown, C. N. Davids, C. J. Lister, and D. Seweryniak

Phys. Rev. C 59, 59-70 (1999)

Study of the ${ }^{56} \mathrm{Ni}(\mathrm{d}, \mathrm{p})^{57} \mathrm{Ni}$ Reaction and the Astrophysical ${ }^{56 \mathrm{Ni}}(\mathrm{p}, \gamma){ }^{57} \mathrm{Cu}$ Reaction Rate

K. E. Rehm, F. Borasi, C. L. Jiang, D. Ackermann, I. Ahmad, B. A. Brown,

F. Brumwell, C. N. Davids, P. Decrock, S. M. Fischer, J. Gorres, J. Greene,

G. Hackman, B. Harss, D. Henderson, W. Henning, R. V. F. Janssens, G. McMichael,

V. Nanal, D. Nisius, J. Nolen, R. C. Pardo, M. Paul, P. Reiter, J. P. Schiffer, D. Seweryniak,

R. E. Segel, M. Wiescher, and A. H. Wuosmaa

Phys. Rev. Lett. 80, 676-679 (1998)

Proton Radioactivity from Highly Deformed Nuclei

C. N. Davids, P. J. Woods, D. Seweryniak, A. A. Sonzogni, J. C. Batchelder,

C. R. Bingham, T. Davinson, D. J. Henderson, R. J. Irvine, G. L. Poli,

J. Uusitalo, and W. B. Walters

Phys. Rev. Lett. 80, 1849-1852 (1998)

$\beta^{+}$Decay Partial Half-Life of ${ }^{54} \mathrm{Mn}$ and Cosmic Ray Chronometry

A. H. Wuosmaa, I. Ahmad, S. M. Fischer, J. P. Greene, G. Hackman, V. Nanal, G. Savard, J. P. Schiffer, P. Wilt, Sam M. Austin, B. A. Brown, S. J. Freedman, and J. J. Connell

Phys. Rev. Lett. 80, 2085-2088 (1998)

Three-Laboratory Measurement of the ${ }^{44}$ Ti Half-Life

I. Ahmad, G. Bonino, G. C. Castagnoli, S. M. Fischer, W. Kutschera, and M. Paul

Phys. Rev. Lett. 80, 2550-2553 (1998)

Hackman et al. Reply

G. Hackman, T. L. Khoo, M. P. Carpenter, T. Lauritsen, A. Lopez-Martens,

I. J. Calderin, R. V. F. Janssens, D. Ackermann, I. Ahmad, S. Agarwala,

D. J. Blumenthal, S. M. Fischer, D. Nisius, P. Reiter, J. Young, H. Amro,

E. F. Moore, F. Hannachi, A. Korichi, I. Y. Lee, A. O. Macchiavelli, T. Dossing, and T. Nakatsukasa

Phys. Rev. Lett. 80, 4611 (1998) 
Large Ion Crystals in a Linear Paul Trap

M. Drewsen, C. Brodersen, L. Hornekaer, J. S. Hangst, and J. P. Schiffer Phys. Rev. Lett. 81, 2878-2881 (1998)

Fusion Cross Sections for the Proton Drip Line Nucleus ${ }^{17} \mathrm{~F}$ at Energies Below the Barrier

K. E. Rehm, H. Esbensen, C. L. Jiang, B. B. Back, F. Borasi, B. Harss,

R. V. F. Janssens, V. Nanal, J. Nolen, R. C. Pardo, M. Paul, P. Reiter, R. E. Segel,

A. Sonzogni, J. Uusitalo, and A. H. Wuosmaa

Phys. Rev. Lett. 81, 3341-3344 (1998)

Ground-State Band and Deformation of the $Z=102$ Isotope ${ }^{254}$ No

P. Reiter, T. L. Khoo, C. J. Lister, D. Seweryniak, I. Ahmad, M. Alcorta,

M. P. Carpenter, J. A. Cizewski, C. N. Davids, G. Gervais, J. P. Greene,

W. F. Henning, R. V. F. Janssens, T. Lauritsen, S. Siem, A. A. Sonzogni, D. Sullivan,

J. Uusitalo, I. Wiedenhoever, N. Amzal, P. A. Butler, A. J. Chewter, K. Y. Ding, N. Fotiades,

J. D. Fox, P. T. Greenlees, R.-D. Herzberg, G. D. Jones, W. Korten, M. Leino, and K. Vetter Phys. Rev. Lett. 82, 509-512 (1999)

Lifetime Measurements of Superdeformed Bands in ${ }^{142} \mathrm{Sm}$ : A Test of the Additivity Principle for Quadrupole Moments

G. Hackman, R. V. F. Janssens, E. F. Moore, D. Nisius, I. Ahmad,

M. P. Carpenter, S. M. Fischer, T. L. Khoo, T. Lauritsen, and P. Reiter

Phys. Lett. B416, 268-273 (1998)

Opening Up the A $180 \mathrm{~K}$-Isomer Landscape: Inelastic Excitation of New Multi-Quasiparticle

Yrast Traps

C. Wheldon, R. D'Alarcao, P. Chowdhury, P. M. Walker, E. Seabury, I. Ahmad,

M. P. Carpenter, D. M. Cullen, G. Hackman, R. V. F. Janssens, T. L. Khoo,

D. Nisius, C. J. Pearson, and P. Reiter

Phys. Lett. B425, 239-245 (1998)

Spin $\sim 60 \mathrm{~h} /$ in 156Dy: Competition Between Collective and Terminating

Structures at Very High-Spin

F. G. Kondev, M. A. Riley, R. V. F. Janssens, J. Simpson, A. V. Afanasjev,

I. Ragnarsson, I. Ahmad, D. J. Blumenthal, T. B. Brown, M. P. Carpenter,

P. Fallon, S. M. Fischer, G. Hackman, D. J. Hartley, C. A. Kalfas, T. L. Khoo, T. Lauritsen,

W. C. Ma, D. Nisius, J. F. Sharpey-Schafer, and P. G. Varmette

Phy. Lett. B437, 35-43 (1998)

Competing $\mathrm{T}=0$ and $\mathrm{T}=1$ Structures in the $\mathrm{N}=\mathrm{Z}$ Nucleus $62 / 31 \mathrm{Ga}$

S. M. Vincent, P. H. Regan, D. D. Warner, R. A. Bark, D. Blumenthal,

M. P. Carpenter, C. N. Davids, W. Gelletly, R. V. F. Janssens, C. D. O'Leary, C. J. Lister, J. Simpson, D. Seweryniak, T. Saitoh, J. Schwartz, S. Tormaninen, O. Juillet, F. Nowacki, and $P$. Van Isacker

Phys. Lett. B437, 264-272 (1998) 
E2 Polarization Charge in ${ }^{102} \mathrm{Sn}$

M. Lipoglavsek, D. Seweryniak, C. N. Davids, C. Fahlander, M. Gorska,

R. V. F. Janssens, J. Nyberg, J. Uusitalo, W. B. Walters, I. Ahmad, J. Blomqvist,

M. P. Carpenter, J. A. Cizewski, S. M. Fischer, H. Grawe, G. Hackman, M. Huhta,

C. J. Lister, D. Nisius, G. Poli, P. Reiter, J. Ressler, J. Schwartz, and A. Sonzogni

Phys. Lett. B440, 246-250 (1998)

A Beam Vertex Detector Using Scintillating Fibers

B. B. Back, R. R. Betts, A. Gillitzer, W. F. Henning, D. J. Hofman,

V. Nanal, A. H. Wuosmaa, R. Ganz, B. Holzman, D. McLeod, W. R. Binns, and J. W. Epstein

Nucl. Instrum. Methods A412, 191-199 (1998)

Study of Radiative Capture Reactions with Radioactive Ion Beams

K. E. Rehm, C. L. Jiang, M. Paul, D. Blumenthal, L. A. Daniel, C. N. Davids,

P. Decrock, S. M. Fischer, D. Henderson, C. Lister, J. Nickles, J. Nolen,

R. C. Pardo, J. P. Schiffer, D. Seweryniak, and R. E. Segel

Nucl. Instrum. Methods A418, 355-364 (1998)

Non-Yrast States of ${ }^{132} \mathrm{Ce}$ Populated in $\beta$-Decay

A. Gade, I. Wiedenhoever, T. Diefenbach, A. Gelberg, M. Luig, H. Meise,

N. Pietralla, M. Wilhelm, T. Otsuka, and P. von Brentano

Nucl. Phys. A643, 225-242 (1998)

Spectroscopy of Rn, Ra and Th Isotopes Using Multi-Nucleon Transfer Reactions

J. F. C. Cocks, D. Hawcroft, N. Amzal, P. A. Butler, K. J. Cann,

P. T. Greenlees, G. D. Jones, S. Asztalos, R. M. Clark, M. A. Deleplanque,

R. M. Diamond, P. Fallon, I. Y. Lee, A. O. Macchiavelli, R. W. MacLeod,

F. S. Stephens, P. Jones, R. Julin, R. Broda, B. Fornal, J. F. Smith, T. Lauritsen,

P. Bhattacharyya, and C. T. Zhang

Nucl. Phys. A645, 61-91 (1999)

Sparse Crystals

John P. Schiffer

Science 279, 675-676 (1998)

Nuclear Physics: The Core of Matter, the Fuel of Stars

John Schiffer

BPA News, 9-11 (1998)

Nuclear Structure Information Derived from Proton Radioactivity Measurements

C. N. Davids

Proceedings of the Sixth International Conference on Nucleus-Nucleus

Collisions, Gatlinburg, TN, 2-6 June 1997; Nucl. Phys. A630, 321c-327c

(1998) 
Superdeformed Shapes and Configurations in Thallium Nuclei

W. Reviol, W. F. Mueller, L. L. Riedinger, B. H. Smith, M. P. Carpenter,

S. M. Fischer, R. V. F. Janssens, T. L. Khoo, T. Lauritsen, D. T. Nisius,

D. Seweryniak, and E. F. Moore

Proceedings of the Sixth International Conference on Nucleus-Nucleus

Collisions, June 2-6, 1997, Gatlinburg, TN; Nucl. Phys. A630, 434c-441c (1998)

Spin Distributions for ${ }^{64} \mathrm{Ni}+{ }^{100} \mathrm{Mo}$ with the Argonne/Notre Dame BGO-Array

D. Ackermann, B. B. Back, R. R. Betts, M. Carpenter, L. Corradi,

S. M. Fischer, R. Ganz, S. Gil, G. Hackman, D. J. Hofman, R. V. F. Janssens,

T. L. Khoo, G. Montagnoli, V. Nanal, F. Scarlassara, M. Schlapp, D. Seweryniak,

A. M. Stefanini, and A. H. Wuosmaa

Proceedings of the Sixth International Conference on Nucleus-Nucleus

Collisions, Gatlinburg, TN, 2-6 June 1997; Nucl. Phys. A630, 442c-448c (1998)

Ordering in Classical Coulombic Systems

John P. Schiffer

Proceeding of the International Conference on Strongly Coupled Coulomb

Systems, Boston College, Chestnut Hill, MA, August 3-10, 1997, eds. Kalman

et al. (Plenum Press, New York, 1998) pp. 43-48

Measurements with Radioactive Beams at ATLAS

K. E. Rehm

Proceedings of the Second Oak Ridge Symposium on Atomic and Nuclear

Astrophysics, Oak Ridge, TN, 2-6 December 1997; pp. 503-509 (1998)

Mass Measurements of Proton-Rich Medium Mass Nuclides

D. S Brenner, B. D. Foy, C. J. Barton, C. N. Davids, D. Seweryniak, D. Blumenthai,

R. L. Gill, N. V. Zamfir, and D. D. Warner

Proceedings of the ENAM98 (Exotic Nuclei and Atomic Masses) Conference, June 23-27, 1998, Bellaire, MI, eds. Bradley M. Sherrill, David J. Morrissey, and Cary N. Davids, AIP Conference Proceedings 455 (Woodbury;

NY 1998) pp. 102-105

Status of the Canadian Penning Trap Mass Spectrometer at the Argonne

National Laboratories

K. S. Sharma, R. C. Barber, F. Buchinger, J. E. Crawford, X. Feng,

H. Fukutani, S. Gulick, G. Hackman, J. C. Hardy, D. Hofman, J. K. P. Lee,

P. Martinez, R. B. Moore, G. Savard, D. Seweryniak, and J. Uusitalo

Proceedings of the ENAM98 (Exotic Nuclei and Atomic Masses) Conference, June 23-27, 1998, Bellaire, MI, eds. Bradley M. Sherrill, David J.

Morrissey, and Cary N. Davids, AIP Conference Proceedings 455 (Woodbury, NY 1998) pp. 130-133 
Proton Radioactivity From Highly Deformed Nuclei

A. A. Sonzogni, C. N. Davids, P. J. Woods, D. Seweryniak, J. C. Batchelder,

C. R. Bingham, T. Davinson, D. J. Henderson, R. J. Irvine, G. L. Poli,

J. Uusitalo, and W. B. Walters

Proceedings of the ENAM98 (Exotic Nuclei and Atomic Masses) Conference,

June 23-27, 1998, Bellaire, MI, eds. Bradley M. Sherrill, David J. Morrissey, and

Cary N. Davids, AIP Conference Proceedings 455 (Woodbury,

NY 1998) pp. 367-370

Proton Decay of the Closed Neutron Shell Nucleus ${ }^{155} \mathrm{Ta}$

J. Uusitalo, C. N. Davids, P. J. Woods, D. Seweryniak, A. A. Sonzogni,

J. C. Batchelder, C. R. Bingham, T. Davinson, J. DeBoer, D. J. Henderson,

H. J. Maier, J. Ressler, R. Slinger, and W. B. Walters

Proceedings of the ENAM98 (Exotic Nuclei and Atomic Masses) Conference, June 23-27, 1998, Bellaire, MI, eds. Bradley M. Sherrill, David J. Morrissey, and Cary N. Davids, AIP Conference Proceedings 455 (Woodbury, NY 1998) pp. 375-378

In-Beam $\gamma$-Ray Spectroscopy in the Vicinity of ${ }^{100} \mathrm{Sn}$

Dariusz Seweryniak

Proceedings of the ENAM98 (Exotic Nuclei and Atomic Masses) Conference, June 23-27, 1998, Bellaire, MI, eds. Bradley M. Sherrill, David J. Morrissey, and Cary N. Davids, AIP Conference Proceedings 455 (Woodbury,

NY 1998) pp. 415-421

Nuclear Structure Information From Recoil Decay Tagging Experiments

M. Leino, R. Julin, J. F. C. Cocks, P. A. Butler, O. Dorvaux, K. Eskola,

P. T. Greenlees, P. Jones, S. Juutinen, K. Helariutta, H. Kankaanpaa, H. Kettunen,

P. Kuusiniemi, M. Muikku, R. D. Page, P. Rahkila, A. Savelius, W. H. Trzaska, and

J. Uusitalo

Proceedings of the ENAM98 (Exotic Nuclei and Atomic Masses) Conference, June 23-27, 1998, Bellaire, MI, eds. Bradley M. Sherrill, David J. Morrissey, and Cary N. Davids, AIP Conference Proceedings 455 (Woodbury, NY 1998) pp. 422-429

In-Beam $\gamma$-Ray Spectroscopy in the Ground-State Proton Emitter ${ }^{113} \mathrm{Cs}$

C. J. Gross, Y. A. Akovali, C. Baktash, J. C. Batchelder, C. R. Bingham,

M. P. Carpenter, C. N. Davids, T. Davinson, D. Ellis, A. Galindo-Uribarri,

T. N. Ginter, R. Grzywacz, R. V. F. Janssens, J. W. Johnson, F. Liang, C. J. Lister, J. Mas, B. MacDonald, S. D. Paul, A. Piechaczek, D. C. Radford, W. Reviol, K. Rykaczewski, W. Satula, D. Seweryniak, D. Shapira, K. S. Toth, W. Weintraub, P. J. Woods, C.-H. Yu, E. F. Zganjar, and J. Uusitalo

Proceedings of the ENAM98 (Exotic Nuclei and Atomic Masses) Conference, June 23-27, 1998, Bellaire, MI, eds. Bradley M. Sherrill, David J. Morrissey, and Cary N. Davids, AIP Conference Proceedings 455 (Woodbury, NY 1998) pp. 444-449 
Probing the Structure of the $N=Z=31$ Nucleus ${ }_{31}^{62} \mathrm{Ga}$

S. M. Vincent, P. H. Regan, D. D. Warner, W. Gelletly, J. Simpson, R. Bark,

D. Blumenthal, M. P. Carpenter, C. N. Davids, D. J. Henderson,

R. V. F. Janssens, C. J. Lister, D. Nisius, C. D. O'Leary, D. J. Pearson, T. Saitoh,

J. Schwartz, D. Seweryniak, and S. Tormanen

Proceedings of the ENAM98 (Exotic Nuclei and Atomic Masses) Conference, June 23-27, 1998, Bellaire, MI, eds. Bradley M. Sherrill, David J.

Morrissey, and Cary N. Davids, AIP Conference Proceedings 455 (Woodbury, NY 1998) pp. 450-455

Spectroscopy of $\mathrm{N}=82,83{ }^{136,137} \mathrm{Xe}$ Isotopes from ${ }^{248} \mathrm{Cm}$ Fission

P. Bhattacharyya, C. T. Zhang, P. J. Daly, Z. W. Grabowski, R. Broda,

B. Fornal, I. Ahmad, T. Lauritsen, L. R. Morss, W. R. Phillips, J. L. Durell, M. J. Leddy,

A. G. Smith, W. Urban, B. J. Varley, N. Schulz, E. Lubkiewicz, M. Bentaleb, and J. Blomqvist

Proceedings of the ENAM98 (Exotic Nuclei and Atomic Masses) Conference,

June 23-27, 1998, Bellaire, MI, eds. Bradley M. Sherrill, David J. Morrissey, and

Cary N. Davids, AIP Conference Proceedings 455 (Woodbury,

NY 1998) pp. 470-473

Alpha Decay of the $h_{9 / 2}$ Ground and $s_{1 / 2}$ Intruder States in Light Bi and At Isotopes

C. R. Bingham, J. C. Batchelder, J. A. Cizewski, C. N. Davids, R. J. Irvine,

W. Reviol, D. Seweryniak, K. S. Toth, W. B. Walters, J. Wauters, J. L. Wood,

X. J. Xu, J. Uusitalo, and E. F. Zganjar

Proceedings of the ENAM98 (Exotic Nuclei and Atomic Masses) Conference,

June 23-27, 1998, Bellaire, MI, eds. Bradley M. Sherrill, David J. Morrissey, and

Cary N. Davids, AIP Conference Proceedings 455 (Woodbury,

NY 1998) pp. 474-477

Evolution of Collective Motion in Light Polonium Nuclei

J. A. Cizewski, K. Y. Ding, N. Fotiades, D. P. McNabb, W. Younes, R. Julin,

M. Leino, J. Cocks, P. Greenlees, K. Helariutta, P. Jones, S. Juutinen,

A. Kankaanpaa, H. Kettunen, P. Kuusiniemi, M. Muikku, P. Rahkila, A. Savelius,

C. N. Davids, R. V. F. Janssens, D. Seweryniak, M. P. Carpenter,

H. Amro, P. Decrock, P. Reiter, D. Nisius, L. T. Brown, S. Fischer, T. Lauritsen, J. Wauters,

C. R. Bingham, M. Huyse, and A. Andreyev

Proceedings of the ENAM98 (Exotic Nuclei and Atomic Masses) Conference, June 23-27, 1998, Bellaire, MI, eds. Bradley M. Sherrill, David J. Morrissey, and Cary N. Davids, AIP Conference Proceedings 455 (Woodbury,

NY 1998) pp. 486-489

High-Spin States in ${ }^{71} \mathrm{As},{ }^{72} \mathrm{Se}$, and ${ }^{72} \mathrm{Br}$

N. Fotiades, J. A. Cizewski, C. J. Lister, C. N. Davids, R. V. F. Janssens,

D. Seweryniak, M. P. Carpenter, T. L. Khoo, T. Lauritsen, D. Nisius, P. Reiter, J. Uusitalo,

I. Wiedenhoever, A. O. Macchiavelli, and R. W. McLeod

Proceedings of the ENAM98 (Exotic Nuclei and Atomic Masses) Conference,

June 23-27, 1998, Bellaire, MI, eds. Bradley M. Sherrill, David J. Morrissey, and

Cary N. Davids, AIP Conference Proceedings 455 (Woodbury,

NY 1998) pp. 506-509 
Fine Structure in ${ }^{192} \mathrm{Po} \alpha$-Decay and Shape Coexistence in ${ }^{188} \mathrm{~Pb}$

R. D. Page, R. G. Allatt, T. Enqvist, K. Eskola, P. T. Greenlees, P. Jones, R. Julin,

P. Kuusiniemi, M. Leino, and J. Uusitalo

Proceedings of the ENAM98 (Exotic Nuclei and Atomic Masses) Conference, June 23-27, 1998, Bellaire, MI, eds. Bradley M. Sherrill, David J. Morrissey, and Cary N. Davids, AIP Conference Proceedings 455 (Woodbury, NY 1998) pp. 564-567

Single-Particle and Cluster Levels in ${ }^{107,108,109} \mathrm{Sn}$ Populated in the Decay of $107,108,109 \mathrm{Sb}$ Jennifer Jo Ressler, Darek, Seweryniak, Louis Conticchio, Diana Ciurczak, Joseph Swider, Heikki Penttila, William B. Walters, Jan Wauters, Carrol Bingham, Robert de Haan, Brian Foy, and Cary N. Davids Proceedings of the ENAM98 (Exotic Nuclei and Atomic Masses) Conference, June 23-27, 1998, Bellaire, MI, eds. Bradley M. Sherrill, David J. Morrissey, and Cary N. Davids, AIP Conference Proceedings 455 (Woodbury, NY 1998) pp. 574-580

Coulomb Excitation of a ${ }^{78} \mathrm{Rb}$ Radioactive Beam

J. Schwartz, C. J. Lister, D. H. Henderson, S. M. Fischer, P. Reiter,

A. Aprahamian, J. A. Cizewski, C. N. Davids, R. deHaan, R. V. F. Janssens,

D. Nisius, D. Seweryniak, and S. M. Vincent

Proceedings of the ENAM98 (Exotic Nuclei and Atomic Masses) Conference, June 23-27, 1998, Bellaire, MI, eds. Bradley M. Sherrill, David J. Morrissey, and Cary N. Davids, AIP Conference Proceedings 455 (Woodbury,

NY 1998) pp. 589-593

Study of the High-j States in ${ }^{249} \mathrm{Cm}$

I. Ahmad, B. B. Back, A. Bacher, G. P. A. Berg, R. R. Chasman, C. C. Foster, J. P. Greene, T. Ishii, W. R. Lozowski, L. R. Morss, W. Schmitt,

E. J. Stephenson, and T. Yamanaka

Proceedings of the ENAM98 (Exotic Nuclei and Atomic Masses) Conference, June 23-27, 1998, Bellaire, MI, eds. Bradley M. Sherrill, David J. Morrissey, and Cary N. Davids, AIP Conference Proceedings 455 (Woodbury, NY 1998) pp. 682-685

In-Beam Gamma-Ray Spectroscopy in the Vicinity of ${ }^{100} \mathrm{Sn}$

D. Seweryniak

2nd International Conference on Exotic Nuclei and Atomic Masses (ENAM'98), June 23-27, 1998, Bellaire, MI, Book of Abstracts, p. C5 (1998)

Competing $\mathrm{T}=0$ and $\mathrm{T}=1$ Structures in the $\mathrm{N}=\mathrm{Z}$ Odd-Odd Nucleus Gallium-62 S. M. Vincent, P. H. Regan, D. D. Warner, R. Bark, D. J. Blumenthal, M. P. Carpenter, R. A. Cunningham, C. N. Davids, W. Gelletly, D. Henderson, R. V. F. Janssens, O. Juillet, C. J. Lister, S. Mohammadi, D. Nisius, C. D. O'Leary, C. J. Pearson, T. Saitoh, J. Schwartz, D. Seweryniak, J. Simpson, S. Tormanen, and P. Van Isacker 2nd International Conference on Exotic Nuclei and Atomic Masses (ENAM98), June 23-27, 1998, Bellaire, MI, Book of Abstracts, p. C10 (1998) 
Mass Measurements of Proton-Rich Medium Mass Nuclides

D. S. Brenner, B. D. Foy, C. N. Davids, D. Seweryniak, D. Blumenthal,

R. L. Gill, N. V. Zamfir, D. D. Warner, and C. J. Barton

2nd International Conference on Exotic Nuclei and Atomic Masses (ENAM'98),

June 23-27, 1998, Bellaire, MI, Book of Abstracts, p. PA5 (1998)

Status of the Canadian Penning Trap Mass Spectrometer at the Argonne

National Laboratory

K. S. Sharma, H. Fukutani, R. C. Barber, G. Savard, G. Hackman, J. Uusitalo,

F. Buchinger, J. E. Crawford, X. Feng, S. Fulick, J. K. P. Lee, P. Martinez,

R. B. Moore, and J. C. Hardy

2nd International Conference on Exotic Nuclei and Atomic Masses (ENAM'98),

June 23-27, 1998, Bellaire, MI, Book of Abstracts, p. PA16 (1998)

Continuum Excitation of Neutron-Rich Oxygen Isotopes

T. Aumann, K. Boretzky, D. Cortina, J. Cub, W. Dostal, B. Eberlein,

Th. W. Elze, H. Emling, H. Geissel, A. Gruenschloss, M. Hellstroem, J. Holeczek,

R. Holzmann, S. Ilievski, N. Iwasa, M. Kaspar, A. Kleinboehl, J. V. Kratz, R. Kulessa,

Y. Leifels, A. Leistenschneider, E. Lubkiewicz, G. Muenzenberg, P. Reiter, M. Rejmund, .

C. Scheidenberger, Ch. Schlegel, H. Simon, J. Stroth, K. Suemmerer, E. Wajda, W. Walus, and S. Wan

2nd International Conference on Exotic Nuclei and Atomic Masses (ENAM98),

June 23-27, 1998, Bellaire, MI, Book of Abstracts, p. PB2 (1998)

Proton Radioactivity From Highly Deformed Nuclei

A. A. Sonzogni, C. N. Davids, P. J. Woods, D. Seweryniak, J. C. Batchelder,

C. R. Bingham, T. Davinson, D. J. Henderson, R. J. Irvine, G. L. Poli,

J. Uusitalo, and W. B. Walters

2nd International Conference on Exotic Nuclei and Atomic Masses (ENAM98),

June 23-27, 1998, Bellaire, MI, Book of Abstracts, p. PB44 (1998)

Proton Emission from the Closed Neutron Shell Nucleus ${ }^{155} \mathrm{Ta}$

J. Uusitalo, C. N. Davids, P. J. Woods, D. Seweryniak, A. A. Sonzogni,

J. C. Batchelder, C. R. Bingham, T. Davinson, D. J. Henderson, and W. B. Walters

2nd International Conference on Exotic Nuclei and Atomic Masses (ENAM98),

June 23-27, 1998, Bellaire, MI, Book of Abstracts, p. PB48 (1998)

Spectroscopy of $\mathrm{N}=82,83$ 136,137Xe Isotopes From ${ }^{248} \mathrm{Cm}$ Fission

P. Bhattacharyya, C. T. Zhang, B. Fornal, R. Broda, P. J. Daly,

Z. W. Grabowski, I. Ahmad, T. Lauritsen, L. R. Morss, W. R. Phillips, J. L. Durell,

M. J. Leddy, A. G. Smith, W. Urban, B. J. Varley, N. Schulz, E. Lubkiewicz, M. Bentaleb, and J. Blomqvist

2nd International Conference on Exotic Nuclei and Atomic Masses (ENAM'98),

June 23-27, 1998, Bellaire, MI, Book of Abstracts, p. PC3 (1998)

Alpha Decay of the $h_{9 / 2}$ Ground and $s_{1 / 2}$ Intruder States in Light Bi and At Isotopes

C. R. Bingham, J. C. Batchelder, C. N. Davids, R. J. Irvine, W. Reviol,

D. Seweryniak, K. S. Toth, W. B. Walters, J. Wauters, J. L. Wood, X. J. Xu,

J. Uusitalo, and E. F. Zganjar

2nd International Conference on Exotic Nuclei and Atomic Masses (ENAM98),

June 23-27, 1998, Bellaire, MI, Book of Abstracts, p. PC4 (1998) 
High-Spin States in Neutron-Deficient ${ }^{71} \mathrm{As}$ and ${ }^{72} \mathrm{Se}$

N. Fotiades, J. A. Cizewski, C. J. Lister, C. N. Davids, R. V. F. Janssens,

D. Seweryniak, M. P. Carpenter, T. L. Khoo, T. Lauritsen, D. Nisius, P. Reiter, J. Uusitalo,

I. Wiedenhoever, A. O. Macchiavelli, and R. McLeod

2nd International Conference on Exotic Nuclei and Atomic Masses (ENAM98),

June 23-27, 1998, Bellaire, MI, Book of Abstracts, p. PC19 (1998)

Coulomb Excitation of a ${ }^{78} \mathrm{Rb}$ Radioactive Beam

J. Schwartz, C. J. Lister, D. H. Henderson, S. M. Fischer, P. Reiter,

A. Aprahamian, J. A. Cizewski, C. N. Davids, R. deHaan, R. V. F. Janssens,

D. Nisius, D. Seweryniak, and S. M. Vincent

2nd International Conference on Exotic Nuclei and Atomic Masses (ENAM'98),

June 23-27, 1998, Bellaire, MI, Book of Abstracts, p. PC45 (1998)

Single-Particle Orbitals in Trans-Fermium Nuclei

I. Ahmad, B. B. Back, R. R. Chasman, P. G. Greene, T. Ishii, L. R. Morss,

G. P. A. Berg, A. Bacher, C. C. Foster, W. R. Lozowski, W. Schmitt,

E. J. Stephenson, and T. Yamanaka

2nd International Conference on Exotic Nuclei and Atomic Masses (ENAM98),

June 23-27, 1998, Bellaire, MI, Book of Abstracts, p. PD2 (1998)

Spectroscopy of ${ }^{187} \mathrm{~Pb}$

A. M. Baxter, A. P. Byrne, G. D. Dracoulis, P. M. Davidson, T. McGoram,

P. H. Regan, K. Chandler, C. Wheldon W. Gelletly, R. Julin, P. Greenlees,

K. Helariutta, P. Jones, S. Juutinen, M. Leino, M. Muikku, and R. V. F. Janssens

Nuclear Structure '98, August 10-15, 1998, Gatlinburg, TN, Book of

Abstracts, p. 9 (1998)

Study of Excited States in ${ }^{167} \mathrm{Ir}$ : Probing States Beyond the Proton Drip Line

M. P. Carpenter, R. V. F. Janssens, T. L. Khoo, D. Seweryniak, A. Sonzogni,

I. Ahmad, L. T. Brown, C. N. Davids, G. Hackman, T. Lauritsen, C. J. Lister,

P. Reiter, S. Siem, J. Uusitalo, I. Wiedenhoever, P. J. Woods, J. A. Cizewski, W. Reviol,

L. L. Riedinger, S. M. Fischer, J. J. Ressler, W. B. Walters, and D. G. Sarantites

Nuclear Structure '98, August 10-15, 1998, Gatlinburg, TN, Book of

Abstracts, p. 13 (1998)

Multi-Qp K-Isomers in Neutron-Rich Hf Nuclei via Inelastic Excitation and Transfer with Heavy Beams

R. D'Alarcao, P. Chowdhury, E. H. Seabury, P. M. Walker, C. Wheldon,

I. Ahmad, M. P. Carpenter, G. Hackman, R. V. F. Janssens, T. L. Khoo,

C. J. Lister, D. Nisius, P. Reiter, D. Seweryniak, and I. Wiedenhoever

Nuclear Structure '98, August 10-15, 1998, Gatlinburg, TN, Book of

Abstracts, p. 22 (1998)

Excited States in 155,157Lu Using Recoil-Decay Tagging of Gammasphere

K. Y. Ding, D. Seweryniak, J. A. Cizewski, H. Amro, L. T. Brown,

M. P. Carpenter, C. N. Davids, N. Fotiades, G. Hackman, R. V. F. Janssens,

T. Lauritsen, C. J. Lister, A. O. Macchiavelli, D. Nisius, P. Reiter, S. Siem, J. Uusitalo, and I Wiedenhover

Nuclear Structure '98, August 10-15, 1998, Gatlinburg, TN, Book of

Abstracts, p. 28 (1998) 
In-Beam $\gamma$-Ray Spectroscopy in the Ground-State Proton Emitter ${ }^{113} \mathrm{Cs}$

C. J. Gross, Y. A. Akovali, C. Baktash, J. C. Batchelder, C. R. Bingham,

M. P. Carpenter, C. N. Davids, T. Davinson, D. Ellis, A. Galindo-Uribarri,

T. N. Ginter, R. Grzywacz, R. V. F. Janssens, J. W. Johnson, F. Liang, C. J. Lister, J. Mas,

B. MacDonald, S. D. Paul, A. Piechaczek, D. C. Radford, W. Reviol, K. Rykaczewski,

W. Satula, D. Seweryniak, D. Shapira, K. S. Toth, W. Weintraub, P. J. Woods, C.-H. Yu,

E. F. Zganjar, and J. Uusitalo

Nuclear Structure '98, August 10-15, 1998, Gatlinburg, TN, Book of

Abstracts, p. 42 (1998)

New Study of the Decay From Superdeformed States in ${ }^{194} \mathrm{~Pb}$

F. Hannachi, A. Korichi, P. Paris, C. F. Liang, A. P. Lopez-Martens, M. Bergstrom, T. Dossing, B. Herskind, P. Varmette, T. L. Khoo, I. Ahmad,

M. Carpenter, S. Fischer, G. Hackman, R. V. F. Janssens, T. Lauritsen, D. Nisius, P. Reiter, M. Meyer, A. Astier, S. Perries, N. Redon, H. Hubel, A. Gorgen, A. Jansen, G. Schonwasser, A. Wilson, C. W. Beausang, K. Hauschild, D. Bazzacco, W. Krolas, S. Lunardi, C. A. Ur, and Z. Podolyak

Nuclear Structure '98, August 10-15, 1998, Gatlinburg, TN, Book of

Abstracts, p. 48 (1998)

Systematics of the Signature Splitting Phenomenon in the $\pi \mathrm{h}_{11 / 2}$ Bands for Odd- $\mathrm{A}_{\mathrm{H}} \mathrm{H} 160$ Nuclei

D. J. Hartley, M. A. Riley, T. B. Brown, F. G. Kondev, D. E. Archer,

J. Doring, P. Fallon, S. M. Fischer, R. V. F. Janssens, R. A. Kaye, R. W. Laird, W. C. Ma, D. Nisius, T. Petters, J. Pfohl, A. M. Richmond, R. K. Sheline, J. Simpson, and S. L. Tabor Nuclear Structure '98, August 10-15, 1998, Gatlinburg, TN, Book of

Abstracts, p. 49 (1998)

Magnetic Moments of High-Spin Normal-Deformed and Superdeformed States in ${ }^{193,194} \mathrm{Hg}$

M. Hass, R. H. Mayer, N. Benczer-Koller, C. Broude, J. A. Cizewski,

J. Holden, R. V. F. Janssens, G. Kumbartzki, T. Lauritsen, I. Y. Lee,

A. O. Macchiavelli, D. P. McNabb, M. Satteson, and L. Weissman

Nuclear Structure '98, August 10-15, 1998, Gatlinburg, TN, Book of

Abstracts, p. 50 (1998)

Competition Between Collective and Terminating Structures in ${ }^{156} \mathrm{Dy},{ }^{159} \mathrm{Er}$ and ${ }^{160}$ Er: Nuclear Behavior at Spin 50-60 h4

F. G. Kondev, M. A. Riley, J. Simpson, R. V. F. Janssens, A. V. Afanasjev,

I. Ragnarsson, D. J. Hartley, T. B. Brown, M. P. Carpenter, P. Fallon,

S. M. Fischer, C. A. Kalfas, T. L. Khoo, T. Lauritsen, W. C. Ma,

J. F. Sharpey- Schafer, and J. C. Lisle

Nuclear Structure '98, August 10-15, 1998, Gatlinburg, TN, Book of

Abstracts, p. 64 (1998)

Experimental Determination of the Excitation Energy of SD Bands in ${ }^{192,194} \mathrm{Hg}$

by Analysis of the Decay Quasi-Continuum Gamma Rays

T. Lauritsen, T. L. Khoo, A. Lopez-Martens, I. Ahmad, H. Amro, S. Berger,

I. Calderin, M. P. Carpenter, S. M. Fischer, G. Hackman, R. V. F. Janssens, D. T. Nisius,

F. Hannachi, A. Korichi, E. F. Moore, and P. Reiter

Nuclear Structure '98, August 10-15, 1998, Gatlinburg, TN, Book of

Abstracts, p. 75 (1998) 
Differential Quadrupole Moment Determinations in Superdeformed 151,152,153Dy

D. T. Nisius, R. V. F. Janssens, I. Ahmad, H. Amro, M. P. Carpenter,

S. M. Fischer, G. Hackman, T. L. Khoo, T. Lauritsen, P. Reiter, E. F. Moore, B. Haas,

P. Fallon, R. M. Clark, and R. Krucken

Nuclear Structure '98, August 10-15, 1998, Gatlinburg, TN, Book of

Abstracts, p. 94 (1998)

Structure and Formation Mechanisms of the Transfermium Isotope ${ }^{254}$ No

P. Reiter, T. L. Khoo, C. J. Lister, I. Ahmad, B. B. Back, M. P.

Carpenter, C. N. Davids, W. H. Henning, R. V. F. Janssens, T. Lauritsen,

D. Seweryniak, J. Uusitalo, I. Wiedenhover, P. A. Butler, N. Amzal, A. J. Chewter,

P. T. Greenlees, R. D. Herzberg, G. Jones, R. Julin, M. Leino, K. Vetter, P. Fallon, I. Y. Lee,

A. Macchiavelli, S. Siem, W. Korten, Y. Le Coz, J. Cizewski. K-Y. Ding, and N. Fotiades

Nuclear Structure '98, August 10-15, 1998, Gatlinburg, TN, Book of

Abstracts, p. 108 (1998)

Shape-Competition in the Mercury-Lead Region: the Case of ${ }^{183} \mathrm{Tl}$ and ${ }^{188} \mathrm{~Pb}$

W. Reviol, L. L. Riedinger, C. R. Bingham, W. Weintraub, R. Wadsworth,

A. N. Wilson, D. Jenkins, N. Kelsall, M. P. Carpenter, R. V. F. Janssens,

D. Seweryniak, J. Uusitalo, I. Wiedenhover, C. Baktash, J. Batchelder,

A. Galindo- Uribarri, C. J. Gross, S. D. Paul, D. C. Radford, K. Rykaczewski, D. Shapira,

K. S. Toth, C. H. Yu, S. Juutinen, K. Helariutta, J. Cizewski, T. N. Ginter, B. MacDonald, and A. Piechaczek

Nuclear Structure '98, August 10-15, 1998, Gatlinburg, TN, Book of

Abstracts, p. 109 (1998)

Five-Quasiparticle Bands in ${ }^{127} \mathrm{Ba}$

A. Schmidt, G. Alexius, O. Vogel, R. S. Chakrawarthy, A. Dewald, D.

Bazzacco, P. von Brentano, A. Gizon, J. Gizon, S. Lunardi, D. R. Napoli, P. Pavan, C. Rossi

Alvarez, and I. Wiedenhoever

Nuclear Structure '98, August 10-15, 1998, Gatlinburg, TN, Book of

Abstracts, p. 115 (1998)

Three-Quasiparticle Bands in ${ }^{123} \mathrm{Xe}$

A. Schmidt, I. Schneider, H. Meise, I. Wiedenhoever, O. Stuch, K. Jessen, D. Weisshaar,

C. Schumacher, P. von Brentano, G. Sletten, B. Herskind, M. Bergstrom, and J. Wrzesinski

Nuclear Structure '98, August 10-15, 1998, Gatlinburg, TN, Book of

Abstracts, p. 116 (1998)

Coulomb Excitation of a ${ }^{78} \mathrm{Rb}$ Radioactive Beam

J. Schwartz, C. J. Lister, D. H. Henderson, S. M. Fischer, P. Reiter, A. Aprahamian,

J. A. Cizewski, C. N. Davids, R. deHaan, R. V. F. Janssens, D. Nisius, D. Seweryniak, and S. M. Vincent

Nuclear Structure '98, August 10-15, 1998, Gatlinburg, TN, Book of

Abstracts, p. 118 (1998)

E2 Polarization Charge and Core Excitations in ${ }^{102} \mathrm{Sn}$

D. Seweryniak, M. Lipoglavsek, C. N. Davids, C. Fahlander, M. Gorska,

R. V. F. Janssens, J. Nyberg, J. Uusitalo, W. B. Walters, I. Ahmad, J. Blomqvist,

M. P. Carpenter, J. A. Cizewski, S. M. Fischer, H. Grawe, G. Hackman, M. Huhta,

T. L. Khoo, C. J. Lister, D. Nisius, G. Poli, P. Reiter, J. Ressler, J. Schwartz, S. Siem,

A. Sonzogni, and I. L. Wiedenhoever

Nuclear Structure '98, August 10-15, 1998, Gatlinburg, TN, Book of Abstracts, p. 119 (1998) 
Decay Out of a Superdeformed Band in ${ }^{191} \mathrm{Hg}$

S. Siem, P. Reiter T. L. Khoo, I. Ahmad, H. Amro, I. Calderin,

M. P. Carpenter, T. Dossing, D. Gassmann, S. M. Fischer, U. Garg, G. Hackman,

F. Hannachi, R. V. F. Janssens, B. Kharraja, A. Korichi, T. Lauritsen, I. Y. Lee,

A. Lopez-Martens, A. O. Macchiavelli, E. F. Moore, D. Nisius, and C. Schuck

Nuclear Structure '98, August 10-15, 1998, Gatlinburg, TN, Book of

Abstracts, p. 122 (1998)

Coincidence RDDS Lifetime Measurement in 130Ba with an EUROBALL Cluster Detector Array

O. Stuch, K. Jessen, A. Dewald, R. Kuhn, R. Krucken, R. Peusquens, H. Tiesler, D. Weil,

I. Wiedenhoever, K. O. Zell, P. von Brentano, C. Ender, T. Hartlein, F. Kock, O. Koschorrek,

P. Reiter, and D. Schwalm

Nuclear Structure '98, August 10-15, 1998, Gatlinburg, TN, Book of

Abstracts, p. 128 (1998)

The $\alpha$-Decay Rates of $180,182,184 \mathrm{~Pb}$ and the $\mathrm{Z}=82$ Closure

K. S. Toth, C. R. Bingham, J. C. Batchelder, L. T. Brown, L. F. Conticchio,

C. N. Davids, R. J. Irvine, D. Seweryniak, D. M. Moltz, W. B. Walters, J. Wauters, and E. F. Zganjar

Nuclear Structure '98, August 10-15, 1998, Gatlinburg, TN, Book of

Abstracts, p. 139 (1998)

Search for Two-Phonon Octupole Vibrational States in ${ }^{208} \mathrm{~Pb}$

K. Vetter, A. O. Macchiavelli, H. Amro, S. J. Asztalos, B. Busse,

R. M. Clark, D. Cline, M. A. Deleplanque, R. M. Diamond, P. Fallon, R. Gray,

R. V. F. Janssens, R. Krucken, I. Y. Lee, R. W. MacLeod, G. J. Schmid, M. W. Simon,

F. S. Stephens, and C. Y. Wu

Nuclear Structure '98, August 10-15, 1998, Gatlinburg, TN, Book of

Abstracts, p. 143 (1998)

Detailed DCO-Analysis from $4 \pi$-Spectrometers

I. Wiedenhoever, R. V. F. Janssens, M. P. Carpenter, O. Vogel, H. Klein, A. Dewald, and $P$. von Brentano

Nuclear Structure '98, August 10-15, 1998, Gatlinburg, TN, Book of

Abstracts, p. 148 (1998)

Unsafe Coulomb Excitation of ${ }^{240} \mathrm{Pu}$ and ${ }^{244} \mathrm{Pu}$

I. Wiedenhoever, G. Hackman, R. V. F. Janssens, I. Ahmad, J. P. Greene,

H. Amro, M. P. Carpenter, D. T. Nisius, P. Reiter, T. Lauritsen, C. J. Lister, T. L. Khoo,

S. Siem, J. Cizewski, D. Seweryniak, J. Uusitalo, A. O. Macchiavelli, P. Chowdhury,

E. H. Seabury, D. Cline, and C. Y. Wu

Nuclear Structure '98, August 10-15, 1998, Gatlinburg, TN, Book of

Abstracts, p. 149 (1998)

The PHOBOS Experiment at RHIC

B. B. Back for the PHOBOS collaboration

Abstracts of Contributed Papers, International Nuclear Physics Conference

(INPC98), August 24-28, Paris, France, p. 231 (1998) 
Alpha-Decay Studies of the Lightest Po Isotopes Characterizing Intruder States in Neutron-Deficient $\mathrm{Pb}$ Nuclei

A. N. Andreyev, N. Bijnens, M. Huyse, P. Van Duppen, M. Leino, J. F. Cocks,

T. Enqvist, P. T. Greenlees, S. Juutinen, K. Helariutta, R. Julin,

H. Kankaanpaa, P. Kuusiniemi, M. Muikku, A. Savelius, W. H. Trzaska, J. Uusitalo,

C. N. Davids, R. V. F. Janssens, D. Seweryniak, M. P. Carpenter, H. Amro, P. Decrock,

P. Reiter, D. Nisius, L. T. Brown, S. Fischer, T. Lauritsen, J. Wauters, C. R. Bingham,

J. A. Cizewski, K. Y. Ding, N. Fotiades, and D. P. McNabb

Abstracts of Contributed Papers, International Nuclear Physics Conference

(INPC98), August 24-28, Paris, France, p. 262 (1998)

A Study of the $h_{9 / 2}$ and $s_{1 / 2}$ Intruder States in $\mathrm{Tl}$ and $\mathrm{Bi}$ Isotopes through Alpha Decay Systematics

C. R. Bingham, J. C. Batchelder, C. N. Davids, T. N. Ginter, C. J. Gross,

R. Grzywacz, R. J. Irvine, B. McDonald, A. Piechaczek, W. Reviol, K. Rykaczewski,

D. Seweryniak, K. S. Toth, W. B. Walters, J. Wauters, J. L. Wood, X. J. Xu, J. Uusitalo, and E. F. Zganjar

Abstracts of Contributed Papers, International Nuclear Physics Conference

(INPC98), August 24-28, Paris, France, p. 278 (1998)

Multi-Quasiparticle K-Isomers in Neutron-Rich Hafnium Nuclei At and Beyond the Stability Line

P. Chowdhury, R. D'Alarcao, E. H. Seabury, P. M. Walker, C. Wheldon,

I. Ahmad, M. P. Carpenter, G. Hackman, R. V. F. Janssens, T. L. Khoo, D. Nisius, and P. Reiter

Abstracts of Contributed Papers, International Nuclear Physics Conference

(INPC98), August 24-28, Paris, France, p. 295 (1998)

The Performance of Gammasphere at ATLAS

C. J. Lister, M. P. Carpenter, C. N. Davids, R. V. F. Janssens, T. L. Khoo,

T. Lauritsen, D. T. Nisius, P. Reiter, D. Seweryniak, J. Uusitalo,

I. Wiedenhoever, P. Fallon, A. O. Macchiavelli, I. Y. Lee, J. A. Cizewski,

N. Fotiades, and E. H. Seabury

Abstracts of Contributed Papers, International Nuclear Physics Conference

(INPC98), August 24-28, Paris, France, p. 365 (1998)

Study of the Strength Distribution of the Primary $\gamma$ Rays in the Decay From Superdeformed States in ${ }^{194} \mathrm{Hg}$

A. Lopez-Martens, T. Dossing, T. L. Khoo, D. Ackermann, I. Ahmad,

M. P. Carpenter, S. M. Fischer, G. Hackman, R. V. F. Janssens, A. Korichi,

C. J. Lister, T. Lauritsen, and D. Nisius

Abstracts of Contributed Papers, International Nuclear Physics Conference

(INPC98), August 24-28, Paris, France, p. 367 (1998)

Superdeformation and Shape-Coexistence Phenomena in Light Thallium Nuclei

W. Reviol, L. L. Riedinger, B. H. Smith, M. P. Carpenter, S. M. Fischer,

R. V. F. Janssens, T. L. Khoo, T. Lauritsen, and D. Nisius

Abstracts of Contributed Papers, International Nuclear Physics Conference

(INPC98), August 24-28, Paris, France, p. 411 (1998) 
Average Magnetic Moments of Pre-Yrast States

L. Weissman, R. H. Mayer, N. Benczer-Koller, C. Broude, J. A. Cizewski, M. Hass, J. Holden, R. V. F. Janssens, G. Kumbartzki, T. Lauritsen, I. Y. Lee, A. O. Macchiavelli, D. P. McNabb, and M. Satteson Abstracts of Contributed Papers, International Nuclear Physics Conference (INPC98), August 24-28, Paris, France, p. 456 (1998)

Viscosity in the Fission of Hot Nuclei

B. B. Back

Abstracts of Contributed Papers, International Nuclear Physics Conference (INPC98), August 24-28, Paris, France, p. 559 (1998)

The $\beta^{+}$Decay of ${ }^{54} \mathrm{Mn}$ and Cosmic Ray Chronometry

A. H. Wuosmaa, I. Ahmad, Sam M. Austin, B. A. Brown, J. J. Connell,

S. M. Fischer, S. J. Freedman, G. Hackman, V. Nanal, G. Savard, J. P. Schiffer, and P. Wilt Abstracts of Contributed Papers, International Nuclear Physics Conference (INPC98), August 24-28, Paris, France, p. 701 (1998)

Weak-Interaction Studies with an On-Line Penning Trap Mass Spectrometer G. Savard, R. C. Barber, F. Buchinger, J. E. Crawford, X. Feng, S. Gulick, G. Hackman, J. C. Hardy, J. K. P. Lee, R. B. Moore, K. S. Sharma, and J. Uusitalo

Abstracts of Contributed Papers, International Nuclear Physics Conference

(INPC98), August 24-28, Paris, France, p. 731 (1998)

Injection of Reaction Recoils in a Penning Trap Mass Spectrometer G. Savard, R. C. Barber, F. Buchinger, J. E. Crawford, X. Feng, S. Gulick, G. Hackman, J. C. Hardy, J. K. P. Lee, R. B. Moore, K. S. Sharma, and J. Uusitalo

Abstracts of Contributed Papers, International Nuclear Physics Conference

(INPC98), August 24-28, Paris, France, p. 783 (1998)

DCO Analysis with $4 \pi$ Spectrometers: The M1 Band in ${ }^{128} \mathrm{Ba}$

I. Wiedenhover, R. V. F. Janssens, O. Vogel, H. Klein, A. Dewald,

P. Von Brentano, D. Bazzaco, C. Rossi Alvarez, and G. DeAngeles

Proceedings of the 1998 Joint APS/AAPT April Meeting, Columbus, OH, April

18-21, 1998; Bull. Am. Phys. Soc. 43, 1099 (1998)

Status of a New Measurement of the Partial $0^{+} \rightarrow 0^{+}$Half Life of ${ }^{10} \mathrm{C}$ with Gammasphere

B. K. Fujikawa, S. J. Asztalos, C. J. Bowers, Y.-D. Chan, R. M. Clark,

M.-A. Deleplanque-Stephens, P. Fallon, S. J. Freedman, K. T. Lesko, I.-Y. Lee, L. J. Lis,

A. O. Machiavelli, R. W. Macleod, J. L. Mortara, C. L. Norris, C. E. Okada, M. C. Perillo-Isaac. J. C. Reich, D. W. Ross, M. A. Rowe, A. Schuelke, S.-Q. Shang, J. Stalnaker, F. S. Stephens, R. G. Stokstad, P. A. Vetter, E. G. Wasserman, and J. P. Greene Proceedings of the 1998 Joint APS/AAPT April Meeting, Columbus, OH, April 18-21, 1998; Bull. Am. Phys. Soc. 43, 1109 (1998 
New Isomer in ${ }^{80} \mathrm{Y}$

J. Doring, H. Schatz, A. Aprahamian, R. C. de Haan, J. Gorres, M. Wiescher,

W. B. Walters, J. Rikovska, L. T. Brown, C. N. Davids, C. J. Lister,

D. Seweryniak, and B. Foy

Proceedings of the 1998 Joint APS/AAPT April Meeting, Columbus, OH, April

18-21, 1998; Bull. Am. Phys. Soc. 43, 1133 (1998)

In-Beam Spectroscopy of ${ }^{68} \mathrm{Se}$

S. M. Fischer, D. P. Balamuth, P. Hausladen, C. J. Lister, D. J. Blumenthal,

J. Schwartz, M. J. Leddy, D. G. Sarantites, and M. Devlin

Proceedings of the 1998 Joint APS/AAPT April Meeting, Columbus, OH, April 18-21, 1998; Bull. Am. Phys. Soc. 43, 1134 (1998)

Study of ${ }^{187} \mathrm{Bi} \alpha$ Decay

J. C. Batchelder, K. S. Toth, C. R. Bingham, J. Wauters, E. F. Zganjar,

C. N. Davids, D. Seweryniak, R. J. Irvine, and W. B. Walters

Proceedings of the 1998 Joint APS/AAPT April Meeting, Columbus, OH, April 18-21, 1998; Bull. Am. Phys. Soc. 43, 1172 (1998)

Location of the $1 / 2+$ Intruder State in ${ }^{195}$ At by Means of $\alpha$ Decay

C. R. Bingham, W. Reviol, X.-J. Xu, J. C. Batchelder, K. S. Toth,

D. Seweryniak, J. Uusitalo, J. L. Wood, and J. A. Cizewski

Proceedings of the 1998 Joint APS/AAPT April Meeting, Columbus, OH, April 18-21, 1998; Bull. Am. Phys. Soc. 43, 1172 (1998)

$\alpha$-Decay Properties of ${ }^{180} \mathrm{Tl}$ and ${ }^{179} \mathrm{Tl}$

K. S. Toth, G. R. Bingham, X. J. Xu, J. Wauters, J. C. Batchelder,

L. F. Conticchio, W. B. Walters, C. N. Davids, and D. Seweryniak

Proceedings of the 1998 Joint APS/AAPT April Meeting, Columbus, OH, April 18-21, 1998; Bull. Am. Phys. Soc. 43, 1172 (1998)

Inelastic Excitation of Multi-Quasiparticle K-Isomers in ${ }^{180,181} \mathrm{Hf}$ Nuclei

R. D'Alarcao, E. H. Seabury, P. Chowdhury, P. M. Walker, C. Wheldon,

I. Ahmad, M. Carpenter, G. Hackman, R. V. F. Janssens; T. L. Khoo, D. Nisius, and P. Reiter Proceedings of the 1998 Joint APS/AAPT April Meeting, Columbus, OH, April

18-21, 1998; Bull. Am. Phys. Soc. 43, 1173 (1998)

Shape Coexistence and Band Termination in $N=89155 \mathrm{Dy}$

T. B. Brown, M. A. Riley, R. V. F. Janssens, I. Ahmad, D. J. Blumenthal,

M. P. Carpenter, P. Fallon, S. M. Fischer, G. Hackman, D. J. Hartley, C. Kalfas, T. L. Khoo,

F. G. Kondev, T. Lauritsen, D. Nisius, W. C. Ma, J. Simpson, J. F. Sharpey-Schafer,

A. Afanasiev, and I. Ragnarsson

Proceedings of the 1998 Joint APS/AAPT April Meeting, Columbus, OH, April

18-21, 1998; Bull. Am. Phys. Soc. 43, 1207 (1998)

Lifetime Measurements of Superdeformed Bands in ${ }^{142} \mathrm{Sm}:$ A Test of the

Additivity Principle for Quadrupole Moments

G. Hackman, R. V. F. Janssens, D. Nisius, I. Ahmad, M. P. Carpenter,

S. M. Fischer, T. L. Khoo, T. Lauritsen, and P. Reiter

Proceedings of the 1998 Joint APS/AAPT April Meeting, Columbus, OH, April

18-21, 1998; Bull. Am. Phys. Soc. 43, 1207 (1998) 
Quasicontinuum Gammà-Ray Study in ${ }^{154} \mathrm{Dy}$

W. C. Ma, P. G. Varmette, T. L. Khoo, T. Lauritsen, I. Ahmad, D. Blumenthal, M. P. Carpenter, B. Crowell, S. M. Fischer, D. Gassmann, R. V. F. Janssens,

D. Nisius, V. Martin, J. L. Egido, P. K. Bhattacharyya, C. T. Żhang, P. J. Daly, Z. W. Grabowski, J. H. Hamilton, and A. V. Ramayya

Proceedings of the 1998 Joint APS/AAPT April Meeting, Columbus, OH, April

18-21, 1998; Bull. Am. Phys. Soc. 43, 1207 (1998)

Multi-Quasiparticle Isomers and High-K Structures in ${ }^{174} \mathrm{~W}$

E. H. Seabury, P. Chowdhury, I. Ahmad, M. Carpenter, S. Fischer,

R. V. F. Janssens, T. L. Khoo, T. Lauritsen, C. J. Lister, and D. Seweryniak

Proceedings of the 1998 Joint APS/AAPT April Meeting, Columbus, OH, April

18-21, 1998; Bull. Am. Phys. Soc. 43, 1223 (1998)

Superdeformation Studies at the Edge of the Mass 190 Region

L. L. Riedinger, W. Reviol, B. H. Smith, M. P. Carpenter, S. M. Fischer,

R. V. F. Janssens, T. L. Khoo, T. Lauritsen, D. Nisius, E. F. Moore, and P. Fallon

Proceedings of the 1998 Joint APS/AAPT April Meeting, Columbus, OH, April

18-21, 1998; Bull. Am. Phys. Soc. 43, 1223 (1998)

Unresolved $\boldsymbol{\gamma}$-Ray Decay of Yrast SD Bands in ${ }^{192,194} \mathrm{~Pb}$

D. P. McNabb, J. A. Cizewski, K. Y. Ding, N. Fotiades, T. L. Khoo,

T. Lauritsen, W. Younes, D. C. Archer, R. W. Bauer, J. A. Becker, L. A. Bernstein,

K. Hauschild, R. M. Clark, M. A. Deleplanque, R. M. Diamond, P. Fallon, I. Y. Lee,

A. O. Macchiavelli, R. W. Macleod, and F. S. Stephens

Proceedings of the 1998 Joint APS/AAPT April Meeting, Columbus, OH, April

18-21, 1998; Bull. Am. Phys. Soc. 43, 1223 (1998)

ICEBall 2: An Array of Six Mini-Orange Spectrometers for Internal Electron Conversion Spectroscopy

J. X. Saladin, I. Birriel, P. Cottle, I. Y. Lee, M. R. Maier, and R. V. F. Janssens

Proceedings of the 1998 Joint APS/AAPT April Meeting, Columbus, OH, April

18-21, 1998; Bull. Am. Phys. Soc. 43, 1224 (1998)

The Performance of Gammasphere at ATLAS

C. J. Lister, M. P. Carpenter, C. N. Davids, R. V. F. Janssens, T. L. Khoo,

T. Lauritsen, D. T. Nisius, P. Reiter, D. Seweryniak, J. Uusitalo,

I. Wiedenhoever, P. Fallon, A. O. Macchiavelli, I. Y. Lee, J. Cizewski, and E. H. Seabury

Proceedings of the 1998 Joint APS/AAPT April Meeting, Columbus, OH, April

18-21, 1998; Bull. Am. Phys. Soc. 43, 1224 (1998)

Measurements of the Partial $\left.0^{+}\right\} 0^{+}$Half Life of ${ }^{10} \mathrm{C}$ with Gammasphere

B. K. Fujikawa, S. J. Asztalos, C. J. Bowers, Y.-D. Chan, R. M. Clark,

M.-A. Deleplanque-Stephens, P. Fallon, S. J. Freedman, K. T. Lesko, I.-Y. Lee, L. J. Lising,

A. O. Macchiavelli, R. W. Macleod, J. L. Mortara, C. L. Norris, C. E. Okada,

M. C. Perillo-Isaac, J. C. Reich, D. W. Ross, M. A. Rowe, A. Schuelke, S.-Q. Shang.

J. Stalnaker, F. S. Stephens, R. G. Stokstad, P. A. Vetter, E. G. Wasserman, and J. P. Greene

1998 Fall Meeting of the Division of Nuclear Physics of the American

Physical Society, 28-31 October 1998, Santa Fe, NM; Bull. Am. Phys. Soc.

43, 1532 (1998) 
Test of Time Reversal Invariance in the $\beta$-Decay of ${ }^{56} \mathrm{Co}$

J. L. Mortara, S. Davis, S. J. Freedman, and R. Janssens

1998 Fall Meeting of the Division of Nuclear Physics of the American

Physical Society, 28-31 October 1998, Santa Fe, NM; Bull Am. Phys. Soc.

43, 1532 (1998)

Population of Low-Spin States in ${ }^{187} \mathrm{Tl}$ by $\alpha$ Decay of ${ }^{191} \mathrm{Bi}$

C. R. Bingham, W. Reviol, J. Wauters, X.-J. Xu, J. C. Batchelder,

K. S. Toth, D. Seweryniak, J. Uusitalo, J. L. Wood, and J. A. Cizewski

1998 Fall Meeting of the Division of Nuclear Physics of the American

Physical Society, 28-31 October 1998, Santa Fe, NM; Bull Am. Phys. Soc.

43, 1533 (1998)

Measurement of the ${ }^{17} \mathrm{~F}(\mathrm{p}, \alpha){ }^{14} \mathrm{O}$ Reaction with a Radioactive ${ }^{17} \mathrm{~F}$ Beam

B. Harss, R. C. Pardo, K. E. Rehm, C. L. Jiang, R. V. F. Janssens,

J. P. Schiffer, J. Uusitalo, I. Wiedenhover, J. Blackmon, A. Chen, P. Parker, M. Paul, and R. E. Segel

1998 Fall Meeting of the Division of Nuclear Physics of the American

Physical Society, 28-31 October 1998, Santa Fe, NM; Bull Am. Phys. Soc.

43, 1536 (1998)

Development of a ${ }^{3} \mathrm{He}$ Gas Target for Experiments with Radioactive Beams

C. L. Jiang, K. E. Rehm, I. Ahmad, F. Brumwell, J. Greene, B. Harss,

D. Henderson, W. F. Henning, R. V. F. Janssens, G. McMichael, R. C. Pardo,

P. Reiter, J. P. Schiffer, D. Seweryniak, A. Sonzogni, J. Uusitalo,

I. Wiedenhoever, A. H. Wuosmaa, B. Zabransky, M. Paul, R. E. Segel, J. Goerres,

M. Wiescher, and D. Ackermann

1998 Fall Meeting of the Division of Nuclear Physics of the American

Physical Society, 28-31 October 1998, Santa Fe, NM; Bull Am. Phys. Soc.

43,1542 (1998)

Gamma-Ray Spectroscopy of Proton Drip-Line Nuclei

Dariusz Seweryniak

1998 Fall Meeting of the Division of Nuclear Physics of the American

Physical Society, 28-31 October 1998, Santa Fe, NM; Bull Am. Phys. Soc.

43,1545 (1998)

Three-Particle States in Exotic Fission Product ${ }^{135} \mathrm{Sb}$

P. Bhattacharyya, C. T. Zhang, B. Fornal, P. J. Daly, Z. W. Grabowski,

I. Ahmad, T. Lauritsen, L. R. Morss, W. R. Phillips, J. L. Durell, M. J. Leddy, A. G. Smith,

W. Urban, B. J. Varley, N. Schulz, E. Lubkiewicz, M. Bentaleb, and J. Blomqvist

1998 Fall Meeting of the Division of Nuclear Physics of the American

Physical Society, 28-31 October 1998, Santa Fe, NM; Bull. Am. Phys. Soc.

43, 1550, (1998)

Proton Decay from Deformed Rare Earth Nuclei

A. A. Sonzogni, C. N. Davids, D. Seweryniak, D. J. Henderson, J. Uusitalo,

P. J. Woods, T. Davinson, W. B. Walters, and J. A. Cizewski

1998 Fall Meeting of the Division of Nuclear Physics of the American

Physical Society, 28-31 October 1998, Santa Fe, NM; Bull. Am. Phys. Soc.

43, 1558 (1998) 
The Identification of the Low Spin Structure of Excited States in ${ }^{113} \mathrm{Cs}$

C. J. Gross, J. C. Batchelder, J. W. Johnson, Y. A. Akovali, C. Baktash, A. Galindo-Uribarri, F. Liang, S. D. Paul, D. C. Radford, K. Rykaczewski, D. Shapira, K. S. Toth, C.-H. Yu, T. N. Ginter, C. R. Bingham, D. Ellis, R. Grzywacz, W. Reviol, W. Weintrab, M. P. Carpenter, C. N. Davids, R. V. F. Janssens, C. J. Lister, D. Seweryniak, J. Uusitalo, A. Piechaczek, E. F. Zganjar, T. Davinson, P. J. Woods, J. Mas, W. Satula, and B. MacDonald 1998 Fall Meeting of the Division of Nuclear Physics of the American Physical Society, 28-31 October 1998, Santa Fe, NM; Bull Am. Phys. Soc. 43, 1558 (1998)

Proton Decay of the Closed Neutron Shell Nucleus ${ }^{155} \mathrm{Ta}$ J. Uusitalo, C. N. Davids, D. Seweryniak, A. A. Sonzogni, D. J. Henderson, P. J. Woods, T. Davinson, R. Slinger, W. B. Walters, J. Ressler, J. C. Batchelder, C. R. Bingham, J. Deboer, and H. J. Maier 1998 Fall Meeting of the Division of Nuclear Physics of the American Physical Society, 28-31 October 1998, Santa Fe, NM; Bull. Am. Phys. Soc. 43, 1559 (1998)

Mass Study of the Neutron Deficient Nucleus ${ }^{68} \mathrm{Se}$ T. Brown, C. Davids, H. Penttila, D. Blumenthal, C. Lister, B. Busse, J. Batchelder, W. Walters, L. Conticchio, D. Mustillo, and A. V. Ramayya 1998 Fall Meeting of the Division of Nuclear Physics of the American Physical Society, 28-31 October 1998, Santa Fe, NM; Bull Am. Phys. Soc. 43, 1561 (1998)

The Mass of ${ }^{36} \mathrm{Ca}$ and the Isobaric Mass Multiplet Equation

A. Komives, A. Garcia, D. Peterson, D. Bazin, J. Caggiano, B. Sherrill, N. Alahari, A. Bacher, W. Lozowski, J. Greene, and E. G. Adelberger 1998 Fall Meeting of the Division of Nuclear Physics of the American Physical Society, 28-31 October 1998, Santa Fe, NM; Bull Am. Phys. Soc. 43, 1561 (1998)

Vibrational Excitations in the $A=80$ Region of Nuclei

A. Aprahamian, J. Doring, R. C. De Hann, J. Gorres, M. Wiescher, C. J. Lister, C. N. Davids, D. Seweryniak 1998 Fall Meeting of the Division of Nuclear Physics of the American Physical Society, 28-31 October 1998, Santa Fe, NM; Bull Am. Phys. Soc. 43, 1562 (1998)

High-Spin Spectroscopy in the Very Neutron-Deficient Barium Region J. F. Smith, S. J. Freeman, J. J. Leddy, C. J. Chiara, D. B. Fossan, D. R. LaFosse, K. Starosta, M. Devlin, F. Lerma, D. G. Sarantites, J. N. Wilson, R. Wadsworth, A. N. Wilson, R. V. F. Janssens, and D. Seweryniak 1998 Fall Meeting of the Division of Nuclear Physics of the American Physical Society, 28-31 October 1998, Santa Fe, NM; Bull Am. Phys. Soc. 43, 1571 (1998) 
High-Spin States in Neutron-Deficient A 72 Nuclei

N. Fotiades, J. A. Cizewski, C. J. Lister, C. N. Davids, R. V. F. Janssens,

D. Seweryniak, M. P. Carpenter, T. L. Khoo, T. Lauritsen, D. Nisius, P. Reiter, J. Uusitalo,

I. Wiedenhoever, and A. O. Macchiavelli

1998 Fall Meeting of the Division of Nuclear Physics of the American

Physical Society, 28-31 October 1998, Santa Fe, NM; Bull Am. Phys. Soc.

43, 1571 (1998)

Studies of Neutron-Deficient $Z=57-59$ Nuclei

D. R. LaFosse, C. J. Chiara, D. B. Fossan, K. Starosta, J. F. Smith,

M. Devlin, F. Lerma, D. G. Sarantites, J. N. Wilson, R. Wadsworth, A. N. Wilson,

R. V. F. Janssens, and D. Seweryniak

1998 Fall Meeting of the Division of Nuclear Physics of the American

Physical Society, 28-31 October 1998, Santa Fe, NM; Bull Am. Phys. Soc.

43, 1571 (1998)

Excited States in ${ }^{155,156,157}$ Lu Using Recoil-Decay Tagging with Gammasphere

K. Y. Ding, J. A. Cizewski, N. Fotiades, D. Seweryniak, H. Amro,

M. P. Carpenter, C. N. Davids, R. V. F. Janssens, T. Lauritsen, C. J. Lister, D. Nisius,

P. Reiter, J. Uusitalo, and A. O. Macchiavelli

1998 Fall Meeting of the Division of Nuclear Physics of the American

Physical Society, 28-31 October 1998, Santa Fe, NM; Bull Am. Phys. Soc.

43, 1571 (1998)

How Far From Stability Can We Go Using Gammasphere and the FMA?

C. J. Lister

1998 Fall Meeting of the Division of Nuclear Physics of the American

Physical Society, 28-31 October 1998, Santa Fe, NM; Bull. Am. Phys. Soc

43, 1571 (1998)

Structure and Formation Mechanism of the Transfermium Isotope ${ }^{254}$ No

P. Reiter, T. L. Khoo, C. J. Lister, I. Ahmad, M. P. Carpenter,

C. N. Davids, W. H. Henning, R. V. F. Janssens, T. Lauritsen, D. Seweryniak,

S. Siem, J. Uusitalo, I. Wiedenhoever, J. A. Cizewski, K. Y. Ding, N. Fotiades, P. A. Butler, N. Amzal, A. J. Chewter, T. P. Greenlees, R. D. Herzberg, G. Jones, K. Vetter, W. Korten, and M. Leino

1998 Fall Meeting of the Division of Nuclear Physics of the American

Physical Society, 28-31 October 1998, Santa Fe, NM; Bull. Am. Phys. Soc.

43, 1572 (1998)

Recoil Decay Tagging Studies of the $\mathrm{N}=84$ Isotone ${ }^{157} \mathrm{Ta}$

J. Uusitalo, D. Seweryniak, M. P. Carpenter, C. N. Davids, R. V. F. Janssens, T. Lauritsen, C. J. Lister, D. Nisius, P. Reiter, J. A. Cizewski, K. Y. Ding, N. Fotaides, and A. O. Macchiavelli

1998 Fall Meeting of the Division of Nuclear Physics of the American

Physical Society, 28-31 October 1998, Santa Fe, NM; Bull. Am. Phys. Soc.

43, 1572 (1998) 
Study of Excited States in ${ }^{167}$ Ir: Probing States Beyond the Proton Drip Line

M. P. Carpenter, R. V. F. Janssens, T. L. Khoo, D. Seweryniak, A. Sonzogni, I. Ahmad, L. T. Brown, C. N. Davids, G. Hackman, T. Lauritsen, C. J. Lister, P. Reiter, S. Siem, J. Uusitalo, I. Wiedenhoever, P. J. Woods, J. A. Cizewski, W. Reviol,

L. L. Riedinger, S. M. Fischer, J. J. Ressler, W. B. Walters, D. G. Sarantites, and S. Heany 1998 Fall Meeting of the Division of Nuclear Physics of the American

Physical Society, 28-31 October 1998, Santa Fe, NM; Bull. Am. Phys. Soc.

43, 1572 (1998)

Unsafe Coulomb Excitation of ${ }^{240} \mathrm{Pu}$ and ${ }^{244} \mathrm{Pu}$

I. Wiedenhoever, G. Hackman, R. V. F. Janssens, I. Ahmad, J. P. Greene,

H. Amro, M. P. Carpenter, D. T. Nisius, P. Reiter, T. Lauritsen, C. J. Lister, T. L. Khoo,

S. Siem, J. Cizewski, D. Seweryniak, J. Uusitalo, A. O. Macchiavelli, P. Chowdhury,

E. H. Seabury, D. Cline, and C. Y. Wu

1998 Fall Meeting of the Division of Nuclear Physics of the American

Physical Society, 28-31 October 1998, Santa Fe, NM; Bull. Am. Phys. Soc.

43, 1573 (1998)

Testing Gammasphere at ATLAS Between Beam Bursts

R. D'Alarcao, P. Chowdhury, E. H. Seabury, M. Carpenter, G. Hackman,

R. V. F. Janssens, T. L. Khoo, T. Lauritsen, C. J. Lister, P. Reiter, D. Seweryniak,

and I. Wiedenhoever

1998 Fall Meeting of the Division of Nuclear Physics of the American

Physical Society, 28-31 October 1998, Santa Fe, NM; Bull Am. Phys. Soc.

43, 1573 (1998)

Coulomb Excitation of a ${ }^{78} \mathrm{Rb}$ Radioactive Beam

J. Schwartz, C. J. Lister, D. Henderson, S. M. Fischer, P. Reiter, C. N. Davids,

R. V. F. Janssens, D. Nisius, D. Seweryniak, A. Aprahamian, and R. De Hann

1998 Fall Meeting of the Division of Nuclear Physics of the American

Physical Society, 28-31 October 1998, Santa Fe, NM; Bull Am. Phys. Soc.

43, 1574 (1998)

Fusion Cross Sections for the Proton Drip Line Nucleus ${ }^{17} \mathrm{~F}$ at Energies Below the Barrier

K. E. Rehm, H. Esbensen, C. L. Jiang, B. B. Back, F. Borasi, B. Harss,

R. V. F. Janssens, V. Nanal, J. Nolen, R. C. Pardo, P. Reiter, A. Sonzogni,

J. Uusitalo, and A. H. Wuosmaa

1998 Fall Meeting of the Division of Nuclear Physics of the American

Physical Society, 28-31 October 1998, Santa Fe, NM; Bull Am. Phys. Soc.

43, 1574 (1998)

Magnetic Rotation in the Cd-Sn Region

R. M. Clark, S. J. Asztalos, B. Busse, M. A. Deleplanque, R. M. Diamond,

P. Fallon, R. Krucken, G. Lane, I. Y. Lee, A. O. Macchiavelli, R. W. MacLeod, G. J. Schmid,

F. S. Stephens, K. Vetter, D. Ward, D. Jenkins, N. Kelsall, R. Wadsworth, C. Parry,

A. N. Wilson, C. Chiara, D. B. Fossan, D. R. LaFosse, J. Sears, J. Smith, K. Starosta,

S. Juutinen, M. P. Carpenter, R. V. F. Janssens, T. Lauritsen, D. Seweryniak,

I. Wiedenhoever, M. Devlin, D. G. Sarantites, and J. N. Wilson

1998 Fall Meeting of the Division of Nuclear Physics of the American

Physical Society, 28-31 October 1998, Santa Fe, NM; Bull Am. Phys. Soc.

43, 1585 (1998) 
Prompt Spectroscopy of Odd-A $173,175 \mathrm{~W}$ Nuclei

E. H. Seabury, I. Shestakova, P. Chowdhury, I. Ahmad, M. Carpenter, S. Fischer,

R. V. F. Janssens, T. L. Khoo, T. Lauritsen, C. J. Lister, and D. Seweryniak

1998 Fall Meeting of the Division of Nuclear Physics of the American

Physical Society, 28-31 October 1998, Santa Fe, NM; Bull Am. Phys. Soc.

43, 1585 (1998)

The Two Phonon Octupole Vibrational States in ${ }^{208} \mathrm{~Pb}$

H. Amro, R. V. F. Janssens, I. Ahmad, M. P. Carpenter, S. Fischer,

G. Hackman, W. Henning, T. L. Khoo, T. Lauritsen, C. J. Lister, D. T.

Nisius, E. F. Moore, S. J. Sanders, P. E. Garrett, S. W. Yates, M. Yeh,

A. O. Macchiavelli, and K. Vetter

1998 Fall Meeting of the Division of Nuclear Physics of the American

Physical Society, 28-31 October 1998, Santa Fe, NM; Bull. Am. Phys. Soc.

43, 1586 (1998)

The Decay Out of a Superdeformed Band in ${ }^{191} \mathrm{Hg}$

S. Siem, P. Reiter, T. L. Khoo, M. P. Carpenter, T. Lauritsen, I. Ahmad,

H. Amro, I. Calderin, S. Fischer, G. Hackman, R. V. F. Janssens, D. T. Nisius, D. Gassmann,

T. Dossing, U. Garg, B. Kharraja, F. Hannachi, A. Korichi, A. Lopez-Martens, C. Schuck,

I. Y. Lee, A. O. Macchiavelli, and E. F. Moore

1998 Fall Meeting of the Division of Nuclear Physics of the American

Physical Society, 28-31 October 1998, Santa Fe, NM; Bull. Am. Phys. Soc.

43, 1586 (1998)

RDM Lifetime Measurements in ${ }^{191} \mathrm{Hg}$ Using the Gammasphere Plunger

H. Jin, B. Kharraja, U. Garg, S. S. Ghugre, M. P. Carpenter, S. Kharraja,

R. V. F. Janssens, T. L. Khoo, T. Lauritsen, D. Nisius, R. Kaczarowski,

I. M. Govil, R. Kruecken, A. Machiavelli, and R. MacLeod

1998 Fall Meeting of the Division of Nuclear Physics of the American

Physical Society, 28-31 October 1998, Santa Fe, NM; Bull Am. Phys. Soc.

43, 1586 (1998)

$\pi \mathrm{h}_{9 / 2} \mathrm{Ox} v \mathrm{i}_{13 / 2}$ Bands in Doubly Odd Tl Nuclei

W. Reviol, L. L. Riedinger, B. H. Smith, M. P. Carpenter, S. M. Fischer,

R. V. F. Janssens, and D. Nisius

1998 Fall Meeting of the Division of Nuclear Physics of the American

Physical Society, 28-31 October 1998, Santa Fe, NM; Bull Am. Phys. Soc.

43,1586 (1998)

Ordering Phenomena in Finite, Cold, One-Component Plasmas

John P. Schiffer

1998 Division of Plasma Physics Fall Meeting of the American Physical

Society, New Orleans, LA, November 16-20, 1998; Bull. Am. Phys. Soc. 43, (1998)

Nuclear Reactions: Exploring Un-Established Nuclear Regions With Established Tools

W. Henning

A Transparency Collection of the Proceedings of the Workshop on the

Experimental Equipment for an Advanced ISOL Facility, Lawrence Berkeley

National Laboratory, Berkeley, CA, July 22-25, 1998, edited by I. Y. Lee,

Lawrence Berkeley National Laboratory Report LBNL-42138, pp. $63-94$ (1998) 
Neutron Detectors

C. Lister

A Transparency Collection of the Proceedings of the Workshop on the

Experimental Equipment for an Advanced ISOL Facility, Lawrence Berkeley National Laboratory, Berkeley, CA, July 22-25, 1998, edited by I. Y. Lee, Lawrence Berkeley National Laboratory Report LBNL-42138, pp. 341-370 (1998)

Focal Plane Detectors

C. Davids

A Transparency Collection of the Proceedings of the Workshop on the Experimental Equipment for an Advanced ISOL Facility, Lawrence Berkeley National Laboratory, Berkeley, CA, July 22-25, 1998, edited by I. Y. Lee, Lawrence Berkeley National Laboratory Report LBNL-42138, pp. 483-502 (1998)

\section{Operation and Development of ATLAS}

Status of RNB Facilities in North America

Jerry A. Nolen

Proceedings of the ENAM98 (Exotic Nuclei and Atomic Masses) Conference, June 23-27, 1998, Bellaire, MI, eds. Bradley M. Sherrill, David J. Morrissey, and Cary N. Davids, AIP Conference Proceedings 455 (Woodbury, NY 1998) pp. 952-959

Radioactive Ion Beam Accelerator Concept Based on Linear Accelerators

Jerry Nolen

Proceedings of the 1998 Joint APS/AAPT April Meeting, Columbus, OH, April 18-21, 1998; Bull. Am. Phys. Soc. 43, 1205 (1998)

RIB Linac for Large Mass to Charge Ratios

Kenneth Shepard

Proceedings of the 1998 Joint APS/AAPT April Meeting, Columbus, OH, April 18-21, 1998; Bull. Am. Phys. Soc. 43, 1205 (1998)

\section{Medium Energy Nuclear Physics}

Measurement of the Flavor Asymmetry in the Nucleon Sea

E. A. Hawker et al. (Fermilab experiment collaboration)

Phys. Rev. Lett. 80, 3715-3718 (1998)

Quasifree (e,e'p) Reactions and Proton Propagation in Nuclei

D. Abbott et al. (E89-12 collaboration)

Phys. Rev. Lett. 80, 5072-5076 (1998)

Effective Neutron Targets

H. Gao

Proceedings of the XVth International Conference on Few-Body Problems in Physics, Groningen, The Netherlands, 22-26 July 1997;

Nucl. Phys. A631, 170c-189c (1998) 
Associated $\Lambda$ Production at Jefferson Lab

Gabriel Niculescu for the E93-18 Collaboration

Proceedings of the International Conference on Hypernuclear and Strange

Particle Physics, Brookhaven National Laboratory, Upton, NY, 13-18, 1997;

Nucl. Phys. A639, 189c-196c (1998)Kaon Electroproduction on Deuterium

J. Reinhold for the E91-16 Collaboration

Proceedings of the International Conference on Hypernuclear and Strange

Particle Physics, Brookhaven National Laboratory, Upton, NY, 13-18, 1997;

Nucl. Phys. A639, 197c-204c (1998)

Feasibility Test Run of ${ }^{12} \mathrm{C}\left(\mathrm{e}, \mathrm{e}^{\prime} \mathrm{K}^{\prime}\right)$ Reaction at Thomas Jefferson National Accelerator Facility

Wendy L. Hinton (for the E93-18 and E91-16 Collaborations)

Proceedings of the International Conference on Hypernuclear and Strange

Particle Physics, Brookhaven National Laboratory, Upton, NY, 13-18 October

1997, eds. D. J. Millener and R. E. Chrien;

Nucl. Phys. A639, 205c-208c (1998)

Counting Rare Noble Gas Atoms with a MOT

Z.-T. Lu, D. S. Lin, K. Bailey, and L. Young

1998 Annual Meeting of the Division of Atomic, Molecular and Optical

Physics (DAMOP) of the American Physical Society, Santa Fe, NM, 27-30 May 1998;

Bull. Am. Phys. Soc. 43, 1333 (1998)

Electroproduction of Kaons on Deuterium

D. Koltenuk for TJNAF E91-16 Collaboration

1998 Fall Meeting of the Division of Nuclear Physics of the American

Physical Society, 28-31 October 1998, Santa Fe, NM; Bull Am. Phys. Soc. 43, 1547 (1998)

Single Pion Electroproduction and Nuclear Pions

H. E. Jackson

1998 Fall Meeting of the Division of Nuclear Physics of the American

Physical Society, 28-31 October 1998, Santa Fe, NM; Bull Am. Phys. Soc. 43, 1546 (1998)

d- $/ \mathrm{u}^{-}$Asymmetry and the Origin of the Nucleon Sea

J. C. Peng et al., (FNAL E866/NuSea Collaboration)

Phys. Rev. D 58, 092004-1 to 092004-5 (1998)

Nuclear Tensor Polarization in a Laser-Driven Polarized Deuterium Internal Target

J. A. Fedchak, K. Bailey, W. J. Cummings, H. Gao, R. J. Holt, C. E. Jones,

R. S. Kowalczyk, T. O'Neill, and M. Poelker

Nucl. Instrum. Methods A417, 182-196 (1998)

The HERMES Spectrometer

$\mathrm{K}$. Ackerstaff et al. (and the HERMES Collaboration)

Nucl. Instrum. Methods A417, 230-265 (1998)

Longitudinal and Transverse Cross Sections in ${ }^{1} \mathrm{H}\left(\mathrm{e}, \mathrm{e}^{\prime} \mathrm{K}^{+}\right) \Lambda$ Reaction

K. Bailey, D. Geesaman, D. Potterveld, B. Zeidman, G. Niculescu et al. (E9318 Collaboration)

Phys. Rev. Lett. 81, 1805-1808 (1998) 
Measurements of Deuteron Photodisintegration up to $4.0 \mathrm{GeV}$

J. Arrington, D. Geesaman, T. O’Neill, D. Potterveld, B. Zeidman,

C. Bochna et al. (E8912 Colaboration)

Phys. Rev. Lett. 81, 4576-4579 (1998)

Determination of the Deep Inelastic Contribution to the Generalized Gerasimov Drell Hearn Integral for the Proton and the Neutron.

D. DeSchepper, H. Jackson, T. O'Neill, D. Potterveld,

K. Acherstaff et al. (HERMES Collaboration)

Phys. Lett. B444, 531-538 (1998)

Measurement of the Proton Spin Structure Function $\mathrm{GP}_{1}$ with a pure Hydrogen Target

D. DeSchepper, H. Jackson, T. O'Neill, D. Potterveld,

K. Acherstaff et al. (HERMES Collaboration)

Phys. Lett. B442, 484-492 (1998)

The Flavor Asymmentry of the Light Quark Sea from Semi-inclusive Deep Inelastic Scattering

D. DeSchepper, H. Jackson, T. O'Neill, D. Potterveld,

K. Acherstaff et al. (HERMES Collaboration)

Phys. Rev. Lett. 81, 5519-5523 (1998)

Workshop Summary: Physics and Instrumentation with 6-12 GeV Beams

D. F. Geesaman

Proceedings of the JLAB Workshop on Physics and Instrumentation with 6-12 GeV Beams, (ed. Steve Dytman, Howard Fenker and Phil Roos) 199, (1998)

\section{Theoretical Nuclear Physics}

Can the Magnetic Moment Contribution Explain the Ay Puzzle?

V. G. J. Stoks

Phys. Rev. C 57, 445-447 (1998)

Some Foundational Aspects of Quantum Computers and Quantum Robots

Paul Benioff

Superlattices and Microstructures 23, 407-417 (1998)

Quantum Effects Clinch Wolf Prize

Murray Peshkin and Lev Vaidman

Physics World 11, 51 (1998)

The Isoscalar S-Wave $\pi-N$ Scattering Length $\mathrm{a}^{+}$from $\pi$-Deuteron Scattering

S. R. Beane, V. Bernard, T.-S. H. Lee, and Ulf-G. Meissner

Phys. Rev. C 57, 424-426 (1998)

Semileptonic Decays of Heavy Mesons

M. A. Ivanov, Yu. L. Kalinovsky, P. Maris, and C. D. Roberts

Phys. Lett. B416, 29-35 (1998) 
Quantum Monte Carlo Calculations for Light Nuclei

R. B. Wiringa

Proceedings of the XVth International Conference on Few-Body Problems in

Physics, Groningen, The Netherlands, 22-26 July 1997; Nucl. Phys. A631, 70c-90c (1998)

Difference in Stopping Power for Protons and Deuterons of a Given Speed

Hans Bichsel and Mitio Inokuti

Nucl. Instrum. Methods B134, 161-164 (1998)

Nuclear Breakup of Borromean Nuclei

G. F. Bertsch, K. Hencken, and H. Esbensen

Phys. Rev. C 57, 1366-1377 (1998)

Chiral Constituent Quark Model Predictions of Electroproduction of the $\Delta$ on the Nucleon and ${ }^{3} \mathrm{He}$

T.-S. H. Lee

Proceedings of the International Conference on Quark-Lepton Nuclear

Physics, Research Center for Nuclear Physics (RCNP), Osaka University, Osaka, Japan, 21-24 May 1997; Nucl. Phys. A629 180c-189c (1998)

Heavy- to Light-Meson Transition Form Factors

M. A. Ivanov, Yu. L. Kalinovsky, P. Maris, and C. D. Roberts

Phys. Rev. C 57, 1991-2003 (1998)

Coupled-Channels Analysis of ${ }^{58} \mathrm{Ni}+{ }^{124} \mathrm{Sn}$ Reactions

H. Esbensen, C. L. Jiang, and K. E. Rehm

Phys. Rev. C 57, 2401-2408 (1998)

Models of Quantum Turing Machines

Paul Benioff

Fortschr. Phys. 46, 423-441 (1998)

Monte Carlo Calculations of Nuclei

Steven C. Pieper

Proceedings of the European Summer School on Microscopic Quantum Many-Body

Theories and Their Applications, Valencia, Spain, 8-19 September 1997, Lecture

Notes in Physics, eds. Jesus Navarro and Arthur Polls (Springer Verlag,

Heidelberg 1998) p. 337-357

Pion Mass and Decay Constant

Pieter Maris, Craig D. Roberts, and Peter C. Tandy

Phys. Lett. B420, 267-273 (1998)

Center-of-Mass Effects on the Quasi-Hole Spectroscopic Factors in the ${ }^{16} \mathrm{O}(\mathrm{e}, \mathrm{e}$ 'p) Reaction

D. Van Neck, M. Waroquier, A. E. L. Dieperink, Steven C. Pieper, and V. R. Pandharipande

Phys. Rev. C 57, 2308-2315 (1998)

Chemical Potential Dependence of $\pi$ and $\rho$ Properties

P. Maris, C. D. Roberts, and S. Schmidt

Phys. Rev. C 57, R2821-R2825 (1998) 
Convariant Quark Model for the Baryons

F. Coester, K. Dannbom, and D. O. Riska

Nucl. Phys. A634, 335-367 (1998)

Comment on "Spin, Parity, Excitation Energies, and Octupole Structure of an Excited

Superdeformed Band in ${ }^{194} \mathrm{Hg}$ and Implications for Identical Bands" by $\mathrm{G}$.

Hackman et al.

R. R. Chasman

Phys. Rev. Lett. 80, 4610 (1998)

Thermodynamic Properties of a Simple, Confining Model

David Blaschke, Craig D. Roberts, and Sebastian Schmidt

Phys. Lett. B425, 232-238 (1998)

Electron Inelastic-Scattering Cross Sections in Liquid Water

Michael Dingfelder, Detlef Hantke, Mitio Inokuti, and Herwig G. Partetkze

Radiation Physics and Chemistry 53, 1-18 (1998)

Weak Capture of Protons by Protons

R. Schiavilla, V. G. J. Stoks, W. Glockle, H. Kamada, A. Nogga, J. Carlson, R. Machleidt, V. R. Pandharipande, R. B. Wiringa, A. Kievsky, S. Rosati, and M. Viviani

Phys. Rev. C 58, 1263-1277 (1998)

Deconfinement at Finite Chemical Potential

A. Bender, G. I. Poulis, C. D. Roberts, S. Schmidt, and A. W. Thomas

Phys. Lett. B432, 263-269 (1998)

Study of Hyperon-Nucleon Interactions with $d\left(e, e^{\prime} \mathrm{K}\right)$ Reactions

T.-S. H. Lee, V. Stoks, B. Saghai, and C. Fayard

Proceedings of the International Conference on Hypernuclear and Strange

Particle Physics, Brookhaven National Laboratory, Upton, NY, 13-18, 1997; Nucl.

Phys. A639, 247c-250c (1998)

Microscopic Calculations of $\Lambda$ Single-Particle Energies

Q. N. Usmani and A. R. Bodmer

Proceedings of the International Conference on Hypernuclear and Strange

Particle Physics, Brookhaven National Laboratory, Upton, NY, 13-18, 1997; Nucl.

Phys. A639, 147c-156c (1998)

Folded-Diagram Effective Interactions for Hypernuclei

Yiharn Tzeng, S. Y. Tsay Tzeng, T. T. S. Kuo, and T.-S. H. Lee

Proceedings of the International Conference on Hypernuclear and Strange

Particle Physics, Brookhaven National Laboratory, Upton, NY, 13-18, 1997; Nucl.

Phys. A639, 165c-168c (1998)

Quantum Robots and Environments

Paul Benioff

Phys. Rev. A 58, 893-904 (1998)

Semiclassical Approximation to Neutron Star Superfluidity Corrected for Proximity Effects

F. Barranco, R. A. Broglia, H. Esbensen, and E. Vigezzi

Phys. Rev. C 58, 1257-1262 (1998) 
Measurement of E2 Transitions in the Coulomb Dissociation of ${ }^{8} \mathrm{~B}$

B. Davids, D. W. Anthony, Sam M. Austin, D. Bazin, B. Blank, J. A. Caggiano, M. Chartier, H. Esbensen, P. Hui, C. F. Powell, H. Scheit, B. M. Sherrill, M. Steiner, and P. Thirolf

Phys. Rev. Lett. 81, 2209-2212 (1998)

Photoproduction of a $\Lambda$ on ${ }^{12} \mathrm{C}$

T.-S. H. Lee, Z.-Y. Ma, B. Saghai, and H. Toki

Phys. Rev. C 58, 1551-1557 (1998)

Analysis of Chiral and Thermal Susceptibilities

D. Blaschke, A. Holl, C. D. Roberts, and S. Schmidt

Phys. Rev. C 58, 1758-1766 (1998)

Reactions of Halo States: Coulomb Excitations

Henning Esbensen

Proceedings of the International School of Heavy Ion Physics, 4th Course:

Exotic Nuclei, Erice, Italy, 11-20 May 1998, eds. Ricardo A. Broglia and P.

Gregers Hansen (World Scientific, Singapore 1998) pp. 71-92

A Solution to Coupled Dyson-Schwinger Equations for Gluons and Ghosts in Landau Gauge Lorenz von Smekal, Reinhard Alkofer, and Andreas Hauck

Ann. Phys. 267, 1-60 (1998)

The New Determination of the $\eta \mathrm{N}$ S-Wave Scattering Length From a Three-Channel, Multi-

Resonance Amplitude Analysis

M. Batinic, I. Dadic, I. Slaus, A. Svard, B. M. K. Nefkens, and T.-S. H. Lee

Physica Scripta 58, 15-18 (1998)

The Landauer Resistance and Band Spectra for the Counting Quantum Turing Machine

Paul Benioff

Proceedings of the Fourth Workshop on Physics and Computations (PhyComp96)

Boston, MA, 22-24 November 1996; Physica D 120, 12-29 (1998)

Leptonic and Semileptonic Decays of Heavy Mesons

C. D. Roberts, M. A. Ivanov, Yu. L. Kalinovsky, and P. Maris

Proceedings of the IVth International Workshop on Progress in Heavy Quark

Physics, University of Rostock, Germany, 20-22 September 1997, eds. M. Beyer,

T. Mannel, and H. Schroder (University of Rostock 1998) pp. 163-166

Differences Between Heavy and Light Quarks

P. Maris and C. D. Roberts

Proceedings of the IVth International Workshop on Progress in Heavy Quark

Physics, University of Rostock, Germany, 20-22 September 1997, eds. M. Beyer,

T. Mannel, and H. Schroder (University of Rostock 1998) pp. 159-162

Deconfinement and Hadron Properties at Extremes of Temperature and Density

David Blaschke and Craig D. Roberts

Proceedings of the International Workshop on QCD at Finite Baryon Density,

Bielefeld, Germany, April 27-30, 1998; Nucl. Phys. A642, 197c-209c (1998) 
Electron Energy Loss Spectrum of Nanowires

G. F. Bertsch, H. Esbensen, and B. W. Reed

Phys. Rev. B 58, 14031-14035 (1998)

Microscopic Calculation of ${ }^{6} \mathrm{Li}$ Elastic and Transition Form Factors

R. B. Wiringa and R. Schiavilla

Phys. Rev. Lett. 81, 4317-4320 (1998)

Pseudovector Components of the Pion, $\pi^{0} \rightarrow \gamma \gamma$, and $F_{\pi}\left(q^{2}\right)$

Pieter Maris and Craig D. Roberts

Phy. Rev. C 58, 3659-3665 (1998)

Non-Perturbative Chiral Approach to $\mathrm{K} p \rightarrow \gamma \mathrm{p}$ Reactions

T.-S. H. Lee, J. A. Oller, E. Oset, and A. Ramos

Nucl. Phys. A643, $402-414$ (1998)

Quantum Monte Carlo Calculations of Light Nuclei

Steven C. Pieper

Proceedings of the ENAM98 (Exotic Nuclei and Atomic Masses) Conference,

Bellaire, MI, eds. Bradley M. Sherrill, David J. Morrissey, and Cary N. Davids,

AIP Conference Proceedings 455 (Woodbury, NY 1998) pp. 399-406

Infrared Behaviour of Propagators and Vertices

F. T. Hawes, P. Maris and C. D. Roberts

Phys. Lett. B440, 353-358 (1998)

Poincaré-Covariant Quark Models of Baryon Form Factors

F. Coester and D. O. Riska

Few-Body Systems 25, 29-43 (1998)

\section{Atomic and Molecular Physics January - September 1998}

Argon KM Photoelectron Satellites

S. H. Southworth, T. LeBrun, Y. Azuma, and K. G. Dyall

Journal of Electron Spectroscopy and Related Phenomena 94, 33-38 (1998)

Manifestations of Nonlocal Exchange, Correlation, and Dynamic Effects in X-Ray Scattering M. Jung, R. W. Dunford, D. S. Gemmell, E. P. Kanter, B. Kraessig, T. W. LeBrun,

S. H. Southworth, L. Young, J. P. J. Carney, L. LaJohn, R. H. Pratt, and P. M. Bergstrom, Jr. Phys. Rev. Lett. 81, 1596-1599 (1998)

First Experiment with Decelerated Bare Uranium Ions Conducted at the ESR Storage Ring T. Stohlker, H. Reich, K. Beckert, F. Bosch, A. Gallus, H. Eickhoff, B. Franzke, T. Kandler, O. Klepper, C. Kozhuharov, G. Menzel, P. H. Mokler, F. Nolden, H. T. Prinz, P. Spadtke, M. Steck. T. Winkler, R. W. Dunford, P. Rymuza, T. Ludziejewski, Z. Stachura, P. Swiat, and A. Warczak

Hyperf. Int. 108, 29 (1997) 
K-Shell Excitation Studied for H- and He-Like Bismuth Ions in Collisions with Low-Z Target Atoms

T. Stohlker, D. C. Ionescu, P. Rymuza, F. Bosch, H. Geissel, C. Kozhuharov, T. Ludziejewski, P. H. Mokler, C. Scheidenberger, Z. Stachura, A. Warczak, and R. W. Dunford

Phys. Rev. A 57, 845 (1998)

Lifetime of the $3 \mathrm{p}^{2} \mathrm{P}_{3 / 2}$ Level in Sodiumlike Bromine (Br XXV)

A. Vasilyev, E. Jasper, H. G. Berry, A. E. Livingston, L. J. Curtis, S. Cheng, and R. W. Dunford

Phys. Rev. A 58, 732 (1998)

Charge-Exchange Cross Sections and Beam Lifetimes for Stored and Decelerated Bare Uranium Ions

T. Stohlker, T. Ludziejewski, H. Reich, F. Bosch, R. W. Dunford, J. Eichler, B. Franzke, C. Kozhuharov, G. Menzel, P. Mokler, F. Nolden, P. Rymuza, Z. Stachura, M. Steck, P. Swiat, A. Warczak, and T. Winkler

Phys. Rev. A 58, 2043 (1998)

Electron Bremsstrahlung in Collision of $223 \mathrm{MeV} / \mathrm{u}$ He-Like Uranium Ions with Gaseous Targets T. Ludziejewski, T. Stohlker, S. Keller, H. Beyer, F. Bosch, O. Brinzanescu, R. W. Dunford, B. Franzke, C. Kozhuharov, D. Liesen, A. E. Livingston, G. Menzel, J. Meier, P. H. Mokler, H. Reich, P. Rymuza, Z. Stachura, M. Steck, L. Stenner, P. Swiat, and A. Warczak

J. Phys. B. 31, 2601 (1998)

Interference Between Electric and Magnetic Amplitudes for K-Shell

T. Stohlker, D. C. Ionescu, P. Rymuza, F. Bosch, H. Geissel, C. Kozhuharov, T. Ludziejewski, P. H. Mokler, C. Scheidenberger, Z. Stachura, A. Warczak, and R. W. Dunford

Phys. Lett. A238, 43 (1998) 